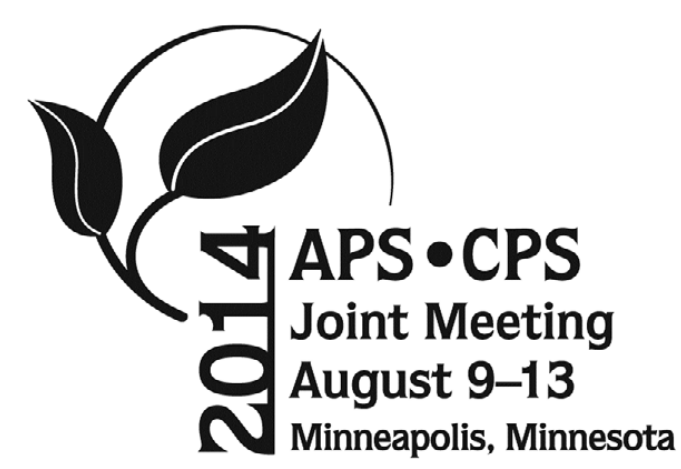

\title{
2014 APS-CPS Joint Meeting Abstracts of Presentations
}

\begin{abstract}
APS-CPS Joint Meeting in Minneapolis, Minnesota, U.S.A., August 9-13, 2014. The abstracts are arranged alphabetically by the first author's name. Recommended format for citing joint meeting abstracts, using the first abstract below as an example, is as follows: Abad, Z. G., Bienapfl, J. C., Balci, Y., Burgess, T., Coffey, M., Martin, F., and Kang, S. 2014. "Online ID tools to Phytophthora: Lucid key, tabular key and sequencing analysis." Progress and demands for accurate identification \& diagnostics. (Abstr.) Phytopathology 104(Suppl. 3):S3.1. http://dx.doi.org/10.1094/PHYTO-104-11-S3.1
\end{abstract}

"Online ID tools to Phytophthora: Lucid key, tabular key and sequencing analysis." Progress and demands for accurate identification \& diagnostics Z. G. ABAD (1), J. C. Bienapfl (1), Y. Balci (2), T. Burgess (3), M. Coffey (4), F. Martin (5), S. Kang (6)

(1) USDA-APHIS-PPQ-S\&T, Beltsville, MD, U.S.A.; (2) Plant Science and Landscape Architecture, University of Maryland, College Park, MD, U.S.A.; (3) Centre of Phytophthora at University of Murdoch, Perth, Australia; (4) World Oomycete and Phytophthora Genetic Resources and University of California, Riverside, CA, U.S.A.; (5) USDA-ARS, Salinas, CA, U.S.A.; (6) Phytophthora Database, Pennsylvania State University, State College, PA, U.S.A. Phytopathology 104(Suppl. 3):S3.1

The genus Phytophthora contains 138 spp., many of which cause significant economic impact to crops, ornamentals, and forests. As of 1999, 59 spp. were described based on morphology and 79 additional spp. have been described since then based on morphological/molecular characters. Globally, many spp. are of regulatory concern, including 29 for the USA ranked by the USDACPHST-Plant Epidemiology and Risk Analysis Laboratory in 2009. In addition, many new species are expected to be described as denoted by "taxon" names in the literature and NCBI, some of which may also be of regulatory concern. Despite the considerable progress made in identification, multigenic phylogenies, and diagnostics, the work in these areas is challenging due to oversights of the types in taxonomic publications and misidentifications submitted to NCBI. The problems are exacerbated especially when dealing with species "complexes." CPHST-BL is leading a collaboration with national and international institutions in Phytophthora research to implement a user-friendly "Online Identification Tools of Phytophthora: Lucid Key, Tabular Key and Sequencing Analysis," based on the type isolates. This is the first resource of this kind developed for plant pathogens. Complete, high-quality reference sequences and a link to Phytophthora Database for BLAST analysis are part of this resource. CPHSTIdentification Technology Program-CO is advising the implementation of the resource to be posted at the web.

The abstracts are published as submitted. They were formatted but not edited at the APS headquarters office.

http://dx.doi.org/10.1094/PHYTO-104-11-S3.1

(C) 2014 The American Phytopathological Society
Phytotoxicity of the mycotoxin ( \pm )-botryodiplodin (Bot) produced by Macrophomina phaseolina to soybean and duckweed in vitro

H. K. ABBAS (1), W. T. Shier (2), T. W. Allen (3), R. E. Baird (4), N. Bellaloui (5), A. M. Butler (1)

(1) USDA ARS, Biological Control of Pests Research Unit, Stoneville, MS, U.S.A.; (2) College of Pharmacy, University of Minnesota, Minneapolis, MN, U.S.A.; (3) Delta Research and Extension Center, Stoneville, MS, U.S.A.; (4) Entomology and Plant Pathology, Mississippi State University, Starkville, MS, U.S.A.; (5) USDA ARS, Crop Genetics Research Unit, Stoneville, MS, U.S.A. Phytopathology 104(Suppl. 3):S3.1

The fungus M. phaseolina causes charcoal rot (CR), a major soybean disease. M. phaseolina also produces a toxin, Bot. Our objective was to determine if phytotoxic responses to Bot are consistent with the toxin's mechanism of soybean root infection. Bot was tested for phytotoxicity in leaf disc assays using leaves from soybean cultivars with different reactions to $\mathrm{CR}$ using one moderately resistant and two susceptible cultivars. Phytotoxicity was defined as brown, necrotic tissue on the edge of leaf discs, when symptoms were absent in controls. Bot phytotoxicity ( 0 to $320 \mu \mathrm{g} / \mathrm{mL}$ ) in leaf disc assays was consistent with the cultivar's reported CR reaction. The resistant cultivar developed symptoms more slowly than the susceptible, but all showed phytotoxicity at $72 \mathrm{~h}$ at greater concentrations. Similar responses to Bot were observed in duckweed, except phytotoxicity occurred at reduced concentrations compared to soybean (>10 $\mu \mathrm{g} / \mathrm{ml}$ Bot). Root toxicity to Bot was measured as soybean seedling lateral and/or tap root growth inhibition in a hydroponic culture medium system containing Bot following four days. Bot caused dosedependent inhibition of both tap and lateral root growth, but lateral root growth was inhibited at reduced Bot concentrations $(4 \mu \mathrm{g} / \mathrm{mL})$ than tap root growth $(23.5 \mu \mathrm{g} / \mathrm{ml})$. The phytotoxic responses to Bot outlined in this research suggest that Bot plays a role in the mechanism of root infection by M. phaseolina.

Occurrence of copper-resistant strains of bacterial spot pathogen and presence of all four species of Xanthomonas in Ontario commercial tomato fields

P. A. ABBASI (1), S. E. Khabbaz (2), L. Zhang (2), B. Weselowski (2)

(1) Atlantic Food and Horticulture Research Centre, Agriculture and AgriFood Canada, Kentville, NS, Canada; (2) Southern Crop Protection and Food Research Centre, Agriculture and Agri-Food Canada, London, ON, Canada Phytopathology 104(Suppl. 3):S3.1

Bacterial spot of tomato is caused by Xanthomonas euvesicatoria, X. vesicatoria, X. perforans, and X. gardneri. Total 105 strains were isolated from 
leaf and fruit samples showing bacterial spot symptoms collected from commercial tomato fields in Ontario during 2011-2012 field seasons. All strains were characterized based on their resistance or sensitivity to copper, pathogenicity on Bonnie Best plants, growth on xanthomonad differential medium, and PCR amplification of estA gene. Strains were separated into species based on PCR amplification of $h r p$ gene followed by restriction digestion and by sequence analysis of $h r p$ gene. In plate assays, only 16.2 and $13.3 \%$ strains were completely sensitive (no growth), and 14.3 and $19.1 \%$ were partially sensitive (grew on 1 and $1.5 \mathrm{mM}$ plates) to copper hydroxide and copper sulfate, respectively. Most of the strains were resistant (grew on 2 or $4 \mathrm{mM}$ plates) to copper hydroxide $(69.5 \%)$ or copper sulfate $(67.6 \%)$. Restriction digestion of hrp gene product and sequencing revealed presence of all four species $(X$. euvesicatoria $1.9 \%, X$. vesicatoria $3.9 \%, X$. perforans $24 \%$, and $X$. gardneri $37.5 \% ; 32.7 \%$ could not be separated). In greenhouse pathogenicity tests, most of the field strains caused severe (43\%) to highly severe $(25 \%)$ symptoms on Bonny Best tomatoes, whereas only $32 \%$ strains caused moderately severe symptoms. Our results suggest presence of copperresistant strains and all four species of bacterial spot pathogen in Ontario.

Reaction of global spring wheat genotypes for resistance to tan spot Pyrenophora tritici-repentis race 5

S. ABDULLAH (1), S. Ali (1), P. K. Singh (2), K. Glover (1), J. S. Rohila (1) (1) South Dakota State University, Brookings, SD, U.S.A.; (2) CIMMYT, Mexico, Mexico

Phytopathology 104(Suppl. 3):S3.2

Tan spot is an important foliar disease of wheat in the USA and other major wheat growing countries in the world. The disease is caused by the fungus Pyrenophora tritici-repentis (Ptr). Eight races have been identified in the fungal population. Of the 8 races, $1-5$ have been observed in the USA. The disease can be managed through fungicide application, resistant cultivars, crop rotation, and/or all three approaches. However, deploying resistant cultivar as a disease management strategy seems more promising because resistance is generally economical and durable. In this study, 299 wheat genotypes developed by CIMMYT were evaluated for their reaction to Ptr race 5. Of these 299 wheat genotypes, 145 previously evaluated with Ptr race 1 and were resistant to tan spot. Two week old seedlings of all 299 genotypes were inoculated with race 5 spores suspension in the greenhouse. Of 299 genotypes evaluated, 111 were resistant to Ptr races 1 and 5.34 were susceptible to race 5 but resistant to race 1,64 were resistant to race 5 but susceptible to race 1 , and 90 were susceptible to both races. In an attempt to identify sources of resistance to multiple leaf diseases, genotypes that showed resistance to Ptr race 1 and 5 are being further evaluated for their reactions to Stagonospora nodorum leaf blotch (SNB) and bacterial leaf streak (BLS).

\section{Prevalence and diversity of viruses infecting Macrophomina phaseolina in} the United States

N. ABOUGHANEM-SABANADZOVIC (1), A. Lawrence (2), T. W. Allen (3), S. Sabanadzovic (4)

(1) Institute for Genomics, Biocomputing and Biotechnology, Mississippi State University, Mississippi State, MS, U.S.A.; (2) Institute for Imaging and Analytical Technologies, Mississippi State University, Mississippi State, MS, U.S.A.; (3) Delta Research and Extension Center, Mississippi State University, Stoneville, MS, Canada; (4) Biochemistry, Molecular Biology, Entomology and Plant Pathology, Mississippi State University, Mississippi State, MS, U.S.A.

Phytopathology 104(Suppl. 3):S3.2

Macrophomina phaseolina is a soil-borne plant pathogenic fungus that causes important diseases in numerous cultivated and non-cultivated plants worldwide. In the spring of 2013 we began an investigation concerning the diversity of viruses infecting populations of this pathogen isolated from several host plants from within the United States and their effects on the reported host plants. To date, we have tested dozens of isolates collected from different hosts (soybean, corn, sweet potato, watermelon, strawberry, etc.) from various states and have identified dsRNA-containing isolates for further in-depth studies. Number and size of dsRNA molecules varied between isolates. These dsRNAs were reverse-transcribed, cloned and sequenced and demonstrated to represent genomes of several mycoviruses belonging to different taxa. At present, we are also investigating the presence of DNA viruses in these fungal isolates using the rolling circle amplification method.

\section{Mycoviruses in Rhizoctonia-like and Southern blight fungi isolated from} different hosts in Mississippi

N. ABOUGHANEM-SABANADZOVIC (1), A. Lawrence (2), M. TomasoPeterson (3), A. Henn (3), S. Sabanadzovic (3)

(1) Institute for Genomics, Biocomputing and Biotechnology, Mississippi State University, Mississippi State, MS, U.S.A.; (2) Institute for Imaging and
Analytical Technologies, Mississippi State University, Mississippi State, MS, U.S.A.; (3) Biochemistry, Molecular Biology, Entomology and Plant Pathology, Mississippi State University, Mississippi State, MS, U.S.A.

Phytopathology 104(Suppl. 3):S3.2

Thirty five isolates of Rhizoctonia spp and Sclerotium rolfsii, isolated from agronomic crops (i.e. corn, soybean, wheat, peanut) and turfgrasses in Mississippi, were examined for the presence of mycoviruses by double stranded RNA (dsRNA) extraction and Rolling Circle Amplification followed by cloning and sequencing, as well as transmission electron microscopy of partially purified preparations. Majority of isolates contained visible amounts of dsRNA molecules of different sizes and concentration. Observed patterns suggested the presence of single and mixed infections in studied fungal isolates. Putative virions were observed in preparations from some dsRNAcontaining isolates. Molecular data on genomes of these viruses indicated the presence of known (i.e. Rhizoctonia solani dsRNA virus 2) and several novel species belonging to different taxa of extant mycoviruses.

\section{Kudzu as a reservoir for soybean viruses}

N. Aboughanem-Sabanadzovic (1), W. Moore (2), T. W. Allen (3), A. Lawrence (4), S. SABANADZOVIC (2)

(1) Institute for Genomics, Biocomputing and Biotechnology, Mississippi State University, Mississippi State, MS, U.S.A.; (2) Biochemistry, Molecular Biology, Entomology and Plant Pathology, Mississippi State University, Mississippi State, MS, U.S.A.; (3) Delta Research and Extension Center, Mississippi State University, Mississippi State, MS, U.S.A.; (4) Institute for Imaging and Analytical Technologies, Mississippi State University, Mississippi State, MS, U.S.A.

Phytopathology 104(Suppl. 3):S3.2

Virus-like symptoms, consisting of mosaic, ringspots, vein feathering, necrosis and leaf deformation, were observed in several kudzu patches during a survey carried out in the summer/fall of 2013 in Mississippi. Laboratory analyses determined the presence of at least two viruses in tested samples. An isolate of Tobacco ring spot virus (TRSV), originally isolated from a sample collected in Kemper County, was partially characterized by mechanical transmission to two soybean varieties, virion purification, cloning and sequencing. Another 11 kudzu patches, collected from 8 different counties, resulted infected by TRSV. The second virus, isolated from a symptomatic kudzu patch from Choctaw County, is apparently a new, borderline species in the genus Potyvirus (fam. Potyviridae) and related to Soybean mosaic virus (SMV). During the characterization, this virus was demonstrated to be pathogenic to soybean where it induces typical mosaic symptoms. In addition to the original source the virus has also been recovered from several other kudzu patches in Northern Mississippi. The results of this study suggest that kudzu could represent an important source for known and yet-to-bediscovered viruses capable of infecting soybean.

Quantification of Septoria musiva in infected Populus clones using qPCR N. D. ABRAHAM (1), M. Acevedo (1), P. Chitrampalam (1), J. LeBoldus (1) (1) North Dakota State University, Fargo, ND, U.S.A.

Phytopathology 104(Suppl. 3):S3.2

Leaf spot and stem canker caused by Septoria musiva limits the use of Populus spp. in agro-forestry. Disease severity rating is tedious and time consuming. The use of quantitative PCR (qPCR) for quantification of fungal biomass in host tissue following infection will greatly increase the speed and accuracy of disease resistance phenotyping. Here we report the use of a multiplexed Taqman qPCR assay to quantify $S$. musiva development in inoculated stems of two resistant and four susceptible genotypes of hybrid poplar. Stems were inoculated by removing the fifth leaf from the shoot apex and placing a plug of sporulating mycelium from four different isolates on the resulting wound. Species specific probes and primers were designed based on conserved gene sequences of, $\beta$ tubulin and eukaryotic translation initiation factor IV, for the pathogen and host respectively. Primers were highly specific and similar amplification efficiencies were obtained for the host $(96 \%)$ and the pathogen (97\%). To compare the qPCR findings to traditional disease resistance assessment, disease severity was evaluated on a 1-5 scale at 1, 4 and 7 weeks post inoculation. Total genomic DNA was then extracted from each canker for the qPCR assay. The ratio of fungal DNA to host DNA was used as a measure of resistance and correlated to the disease severity score. This assay showed significant differences in the level of resistance among the different genotypes.

Characterization of Clavibacter michiganensis subsp. nebraskensis strains from epiphytic and symptomatic infections of maize in Iowa

J. ACHARYA (1), G. Y. Mbofung (1), A. Ahmad (1), A. E. Robertson (1)

(1) Iowa State University, Ames, IA, U.S.A.

Phytopathology 104(Suppl. 3):S3.2 
Clavibacter michiganensis subsp. nebraskensis (Cmn), the causal agent of Goss's bacterial wilt and leaf blight in corn is a Gram-positive bacterium that has an epiphytic (asymptomatic) phase and a disease phase (symptomatic). The role of epiphytic phase in disease development is not clearly understood. Currently, a bioassay is the best method for accurate identification Cmn and this can take several weeks. The objectives of this study were to i) compare morphology and pathogenicity of 37 putative isolates of $\mathrm{Cmn}$ recovered from asymptomatic and diseased corn leaves collected from fields in Iowa and Nebraska, and ii) validate two published methods for identification of Cmn; subspecies-specific primers, and a PCR-RFLP method. Colony morphology for 33 of the isolates was orange mucoid which is characteristic of Cmn. Twenty-eight of the 33 isolates were pathogenic on corn. A 215 bp subspecies-specific band was amplified from some of our isolates of Cmn and also from two isolates of C. michiganensis subsp. michiganensis. Amplification of two housekeeping genes recA and rpoD digested with Bst $\mathrm{UI}$ sucessfully identified our Cmn isolates. These data indicate that epiphytic population of Cmn could serve as a source of inoculum for Goss's wilt and leaf blight in corn, and PCR-RFLP method could be used to accurately identify $\mathrm{Cmn}$.

\section{The status of soybean cyst nematode, Heterodera glycines, in South Dakota} K. ACHARYA (1), E. Byamukama (1), C. Tande (1)

(1) South Dakota State University, Brookings, SD, U.S.A.

Phytopathology 104(Suppl. 3):S3.3

The soybean cyst nematode ( $\mathrm{SCN}$ ) is the most important soybean production constraint in South Dakota. SCN was first detected in South Dakota in 1996 in Union County, and has spread to 28 counties as of 2013. The counties that have been found with SCN span the eastern part of the state. Since the start of keeping records of SCN testing for soil samples submitted to the South Dakota State University Plant Diagnostic Clinic in 2003, over 4,578 soil samples have been analyzed for SCN. Of these, $33 \%$ (field incidence) were found to have SCN. Turner County had the highest number of samples (50\%) found with SCN and the top four counties with the highest SCN positive samples are from the south-east corner of the state. The years 2005 and 2012 had the highest SCN population (3,124 and 2,245 eggs and second juvenile counts per $100 \mathrm{~cm}^{3}$ of soil, respectively). Although the samples submitted to the clinic came from growers voluntarily and therefore may not be the true representation of SCN incidence within the state, these samples still indicate the wide distribution and the relatively high SCN field incidence in South Dakota. This underscores the importance of SCN in soybean production in South Dakota. Current efforts being promoted in the management of SCN are continued monitoring of SCN through free soil testing sponsored by the South Dakota Soybean Research and Promotion Council, use of crop rotation, and planting resistant varieties.

Trunk-injected potassium phosphites and acibenzolar-S-methyl induce SAR in apple trees allowing control of fire blight (Erwinia amylovora) S. G. AĆIMOVIĆ (1), Q. Zeng (1), G. C. McGhee (1), J. C. Wise (1), G. W. Sundin (1)

(1) Michigan State University, East Lansing, MI, U.S.A

Phytopathology 104(Suppl. 3):S3.3

Trunk injection of Systemic Acquired Resistance (SAR) inducers as an in planta delivery approach could enhance their effect in fire blight control. PRprotein gene expression indicating SAR should be achieved after injection of SAR inducers on mature apple trees. After 1-2 injections of acibenzolar-Smethyl (ASM; Actigard) or two injections of potassium phosphites (PJ; Phosphojet), using 4 replicates per treatment, inoculated Gala trees were evaluated for blossom and shoot blight and for PR-protein gene expression in leaves and flowers. In year 1, ASM and PJ significantly reduced blossom blight incidence by $37.7-42.2 \%$ and $55.9 \%$, respectively. Shoot blight incidence was reduced $70.8 \%$ by PJ only. In year 2, ASM and PJ reduced blossom blight by $19.1-21.1 \%$ and $25.1 \%$, respectively, and shoot blight by $27-31 \%$ and $23.4 \%$, respectively. In year 2, PJ significantly upregulated expression of the PR-1, PR-2 and PR-8 protein genes. ASM upregulated these genes in leaves consistently, in both years. Thus, injected PJ and ASM induce SAR in leaves on mature apple trees but at different times and with different persistence of the effect. SAR activity was not detected in flowers, probably due to reduced accumulation of injected compounds in them, which was driven by their lower transpiration compared to the leaves. With proper dosing and more time for translocation and accumulation in the canopy, trunk-injected SAR inducers could serve as an effective option for fire blight control.

Development of a real-time PCR assay to detect Sugarcane Yellow Leaf Virus (SCYLV) in sugarcane in Colombia

Y. C. Acosta (1), M. Cadavid (1), C. Cardozo (1), J. C. Angel (1), J. I. Victoria (1), C. A. ANGEL (1)
(1) Colombian Sugarcane Research Center - CENICAÑA, Cali, Colombia Phytopathology 104(Suppl. 3):S3.3

Sugarcane is an important crop in Colombia used to produce sugar, ethanol, panela, and electricity. It is affected by diseases including the Sugarcane Yellow Leaf Virus (SCYLV), a ssRNA Polerovirus known by a high incidence worldwide and variable effects on yield. It is transmitted by aphids, but mostly through infected cane seed setts. CENICAÑA detected SCYLV in Colombia in 1998, and uses Tissue Blot Enzyme Immunoassay (TBIA) and ReverseTranscription PCR (RT-PCR) to evaluate nurseries and commercial fields. However, low viral titres and detection limits could generate false negative results. To perform a faster and more sensitive diagnosis, a real time PCR (RT-qPCR) reaction was developed ampliying a SCYLV 147bp fragment from the ORF $0-1$, testing the plant genes O-Met, Actin, $\beta$-Tubulin, and $G A P D H$ as reference. The SCYLV dynamic range was from $1 \mathrm{X}$ (100 ng total plant RNA) until $1 \times 10^{-5}$; and for $\beta$-Tubulin until $1 \times 10^{-4}$. No significant differences $(\alpha=0.05)$ on $\beta$-Tubulin expression for both healthy and infected samples were obtained, but there were differences for SCYLV as expected. $\beta$ Tubulin did not vary in samples from the same cane variety and between varieties, as required for a reference gene. SCYLV was detected in $1.0 \mathrm{pg}$ of total RNA from infected samples. Higher sensitivity for RT-qPCR was validated evaluating the same samples with TBIA and RT-PCR. Absolute quantification was attempted identifying the limit of detection based on the number of SCYLV genome copies.

Polyoxin-D, a new, exempt-from-tolerance postharvest treatment for control of gray mold of fruit crops

J. ADASKAVEG (1), H. Forster (1), Y. Luo (1), D. Cary (1)

(1) University of California, Riverside, CA, U.S.A.

Phytopathology 104(Suppl. 3):S3.3

New postharvest fungicides are needed because decays developing after harvest continue to cause significant losses in many fruit crops. In many instances only one fungicide is registered or export tolerances limit use of available treatments to a single product. Gray mold caused by Botrytis cinerea affects many commodities, and additional treatments are necessary to manage resistance development in the pathogen. We tested the efficacy of polyoxin-D against gray mold in laboratory and experimental packingline trials. On sweet cherry, peach, and nectarine, the incidence of decay was $<5 \%$ when aqueous solutions at 50 to $200 \mathrm{mg} / \mathrm{L}$ were applied 12 to $14 \mathrm{~h}$ after inoculation, whereas it was $>80 \%$ in untreated fruit. On apple, pear, and pomegranate, the efficacy of polyoxin-D was more variable and depended highly on fruit cultivar and treatment time after inoculation. Still, gray mold incidence was significantly reduced from that of the control. Thus, polyoxin-D was shown to be a highly effective gray mold treatment when used at low rates. Baseline sensitivity ranged from 0.011 to $0.264 \mathrm{mg} / \mathrm{L}$ (avg. $0.063 \mathrm{mg} / \mathrm{L}$ ) for $B$. cinerea. It was less effective against brown rot of stone fruits caused by Monilinia fructicola and this correlated with a reduced in vitro sensitivity $(0.74$ to $11.2 \mathrm{mg} / \mathrm{L}-$ avg. $5.58 \mathrm{mg} / \mathrm{L}$ ). Polyoxin-D is exempt from tolerance in the United States, is compatible in fungicide mixtures, and it is scheduled for postharvest registration for commercial use.

High fungicide insensitivity and reciprocity of iprodione sensitivity in Botrytis cinerea populations from berry crops in the Pacific Northwest J. E. ADASKAVEG (1), H. Forster (1), C. Clemens (2), S. Midboe (3)

(1) University of California, Riverside, CA, U.S.A.; (2) Syngenta Crop Protection, Richland, WA, U.S.A.; (3) Whatcom Farmers Coop, Bellingham, WA, U.S.A.

Phytopathology 104(Suppl. 3):S3.3

High losses due to gray mold in berry crops in the Pacific Northwest led to an evaluation of sensitivity to commonly used fungicides in Botrytis cinerea populations. Isolates from raspberry fruit in nine locations collected in summer 2012 were resistant to fenhexamid $\left(\mathrm{EC}_{50}>0.5 \mathrm{mg} / \mathrm{L}\right)$, cyprodinil $\left(\mathrm{EC}_{50}>0.5 \mathrm{mg} / \mathrm{L}\right)$ and boscalid $\left(\mathrm{EC}_{50}>8 \mathrm{mg} / \mathrm{L}\right)$ at $100 \%, 0$ to $50 \%$, and 0 to $44.4 \%$, respectively. Resistance to iprodione was low $\left(\mathrm{EC}_{50}<1\right)$ to moderate $\left(\mathrm{EC}_{50} 1\right.$ to $2 \mathrm{mg} / \mathrm{L}$ ) and occurred in 0 to $40 \%$ of the isolates. The distribution and incidence of resistance for all fungicides was similar in 2013 samplings of overwintering sclerotia of the pathogen in the same locations. In two fields where fenhexamid was not used previously, however, only 14.3 to $18.7 \%$ of the isolates were resistant to this fungicide. Iprodione was not used since 1997 due to high resistance and crop failures. The low to moderate levels of resistance indicated that iprodione could be used again in a rotation once per season. In 2013, incidence of moderate to high resistance at nine raspberry, blueberry, and strawberry fields ranged from 0 to $28.8 \%, 0$ to $71.4 \%$, and 16.7 to $71.4 \%$ for fenhexamid, cyprodinil, and boscalid, respectively. No resistance to fludioxonil and chlorothalonil was found in any of the samplings. These results demonstrate regional differences in fungicide sensitivity among $B$. 
cinerea populations that probably reflect differences in fungicide usage patterns among growers.

Root-knot nematode suppression in tomato with selected organic amendments in comparison to carbofuran

O. K. ADEKUNLE (1)

(1) Obafemi Awolowo University, Ile-Ife., Ile-Ife, Nigeria

Phytopathology 104(Suppl. 3):S3.4

A two-year field study was conducted in 2011 and 2012 in the tropical rainforest zone of Nigeria to investigate suppression of population densities of Meloidogyne incognita with compost, poultry manure and Neem leaves in comparison to carbofuran. The experimental field was naturally infested with $M$. incognita and this had resulted in wilting of tomato seedlings, floral abortion and poor yield in past cropping seasons. The highest reduction in $M$. incognita population density was recorded in plots amended with compost at $10 \mathrm{t} /$ ha which was not significantly lower than $M$. incognita population densities in plots amended with carbofuran at $3.0 \mathrm{~kg} /$ ha and $1.5 \mathrm{~kg}$ a.i / ha, poultry manure at $10 \mathrm{t} / \mathrm{ha}$ and $5 \mathrm{t} / \mathrm{ha}$ and Neem leaves at $10 \mathrm{t} / \mathrm{ha}$. The reduction in nematode population in amended plots were significantly lower than that in control plots. Cumulative tomato fruit yield was highest in plots amended with carbofuran at $3.0 \mathrm{~kg}$ a.i/ha, but this was not significantly higher than yield from plots amended with compost, poultry manure and neem leaves at $10 \mathrm{t} / \mathrm{ha}$. The fruit yield in amended plots was significantly higher than in control plots. Results were consistent in both years of the study indicating that the organic amendments had potentials to suppress root-knot nematode population associated with tomato thereby ensuring sustainable tomato production.

\section{Differential colonization of Solanum sisymbriifolium by Ralstonia solanacearum \\ M. AFROZ (1) \\ (1) Ohio State University, OARDC, Wooster, OH, U.S.A. \\ Phytopathology 104(Suppl. 3):S3.4}

Grafting desired scions of tomato and eggplant onto disease-resistant rootstocks is a tactic used to manage bacterial wilt caused by Ralstonia solanacearum in South Asia. Solanum sisymbriifolium is frequently used as a rootstock, but plants grafted onto this species have failed recently in some locations in Bangladesh and Nepal. To determine the susceptibility of $S$. sisymbriifolium plants to colonization by $R$. solanacearum, eight strains from wilted tomato or eggplant grafted onto $S$. sisymbriifolium rootstocks, or wilted non-grafted plants in Bangladesh and Nepal were used to challenge $S$. sisymbriifolium seedlings. Plants were inoculated by root drenching and stems were cut $0.5 \mathrm{~cm}$ above the soil line 5, 12 and 20 days post-inoculation (dpi), then placed in $500 \mu \mathrm{l}$ sterilized distilled water for $24 \mathrm{hrs}$. Populations of $R$. solanacearum in the bacterial ooze determined by quantitative real-time PCR (qPCR) were strongly positively correlated with populations determined by dilution plating. All eight strains were recovered from inoculated plants. $R$. solanacearum populations remained relatively low until 20 dpi. There were significant differences in numbers of bacteria detected in ooze among the eight strains. None of the $S$. sisymbriifolium plants wilted up to $36 \mathrm{dpi}$, although tomato 'OH7814' seedlings inoculated with the same $R$. solanacearum strains began wilting at $7 \mathrm{dpi}$.

\section{Seasonality of canker induction and expansion by Neofabraea perennans} and Cryptosporiopsis kienholzii in apple trees

C. AGUILAR (1), M. Mazzola (2), C. L. Xiao (3)

(1) WSU Tree Fruit Research \& Extension Center, Wenatchee, WA, U.S.A.;

(2) USDA-ARS Tree Fruit Research Lab, Wenatchee, WA, U.S.A.; (3) USDA-ARS, Parlier, CA, U.S.A.

Phytopathology 104(Suppl. 3):S3.4

In the U.S. Pacific Northwest, bull's-eye rot is a major postharvest disease of apples and other pome fruit. Four closely related fungi belonging to Neofabraea or related genera are known to cause this disease. Depending upon species, these fungi overwinter either as saprophytes of organic plant debris on the orchard floor, or as mycelium in cankers of infected trees. $N$. perennans and recently described Cryptosporiopsis kienholzii are two bull'seye rot-causing pathogens that are prevalent in pome fruit production areas of central and eastern Washington State. While $N$. perennans is known to cause perennial canker in apple trees, the canker-inducing status of $C$. kienholzii and its survival in the orchard remains unknown. Twigs of 'Red Delicious' and 'Fuji' apple trees were inoculated in the orchard with mycelial plugs of either $N$. perennans or C. kienholzii throughout the year for two years and monitored for canker expansion on a monthly basis up to six months post inoculation. Twigs were harvested on the final month of measurement, during which reisolation of fungi from diseased twigs was attempted. Preliminary data from this experiment suggests rapid growth and expansion for cankers induced by either species during the months of April and October. Inoculations conducted during hot summer and freezing cold winter months seemed to be the least conducive for canker growth. However, both fungi were capable of surviving as mycelium in twigs throughout the year.

A filamentous phage XacF1 causes loss of virulence to Xanthomonas axonopodis $p v$ citri, the causative agent of citrus canker disease

A. A. AHMAD (1), A. Askora (2), T. Kawasaki (1), M. Fujie (3), T. Yamada (1) (1) Department of Molecular Biotechnology, Graduate School of Advanced Science of Matter, Hiroshima University, Higashihiroshima, Japan; (2) Department of Molecular Biotechnology, Graduate School of Advanced Science of Matter, Hiroshima University, Japan/Department of Microbiology, Faculty of Science, Zagazig university, Egypt., Higashihiroshima, Japan; (3) Department of Molecular Biotechnology, Graduate School of Advanced Science of Matter, Hiroshima University, Higashihiroshima, Japan

Phytopathology 104(Suppl. 3):S3.4

In this study, a filamentous phage designated $\mathrm{XacF} 1$, which can infect Xanthomonas axonopodis pv citri (Xac) strains was isolated and characterized. Electron microscopy showed that XacF1 is a member of the family Inoviridae and was about $1000 \mathrm{~nm}$ long, and $7 \mathrm{~mm}$ in width. The genome of XacF1 is 7325 nucleotides long, containing 13 predicted open reading frames (ORFs) with significant homology to Ff like phage proteins, such as ORF1 (pII), ORF2 (pV), ORF6 (pIII), and ORF8 (pVI). XacF1 showed a relatively wide host range infecting 7 out of 11 strains tested in this study. Frequently, XacF1 was found to be integrated in the genome of Xac strains. This integration was occurred at the host dif site $(a t t B)$ mediated by the host XerC/D recombination system. The attP sequence was coincided with that of Xanthomonas phage Cf1c. Interestingly, infection by XacF1 phage caused several cultural and physiological changes to the bacterial host cells, including lower levels of extracellular polysaccharides (EPS) production, slower growth rate of the host, and a dramatically reduction in the virulence of the bacterial host. Especially the latter change suggested possible utilization of XacF1 as a biological control agent against citrus canker disease.

Population genetic structure of the rice brown spot pathogen Bipolaris oryzae in Iran

A. AHMADPOUR (1), M. Javan-Nikkhah (2), M. R. Naghavi (3), F. Padasht Dehkaei (4), Y. Leng (1), K. Puri (1), C. Castell-Miller (5), S. Zhong (1)

(1) Department of Plant Pathology, North Dakota State University, Fargo, ND, U.S.A.; (2) Department of Plant Protection, University College of Agriculture and Natural Resources, University of Tehran, Karaj, Iran, Karaj, ND, Iran; (3) Department of Biotechnology, University College of Agriculture and Natural Resources, University of Tehran, Karaj, Iran, Karaj, ND, Iran; (4) Rice Research Institute of Iran, Rasht, Iran, Rasht, ND, Iran; (5) Department of Plant Pathology, University of Minnesota, St. Paul, MN, U.S.A.

Phytopathology 104(Suppl. 3):S3.4

Brown spot, caused by the fungus Bipolaris oryzae, is one of the most important diseases of rice (Oryza sativa) worldwide. However, the population biology of $B$. oryzae is poorly understood. In this study, B. oryzae isolates from Iran $(n=299)$, the Philippines $(n=20)$, USA $(n=20)$, Japan $(n=8)$ and Bangladesh $(n=6)$ were analyzed for genetic variation using 12 simplesequence repeat (SSR) markers developed from the genome sequence of the fungus. STRUCTURE revealed that Iranian isolates were clustered into three populations corresponding to the three sampled regions (Northwest, Northeast and South) of Iran. The isolates from the Philippines, Japan and Bangladesh grouped with Northwest and Northeast populations, while isolates from the USA grouped with the South population. In total, 330 unique haplotypes were identified among the $353 \mathrm{~B}$. oryzae isolates, and gene diversity $(\mathrm{H}=0.70$ 0.75 ) and genotypic diversity were high in the three populations. Genetic variation was $84 \%$ among $B$. oryzae individuals within a population and $16 \%$ among populations. Genetic differentiation was high $(\theta=0.155, P<0.001)$, with corrected $\mathrm{G}^{\prime \prime}{ }_{\text {st }}=0.58$, whereas gene flow $(\mathrm{Nm})$ ranged from 2.22 to 3.32 among the three populations. Linkage disequilibrium tests did not support the hypothesis of random mating at the subpopulation level, but both mating types (MAT1-1 and MAT1-2) were found in all sampled areas suggesting sexual recombination is possible.

\section{Sensitivity of Rhizoctonia solani to SDHI and DMI fungicides}

O. AJAYI (1), C. A. Bradley (2)

(1) University of Illinois At Urbana-Champaign, Urbana, IL, U.S.A.; (2) University of Illinois, Urbana, IL, U.S.A.

Phytopathology 104(Suppl. 3):S3.4

Rhizoctonia solani, is an important seedling pathogen of soybean in the United States. Seed treatments in the demethylation inhibitor (DMI) and succinate dehydrogenase inhibitor (SDHI) fungicide classes are used to manage seedling disease caused by $R$. solani. Baseline sensitivities of $R$. 
solani to two SDHI fungicides (penflufen and sedaxane) and two DMI fungicides (ipconazole and prothioconazole) were determined in in vitro assays. Baseline isolates originated from four different states (IL, OH, ND, $\mathrm{MN}$ ) and had been isolated prior to 2000. Isolates recovered in 2012 and 2013 from Illinois were tested for sensitivity to SDHI fungicides. The effective concentration of the fungicide that inhibited mycelial growth by $50 \%\left(\mathrm{EC}_{50}\right)$ was determined for each isolate. For the baseline isolates, the range of $\mathrm{EC}_{50}$ values for penflufen, sedaxane, ipconazole, and prothioconazole was 0.01$0.49,0.02-0.49,0.21-5.47$, and $0.20-5.58 \mathrm{ug} / \mathrm{ml}$, respectively, with respective means of $0.07,0.09,1.74$, and $2.15 \mathrm{ug} / \mathrm{ml}$. For the 2012 and 2013 isolates, the mean $\mathrm{EC}_{50}$ values were 0.09 and $0.16 \mathrm{ug} / \mathrm{ml}$, and ranges were 0.01-0.48 and $0.04-0.45 \mathrm{ug} / \mathrm{ml}$, respectively for penflufen and sedaxane. The establishment of the baseline sensitivities to these fungicides serves as a first step in monitoring fungicide resistance development in populations of $R$. solani affecting soybean in the north central states.

Host-dependent symptom development as affected by Tomato bushy stunt virus encoded silencing suppressor

A. AKBASSOVA (1), G. Mukiyanova (1), M. Sutula (1), M. J. Pozo (2), R. Omarov (1)

(1) L.N. Gumilyov Eurasian National University, Astana, Kazakhstan; (2) Estacion Experimental del Zaidin (CSIC), Granada, Spain

Phytopathology 104(Suppl. 3):S3.5

Virus derived proteins named as suppressors of RNA interference (RNAi) have distinct biochemical properties, which allow them to block host defense pathways during infection. Tomato bushy stunt virus encoded P19 protein is a major pathogenicity factor, required for symptom development and elicitation of a hypersensitive response in various hosts. Structural properties of P19 protein enable it to act as a potent suppressor of defensive RNAi during viral infection. Molecular mode of P19 RNAi suppressing activity is based on protein ability to bind size-selectively virus derived siRNAs. Inoculation of tomato plants with TBSV transcripts showed that the ability of the virus to infect plants varied between different cultivars. For example, inoculations of tomato cultivars Pera and Better Boy did not result in any noticeable symptoms on plants. Moreover, inoculated plants did not show any viral protein (P19) accumulation. However, the inoculation of cv. Money Maker resulted in readily detectable levels of P19 accumulation in leaves and roots of plants. Interestingly, despite P19 protein accumulation no significant symptoms were detected on plants. It is possible that P19 structural alterations in planta may affect its suppressor activity during infection. Therefore, tomato plants "resistance" to TBSV infection may be associated with specific defensive mechanisms against viral suppressors of RNAi.

Evaluation of quantitative PCR for detection of Candidatus Liberibacter asiaticus in composite plant DNA samples

O. J. ALABI (1), M. Kunta (2)

(1) Texas AgriLife Research, Weslaco, TX, U.S.A.; (2) Texas A\&M University Kingsville, Weslaco, TX, U.S.A.

Phytopathology 104(Suppl. 3):S3.5

Recent finds of huanglongbing (HLB; citrus greening) in commercial groves and residential areas in South Texas have necessitated intensification of surveillance efforts to ensure early detection of Candidatus Liberibacter asiaticus (CLas) as a precursor to implementing quarantine measures. Since the probability of HLB detection over a given area is directly proportional to the number of tree samples tested in epidemiological studies, we evaluated the sensitivity of real-time qPCR for detection of CLas in composite (pooled) plant DNA samples. One unit DNA from a range of known positive samples with $\mathrm{Ct}$ values 25,30 and $32\left(1.128 \times 10^{9}, 4.17 \times 10^{7}\right.$ and $1.114 \times 10^{7} \mathrm{CLas}$ genomes/g HLB-infected fresh tissue, respectively) were spiked into increments of five (up to 100) units of DNA from known negative samples. Resulting composite DNA were then used as template in qPCR assays. Subsequently, the optimal composite ratio was validated using DNA extracts from field survey samples and results compared with individual qPCR tests performed on each sample. Our results show that CLas is consistently detectable when at least one unit of DNA extract from a positive tree is pooled with five units of DNA from a healthy tree $\left(3.713 \times 10^{6}\right.$ CLas genomes $/ \mathrm{g}$ HLB-infected fresh tissue). The use of composite DNA samples will enable a cost-effective quick screen of large number of survey samples in disease surveys.

Is Cercospora kikuchii the causal agent of Cercospora leaf blight and purple seed stain in the Gulf South?

S. ALBU (1), P. P. Price (1), V. Doyle (2), R. Schneider (1)

(1) Louisiana State University Agricultural Center, Baton Rouge, LA, U.S.A.;

(2) Louisiana State University, Baton Rouge, LA, U.S.A.

Phytopathology 104(Suppl. 3):S3.5
Cercospora kikuchii is considered to be the causal agent of Cercospora leaf blight (CLB) and purple seed stain (PSS), two economically important diseases of soybean (Glycine max) in the Gulf South. Identification of $C$. kikuchii is predominantly based on host association, cercosporin production and conidial morphology. However, these characters are unreliable for differentiating closely related species. Cercosporin production is variable among isolates, and it is difficult to induce sporulation in vitro. Recent molecular phylogenetic studies have shown that multiple cryptic species of Cercospora are capable of infecting a single host. Furthermore, previous studies determined that there is genetic diversity among cercosporoid soybean pathogens. Therefore, the etiology of CLB and PSS remains in question. In this study, we used a multilocus phylogenetic approach to determine if fungal isolates responsible for CLB and PSS are monophyletic. We addressed the utility of actin, calmodulin, elongation factor, histone and the internal transcribed spacer region for evaluating intraspecific relationships among Cercospora isolates collected from symptomatic soybean seeds and leaves. The multilocus phylogeny showed that none of the isolates collected in this study clustered within the clade containing the type strain of C. kikuchii. These results suggest that $C$. kikuchii is not the pathogen responsible for causing CLB and PSS in Louisiana and other gulf states.

Genotypic characterization of Pyrenophora tritici-repentis isolates from South Dakota

S. Ali (1), S. ABDULLAH (1), J. S. Rohila (1)

(1) South Dakota State University, Brookings, SD, U.S.A.

Phytopathology 104(Suppl. 3):S3.5

The fungus Pyrenophora tritici-repentis (Ptr), causes tan spot, is an important foliar pathogen of wheat worldwide. The fungal isolates have been grouped into eight races based on their ability to induce two distinct symptoms, necrosis and chlorosis, on appropriate wheat differential genotype. These two distinct symptoms are due to the three host-selective effectors (toxins) Ptr ToxA, ToxB, and ToxC produced by the fungus. In this study, 59 Ptr isolates recovered from wheat and rye were characterized to determine if they contain Ptr ToxA and/or Ptr ToxB genes. DNA of all 59 isolates was recovered and PCR was performed, using Ptr ToxA and Ptr ToxB specific primers. Ptr ToxA gene was amplified in 58 isolates; whereas, both ToxA and ToxB genes were amplified in only one isolate. All 59 isolates are being phenotyped for their race identification.

Comparison of soil microbial community between organically and conventionally managed golf courses using pyrosequencing-based metagenomics

E. ALLAN (1), D. Manter (2), G. Jung (1)

(1) University of Massachusetts Amherst, Amherst, MA, U.S.A.; (2) USDA Agricultural Research Service, Fort Collins, CO, U.S.A.

Phytopathology 104(Suppl. 3):S3.5

Soil microbes influence water retention, nutrient capacity, and influence plant health through competition/antagonism with potential pathogens, upregulation of plant immune systems, and symbiotic relationships with plants, e.g. mycorrhizal fungi in nutrient acquisition. Increased biodiversity seems to support these relationships and organic management practices increase biodiversity. We used next generation pyrosequencing of $16 \mathrm{~S}$ and $18 \mathrm{~S}$ rDNA to characterize the bacterial and fungal populations of an organic and two conventional golf courses to determine how these management practices affect soil microbes. Soil cores were taken from the rough, fairway, and putting green. There were more observed bacterial species, greater species diversity, and greater species evenness on the conventional courses than the organic course. The organic course had higher fungal species abundance. The putting greens had lower fungal abundance and fewer observed fungal species than the fairways and roughs. Although all of the courses and locations were dominated by alphaproteobacteria; the composition of the alphaproteobacteria was significantly different among courses and the locations. The results of this study will increase understanding of how organic management influences golf course soils and will help direct future studies to understand how they affect turf health and the best way to utilize them in disease control.

Developing germplasm resources to identify the genetic basis of resistance to common scab in potato

C. ALLEN (1), A. Charkowski (1), S. Jansky (2)

(1) University of Wisconsin-Madison, Madison, WI, U.S.A.; (2) USDA-ARS, Madison, WI, U.S.A.

Phytopathology 104(Suppl. 3):S3.5

Common scab, caused mainly by the soil-borne bacterium Streptomyces scabies, produces lesions on potato tubers, reducing tuber quality and profitability. Methods to manage common scab are often expensive, impractical, and can be ineffective. Therefore, creating cultivars that are 
resistant to common scab has been cited as the best method to control the disease. 524-8, an inbred diploid line of the wild potato species Solanum chacoense, exhibits significant and consistent resistance to common scab. M6, a $S$. chacoense inbred line with high genetic similarity to 524-8, is susceptible to common scab. These two closely related lines offer a great opportunity to examine the factors that contribute to resistance or susceptibility to common scab. Recombinant inbred lines (RILs) of 524-8 x M6 are being created by self-pollinating successive generations of the F2 population to achieve homozygosity. The RILs will then be phenotyped and genotyped to identify the genetic regions associated with scab resistance.

Occurrence of the cassava pathogen Sphaceloma manihoticola in a reservoir host, Bermuda grass (Cynodon dactylon) in Barbados

A. T. ALLEYNE (1), J. M. Gilkes (2)

(1) University of the West Indies, Bridgetown, Barbados; (2) University of the West Indies, Cave Hill Campus, Bridgetown, Barbados

Phytopathology 104(Suppl. 3):S3.6

Cassava (Manihot esculenta Crantz), is an important tropical root crop which increasingly is adversely affected by Super-elongation disease (SED). The causal agent, Sphaceloma manihoticola produces necrotic leaf spots and exaggerated stem elongation; the latter due to overproduction of the hormone gibberellin A4 (GA4). Potential reservoir sources of the pathogen may include infected planting material and nearby weeds growing in cultivated fields. This study aimed to determine whether the cassava pathogen, S. manihoticola is present in common weeds found in cassava growing areas in Barbados. The Spm 1 molecular marker which targets the Smp450-2 gene and codes for GA4 in S. manihoticola was used to determine the presence of the pathogen. Weed samples from fields infected with SED were collected and DNA was extracted and amplified with $S p m 1$ primers. The $S p m 1$ primer pair amplified a $600 \mathrm{bp}$ fragment in the grassy weed Cynodon dactylon (Bermuda grass). However, DNA amplified with Spm1 from Convolvulus arvensis (Bindweed) and other Euphorbiaceae species did not produce this fragment. This study is the first to apply the Spm1 primer to determine the presence of $S$. manihoticola in potential reservoir sources of the pathogen. These results show that weed maintenance is an important factor in managing, and preventing the spread of SED and may assist in the development of new disease management tools.

Quantification of disease severity in field and laboratory grown cassava by application of molecular markers for measuring Super-elongation disease

A. T. ALLEYNE (1), J. M. Gilkes (1)

(1) The University of the West Indies, Bridgetown, Barbados

Phytopathology 104(Suppl. 3):S3.6

The fungal pathogen $S$. manihoticola produces large amounts of gibberellin $\mathrm{GA}_{4}$ in late stages of Super elongation disease (SED), due to the expression of the Smp 450-2 gene in infected cassava plants. Laboratory grown cassava plants were inoculated with a suspension of $S$. manihoticola; DNA was extracted over a 21 day period and then amplified via PCR using SPM primers designed from the Smp450-2 gene. In laboratory grown cassava, late stage SED was not observed although this was evident in field grown cassava. However SPM primers were able to amplify the same 600bp fragment in DNA extracted from both laboratory grown cassava and field cultivated cassava plants; although late stages of SED were not observed in planta. In addition, field grown cassava displaying differential SED symptoms such as cankers on stems and necrotic leaf lesion produced variations in band intensities when DNA from these tissues were amplified with SPM primers. This may indicate the ability of this method to measure disease severity in cassava showing symptom of SED. Quantification of disease severity in cassava by use of molecular markers is important in management of local planting material and in continuous assessment of SED tolerant varieties of cassava which are widely distributed in the Caribbean region.

Resistance of Brazilian cultivars to ratoon stunting disease measured by sugarcane leaves

J. ALMEIDA (1), A. Urashima (1)

(1) Universidade Federal de Sao Carlos, Araras, Brazil

Phytopathology 104(Suppl. 3):S3.6

Ratoon stunting disease (RSD) is an important disease of sugarcane worldwide because of yield loss in all cultivars, no external symptoms, no possibility of control once the disease is detected in fields. Leifsonia xyli subsp. xyli (Lxx), xylem-limited fastidious bacterium that spreads within a field exclusively by implements. The rate and distance of RSD transmission and yield loss are positively correlated with bacterial population in vascular bundles. Therefore, the knowledge of cultivar resistance is essential to know the rate of disease progress over time and space. Resistance is determined by bacterium titer in xylem, measured by destructive assay of internodes. Little information is available on reaction of Brazilian cultivars despite widespread dissemination of RSD. This work examined resistance of important cultivars to RSD by a non-conventional procedure, ie, by the Lxx spread in leaves of cultivars. Leaf (+5) of RB835054, RB855156, RB855453, RB855536, RB867515, RB92579, RB935744, RB966928, eight month, second ratoon were selected and DNA was extracted from basal leaf tissue. PCR reaction was run with primers $\mathrm{Cxx} 1$ and $\mathrm{Cxx} 2$. Resistance evaluated by percentage of Lxx detection. Results of this work showed RB835054 as the most susceptible since Lxx was detected in all three replications, then RB92579, RB966928 detected in two, and RB867515, RB935744 in one. RB855156, RB855453 and RB855536 were the most resistant since no Lxx was detected in any replications.

From research stations to seed fields: Survey, detection and identification of sweetpotato viruses in North Carolina

C. V. Almeyda (1), T. Abernethy (1), J. A. Abad (2), Z. PESIC (1)

(1) North Carolina State University, Raleigh, NC, U.S.A.; (2) USDA, APHIS PPQ FO PGQP, Beltsville, MD, U.S.A.

Phytopathology 104(Suppl. 3):S3.6

Limited information on the status of sweetpotato viruses in $\mathrm{NC}$ led us to conduct field surveys in research stations (2012) and seed fields (2013), using biological, serological and molecular detection methods. Susceptible indicator plants (Ipomoea setosa) were placed in sweetpotato fields and tested for the presence of viruses by NCM-ELISA, multiplex PCR, multiplex and quantitative RT-PCR. Additionally, naturally infected sweetpotato samples were collected and virus tested using the same procedures. Results from research stations showed that single and mixed viral infections were found in $34 \%$ and $66 \%$ of the indicator plants, respectively; with potyviruses mixed infections being the most common. The same trend was observed in sweetpotato samples. Sweetpotato feathery mottle virus (SPFMV) was the most frequent followed by Sweetpotato virus G (SPVG), Sweetpotato virus $C$ (SPVC), Sweetpotato leaf curl virus (SPLCV), Sweetpotato chlorotic stunt virus (SPCSV) and Sweetpotato virus 2 (SPV2). Results from seed fields showed that most of the indicator plants and sweetpotatoes were non-infected and within the few infected, potyvirus single infections were the most predominant. No presence of SPLCV and SPCSV was observed. This study showed evidence of the wide occurrence of potyviruses and a begomovirus affecting sweetpotatoes in $\mathrm{NC}$ in research stations and the low incidence of potyviruses in seed fields.

Two begomoviral species causing yellow leaf curl disease of tomato: Distinct spatial distribution patterns in the Arabian Peninsula

M. A. AL-SALEH (1), J. K. Brown (2), A. M. Idris (3)

(1) King Saud University, Riyadh, Saudi Arabia; (2) University of Arizona, Tucson, AZ, U.S.A.; (3) King Abdullah University of Science and Technology, Thuwal, Saudi Arabia Phytopathology 104(Suppl. 3):S3.6

Tomato leaf samples were collected from tomato plants exhibiting yellowing and leaf curl symptoms, grown in the field or in a protected environment, in the Arabian Peninsula. The total DNA was isolated, and subjected to rolling circle amplification (RCA). The RCA products were digested with a suite of restriction endonucleases to identify enzymes that linearize begomoviral genomes, and thereby facilitate cloning. The cloned inserts $(\sim 2.8 \mathrm{kbp})$ were sequenced bi-directionally, assembled, and were blasted against sequences available in the NCBI database to identify the closest begomovirus relatives, and select reference isolates for the phylogenetic analyses. Results indicated that Tomato leaf curl Sudan virus (ToLCSDV) was the predominant begomovirus species in the west coast and central regions, that include AlHufuf, Asir, Hail, Jazan, Mecca, Riyadh, Yanbu, Yemen, and southwestern Oman. In contrast Tomato yellow leaf curl virus (TYLCV) predominated in Qassim and Tabuk, Saudi Arabia, and in Kuwait, Oman, Qatar and UAE. In Saudi Arabia, two strains of TYLCV were identified in Qassim, one that was more related to a TYLCV strain from Iran, and the other from Tabuk, whose closest relative was TYLCV from Jordan. The high, shared nucleotide identities of the tomato-infecting begomoviruses from Qassim and Tabuk with previously reported strains of TYLCV from the neighboring countries of Jordan and Iran, suggest that isolates in Saudi Arabia are recent introductions.

Impacts of crop rotation on disease-suppressive Trichoderma and Streptomyces densities

N. ALTIER (1), A. Villar (2), P. Vaz Jauri (3), L. K. Kinkel (4), C. A. Perez (2)

(1) INIA Las Brujas, Canelones, Uruguay; (2) Departamento Proteccion Vegetal, EEMAC, Facultad Agronomia, Universidad de la Republica, Uruguay, Paysandu, Uruguay; (3) INIA Las Brujas, Rincon del Colorado, Uruguay; (4) University of Minnesota, St. Paul, MN, U.S.A.

Phytopathology 104(Suppl. 3):S3.6 
Soil-borne and stubble-borne pathogens are a primary limitation in no-till cropping in Uruguay. Indigenous microbial communities can naturally reduce pathogen inoculum density of these plant pathogens. It has been widely documented that soil microbial communities are modified by agricultural practices. Thus, the objective of our work was to evaluate the impact of different crop sequences on native Trichoderma spp. and Streptomcyes spp., as potentially pathogen-suppressive organisms. Samples were collected from a field experiment with crop rotations 1- to 4-years long, established in 1999. Streptomyces and Trichoderma densities were quantified in soil samples collected from all plots and Trichoderma density was also quantified on stubble. Both Trichoderma and Streptomyces densities were significantly affected by the cropping history. Interestingly, higher soil densities of both groups of microorganisms were found in the 4-year rotation. In addition, barley debris showed the highest Trichoderma density. Our results give insight into how crop rotation influences microbial populations and suggest the importance of crop rotations designed to enhance disease suppressiveness.

The metabolism of carbohydrates in roots of cassava (Manihot esculenta Crantz) infected with frogskin disease

E. Alvarez (1), J. M. Pardo (2), D. Dufour (3), J. L. Moreno (2), E. ALVAREZ (2)

(1) CIAT, Cali, Colombia; (2) CIAT, Palmira, Colombia; (3) CIRAD-CIAT, Palmira, Colombia

Phytopathology 104(Suppl. 3):S3.7

Cassava roots with symptoms of frogskin disease (CFSD) characteristically possess longitudinal "lips" and a peel that has a cork-like aspect. Yields, comprising bulked and starchy roots, are low. Previous studies reported that the phytoplasma 16 SrIII-L is associated with CFSD. However, the plantpathogen interaction and the pathogenic effects on the host are unknown. The study's objective was therefore to discover the changes occurring in the metabolism of carbohydrates in cassava roots, both in the presence and absence of symptoms. Samples were taken from healthy and diseased roots of two cassava varieties: Valencia and Señorita. The diseased roots were selected for different levels of severity of attack. Parameters were dry matter content and concentrations of organic acids, reducing sugars, and total sugars, using HPLC liquid chromatography. Malic acid was found to be more highly concentrated in diseased roots, correlating with the amounts of reducing and total sugars, particularly fructose $\left(R^{2}=95 \%\right)$. Because of the small quantity of stored starch, the percentage of dry matter in diseased roots is less than that found in healthy roots. That is, in the diseased roots, starch probably converts into fructose and glucose during the Krebs cycle. These findings contribute towards understanding the pathogenic effects of phytoplasma 16 SrIII-L in cassava roots attacked by CFSD. They are also consistent with reports on phytoplasmas attacking other plant species.

First report of hypovirulence between a reovirus and phytoplasma 16SrIII-L associated with frogskin disease of cassava (Manihot esculenta Crantz)

E. ALVAREZ (1), J. M. Pardo (1)

(1) CIAT, Cali, Colombia

Phytopathology 104(Suppl. 3):S3.7

Frogskin disease attacks cassava roots, giving them a cork-like appearance and covering them with longitudinal "lips". It reduces yield by as much as $90 \%$. Causal agents of the disease are believed to be phytoplasma 16SrIII-L and a reovirus (CFSV). However, for more than 20 years, the role that each agent plays in the disease was unknown. The qPCR technique, involving $\mathrm{TaqMan}^{\circledR}$ probes, was used to evaluate the presence of both phytoplasma and reovirus in 158 stakes (vegetative seed) cut from cassava plants infected with frogskin disease. In $41.77 \%$ of samples, only the phytoplasma was detected and, in $13.29 \%$, only the virus was found. Both microorganisms were found together in another $22.52 \%$, while, for the remaining $22.42 \%$, neither microorganism was detected. The stakes were then planted in a screenhouse and harvested after 8 months of growth. Plants in which both reovirus and phytoplasma were detected showed a yield increase of $20 \%$, a reduced level of severity by $30 \%$, and fewer diseased roots, compared with those plants in which only the phytoplasma was detected. The finding that the phytoplasma's pathogenicity should weaken in roots carrying both reovirus and phytoplasma suggested the phenomenon of hypovirulence. Viruses with double-stranded RNA (dsRNA) are extensively found in association with hypovirulence of fungi. However, this is the first report of a virus with dsRNA causing hypovirulence in a phytoplasma.

Detection and identification of 'Candidatus Phytoplasma asteris'-related phytoplasma associated with a witches' broom disease of cassava in Cambodia

E. ALVAREZ (1), J. M. Pardo (2), M. J. Truke (2)
(1) CIAT, Cali, Colombia; (2) CIAT, Palmira, Colombia Phytopathology 104(Suppl. 3):S3.7

Cassava witches' broom (CWB) is an important disease of Manihot esculenta Crantz that is widely cultivated across South East Asia. In 2012, cassava farms were affected in Cambodia with significant crop losses up to $50 \%$ and reductions in yield and starch content. The disease was observed in Kampong Cham, Kratie and Prey Veng provinces of Cambodia affecting the availability of clean planting material. Understanding the etiology of this disease is critical for managing infection by the pathogen. Restriction fragment length polymorphism analyses of nested PCR-amplified fragments from Cambodian CWB phytoplasma isolates and representative phytoplasmas from other phytoplasma groups using MseI restriction enzyme indicated that the cassava phytoplasma isolates are related to the aster yellows (16SrI) group. Sequence analyses of partial 16S rRNA fragments showed that Cambodian CWB phyoplasmas have $99 \%$ to $100 \%$ similarity with 'Candidatus phytoplasma Asteris' (16SrI) phytoplasma group. To our knowledge, this is the first report of cassava infection with a phytoplasma related to the 16SrI group in Cambodia.

Identification of defense-related genes in buffalograss associated with Curvularia inaequalis infection using high throughput sequencing

B. S. AMARADASA (1), K. Amundsen (1)

(1) University of Nebraska-Lincoln, Lincoln, NE, U.S.A.

Phytopathology 104(Suppl. 3):S3.7

Buffalograss (Bouteloua dactyloides) is a low maintenance United States native turfgrass species with exceptional drought, heat and cold tolerance. Leaf spot caused by Curvularia inaequalis negatively impacts buffalograss visual quality. Two leaf spot susceptible and two resistant buffalograss lines were challenged with $C$. inaequalis. Total RNA was extracted from treated and untreated leaf samples when susceptible lines showed symptoms. Transcriptome sequencing was carried out and differential gene expression evaluated between resistance and susceptible lines. On average, 27 million raw sequencing reads were produced per sample. More than $86 \%$ of the sequencing reads mapped to an existing buffalograss cv. Prestige reference transcriptome. De novo assembly of unmapped reads was merged with the original Prestige reference to produce a more complete transcriptome. Upregulated genes were identified by comparative analysis of inoculated resistance lines and inoculated susceptible lines at $\mathrm{P}<0.01$. Uninoculated samples were also analyzed to identify upregulated genes at basal level in resistant lines compared to susceptible lines. There were 3544 differentially expressed transcripts between the two groups when challenged with the pathogen and 1076 in its absence. There were 180 transcripts identified with sequence similarity to known defense-related genes in other organisms. In future studies, selected defense genes will be used to confirm expression in buffalograss lines.

Molecular phylogenetic relationships, haplotype diversity and aggressiveness of Fusarium graminearum isolates causing head blight in wheat

C. C. AMARASINGHE (1), D. Fernando (1), B. J. Sharanowski (2)

(1) Department of Plant Science, University of Manitoba, Winnipeg, MB, Canada; (2) Department of Entomology, University of Manitoba, Winnipeg, MB, Canada

Phytopathology 104(Suppl. 3):S3.7

The Fusarium graminearum species complex (FGSC) is a group of mycotoxigenic fungi that cause - Fusarium head blight (FHB), a major disease in cereal crops worldwide. The objective of our study was to examine the frequency and genetic diversity of a collection of 143 FGSC isolates recovered from major wheat producing regions worldwide. Sequence analysis of the TRI5 gene resolved 10 haplotypes within the FGSC species representing $F$. graminearum sensu stricto $15 \mathrm{ADON}, F$. graminearum s.s $3 \mathrm{ADON}, F$. graminearum s.s NIV, $F$. asiaticum $3 \mathrm{ADON}, F$. asiaticum NIV, $F$. meridionale/ $F$. cortaderiae NIV, $F$. boothii $15 \mathrm{ADON}, F$. acaciae-mearnsii NIV and $F$. vorosii $15 \mathrm{ADON}$. Among the examined isolates Haplotype $1, F$. graminearum s.s $15 \mathrm{ADON}$ isolates were common. According to the aggressiveness test $3 \mathrm{ADON}$ isolates of $F$. asiaticum showed the highest disease severity at $10 \mathrm{dpi}$, both in susceptible and resistant wheat cultivars, followed by $3 \mathrm{ADON}$ isolates of $F$. graminearum s.s. Aggressiveness of $15 \mathrm{ADON} F$. graminearum s.s isolates were higher than the $F$. asiaticum NIV isolates. The results of this study also revealed that TRI5 gene can be used to identify different haplotypes of FGSC based on the type of mycotoxins they produce. This study confirms the importance of understanding the FHB pathogen species limits, chemotype diversity and global geographic distribution in designing effective plant disease management, quarantine and plant breeding strategies to control FHB. 
Evaluation of rotational programs to extend the life of new succinatedehydrogenase inhibitors for control of Botrytis cinerea in strawberry A. AMIRI (1), A. Zuniga (1), N. A. Peres (1)

(1) University of Florida, GCREC, Wimauma, FL, U.S.A.

Phytopathology 104(Suppl. 3):S3.8

Fluopyram (Fluop) and penthiopyrad (Penth) are two new succinatedehydrogenase inhibitors (SDHIs) developed for the control of Botrytis cinerea. To prevent or delay selection for resistance to the new SDHIs, we evaluated multiple rotational and tank-mix programs i.e. Fluop and Penth with Captan (Cap), thiram (Thir), fenhexamid (fenh) or fludioxonil (Flud) in two strawberry fields for two seasons. The SDHIs were self-rotated, rotated with one of the four other fungicides, or tank-mixed with Cap, Thir or Fenh on a weekly basis for up to four applications per season. Fruit were harvested weekly from January to March to evaluate gray mold incidence and yield. Isolates of $B$. cinerea were tested for their sensitivity to Fluop and Penth. In 2013, the Fluop-Fenh rotation was the most effective against gray mold but selected more for resistance to both SDHIs. Fluop, Flud, and Cap rotated with Fluop were effective controlling the disease and selected less for resistance to Fluop. A similar trend was observed in 2014 but with some disparities between fields. The rotation Fluop-Flud, the tank mixtures Fluop-Fenh, FluopThir, and Penth-Flud were the most effective. Isolates collected in 2014 are being tested for fungicide sensitivity. The rotation of the new SDHIs with Flud and tank mixing with the multi-site fungicides should provide good disease control and alleviate risks of fungicide resistance in the gray mold fungus.

First case of gray mold caused by Botrytis ricini (Amphobotrys ricini) on strawberry in United States

A. AMIRI (1), R. B. Onofre (1), N. A. Peres (1)

(1) University of Florida, Wimauma, FL, U.S.A.

Phytopathology 104(Suppl. 3):S3.8

In a study aimed to investigate the population dynamic of Botrytis cinerea on strawberry flowers and leaves, samples were collected monthly between November 2013 and February 2014 from two strawberry fields in Hillsborough County, Florida. Flowers and leaves were surface-sterilized in $0.5 \%$ sodium hypochlorite for $2 \mathrm{~min}$, rinsed twice in sterile water, placed in clean plastic box containing $100 \mathrm{ml}$ of water, and frozen overnight. Samples were then incubated at $22^{\circ} \mathrm{C}$ for 5 days and checked for the presence of Botrytis spp. using a stereoscope and isolates were purified. Conidiophores and conidia with shapes and color different from typical $B$. cinerea have been observed on flowers and leaves. Isolates grew slower compared to typical $B$. cinerea isolates on general isolation (GI) and potato dextrose agar (PDA) media. These isolates were initially characterized as B. ricini (Amphobotrys ricini) using morphological characteristics. DNA was isolates from five isolates and used for molecular detection using three nuclear genes i.e. HSP60, RPB2, and G3PDH. Gene bank blast showed 98\% similarity of our sequences with sequences of $B$. ricini for all three genes. To verify the ability of $B$. ricini to cause gray mold, symptomatic fruit were collected from January to March of 2014 from the same fields. Isolates were purified and more than 10 isolates were characterized as $B$. ricini. This is the first report of $B$. ricini causing gray mold on strawberry in North America.

\section{The melon Prv gene conferring PRSV resistance is a member of a TIR-} NBS-LRR gene pair expressing multiple splice variants

A. AMITZUR (1), M. Normantovich (1), I. Kovalski (1), R. Perl-Treves (1)

(1) Bar-Ilan University, Ramat Gan, Israel

Phytopathology 104(Suppl. 3):S3.8

We have mapped, using molecular markers, the genomic locus for melon resistance towards two pathogens: Fusarium oxysporum f.sp. melonis races 0 and 1 , and the papaya ring spot virus (PRSV). High resolution mapping and chromosome walking enabled the identification and cloning of a pair of genes that control, respectively, the two resistances. We have sequenced the $P r v^{1}$, $P r v^{2}$ and prv alleles, specifying an immune resistance mode, necrotic resistance mode and susceptibility, respectively. Prv encodes a protein of the NBL (nucleotide binding site-leucine rich repeat) family. Proteins of this large and diverse family act as receptors of the plant immune system, responsible for sensing specific pathogens in the "gene for gene" interaction. We report extensive allelic polymorphism in the locus, indicative of diversifying selection. The gene's expression pattern was studied using a promoter-reporter system in transgenic melon roots. Positional cloning of Prv raises exciting questions about recognition of specific viral proteins by the $R$ protein, the possible relations between the two adjacent $R$-genes, and between multiple splice-variants found for the Prv transcripts. Some of the variants encode an additional, c-terminal nucleotide-binding domain whose function is presently unknown.
Variation in Streptomyces densities and inhibitory capacities from diverse natural habitats in New Zealand

J. ANDERSON (1), D. Schlatter (1), L. Otto-Hanson (1), L. Kinkel (1)

(1) University of Minnesota, St. Paul, MN, U.S.A.

Phytopathology 104(Suppl. 3):S3.8

Indigenous soil bacteria have significant potential to suppress plant pathogens, yet the ecology and biogeography of suppressive populations remains poorly understood. New Zealand is an attractive target for exploring the structure and dynamics of inhibitory phenotypes because of its remote location and diverse non-disturbed natural habitats. We characterized inhibitory capacities and nutrient use preferences of indigenous Streptomyces isolated from 10 soils across New Zealand using a modified Herr's assay against two wellcharacterized Streptomyces isolates. Biolog SFP-2 plates were used to characterize nutrient use of each isolate. Streptomyces densities and densities of inhibitory Streptomyces varied significantly among locations $(\mathrm{p}<0.0001 \&$ $\mathrm{p}<0.0001$, respectively). The proportion of antagonistic Streptomyces also differed significantly among locations $(\mathrm{p}<0.0001)$, as did mean inhibition zone sizes $(\mathrm{p}<0.0001)$. The location with lowest densities had the highest proportion of inhibitors ( $>50 \%$ of Streptomyces density was antagonistic) and significantly larger mean inhibition zone sizes than all other locations. Variation in nutrient use and inhibitory phenotypes among locations suggests that habitats vary in the dynamics of selection for antagonistic phenotypes. These data shed light on the ecology and evolution of New Zealand Streptomyces populations and provide important insight into biogeographic patterns of Streptomyces in natural habitats.

Fungicides as a first line of defense for effective control of stem rust in hard red spring wheat

A. ANDHIKARI (1), R. Dill-Macky (1), J. J. Wiersma (2), M. J. Smith (2)

(1) University of Minnesota, St. Paul, MN, U.S.A.; (2) University of Minnesota, Crookston, MN, U.S.A.

Phytopathology 104(Suppl. 3):S3.8

Stem rust (SR), caused by Puccinia graminis f.sp. tritici, is a devastating disease of wheat, and the only disease of wheat that can cause a $100 \%$ yield loss. Breeding against virulent races such as Ug99 (TTKS) is a slow process, so there may be time between the arrival of race TTKS in North America and the deployment of resistance genes in adapted wheat cultivars. Fungicides are likely to provide the first line of defense against races like TTKS. Field experiments in Crookston and St. Paul, MN aimed to determine the efficacy of eight fungicides, each applied at one of two times. Fungicides (Caramba, Prosaro, Proline, Priaxor, Folicur, Tilt, Twinline, Headline) were applied to the SR susceptible hard red spring wheat cv. 'Max' at flag leaf (Feekes growth stage [FGS] 9) or the beginning of flowering (FGS 10.5.1). The experimental design was a randomized complete block design with four replicates. An untreated control was included in each experiment. Plants were needle inoculated with SR race QFCS at FGS 9. The Crookston site was also inoculated with a spore suspension at FGS 9. All fungicides tested reduced SR incidence and severity $(\mathrm{P}=0.0001$ and 0.04$)$ at Crookston. All fungicides reduced SR incidence $(\mathrm{p}=0.0001)$ and reduced yield losses compared to the untreated control $(\mathrm{p}=0.0001)$ in St. Paul. These results suggest that fungicides, including Caramba and Prosaro, may be used according to their label to reduce the impact of SR in wheat.

The post-transcriptional regulator $\operatorname{rsm} A / \operatorname{csr} A$ activates the T3SS in Xanthomonas citri by stabilizing the master regulator 5' UTR of hrpG

M. ANDRADE (1), S. C. Farah (2), N. Wang (1)

(1) Department of Microbiology and Cell Science, CREC, University of Florida, Lake Alfred, FL, U.S.A.; (2) Department of Biochemistry, Institute of Chemistry, University of São Paulo, Sao Paulo, Brazil

Phytopathology 104(Suppl. 3):S3.8

In this study, we demonstrated that $\operatorname{rsmA}$ not only is required for the full virulence of the phytopathogenic bacterium Xanthomonas citri subsp. citri (XCC) but also contributes to triggering the hypersensitive response (HR) in non-host plants. Deletion of $r \sin A$ resulted in a reduction of virulence in the host plant sweet orange and weakened HR in the non-host plant Nicotiana benthamiana. Microarray, qRT-PCR, Western-blotting, and GUS assays indicated that RsmA regulates the expression of the type 3 secretion system (T3SS) at both transcriptional and post-transcriptional levels. Here, we show that RsmA activates the expression of T3SS-encoding hrp/hrc genes by directly binding to the $5^{\prime}$ untranslated region (UTR) of $h r p G$, the master regulator of the $h r p / h r c$ genes, and another T3SS-associated $h r p D$ in XCC. RsmA stabilizes $h r p G$ mRNA, leading to increased accumulation of HrpG proteins and subsequently, the activation of $h r p / h r c$ genes. The activation of the $h r p / h r c$ genes by RsmA via HrpG was further supported by the observation that ectopic overexpression of $h r p G$ in an $\operatorname{rsm} A$ mutant restored its full-ability to cause disease in host plants and trigger HR in non-host 
plants. Taken together, these data revealed that RsmA activates T3SS by acting as a positive regulator of $h r p G$ and that this regulation is critical to the pathogenicity of XCC.

Spatial variation analysis of Ceratocystis manginecans incidence in Pakistan

R. ANJUM (1), E. M. Goss (2), I. A. Khan (3), S. T. Sahi (3), I. U. Haq (3), H. R. Ahmad (3)

(1) University of Florida, Gainesville, Gainesville, FL, U.S.A.; (2) Department of Plant Pathology, University of Florida, Gainsvile, FL, U.S.A.; (3) University of Agriculture, Faisalabad, 38040, Pakistan, Faisalabad, Pakistan

Phytopathology 104(Suppl. 3):S3.9

Ceratocystis manginecans sp. nov. is a cause of mango quick decline (MQD) and has become a major problem in mango production in Pakistan. MQD, as its name suggests, causes rapid decline of mango trees within a couple of days due to systemic infection by C. manginecans. Symptoms of MQD-infected mango trees are chlorosis, wilting of leaves, gum exudation with a rotten smell, bark splitting and subsequent browning of the whole tree leads to eventual death. The disease incidence in Pakistan has not previously been quantified. To assess the prevalence and incidence of this disease, spatial variation analysis was conducted by GPS tagging, surveying and sampling of various mango orchards of the southern Punjab districts: Khanewal (KHW), Multan (MLT), Muzaffergarh (MZG), Rahim Yar Khan (RYK); and northern Punjab districts: Sialkot (SKT), Gujrat (GRT) of Pakistan. On the basis of spatio-variational analysis the districts of southern Punjab; KHW 83.21\%, MLT 75\%, MZG $67.86 \%$, RYK $71.54 \%$ are highly effected with MQD incidence while districts of northern Punjab are not affected yet according to the sampling points.

\section{Sensitivity of Rhizoctonia solani to fungicides}

S. I. ARABIAT (1), M. Khan (2)

(1) NDSU, Fargo, ND, U.S.A.; (2) NSDU and University of Minnesota, Fargo, ND, U.S.A.

Phytopathology 104(Suppl. 3):S3.9

Rhizoctonia crown and root rot (RCRR) is a destructive disease of sugarbeet caused by Rhizoctonia solani. Fungicides application is an important tactic to control $R$. solani. Recently, $R$. solani AG 1 was reported to have developed resistance to azoxystrobin. Since azoxystrobin has been the most widely used fungicide for controlling $R$. solani on sugarbeet since the late 1990s, it is pertinent to evaluate the sensitivity of $R$. solani AG 2-2 to fungicides labeled for controlling this pathogen. Sensitivity to fungicides was determined using mycelium radial growth assay, and in greenhouse studies. Baseline $\mathrm{EC}_{50}$ values mean were $0.3,0.2,0.9,4.8$, and $97.1 \mu \mathrm{g} / \mathrm{ml}$, and non-baseline isolates were $0.9,0.2,0.6,296.0$, and $341.7 \mu \mathrm{g} / \mathrm{ml}$ for pyraclostrobin, penthiopyrad, prothioconazole, azoxystrobin, and trifloxystrobin respectively. Frequency of isolates with $\mathrm{EC}_{50}$ values $>10 \mu \mathrm{g} / \mathrm{ml}$ for azoxystrobin and trifloxystrobin increased in non-baseline isolates $(80 \%)$ compared with baseline isolates $(25 \%)$. Although the tested fungicides varied in their $\mathrm{EC}_{50}$ values for the same isolate, all fungicides were only effective at high concentrations in controlling RCRR. The data indicated that $R$. solani was still sensitive to azoxystrobin although it was widely used in sugarbeet production. Penthiopyrad had low $\mathrm{EC}_{50}$ values, and showed no cross sensitivity with azoxystrobin, which makes it a good choice to be used with azoxystrobin in RCRR disease management and for fungicide resistance management.

\section{Decaying leaf litter supports Phytophthora ramorum and endemic Phytophthora species in streams \\ K. ARAM (1), D. M. Rizzo (1) \\ (1) University of California, Davis, CA, U.S.A. \\ Phytopathology 104(Suppl. 3):S3.9}

In surface waters, leaf litter is a potential substrate for persistence and propagation of introduced tree pathogen Phytophthora ramorum and putatively endemic and saprobic species grouped in ITS clade 6. Previous research showed that $P$. ramorum readily colonizes fresh leaf tissue in streams, but is not effective at colonizing leaves killed by freezing or drying in the lab before exposure in streams. In contrast, P. ramorum was recovered abundantly from significantly degraded natural leaf litter. "Clade 6" species occurred on all leaf types. We asked how these species might compete for fresh leaf tissue introduced into streams, how long they would persist and whether they could sporulate from progressively more decayed leaf tissue. $P$. ramorum-infected and uninfected bay laurel (Umbellularia californica) leaves were incubated in two forest streams and sampled at 8 intervals over 16 weeks. Leaves were evaluated for Phytophthora colonization through isolations and morphological identification. P. ramorum and "clade 6" taxa quickly colonized leaves in streams and persisted throughout the full duration of in-stream incubation despite loss of as much as $70 \%$ of leaf mass due to decay. Both could be baited from leaf samples over the entire 16 weeks, demonstrating sporulation potential despite substantial decay and biomass loss. This indicates that bay laurel leaf litter can serve as a persistent source of P. ramorum and "clade 6" Phytophthora inoculum in infested streams.

Resistance in mango against infection by Ceratocystis fimbriata

L. ARAUJO (1), W. M. S. Bispo (1), I. S. Cacique (1), W. R. Moreira (1), F. A. Rodrigues (1)

(1) Vicosa Federal University, Vicosa, Brazil

Phytopathology 104(Suppl. 3):S3.9

This study was designed to characterize and describe host cell responses of stem tissue to mango wilt disease caused by the fungus Ceratocystis fimbriata in Brazil. Disease progress was followed, through time, in inoculated stems for two cultivars, Ubá (field resistant) and Haden (field susceptible). Stem sections from inoculated areas were examined using fluorescence light microscopy, transmission and scanning electron microscopy coupled with energy dispersive X-ray microanalysis. Tissue from Ubá colonized by $C$. fimbriata had stronger autofluorescence than those from Haden. The X-ray microanalysis revealed that the tissue of Ubá had higher levels of insoluble sulfur and calcium than those of the Haden. Scanning electron microscopy revealed that fungal hyphae, chlamydospores (aleurioconidia) and perithecialike structures of $C$. fimbriata were more abundant in Haden relative to Ubá. At the ultrastructural level, pathogen hyphae had grown into the degraded walls of parenchyma, fiber cells and xylem vessels in the tissue of Haden. However, in Ubá, plant cell walls were rarely degraded and hyphae were often surrounded by dense amorphous granular materials and hyphae appeared to have died. Taken together, the results of study characterize the susceptible and resistant basal cell responses of mango stem tissue to infection by $C$. fimbriata.

The use of calcium and manganese foliar nutrition to induce resistance to Sclerotinia sclerotiorum

A. ARFAOUI (1), A. El Hadrami (2), F. Daayf (3), L. Adam (3)

(1) University of Manitoba, Winnipeg, MB, Canada; (2) Omex Agriculture Inc., Winnipeg, MB, Canada; (3) Department of Plant Science, Winnipeg, MB, Canada

Phytopathology 104(Suppl. 3):S3.9

White mold caused by Sclerotinia sclerotiorum (Lib.) de Bary impedes yield and quality in a variety of field crops and vegetables. The efficiency of currently used fungicides to control this disease remains insufficient. We tested foliar formulations containing calcium and manganese in controlling white mold in canola and soybean, using isolates of S. sclerotiorum with different levels of aggressiveness. The addition of oxalic acid or potassium oxalate increased the lesion size and the overall pathogenicity of weakly aggressive isolates. Pre-treatment of leaf tissues with $\mathrm{Ca} / \mathrm{Mn}$ formulations substantially reduced the hyphal growth of highly-aggressive isolates and the size of the lesions. In parallel, we revealed the suppression of ROS production and a delay in the activation of related-enzymes in response to highlyaggressive isolates as compared to the weakly-aggressive ones. Application of calcium/manganese-based formulations two days prior to inoculation restored the early production of ROS around the infection site and increased the activity of several defense-related enzymes. The analysis of phenolics and known phytoalexins revealed a differential change in the profile in response to weakly- versus highly-aggressive isolates and in response to the pre-treatment with $\mathrm{Ca} / \mathrm{Mn}$, suggesting the potential of these nutrients in activating host defense responses to this and other diseases.

Comparative sequence-based analysis of Fusarium proliferatum populations from seven maize genotypes

M. ARIF (1), G. Busot (1), F. Iriarte (1), T. Fischer (1), J. P. Stack (1)

(1) Kansas State University, Manhattan, KS, U.S.A.

Phytopathology 104(Suppl. 3):S3.9

Pathogenic to many plant species and endophytic in others, Fusarium proliferatum is dispersed globally in seed of many crop plants, often in a cryptic, non-symptomatic state. $F$. proliferatum isolates vary in mycotoxin production, host range and can be detected in a vast array of habitats. Understanding the genetic complexity among and within populations is critical to understanding the significance of their dispersal and to developing effective detection protocols and mitigation measures. A comparative sequence-based genetic analysis of $F$. proliferatum populations from seven maize genotypes differing in insect resistance and herbicide tolerance was undertaken using two techniques. Inter-Simple Sequence Repeats (ISSR) analysis using ten primer sequences and multi-locus sequence typing using 3 genes was used to characterize over 100 isolates of $F$. proliferatum and closely related Fusarium species. Outgroup populations included $F$. 
verticillioides, $F$. subglutinans, $F$. fujikuroi, $F$. thapsinum, and $F$. adyazi. Analyses indicated that each maize genotype was host to genetically diverse populations of $F$. proliferatum. In a few instances, genetically identical and nearly identical $F$. proliferatum isolates occurred in more than one corn genotype. Phylogenetic analysis indicated that F. proliferatum isolates clustered tightly and independently from all outgroup populations.

Detection of the G143A mutation associated with QoI resistance in Cercospora sojina using a real time TaqMan SNP assay

E. ARNAO (1), E. Stumpf (1), A. Subedi (1), B. H. Bluhm (2), C. Bradley (3), A. Fakhoury (1)

(1) Southern Illinois University, Carbondale, IL, U.S.A.; (2) University of Arkansas, Fayetteville, AR, U.S.A.; (3) University of Illinois, Urbana, IL, U.S.A.

Phytopathology 104(Suppl. 3):S3.10

The management of Frogeye Leaf Spot, caused by Cercospora sojina in soybean, is primarily based on the use of genetic resistance in soybean and the application of fungicides, including Quinone outside inhibitors (QoI). However, resistance to this class of fungicides in some important plant pathogens has been reported to develop in a relatively short time. Molecular studies have revealed that the resistance to QoI fungicides is often conferred to a point mutation in the $C$. sojina cytochrome b $(c y t b)$ gene resulting in a glycine to alanine substitution at position 143. Recently, isolates of $C$. sojina with resistance to strobirulins fungicides have been reported for the first time in soybean fields in the USA. In this study, we report the development of a real time QPCR assay to detect the G143A mutation in C. sojina. The assay was used to screen $114 C$. sojina isolates collected from two commercial soybean production fields of Illinois. The screen revealed the presence of the G143A mutation in $62.28 \%$ of the isolates.

Integrated pest management of sting nematode (Belonolaimus longicaudatus) on bermudagrass

S. ARYAL (1), W. T. Crow (1), R. McSorley (1), R. M. Giblin-Davis (1), K. E. Kenworthy (1)

(1) University of Florida, Gainesville, FL, U.S.A.

Phytopathology 104(Suppl. 3):S3.10

Use of bermudagrass cultivars tolerant to Belonolaimus longicaudatus is vital for sustainable turf management in sandy soils of the southeast US. Identification and development of tolerant bermudagrass and new IPM programs for plant-parasitic nematodes on golf courses are possible. A multiyear field experiment including five bermudagrass genotypes and four different nematicide regimes in a split-plot design with five replications was initiated in fall, 2011. The five bermudagrass genotypes evaluated were 'Tifway', two commercial cultivars ('TifSport' and 'Celebration') that were identified as tolerant to B. longicaudatus, and two experimental germplasm. Nematode samples were collected at three-month intervals to monitor the population density of $B$. longicaudatus. Mixed models analysis and comparison of least squares means indicated no significant differences among the genotypes. However, there were significant treatment responses on nematode population level after August, $2013(P \leq 0.05)$. A calendar-based IPM program rotating Bacillus firmus strain I-1582, Furfural, Brassica juncea, and Abamectin significantly reduced the nematode population density compared to a monitoring-based IPM and 1,3-dichloropropene. In the untreated control, nematode population densities were significantly higher than the other treatments. Work is ongoing for one more year to follow these trends and to determine long-term performance of these germplasm and pest management program entries.

Prevalence and distribution of Aspergillus section Flavi in maize and groundnut fields and aflatoxin contamination in Mozambique

J. Augusto (1), J. Atehnkeng (2), J. Akello (3), P. Cotty (4), R. Bandyopadhyay (2)

(1) International Institute of Tropical Agriculture (IITA), Nampula, Mozambique; (2) International Institute of Tropical Agriculture (IITA), Ibadan, Nigeria; (3) International Institute of Tropical Agriculture (IITA), Lusaka, Zambia; (4) USDA/ARS, Tucson, AZ, U.S.A.

Phytopathology 104(Suppl. 3):S3.10

High prevalence of liver cancer in Mozambique has been linked to intake of aflatoxin contaminated foods. Aflatoxins have also adversely impacted exports of Mozambican groundnuts. The extent of aflatoxin contamination of groundnut and maize, two aflatoxin-prone crops, and field population of Aspergilli is not clear. Geo-referenced 305 soil samples were collected in maize and groundnut farmers' fields at harvest in 2013 in different agroecological zones (AEZ) to determine inoculum density and distribution of Aspergillus section Flavi. At least 300 geo-referenced samples of each maize and groundnut were collected at harvest and during storage for aflatoxin mapping. Soil inoculum of Aspergilli was medium to high (30-2,000 CFU/g) in the hot, humid, low to medium altitude $(50-600 \mathrm{~m})$ AEZ of Manica, Zambezia and Nampula provinces, and low $(0-30 \mathrm{CFU} / \mathrm{g})$ in the wet, high altitude $(>1,000 \mathrm{~m}) \mathrm{AEZ}$ of Tete province, irrespective of crop. Aspergillus flavus $\mathrm{L}$-strain and $A$. parasiticus were the most frequently isolated Aspergilli but $\mathrm{S}$-strain and A. tamari were also isolated. Aflatoxin levels were high in groundnut $(0-5,674 \mathrm{ppb})$ compared to maize $(0-687 \mathrm{ppb})$ in all locations but aflatoxin levels were similar at harvest and storage for both crops. Nampula and Zambezia had the highest aflatoxin levels for both crops. The high occurrence of aflatoxin in these two provinces in this study suggests that they are potentially aflatoxin-hot spot areas and aflatoxin mitigation options are needed.

Molecular diagnostic tools for detection and identification of sweet potato viruses

A. S. AVELAR (1), J. K. Brown (1)

(1) University of Arizona, School of Plant Sciences, Tucson, AZ, U.S.A.

Phytopathology 104(Suppl. 3):S3.10

Sweet potato is one of the 10 most important food crops worldwide, and is a host of at least thirty plant viruses. Little is known about the particular viruses present in sweet potato crops in Central America, a primary origin of the genus, Ipomoea, making cost-effective, molecular assays for virus detection and identification essential. PCR and RT-PCR primers were designed and validated for all sweet potato viruses classified in the Closteroviridae, Geminiviridae, Flexiviridae, and Potyviridae, For primer design, the genome sequences of all sweet potato-infecting viruses (available in GenBank) were aligned. Primers were designed around the most conserved regions of the heat shock or coat protein gene. Genus-specific primers were designed for begomoviruses and potyviruses, and virus-specific primers were designed for Sweet potato chlorotic fleck virus (SPCFV), Sweet potato chlorotic stunt virus (SPCSV), Sweet potato feathery mottle virus (SPFMV), and Sweet potato mild mottle virus (SPMMV). Primers were validated using positive and negative controls e.g. virus-infected or virus-free leaf tissue, and then used to screen field, greenhouse, or tissue culture samples. The amplicons were cloned, and viral identity confirmed by DNA sequencing and comparative analysis. All viruses tested here were shown to be detectable using the respective primers under optimized conditions, indicating that they will provide for reliable virus detection and identification.

Management of Phytophthora blight (Phytophthora capsici) of pepper in Illinois

M. BABADOOST (1), C. Pavon (1), S. Z. Islam (2)

(1) University of Illinois, Urbana, IL, U.S.A.; (2) Syngenta Seeds, Inc., Naples, FL, U.S.A.

Phytopathology 104(Suppl. 3):S3.10

Phytophthora blight, caused by Phytophthora capsici, is one of the most important diseases of peppers in Illinois. P. capsici can infect pepper plants at all growth stages, causing seedling death, root rot, crown rot, stem blight, leaf spot, and fruit rot. To develop effective strategies for management of $P$. capsici in peppers, three approaches were evaluated, which included: (i) using resistant cultivars, (ii) crop rotation, and (iii) fungicide application. To identify resistant pepper cultivars to $P$. capsici, accessions/cultivars of bell pepper were tested in the greenhouse and field, and several cultivars were found resistant/tolerant to $P$. capsici. To establish effective crop rotation for management of $P$. capsici, host-range of the pathogen was determined and survival of the pathogen in soil textures and soil depths were investigated. A 4-year crop rotation with nonhost plants and effective weed control are recommended for management of Phytophthora blight of peppers. More than 40 fungicides with potential effects were evaluated for their efficacy for control of P. capsici. Ametoctradin + dimethomorph (Zampro 525F), captan (Maestro 80DF), cyazofamid (Ranman 400SC), dimethomorph (Forum 4.16 SC), famoxadone + cymoxanil (Tanos 50WDG), fluazinam (Omega 500F), fluopicolide (Presidio 4SC), mandipropamid (Revus 2.09SC), and mefenoxam (Ridomil Gold Copper 65WP, Ridomil Gold EC 4SC) fungicides were effective for control of $P$. capsici in pepper.

Effect of molybdo-priming on the tolerance of crested wheatgrass to biotic stress caused by leaf rust

O. N. BABENKO (1), Z. Alikulov (1)

(1) L.N. Gumilyov Eurasian National University, Astana, Kazakhstan Phytopathology 104(Suppl. 3):S3.10

Leaf rust (LR) is one of the most widespread diseases of crested wheatgrass (Agropyron cristatum (L.) Beauv. s.l.) in the Kazakhstan. Molybdoenzymes, nitrate reductase (NR), aldehyde oxidase (AO), xanthine dehydrogenase $(\mathrm{XDH})$, play important role in the tolerance of plants to pathogens. The main factor limiting their activity is molybdenum (Mo) content in soil. However, 
the soil in the Kazakhstan is deficient in Mo. At present, pre-sown seed priming regarded as an essential strategy for plant adaptation to stresses. We investigated the effect of seed priming with Mo or tungsten (W) on the molybdoenzymes' activity of Agropyron under LR infection. In the stage of two leaves, plants inoculated with LR urediniospores (TTT/QJ pathotype). $\mathrm{XDH}$ and $\mathrm{AO}$ activities were determined by native gel electrophoresis, but NR activity was determined by in vivo assay. Determination of total hydrogen peroxide carried out spectrophotometrically. Results showed that the Mopriming increased activity of molybdoenzymes and did not change the total content of hydrogen peroxide in plant tissues, thereby increasing plant resistance to LR. At the same time W-priming had inversely effect. Improvement of molybdoenzymes' activity by seed priming deserves special attention, as understanding the mechanisms of their functioning in stress conditions have not only fundamental, but also practical significance to the phytopathology.

\section{Effect of pre-sown molybdo-priming on the tolerance of wheat to leaf rust} infection

O. N. BABENKO (1), Z. Alikulov (1)

(1) L.N. Gumilyov Eurasian National University, Astana, Kazakhstan

Phytopathology 104(Suppl. 3):S3.11

Leaf rust (LR) caused by the fungus Puccinia triticiana Eriks. is a major pathogen of spring wheat (Triticum aestivum) in the Northern Kazakhstan. Molybdoenzymes, such as nitrate reductase (NR), aldehyde oxidase (AO), xanthine dehydrogenase $(\mathrm{XDH})$, play important role in plant resistance to abiotic and biotic stresses. It is well known, the soils of Kazakhstan is deficient in Mo and it is limiting factor for activity of molybdoenzymes. However, pre-sown seed priming regarded as an essential strategy for plant adaptation to stresses. We investigated the effect of seed priming with Mo or tungsten (W) on the molybdoenzymes' activity of wheat under LR infection. The week-old seedlings were inoculated by LR urediniospores. XDH and AO activities were determined by native gel electrophoresis. NR activity was determined by in vivo assay. Determination of total hydrogen peroxide carried out spectrophotometrically. Results showed that the Mo-priming increased activity of $\mathrm{AO}$ and $\mathrm{XDH}$, decreased NR activity and did not change the total content of hydrogen peroxide in plant tissues, thereby increasing plant resistance to LR. The W-priming had inversely effect. Hence, our results suggested that the Mo-priming might play an important role in the process of plant tolerance to leaf rust.

Characterization of two novel Didymella bryoniae isolates from watermelon in Florida and Georgia

B. BABU (1), Y. W. Kefialew (2), P. F. Li (3), X. P. Yang (3), S. George (4), E. Newberry (5), N. Dufault (5), J. Marois (4), M. L. Paret (4)

(1) North Florida Research \& Education Ctr, Quincy, FL, U.S.A.; (2) Ethiopian Institute of Agricultural Research, Gambella, Ethiopia; (3) Jiangsu Academy of Agricultural Sciences, Jiangsu, China; (4) NFREC, University of Florida, Quincy, FL, U.S.A.; (5) University of Florida, Gainsville, FL, U.S.A. Phytopathology 104(Suppl. 3):S3.11

Gummy stem blight (GSB) caused by Didymella bryoniae (anamorph, Phoma cucurbitacearum) is a major fungal disease of watermelon and other cucurbits. Thirty five isolates of Didymella spp. and Phoma spp. associated with GSB on watermelon, Canary melon, muskmelon and squash from Florida and Georgia were characterized based on morphology on culture media, pathogenicity assay, genetic characterization using ITS sequence analysis, Random Amplified Polymorphic DNA (RAPD) analysis and PCR-Restriction Fragment Length Polymorphism (RFLP). All the isolates were pathogenic on watermelon cv. Melody, but to a varying degree. RAPD and ITS sequence analysis provided genetic variability between the isolates, but PCR-RFLP did not show any variability. Nucleotide sequences analysis and phylogenetic clustering of the ITS region showed the presence of two isolates DB-05 and DB-33, which showed an higher identity to the D. bryoniae isolates from China. These two isolates possessed a single nucleotide substitution of A to $G$ at position 131 of the ITS-1 region. Even though substitutions in this ITS-1 region have also been previously reported in $D$. bryoniae isolates from other locations, this is the first description of an isolate with this unique substitution in Florida and Georgia. The present study brings insights into the current genetic profile of $D$. bryoniae isolates in Florida and Georgia, and its similarity with international isolates.

Polymorphic avr genes in isolates of two races of Passalora fulva from Argentina

P. A. BALATTI (1), R. Medina (1), C. Rollan (1), S. M. Lopez (2), E. M. Franco (1), M. C. Saparrat (3), B. L. Ronco (1)

(1) Centro de Investigaciones de Fitopatologia Facultad de Ciencias Agrarias y Forestales Universidad Nacional de La Plata, La Plata, Argentina; (2)
Facultad de cienciasAgrarias y Forestales, La Plata, Argentina; (3) INFIVECONICETFacultad de Ciencias Agrarias y Forestales, Universidad Nacional de La Plata, La Plata, Argentina

Phytopathology 104(Suppl. 3):S3.11

The purpose of this work was to identify based on morphological and molecular tools the etiological agent of diseased tomatoes with leaf mould symptoms. Diseased plants were collected mainly from greenhouses within the most important production area of tomato in Argentina, the Cinturón Hortícola of Gran La Plata, Buenos Aires province, Argentina. Isolates identity was confirmed by morphological characteristics as well as the Internal Transcribed spacer sequence. Avr genes were amplified and sequenced by means of a modification of the protocol described by Stergiopoulos et al (2007). The amplicons obtained were sequenced and deposited in the NCBI GenBank. Even though P. fulva isolates were collected from different tomato cultivars and different farms, they belong either to race 0 (avr2, avr4, avr4E and $a v r 9)$ or race 2 (avr4, avr4E and avr 9 ) The avr4 sequences included transitions, transversions and insertions. While the two other genes of the isolates from Argentina avr 4E and avr 9, presented transitions and transversions and only one transversion, respectively. Although we have not studied yet the biological implications of these changes, we found that though the number of races identified by now is only two, the population of the fungus appears to be evolving therefore new races or more virulent isolates might arise. This work was supported by the Fac. Cs. Agrs. Y Ftales UNLP, Comisión de Investigaciones Científicas (CICBA) and PICT 2012-2760.

Impact of Fusarium stalk rot and charcoal rot on yield parameters of grain sorghum

A. Y. BANDARA (1), D. K. Weerasooriya (1), C. R. Little (1)

(1) Kansas State University, Manhattan, KS, U.S.A.

Phytopathology 104(Suppl. 3):S3.11

Stalk rots are among the most prevalent diseases of sorghum. However, no quantitative crop loss assessment has been reported in relation to these diseases. The objective of this study was to test the effects of Fusarium stalk rot and charcoal rot on grain sorghum yield parameters when plants were inoculated at two growth stages. Four genotypes were tested in the greenhouse and field, against three Fusarium spp. ( $F$. thapsinum, F . proliferatum, $F$. andiyazi) and Macrophomina phaseolina (MP) at GS1 (30 d after emergence) and GS3 (14 d after flowering). Panicles harvested at physiological maturity were measured for total seed weight (TSW), 100-seed weight (SW), and number of seeds per panicle (TNSP). Disease severity was measured by splitting stems and measuring lesion length and number of nodes crossed by the lesion. Pathogens significantly decreased TSW over the control and at GS1 and GS3. There was a greater reduction of TSW at GS1 (50\%) than GS3 $(30 \%)$. Inoculation at GS1 reduced TNSP while GS3 inoculations reduced SW. Fusarium species caused higher disease severity when inoculated at GS1 compared to MP, demonstrating a greater impact of Fusarium stalk rot compared to charcoal rot at early vegetative stages. Correlations between yield parameters and disease severity traits were insignificant within GS1 and GS3. These findings stress the importance of concurrent evaluation for disease severity (resistance) and tolerance when screening germplasm for stalk rot diseases.

Identifying novel interacting proteins of Maize mosaic rhabdovirus glycoprotein using the split-ubiquitin membrane-based yeast two hybrid system

K. BARANDOC-ALVIAR (1), D. Rotenberg (1), A. E. Whitfield (1)

(1) Kansas State University, Manhattan, KS, U.S.A.

Phytopathology 104(Suppl. 3):S3.11

The corn planthopper, Peregrinus maidis, transmits Maize mosaic rhabdovirus (MMV) in a persistent-propagative manner. Identification of insect molecules that interact with viral proteins to mediate internalization and movement of virus in host tissues is essential for defining vector-virus relationship. To identify receptors and other proteins interacting with MMV glycoprotein $(\mathrm{G})$, we used the split-ubiquitin membrane-based yeast two hybrid system $(\mathrm{MbY} 2 \mathrm{H})$ for in vivo detection of membrane protein interactions. In our assay, MMV G served as the bait and a P. maidis cDNA library served as the prey. The initial screen identified 474 potential interactors and confirmation screening revealed 110 true interactors. Sequencing of 48 candidates revealed 19 unique proteins interact with MMV G. Sixty three percent of the sequences had significant similarity with annotated protein sequences from other organisms while $37 \%$ were hypothetical proteins or had no significant match. Annotated putative interactors had predicted conserved domains associated with lipid binding, oxidoreductase activity, GTP binding, hydrogen ion transmembrane transporter activity, and chromatin-mediated transcriptional regulation. Identification and characterization of additional interacting proteins is in 
progress. Collectively, knowledge gained from this study will enable design of new technologies to disrupt the molecular interaction between virus and vector, and potentially inhibit virus transmission.

Comparing populations of epiphytic bacteria in Pennsylvania's organic and conventional stone fruit orchards as it relates to bacterial spot management

S. BARDSLEY (1), M. Jimenez-Gasco (1)

(1) Penn State University, University Park, PA, U.S.A

Phytopathology 104(Suppl. 3):S3.12

Bacterial spot of stone fruit (caused by Xanthomonas arboricola pv. pruni) remains the most important bacterial disease of peach and nectarine in the eastern US and has greatly hindered the establishment of organic stone fruit orchards in this region. Despite that, no bacterial spot was found in two organic peach orchards in Adams County, PA. The objectives of this research were to monitor and identify populations of bacterial epiphytes in organic and conventional stone fruit orchards, including bacterial epiphytes resistant to the antibiotic oxytetracycline, the primary antibiotic used in conventional management. Samples were taken from 6 conventional and 2 organic stone fruit orchards. Bacteria growing on media amended with 10 and $25 \mathrm{mg} / \mathrm{L}$ oxytetracycline were recovered from all orchards. Comparisons made between the overall bacterial populations obtained from organic and conventional orchards, based on morphological characteristics, demonstrated that bacterial populations from organic and conventional orchards were completely different. Gram positive bacterial colonies and yeasts predominated populations recovered from the two organic orchards while the majority of bacterial colonies recovered from the conventional orchards were Gram negative.

Grower perceptions of preventative practices for management of trunk diseases of grape

K. BAUMGARTNER (1), R. Travadon (2), V. Hillis (2), J. Kaplan (3), M. Cooper (2), M. Lubell (2)

(1) USDA ARS, Davis, CA, U.S.A.; (2) UC Davis, Davis, CA, U.S.A.; (3) California State University, Sacramento, Sacramento, CA, U.S.A.

Phytopathology 104(Suppl. 3):S3.12

Trials on prevention of trunk diseases (e.g., Eutypa dieback) show that three practices [delayed pruning, double pruning, pruning-wound protectants] prevent pruning-wound infections by $25-100 \%$. Nonetheless, they are often not adopted until disease incidence is $>20 \%$ in mature vineyards. There are no eradicative controls. Our goal is thus to encourage adoption in young vineyards. We surveyed 350 grape growers in five California regions (NapaSonoma, Central Coast, N. San Joaquin, S. San Joaquin, N. California) using the audience-response software Turning Point, in extension meetings. Growers answered questions on practice usage (never to always), vineyard age when practice was adopted $(0-3,4-7,8-12$, and $13+$ years), and perceptions of disease-control efficacy and cost-effectiveness (ineffective to effective). In all but one region (Napa-Sonoma), preventative practices were adopted in vineyards $>8$ years old, with mean disease incidence of $15 \%$. Delayed pruning was most common except in Sonoma, where pruning-wound protectants were most common. Growers who adopt a practice in vineyards $<8$ years old also had positive perceptions of efficacy and cost-effectiveness, suggesting that timing the practice before symptoms appear does indeed maintain yields and is cost-effective. With a clear understanding of their usage and perceptions, we will develop new extension tools that better communicate to growers the case for preventing trunk diseases in young vineyards.

Management of bacterial wilt in muskmelon with perimeter trap cropping F. BAYSAL-GUREL (1), M. M. Gardiner (1), C. Welty (2), M. L. Gleason (3), S. A. Miller (1)

(1) The Ohio State University, Wooster, OH, U.S.A.; (2) The Ohio State University, Columbus, OH, U.S.A.; (3) Iowa State University, Ames, IA, U.S.A. Phytopathology 104(Suppl. 3):S3.12

Bacterial wilt (BW) is a destructive disease of cucurbits. The cucumber beetles (CB) that vector the causal agent, Erwinia tracheiphila, feed preferentially on different cucurbit species. A perimeter trap crop (PTC) planted around main crop (MC) may preferentially attract $\mathrm{CB}$, which can be controlled in the PTC by insecticide applications, reducing the number of applications to the MC. The efficacy of buttercup squash 'Space Station' as a PTC for management of BW in muskmelon was evaluated in field trials conducted in Wooster, OH. PTC consisted of two rows of squash plants surrounding $50 \times 50 \mathrm{ft}$ muskmelon subplots. Subplots without PTC were surrounded by annual ryegrass border with the same dimensions as the PTC. The two subplots were separated by at least $50 \mathrm{ft}$ on each of two farms. BW pressure was low and there were no significant differences in disease incidence over the season between muskmelons with or without PTC. Incidence of BW reached $44.4 \%$ in one of the two PTC. There were no significant differences in the number and weight of marketable muskmelons from plots with or without PTC. The squash PTC received on average 7 Sevin XLR PLUS foliar applications and 2 Admire Pro 4.6F drench applications to control CB and squash bug populations. However, on average, 2.5 foliar applications of Sevin XLR PLUS were made in muskmelons without PTC, whereas 1.5 applications were made in muskmelons with PTC, indicating that $\mathrm{PTC}$ reduced reliance on insecticides in the MC.

Evaluation of timing of row cover removal in management of bacterial wilt and Alternaria leaf spot of muskmelon

F. BAYSAL-GUREL (1), M. M. Gardiner (1), C. Welty (2), M. Gleason (3), S. A. Miller (1)

(1) The Ohio State University, Wooster, OH, U.S.A.; (2) The Ohio State University, Columbus, OH, U.S.A.; (3) Iowa State University, Ames, IA, U.S.A.

Phytopathology 104(Suppl. 3):S3.12

Bacterial wilt (BW) (Erwinia tracheiphila) is an important cucumber beetlevectored disease of cucurbits in the North Central Region of the US. Row covers can provide a barrier to the beetles and reduce the incidence of bacterial wilt, but are also a barrier to pollinators and beneficial insects. The effect of row cover removal timing (at anthesis, 10 days after anthesis, or 10 days after row cover ends opened at anthesis) on the occurrence of BW and Alternaria leaf spot (ALS) in muskmelon was tested in 2011 and 2012. In 2011, the area under the disease progress curve (AUDPC) for ALS was significantly lower in covered plots than in non-covered control plots, but the timing of cover removal did not influence AUDPC or disease severity. Noncovered plots had more marketable fruit than covered plots, primarily the result of aphid infestations in covered plots. BW was not observed in 2011 in this experiment. In 2012, BW incidence was moderate, and covered plots had significantly fewer wilted plants and lower AUDPC than non-covered plots. ALS severity and season-long disease progress was significantly lower in plots with row covers than in the non-covered plots. However, there were no significant differences in BW incidence or ALS severity and disease progress among the row-cover treatments. Plots with the row cover removed at anthesis yielded more marketable fruit than those with row covers removed 10 days after anthesis or without cover.

Fighting back against blackleg: Utilization of high throughput RNA-seq to characterize the blackleg-canola pathosystem

M. G. BECKER (1), I. J. Girard (2), T. de Kievit (2), D. Fernando (2), M. F. Belmonte (2)

(1) University of Manitoba, Ile Des Chenes, MB, Canada; (2) University of Manitoba, Winnipeg, MB, Canada

Phytopathology 104(Suppl. 3):S3.12

Leptosphaeria maculans (blackleg) is a devastating fungal pathogen of Brassica napus (canola) and causes millions of dollars in crop damage and loss to growers around the globe every year. Canola has a hybrid genome between Brassica rapa and Brassica oleracea. Together with a large genome size, poor annotation, and abundance of homologous genes this presents unique challenges when analyzing global RNA populations using next generation sequencing strategies. Cotyledons of canola cultivars that demonstrated either susceptibility or qualitative resistance to Blackleg were captured $0,3,7$, and 10 days during the infection process from the earliest stages of fungal infection to complete cotyledon devastation. Using low-cost cDNA library protocols, the transcriptomes of blackleg-infected canola cotyledons were sequenced using the Illumina Hi-Seq platform. We then profiled global RNA populations at the cellular level by capturing individual cells and tissues directly at the site of infection using cutting-edge laser microdissection methods. Our work provides the most comprehensive global RNA profiling dataset to date and provides a resource for the identification of novel genes and gene products associated with qualitative resistance. Challenges associated with the processing and bioinformatics of the canola transcriptome and possible solutions will be discussed.

The biochemistry of an extract with zoosporicidal activity from late blight infected Petunia $\times$ hybrida

M. C. BECKTELL (1)

(1) Colorado Mesa University, Grand Junction, CO, U.S.A.

Phytopathology 104(Suppl. 3):S3.12

Late blight of petunias, caused by the oomycete pathogen Phytophthora infestans, is not a novel disease, but there is limited knowledge on this hostpathogen system. It has been established that petunias are less susceptible than the more common potato and tomato hosts. It is also known, based on previous work in our lab, that leachates from petunia late blight lesions cause lysis of $P$. infestans zoospores. The objectives of our current work were to get the lysing agent into a stable, storable form, and to elucidate the identity of the 
lysing agent. Five to six week old petunias (cv. White Madness) were inoculated with a US-11 isolate of $P$. infestans. Five DAI, infected petunia leaves were washed in water and the aqueous leachate was filtered, tested for activity, then extracted into dichloromethane via liquid-liquid partitions and concentrated. The resulting filtrate crude extract (FCE) was resuspended in water and added to a suspension of healthy zoospores. Lysis of zoospores within 1-4 minutes confirmed the presence of the lysing agent in the FCE from petunia late blight lesions. Furthermore, the stability of the FCE allowed for further analyses. The FCEs from six separate inoculation experiments were analyzed via LCMS-ELSD and ${ }^{1} \mathrm{H}$ and ${ }^{13} \mathrm{C}$ NMR. The LCMS and NMR data suggest the presence of structurally interesting terpenes with masses between 300-800 amu. Work to elucidate the identity of the terpenes and other potential compounds of interest are ongoing. This work will provide insights into the petunia-late blight interaction, of which little is known.

Microbial community structure in the Poa annua rhizosphere is affected by nitrogen and potassium

L. BEIRN (1), C. J. Schmid (1), J. W. Hempfling (1), J. A. Murphy (1), B. B. Clarke (1), J. A. Crouch (2)

(1) Rutgers University, New Brunswick, NJ, U.S.A.; (2) USDA-ARS, Systematic Mycology \& Microbiology Lab, Beltsville, MD, U.S.A.

Phytopathology 104(Suppl. 3):S3.13

Nitrogen $(\mathrm{N})$ and potassium $(\mathrm{K})$ are vital for maintaining healthy turfgrass, but little is known about whether these nutrients affect pathogenic or beneficial microorganisms in the rhizosphere. The objectives of this study were to evaluate the composition, diversity, and distribution of microorganisms in the rhizosphere of Роа аппиа turf. Three $16 \mathrm{~mm} \times 51 \mathrm{~mm}$ soil cores were sampled from four replicated plots receiving: (1) 100,132 , or $200 \mathrm{~kg} \mathrm{~N} \mathrm{ha}^{-1}$ $\mathrm{yr}^{-1}$; (2) $200 \mathrm{~kg} \mathrm{~K}_{2} \mathrm{O} \mathrm{ha}^{-1} \mathrm{yr}^{-1}$; or (3) $132 \mathrm{~kg} \mathrm{~N} \mathrm{ha}^{-1} \mathrm{yr}^{-1}$ and $200 \mathrm{~kg} \mathrm{~K}_{2} \mathrm{O} \mathrm{ha}^{-1}$ $\operatorname{yr}^{-1}\left(\mathrm{~N}=\right.$ urea $\left.\left[\mathrm{CO}\left(\mathrm{NH}_{2}\right)_{2}\right], \mathrm{K}=\mathrm{KCl}\right)$. Organism-specific DNA regions were PCR amplified from fungi (ITS), bacteria and archaea (16s). Multiplexed next-generation Illumina sequencing generated $2.3 \times 10^{7}$ paired-end reads (avg. $1.38 \times 10^{5} \mathrm{seq} / \mathrm{sample}$ ). The QIIME pipeline picked $7.2 \times 10^{5}$ OTUs, $4.1 \%$ of which were identified as archaea, $26.7 \%$ bacteria, and $29.7 \%$ fungi. Alpha diversity metrics showed that diversity was highest in low and high $\mathrm{N}$ treatments. DCA plots and Kruskal-Wallis ANOVAs revealed significant differences in microbial community structure across all treatments, particularly amongst archaea and bacteria. For example, ammonium oxidizers in the Crenarchaeales dominated K-based treatments, whereas intermediate and high rates of $\mathrm{N}$ favored ammonium oxidizers in the Nitrososphareales. These data show that $\mathrm{N}$ and $\mathrm{K}$ treatments can affect the composition and structure of rhizosphere microbial communities in $P$. апnиа turf.

Characterization of new streptomycin resistant Erwinia amylovora strains in New York orchards

K. BEKOSCKE (1), S. Villani (1), K. Cox (1)

(1) Cornell University, Geneva, NY, U.S.A.

Phytopathology 104(Suppl. 3):S3.13

Resistance to streptomycin in Erwinia amylovora was first observed in the United States in the 1970's, but was not present in New York until 2002. Currently, the presence of streptomycin resistant (SmR) E. amylovora in New York apple orchards is largely unknown. The objective of this research is to understand the prevalence and characterize SmR isolates of E. amylovora present in New York apple orchards. Isolates of E. amylovora were collected from 43 and 32 of New York apple orchards, in $2012(n=175)$ and $2013(n=$ 320 ) respectively, and were assessed for resistance to streptomycin. Twentyfour isolates from 16 orchards were found to have streptomycin resistance. PCR and Sanger sequencing based screening was used to characterize the genetic basis of streptomycin resistance. Strain tracking of SmR resistant isolates using PCR based CRISPR sequencing technology is currently underway and will be discussed.

Plant pathology based framework for novel high school student outreach program

A. BERNERT (1), L. Santamaria (2), A. Schauer (3)

(1) Oregon State University, Corvallis, OR, U.S.A.; (2) Oregon State University, Aurora, OR, U.S.A.; (3) CREST Program Coordinator West Linn Wilsonville School District, Wilsonville, OR, U.S.A.

Phytopathology 104(Suppl. 3):S3.13

In the West Linn-Wilsonville school district, there is limited agricultural education at the high school level. Many students graduate with a poor understanding of agriculture and the career opportunities available in this field. Oregon State University -NWREC developed a high school internship program using plant pathology as a vector for agriculture education and scientific research training. A novel outreach program was created to fill this agriculture education gap while also providing valuable skills training in scientific research. The program was organized in three separate phases. Phase 1 consisted of hands on training, field trips, safety training, and journal reading exercises. Phase 2 allowed students to apply their new skills in guided research projects. Phase 3 shifted to developing an independent project by requiring students to write a project proposal, conduct experimentation, and present their findings at the local science fair competition. Students significantly improved their knowledge in plant pathology, agriculture, and laboratory techniques based on preliminary and post written assessment scores $(p<0.01)$. This is an effective, plant pathology based program framework that can be adopted by other plant pathology programs to increase student interest and education in agriculture and science. Exposure of these themes at the high school level is an ideal method to gain new, talented generations in the advancement of this vital field.

Characterization of plant pathogenic and non-pathogenic strains of Serratia marcescens using rep-PCR and multilocus sequence typing

K. BESLER (1), E. Little (1)

(1) University of Georgia, Athens, GA, U.S.A.

Phytopathology 104(Suppl. 3):S3.13

Cucurbit yellow vine disease (CYVD) caused by the squash bug (Anasa tristis) transmitted bacterium Serratia marcescens was first observed in Oklahoma and Texas in 1988. In 2012, CYVD was confirmed in Georgia where it causes significant losses in organic squash production. S. marcescens is a highly diverse species found in many ecological niches. Genetic diversity of CYVD strains and non-CYVD strains of $S$. marcescens was compared using repetitive extragenic palindromic PCR (rep-PCR) and multilocus sequence typing (MLST). For the rep-PCR, the DNA of each strain was subjected to PCR using the BOX-A1R primers. Products were resolved on an agarose gel, bands were scored using BioNumerics software, and a phylogenetic tree was generated. Results indicated that CYVD strains formed a cluster separate from non-CYVD strains, although there was intra-species variability. In order to better understand this genetic variation, MLST was performed on the DNA from the same strains. PCR primers were designed to amplify DNA fragments from seven housekeeping genes. PCR products of these gene fragments were purified and sequenced. Sequences were aligned and an allelic profile was determined for each strain. An eBURST analysis was performed and a phylogenetic tree was generated. Results of this study identify similarities between the two methods in determining genetic relatedness and in inferring the evolutionary origins of CYVD strains.

Infection and colonisation of pyrethrum leaves by Stagonosporopsis tanaceti

M. A. H. BHUIYAN (1), T. Groom (2), P. W. J. Taylor (1)

(1) The University of Melbourne, Melbourne, Australia; (2) Botanical Resources Australia Pty Ltd, Tasmania, Australia

Phytopathology 104(Suppl. 3):S3.13

Pyrethrum (Tanacetum cinerariifolium) plants are used to produce biopesticide pyrethrins commercially in Australia. Ray blight caused by Stagonosporopsis tanaceti is a major disease of pyrethrum. Successful implementation of disease control methods depends on knowledge of the infection process by the pathogen. Histopathology is an important tool in studying host-pathogen relationships especially with biological stains that differentiate the pathogen from host cell tissues. The first fully expanded leaves were inoculated with a spore suspension of $S$. tanaceti and incubated in high humidity to induce spore germination and infection. Leaf sections were sampled, fixed in FAA, embedded with paraffin and sectioned $(6 \mu \mathrm{m})$ using a rotary microtome. Sections were then stained with the modified Johansen's quadruple stain technique. Adhesion of conidia to the leaf surface was followed by direct penetration of the cuticle by germ tubes from $3-12$ hours after inoculation. Infection hyphae penetrated the epidermis within $1-2$ days after inoculation (dai), and mesophyll tissues within 3-7 dai. Secondary infection of adjacent mesophyll cells then occurred leading to disintegration of the colonized cells. Pycnidia formation, maturation and spore release occurred between 7-9 dai in the cells of both the epidermal and mesophyll tissues. Although hyphae were detected in the leaf petioles no vascular tissue was colonized.

Transformation of Fusarium secorum, a novel pathogen causing Fusarium yellowing decline of sugar beet

Z. BIAN (1), G. Secor (1), M. D. Bolton (2)

(1) North Dakota State University, Fargo, ND, U.S.A.; (2) USDA-ARS, Fargo, ND, U.S.A.

Phytopathology 104(Suppl. 3):S3.13

Sugar beet is an important source of sucrose for human consumption throughout the world. The sugar beet growing areas of ND and MN constitute the largest sugar beet production area in the United States. A new disease of sugar beet was recently found in in the Red River Valley of MN and ND. This 
disease can cause discoloration of petiole vascular, seedling infection and rapid death of plants early in the season and has been named Fusarium yellowing decline. We have shown previously that Fusarium yellowing decline is caused by a novel pathogen named Fusarium secorum, a member of the Gibberella fujikuroi species complex (GFSC). To study pathogenesis of $F$. secorum, we developed a transformation protocol using polyethylene glycol (PEG)-mediated transformation. High-quality protoplasts $\left(10^{8} \mathrm{ml}^{-1}\right)$ were incubated with $20 \mu \mathrm{g}$ of linearized plasmid DNA containing the hygromycin phosphotransferase (hpt) gene. Putative transformants grew on selective media containing $75 \mu \mathrm{g} \mathrm{ml}^{-1}$ hygromycin, which was previously shown to be a concentration that inhibited growth of wild-type $F$. secorum. PCR and Southern blotting will be used to confirm integration and copy number of $h p t$ in putative transformants. Fusarium yellowing decline has been increasing in incidence since first identified in 2006. A transformation system will be a valuable tool to characterize putative effectors and gain insight into the pathology of this novel and important sugar beet pathogen.

Development and evaluation of molecular methods for species-specific detection of Phytophthora tentaculata

J. C. BIENAPFL (1), Z. G. Abad (1), M. K. Nakhla (1)

(1) USDA-APHIS-PPQ-S\&T, Beltsville, MD, U.S.A.

Phytopathology 104(Suppl. 3):S3.14

Phytophthora spp. cause major losses in agricultural crops, including nurseries, and devastate natural ecosystems worldwide. In 2009, CPHSTPERAL generated a list of 29 Phytophthora spp. exotic to the USA that are of concern due to the potential damage they can cause if introduced. Phytophthora tentaculata, ranked $\# 5$ on this list, has been reported as a root and stalk rot pathogen of chrysanthemum, larkspur, and verbena in Germany and the Netherlands; verbena, lavender, and cotton lavender in Spain; chicory, oregano, and African daisy in Italy; and Aucklandia and celery in China. $P$. tentaculata was first detected in California by CDFA in 2012 from nurserygrown sticky monkeyflower with root rot that was later confirmed by our lab. Four subsequent confirmations have occurred since that time. To enhance efforts in pest detection of Phytophthora spp. of concern, we designed a species-specific conventional PCR assay that targets the beta-tubulin region and a real-time PCR assay that targets the ITS region. Genomic DNA extracted from the ex-type of $P$. tentaculata, as well as from other Phytophthora spp. in clade 1 , has been used to test the specificity of both assays. Preliminary results indicate both assays are specific for $P$. tentaculata. Additional tests are underway using DNA from other Phytophthora spp. commonly reported from agricultural crops and forests in the USA to further test the specificity of the assays, and results for assay validation will be presented.

Prevalence and characterization of culturable acetoin-producing rhizobacteria in organic no-till vegetable production

E. BIETILA (1), A. Charkowski (1), E. Silva (1)

(1) UW Madison, Madison, WI, U.S.A.

Phytopathology 104(Suppl. 3):S3.14

Farming practices which conserve soil biodiversity can promote rhizobacterial activity beneficial to crop plants. Acetoin, a volatile organic compound produced by rhizobacteria is implicated in growth promotion and induced systemic response in plants, perhaps through inhibition of the ethylene pathway. Genes required for this pathway also contribute to pathogenesis of soft rot enterobacteria. Due to its benefits in plant growth and involvement in plant disease in laboratory assays, it is important to assess the relative abundance of native organisms producing this substance in agricultural soils. Cultures were sampled from rhizosphere soil, the rhizoplane, and endophytes of bean and peppers grown in no-till rye and cultivated bare ground. Bacteria producing acetoin were common in both field treatments, with acetoin detected in $18 \%$ of cultured bacteria using the Voges-Proskauer test. A higher percentage of rhizosphere isolates produced acetoin $(27 \%)$ than bulk soil isolates $(14 \%)$. Both gram-negative and positive isolates were found to produce acetoin and of the 176 acetoin-producing isolates, 111 exhibited protease activity on $1 \%$ milk agar plates, 56 exhibited pectate-lyase activity on PGA plates. The abundance of native acetoin-producing rhizobacteria in bulk field soil and in rhizosphere samples suggests that this volatile compound is playing an important role for multiple bacterial species during plant-microbe interactions under field conditions.

Biosynthesis of the virulence-associated COR-like metabolites in the potato scab pathogen Streptomyces scabies

D. R. BIGNELL (1), Y. Li (1), M. S. Altowairish (1)

(1) Memorial University, St. John's, NF, Canada

Phytopathology 104(Suppl. 3):S3.14

Streptomyces scabies is the best characterized and most widely distributed bacterial species that causes potato scab disease. Among the virulence factors that are produced by this organism are the COR-like metabolites, which resemble the coronatine (COR) phytotoxin produced by several pathovars of the plant pathogenic bacterium Pseudomonas syringae. Although the $S$. scabies COR-like metabolites are composed of the same coronafacic acid (CFA) polyketide moiety as in COR, the biosynthetic gene cluster for producing CFA differs in the two pathogens in that the $S$. scabies gene cluster contains four additional genes that are predicted to encode biosynthetic enzymes. The objective of this study was to determine whether these novel genes participate in the biosynthesis of the COR-like metabolites in S. scabies. Gene deletion mutants were constructed for each of the four genes in $S$. scabies $87-22$, and the effect of each deletion on metabolite production was assessed using HPLC and plant bioassays. The results of our study show that three of the four genes are essential for normal production of the $S$. scabies COR-like metabolites and suggest that the biosynthesis of CFA in $S$. scabies may be distinct from that in P. syringae.

Reaction of half sib families of Medicago sativa to three races of Aphanomyces euteiches

A. BILGRI (1), V. Kartanos (1), D. Rouse (1)

(1) UW-Madison, Madison, WI, U.S.A.

Phytopathology 104(Suppl. 3):S3.14

Aphanomyces euteiches Drechs. was recognized as a major problem of alfalfa in the 1980s. Varieties of alfalfa initially found to be resistant to A. euteiches became susceptible during the 1990s. New resistant varieties were subsequently deployed. At that time two races of the pathogen were recognized on alfalfa. In 2010 and 2011 isolates of A. euteiches were obtained from field soils where race 2 resistant alfalfa had expressed severe symptoms of root rot. These isolates were tested against multiple varieties with high levels of race 2 resistance in a standard seedling assay. In most cases severe root rot was observed. It was concluded that these isolates were representatives of race 3. 'WAPH-5' is a germplasm line that has resistance to all three races. In order to understand the genetic basis for resistance, 93 WAPH-5 half-sibling families were generated. The families were screened against isolates from each of the three known races in a standard seedling assay using a mycelial suspension. Of eight possible combinations of resistant or susceptible reactions for each of three races, five combinations were found. These included families that were resistant to race 3 but not race 2 and vice versa. The differential interaction between half sib families and isolates of $A$. euteiches supports the designation of recently obtained isolates as race 3 . The genetic basis of this resistance is being investigated.

Optimization of nucleic acid extraction from field and bulk samples for sensitive direct detection of plant pests

G. J. BILODEAU (1), G. P. Robideau (1)

(1) Canadian Food Inspection Agency, Ottawa, ON, Canada

Phytopathology 104(Suppl. 3):S3.14

The quality/yield of a DNA extraction is the key in direct detection of quarantine and invasive species in field and bulk samples. We evaluated different nucleic acid extraction methods and optimized them for sensitive direct detection in order to obtain the best DNA and to understand the limit of detection for metagenomic analysis. Real-time PCR was employed as a tool for detection and quantification of target organisms to determine the best extraction method. Internal controls and specific qPCR assays targeting Phytophthora (Oomycetes), Verticillium (Fungi) and aphids (Insecta) were used to determine PCR inhibition (quality) and DNA yield. Various soil and water samples were collected and spiked with the target organisms to evaluate the best DNA extraction procedure. As many as six DNA extraction methods were tested, as well as magnetic bead purification and chemical flocculation to remove PCR inhibitors. Our results clearly indicate that the methods giving the highest DNA concentration do not always provide the best quality of DNA, and the ones with the best quality do not provide the highest yield. Removal of PCR inhibitors such as humic acids usually requires addition of a chemical flocculant during the extraction, or a post-extraction purification step such as magnetic bead-based purification. In addition to traditional DNA extraction methods, microfluidics devices have been developed and evaluated as a potential new tool.

Photosynthetic performance and carbohydrate metabolism of mango cultivars with different levels of resistance to Ceratocystis fimbriata infection W. M. S. BISPO (1), L. Araujo (1), R. T. Avila (1), F. M. DaMatta (1), F. A. Rodrigues (1)

(1) Universidade Federal de Vicosa, Vicosa, Brazil

Phytopathology 104(Suppl. 3):S3.14

The mango wilt, caused by Ceratocystis fimbriata, is one of the most problematic diseases affecting mango production worldwide and little information is available about how this disease affect mango physiology. The 
relationships between disease severity, gas exchange and chlorophyll $a$ fluorescence parameters, carbohydrate concentrations and the activity of carbon metabolism enzymes were evaluated over 21 days on plants from resistant (Ubá) and susceptible (Palmer) cultivars. The greater disease severity for plants from cv. Palmer contributed to the reduced photosynthetic performance and changes at photochemical level, in addition to decreased leaf concentration of starch and increases in hexoses concentration as disease progressed. The alterations in carbohydrates concentrations were in accordance with the reduced activity of ADP-glucose pyrophosphorylase and increased activity of invertases. There was no alterations in carbohydrate concentrations and enzyme activity on leaves of plants from cv. Ubá, but reduced starch concentrations were found in stem tissues of these plants, suggesting an increased remobilization of reserves for the production of defense compounds at the infection sites. The greater ability of plants from cv. Ubá to act locally at the infection sites appears to be crucial for their higher resistance, favoring, therefore, the postponement of the xylem colonization and the onset of disease symptoms.

Effect of cultivar and fungicide on Fusarium mycotoxins in wheat straw K. M. BISSONNETTE (1), K. A. Ames (1), Y. Dong (2), F. L. Kolb (1), C. A. Bradley (1)

(1) University of Illinois, Urbana, IL, U.S.A.; (2) University of Minnesota, St. Paul, MN, U.S.A.

Phytopathology 104(Suppl. 3):S3.15

Management of Fusarium graminearum-associated mycotoxins in wheat grain has been evaluated extensively, but little is known about management of mycotoxins in straw tissue. From 2011 to 2013, research trials were conducted in Illinois to determine fungicide or cultivar effects on $F$. graminearumassociated mycotoxins in wheat straw. The first trial tested the effect of fungicides at 3 locations. Mycotoxin concentration ranges for DON, 3ADON, 15ADON, NIV, and ZEA were 0.2-154.5, 0-10.1, 0-29.8, 0-5.2, and 0-2.5 ppm, respectively, across all locations and years. No fungicides decreased mycotoxin levels compared to the non-treated control. The second trial compared a susceptible versus a moderately resistant cultivar with or without a fungicide at 3 locations. Ranges of DON, 3ADON, 15ADON, NIV, and ZEA in these trials were $0-45.3,0-3.3,0-15.7,0-2.3$, and $0-5.5 \mathrm{ppm}$, respectively, across all locations and years. In 2011 and 2013, 15ADON was significantly higher in straw from the untreated, susceptible cultivar compared to all other treatments, but no other significant differences were observed. The final trial was a cultivar evaluation trial conducted at one location. In this trial, ranges of DON, 3ADON, 15ADON, NIV, and ZEA were 0.1-135.2, 0-20.0, 0$25.5,0-1.9$, and $0-1.0 \mathrm{ppm}$, respectively across all years. Management practices typically used to manage Fusarium mycotoxins in wheat grain generally were not effective in managing mycotoxins in straw.

\section{Microsclerotia production by the mycoparasitic fungus, Coniotyrium} minitans, in a shaken liquid culture

N. BITSADZE (1), J. A. Strauss (2), H. R. Dillard (3)

(1) Agricultural University of Georgia, Athens, GA, U.S.A.; (2) Cornell University, Geneva, NY, U.S.A.; (3) University of California, Davis, CA, U.S.A. Phytopathology 104(Suppl. 3):S3.15

Coniotyrium minitans is a well-known biocontrol agent of the sclerotia of Sclerotinia sclerotiorum, a fungal pathogen with worldwide distribution. $C$. minitans is available commercially in a conidia-based formulation. However, the viability of thin-walled spores and hyphal units can decline under certain environmental conditions. It was discovered that $C$. minitans is able to produce melanised, compact hyphal-aggregates of microsclerotia in a shaken liquid culture of basal salts medium supplemented with trace metals and vitamins. Six liquid media were tested with various combinations of fructose and acid hydrolyzed casein. The best results for obtaining melanised microsclerotia were obtained with a carbon to nitrogen $(\mathrm{C}: \mathrm{N})$ ratio of $30: 1$ and $50: 1$. The production of a microsclerotia based formulation is a significant technological advancement because it would contain a biocontrol agent with persistent infective propagules that are capable of surviving for long periods of time in diverse environmental conditions and are capable of producing an abundance of conidia.

\section{Managing black shank of tobacco with a novel fungicide in on-farm studies} R. BITTNER (1), A. Mila (1)

(1) North Carolina State University, Raleigh, NC, U.S.A.

Phytopathology 104(Suppl. 3):S3.15

Black shank, caused by Phytophthora nicotianae, is one of the most destructive soilborne diseases of tobacco (Nicotiana tabacum L.) in North Carolina (NC). Chemical control can be an effective management tool when combined with other practices. Currently, only mefenoxam (Ridomil Gold SL) is available to tobacco growers for black shank management.
Oxathiapiprolin is a new fungicide with a novel mode of action that has shown efficacious activity against oomycetes. The efficacy of oxathiapiprolin on black shank incidence was investigated in studies conducted in 2012 and 2013 in three naturally infested fields in NC. Oxathiapiprolin treatments were applied to the soil on the day of transplanting tobacco to the field (transplant), 2-4 weeks after transplant (first cultivation), and 7-9 weeks after transplant (layby). Treatments with three applications of oxathiapiprolin at a rate of 0.07 $\mathrm{kg}$ a.i./ha provided control ranging from 55 to $83 \%$ when compared to the untreated controls, whereas three applications of mefenoxam at $0.56 \mathrm{~kg}$ a.i./ha provided control ranging only from 3 to $55 \%$. The efficacy of rotating applications of oxathiapiprolin with mefenoxam at transplant, first cultivation, and layby was also examined. In general, when mefenoxam and oxathiapiprolin were rotated, disease incidence was lower than the untreated control. These field studies demonstrate that oxathiapiprolin is efficacious for the control of the black shank disease.

In vitro studies of a novel fungicide against the black shank pathogen, Phytophthora nicotianae

R. BITTNER (1), A. Mila (1)

(1) North Carolina State University, Raleigh, NC, U.S.A.

Phytopathology 104(Suppl. 3):S3.15

Oxathiapiprolin is a new fungicide with a novel mode of action that has been shown to be efficacious against oomycetes. In vitro assays were conducted with four isolates of Phytophthora nicotianae to examine the effect of oxathiapiprolin on mycelial growth, sporangia production, zoospore motility, and zoospore germination. At each life cycle stage, all isolates were highly sensitive to low concentrations of oxathiapiprolin. $\mathrm{EC}_{50}$ values ranged from 0.0039 to $0.0049 \mu \mathrm{g}$ a.i. $/ \mathrm{ml}$ for mycelial growth, 0.00052 to $0.00081 \mu \mathrm{g}$ a.i. $/ \mathrm{ml}$ for sporangia productions, 0.0035 to $0.0051 \mu \mathrm{g}$ a.i. $/ \mathrm{ml}$ for encysted zoospore germination, and 0.0055 to $0.0166 \mu \mathrm{g}$ a.i. $/ \mathrm{ml}$ for zoospore motility. In addition, 60 tobacco isolates of $P$. nicotianae, along with an ornamental isolate resistant to mefenoxam at $100 \mu \mathrm{g}$ a.i. $/ \mathrm{ml}$, were examined for sensitivity to oxathiapiprolin at $1 \mu \mathrm{g}$ a.i. $/ \mathrm{ml}$. Mycelial growth was only observed in one isolate originating from tobacco. These results suggest that oxathiapiprolin is a highly efficacious fungicide against $P$. nicotianae, the cause of black shank disease of tobacco.

How many standard area diagram sets are needed for accurate disease severity assessment?

C. H. BOCK (1), M. W. Hotchkiss (1), B. W. Wood (1)

(1) USDA-ARS-SEFTNRL, Byron, GA, U.S.A.

Phytopathology 104(Suppl. 3):S3.15

Standard area diagram sets (SADs) are widely used in plant pathology: a rater estimates disease severity by comparing an unknown sample to actual severities in the SADs and interpolates an estimate as accurately as possible (although some SADs have been developed for categorizing disease too). Most SADs have 3 to 10 images. We compared SADs with 3, 5, 7 and 10 images of known pecan scab severity on valves of pecan fruit; 12 raters used these to 1) estimate disease by interpolation, and 2) apply the image disease value to the sample as if it were a category. Raters also assessed the disease without any SADs. Agreement (Lin's Concordance Correlation Coefficient, $\left.\rho_{c}\right)$ increased for raters using $3,5,7$ or 10 SADs $\left(\rho_{c}=0.895\right.$ to 0.933$)$ compared to estimates without SADs $\left(\rho_{c}=0.868\right)$ when interpolating an estimate. When based on categories, the estimates using 3,5 or 7 SADs $\left(\rho_{c}=\right.$ 0.554 to 0.838 ) had less agreement to actual values compared to using the 10 SADs $\left(\rho_{c}=0.916\right)$, or when not using SADs $\left(\rho_{c}=0.868\right)$. Inter-rater reliability followed a similar pattern. Specific disease severity ranges over which SADs are chosen in relation to the range of severity in a sample might affect agreement. These results suggest as few as 3 SADs improve a rater's ability to estimate disease by interpolation, and add to the evidence that category scales do not provide an accurate basis for estimating disease compared with a rater estimating to the nearest percent severity.

A comparison of raters and disease assessment methods for estimating disease severity for purposes of hypothesis testing

C. H. BOCK (1), M. El Jarroudi (2), L. A. Kouadio (3), C. Mackels (2), K.-S. Chiang (4), P. Delfosse (5)

(1) USDA-ARS-SEFTNRL, Byron, GA, U.S.A.; (2) Université de Liège, Department of Environmental Sciences and Management, Arlon, Belgium; (3) Agriculture and Agri-Food Canada Lethbridge Research Centre, Lethbridge, AB, Canada; (4) Division of Biometrics, Department of Agronomy, National Chung Hsing University, Taichung, Taiwan; (5) Centre de Recherche PublicGabriel Lippmann, Environment and Agro-biotechnologies Department, Belvaux, Luxembourg

Phytopathology 104(Suppl. 3):S3.15 
Assessment of disease severity is most often made visually, and estimates can be inaccurate. Nearest percent estimates (NPEs) of Septoria leaf blotch on leaves of winter wheat by four raters (R1-R4) assessing non-treated (NT) and fungicide-treated (FT) plots were compared to true values using Lin's concordance correlation coefficient $\left(\rho_{c}\right)$ on two dates in 2006 and 2007. Estimates were converted to Horsfall-Barratt (HB) mid-points and again compared for accuracy and precision. Estimates of severity from FT and NT plots were analyzed to ascertain effects of rater using both the NPE and HB values. Regardless of method, all raters showed a range of agreement with true values on FT and NT plots $\left(\rho_{c}=0\right.$ to 1$)$. Use of the HB scale most often reduced agreement ( $84.4 \%$ of the time), and did not improve rater-associated bias of treatment mean severity estimates. Consequently, estimates of mean severity differed significantly among raters and from true values $(F=126$ to $1260, \mathrm{P}=0.002$ to $<0.0001)$. However, a comparison of treatment effects showed that the true values and R1 to R4 estimates all demonstrated significant effects of fungicide ( $\mathrm{F}=101$ to $1952, \mathrm{P}=0.002$ to $<0.00001$ ). Ranking of raters differed on one occasion when HB values were used. These results demonstrate the effect of the HB scale, and the need for accurate disease assessment to minimize over or underestimates compared to true severity so as to minimize the potential for type II errors.

Characteristics of genetic variability among isolates of Fusicladium species from peach, almond and pecan in the USA

C. H. BOCK (1), C. Chen (1), P. M. Brannen (2), J. E. Adaskaveg (3), M. W. Hotchkiss (1), M. Brewer (2), B. W. Wood (1)

(1) USDA-ARS-SEFTNRL, Byron, GA, U.S.A.; (2) University of Georgia, Athens, GA, U.S.A.; (3) Department of Plant Pathology and Microbiology, University of California, Riverside, Riverside, CA, U.S.A.

Phytopathology 104(Suppl. 3):S3.16

Peach and almond scab, caused by Fusicladium carpophilum, and pecan scab, caused by $F$. effusum are serious diseases of their host crops, causing yield loss and downgrading of fruit. Genetic diversity of these pathogens has not previously been compared, but helps characterize pathogen variability and can aid with resistance breeding. The aim of this study was to use UP-PCR and RAPDs to compare variability within and among isolates from different hosts. A total of 18 isolates of $F$. carpophilum from peach (from the southeastern US), 12 from almond (from California), and 21 isolates of $F$. effusum (from the southeastern US) were screened against 9 RAPD and 5 UP-PCR markers. Combined marker results showed a low incidence of polymorphism among peach isolates $(4.2 \%$ of markers), but a higher incidence of polymorphism among isolates from almond (42.0\%) and isolates of $F$. effusum from pecan $(61.0 \%)$. The Dice coefficient of similarity ranged from 0.9323 to 1.0000 for isolates of $F$. carpophilum from peach, 0.2143 to 0.9756 for almond isolates, and 0.5283 to 0.9200 for $F$. effusum. UPGMA bootstrap analysis (1000 runs) gave a high node value $(100 \%)$ differentiating all isolates of $F$. carpophilum from $F$. effusum, and a moderate node value $(68 \%)$ differentiating peach and almond isolates of $F$. carpophilum. The results suggest some divergence among isolates of $F$. carpophilum from almond in California and peach in the southeastern US.

\section{Populations dynamics of Xanthomonas citri pv citri in lesions on cankered} grapefruit, leaves and twigs

C. H. BOCK (1), A. B. Kriss (2), T. R. Gottwald (2), J. H. Graham (3), G. H. Poole (2)

(1) USDA-ARS-SEFTNRL, Byron, GA, U.S.A.; (2) USDA, ARS, US Horticultural Research Laboratory, Fort Pierce, FL, U.S.A.; (3) University of Florida, Citrus Research and Education Center, Lake Alfred, FL, U.S.A. Phytopathology 104(Suppl. 3):S3.16

Quarantine restrictions on movement of fresh fruit from areas with endemic Asiatic citrus canker (caused by Xanthomonas citri subsp. citri (Xcc)) is a major trade issue for commercial citrus producers within the USA and internationally. Two studies were conducted to evaluate initial assertions that viable Xcc populations in fruit lesions declined to near zero with time after harvest and that populations in symptomatic fruit exposed to packingline treatments may have further reductions. First, monthly assessments of bacterial populations in lesions on fruit, leaves, and shoots were conducted from June to January during the 2009/10 and 2010/11 growing seasons. The resulting data were fit with a two-component (generalized gamma-binomial) mixed effects model as data were highly skewed and zeroinflated (non-active lesions). Results confirm that active lesions on fruit, as opposed to leaves and shoots, declined over the study period. Second, reduction in Xcc populations from packingline processed versus nonprocessed fruit was investigated weekly in two studies (13 Nov $2013-11$ Dec 2013 and 29 Jan 2014 - 26 Feb 2014). In both studies, the proportion of packingline processed fruit with active lesions and the population in active lesions decreased over time. However, there was not a consistent significant difference in either the proportion of active lesions or the populations of Xcc between fruit processed through the packingline versus non-processed fruit.

Suppression of pecan and peach pathogens using metabolites or broths from symbiotic bacteria obtained from the guts of entomopathogenic nematodes

C. H. BOCK (1), D. I. Shapiro-Ilan (1), M. W. Hotchkiss (1)

(1) USDA-ARS, Southeastern Fruit and Tree Nut Research Laboratory, Byron, GA, U.S.A.

Phytopathology 104(Suppl. 3):S3.16

Concentrated metabolites from the bacteria Xenorhabdus spp. and Photorhabdus spp. have previously been shown to suppress growth of peach and pecan pathogens in vitro, and reduce disease on detached leaves or terminals. The objectives of this study were 1) determine if bacterial broths (in addition to the metabolites) have suppressive ability, and 2) determine if metabolites or bacterial broths are active against pathogens in a soil medium. The pecan pathogens Fusicladium effusum and Phytophthora cactorum, and the peach pathogen Armillaria tabescens were tested for sensitivity to bacterial broths or concentrated metabolites. Treatments were applied to lesions of $F$. effusum on pecan terminals to ascertain effects on sporulation, to A. tabescens in soil to determine survival of mycelia, and pecan leaves inoculated with $P$. cactorum to ascertain effects on lesion development. The Xenorhabdus bovienii metabolite was as efficacious as the fungicide febuconazole in reducing sporulation of $F$. effusum. The Photorhabdus luminescens metabolite treatment reduced sporulation, but the bacterial broths had no effect. All bacterial broth and metabolite treatments suppressed lesions of $P$. cactorum. Only the $P$. luminescens metabolite suppressed $A$. tabescens. The results provide a basis for further research on the use of Xenorhabdus and Photorhabdus metabolites or bacterial broth for suppression of pecan and peach diseases.

Comparative genomics of Ralstonia solanacearum strain P781 that infects Mandevilla and Dipladenia plants

A. M. BOCSANCZY (1), J. M. F. Yuen (1), A. J. Palmateer (2), D. J. Norman (1)

(1) University of Florida Mid-Florida Research and Education Center, Apopka, FL, U.S.A.; (2) University of Florida Tropical research and Education Center, Homestead, FL, U.S.A.

Phytopathology 104(Suppl. 3):S3.16

Ralstonia solanacearum causes Bacterial Wilt in more than 200 species of plants. Recently, we isolated $R$. solanacearum strains from production of Mandevilla and Dipladenia in Florida. Many of the cultivars infected are now crosses of both genera, referred as 'Sundavilla'. We confirmed the strains as biovar 3. A limited host range test was performed on tomato, geranium, Dipladenia sanderi, and Mandevilla boliviensis with biovar 3 control strain GMI1000 along with three isolated strains. GMI1000 was pathogenic and highly virulent on tomato plants, while weakly virulent on the other hosts. The other strains were highly virulent on all hosts. Host specificity of bacterial pathogens has been linked to type III effectors. We seek to identify type III effectors that may restrict the host range of GMI1000 to solanaceous plants. The genome of strain P781 selected from the group of three strains was sequenced using the Illumina paired-end technology. More than 9 million reads were assembled in 262 contigs. Preliminary genome annotation and assembly in gapped molecules was done. Phylogenetic closeness to GMI1000 was confirmed by comparison of the endoglucanase gene sequence with $R$. solanacearum genome sequences available. Currently, the genome sequence is in process of manual curation and identification of type III effectors. Comparative genomics of the annotated sequence of P781 with GMI1000 genome is expected to reveal potential host specificity factors.

QoI-insensitive Powdery Mildew Erysiphe polygoni on sugar beet impact, challenges and control

M. D. Bolton (1), O. T. NEHER (2)

(1) USDA-ARS, Northern Crop Science Laboratory, Fargo, ND, U.S.A.; (2) The Amalgamated Sugar Company LLC, Boise, ID, U.S.A.

Phytopathology 104(Suppl. 3):S3.16

Quinone outside inhibitor $\left(\mathrm{Q}_{0} \mathrm{I}\right)$ fungicides pyraclostrobin and trifloxystrobin have been important tools to manage powdery mildew since registration in 2002 and 2005, respectively. In 2011-2013, researcher in Idaho reported poor disease control despite $\mathrm{Q}_{\mathrm{O}} \mathrm{I}$ applications. The lack of significant disease reduction resulted in experiments that were conducted to clone a partial fragment of the E. polygoni cytochrome $b$ (cytb) gene to gain insight into the 
molecular basis of $\mathrm{Q}_{0} \mathrm{I}$ resistance. PCR products derived from six $\mathrm{Q}_{\mathrm{o}} \mathrm{I}$-treated samples and six untreated samples were sequenced directly. Without exception, all $\mathrm{Q}_{\mathrm{O}} \mathrm{I}$-treated samples harbored a point mutation that encoded a G143A mutation compared with $c y t b$ sequence from untreated samples. This is the first report of $\mathrm{Q}_{\mathrm{O}} \mathrm{I}$ resistance and the associated cytb G143A mutation in E. polygoni in the United States. The consistent loss of disease control associated with a G143A mutation despite $\mathrm{Q}_{\mathrm{O}} \mathrm{I}$ applications forces growers to implement integrated pest management (IPM) practices. The use of partially resistance varieties, scouting and timely fungicide applications, as well as the judicious use of $\mathrm{Q}_{\mathrm{O}} \mathrm{I}$ fungicides and monitoring for fungicide resistance will be necessary to ensure $\mathrm{Q}_{0} \mathrm{I}$ fungicides remain efficacious for powdery mildew control.

First detection of Phytophthora parasitica from Mediterranean fan palm in the United States

R. A. BOMBERGER (1), X. Duranovic (2), S. Wang (1)

(1) Nevada Dept of Agric, Sparks, NV, U.S.A.; (2) Nevada Dept of Agriculture, Las Vegas, NV, U.S.A.

Phytopathology 104(Suppl. 3):S3.17

A lethal disease, causing leaf basal rot and wilting of Mediterranean fan palm (Chamaerops humilis), was first noticed in several nurseries in Nevada in November 2013. Infected young plants showed initial symptoms of dark brown lesions on the leaf base and then complete rot of internal basal leaves. To isolate Phytophthora, fresh plant tissue was placed onto PARP medium and incubated at $22^{\circ} \mathrm{C}$ in the dark. Phytophthora was isolated and transferred to corn meal agar and V8 juice agar for further morphological identifications. On corn meal agar, the isolate produces abundant sporangia with dimension of 31.4 to $68.7 \mu \mathrm{m}$ long $\times 29.5$ to $49.1 \mu \mathrm{m}$ wide. To further confirm the isolate identity, regions of rDNA including partial $18 \mathrm{~S}$ ribosomal RNA gene, ITS1, $5.8 \mathrm{~S}$ ribosomal RNA gene, ITS2, and partial 28S ribosomal RNA gene were amplified, sub-cloned into the pGem- $\mathrm{T}^{\circledR}$ vector, and then sequenced. A consensus DNA sequence of 892-bp PCR fragment (Accession No. KJ549640) was obtained by aligning 3 cloned sequences. BLASTn search and sequence alignments revealed $99 \%$ homology to the corresponding regions of P. parasitica strain TARI 24057 (Accession No. GU111669.1). The morphological observations and molecular data suggest that the isolate from Mediterranean fan palm belong to $P$. parasitica. $P$. parasitica was first reported from C. humilis in Italy and then in Iran. To our knowledge, this is the first detection of $P$. parasitica from Mediterranean fan palm in the United States.

Longevity of Uromyces transversalis urediniospores under various environmental conditions

M. R. BONDE (1), S. E. Nester (2), C. L. Palmer (3), J. M. Revell (4)

(1) USDA ARS, Frederick, MD, U.S.A.; (2) USDA-ARS, FDWSRU, Frederick, MD, U.S.A.; (3) IR-4, Rutgers University, Princeton, NJ, U.S.A.; (4) Hood College, Frederick, MD, U.S.A.

Phytopathology 104(Suppl. 3):S3.17

Uromyces transversalis (Thum.) Winter, causal agent of gladiolus rust, is a regulated quarantine-significant plant pathogen first discovered in the U.S. in April, 2006. Since this initial discovery in Florida, the disease has also been detected in California. In order to determine the likelihood of establishment of the pathogen in other areas of the country, we initiated a study to determine effects of environment on urediniospore longevity. In a set of three experiments, urediniospores were maintained 1) buried in damp soil (equal wt. soil and water) in the greenhouse $\left(22\right.$ to $\left.\left.26^{\circ} \mathrm{C}\right), 2\right)$ not buried and kept dry in the greenhouse, 3 ) buried in damp soil in a cold room $\left(5^{\circ} \mathrm{C}\right)$, and 4) not buried and kept dry in the cold room. At weekly intervals, urediniospores were recovered from each environment and tested for viability on $1.0 \%$ water agar. Results from the study showed that urediniospores remained viable for a maximum of 21 days in damp soil in the greenhouse. However, a few urediniospores remained viable up to 77 days when dry in the greenhouse, 70 days when dry in the cold room, and 63 days when buried in moist soil in the cold room. We concluded that under normal conditions, in which urediniospores must pass through a warm period in the presence of moisture, urediniospore longevity is less than 21 days. The effects of environment on other aspects of the disease are being evaluated.

Sequence analysis of the Beet necrotic yellow vein virus $\mathrm{P} 25$ pathogenicity factor in Turkey

K. BORNEMANN (1), N. D. Kutluk Yilmaz (2), M. F. Khan (3), M. D. Bolton (4)

(1) North Dakota State University, Fargo, ND, U.S.A.; (2) University of Ondokuz Mayis, Agriculture Faculty, Department of Plant Protection, Samsun, Turkey; (3) North Dakota State University and University of Minnesota, Fargo, ND, U.S.A.; (4) USDA-ARS, Fargo, ND, U.S.A.

Phytopathology 104(Suppl. 3):S3.17
Turkey is one of the largest beet sugar producing countries in the world. Rhizomania is a devastating disease of sugar beet caused by Beet necrotic yellow vein virus (BNYVV) and is widely present in Turkey. In the past, disease management was obtained by using hybrids with resistance genes $R z 1$ or Rz2. Recently, BNYVV strains with four RNA components were identified that are able to overcome $R z l$-mediated resistance. All $R z l$-resistant strains described so far possess an A67V amino acid exchange within the RNA3encoded P25 pathogenicity factor. To identify the extent that the A67A mutation or other mutations occur in P25, 638 soil samples were collected throughout the country and analyzed for the presence of the virus. Positive samples were taken for a resistance test with $R z 1, R z 2$, and $R z 1+R z 2$ resistant sugarbeet cultivars and virus titer was measured with ELISA. Samples were also analyzed for the composition of P25. One sample showed high virus titers over all treatments. P25 tetrad composition showed an ASHG tetrad in susceptible plants and ACHG in $R z 2$ and $R z 1+R z 2$ resistant plants. Nine samples were identified carrying an additional RNA component with the P26 pathogenicity factor and ACHG tetrad composition as well as high virus titers. P26 has so far been identified in France, England, and Kazakhstan, and has only been associated with the SYHG tetrad. However, sequencing results showed a high variability of the P25 tetrad with newly identified compositions.

A quantitative real-time PCR assay for detection of Ephelis japonicaon ornamental grasses

L. E. BOSTIC (1), D. M. Benson (1), K. Ivors (1)

(1) North Carolina State University, Raleigh, NC, U.S.A.

Phytopathology 104(Suppl. 3):S3.17

The fungus Ephelis japonica causes black choke disease on a wide range of warm and cool season ornamental grasses and grows epiphytically along tillers, leaves and flowers resulting in a black stroma encapsulating the flower and impacting plant aesthetics. Ornamental grasses are routinely propagated by plant division; the use of asymptomatic stock plants can result in rapid spread of the pathogen throughout a production facility. Before visible choke symptoms develop, the fungus is difficult to detect. In this study, a real-time quantitative PCR (qPCR) assay was developed to detect the pathogen in Miscanthus sinensis and Pennisetum alopecuroides grass cultivars prior to symptom development. The primer set LEB1 and LEB2 and fluorescent probe LEBOH were designed from the ITS $4 / 5 \mathrm{rDNA}$ region of E. japonica using the sequences of three reference isolates. This assay was validated with plant isolations. Specificity was confirmed using the DNA extracts of two fungi, Myriogenospora atramentosa and Magnaporthe grisea, commonly found on the same grass hosts. The assay was optimized with genomic DNA of $E$. japonica and was able to detect target DNA at concentrations as low as $100 \mathrm{fg}$. This method can be used to screen M. sinensis and P. alopecuroides stock plants for detection of early stages of black choke disease, saving time and money for ornamental grass producers.

Aflatoxin in corn: Does drought tolerance contribute to decreased contamination?

K. BOWEN (1), A. Hagan (1)

(1) Auburn University, Auburn, AL, U.S.A.

Phytopathology 104(Suppl. 3):S3.17

Extended periods without rain and high temperatures are associated with aflatoxin contamination of corn. These conditions often prevail during the corn growing season in Alabama. While no commercial hybrids are available with aflatoxin resistance, plant characteristics of available hybrids might contribute to minimization of the risk of aflatoxin contamination. Corn hybrids, with various drought tolerance ratings, relative maturity and $\mathrm{Bt}$ technology traits, but adapted to southeastern production, were grown at 3 to 5 locations in Alabama, from 2009 through 2012, and assayed for aflatoxin content. Aflatoxin contamination was problematic at 2 or more locations in 2010, 2011 and 2012. None of the plant characteristics (drought tolerance, relative maturity, $\mathrm{Bt}$ traits) consistently contributed to reduced aflatoxin contamination.

Bacterial antagonists as a biological solution for control of potato late blight disease

S. M. BOYETCHKO (1), P. Audy (2), H. S. Choo (1), R. Desgagnes (2), K. Sawchyn (1), K. L. Conn (3)

(1) Agriculture and Agri-Food Canada, Saskatoon, SK, Canada; (2) Agriculture and Agri-Food Canada, Quebec City, QC, Canada; (3) Agriculture and Agri-Food Canada, London, ON, Canada

Phytopathology 104(Suppl. 3):S3.17

Potato late blight caused by Phytophthora infestans (Mont.) de Bary is the world's single most economically important food crop disease, requiring multiple applications of chemical fungicides annually. Antagonistic bacteria 
show potential as biopesticides while mitigating chemical pesticide load in soil and water. Two isolates of $P$. infestans (US8 and US23) were studied to determine the impact of forty-five bacterial strains to inhibit/suppress the pathogen. Fifteen bacterial strains significantly inhibited the pathogen by $95 \%$. Twenty-three bacterial strains were further assessed for their ability to reduce the disease severity. Potato leaves freshly harvested and rooted in $10 \%$ Hoagland's solution were treated with either bacterial filtrate alone, whole bacterial culture (cells and filtrate) or autoclaved bacterial cultures $2 \mathrm{~h}$ prior to inoculation with the pathogen $\left(10^{4}\right.$ sprorangia $\left./ \mathrm{ml}\right)$. They were maintained at $18^{\circ} \mathrm{C}$ under a $16 \mathrm{~h}$ photoperiod, and sprayed daily with distilled water to mimic dew and rain for optimum disease development. Disease severity was expressed as \% leaf surface exhibiting disease symptoms after 7 and 10 days. At least 3-5 bacterial strains reduced disease severity by close to $100 \%$ when whole bacterial cultures were applied while application of filtrate alone decreased disease by $70-80 \%$. Promising bacterial strains belonging to several distinct species for biological control have now been selected as leading biopesticide candidates.

\section{Botryosphaeria spp. associated with dieback and stem canker of} blackberry in Michoacán, Mexico

J. Boyzo-Marin (1), A. REBOLLAR-ALVITER (2), H. V. Silva-Rojas (3), L. F. Ceja-Torres (4), B. Uribe-Cortés (3), R. Moncayo-Estrada (5)

(1) CIIDIR-IPN, Jiquilpan, México, Morelia, Mich, Mexico; (2) Centro Regional Morelia, Univ Autonoma Chapingo, Morelia, Mich, Mexico; (3) Laboratorio de Biotecnología de Semillas, Colegio de Postgraduados, Montecillo, Texcoco, México, Mexico; (4) CIIDIR-IPN, Jiquilpan, México, Jiquilpan, Michoacan, Mexico; (5) CIIDIR-IPN, Jiquilpan, Michoacan, Mexico

Phytopathology 104(Suppl. 3):S3.18

Dieback and stem cankers of blackberry (Rubus sp.) are becoming common in Mexico. The objectives were to identify the causal agents of stem cankers and dieback of blackberry and to know their pathogenicity. Plants from 38 commercial plots distributed in different regions of Michoacan where surveyed. Symptomatic tissue was collected and cultured on PDA media. From these isolations, 123 isolates were obtained and characterized based on conidia and colony morphology. ITS region of rDNA, $\beta$-tubulin and elongation factor EF1- $\alpha$ were amplified by PCR using primers ITS5/ITS4, $\mathrm{Bt} 1 \mathrm{a} / \mathrm{Bt} 1 \mathrm{~b}$ and EF1f/EF1r, respectively. PCR-products were sequenced and compared with those deposited in Genbank. Fungal identification was based on the maximum identity with published sequences. The pathogenicity tests were conducted on 6-month blackberry plants cv tupi. Results indicated that isolates obtained belonged to Lasiodiplodia pseudo-theobromae (24.1\%), Lasiodiplodia sp. (10.2\%), Lasiodiplodia sp. (7.4\%), L. theobromae (7.4\%), L. hormozganensis (2.8\%), L. parva (1.9\%), Neofusicoccum parvum (43.5\%), N. kwanbonambiense $(0.9 \%), \quad N$. ribis $(0.9 \%)$ and Pseudofusicoccum stromaticum $(0.9 \%)$. Different fungal species induced similar symptoms on blackberry plants, but there were differences in pathogenicity. N. parvum and L. pseudo-theobromae were frequently associated with dieback and stem cankers on blackberries in Michoacan.

Development and validation of standard area diagrams as assessment aids for estimating the severity of citrus canker on unripe oranges

R. Braido (1), A. M. O. Gonçalves-Zuliani (2), S. A. Carvalho (3), J. Belasque (4), C. H. Bock (5), W. M. NUNES (6)

(1) AMEA, Maringa, Brazil; (2) Universidade Estadual de Maringa, Maringa, Brazil; (3) Instituto Agronomico de Campinas, Cordeiropolis, Brazil; (4) Universidade de Sao Paulo, Piracicaba, SP, Brazil; (5) USDA-ARSSEFTNRL, Byron, GA, U.S.A.; (6) Universidade Estadual de Maringa, Maringa, Parana, Brazil

Phytopathology 104(Suppl. 3):S3.18

Canker (caused by Xanthomonas citri subsp. citri) is an important disease of citrus in Brazil and elsewhere in the world, and can cause severe disease on the fruit. The severity of citrus canker of fruit must often be estimated visually. The objective of this research was to construct and validate standard area diagram sets (SADs) as assessment aids for raters to improve the accuracy and reliability of visual estimates of citrus canker on unripe fruit of sweet orange. Two SADs were constructed. A 5-diagram SADs had five severities depicted $(0.5,2.0,8.0,27.0$ and $40.0 \%)$ and a 6-diagram SADs had six severities depicted $(0.5,1.0,3.0,9.0,20.0$ and $40.0 \%)$. Fifteen raters evaluated 40 images of cankered, unripe fruit. Both the 5- and 6-diagram SADs significantly improved the accuracy and reliability of estimates. Agreement, measured by Lin's concordance correlation coefficient was 0.220 to 0.913 when not using SADs, 0.814 to 0.955 when using 5-diagram SADs, and 0.863 to 0.925 when using 6-diagram SADs. The 5-diagram SAD set was significantly more accurate and reliable compared to the 6-diagram set. Possible reasons for this are discussed. Based on the results, the 5-diagram
SADs is preferable to use. Although the SAD was developed for sweet orange, it doubtless has applicability to other citrus, including grapefruit. These SADs should be useful for research and disease management where accurate and reliable estimates of the severity of citrus canker are sought.

Thaxtomin A did not control Phytophthora plurivora in beech seedlings S. C. BRAND (1), S. F. Pascholati (1), W. Oßwald (2), F. Fleischmann (2)

(1) Luiz of Queiroz College of Agriculture - University of São Paulo, Piracicaba, Brazil; (2) Technical University of Munich, Freising, Germany Phytopathology 104(Suppl. 3):S3.18

Phytophthora plurivora is responsible for the decline of European Beech and until now no effective treatment against this pathogen is available. Thaxtomin A, a toxin produced by Streptomyces scabies, has been show to induce resistance in plants. Thus, the objective was to investigate if thaxtomin was able to induce resistance against the root pathogen P. plurivora in beech. Plants three months old were placed inside Falcon tubes containing 10 or 25 $\mu \mathrm{g} \mathrm{mL} \mathrm{m}^{-1}$ (first experiment) and 50 or $100 \mu \mathrm{g} \mathrm{mL}^{-1}$ thaxtomin A (second experiment). After 3 days, the thaxtomin solution was removed and the plants were inoculated with P. plurivora and evaluated for water consumption, chlorophyll fluorescence, disease incidence and pathogen DNA in the root system. Beech plants treated with 10 and $25 \mu \mathrm{g}$ of thaxtomin and inoculated with the pathogen showed a reduction in water uptake when compared to the treatment only with $P$. plurivora and an increase in disease incidence as well as in the amount of $P$. plurivora DNA. Chlorophyll fluorescence was reduced in the plants treated with $10 \mu \mathrm{g}$ and inoculated with the pathogen. On the other hand, plants treated with 50 and $100 \mu \mathrm{g}$ of thaxtomin and inoculated with the pathogen did not show differences in the evaluated parameters when compared to plants only inoculated with the pathogen. Finally, it is concluded that thaxtomin A, applied through the root system, does not protect beech plants from root rot caused by $P$. plurivora.

Effect of Rhizoctonia solani inoculum density and sugar beet cultivar susceptibility on development of crown and root rot

J. R. BRANTNER (1)

(1) University of Minnesota, Crookston, MN, U.S.A.

Phytopathology 104(Suppl. 3):S3.18

Rhizoctonia crown and root rot (RCRR) of sugar beet is controlled by fungicides if applied before infection. A field study was established in Crookston, MN to determine how inoculum density and host susceptibility influence onset and severity of disease and yield. The goal is to define the need for, and timing of, fungicide applications based on inoculum potential and variety susceptibility. Barley grain infested with $R$. solani was hand spread across plots at $0,20,40$, or $60 \mathrm{~kg} / \mathrm{ha}$ and incorporated into the top 10 $\mathrm{cm}$ of soil. Susceptible, moderately susceptible, and partially resistant sugar beet cultivars were sown in a randomized block design with four replicates on May 9. Stand counts were recorded weekly from May 23 to September 5. Yield, quality, and RCRR severity were determined on September 23. Disease developed at emergence at inoculum densities of 20,40 , and $60 \mathrm{~kg} / \mathrm{ha}$ with the susceptible and moderately susceptible cultivars, but only at $60 \mathrm{~kg} / \mathrm{ha}$ with the partially resistant cultivar. Inoculum density by cultivar interactions were significant $(P=0.05)$ for stand counts, RCRR rating, and yield. Disease severity was greater with the susceptible and moderately susceptible cultivars compared to the resistant cultivar. Fields planted with a susceptible cultivar need a full-season control strategy including fungicide applications at planting and postemergence, while a partially resistant variety may only require fungicides under high inoculum densities.

Race structure characterization and genetic diversity analysis of Puccinia striiformis f. sp. tritici in Saskatchewan

G. BRAR (1), R. Kutcher (1), Y.-B. Fu (2)

(1) University of Saskatchewan, Saskatoon, SK, Canada; (2) Plant Gene Resources of Canada, Agriculture and Agri-Food Canada, Saskatoon Research Centre, Saskatoon, SK, Canada

Phytopathology 104(Suppl. 3):S3.18

Stripe rust of wheat, caused by Puccinia striiformis f. sp. tritici (Pst) is one of the most devastating diseases of wheat world-wide. Selection pressure on the pathogen population may result in a rapid shift to races virulent on wheat genotypes with specific resistance genes. For successful disease management, it's important to monitor the virulence spectrum of the pathogen. The purpose of this research was to characterize the race structure of Pst in Saskatchewan and to determine genotypic diversity of the pathogen population. Race characterization was performed by inoculating 27 near-isogenic wheat lines, four supplemental lines and one triticale with 24 genetically uniform Pst isolates from Saskatchewan and other provinces of western Canada. Whole genome of the pathogen isolates was sequenced after DNA extraction, using the illumina MiSeq platform and polymorphisms were assessed by single 
nucleotide polymorphism (SNP) markers. Characterization of Pst isolates identified 18 races of the pathogen. Genes Yr1, Yr5, Yr15 and YrSP conditioned resistance against all races. Variation for virulence was observed among isolates on Yr10, Yr24 and YrSu. Genotypic diversity was not observed to be related to geographical location or virulence phenotypes of the isolates. The SNP data revealed little variation in the Pst population, suggesting clonal lineage, which means they were derived from one another by step-wise mutation.

Characterization of apoptotic-like cell death players in Aspergillus nidulans M. BREUNIG (1), S. Jain (1), M. Kabbage (1)

(1) University of Wisconsin-Madison, Madison, WI, U.S.A.

Phytopathology 104(Suppl. 3):S3.19

Apoptosis is a type of programmed cell death (PCD) that plays an essential role in the biology of many organisms. It involves the precise cooperation of tightly controlled processes mediated by cellular proteases that lead to the organized dismantlement of the cell. Increasing evidence suggest that apoptotic-like cell death occurs in widely divergent species, but the molecular components in many cases remain unclear. Indeed, core regulators of cell death, including Bcl-2 family members, have been identified and are well characterized in animals ranging from Caenorhabditis elegans to mammals. However, this primary sequence conservation does not extend to fungi, and bioinformatic analyses of fungal genomes have not revealed any apparent homologs to these core apoptotic players. Here we use Aspergillus nidulans as a model filamentous fungus to gain a better understanding of apoptotic-like cell death in fungi. A functional screen was performed to assess the ability of an $A$. nidulans cDNA library to rescue yeast cells from cell death imposed by $B A X$, a mammalian pro-apoptotic Bcl-2 family member. This screen identified $20 \mathrm{~A}$. nidulans genes that rescued yeast cells and reverted the $B A X$ induced cell death phenotype. Among these, two previously uncharacterized genes were chosen for further studies.

\section{Mapping adult plant resistance to Puccinia graminis f.sp. tritici race Ug99 which reduces disease severity and infection response}

J. BRIGGS (1), M. Rouse (2), S. Bhavani (3), C. Hiebert (4)

(1) University of Minnesota, St. Paul, MN, U.S.A.; (2) USDA-ARS, St. Paul, MN, U.S.A.; (3) CYMMIT, Nairobi, Kenya; (4) Agriculture and Agri-Food Canada, Winnipeg, Canada

Phytopathology 104(Suppl. 3):S3.19

Stem rust can be controlled through targeted deployment of major and minor host resistance genes. Puccinia graminis f.sp. tritici race TTKSK (Ug99) and its variants are virulent to most widely deployed major resistance genes. Minor genes contributing to adult plant resistance (APR) phenotypes have remained effective in reducing stem rust severity. Combinations of APR and major resistance genes are considered likely to be more durable than major genes alone. APR was observed in the mid-20th century Ecuadorian bread wheat cultivar Morocho Blanco (PI 286545) during field testing at Njoro, Kenya, and St. Paul, MN. Morocho Blanco is susceptible as a seedling to race TTKSK. To map APR, a doubled haploid mapping population was created from LMPG- $6 \times$ Morocho Blanco, the first parent being susceptible. Eighty eight lines were genotyped with 90,000 SNP markers from a custom Illumina Infinium assay. Stem rust infection responses and severities were assessed on adult plants grown at Njoro, Kenya, in 2013. Two QTL significantly reducing disease severity were located on chromosomes 2BS and 6AS using both CIM and G-Model mapping approaches. The QTL on 6AS also significantly reduced infection response. Further studies using single race nurseries will identify the association between pathogen race and infection response. These QTL provide a valuable resource for developing durable stem rust resistance, especially the locus on 6AS which reduces infection response and severity.

Comparative analysis of differential gene expression in response to rust pathogens

J. BRIGGS (1), J. Garbe (1), M. Rouse (2), J. Kurle (1)

(1) University of Minnesota, St. Paul, MN, U.S.A.; (2) USDA-ARS, St. Paul, MN, U.S.A.

Phytopathology 104(Suppl. 3):S3.19

The presence of plant pathogens alters the host transcriptome throughout the infection process. To gain an understanding of host-rust pathogen interactions, we examined susceptible host differential expression early in the infection process in barley, soybean, and maize with and without rust infection. Total RNA was extracted from 6-10 bulked plants at 6, 12, 24, and 72 hours post inoculation (hpi) and was sequenced using the Illumina HiSeq platform at 20 million 100 bp paired-end reads per sample. Two biological replicates were used per time point. Transcriptomes were assembled via genome-guided assembly using the Galaxy program. Peak differential expression was observed in Morex at 12 and 72 hpi with a reduction in differential expression occurring at $24 \mathrm{hpi}$ and no differential expression at $6 \mathrm{hpi}$. Peak differential expression occurred in Williams 82 at 6,12 , and $72 \mathrm{hpi}$; a reduction in differential expression was also observed at 24 hpi. Currently the maize transcriptome is undergoing analysis. Sets of homologous and similarly differentially expressed genes were identified between Morex and Williams 82. Results from this study not only illuminate our understanding of host-rust pathogen interactions, but also provide candidate host (susceptibility) genes that are necessary for successful rust colonization. Manipulation of these 'susceptibility' genes may lead to novel and broad-spectrum rust resistance.

Selective isolating of Ceratocystis fimbriata

R. A. S. BRITO (1), G. P. Cavalcante (1), L. A. Maffia (1)

(1) Departamento de Fitopatologia, Universidade Federal de Viçosa, Viçosa, Brazil

Phytopathology 104(Suppl. 3):S3.19

In a research program of Ceratocystis wilt of mango, there is a need to sample the pathogen, C. fimbriata, from infested soil and diseased tissue. Therefore the traditional use of baits of carrot slices, that produces variable results, was compared to the use of a semi-selective medium. To prepare the baits, carrot slices were immersed in sodium hypochlorite $2 \%$ for $3 \mathrm{~min}$, washed three times in sterile distilled water and immersed in a solution of $200 \mathrm{mg}$ chloramphenicol. $\mathrm{L}^{-1}$ for $5 \mathrm{~min}$. The semi-selective medium was the V8-A (Moutia \& Saumtally, 2001, Proceedings of the 4th Annu. Meet. of Agric. Sci., Mauritius, 1999, p. 75-82) modified (V8-AM). To prepare 1L of V8AM: $200 \mathrm{ml} \mathrm{V8} \mathrm{juice,} 1 \mathrm{~g}$ calcium carbonate, $20 \mathrm{~g}$ agar, $0.16 \mathrm{~g}$ Ox-bile, $0.04 \mathrm{~g}$ streptomycin sulfate, $100 \mathrm{mg}$ tetracycline hydrochloride, and $10 \mathrm{mg}$ Fluazinan, $\mathrm{pH}$ 6.35. The antibiotics, fungicide and Ox-bile were added after autoclaving at $45^{\circ} \mathrm{C}$. From both soil samples artificially infested with conidia and stem fragments of diseased mango tree, we attempted to isolate $C$. fimbriata by using the baits and the V8-AM. From the soil samples, contaminants occurred in either method; the pathogen was not recovered from the baits, but it was possible to count the number of colonies on the V8-AM on Petri dishes. From the diseased fragments, both methods recovered the pathogen. The V8-AM is been used for selective isolating of $C$. fimbriata in our laboratory, from both soil samples and diseased plant tissue.

Distribution of azoxystrobin resistant Cercospora sojina throughout soybean production fields in Mississippi

N. BROCHARD (1), J. Standish (1), M. Tomaso-Peterson (1), T. W. Allen (2) (1) Mississippi State University, Mississippi State, MS, U.S.A.; (2) Delta Research and Extension Center, Stoneville, MS, U.S.A.

Phytopathology 104(Suppl. 3):S3.19

A survey of Mississippi (MS) soybean fields occurred during the 2013 growing season to identify fields exhibiting symptoms of frogeye leaf spot (FLS) caused by Cercospora sojina Hara. Quinone outside Inhibitor (QoI) fungicides are used to control FLS but resistance to this class of fungicides has been confirmed in C. sojina isolates from MS. The objectives of this study were to survey MS soybean fields symptomatic for FLS, amass a sample distribution of $C$. sojina for live and fungarium specimens, and to map the distribution of QoI resistant isolates of $C$. sojina. Leaf samples showing symptoms of FLS were collected from over 100 fields located in 49 soybean producing counties throughout MS. Coordinates were taken from each field where a sample was collected for future reference of FLS activity in MS. The cropping, fungicide, and irrigation history was also compiled throughout the survey. A sample is defined as FLS symptomatic leaves originating from an individual field. As a result of the survey, a mono-conidial isolation was made from each sample to compile a collection of more than $100 \mathrm{C}$. sojina isolates. Isolates were evaluated for QoI resistance and results were referenced back to the sample origin.

Alternative applications of pecan fungicides and implications for disease management

K. BROWN (1), T. Brenneman (2)

(1) University of Georgia, Baconton, GA, U.S.A.; (2) University of Georgia, Tifton, GA, U.S.A.

Phytopathology 104(Suppl. 3):S3.19

Pecan scab, caused by Fusicladium effusum, is a common disease of pecans (Carya illinoinensis) in Georgia, especially during wet years. Fungicides applied by air-blast sprayers are the primary means of control. In 2013, alternative methods such as soil-applied granulars and injection into drip irrigation was evaluated. Treatments included 3 rates of Reliant (K salts of phosphorous acid) granules applied adjacent to emitters. In a separate trial, drip irrigation treatments included Luna Priviledge $(6.8 \mathrm{oz}$. twice, early season) with and without post-pollination applications of Super Tin/Elast via. air blast sprayer. The Reliant granules showed small reductions in leaf and nut 
scab versus nontreated trees, but had no effect on defoliation. Drip applications of Luna Priviledge pre-pollination reduced leaf scab incidence and severity versus nontreated plots, but did not affect late season defoliation. Nut scab severity in July was lower with Luna Priviledge, but not at harvest. Nut size and quality were larger from Super Tin/Elast cover sprays, but not from the drip applications of Luna Priviledge. Frequent rains resulted in severe scab epidemics in 2013. The efficacy of both drip and soil-applied treatments may have greater control during a dryer grower season.

Potato psyllid and Asian citrus psyllid acquisition patterns, in relation to gene expression profiles

J. K. BROWN (1), T. Fisher (1)

(1) University of Arizona, Tucson, AZ, U.S.A.

Phytopathology 104(Suppl. 3):S3.20

The circulative, propagative transmission pathway of Candidatus Liberibacter in psyllid vectors requires circulation across gut and salivary gland barriers. The bacteria encounter potentially hostile environments there and in the hemolymph. Although poorly understood mechanistically, such co-evolved interactions are mediated by bacterial and psyllid 'effectors'. $\mathrm{Ca}$. Liberibacter transmission by Asian citrus psyllid (ACP) Diaphorina citri Kuwayama and potato psyllid (PoP) Bactericera cockerelli Sulc. exhibit distinct acquisition features, in that PoP adults and nymphs can both acquire CLso, but adult transmission frequency is higher $(30 \%)$ than for nymphs. In contrast, CLas acquisition by ACP occurs only by nymphs $(<5$ th) prior to transmission, which involves only adults (Inoue et al., 2009). For both, the abilities of $\mathrm{Ca}$. Liberibacter to overcome host defenses and systemically invade are essential for acquisition. To this end, the transcriptome of ACP and PoP nymphs and adults, and midgut and salivary glands were obtained. Many psyllid genes were differentially expressed as a result of $\mathrm{Ca}$. Liberibacter infection and systemic invasion e.g. ACP vs. PoP nymph GO categories, at 52 and $14 \%$, and 22 and $15 \%$ for adults, respectively. The predicted gene functions and associated altered expression patterns were correlated with psyllid host responsiveness to $\mathrm{Ca}$. Liberibacter attack and counter-attack, and psyllid defenses mounted against the bacterial invasion.

Evaluating the potential of anaerobic soil disinfestation for the control of Prunus replant disease

G. T. BROWNE (1), L. S. Schmidt (1), M. T. Devengenzo (2)

(1) USDA-ARS, CPGRU, Davis, CA, U.S.A.; (2) UC Davis, Davis, CA, U.S.A.

Phytopathology 104(Suppl. 3):S3.20

Productivity of replanted almond and stone fruit orchards is suppressed by Prunus replant disease (PRD), a poorly understood soilborne complex. Pythium ultimum (Pult) can contribute to PRD. Preplant soil fumigation prevents PRD but is increasingly regulated and costly. We tested the potential of a non-fumigant alternative, anaerobic soil disinfestation (ASD), for control of PRD. In the first of two almond replant trials, preplant ASD was initiated after a sudan grass rotation as follows: dry rice bran $\left(20\right.$ metric tons ha $\left.{ }^{-1}\right)$ was incorporated into a $15-\mathrm{cm}$ soil depth, covered with clear plastic film, and irrigated continuously for $50 \mathrm{~h}\left(25 \mathrm{~cm}\right.$ water, 1 drip emitter per $\left.930 \mathrm{~cm}^{2}\right)$. ASD was maintained for $6 \mathrm{wk}$, irrigating daily for $1 \mathrm{~h}(0.5 \mathrm{~cm}$ water $)$. Molasses was drip applied ( 9 metric tons $\mathrm{ha}^{-1}$ ) $3 \mathrm{wk}$ after the bran. In the second trial, ASD was implemented similarly, except without sudan grass and molasses. Both trials included non-treated and preplant-fumigated (Telone $\mathrm{C} 35,600 \mathrm{~kg} \mathrm{ha}^{-1}$ ) controls. All treatments were applied to replicate $3 \times 27-\mathrm{m}$ plots. Bags of soil (30-ml) infested with Pult were buried at 30 and $45 \mathrm{~cm}$ in each plot before treatment and assayed for Pult viability afterwards. In both trials, fumigation and ASD eradicated the inoculum, whereas Pult survived in the non-treated controls (means 3180 to $6383 \mathrm{cfu} / \mathrm{g}$ soil). ASD has the potential to suppress contributions of Pult to PRD. Data reflecting almond tree responses to the treatments will be presented.

Cellular localization of proteins involved in trichothecene biosynthesis in Fusarium graminearum

K. L. BROZ (1), H. C. Kistler (2)

(1) USDA ARS Cereal Disease Laboratory, St. Paul, MN, U.S.A.; (2) USDA ARS Cereal Disease Laboratory/ University of Minnesota, St. Paul, MN, U.S.A. Phytopathology 104(Suppl. 3):S3.20

Several species of the fungus Fusarium colonize wheat and barley, producing mycotoxins that contaminate the grain and render it unsuitable for consumption. Among the most destructive of these species is $F$. graminearum, which causes Fusarium head blight disease and produces harmful trichothecene mycotoxins such as deoxynivalenol (DON). By fluorescent labeling enzymes involved in DON synthesis we have determined that these proteins are directed to the membrane of $\sim 3 \mu \mathrm{m}$ spherical vesicles called "toxisomes" or "TAO" (terpene assembly organelles) that appear to be the site of trichothecene synthesis. An enzyme of the mevalonate pathway, HMG CoA reductase also localizes to TAO. Using Fluorescence Resonance Energy Transfer (FRET) between fluorescently tagged enzymes, we infer that HMG CoA reductase and DON biosynthetic enzymes may form a multienzyme complex. Assembly of DON on a multienzyme complex contained within the TAO may promote pathway efficiency by channeling pathway intermediates and localizing precursor molecules. The toxic product and intermediates of DON synthesis also may be sequestered within the TAO, preventing selfinhibition. Cell-free preparations of fluorescently labeled TAO are being sorted using flow cytometry with the goal of directed proteomic analysis of this newly discovered organelle. We propose that DON synthesis requires a complex developmental process that ultimately determines the outcome of the plant disease interaction.

Switchgrass extractives have potential as a value-added biopesticide against plant pathogens and foodborne pathogens

A. BRUCE (1), B. H. Ownley (1), N. Labbe (1), K. D. Gwinn (1), D. H. D’Souza (1), N. Moustaid-Moussa (2)

(1) University of Tennessee, Knoxville, TN, U.S.A.; (2) Texas Tech University, Lubbock, TX, U.S.A.

Phytopathology 104(Suppl. 3):S3.20

A fraction of switchgrass biomass, known as 'extractives', has compounds that inhibit biomass conversion to biofuels. Development of 'extractives' as biopesticides would make switchgrass biomass a more economically competitive biofuel feedstock. The aim of this study was to evaluate fractions of switchgrass extractives [aqueous, butanol, ethyl acetate, and petroleum ether (PET)] against bacterial pathogens using agar diffusion disk assays on tryptic soy agar (TSA). Fractions were dried under $\mathrm{N}_{2}$ and rehydrated in water, at $1 \mathrm{X}$ and 10X rates. Agrobacterium tumefaciens (AT), Clavibacter michiganensis subsp. michiganensis (CMM), C. m. subsp. nebraskensis (CMN), Erwinia amylovora (EA), Pseudomonas syringae pv. tabaci (PsTab), P. s. pv. tomato (PsTom), Xanthomonas axonopodis pv. malvacearum (XA), and X. perforans (XP), were spread on TSA, and sterile blank disks, soaked in extractives solutions, were placed on the TSA plates. Zones of inhibition were greatest with the ethyl acetate fraction, followed by butanol and PET; the aqueous fraction had the least inhibition. Bacterial sensitivity to the $1 \mathrm{X}$ fractions varied: AT, EA, PsTab, and PsTom were the least sensitive, and CMM, CMN, $\mathrm{XA}$, and XP were the most inhibited. Except for PsTab, inhibition was greater with the 10X rate of ethyl acetate and butanol fractions. In similar tests with Salmonella enterica, serovars Enteritidis and Typhimurium, the ethyl acetate and butanol fractions had the greatest antimicrobial activity.

Quantification of fungal biomass in maize, wheat and soybean seedlings infected with wild-type and Tri6-deletion mutants of Fusarium graminearum

T. BRUNS (1), G. Munkvold (1)

(1) Iowa State University, Ames, IA, U.S.A.

Phytopathology 104(Suppl. 3):S3.20

Fusarium graminearum is a fungal pathogen of cereals and other crops, causing significant losses in grain yield and quality. $F$. graminearum produces deoxynivalenol (DON), a mycotoxin that can act as a virulence factor for head blight of wheat. The contribution of DON to seedling diseases caused by $F$. graminearum is not yet clear. To investigate the role of DON in the ability of $F$. graminearum to colonize plant seedling tissues, a quantitative PCR assay was developed using primers designed to amplify a portion of the ITS region. Crown, root, or hypocotyl tissue samples were taken at intervals up to 7 days after seedling inoculation and DNA was extracted for analysis using qPCR. To determine the impact of DON production on fungal colonization of the plant tissue, a Tri6 deletion mutant that does not produce DON was compared to the wild-type strain. Generally, maize inbreds and wheat cultivars infected with the wild-type strain contained more fungal biomass compared to plants infected with the Tri6 deletion mutant. In hybrid maize, the results differed among hybrids but the wild-type had significantly more biomass than the mutant in tissues of the most susceptible hybrid. In soybean, the wild-type showed increased fungal biomass compared to the Tri6 mutant in only 1 of 4 cultivars. DON or other Tri6-mediated metabolites appear to contribute to seedling disease caused by $F$. graminearum, but the effects differ among host genotypes.

Spatio-temporal dynamics of Ceratocystis wilt of mango and association of the disease with potential vectors

W. Bucker Moraes (1), L. A. MAFFIA (1), V. C. Garnica (1), A. G. C. Souza (2), G. R. Santos (3)

(1) Departamento de Fitopatologia, Universidade Federal de Viçosa, Viçosa, Brazil; (2) EMBRAPA Milho e Sorgo, Sete Lagoas, Brazil; (3) Departamento de Estatística, Universidade Federal de Viçosa, Viçosa, Brazil

Phytopathology 104(Suppl. 3):S3.20 
Ceratocystis wilt (CW), caused by $C$. fimbriata, is a destructive disease on mango. Disease epidemiology is poorly understood, and we aimed to describe its spatio-temporal dynamics and to evaluate the association of the disease with beetles and termites. Disease progress was followed over 15 months in five experimental units, two in Itaocara-Rio de Janeiro State (RJ1 and RJ2) and three in Frutal-Minas Gerais State (MG1, MG2, and MG3). In Itaocara, higher values of disease intensity occurred in foci, intermediate values were in different plots positions; and $\mathrm{CW}$ was spread to distant locations of the initial foci. In Frutal, CW was only observed in foci of higher intensity; and CW only progressed in the initial foci. It was found no spatial association of $\mathrm{CW}$ with termites; the disease was spatially associated with beetles in most units. In Itaocara, the beetles were detected from 0 to 171 days before $\mathrm{CW}$ was detected; in Frutal, the insects occurred from 206 to 451 days after disease detection. It was concluded that the spatio-temporal dynamics of $\mathrm{CW}$ of mango differed between regions because of the dispersal mechanisms of the pathogen. In Itaocara, the beetles disperse the pathogen from the initial foci, whereas in Frutal pathogen dispersal is probably related to soil movement through cultural practices.

Development of Tioxazafen as a next-generation seed treatment nematicide G. J. BUNKERS (1), M. S. South (1), J. Williams (1), J. McCarter (1)

(1) Monsanto Company, St. Louis, MO, U.S.A.

Phytopathology 104(Suppl. 3):S3.21

Tioxazafen is Monsanto's new broad spectrum seed treatment nematicide that is designed to provide consistent broad spectrum control of nematodes in corn, soy, and cotton. This new nematicide has been found to exhibit excellent activity on soybean cyst, root knot, and reniform nematodes in soy; lesion, root knot, and needle nematodes in corn; reniform and root knot nematodes in cotton. This class of nematicide chemistry has demonstrated equal or better performance in greenhouse and field trials vs. competitive treatments. Regulatory approvals are pending with submissions on track.

Siderophore-mediated iron uptake is essential for full virulence in the bacterial phytopathogen, Pantoea stewartii subsp. stewartii

L. BURBANK (1), C. Roper (1)

(1) University of California, Riverside, Riverside, CA, U.S.A.

Phytopathology 104(Suppl. 3):S3.21

Iron is a key micronutrient for microbial growth but is often present in low concentrations or in unavailable forms. Many microorganisms overcome this challenge by producing siderophores, which are ferric-ion chelating compounds that enable the solubilization and acquisition of iron in a bioactive form. Pantoea stewartii subsp. stewartii, the causal agent of Stewart's wilt of sweet corn, produces a siderophore under iron-limiting conditions. The proteins involved in biosynthesis and export of this siderophore are encoded by the iucABCDiutA operon, which is homologous to the aerobactin biosynthetic gene cluster found in a number of enteric pathogens. Siderophore production in $P$. stewartii is induced under iron limiting conditions and the ferric uptake regulator, Fur, represses siderophore production under ironreplete conditions. Induction of flagellar-mediated surface motility, a critical aspect of xylem colonization and virulence, requires siderophore production and uptake and is correlated with iron availability. Most importantly, Stewart's wilt symptom development, host colonization, and systemic movement in the xylem are all reliant on a functional siderophore-dependent iron uptake pathway.

Genome-informed identification of diagnostic sequences in nonpathogenic Pseudomonas syringae

G. BUSOT (1), M. Arif (1), J. P. Stack (1)

(1) Kansas State University, Manhattan, KS, U.S.A.

Phytopathology 104(Suppl. 3):S3.21

Specific populations of the kiwi fruit pathogen, $P$. syringae pathovar actinidiae $(P s a)$, have been identified as quarantine pathogens in some countries. Next-generation sequencing was used to identify $P s a$-specific DNA sequences with which to develop accurate diagnostic tools. Non-pathogenic plant-associated $P$. syringae strains may be a potential source of false positives in some sample matrices and with some diagnostic tools. Two commercially available non-pathogenic strains of $P$. syringae (ESC10 \& ESC11) were used to explore genomic differences among pathogenic and nonpathogenic populations of $P$. syringae and to determine the potential of false positive interference in Psa diagnostics. Genomic DNA was isolated and paired end libraries prepared and sequenced using Illumina MiSeq platform (2X300bp). De novo assembly was performed using MiSeq Reporter. The genome assembly of the two non-pathogenic strains resulted in $6.1 \mathrm{Mb}$ and $5.8 \mathrm{Mb}$ represented by 413 and 562 contigs respectively. Unique and core regions were identified by comparison to published genomes. In one of the non-pathogenic $P$. syringae strains (ESC11), the pathogenicity island (PAI) reported in P. syringae pv. syringae B728a was absent. In the other nonpathogenic strain (ESC10), the PAI is present but six genes within the island were absent. The potential for false positive diagnostic tests with some nonpathogenic populations of $P$. syringae may exist.

\section{Integrated disease management of sugarcane viral and bacterial diseases} in Colombia

M. Cadavid (1), J. C. Angel (1), M. L. Guzman (1), F. Angel (1), J. I. Victoria (1), C. A. ANGEL (1)

(1) Colombian Sugarcane Research Center - CENICAÑA, Cali, Colombia Phytopathology 104(Suppl. 3):S3.21

Colombia has approximately 550,000 hectares in Sugarcane, being the 3rd most important crop, $55 \%$ used for panela production along the country, and $45 \%$ for sugar, ethanol, and electricity production, mostly located along the Cauca river valley. Colombia has the highest yield worldwide in tonnes of cane per hectare, and is 13th in total sugar production. The most important bacterial diseases are Leaf Scald Disease (Xanthomonas albilineans), and Ratoon Stunting Disease (Leifsonia xyli subsp. xyli), and the viral diseases are Sugarcane mosaic virus (Potyvirus), Sugarcane yellow leaf virus (Polerovirus), and Sugarcane bacilliform virus (Badnavirus), most of them with potential economic impact. The Plant Pathology Area and Variety Program of CENICAÑA have developed a complete integrated disease management approach, diagnosing, preventing, and controlling these diseases, also avoiding introduction of exotic pathogens. This work will present details and data how serological and molecular diagnostic assays, closed and open quarantine systems to exchange germplasm, in vitro tissue culture, healthy cane seed distribution, extensive surveys, and breeding for resistance, have been the key strategies to keep competitive and sustainable the Colombian sugarcane agro-industry. Research, services, and extension activities have allowed most farmers and sugarmills to keep losses due to bacterial and viral diseases under the economic importance threshold for the last decade.

Risk assessment and resistance molecular mechanism of Magnaporthe oryzae to three DMIs

M. Cai (1), B. Li (1), D. Lin (1), X. LIU (1)

(1) China Agricultural University, Beijing, China

Phytopathology 104(Suppl. 3):S3.21

Rice blast is a devastating disease worldwide. DMIs are the main chemicals used to control this disease. Sensitivities to prochloraz, difenoconazole and epoxiconazole of 105 Magnaporthe oryzae isolates in China were determined with mean $\mathrm{EC}_{50}$ values of $0.10 \pm 0.02 \mu \mathrm{g} / \mathrm{mL}, 1.75 \pm 0.46 \mu \mathrm{g} / \mathrm{mL}$ and $0.30 \pm 0.10$ $\mu \mathrm{g} / \mathrm{mL}$, respectively. Four stable resistant mutants were generated by epoxiconazole-selection. The resistance factors ranged from 5 to 72 folds. Compared to parent isolates, fitness penalty was affected in all mutants according to mycelial growth rate, sporulation and virulent, but with no significant difference in spore germination. Y126F mutation was found in CYP51A in high resistant mutants, while I125L was found in low resistant mutants, and no mutation was found in moderate mutants. Amino acids of CYP51B were identical in all mutants and parent isolates. cyp51A was overexpressed in all tested isolates when treated by epoxiconazole, but was higher in mutants. Docking of epoxiconazole, prochloraz or difenoconazole into the binding site in the CYP51 crystal structure 3JUS showed different DMIs were combined with different amino acids in the binding pocket. After mutagenesis of Y145F (Y126F in M. oryzae), the hydrogen bond between Y145 and heme, $\pi-\pi$ connection between fungicides and amino acids were disappeared; hydrophobic interaction and van der walls weaken down. Taken together, point mutation $\mathrm{Y} 126 \mathrm{~F}$ is one of the resistance mechanisms to azole fungicides in M. oryzae.

Geographic variation in the mycangial mycoflora of Xyleborus glabratus in Florida

A. S. CAMPBELL (1), R. C. Ploetz (1), P. E. Kendra (2), W. S. Montgomery (2)

(1) University of Florida, Homestead, FL, U.S.A.; (2) Subtropical Horticulture Research Station, USDA-ARS, Miami, FL, U.S.A.

Phytopathology 104(Suppl. 3):S3.21

Laurel wilt kills Persea borbonia and other American members of the Lauraceae. It is caused by Raffaelea lauricola, a fungal symbiont of an exotic ambrosia beetle, Xyleborus glabratus. R. lauricola and other fungi were quantified in X. glabratrus collected from three locations in Florida. A MiamiDade Co. (MDC) site, $25.8^{\mathrm{N}}$, was populated by P. palustris, a Highlands Co. (HC) site, $27.9^{\circ} \mathrm{N}$, by $P$. borbonia, $P$. humulis and $P$. palustris, and an Alachua Co. (AC) site, $29.8^{\mathrm{N}}$, by P. borbonia. Mycangia from individual females were macerated and plated on CSMA and $1 / 2$ PDA. Seven putative taxa were identified based on identical LSU genotypes and colony phenotypes. With the exception of $R$. lauricola and Zygozyma oligophaga, it was not possible to match LSU genotypes/sequences with those deposited in 
GenBank, as the remaining taxa were 98 to $99 \%$ similar to two or more species of Fragosphaeria, Leptographium, Grosmannia and/or Ophiostoma. The numbers of $R$. lauricola colony forming units (CFUs) in X. glabratrus varied by location $(\mathrm{p}<0.0003)$, as beetles from MDC had more CFUs $(3,532)$ than those from $\mathrm{HC}(2,593)$ and $\mathrm{AC}(1,829)$. The numbers of fungal species also differed by location ( $\mathrm{p}<.0001)$, as $80 \%$ of the individuals from $\mathrm{AC}$ and MDC carried $\geq 3$ species, compared to $31 \%$ of those from HC. The results indicate that climate and vegetation may affect fungal diversity and the relative abundance of $R$. lauricola in the mycangia of $X$. glabratus.

\section{Pathotype shifts in Plasmodiophora brassicae as a result of infection with mixed isolates}

T. CAO (1), V. P. Manolii (1), S. F. Hwang (2), S. E. Strelkov (1)

(1) University of Alberta, Edmonton, AB, Canada; (2) Crop Diversification Centre North, Alberta Agriculture and Rural Development, Edmonton, AB, Canada

Phytopathology 104(Suppl. 3):S3.22

Clubroot, caused by Plasmodiophora brassicae, has become widespread on canola (Brassica napus) in Alberta, Canada, where it is managed mainly by cropping resistant cultivars. Proper resistance stewardship requires understanding of the effect of genetic resistance on P. brassicae population dynamics and pathotype composition. Greenhouse studies were conducted to examine shifts in pathotype composition following inoculation of isolates representing Williams' pathotypes 3 and 6 onto the canola ' $45 \mathrm{H} 26$ ' and the Chinese cabbage (B. rapa var. pekinensis) 'Granaat'. The pathotypes were inoculated in a 50:50 mixture onto each host, with the resulting galls assessed for pathotype designation on Williams' differentials. Controls were inoculated with pathotypes 3 or 6 in isolation. Of galls developing on ' $45 \mathrm{H} 26$ ' after inoculation with the pathotype mix, $84 \%$ were classified as pathotype 3, whilst the remainder were classified as pathotypes $5(7 \%), 2(5 \%)$ or $6(4 \%)$. Of galls developing on 'Granaat' after inoculation with the mix, $61 \%$ were classified as pathotype $3,26 \%$ as pathotype $6,7 \%$ as pathotype $5,4 \%$ as pathotype 2 , and $2 \%$ as pathotype 8 . No shifts in pathotype were detected following inoculation of either host with pathotypes 3 or 6 in isolation. Pathotype 3 appears to be more competitive than pathotype 6 . The recovery of additional pathotypes beyond those inoculated in the mixtures indicates possible genetic exchange or interaction among the pathotypes.

\section{Dickeya spp. associated to imported and ware potato tubers in Brazil}

Y. F. Cardoza (1), V. DUARTE (2), C. A. Lopes (3)

(1) Universidade Federal do Rio Grande do Sul, Porto Alegre, RS, Brazil; (2) Agronomica - Laboratorio de Diagnostico Fitossanitario, Porto Alegre, RS, Brazil; (3) EMBRAPA, Brasília, DF, Brazil

Phytopathology 104(Suppl. 3):S3.22

Blackleg and soft rot, caused by pectobacteria, are top-ranked diseases of potato in Brazil. Recently, D. solani has emerged as a major threat to potato production in Europe, from where most of the seed potatoes are imported by Brazil. In this context, the aim of this study was to detect and identify Dickeya spp. in ware (109), certified (111), and imported (52) potato samples. In a preliminary survey Dickeya spp. were detected in $54.9 \%$ of 206 samples of national and imported potato tubers, but not identified to the species level. In our studies, D. dianthicola was identified by $\mathrm{qPCR}$ with primers and TaqMan ${ }^{\circledR}$ probe (DIA-A) with total DNA obtained through Whatman FTA $^{\circledR}$ Cards technology. Concomitantly, bacterial isolates grown on CPG and NGM media were characterized by biochemical tests, PCR with primers ADE1/ADE2, specific to Dickeya, and sequence analysis of $d n a X$ and $m d h$ genes. Nineteen out of 258 pectobacteria-like colonies, from ware potato samples, yielded the expected $420 \mathrm{bp}$ PCR fragment with ADE1/ADE2 primers, as well as matched the biochemical and tinctorial traits of the genus. Phylogenetic analysis of concatenated partial sequences of $d n a X$ and $m d h$ genes identified the isolates as $D$. dadantii. $D$. dianthicola was detected in 6.4 and $1.8 \%$ of in ware and certified potato samples, respectively, which indicates a low prevalence of this species associated to potato tubers in Brazil. Strategies to detect and identify other Dickeya spp. are underway.

Combined influence of airborne inoculum concentration and weather on incidence of raspberry gray mould

O. CARISSE (1), A. Lefebvre (1), D. M. Tremblay (1)

(1) Agric and AgriFood Canada, St-Jean-sur-Richelieu, QC, Canada Phytopathology 104(Suppl. 3):S3.22

Grey mould (Botrytis cinerea), one of the most important fruit disease of raspberry, is primarily controlled with fungicides. The aim of this study was to investigate the combined influence of airborne inoculum and weather on incidence of fruit rot in order to improve management of the disease. The incidence of Botrytis fruit rot was monitored at two sites, in unsprayed field plots during three successive years together with meteorological data and numbers of conidia in the air. Concentrations of conidia were monitored using a Burkard volumetric sampler and rotating-arms samplers. The number of $B$. cinerea in air samples was determined with a real-time qPCR assay. The coefficient of correlation between the volumetric and rotating-arms samplers placed at $45 \mathrm{~cm}$ from the ground was significant $(\mathrm{r}=0.84)$. A diurnal pattern of conidial release was observed. Correlation between fruit rot incidence and weekly mean airborne conidia concentration was significant and positive. Of the weather variables examined, vapor pressure deficit and temperature during flowering had the greatest effect on fruit rot. Three sets of models were developed to predict fruit rot from inoculum only, weather variables only, and from both occurring 3,2 , and 1 weeks before flowering, and at 10, 50, and $80 \%$ flowering. The accuracy of the inoculum-weather based models increased with decreasing time period before flowering.

\section{Comparison of sampling methods for incidence of fungicide resistance} O. CARISSE (1)

(1) Agric \& Agri-Food Canada, St-Jean-sur-Richelieu, QC, Canada Phytopathology 104(Suppl. 3):S3.22

The single nucleotide polymorphism (SNPs) related to resistance to Succinate dehydrogenase inhibitors (SDHIs) in Botrytis cinerea populations are considered spatially aggregated. A new strategy of sampling is proposed to predict mutant's distribution which could provide a more efficient sampling in comparison with random sampling. Adaptive Cluster Sampling (ACS) was evaluated with a set of $400 \mathrm{~B}$. cinerea samples tested for the presence of SNPs. The $s d h$ gene was amplified, the nucleotide sequences were determined by the dideoxynucleotide chain-termination method and the presence of SNPs (P225F, N230I and H272L/R/Y) was determined. These data were used to stochastically generate data sets with different incidences (5 to 60\%) and different level of aggregation ( $\theta$ of $0.1,0.15$, and 0.20 ). Best sampling procedures when SNP incidence was below $30 \%$ was the ACS. Considering that the presence of SNP can only be determined after a laboratory procedure, the only practical application of ACS is for selecting the samples that will be processed. In that case, a large number of geo-referenced samples can be collected and an initial sample randomly selected and tested for resistance. If the sample is positive, other samples collected in the vicinity of this initial sample will be tested; if the sample is negative, another sample will be selected at random. This sampling approach can be used to detect and monitor low incidence and emerging mutations.

Morphological and molecular characterization of Laimaphelenchus sp. and Aphelenchoides parietinus endophytic within trees, and a new Bursaphelenchus from palm seeds

L. K. CARTA (1), Z. A. Handoo (1)

(1) USDA-ARS Nematology Laboratory, Beltsville, MD, U.S.A.

Phytopathology 104(Suppl. 3):S3.22

Bursaphelenchus, Aphelenchoides and Laimaphelenchus are nematode genera that contain many fungal-feeding species and some plant parasitic species. One species each of the last two genera were found living endophytically within trees in Idaho by George Newcombe, University of Idaho, Moscow, ID, without causing obvious symptoms of disease. Laimaphelenchus cf. heidelbergi was isolated in large numbers within surface-sterilized Populus trichocarpa (poplar) leaves, and Aphelenchoides parietinus from Pinus monticola roots. We characterized these nematodes morphometrically and anatomically with a polarizing microscope. Nematodes were also sequenced using partial sequences of 28S D2-D3 rDNA, and 18S rDNA. When placed in phylogenetic context with other 18S Laimaphelenchus sequences from GenBank, this species of Laimaphelenchus did not group with three other species of Laimaphelenchus but instead with three uncharacterized species. Neither Laimaphelenchus nor Aphelenchoides were found within clades containing plant-pathogenic taxa. Although Bursaphelenchus sp. was found from seeds of parlor palm Chamaedorea elegans from Mexico via APHIS in 2012, its closest relative $B$. arthuroides is not known to be plant-pathogenic. It was characterized microscopically and molecularly with ribosomal genes and was not closely related to either plant-pathogenic Bursaphelenchus cocophilus or B. xylophilus vectored by beetles.

Functional analysis of Cowpea mild mottle virus Triple Gene Block protein 1 and Cysteine-rich protein as potential RNA-silencing suppressors

S. L. CARVALHO (1), D. F. Milanesi (1), C. M. Carvalho (1)

(1) Universidade Federal de Vicosa, Vicosa, Brazil

Phytopathology 104(Suppl. 3):S3.22

RNA-silencing suppression (RSS) is one of the most important counteraction strategies of viruses against plant defences. The Cowpea mild mottle virus (CpMMV) encodes two candidate RSS proteins in its genome: Triple Gene Block protein 1 (TGB1) and Cysteine-rich protein (CRP). To determine whether these two proteins have RSS activity, an Agrobacterium-mediated 
transient co-expression assay was conducted using Nicotiana benthamiana plants. The proteins were cloned on a binary vector, pK7WG2, and then transformed into A. tumefaciens strain GV3101. Equal amounts of an Agrobacterium culture containing GFP and the cultures containing the candidates were mixed before infiltration. The $\mathrm{OD}_{600}$ of each culture was 1.0. The empty vector and HcPro were used as negative and positive controls, respectively. GFP expression was analyzed daily using a UV lamp and the leaves were photographed. The agroinfiltrated patches were collected at 3 and 5 days post-agroinfiltration (dpa) and total protein was extracted from these samples. Western blot analysis was carried out using antiserum against GFP and Coomassie brilliant blue was used as loading control. Slight supression of GFP RNA silencing by TGB1 and CRP was detected by 5 dpa when compared to the negative control. The western blot analysis confirm the results of the visual evaluation. The results indicated that the TGB1 and CRP may act as a suppressor of local RNA silencing.

\section{Identification of fungal pathogens isolated from blueberry gardens in} Turkey

C. CASTELL-MiLleR (1), M. Figueroa (1), B. Steffenson (1), Z. Mert (2), A. Karakaya (3), A. Celik Oğuz (3), T. Dil (3)

(1) University of Minnesota, St. Paul, MN, U.S.A.; (2) Central Research Institute for Field Crops, Ankara, Turkey; (3) Ankara University, Faculty of Agriculture, Department of Plant Protection, Ankara, Turkey

Phytopathology 104(Suppl. 3):S3.23

Blueberry bushes are perennial plants grown in many countries across the world. Recently, there has been an increase in blueberry production and acreage in the northeastern region of Turkey, specifically Rize province. However, fungal diseases hinder further expansion of the crop. We conducted a disease survey in the three blueberry production areas of Rize province, Yenidoğan, Ambarlı, and Ihlamurlu villages. Morphological-based identification indicated that the most common fungal pathogens were Botrytis sp., Colletotrichum sp., Pestalotiopsis sp., Phomopsis sp., Phyllosticta sp., Alternaria sp., Epicoccum sp. and Curvularia inaequalis. Pathogenicity tests using representative strains confirmed that all those species caused disease in wounded blueberry leaves and/or twigs. To identify the species further we are utilizing amplification of the universal fungal barcode of the nuclear ribosomal DNA internal transcribed spacers 1 and 2. Using this molecular approach we validated Collectotrichum gloeosporioides, the causal agent of anthracnose, as one of the pathogenic strains. Coupling both morphological and molecular barcode based tools will allow the correct identification of fungal pathogens affecting blueberry orchards. This will lead to design better and integrated disease control measurements and favor a profitable expansion of blueberry as a crop in Turkey.

\section{Assembly and annotation of the wildrice transcriptome challenged by Cochliobolus miyabeanus, the fungal brown spot pathogen}

C. CASTEL-MILLER (1), J. Gutierrez-Gonzalez (2), M. Figueroa (1), D. F. Garvin (2), D. A. Samac (2)

(1) University of Minnesota, St. Paul, MN, U.S.A.; (2) USDA ARS, St. Paul, MN, U.S.A.

Phytopathology 104(Suppl. 3):S3.23

American wildrice (Zizania palustris) is an aquatic cereal that is harvested from natural stands and commercial paddies for its gourmet grain. Fungal brown spot (FBS), caused by Cochliobolus miyabeanus, is the most important disease that inflicts annual yield losses in this crop. The development of resistant wildrice cultivars against FBS has been hampered by the lack of characterization of defense responses in this pathosystem. To alleviate this gap in knowledge we conducted RNA-seq analysis to obtain the transcriptional profile of mock- and C. miyabeanus-inoculated plants (cultivar Itasca C-12) at 48 hours after inoculation. Single-end cDNA sequencing of multiplexed, $50 \mathrm{bp}$ reads was conducted with an Illumina HiSeq2000. A total of 350 million reads were used as input for the transcriptome-specialized Trinity de-novo assembler, which yielded over 78,000 transcript isoforms with an average length of $681 \mathrm{bp}$. Transcripts were annotated based on a Blast search against the GenBank non-redundant protein database. Transcripts associated with PAMP-triggered and effector-triggered immunity were identified and analyses to compare gene expression are underway. This assembly constitutes the first comprehensive transcriptome study in wildrice and provides a valuable resource for enhancing disease resistance and other important traits. Transcripts involved in preventing disease progression may be useful in marker development for parent selection in wildrice breeding programs.

Cyclic di-GMP regulates biofilm formation in Erwinia amylovora through cellulose biosynthesis

L. F. CASTIBLANCO (1), G. W. Sundin (1)
(1) Michigan State University, East Lansing, MI, U.S.A.

Phytopathology 104(Suppl. 3):S3.23

Cyclic di-GMP (c di-GMP) is a ubiquitous bacterial secondary messenger molecule that regulates a broad range of cellular processes including exopolysaccharide biosynthesis, biofilm formation, motility, virulence, adhesion, cell cycle and differentiation, among others. After the perception of environmental signals, the intracellular concentration of c di-GMP is modified by enzymatic synthesis or degradation. Subsequent downstream signal transduction and phenotypic modulation is achieved by binding of $\mathrm{c}$ di-GMP to specific receptor molecules. Although activation of biofilm formation by high intracellular c di-GMP levels has been previously reported in Erwinia amylovora, the molecular mechanism involved in the modulation of this cellular process have not been characterized. Using gene overexpression and site-directed mutagenesis analyses, we investigated the role of c di-GMP on cellulose biosynthesis and identified one candidate receptor protein, EAM_3387 (BcsA), that encodes the cellulose synthase catalytic subunit and contains the $\mathrm{c}$ di-GMP binding domain PilZ. We determined that posttranslational activation of cellulose biosynthesis by binding of $\mathrm{c}$ di-GMP to BcsA mediates the c di-GMP-dependent activation of biofilm formation in E. amylovora. Our results present evidence of the molecular signaling mechanism employed by E. amylovora to regulate late stage infection phenotypes such as biofilm formation through c di-GMP.

Predominance of resistance to QoI fungicides in populations of the wheat blast pathogen Magnaporthe oryzae from Brazil

V. L. CASTROAGUDIN (1), J. T. A. Reges (2), S. C. Oliveira (3), J. L. N. Maciel (4), B. A. McDonald (5), P. C. Ceresini (6)

(1) UNESP Univ Estadual Paulista. CNPq 504073/2011-7., Ilha Solteira, Brazil; (2) UNESP Univ Estadual Paulista., Ilha Solteira, Brazil; (3) UNESP Univ Estadual Paulista. FAPESP 2013/03116-0., Ilha Solteira, Brazil; (4) EMBRAPA Wheat / Trigo, Passo Fundo, RS, Brazil; (5) ETH Zurich, Zurich, Switzerland; (6) UNESP Univ Estadual Paulista. FAPESP 2013/10655-4. CNPq 307361/2012-8., Ilha Solteira, Brazil

Phytopathology 104(Suppl. 3):S3.23

Wheat blast caused by Magnaporthe oryzae is a threat to wheat cultivation across central-southern Brazil. Its control relies strongly on strobilurin fungicides (Quinone oxidase inhibitors, QoI). Resistance to QoI (QoI-R) has been reported for several pathosystems worldwide. Here, we report the occurrence and distribution of QoI-R in populations of $M$. oryzae from Brazilian wheat fields $(\mathrm{N}=316$, of which 189 derived from wheat and 91 from weed species within fields sampled in 2012, and 36 from wheat sampled in 2005). Sequencing of cytochrome $B$ gene (cytB) distinguished nine haplotypes, four of which contained the G143A mutation linked to QoI-R. While the haplotype H1 (resistant to QoI) predominated (71\% of the isolates), the haplotype H9 (sensitive to QoI) was in $20 \%$ of the isolates. The other seven haplotypes were detected only in weeds isolates. While $92 \%$ of the wheat-derived isolates and $48 \%$ of the 2012 weed-derived had the G143A substitution, only $32 \%$ of the 2005 isolates showed this mutation. The QoI-R isolates represented distinct multilocus SSR genotypes, indicating multiple origins. Phenotypic data from in vitro assays (based on sensitivity to azoxystrobin at $10 \mathrm{ppm}$ ) fully supported the $c y t B$ data. This is the first report of the occurrence and widespread distribution of the $c y t B$ allele conferring QoI-R in populations of the wheat blast pathogen. These findings point out the need to reexamine the use of strobilurins for managing wheat blast in Brazil.

\section{Assessment of Ceratocystis fimbriata translocation in mango plants using} PCR

G. P. CAVALCANTE (1), R. A. S. Brito (1), L. A. Maffia (1)

(1) Departamento de Fitopatologia, Universidade Federal de Viçosa, Viçosa, Brazil

Phytopathology 104(Suppl. 3):S3.23

Ceratocystis fimbriata is the causal agent of mango wilt and responsible for devastating mango orchards in Brazil. The elimination of infected branches is based on visual symptoms, but previous studies showed the pathogen can be present even in asymptomatic tissues; therefore we investigated the translocation of C. fimbriata in mango plants and detection of the pathogen in asymptomatic regions using PCR. One year old mango plants were inoculated with $C$. fimbriata using mycelial discs deposited on the base of the stem and after the first pair of leaves, and the development of upward and downward symptoms was observed. After one month, stem samples were collected at the inoculation point and equally spaced by $4 \mathrm{~cm}$ along the stem where the symptom was observable. The assessment of wooden tissue discoloration in the inoculated mango plants based on upward and downward movement of $C$. fimbriata in the wood showed the pathogen moved, respectively, an average of 9.87 and $7.7 \mathrm{~cm}$ in one month, with no significant differences in the rate of tissue colonization in opposite directions. For the upward and downward 
movements evaluated using PCR we observed that though some tissues were not visually discolored the pathogen was present, colonizing up to $20 \mathrm{~cm}$ in evaluated plants. Our results show the pathogen can be found far beyond the place where the symptom was visually observed, therefore extra care should be taken when recommending pruning.

\section{Antagonist effect of Trichoderma harzianum against Sclerotium cepivorum} and Sclerotium rolfsii

L. F. CEJA-TORRES (1), E. Zuñiga-Mendoza (2)

(1) Instituto Politecnico Nacional (CIIDIR-IPN-Unidad-Michoacan), Jiquilpan, Mexico; (2) CIIDIR-IPN-Unidad-Michoacan, Jiquilpan, Mexico

Phytopathology 104(Suppl. 3):S3.24

In the present research was evaluated in vitro the antagonistic capacity of Trichoderma strain isolated from soil of the Cienega de Chapala region against Sclerotium rolfsii and Sclerotium cepivorum pathogens causing rot in onions. Pathogens were obtained from sclerotia present in infected onions. In vitro tests were conducted using dual culture, to obtain the percentage of radial growth inhibition every $24 \mathrm{hrs}$. Percent inhibition in sclerotia production and the degree of antagonism of Trichoderma and sclerotia parasitism was also obtained; four replicates were taken in each test. The antagonistic strain isolated from soil of the Cienega de Chapala region was identified at the molecular level as Trichoderma harzianum, which achieved a percentage of radial growth inhibition to $S$. rolfsii $17.4 \%$ at $48 \mathrm{hrs}$ of confrontation and to S. cepivorum $22.2 \%$ at $72 \mathrm{hrs}$. In relation to the percentage of inhibition in sclerotia production, statistical analysis showed significant differences $(\mathrm{P} \leq 0.05)$ for both pathogens regarding witnesses, it was however higher against $S$. cepivorum $95.1 \%$ with $S$. rolfsii $78.1 \%$. Furthermore, T. harzianum showed great ability to parasitize sclerotia of both pathogens, however higher against $S$. cepivorum percentages were $90 \%$. The antagonistic capacity provided $T$. harzianum was 1 and 2 on a scale of Bell to confront against $S$. cepivorum and $S$. rolfsii respectively.

\section{The origins of emerging populations of the Brachiaria collar rot pathogen (Rhizoctonia solani AG-1 IA) from Brazilian Amazon and Colombian Llanos}

P. C. CERESINI (1), E. Chavarro Mesa (2), L. M. Ramos Molina (3), M. Zala (4), B. A. McDonald (4)

(1) UNESP Univ Estadual Paulista. FAPESP 2011/50150-3 and 2013/106554; CNPq 307361/2012-8., Ilha Solteira, Brazil; (2) UNESP Univ Estadual Paulista. FAPESP 2011/23050-8., Jaboticabal, Brazil; (3) UNESP Univ Estadual Paulista., Jaboticabal, Brazil; (4) ETH Zurich, Zurich, Switzerland Phytopathology 104(Suppl. 3):S3.24

In the early 1990's Rhizoctonia solani AG-1 IA emerged as an important Brachiaria pathogen in South America. This fungus emerged for the first time in Colombia after rice cropping (a highly susceptible host for the fungus) was displaced by Brachiaria cultivation in response to increasing demand for extensive cattle raising. Brachiaria is an extremely important forage in tropical Latin America. In Brazil, the fungus was described as a Brachiaria pathogen in 1999, but only recently has been reported as an important forage pathogen in the Amazon. In this study we asked the following question: Was the emergence of this disease due to host-shifts or host-jumps occurred between geographically overlapping host species? To address this question, sympatric host-distinct populations of $R$. solani AG-1 IA from Brachiaria and rice in Colombia or Brachiaria and soybean in Brazil ( $\mathrm{N}=335$ isolates) were genotyped using nine microsatellite (SSR) loci. Patterns of historical assymetrical migration between pathogen populations indicated the probable origin of current populations that infect Brachiaria in Colombia was from populations that originally infected rice. In Brazil, in contrast, we could not establish such a clear asymmetric migration route since migration rates among populations were all similar in magnitude (average of 12.8 migrants/ generation). However, these high migration rates suggested that the pathogen had the ability to jump between a Poaeceae and a Fabaceae host.

Identification of QTL for resistance to Sclerotinia stem rot in a wild perennial relative of cultivated soybean

S. CHANG (1), C. S. Thurber (1), P. J. Brown (1), G. L. Hartman (2), L. L. Domier (2)

(1) Department of Crop Sciences, University of Illinois, Urbana, IL, U.S.A.; (2) Department of Crop Sciences, University of Illinois; USDA-Agricultural Research Services, Urbana, IL, U.S.A.

Phytopathology 104(Suppl. 3):S3.24

Soybean (Glycine max), like many cultivated crops, has a relatively narrow genetic base and lacks diversity for some economically important traits, including Sclerotinia stem rot (also known as white fold), which is caused by Sclerotinia sclerotiorum. Unlike soybean, Glycine latifolia, a perennial wild relative of soybean in the subgenus Glycine, shows high levels of resistance to
SSR. To generate molecular resources for gene mapping and identification in G. latifolia, single nucleotide polymorphism (SNP) markers were generated by high-throughput sequencing. In $G$. latifolia populations of $186 \quad \mathrm{~F}_{2}$ individuals and $90 \mathrm{~F}_{5}$ lines that segregated for resistance to SSR, a set of 3,710 SNP markers formed 20 large linkage groups, 12 of which were collinear with soybean chromosomes. Analysis of the segregation of the SNP markers and phenotypic responses to incubation in oxalic acid (a pathogenicity determinant for S. sclerotiorum) identified a region on G. latifolia linkage group 2 for resistance to oxalic acid that contained 69 genes (including two NBS-LRR genes). Glycine latifolia and other perennial wild relatives of cultivated soybean represent sources of genes that could be beneficial to soybean production, especially when resistance is lacking in the G. $\max$ primary gene pool as with SSR.

Secretory plant cell wall degrading enzyme expression analysis and polygalacturonase modeling of Fusarium virguliforme

H. X. CHANG (1), C. R. Yendrek (2), G. L. Hartman (3)

(1) Department of Crop Sciences, University of Illinois, Urbana, IL, U.S.A.; (2) Institute for Genomic Biology, Urbana, IL, U.S.A.; (3) Department of Crop Sciences, University of Illinois; USDA-Agricultural Research Services, Urbana, IL, U.S.A.

Phytopathology 104(Suppl. 3):S3.24

The secretion of plant cell wall degrading enzymes (PCWDE) is an important process necessary for soilborne pathogens such as Fusarium virguliforme to colonize host roots. Plants respond to PCWDE by enhancing cell walls and increasing defense-related compounds. To understand the complexities of PCWDE, transcript abundance of genes encoding secretory PCWDE produced by $F$. virguliforme was evaluated using RNA-Seq from 5-day and 20-day old cultures. This analysis identified 161 expressed genes encoding proteins belonging to the carbohydrate-active enzymes (CAZy), of which 121 were secretory PCWDE belonging to the carbohydrate esterase (CE), glycoside hydrolase, and polysaccharide lyase (PL) classes. A greater number of CE and PL genes were seen compared to the average reported in the CAZy database, and 27 PCWDE genes were up-regulated in 20-day old cultures compared to only 14 down-regulated genes. Structural modeling and protein-protein docking analysis of one PCWDE, F. virguliforme polygalacturonase (FvirPG), suggested that FvirPG was recognized by polygalaturonase-inhibiting protein (PGIP), which suggests the possibility of developing transgenic soybean plants with exotic PGIPs to reduce root infection caused by $F$. virguliforme.

Biology of foliar and root infection of potato plants by Colletotrichum coccodes in Australia

J. CHANG (1), R. Boer (2), P. W. Crous (3), P. W. Taylor (1)

(1) The University of Melbourne, Melbourne, Australia; (2) Department of Environment and Primary Industries, Melbourne, Australia; (3) CBS Fungal Biodiversity Centre, Utrecht, Netherlands

Phytopathology 104(Suppl. 3):S3.24

Black dot disease is a cosmopolitan disease on potato (Solanum tuberosum L.) which is caused by the fungal pathogen Colletotrichum coccodes. The infection biology of this pathogen was studied using two inoculation methods. For foliar inoculation, inoculum concentrations of $10^{3}, 10^{4}, 10^{5}$ and $10^{6}$ spores $/ \mathrm{mL}$ were used to spray the leaves of potato seedlings established from in vitro culture of cv. Russet Burbank. For root dipping inoculation, roots of cvs. Trent, Nicola, Kennebec and Russet Burbank were dipped into spore suspensions of similar concentrations for 5 mins before transplanting to pasteurised potting soil. Leaves were assessed for disease symptoms at 2week intervals, and the petioles cultured on selected medium (EPAA). Foliar symptoms did not develop on plants inoculated by either method. However, only the old leaves inoculated by spray inoculation with $10^{5}$ and $10^{6}$ spores $/ \mathrm{mL}$ were infected. New leaves inoculated by foliar spray and root dipping remained uninfected at all concentrations. At maturity the below ground plant tissues were cultured on EPAA with the roots, lower stems, stolons and tubers showing infection for both inoculation methods at all spore concentrations. For root dipping inoculation method there was a difference in incidence of tuber infection between cultivars with Kennebec appearing to have more infected tubers than the other cultivars. Thus, resistance in potato cultivars may exist for this disease, which further studies are required.

The bacterial alarmone ppGpp serves as a global signal to regulate type III secretion system and other virulence factors in plant pathogenic bacteria

T. CHATNAPARAT (1), V. Ancona (1), Y. Zhao (1)

(1) University of Illinois, Urbana, IL, U.S.A

Phytopathology 104(Suppl. 3):S3.24

The hypersensitive response and pathogenicity ( $h r p$ ) -type III secretion system (T3SS) is a key pathogenicity factor in Gram-negative bacteria. Despite recent efforts to understand the hrp-T3SS, we have yet to determine which environ- 
mental host-pathogen signals modulate the T3SS and how bacterial pathogens actively acquire nutrients in the apoplast or on leaf surfaces. Stringent response, mediated by the linear nucleotide second messengers ( $\mathrm{p}) \mathrm{ppGpp}$, leads to a swift and massive switch of transcription under nutrient limitation conditions. In this study, we generated and characterized ppGpp-deficient $\left(\mathrm{ppGpp}^{0}\right)$ mutants of $P$. syringae pv. tomoto DC3000 (PstDC3000), P. syringae pv. syringae B728a (PssB728a) and Erwinia amylovora Ea1189. Results showed that the ppGpp ${ }^{0}$ mutants of PstDC3000, PssB728a and Ea1189 were greatly impaired in causing disease on their respective host plants, i.e. tomato, bean and apple. Bacterial growth of these ppGpp mutants in planta was also significantly reduced as compared to wild type strain. In addition, expressions of T3SS and effector genes were barely detected in the $\mathrm{ppGpp}^{0}$ mutants. Moreover, the ppGpp ${ }^{0}$ mutants of PstDC3000 and PssB728a resulted in a diminution of pyoverdin production and were more sensitive to oxidative stress. Our results, for the first time, indicate that ppGpp serves as an internal signal to activate T3SS and other virulence factors in plant pathogenic bacteria.

Global investigation of microRNAs involved in soybean innate immune response to Soybean Mosaic Virus (SMV) infection by deep sequencing

H. CHEN (1), A. Wang (1)

(1) Agriculture and Agri-Food Canada, London, ON, Canada

Phytopathology 104(Suppl. 3):S3.25

MicroRNAs (miRNAs) play a vital role in a wide variety of biological processes in various organisms. To explore if miRNAs are involved in soybean innate immune response to infections by different Soybean Mosaic Virus (SMV) strains G2 and G7, four small RNA cDNA libraries derived from mock-inoculated and SMV-infected leaves in soybean (Glycine Max) cultivar Williams 82 were constructed, sequenced and analyzed on the Illumina MiSeq Platform. We identified 171 miRNAs that were induced or reduced by SMV infection, showing at least two-fold changes in reads between SMV-infected and mock-inoculated plants. Among them, 11 miRNAs were novel miRNAs in soybean. To investigate miRNA-mediated target cleavage events, four corresponding libraries of parallel analysis of RNA ends (PARE) were constructed and sequenced to a total depth of approximately 17 million sequences. Analysis of the PARE and miRNAs data revealed that 460 potential targets of these identified miRNAs were predicted and 111 precise cleavage sites in the predicted miRNA targets were detected. Randomly picked examples of virus-responsive miRNAs were validated by Northern blot analysis and Stem-loop RT-qPCR assay. In addition, an indepth global analysis of these high-throughput sequencing data indicated that a group of miRNAs were differentially expressed in response to different strain of SMV infection.

Effects of long-term corn-soybean crop sequences and nematicide treatments on corn and soybean yields

S. CHEN (1), Y. Bao (2), Z. Grabau (1), S. Xiao (3)

(1) University of Minnesota, Waseca, MN, U.S.A.; (2) University of Minnesota, St. Paul, MN, U.S.A.; (3) Fujian Agriculture and Forestry University, Fuzhou, China

Phytopathology 104(Suppl. 3):S3.25

The effects of corn-soybean crop sequences on the crop yields have been studied extensively. Generally, there is yield penalty for either crop with monoculture or inadequate rotation. However, the mechanisms of the yield penalty have not been fully studied. The objective of this study was to determine if plant-parasitic nematodes are involved in the yield penalty of inadequate crop rotation. Field plots of long-term corn-soybean crop sequences were established in 1982 in Minnesota, USA: (i) five-year rotation of each crop such that both crops are in years $1,2,3,4$, and 5 of monoculture every year; (ii) annual rotation of each crop with both crops planted each year; (iii) continuous monoculture of each crop. Beginning in 2010, half of each plot was treated with granular a nematicide, terbufos in 2010-2011 and aldicarb in 2012-2013. Heterodera glycines, Pratylenchus spp., Helicotylenchus spp., and Xiphinema spp. are major plant-parasitic nematodes in the field. As expected, crop sequence affected soybean and corn yields, which decreased with increasing number of years of monoculture. While nematicide terbufos did not affect the crop yields, aldicarb effectively reduced population densities of the plant-parasitic nematodes and increased soybean and corn yields. However, increase of the yields was not related to initial nematode population density, suggesting the yield penalty of inadequate crop rotation could not be fully explained by the damage caused by nematodes.

The two-component response regulator BfdR links $r p f$ / DSF with biofilm formation of Xanthomonas axonopodis pv. citri

Y. H. Chen (1), T. P. HUANG (1)

(1) Natl Chung-Hsing University, Taichung, Taiwan

Phytopathology 104(Suppl. 3):S3.25
Citrus bacteria canker caused by Xanthomonas axonopodis pv. citri is the most devastating bacterial disease of citrus plants worldwide. In our previous study, the two-component response regulator BfdR (for biofilm formation defective, regulator) mutant showed reduction in the development of biofilm and canker lesions as well as epiphytic growth on the leaf surfaces of citrus plants. BfdR was found to positively control the rpfF expression in XVM2 medium. The results indicated that biofilm formation and bacterial survival of $X$. axonopodis pv. citri on the leaf surfaces of citrus are associated with bacterial virulence and are controlled by BfdR through regulation of $r p f F$. Here, we investigate whether $X$. axonopodis pv. citri BfdR controls biofilm formation via $r p f$ (for $r$ egulation of $p$ athogenicity factors)/ DSF ((for diffusible signal factor) system and its regulatory mechanisms. The results showed that the transcript levels of $r p f B$ and $r p f F$, genes for DSF synthesis, were reduced; while those of $r p f A, r p f C, r p f D$ and $r p f G$ were up-regulated in the $b f d R$ mutant compared to the wild type and the complemented strain. Addition of synthetic DSF in the tryptic soy broth restored biofilm formation on polystyrene and leaf surfaces of citrus by the $b f d R$ mutant to the level by the wild type. In conclusion, our results suggested that $\mathrm{BfdR}$ of $X$. axonopodis pv. citri strain XW19 controls biofilm formation through regulation in $\mathrm{rpf} / \mathrm{DSF}$ system.

\section{Structural and functional diversity of CLAVATA3/ESR (CLE)-like genes} from Globodera nematode species

S. CHEN (1), P. Lang (1), X. Wang (2)

(1) Department of Plant Pathology and Plant-Microbe Biology, Cornell University, Ithaca, NY, U.S.A.; (2) USDA-ARS, Robert W. Holley Center for Agriculture and Health, Ithaca, NY, U.S.A.

Phytopathology 104(Suppl. 3):S3.25

The plant CLAVATA3/ESR (CLE) proteins are a group of signaling peptides that play critical roles in plant growth and development. Effector genes encoding CLE-like proteins have recently been identified in several cyst nematode species including the potato cyst nematode Globodera rostochiensis, a significant quarantine pest that infects the potato crop. Using a comparative genomics approach, we cloned a group of $C L E$ genes from three closely related Globodera species, including G. pallida and G. ellingtonae that infect potato and G. tabacum that infects tobacco. The Globodera CLE genes were found to predominantly encode multiple CLE motifs and a total of thirty-eight unique A-type CLE motif sequences were identified from them. In vitro CLE peptide application assays and overexpression studies in Arabidopsis indicated that these newly identified Globodera CLEs function as peptide mimics of plant CLEs. The alanine scanning experiment on a representative dodeca-CLE peptide identified several residues that are important for peptide activity and efficient binding to a CLV2-like receptor in potato. Transgenic potato plants expressing dsRNA targeting a Globodera CLE gene were found to be less susceptible to G. pallida infection, suggesting an important role of nematodesecreted CLEs in promoting nematode infection on host plants. A better understanding of this CLE mimicry in plant parasitism may lead to the development of novel tools for controlling cyst nematodes.

Manipulation of chromatin configuration for suppression of host immunity by zinc finger effectors (MoZFEs) of rice blast pathogen

Y. CHEN (1), W. Liu (2), Y. Yang (2)

(1) Penn State, Harrisburg, PA, U.S.A.; (2) Penn State, State College, PA, U.S.A.

Phytopathology 104(Suppl. 3):S3.25

Rice blast disease, caused by the fungal pathogen Magnaporthe oryzae, is one of the most devastating plant diseases in the world. The fungal pathogen is known to produce various protein effectors to target host proteins and facilitate pathogenesis and disease development. We have recently identified a family of M. oryzae zinc finger effectors (MoZFEs) and demonstrated their entry into rice cells during the fungal infection. Interestingly, transgenic rice lines expressing MoZFEs exhibited suppression of defense gene expression and increased susceptibility to rice blast disease. To elucidate the MoZFEmediated virulence mechanism, nine putative host targets of MoZFEs were identified from rice by the yeast two-hybrid screening. One of them directly interacts with MoZFEs in plant cell nucleus and is a conserved histone chaperone important for chromatin remodeling. Based on the preliminary data, we hypothesize that MoZFEs target a histone chaperone and likely modify chromatin configuration to suppress defense gene expression and host immunity. Further testings of this hypothesis will help elucidate the molecular mechanism of the rice-M. oryzae interaction and facilitate the development of new strategies to improve rice disease resistance and food security.

Functional analysis of $\mathrm{CfaR}$, a PAS-LuxR family regulator of COR-like metabolite production in the common scab pathogen Streptomyces scabies Z. CHENG (1), D. R. Bignell (1)

(1) Memorial University of Newfoundland, St. John's, NF, Canada

Phytopathology 104(Suppl. 3):S3.25 
Streptomyces scabies is a Gram-positive filamentous bacterium that serves as the main causal agent of potato common scab in North America. Among the virulence factors produced by $S$. scabies are the COR-like metabolites, which resemble the coronatine (COR) phytotoxin produced by Pseudomonas syringae. The COR-like metabolite biosynthetic gene cluster consists of genes encoding biosynthetic enzymes for metabolite production as well as a gene encoding CfaR, which is a PAS-LuxR family transcriptional regulator required for expression of the metabolite biosynthetic genes. The objective of this study was to characterize the DNA binding activity of the CfaR protein. $\mathrm{CfaR}$ was overexpressed and purified in E. coli as a HIS-tagged protein, and gel mobility shift assays with the purified protein showed that $\mathrm{CfaR}$ binds to a sole site in the $c f a l$ promoter region, which drive expression of the COR-like metabolite biosynthetic genes. The DNA binding activity required both the PAS and LuxR domains in the protein as truncated versions of CfaR could not bind DNA. Furthermore, the binding activity was shown to be influenced by small molecule(s) produced by S. scabies. The results of this study provide important insight into how $\mathrm{CfaR}$ regulates the production of the virulenceassociated COR-like metabolites in S. scabies.

Phylogenetic analysis and association mapping for agronomic and quality traits in USDA pea PSP collection

P. CHENG (1), W. Holdsworth (2), Y. Ma (1), M. Mazourek (2), M. Grusak (3), C. Coyne (1), R. McGee (1)

(1) Washington State University, Pullman, WA, U.S.A.; (2) Cornell University, Ithaca, NY, U.S.A.; (3) USDA-ARS Children's Nutrition Research Center, Houston, TX, U.S.A.

Phytopathology 104(Suppl. 3):S3.26

Association mapping is an efficient approach for the identification of the molecular basis of agronomic traits in crop plants. For this purpose, we genotyped and phenotyped individual lines of the USDA pea Pisum singleplant (PSP) collection including accessions from 327 landraces and cultivars of Pisum sativum, 26 P. sativum subsp. elatius, 16 P. sativum var. arvense, 4 $P$. sativum var. pumilio, 3 P. sativum subsp. asiaticum, 3 P. abyssinicum, $2 P$. fulvum, 2 Pisum spp., and $1 P$. sativum subsp. transcaucasicum. These 384 accessions were collected from a total of 64 countries. The accessions were genotyped with 256 informative SNPs using Sequenom MassARRAY. Genetic structure analysis showed that the population was structured into two main groups, corresponding roughly to the cultivated types/landraces and the wild subspecies, with some intermediates. Linkage disequilibrium (LD) of pairwise loci was analyzed. Association analysis between SNP genotypes and 37 valuable traits such as disease resistance, morphological traits, carbohydrate content, and mineral nutrient concentration were performed using a mixed linear model. A total of 123 marker trait associations (MTAs) were detected to be significant with 1-9 markers per trait. Some of these geneanchored SNP markers revealed candidate genes and confirmed quantitative trait loci that have been identified previously. This study showed the potential of using association mapping in pea breeding.

Etiological and genetic characteristics of Colletotrichum gloeosporioides isolated from newly emerging anthracnose of static symptoms on apple W. CHEON (1), S. G. Lee (1), Y. H. Jeon (1)

(1) Department of Bioresource Sciences, Andong National University, 1375 Gyeongdong-Ro, Andong, Gyeongbuk 761-749, Korea, Andong, Korea Phytopathology 104(Suppl. 3):S3.26

Disease occurrence in apple cultivars in 24 apple orchards from 2013 was investigated. Typical anthracnose with progressed symptom was observed all apple orchards. Recently, static symptoms were observed on apples together with typical progress symptoms. In the static symptoms, a small lesion is observed at the beginning of the growth period. The spot doesn't expand further, is remaining static until the harvesting season. We found that the shape and size were different between these two types of the conidia of progressed symptoms fungi were straight, cylindrical, with an obtuse apex. However, the shape and size of the static symptoms fungi conidia were smaller than those of the progressed symptoms fungi. A pathogenicity of these two types was performed on immature apples by inoculating with conidial suspensions. In a progressed symptoms fungus-inoculated apple, anthracnose symptoms were progressed. However, with static symptoms fungal inoculation, there was no observed expansion in the size of static spots. Although the nucleotide sequences of the actin, chitin synthase, $\beta$-tubulin and internal transcribed spacer regions were $100 \%$ consistent, PCR analysis by the specific primers was observed two types isolates consistent Colletotrichum gloeosporioides. RAPD-PCR was showed clearly differentiated subgroups of C. gloeosporioides genotypes. The clustering of these groups was highly related to the symptom types of the individual strains.
Transcription factor Zfp1 required for full pathogenesis in Ustilago maydis

H. K. Cheung (1), K. L. Spence (1), B. J. SAVILLE (2)

(1) Trent University, Peterborough, ON, Canada; (2) Trent University, Ennismore, ON, Canada

Phytopathology 104(Suppl. 3):S3.26

Smut fungi are biotrophs- obtaining nutrients from the plant host to complete their life cycles. To facilitate this they produce effectors, secreted proteins which alter the host to favour fungal growth. While effector function is investigated by many researchers, the control of effector expression has received little attention. The characterization of a putative transcription factor conserved in smut fungi has revealed a potential link to the control of effector expression. This transcription factor, zinc finger protein 1 (Zfp1), is being investigated in Ustilago maydis, the causal agent for common smut of corn. The protein contains a GAL4-like Zn(II)2Cys6 binuclear cluster DNA-binding domain, and a fungal specific transcription factor domain. Microscopic investigation of infection with $z f p 1$ deletion strain showed fungal development is attenuated and remains mostly in the leaf tissue, corresponding with the production of small leaf tumours and reduced anthocyanin. This phenotype is also observed in the $U$. maydis effector tin 2 deletion strains. The transcript level of tin2 and three other effectors appears altered in the $z f p 1$ deletion mutant strains during in planta growth. Additional alteration in expression over the transcriptome is investigated by RNAseq. Complementation by wildtype $z f p 1$ restores pathogenesis and anthocyanin production. This work begins an investigation of the control of effector gene expression in the model fungal pathogen $U$. maydis.

The effects of rater bias on hypothesis testing when using different assessment methods to estimate disease severity

K. S. P. CHIANG (1), C. H. Bock (2), M. E. Jarroudi (3), P. Delfosse (4), I. H. Lee (1), H. I. Liu (1)

(1) National Chung Hsing University, Taichung, Taiwan; (2) USDA-ARSSEFTNRL, Byron, GA, U.S.A.; (3) Université de Liège, Department of Environmental Sciences and Management, Arlon, Belgium; (4) Centre de Recherche Public-Gabriel Lippmann, Environment and Agro-biotechnologies Department, Belvaux, Luxembourg Phytopathology 104(Suppl. 3):S3.26

Bias (over and underestimates) in estimates of disease severity, and the impact of that inaccuracy on hypothesis testing using different disease scales were explored. Nearest percent estimates (NPE), the Horsfall-Barratt (H-B) scale and four different linear category scales $(5 \%$ and $10 \%$ increments, with and without additional grades at low severity) were compared. Actual values (by image analysis) and estimates by 4 different raters of the severity (0 to 100\%) of Septoria leaf blotch on leaves of winter wheat were used to develop distributions for a simulation model. The simulations were based on i) all the 4 raters data combined, ii) only the most accurate rater estimates, and iii) only the most biased rater. Regardless of the effect of rater ability, we found that, there were lower type II error rates with NPEs as compared with the other category scales at severities of 80 to $100 \%$. On the other hand, with lower severities ( 0 to $20 \%$ ), the $5 \%$ and $10 \%$ scales with additional grades had type II error rates comparable to those for the NPEs. Raters who overestimated severity and used the H-B scale had the highest risk of a type II error when the mean disease severity was low $(\leq 10 \%)$. Knowledge of how rater ability and scale type can affect hypothesis testing can be used to improve disease assessment as well as to provide a logical framework for developing standard area diagrams.

Genetic diversity studies and molecular diagnostics of Cacao swollen shoot virus (CSSV)

N. CHINGANDU (1), J. K. Brown (1)

(1) University of Arizona, School of Plant Sciences, Tucson, AZ, U.S.A.

Phytopathology 104(Suppl. 3):S3.26

Cacao swollen shoot virus (CSSV; genus, Badnavirus; family, Caulimoviridae) has become an increasingly important constraint on cocoa production in West Africa, which supplies about $70 \%$ of the world's cocoa beans. To conduct epidemiological studies, and provide support to breeding and disease management programs, the development of sensitive virus detection and identification methods has become essential. Preliminary studies indicated that CSSV isolates are genetically variable and highly divergent, rendering existing diagnostics ineffective. To test the feasibility of PCR for CSSV detection and differentiation of all isolates and strains in cacao plants, endemic hosts, or the mealybug vectors, PCR primers were designed for six viral genome loci based on seven CSSV genome sequences available in GenBank database. Total DNA extracted from cacao leaves and stems was subjected to rolling circle amplification, followed by PCR. Sequences of cloned amplicons were analyzed to determine the extent of CSSV genomic 
diversity. Results indicated that isolates from Cote d'Ivoire cacao plant samples were highly variable in certain genomic regions, and conserved in others, and that no one primer pair amplified CSSV in all cases in which it was present. Thus, complete genome sequencing will be required to reveal the full extent of genomic diversity, upon which more accurate diagnostic tools can be based, and implemented to understand the basis for the recent epidemics in West Africa.

\section{Pathogenicity of Fusarium tricinctum on soybean under field conditions}

P. CHITRAMPALAM (1), B. D. Nelson Jr (1)

(1) North Dakota State University, Fargo, ND, U.S.A.

Phytopathology 104(Suppl. 3):S3.27

Fusarium tricinctum is commonly isolated from soybean roots in North Dakota, but the role of this species in root disease is poorly understood. Pathogenicity of $F$. tricinctum on soybean was evaluated under field conditions in Fargo, ND in 2013. Two F. tricinctum isolates, 91-1-8, and 91319-3 which differ in morphology and mating type were tested. Plastic pots (5L) filled with pasteurized Glyndon sandy loam soil were buried $25 \mathrm{~cm}$ deep in the ground with a portion of the bottom of the pot removed. Barley grain infested with $F$. tricinctum was added and mixed into soil at $340 \mathrm{~g} /$ pot before planting. Fifteen soybean seeds were planted in each pot. Pots without inoculum served as non-inoculated controls. Treatments were arranged in a randomized complete block design with ten replications. Four weeks after planting, plants in each pot were thinned to five plants/pot. Both isolates significantly reduced seed germination. Seed germination at three weeks following planting was 63,37 and $90 \%$ for $91-1-8,91-319-3$ and control, respectively. With isolate $91-1-8$ only $3 \%$ of emerged plants contained trifoliate leaves with an average plant height of $7.3 \mathrm{~cm}$ while in pots with isolate $91-319-370 \%$ of emerged plants had trifoliate leaves with an average plant height of $8 \mathrm{~cm}$. Root biomass was also significantly reduced with both isolates compared to control. Percent yield reduction with isolate 91-1-8 was significantly higher $(90 \%)$ than with isolate $91-219-3$ (40\%) compared to control.

\section{Genetic diversity of Fusarium solani from soybean}

P. CHITRAMPALAM (1), B. D. Nelson (1)

(1) North Dakota State University, Fargo, ND, U.S.A.

Phytopathology 104(Suppl. 3):S3.27

Root rot caused by Fusarium solani is a common soybean disease in the northern Great Plains, but the genetic diversity of the pathogen is unknown. Multiloci pylogeny was performed to determine the genetic diversity in $F$. solani from soybean. In total $93 \mathrm{~F}$. solani isolates were used, of which 89 were from soybean and four from pea. Of the 89 soybean isolates, 74 isolates were from North Dakota and 15 isolates were from southern Minnesota. Three loci, ITS $+28 \mathrm{~S}$ of rDNA, $E F 1$ - $\alpha$, and RNA polymerase II subunit (RPB2) were PCR amplified and sequenced. In total 946, 643, and 1730 nucleotide characters were generated for ITS $+28 \mathrm{~S}, E F 1-\alpha$, and $R P B 2$, respectively. Both homogeneity partition test and $\mathrm{KH}$ test revealed incongruence among loci, and therefore maximum parsimony analysis was separately performed for each data set along with sequences of three clades of Fusarium solani species complex (FSSC) from NCBI. Analysis from all three data sets placed isolates from this study within clade-III of FSSC. In addition, all three data sets revealed two genetic clusters among $F$. solani isolates used in this study, and both clusters contained isolates from both North Dakota and Minnesota and also from pea. Cluster-I contained 39 isolates while cluster-II contained 54 isolates. There was significant variability within each cluster, and the number of sub-clades within each cluster varied depending on the locus. PCR screening for mating types revealed a 1:1 distribution among isolates. Modification of seed germination and induction of defense response of
plants through cold atmospheric plasma technology

S. Y. CHO (1), Y. M. Lee (1), Y. H. Jeon (2)

(1) Andong National University, Andong, Korea; (2) Andong National University, Gyeongbuk, Korea

Phytopathology 104(Suppl. 3):S3.27

Application of physical technology such as ionizing radiation, laser etc. in agriculture are more and more popular, but most of them are radiations and cells of plant seeds are damaged by radiations. One of the next physical resources is plasma which is an ionized state of matter similar to gas. This study was conducted to test the effects of seed germination and induction of defense responses in crop plants. Seeds of hot pepper and cucumber treated with cold atmospheric plasma for different treatment times at $3,000 \mathrm{~V}$ and 20 $\mathrm{kHz}$. Under greenhouse conditions, the germination percentage, germination speed, and seedling length were measured and resulted in a significant increase in germination speed compared with untreated control. We analyzed expression level of defense-related genes in cucumber seedlings to test whether a plant defense response is influenced by the cold atmospheric plasma. The results of real-time reverse transcription-polymerase chain reaction were showed that genes involved in jasmonate/ethylene signaling pathway of induced systemic resistance (ISR) in 20-day-old cucumber seedlings were highly expressed. This result suggests that treatment of the cold atmospheric plasma to cucumber seedlings would be affected the ISR mechanism and induced disease resistance to subsequent pathogen infection. These results demonstrate that cold atmospheric plasma might be a simple, easy-to-handle technique for enhancing seed germination and plant disease resistance.

Pyrosequencing analyses of endophytic bacterial populations in tomato leaves infected by 'Candidatus Liberibacter solanacearum'

N. CLARK (1), T. L. Frigulti (1), J. Bushoven (1), Z. Zheng (2), C. Wallis (3), J. Chen (4)

(1) California State University, Fresno, CA, U.S.A.; (2) South China Agricultural University, Guangzhou, China; (3) USDA-ARS, Parlier, CA, U.S.A.; (4) USDA ARS PWA, Parlier, CA, U.S.A.

Phytopathology 104(Suppl. 3):S3.27

'Candidatus Liberibacter solanacearum' (CLso) is associated with zebra Chip (ZC) disease of potato. The bacterium is currently not culturable and commonly maintained in plant hosts for characterization. This also provides an opportunity to study interactions between CLso and other endophytic bacteria. In this study, CLso was graft-transmitted to tomato plants. A maximum of over 200 -folds titer increase was obtained by maintaining excised tomato leaves in potting soil for 28 days under greenhouse conditions. Metabolism changes in tomato leaves were also monitored. Preliminary results revealed that sucrose levels were over $60 \%$ greater in leaves at date 28 than when initially moved into potting soil. DNA was extracted from CLsoenriched tomato leaves and subjected to pyrosequencing. A total of 100,867 reads averaging $419 \mathrm{bp}$ each was generated. BLAST (Basic Local Alignment Search Tool) analyses, using 43 selected whole genome sequences covering all major categories of prokaryotes in GenBank database as queries, revealed that bacterial sequences similar to CLso, Pseudomonas putida, and Streptomyces davawensis were highly abundant. PCR experiments further confirmed simultaneous co-enrichment of these bacteria. This is the first observation on the association of Pseudomonas-like and Streptomyces-like bacteria with CLso titer increase in tomato plants.

Sentinel Plant Network members in the Northeast and Mid-Atlantic regions use smartphone app for early detection of invasive diseases and insects

D. L. CLEMENT (1), M. K. Malinoski (1), C. T. Bargeron (2), R. L. McCarthy (3)

(1) University of Maryland, Ellicott City, MD, U.S.A.; (2) The University of Georgia, Tifton, GA, U.S.A.; (3) Cornell University, Ithaca, NY, U.S.A.

Phytopathology 104(Suppl. 3):S3.27

The Mid Atlantic Early Detection Network (MAEDN) smartphone app developed by the Center for Invasive Species and Ecosystem Health (Bugwood) increases the possibility of early detection by providing a convenient tool for comparison and reporting. Nationally, pests like sudden oak death, thousand cankers disease, boxwood blight, Asian longhorned beetle and emerald ash borer are causing serious problems for public gardens, the Green Industry and private land owners. Training on how to use the MAEDN app was delivered to northeast and mid-Atlantic public garden professionals who are member of the Sentinel Plant Network. This smartphone app allows users to compare photos and descriptions of signs and symptoms while in the field without any additional tools or equipment. The app allows one to send in a report and pictures along with precise GPS location for further confirmation by regulatory officials. Field ID cards are available which further increase the possibility of accurate early detection. The ID cards which support the app are easily modified and allow users to see compare photos and descriptions of target species leading to fewer false reports.

Surveying high tunnel tomato growers in Wisconsin to identify extension and disease control needs

K. CLEVELAND (1), A. Gevens (1)

(1) University of Wisconsin, Madison, WI, U.S.A

Phytopathology 104(Suppl. 3):S3.27

Over the last several years in Wisconsin, the construction of high tunnels (HTs) for season extension of locally-grown produce has greatly increased. Tomato is a high value specialty crop and is one of the most commonly grown HT crops. Different environmental conditions prevail in a HT than in an openfield setting, requiring unique management. Many recommendations, to date, have come from open-field trials and research from other regions. To assess the production and disease information needs of Wisconsin HT growers, a 
survey was conducted with questions ranging from cultural practices, pest and disease concerns to interests in informational tools. Just under $70 \%$ of growers have been growing tomatoes in HTs for $<5$ years, with just over $60 \%$ of these growers raising $100+$ plants. Over $30 \%$ of the growers identified pest and disease management concerns as particular current challenges. Of disease troubles, over $45 \%$ of grower specified problems with the tomato leaf mold fungus, Fulvia fulva. Most growers (85\%) would find if valuable to have additional web tools for pest and disease control, and $77 \%$ indicated interest in a leaf mold management or forecasting tool that alerted growers to when preventative fungicides would need to be applied. Results indicated that a majority of HT tomato growers are relatively new, they are growing a substantial number of tomato plants, and they are in need of further production and specific disease management information.

\section{Impact of fungicide modes of action on Frogeye Leaf Spot}

A. M. COCHRAN (1), W. J. Jordan (1), K. Lamour (2), C. A. Bradley (3), H. M. Kelly (1)

(1) University of Tennessee, Jackson, TN, U.S.A.; (2) University of Tennessee, Knoxville, TN, U.S.A.; (3) University of Illinois, Urbana, IL, U.S.A.

Phytopathology 104(Suppl. 3):S3.28

Cercospora sojina, the cause of Frogeye Leaf Spot (FLS) on soybean, was first reported to be quinone outside inhibitor (QoI) fungicide resistant in 2010. Evaluating how fungicide modes of action influence selection for QoI resistance could contribute to designing more efficacious FLS management strategies. A FLS susceptible soybean cultivar was grown in four field sites in west Tennessee and one site in southern Illinois. Sites varied in disease pressure and incidence of QoI resistant C. sojina. Quinone outside inhibitor, demethylation inhibitor (DMI), succinate dehydrogenase inhibitor (SDHI), methyl benzimidazole carbamate (MBC), and chloronitrile fungicides were applied at R3. Treatment effects were evaluated by assessing FLS severity (\%), soybean yield (bu/a), C. sojina QoI sensitivity and genetic diversity. FLS severity was negatively correlated to yield and displayed a significant linear regression $(\mathrm{R} 2=0.66$, slope $=-0.36)$. Location and fungicide treatment were main effects on FLS severity and yield. Fungicide significantly effected FLS severity in moderate to high disease pressure locations and yield in high disease pressure locations. Across all locations chloronitrile and QoI fungicides did not significantly reduce FLS severity, while DMI, MBC, and QoI+DMI fungicides consistently had the greatest reduction in FLS severity. More than 11000 candidate SNPs have been identified and a subset will be used to analyze $C$. sojina genetic diversity on a fine scale.

\section{Fungi associated with Arkansas soybean seed}

K. COCHRAN (1), J. Rupe (1), R. Holland (1)

(1) University of Arkansas, Fayetteville, AR, U.S.A.

Phytopathology 104(Suppl. 3):S3.28

Fungal seed pathogens can adversely affect soybean seed quality and performance. Most soybean seed research has concentrated on two fungi, Phomopsis longicolla and Cercospora kikuchii, but many other fungi have been isolated from seed and may also affect seed quality and performance. The extent and diversity of seed infection by these fungi vary depending on the environment, cropping history and cultivar. To determine which fungi infect soybean seed in Arkansas, seed was collected from five tests conducted in Arkansas from 2008-2013. Fifty seed/plot were surface disinfested, plated on PDA, and incubated at room temperature (RT). Fungi growing from the seed were transferred to PDA and incubated at RT until sporulation. Fungi were then identified morphologically and molecularly using ITS primers. Over thirty different species of fungi were recovered from Arkansas soybean seed. The most common were P. longicolla, C. kikuchii, Fusarium spp., Colletotrichum spp., Macrophomina phaseolina, Alternaria spp., Aspergillus spp., Chaetomium spp., and Cladosporium spp.

An agro-climatic approach to determine citrus postbloom fruit drop risk in the São Paulo State, Brazil

A. R. S. COLLETTI (1), P. C. Sentelhas (1)

(1) University of São Paulo, Piracicaba, Brazil

Phytopathology 104(Suppl. 3):S3.28

Postbloom Fruit Drop (PFD), caused by Colletotrichum acutatum and C. gloeosporioides, is a serious problem in most humid citrus production areas of the Americas and can be very severe when the rainfall occurs during the bloom. The objective of the present study was to develop an agro-climatic approach to determine the risk of citrus PFD epidemics. For such purpose, twenty years of daily weather data, from ten representative citrus areas in São Paulo state were analyzed. Based on previous studies, the flowering period was estimated based on degree-days and the accumulated rainfall data from the flowering peak until the final $50 \%$ of flowers was determinate for each year for all locations. Non-linear Gompertz regression was used to fit the observed PDF incidence (\%) to the accumulated rainfall, generating an estimating model. Finally, the percentages of years with PDF incidence in different intervals were obtained in order to define a PDF climatic risk index. The accumulated rainfall of $70.8 \mathrm{~mm}$ between the flowering peak and the last flowering $50 \%$ provided a PDF incidence of $50 \%$. The locations in the south and southwest regions of the state were those with the greatest risk for PDF. According to our findings, traditional citrus areas presented a medium PDF risk, showing the importance of monitoring this disease for management strategies. The present approach can be very useful to predict disease incidence and to develop a new warning system for this disease.

Identifying spinach cultivars with reduced susceptibility to Fusarium wilt B. D. COLLINS (1), M. R. McDonald (1), L. J. du Toit (2), S. Westerveld (3) (1) University of Guelph, Guelph, ON, Canada; (2) Washington State University, Mt Vernon, WA, U.S.A.; (3) Ontario Ministry of Agriculture and Food, Simcoe, ON, Canada

Phytopathology 104(Suppl. 3):S3.28

Fusarium wilt, caused by the soilborne pathogen Fusarium oxysporum f. sp. spinaciae, is a destructive and persistent disease in spinach production. Currently, there are few effective control methods for Fusarium wilt and few partially resistant commercial cultivars available. Field trials were conducted in July and August of 2012 and 2013 in naturally infested soils in Hamilton, Ontario to determine the susceptibility of spinach cultivars to this disease. In 2012, 25 cultivars were screened; eight were screened again in 2013. Disease incidence, disease severity index (DSI), vascular discolouration index (VDI) of the roots, and yield were assessed. In the 2013 trial, disease incidence was $75 \%$ less severe than in 2012, likely due to lower soil temperatures in 2013. The cultivar Norgreen had the greatest DSI in 2012 and 2013 at 38 and 18\%, respectively. The cultivars Unipack 12 and Greyhound also had severe DSI and VDI ratings over the two years. Cultivars C2606, Carmel, Sardinia, POH0438 and Imperial Green had low disease severity ratings both years with DSI ratings $<14 \%$ and VDI ranging from $26-37 \%$. Vascular discolouration and disease severity ratings were positively correlated $(r=0.72)$. Only disease incidence and DSI ratings were negatively correlated with spinach yield $(\mathrm{r}=-$ 0.40 ). Although no spinach cultivars were identified as highly resistant to Fusarium wilt, the differences observed in susceptibility of cultivars could be useful for cultivar selection.

Leveraging technologies to deliver on-farm nematode management solutions

V. C. CONCIBIDO (1)

(1) Monsanto Co, Chesterfield, MO, U.S.A.

Phytopathology 104(Suppl. 3):S3.28

Plant parasitic nematodes are major pests of crops worldwide and can cause substantial economic damage. By their nature, nematodes may cause significant below ground damage not easily observable to growers. Soybean cyst nematode (SCN) (Heterodera glycines Ichinohe) can cause over \$2 B in damage to soybean production in North and South America while root knot nematodes (RKN, Meloidogyne spp.), that parasitize over 2,000 crops species, account for approximately $5 \%$ of global crop loss. Sustainable and effective nematode management is a key focus in Monsanto to deliver superior product options to growers. The technologies currently being used by Monsanto to deliver a robust pipeline of nematode management options, including traditional and molecular breeding, biotechnology, and chemistry will be highlighted.

Plant-induced gene expression in a rice endophyte belonging to the group of plant beneficial Burkholderia

B. G. COUTINHO (1), V. Venturi (1)

(1) ICGEB, Trieste, Italy

Phytopathology 104(Suppl. 3):S3.28

The use of plant growth promoting bacteria as biofertilizers now has important implications on resource management and world nutrition. Burkholderia kururiensis M130 is one of the few rice endophytic diazotrophic bacteria identified so far which is able to enhance growth and yield of rice plants. However, very little is known on how endophytes enter and colonize plants. We have initiated studies on analyzing the changes in gene expression during the interaction with the plant by identifying genes of strain M130 that are differentially regulated in the presence of rice plant extract. The methodology used was via a genetic screen of a promoter probe transposon mutant genome bank and RNAseq analysis. Results indicated that the endophyte undergoes dramatic changes in its gene expression profile in the presence of plant extract regulating $31.1 \%$ of its protein encoding genes by more than 2 -fold. Interestingly, a great number of differentially expressed genes encode membrane transporters and secretion systems, indicating that the 
exchange of molecules is a major factor in the plant environment. Genes related to motility and chemotaxis were also over-represented which suggests the importance of movement and signaling/recognition between bacteria and plant. This work stresses the probable intensive signalling taking place between plants and bacteria and begins to help us understand the adaptation that a beneficial endophyte undergoes in order to colonize and grow in planta.

The effect of copper application on demethylation inhibitor (DMI) sensitivity in a DMI-resistant population of Venturia inaequalis

K. COX (1), S. Villani (1), Z. Frederick (2)

(1) Cornell University, Geneva, NY, U.S.A.; (2) Washington State University, Pullman, WA, U.S.A.

Phytopathology 104(Suppl. 3):S3.29

Demethylation inhibitor (DMI) fungicides are an effective means to manage apple scab caused by Venturia inaequalis. Unfortunately, practical resistance to DMI fungicide chemistries is prevalent in populations in NY and New England states. Management practices that delay the development or impact the magnitude of DMI resistance in $V$. inaequalis populations are highly desired by regional apple producers. Orchard trials were conducted in a New York apple orchard in 2011 and 2012 to determine the impact of delayed dormant applications of copper (after bud break, but prior to green tissue) on the DMI sensitivity of a $V$. inaequalis population with stable resistance to DMI fungicides. Delayed-dormant treatment programs consisted of either an application of copper, manganese, myclobutanil (DMI fungicide), or no application. Fungicide sensitivity to myclobutanil was evaluated for a minimum of $25 \mathrm{~V}$. inaequalis single lesion conidial isolates from each replicated treatment block. In both years, mean percent relative growth of $V$. inaequalis isolates from the copper treatment were significantly $(P<0.05)$ lower than isolates that did not receive a delayed dormant treatment. The effect of the manganese treatment was inconsistent between years, and applications of myclobutanil did not affect DMI sensitivity. These results suggest delayed dormant treatments of copper may have an effect on the DMI sensitivity for a population of $V$. inaequalis.

Polymyxa graminis isolates from Australia: Identification in wheat roots and soil, molecular characterization and wide genetic diversity

B. A. Cox (1), H. Luo (2), R. JONES (3)

(1) University of Western Australia, Crawley, Australia; (2) Department of Agriculture and Food Western Australia, South Perth, Australia; (3) University of Western Australia, South Perth, WA, Australia

Phytopathology 104(Suppl. 3):S3.29

Polymyxya graminis is an important virus vector in cereal crops. In 2011-12, it was identified in wheat root samples from southwest Australia. It's presence was detected by PCR, and confirmed by DNA sequencing of transcribed regions of its ribosomal RNA genes and observing sporosori of characteristic morphology and size in wheat roots. When soil samples were collected from 2/3 locations where $P$. graminis was found and wheat bait plants grown, $P$. graminis was detected in their roots by PCR. Ribosomal DNA sequences of six southwest Australian isolates from wheat roots, and one northeast Australian isolate from barley roots were obtained. When these sequences were compared with others from Genbank, three south-west Australian isolates were classified as $P$. graminis f. sp. temperata (ribotypes Ia and Ib), and three as f. sp. tepida (ribotypes IIa and IIb). The $P$. graminis isolate from northeast Australia was placed into new ribotype VI. When wheat leaf samples from 67 field crops and soil bait plants from two locations with $P$. graminis were tested by RT-PCR for Soil-borne wheat mosaic virus, Soilborne cereal mosaic virus, Wheat spindle streak mosaic virus and furoviruses, no virus was found. This study suggests that soil-borne cereal viruses are likely to become established should they become introduced to Australia.

Vertical profile of Plasmodiophora brassicae resting spores in mineral and muck soils

T. J. CRANMER (1), B. D. Gossen (2), A. Deora (1), M. R. McDonald (1) (1) University of Guelph, Guelph, ON, Canada; (2) Agriculture and AgriFood Canada, Saskatoon, SK, Canada

Phytopathology 104(Suppl. 3):S3.29

Canola (Brassica napus L.) is a major crop in Canada, with an economic value of over $\$ 19$ billion per year. Plasmodiophora brassicae Woronin, the causative agent of clubroot, can cause substantial reduction in yield of canola and is spreading rapidly in western Canada. Resting spores of $P$. brassicae can survive in soil for many years, but information on their vertical distribution in the soil profile is lacking. Vertical soil cores from the soil surface to $53-\mathrm{cm}$ depth were collected by hand from naturally infested mineral soil sites near Bassano Alberta and Milgrove Ontario, and a muck soil ( $70 \%$ organic matter) from the Holland Marsh in Ontario. A multiplex TaqMan qPCR assay, including an internal control based on $G F P u v 1$, was used to quantify resting spore concentration in soils at selected points along the vertical profile. Spore concentrations ranged from $1 \times 10^{3}$ to $6 \times 10^{6} \mathrm{gram}^{-1}$ of dry soil. Resting spores were present throughout the soil profile, with concentrations of $1.0 \times$ $10^{3}$ spores gram $^{-1}$ or more at $45-53 \mathrm{~cm}$ below the surface in each soil type. Resting spores are generally produced near the soil surface, so this observation indicates that spores are likely carried down into the soil profile by movement of water. These results, showing that resting spores can be found well below the plow layer, have important implications for developing future clubroot management techniques.

Role of fungi and oomycetes in marsh wetland seed bank viability

E. CROCKER (1), E. Nelson (1)

(1) Cornell University, Ithaca, NY, U.S.A.

Phytopathology 104(Suppl. 3):S3.29

Many marsh wetland plant species depend on annual establishment by seeds, but it is unclear what role soil pathogens play in seed bank viability. We tested the effect of soil fungi and oomycetes on seed germination and seedling survival for ten plant species, both native and invasive, by treating seeds with oomycete and fungal specific biocides. In addition, we isolated fungi and oomycetes from seed surfaces and tested their pathogenicity and virulence. We found no significant effect of biocide treatment on seed viability in the field for most of the plant species we tested. However, different plant species acquired unique seed-associate pathogens which varied in virulence. In particular, the fungal isolates associated with some invasive species were more virulent than those associated with similar native species, suggesting a potential role for soil pathogens in marsh wetland invasive success.

Sensitivity of Fusarium species from soybean roots to seed treatment fungicides

D. R. CRUZ (1), D. A. Mayfield (1), Z. L. Njus (1), S. Pandey (1), M. S. Beattie (1), L. F. Leandro (1), G. P. Munkvold (1)

(1) Iowa State University, Ames, IA, U.S.A.

Phytopathology 104(Suppl. 3):S3.29

Fungicide seed treatments have become important in soybean due to earlier planting, increased prevalence of reduced tillage, and higher seed prices. Information on the effects of fungicides on Fusarium species causing disease in soybean is needed in order to sustain effective management of these pathogens. The objective of this study was to characterize sensitivity of Fusarium populations from soybean to the commonly used seed treatment fungicides fludioxonil, ipconazole, pyraclostrobin, and trifloxystrobin. Fungicide formulations were incorporated into potato dextrose agar at a range of concentrations ( 0.01 to $100 \mu \mathrm{g} / \mathrm{ml})$. Radial growth and spore germination were visually assessed and measured for representative isolates of $F$. graminearum, $F$. oxysporum, $F$. solani, and $F$. acuminatum from Iowa and surrounding states. These data were compared to results obtained using image analysis software. Mycelial growth inhibition differed significantly among the fungicides and among Fusarium species and strains. Fludioxonil had a stronger effect than ipconazole on radial growth of $F$. graminearum; in contrast, ipconazole strongly inhibited mycelial growth of $F$. oxysporum, $F$. acuminatum and $F$. solani. Spore germination was affected by both trifloxystrobin and pyraclostrobin. EC50 frequency distributions will be generated for each combination of species and fungicide; these will be valuable for tracking potential future changes in sensitivity.

Qualitative plate assay for detection of aflatoxigencity and inhibition of aflatoxigenicity by Aspergillus flavus

K. DAMANN (1), C. DeRobertis (2)

(1) Louisiana State University Agricultural Center, Baton Rouge, LA, U.S.A.; (2) Louisiana State University Agricultural Center Department of Plant Pathology \& Crop Physiology, Baton Rouge, LA, U.S.A.

Phytopathology 104(Suppl. 3):S3.29

Aflatoxin producing isolates of Aspergillus flavus grown 5 days in the dark at $30^{\circ} \mathrm{C}$ on cream of coconut (1:1 with water) impart a highly visible yellow color to the medium when viewed from below. These yellow pigments are known anthraquinone intermediates in the aflatoxin biosynthetic pathway which have been secreted into the medium by the fungus. Non-toxigenic isolates which we have tested fail to secrete these yellow pigments and the medium retains its original off-white color when viewed from below. This simple qualitative test allows rapid discrimination between toxigenic and nontoxigenic isolates. Interestingly, when aflatoxigenic strains are paired with known non-toxigenic biocontrol strains or certain other non-toxigenic isolates the yellow color is abolished and the medium remains an off white color. This appears to be an expression of intraspecific aflatoxin inhibitory ability by the non-producer. This method has utility in rapidly identifying toxigenic isolates and isolates which have intraspecific aflatoxin inhibitory ability. Isolates 
identified as inhibitory are candidates for further testing as non-toxigenic biocontrol strains.

Workshop on plant disease identification and management strategies for smallholder farms in Haiti

L. M. C. DANDURAND (1), G. R. Knudsen (1)

(1) University of Idaho, Moscow, ID, U.S.A.

Phytopathology 104(Suppl. 3):S3.30

Among many Haitian farmers there is relatively little awareness of how plant disease impacts crop yields, and how it can be diagnosed and managed. In November, 2013, a workshop (sponsored by APS OIP) was presented at Université de la Nouvelle Grand'Anse (UNOGA) in Jérémie. The hands-on workshop consisted of eight half-day sessions on disease identification and management, and presentations also were made to farmers in the community of Roseau. Course materials were placed on the internet (small files for low bandwidth) since although printed materials are not readily available in Haiti there is relatively widespread internet access, including via cell phone. An ebook ("Phytopathologie") was published online and is available free of charge. Materials and UNOGA presentations were in French, and talks with community groups were presented in Haitian Kreyol. During the two-week workshop, we covered a range of topics including diseases important to Haitian food crops (banana/plantain, citrus, mango, breadfruit, coffee, rice, corn, sugarcane, cassava) with field trips and laboratory exercises. We brought laboratory equipment and supplies including digital compound and dissecting microscopes, agar, petri dishes, and general lab supplies. Workshop participants were fourth-year undergraduate students in General Agricultural Studies. UNOGA students are recruited with the expectation that they will return to their communities to help solve real-world problems.

\section{Recovery of soil microbial communities after fumigation with time}

S. R. Dangi (1), B. D. Hanson (2), J. GERIK (3)

(1) UC Davis, Parlier, CA, U.S.A.; (2) UC Davis, Davis, CA, U.S.A.; (3) USDA ARS, Parlier, CA, U.S.A.

Phytopathology 104(Suppl. 3):S3.30

Soil fumigants can affect both target and non-target or beneficial microorganisms in soil, but the recovery of soil microbial communities after fumigation is unknown until recently. The present study evaluated the post fumigation recovery of soil microbial communities overtime and the study captured both short-term and long-term changes in microbial communities after continuous fumigation. The study was conducted in the Watsonville area, the dominant strawberry growing region of California, USA. Chronosequences were chosen considering Jenny's state factors: organisms, climate, relief, parent material and time; sites were chosen which were as similar as possible. The chronosequence selected for this study contained four sites defined by number of years since fumigation with methyl bromide; a 15 year old site, a 33 year old site, a 39 year old site, and an organic farm. Soil samples were collected from 0-5, 5-15 and 15-30 cm depths. Phospholipid fatty acid analysis and terminal restriction fragment length polymorphism were used to characterize recovery of soil microbial community structure after fumigation. Our results indicate disparities in soil microbial contents at different fumigated sites and non-fumigated sites, indicating differences in microbial biomass production. For the 0-5, 5-15 and 15-30 cm depths, all bacterial, mycorrhizal and actinomycetes groups were greater in nonfumigated and organic sites as compared to methyl bromide fumigation sites.

To the vector go the spoils: Identifying fungal pathogens associated with walnut twig beetle, a vector of thousand cankers disease complex

D. A. DANIELS (1), R. J. Lord (1), P. A. Wadl (1), K. A. Nix (1), L. M. Vito (1), P. R. Merten (2), M. T. Windham (1), P. L. Lambdin (1), J. F. Grant (1), G. J. Wiggins (1), D. Hadziabdic (1)

(1) University of Tennessee, Knoxville, TN, U.S.A.; (2) USDA Forest Service, Forest Health Protection, Asheville, NC, U.S.A.

Phytopathology 104(Suppl. 3):S3.30

The fungus, Geosmithia morbida, vectored by the walnut twig beetle (WTB), Pityophthorus juglandis, causes mortality in black walnut (Juglans nigra) known as Thousand Cankers Disease (TCD). Infected trees exhibit symptoms similar to drought, making disease identification difficult. Early detection and confirmation of the pathogen is feasible using molecular markers. In 2010, TCD was discovered in Tennessee and has since been detected in four more eastern U.S. states within the native range of black walnut. Our objective was to identify fungal pathogens associated with WTB infestation of black walnut in Tennessee. WTB $(n=180)$ were collected from two symptomatic trees in Knoxville, TN, separated by sex, divided into 3 treatment groups (decapitation, washing, or direct plating), and placed onto antibiotic amended $10 \%$ potato dextrose agar medium. Fungal isolates were identified based on morphology and confirmed by sequencing ITS1 and ITS4 regions.
Additionally, microsatellite loci were used to detect the presence of $G$. morbida. Statistically significant associations between pathogen presence and direct plating method $(P=0.026)$, and between male WTB and G. morbida $(P$ $=0.044$ ) were determined. Although four species of Fusarium, including $F$. solani, were isolated from both female and male WTB, the findings were not significant with respect to sex. It is vitally important to fully understand TCD complex and the role of secondary pathogens in TCD pathogenicity.

Acquired resistance to mefenoxam in sensitive Phytophthora infestans isolates

G. DANIES (1), R. A. Childers (1), K. Myers (1), Z. Fei (1), I. M. Small (1), W. E. Fry (1)

(1) Cornell University, Ithaca, NY, U.S.A.

Phytopathology 104(Suppl. 3):S3.30

The systemic fungicide mefenoxam (metalaxyl) has been an important fungicide in the control of late blight disease caused by Phytophthora infestans since the late 1970's. Unfortunately, insensitive strains have been present since the early 1980's, and mefenoxam use declined. Recently, sensitive populations of $P$. infestans have been prevalent in the United States. The sensitivity or resistance of $P$. infestans to mefenoxam is commonly assessed in vitro by measuring the radial growth of the pathogen in response to fungicide in amended media. We recently observed that sensitive isolates (in three diverse genotypes) exposed to mefenoxam became tolerant of mefenoxam in subsequent exposures. In contrast isolates that were "stably resistant" to mefenoxam in the initial assessment did not become even more resistant upon further exposure. To investigate the mechanism of acquired resistance, we employed single-strand specific RNA-Sequencing to examine differential expression associated with acquired resistance. Many differentially expressed genes were genotype specific, but there was a set of genes differentially expressed in all genotypes. Among these were a phospholipase "Pi-PLD-like-3", two ATP binding cassette superfamily (ABC) transporters, one mannitol dehydrogenase, three CRN and five RxLR effectors, and four conserved hypothetical proteins.

Effect of simultaneous water deficit stress and Meloidogyne incognita infection on cotton yield and fiber quality

R. F. DAVIS (1), H. J. Earl (2), P. Timper (1)

(1) USDA ARS, Tifton, GA, U.S.A.; (2) University of Guelph, Guelph, ON, Canada

Phytopathology 104(Suppl. 3):S3.30

Drought stress and Meloidogyne incognita infection reduce cotton yield. Drought affects fiber quality, but nematode effects on quality are not known. To determine whether nematodes affect quality and whether the combined effects of nematodes and drought on yield and quality are independent, a seven year study was conducted in a field infested with $M$. incognita. A splitplot design was used with the main plot factor as one of three irrigation treatments (non-irrigated, moderate irrigation, and water-replete) and the subplot factor as 0 or $561 /$ ha 1,3-dichloropropene. We prevented water deficit stress in water-replete plots by supplementing rainfall with irrigation. Moderate irrigation plots received half the water applied to water-replete plots. Root galling was greater in non-fumigated plots and in plots receiving the least irrigation, but irrigation did not influence the effect of fumigation on root galling (no irrigation $\times$ fumigation interaction). Lint and seed harvests were reduced in non-fumigated plots and also decreased as the level of irrigation decreased, but irrigation did not influence the effect of fumigation. Nematodes increased micronaire but typically had no effect on other fiber quality elements. Micronaire also was increased by water deficit stress, but the effects from nematodes and water stress were independent. We conclude that the effects of nematode parasitism and water deficit stress on cotton are independent and therefore additive.

Investigating the soil microbiome of two native shrubs with plant growth promoting activity in an intercropping system with millet in Senegal

S. DEBENPORT (1), R. Bayala (2), K. Assigbetse (2), L. Chapuis-Lardy (2), R. P. Dick (3), B. McSpadden Gardener (4)

(1) The Ohio State University, Ballwin, MO, U.S.A.; (2) IRD, Institut de Recherche pour le Developpement, Dakar, Senegal; (3) The Ohio State University, Columbus, OH, U.S.A.; (4) The Ohio State University, Wooster, OH, U.S.A.

Phytopathology 104(Suppl. 3):S3.30

Poor soil quality and yields in the Sahel region of sub-Saharan Africa pose a risk to subsistence farmers. This calls for the need to find both economically and environmentally sustainable methods of improving crop yield. A potentially useful interaction between two native shrubs (Guiera senegalensis and Piliostigma reticulatum) and millet (Pennisetum glaucum) was found to increase crop yield. Two long term study sites containing blocks of millet 
grown alone or intercropped with the shrubs were sampled in Keur Matar and Nioro Senegal. We performed high throughput sequencing on DNA from soils collected from three sample types: the base of shrubs, millet grown in association with those shrubs, and millet grown alone. Amplicon libraries of both 16S and ITS regions were sequenced in order to profile bacterial and fungal communities. Whole community similarities were observed through clustering analysis, and individual OTUs were analyzed for statistically significant associations among sample types. Our data show that, at both sites, the soil communities surrounding shrub and intercropped millet roots are more similar than the communities surrounding millet grown alone. This indicates that these two native shrubs could be influencing the structure of microbial communities in soil within the root zone of adjacent crop plants, which could have an impact on the growth and health of the millet.

Specific expansion and functional importance of a group of Histidine Kinases in genus Fusarium

G. DEIULIO (1), J. Goldberg (2), Q. Zeng (2), L. Guo (1), U. Shrestha (1), L. J. Ma (1)

(1) UMass Amherst, Amherst, MA, U.S.A.; (2) Broad Institute, Cambridge, MA, U.S.A.

Phytopathology 104(Suppl. 3):S3.31

Fungi and their host plants recognize one another on a molecular level. These interactions are mediated by signaling molecules that directly, or indirectly, detect the presence of a pathogen or host plant. Histidine kinases are a group of well conserved signaling receptors that are present in plants, fungi, and bacteria. Previous studies have shown that some histidine kinases are important fungal signaling molecules, having been implicated in osmosensing, reproduction, fungicide resistance, and pathogenicity. We have identified a group of these genes that appear to be uniquely expanded among Fusarium species, defined as FusHKs. Their expression is detected in both axenic condition and in planta. Most interestingly, one FusHK is uniquely expanded in the $F$. oxysporum species complex and its expression is upregulated during infection. Here we present preliminary work identifying the role these genes have in infection using the Fusarium oxysporum f.sp. lycopersici - Solanum lycopersicum pathosystem.

\section{Pythium spp. associated with greenhouse floriculture crops in Michigan} J. DEL CASTILLO-MÚNERA (1)

(1) Michigan State University, East Lansing, MI, U.S.A.

Phytopathology 104(Suppl. 3):S3.31

Michigan ranks third in the U.S. for the wholesale value of floriculture products, with an annual estimated value of $\$ 375.7$ million. Seedling damping-off, and root-rot incited by Pythium spp. are important problems for greenhouse growers. Pythium spp. associated with Michigan's floriculture crops were characterized as a means to improve current management strategies. Potted poinsettias with root rot symptoms were sampled from nine greenhouses located in three Michigan counties during 2011 and 2012. Symptomatic geranium and snapdragon bedding plants were sampled from 11 greenhouses in the same counties, during 2012 and 2013. Pythium isolates were confirmed via morphology and sequencing of the ITS region. A total of 243 Pythium spp. isolates were obtained from poinsettias and 728 isolates from geranium and snapdragon. Seven Pythium spp. were identified, with $P$. irregulare, $P$. ultimum, and P. aphanidermatum the most prevalent species. A subset of isolates was chosen for pathogenicity and mefenoxam sensitivity testing. Five of the species were moderately to highly pathogenic $(\mathrm{P}<0.005)$ to germinating geranium seeds. Most $P$. ultimum and $P$. irregulare isolates tested were intermediate to highly resistant to mefenoxam. All $P$. aphanidermatum isolates were sensitive to mefenoxam. This study suggests that Pythium spp. recovered from the same Michigan greenhouses may vary depending on the host, and that mefenoxam, may not be effective to control P. ultimum.

Effect of timing of application of azoxystrobin and pyraclostrobin on control of blackleg of canola

L. E. DEL RIO MENDOZA (1)

(1) North Dakota State University, Fargo, ND, U.S.A.

Phytopathology 104(Suppl. 3):S3.31

Strains of Leptosphaeria maculans pathogenicity group (PG) 4, which are capable of infecting most commercial canola cultivars planted in North Dakota, are becoming widespread in the region. Until resistance cultivars become available growers will have to rely on fungicide applications to protect their crops. Fungicides like azoxystrobin and pyraclostrobin are recommended to be applied when plants are between the two and four-leaf growth stages; however, this period could last three to four weeks depending on weather. The objective of this study was to determine the effect of fungicide timings of application on control of blackleg. Twelve-days old seedlings of cultivar Westar were inoculated at the cotyledon stage with a spore mixture of five PG-4 isolates and sprayed in separate trials with commercial doses of these compounds at $-2,0,2,4,8$, or 16 days from inoculation. Each trial was replicated and conducted three times in greenhouse conditions using a randomized complete block design. In general, applications of either compound made within eight days from inoculation were effective $(P$ $=0.05)$ in controlling the disease. Applications made at later times resulted in severe damage to plants.

Rust fungi: The intersection of systematics and trade

J. DEMERS (1), M. Romberg (2), L. A. Castlebury (1)

(1) USDA ARS, Beltsville, MD, U.S.A.; (2) USDA APHIS, Beltsville, MD,

U.S.A.

Phytopathology 104(Suppl. 3):S3.31

The economically important rust fungi are among the most common fungal interceptions by the Customs and Border Protection Agency at U. S. ports of entry. Many intercepted specimens are found in baggage or as debris in cargo, complicating identification of the host and place of origin. Rust fungi can be difficult to identify based on morphology, particularly when the host is not known. In this study, a region of the large subunit rDNA was sequenced for 98 specimens that represent 58 species based on morphology. Only $38 \%$ of the species encountered had sequences available in GenBank. Phylogenetic analyses of the generated sequences and those available in GenBank revealed probable cryptic species in two frequently intercepted rusts, Puccinia malvacearum on hollyhock and Puccinia cnici-oleracei on Asteraceae. Host ranges and geographic distributions could change based on more accurate species concepts resulting in potential regulatory consequences. Although DNA sequence data is useful for identification of the host and the rust species, many intercepted species could not be identified based solely on the molecular data available prior to this work. Furthermore, as species are redefined based on molecular data, regulatory policies may need to be amended to reflect these changes. These results indicate a need for accurate molecular identification of rust fungi in quarantine situations and for more accurately identified and vouchered DNA sequences.

Secondary metabolite production in a newly discovered endophyte species of Veratrum californicum

\section{A. D. DENSLEY (1)}

(1) Brigham Young University, Provo, UT, U.S.A.

Phytopathology 104(Suppl. 3):S3.31

Veratrum californicum, a montane perennial of Western America, is known to produce several bioactive alkaloids with teratogenic, antibiotic and antiproliferative properties. The most notable is the anticancer compound cyclopamine, currently undergoing clinical trials. However, research has overlooked the potential for similar analog production by endophytes. Historically, endophytes of a bioactive host plant have proven to be rich sources of novel compounds. In this study, 44 unique endophytes were isolated from fresh plant matter and allowed to grow in potato dextrose broth for one week before undergoing liquid-liquid chemical extraction. Normalphase column chromatography further purified the extracts followed by MS with each fraction. One noteworthy endophyte produced peaks corresponding with several known teratogenic compounds of $V$. californicum. Other unknown compounds of similar masses present likely possess comparable molecular structure. NMR spectroscopy will confirm our results and identify the unknown structures. Subsequent experiments will confirm bioactivity. DNA sequencing indicated a new species of fungi closely related to cadophora luteo-olivacea, a pathogen documented in grapevine roots and Antarctica soils. These pathogenic properties in a sister fungus substantiate the likelihood of novel compound production. Undoubtedly, this new species of fungus serves as an abundant source of secondary metabolites both common and unique to its host plant.

Development of a multiplex qPCR assay for quantification of Plasmodiophora brassicae in soil

A. Deora (1), B. D. GOSSEN (2), S. Amirsadeghi (1), M. R. McDonald (1)

(1) University of Guelph, Guelph, ON, Canada; (2) Agriculture and AgriFood Canada, Saskatoon, SK, Canada

Phytopathology 104(Suppl. 3):S3.31

Physical and chemical components of soils can inhibit detection and quantification of soil-borne plant pathogens by polymerase chain reaction (PCR) assays. An accurate and sensitive assay was developed for quantification of resting spores of Plasmodiophora brassicae Woronin (cause of clubroot on Brassica spp.) using multiplexed TaqMan quantitative realtime PCR (qPCR). The standard curve for genomic DNA of $P$. brassicae was linear $\left(\mathrm{R}^{2}>0.99\right)$ from $10^{3}$ to $10^{8}$ resting spores and the assay detected as few as 200 spores/g of soil. The assay included a competitive internal positive control (CIPC) from the GFPuv1 gene, which was used to quantify inhibition 
of qPCR. Co-amplification of the P. brassicae gDNA and CIPC did not affect the sensitivity, specificity, or reproducibility of the assay. Samples exhibiting high level of inhibition were further diluted 5-or 10-fold and assayed again. In samples with moderate or low level of inhibition, the delay in CIPC amplification was used to correct the concentration of $P$. brassicae resting spores to account for losses from inhibition. The assay, which was suitable for use on a wide range of soil types, could reduce the frequency of false negatives, which can be a problem with conventional qPCR assays.

Effect of saponins on clubroot (Plasmodiophora brassicae) in canola and Shanghai pak choy

A. Deora (1), B. D. Gossen (2), J. Dalton (1), M. R. MCDONALD (1)

(1) University of Guelph, Guelph, ON, Canada; (2) Agriculture and AgriFood Canada, Saskatoon, SK, Canada

Phytopathology 104(Suppl. 3):S3.32

Plasmodiophora brassicae (Woronin) causes clubroot on many members of the Brassicaceae family. No information is available on the efficacy of saponins against clubroot. The present study assessed application of two saponins of plant origin on susceptible canola (Brassica napus L. cv. Westar) and Shanghai pak choy (B. rapa subsp. Chinensis cv. Mei Qing Choi) under controlled conditions. Vaccaria hispanica (cow cockle), a common weed on the Canadian prairies, was the source of saponin applied as a pre-plant drench application at $0,3,9,27$ and $81 \mathrm{~kg} / \mathrm{ha}$. Seed treatment of saponin from cow cockle and Heads Up (source: Chenopodium quinoa) applied at $28 \mathrm{~g} / 100 \mathrm{~kg}$ of seed were also examined. There were differences in clubroot incidence and severity among drench treatments for both crops, but seed treatment had no effect. Both incidence and severity decreased with increasing rate. At the highest rate, clubroot incidence and severity in canola were reduced by $43 \%$ and $52 \%$, respectively. Incidence and severity in Shanghai pak choy were reduced by $46 \%$ and $44 \%$ in one repetition of the study, and by $25 \%$ and $43 \%$ in a second repetition. Although saponins reduced clubroot severity, the rate required to obtain acceptable levels of reduction are not economical for canola production, and likely not economical for Brassica vegetables.

Screening of Pineapple mealybug wilt associated virus (PMWaVs) genome for suppressors of gene silencing

K. K. DEY (1), W. Borth (2), M. Melzer (2), J. Hu (2), M. L. Wang (3)

(1) University of Hawaii At Manoa, Honolulu, HI, U.S.A.; (2) University of Hawaii, Honolulu, HI, U.S.A.; (3) Hawaii Agricultural Research Center, Honolulu, HI, U.S.A.

Phytopathology 104(Suppl. 3):S3.32

Higher plants use post-transcriptional gene silencing (PTGS), an RNAdegradation system, to defend against viral infections. To counteract PTGS, plant viruses have evolved proteins that suppress PTGS. Mealybug wilt of pineapple (MWP), an important disease of pineapple, has been associated with at least three distinct viruses, Pineapple mealybug wilt associated virus-1, -2, and -3 (PMWaV-1, -2 and -3 ). Selected open reading frames (ORF's) of PMWaV-1 and PMWaV-2 were screened for their local and systemic suppressor activities using green fluorescent protein (GFP) in Agrobacteriummediated transient assays in Nicotiana benthamiana. The ability of these proteins to suppress local silencing was assessed 5 days post-infiltration (dpi) by monitoring GFP fluorescence, GFP mRNAs, and GFP-specific small interfering RNAs (si-RNAs) in infiltrated leaves of wild-type $N$. benthamiana . Suppression of systemic silencing was assessed by monitoring suppression of GFP silencing in newer, non-inoculated leaves of transgenic $N$. benthamiana plants that constitutively express GFP. The coat protein (CP), and p20 protein of PMWaV-2 produced greater fluorescence than negative controls in local suppressor assays. Northern analyses showed that this increased GFP fluorescence was correlated with increased levels of GFP mRNA at 5dpi. Interestingly, the $\mathrm{CP}$ suppressed systemic silencing for an extended period in uninfiltrated leaves and also suppressed local silencing in infiltrated leaves.

Development of glasshouse bioassay for assessing resistance to verticillium wilt disease in potato

V. N. DHARJONO (1), N. S. Crump (2), T. Wiechel (3), P. W. J. Taylor (1)

(1) The University of Melbourne, Parkville, Australia; (2) ViCSPA, Toolangi, Australia; (3) Department of Environment and Primary Industries, Bundoora, Australia

Phytopathology 104(Suppl. 3):S3.32

Verticillium dahliae is a major and persistent soil pathogen known to cause verticillium wilt in potato (Solanum tuberosum L.). Resistance in commercial Australian potato cultivars is unknown. The threshold level of inoculum required to establish infection, symptom development and differentiate host reactions needs to be determined before a glasshouse screening trial can be undertaken. Different inoculum concentrations were assessed using root dip inoculation method of seedlings established from tissue culture for susceptible potato cv. Russet Burbank. The seedlings roots were dipped in water as a control and in six different inoculum concentrations $10^{6}, 10^{5}, 10^{4}, 5 \times 10^{3}, 10^{3}$, and $10^{2}$ spores $/ \mathrm{mL}$ for five minutes, followed by transferring plants to potting mixed. Similar experiments were conducted on eggplant seedlings (cv. Black Beauty). The eggplant bioassay was found to be less time consuming and more cost effective compared to tissue culture potato plant bioassay. Severity of foliar symptoms was determined using a $0-5$ visual qualitative scale. An inoculum threshold level of $10^{4}$ spores $/ \mathrm{mL}$ was required to establish infection and wilt symptoms in both eggplant and potato plants, although $V$. dahliae was isolated from petiole tissue plants inoculated with as low as $10^{2}$ spores $/ \mathrm{mL}$. In glasshouse potato cultivar screening trials using $5 \times 10^{4}$ spores $/ \mathrm{mL}$, cultivars Denali showed moderate to high resistance and Catani and Desiree moderate resistance to infection by $V$. dahliae.

Detection of quarantine pathogens with bio-layer interferometry-based BLITZ system

R. DI (1), L. Zhao (2), L. Levy (2)

(1) Rutgers University, New Brunswick, NJ, U.S.A.; (2) USDA-APHISCPHST, Beltsville, MD, U.S.A.

Phytopathology 104(Suppl. 3):S3.32

We have previously used surface plasmon resonance (SPR)-based technology to detect quarantine pathogen Ralstonia solanacearumr race 3 biovar 2 (R3b2) from devitalized cultures and in spiked geranium samples. The portable SPR detection platform, SPIRIT V4, with a monoclonal antibody that is more specific for R3b2 from Forsite Diagnostics Inc., can detect as low as 9 microgram/ml UW551 (R3b2) in PBS buffer and 90 microgram/ml UW551 spiked into the healthy geranium extract. The detection process takes 7 to 30 min. Here, we report the application of an SPR variant technology, bio-layer interferometry (BLI), for the detection of $R$. solanacearum R3b2. BLI provides the advantages of being easier to operate, using cheaper biosensors and requiring less reagents compared to the SPR detection platform evaluated. Our data have shown that as low as 5 microgram/ml UW551 bacterial cells in PBS buffer can be detected by the Forsite $R$. solanacearum monoclonal antibody-coupled biosensor with the BLI-based portable BLITZ device in 10 min. The portability of the BLITZ system makes it possible to use it at the ports and diagnostic labs.

Additional complexity identified in Rose rosette virus, the causal agent of the homonymous disease

P. DI BELLO (1), T. Ho (1), I. E. Tzanetakis (1)

(1) University of Arkansas, Fayetteville, AR, U.S.A.

Phytopathology 104(Suppl. 3):S3.32

Rose rosette virus (RRV) was found perfectly associated with the homonymous disease, but has not been proven as the causal agent of the disorder. To fulfill molecular Koch's postulates eriophyid mites were collected from rosette-affected roses, and transferred to RRV and symptomsfree roses, to selectively transmit the rosette agent. Some roses developed typical RRD symptoms and tested positive for the virus. RNA was extracted from one of these roses, DOP-PCR was performed, and the product was sequenced on a pyrosequencing platform. The majority of the reads matched with high identity to RRV RNAs 1 to 4 . In addition, several reads were found to code for virus-like proteins of negative sense RNA viruses. Those sequences were characterized in depth and determined to be four additional RNA of RRV. No other plant viruses were found in the sequencing data satisfying molecular Koch's postulates, and proving that RRV causes rosette disease.

The population biology of the Colletotrichum truncatum from chili peppers in China

Y. DIAO (1), C. Zhang (1), P. Liu (1), O. G. Mtung'e (1), X. Liu (1)

(1) Department of Plant Pathology, College of Agriculture and Biotechnology, China Agricultural University, Beijing, China

Phytopathology 104(Suppl. 3):S3.32

Colletotrichum truncatum is a major fungal pathogen of peppers and China is one of the main pepper producing countries in the world. However, almost nothing is known about the population biology of this pathogen in China. We obtained and analyzed $266 C$. truncatum isolates from 13 regions representing the main distributions throughout China. Our genotypic analyses using nine microsatellite markers identified high intra-population genetic diversity, evidence of sexual recombination, and significant geographic differentiation. The genetic differentiation was positively correlated with geographical distance, with the southern and northern China populations grouped in two distinct clusters. Interestingly, the pepper-breeding center with the highest host genetic diversity also harbored the most private alleles. Overall, regions with intermediate temperatures had higher pathogen genotype diversity than those with low or high mean temperatures. Our phenotypic analyses largely 
supported the north-south divide and showed that the southern populations were less sensitivity to the fungicide prochloraz than northern populations. However, fungicide resistance was negatively correlated with mycelial growth rate in the absence of the drug, consistent with a cost associated with fungicide resistance. Our results showed the most significant differentiations between northern and southern Chinese populations of $C$. truncatum from chili peppers in both genotypes and phenotypes.

\section{Diaporthe species identified from postharvest rot on kiwifruits during} long term storage

G. A. DIAZ (1), B. A. Latorre (1), S. Jara (1), E. Ferrada (1), P. Naranjo (1), J. P. Zoffoli (1)

(1) Pontificia Univ Catolica de Chile, Santiago, Chile

Phytopathology 104(Suppl. 3):S3.33

Species of Diaporthe (Phomopsis) cause diseases of a wide range of plants including postharvest patch decay of kiwifruit (Actinidia deliciosa) that eventually can affect the entire fruit. Prevalence of $1 \%$ of Diaporthe rot was estimated in Chilean packinghouses in 2013. Recently, D. ambigua was reported in kiwifruit in Chile. However, it has been postulated that several Diaporthe species may be associated with kiwifruit rot in stored fruits. In this study the Diaporthe spp. causing decay after long term storage of kiwifruit were identified. Kiwifruits $(\mathrm{n}=354)$, showing soft rot in controlled atmosphere $\left(5 \% \mathrm{CO}_{2}\right.$ and $\left.2 \% \mathrm{O}_{2}\right)$ at $0^{\circ} \mathrm{C}$ for $90 \mathrm{~d}$, were surface disinfected and small pieces of rotten tissues were placed on PDA for $7 \mathrm{~d}$ at $20^{\circ} \mathrm{C}$. Colonies (n $=17$ ) that developed black pycnidia producing ovoid hyaline and biguttulate conidia were tentatively identified as Diaporthe spp. Species were identified by molecular analysis of the ITS region, beta-tubulin and translation elongation factor 1- $\alpha$. D. novem, D. ambigua, D. australafricana and $D$. viticola were identified, being $D$. novem the most frequently species obtained. Pathogenic was demonstrated in kiwifruits inoculated with mycelium at $0^{\circ} \mathrm{C}$. In conclusion, multiple species of Diaporthe can be associated with soft rot during long term storage. Interestingly, $D$. actinidiae, a commonly found Diaporthe species described in kiwifruit, was not found in this study.

\section{Genetic diversity of Leptosphaeria spp. from winter canola in Oklahoma}

\section{DIAZ (1)}

(1) Oklahoma State University, Stillwater, OK, U.S.A.

Phytopathology 104(Suppl. 3):S3.33

Black leg, caused by the fungus Leptosphaeria maculans, is a widespread disease of winter canola (Brassica napus) in Oklahoma. L. maculans has a single mating type $(M A T)$ locus with two alternate alleles, each needed for isolates to mate. Sexual reproduction is important in the disease cycle because the airborne ascospores are the primary inoculum and genetic variation may lead to new races. In order to determine the frequency of mating types, genomic DNA was isolated from mycelia of 93 isolates of Leptosphaeria spp. from different fields and years. Specific primers for the two alternate forms of the MAT locus were used in a multiplex polymerase chain reaction (PCR). A PCR product of $686 \mathrm{bp}$ was amplified for MAT1-1 isolates, and a $443 \mathrm{bp}$ fragment was amplified for MAT1-2 isolates. MAT1-1 isolates (52\%) and MAT1-2 isolates (48\%) occurred at similar frequencies in the population. Avirulence genes in L. maculans (AvrLm) interact with specific resistance genes in winter canola $(R l m)$. Inoculation of differential cultivars harboring $R \operatorname{lm} 1, R \operatorname{lm} 2$, and $R \operatorname{lm} 3$ has revealed the presence of virulence alleles avrLm1, avrLm2 and avrLm3 in $61 \%$ of the population. PCR analyses are being conducted to confirm the frequency of AvrLml and to determine the frequency of AvrLm4-7 and AvrLm6 in the population in order to better characterize the local race structure of Leptosphaeria spp.

\section{A new virus isolated from blueberry exhibiting a complete fruit drop} symptom

A. DIAZ-LARA (1), K. E. Keller (2), R. R. Martin (2)

(1) Oregon State University, Corvallis, OR, U.S.A.; (2) USDA-ARS Horticultural Crops Research Unit, Corvallis, OR, U.S.A.

Phytopathology 104(Suppl. 3):S3.33

Blueberry (Vaccinium corymbosum) is an important fruit crop in the Pacific Northwest. Recently, a fruit drop symptom has been observed in several fields of cv. Bluecrop, in the Fraser Valley in Washington and British Columbia. Also, it was observed that young leaves showed a transient red coloration during the bloom period and the corolla of the flowers exhibited some red striping. After bloom the plants appear normal until about three weeks prior to harvest, when the fruit drops. Prior to harvest, affected bushes can be identified easily since they stand upright. Previously, a cryptic virus had been characterized that was not associated with the disease, as it was widespread in all production areas. Using Rolling Circle Amplification, enzymatic digestion, cloning and primer walking, a novel virus was isolated and sequenced from samples showing symptoms described above. Using BLAST, it was found that the obtained sequence had a high degree of homology with Dahlia mosaic virus and Cauliflower mosaic virus, both viruses belonging to the family Caulimoviridae. The new virus (provisionally named Blueberry fruit drop associated virus) had a genome of approximately $9500 \mathrm{bp}$, which is the largest caulimovirus known. Detection primers were designed that amplified a $350 \mathrm{bp}$ amplicon and confirmed the presence of the virus from symptomatic plants but not from healthy plants. Unique features of this virus will be discussed along with symptoms in other cultivars.

A variant of Rubus yellow net virus with a different genomic arrangement A. DIAZ-LARA (1), N. J. Mosier (2), K. E. Keller (2), R. R. Martin (2)

(1) Oregon State University, Corvallis, OR, U.S.A.; (2) USDA ARS Horticultural Crops Research Unit, Corvallis, OR, U.S.A.

Phytopathology 104(Suppl. 3):S3.33

Rubus yellow net virus (RYNV) is a member of the family Caulimoviridae, genus Badnavirus. RYNV infects Rubus species causing chlorosis of the tissue along the leaf veins giving an unevenly distributed netted symptom in some cultivars of red and black raspberry. Recently, this virus was isolated and characterized from native $R$. strigosus plants in Alberta, Canada, that showed symptoms similar to those described above. The viral genome contained seven open reading frames with most of them in the sense-strand, including a large polyprotein. This isolate from Canada (RYNV-Ca) is the only complete sequence of RYNV available in GenBank. Here we describe a graft transmissible strain of RYNV from Oregon (named RYNV-OR), which was sequenced using Rolling Circle Amplification, enzymatic digestion, cloning and primer walking, plus resequenced at a $5 \mathrm{X}$ coverage. This sequence was then compared with the RYNV-Ca genome and significant differences were observed. Genomic analysis identified changes in the arrangement of coding regions, promoter elements and motif presents. Genomic structure of RYNV-OR consisted of six ORF's (five ORF's in the sense-strand and one ORF in antisense-strand). ORFs 1, 2, 3, 4 and 6 showed a high degree of homology to RYNV-Ca, on the other hand ORF 5 of the RYNV-OR was quite distinct. Also, the predicted ORF 7 in the RYNV-Ca was absent in the RYNV-OR sequence. These differences that may account for the lack of aphid transmissibility of RYNV-OR.

\section{ChemView: Making data on chemicals publicly available}

M. Doa (1), P. LEWIS (1), S. Canavan (1), Y. Gonzalez (1)

(1) Environmental Protection Agency, Washington, DC, U.S.A

Phytopathology 104(Suppl. 3):S3.33

EPA has implemented an innovative conceptual approach to improve chemical safety and streamline access to information on chemicals regulated under the Toxic Substances Control Act (TSCA) by developing a publicly accessible, easy-to-use online database called ChemView (www.epa gov/chemview). ChemView allows users to view information in both summary form and in detail, including links to documents provided to EPA by the regulated community and others, significantly enhancing access to chemical information in a one-stop shop venue. The information made available in ChemView will be useful for assessing exposures to chemicals, as well as the hazards chemicals pose to human health and the environment. Through ChemView, EPA is now able to provide downstream users of chemicals, those much closer to the consumer with easy access to information they can use to make safer chemical choices. The project entailed identifying, organizing and extracting human health, ecotoxicological, environmental fate and product chemistry data to populate ChemView so users could access at a glance, summary and in-depth information of their choosing. ChemView does not contain any Confidential Business Information (CBI). While the current version of ChemView contains a substantial amount of information, when fully populated, ChemView will contain data for thousands of chemicals regulated under TSCA.

Efficacy of seed treatments on viability of Fusarium oxysporum f. sp. vasinfectum race 4 in infected cotton seed

H. DOAN (1), R. M. Davis (2)

(1) University of California, Davis, CA, U.S.A.; (2) University of California, Davis Department of Plant Pathology, Davis, CA, U.S.A.

Phytopathology 104(Suppl. 3):S3.33

Fusarium wilt caused by Fusarium oxysporum f. sp. vasinfectum (FOV) race 4 is found in an increasing number of fields in California, due at least in part, to seedborne dissemination. The disease can quickly become epidemic as residue from infected plants release numerous propagules into the soil, potentially increasing disease severity in subsequent cotton crops. Detection of the pathogen in seed is possible with race 4-specific primers, but due to the relatively large size of cotton seed and the high plant densities used in cotton production, sampling a seedlot makes seed assays problematic. The aim of this study was to evaluate efficacy of hot water treatments alone or in 
conjunction with fungicides, potato dextrose broth, bleach, or sodium lauryl sulfate treatments on viability of FOV in infected cotton seeds. To determine the best temperatures and times combination to treat seeds, a series of hot water treatments from 55 to $90^{\circ} \mathrm{C}$ for various lengths of time were evaluated. Seed of all tested varieties remained viable and lost no seedling vigor after immersion in hot water at $60^{\circ} \mathrm{C}$ for 20 minutes. In the 20 minutes at $60^{\circ} \mathrm{C}$ hot water bath, seed infection was reduced from $39.2 \%$ to $15.6 \%$. When seed were pre-treated in potato dextrose broth for 1 hour at $23^{\circ} \mathrm{C}(100 \mathrm{rpm})$ followed by 20 minutes at $60^{\circ} \mathrm{C}$ hot Topsin ${ }^{\circledR}$ fungicide bath, seed infection was reduced from $39.2 \%$ to $15.6 \%$.

Screening for plant viruses including TMV, HVX, CMV, TSWV and INSV in ornamental and nursery crops from four states of the United States S. DOBHAL (1), M. Arif (1), J. Olson (2), A. Mendoza-Yerbafría (1), S. Aguilar-Moreno (1), M. Perez-Garcia (1), F. M. Ochoa-Corona (1)

(1) National Institute for Microbial Forensics \& Food and Agricultural Biosecurity, Department of Entomology and Plant Pathology, Oklahoma State University, Stillwater, OK, U.S.A.; (2) Department of Entomology and Plant Pathology, Oklahoma State University, Stillwater, OK, U.S.A.

Phytopathology 104(Suppl. 3):S3.34

Tobacco mosaic virus (TMV), Hosta virus X (HVX), Cucumber Mosaic Virus (CMV), Tomato spotted wilt virus (TSWV), and Impatiens necrotic spot virus (INSV) are a few of the major viruses that infect ornamental and nursery plants. These viruses cause significant losses that impact growers in the ornamental industry. Often a single plant is co-infected with multiple viruses which make identification of virus a difficult task. To address this, an end point multiplex RT-PCR was developed (Phytopathology 103:S2.9). The sensitivity of this method for detection is $100 \mathrm{fg}$ for TMV, $1 \mathrm{fg}$ for HVX, 10 fg for CMV, $10 \mathrm{pg}$ for TSWV and INSV. Forty six plant samples which include 25 different host species from different locations from Oklahoma, Texas, Illinois and Pennsylvania were screened. Among the samples tested, 30 were found to be positive for the plant viruses. However, nine samples were found to be infected with more than one virus and seven samples were found to be co-infected with two different viruses. One sample (Lobelia) was found to be co-infected with four (TMV, CMV, TSWV and INSV) and Petunia was co-infected three (TMV, TSWV and INSV) viruses. None of the samples tested positive for all five viruses simultaneously. Our results have important implications in providing an effective method for detection of mixed target infections in a host species. This method has a potential application in disease management and epidemiological studies.

Development of endpoint RT-PCR for Rose Rosette virus and screening of Rosa spp. from eight states of the United States

S. DOBHAL (1), J. Olson (2), M. Arif (1), J. A. Garcia Suarez (1), F. M. Ochoa-Corona (1)

(1) National Institute for Microbial Forensics \& Food and Agricultural Biosecurity, Department of Entomology and Plant Pathology, Oklahoma State University, Stillwater, OK, U.S.A.; (2) Department of Entomology and Plant Pathology, Oklahoma State University, Stilllwater, OK, U.S.A.

Phytopathology 104(Suppl. 3):S3.34

Rose rosette disease is a recalcitrant disorder associated with a newly characterized Emaravirus (Rose rosette virus, RRV). Rose rosette disease has progressively spread through the continental United States and may be devastating to most cultivated Rosa spp. Early detection of this disease is important for control measures. Sequences of the nucleocapsid protein gene located in RNA 3 of RRV were retrieved from NCBI and aligned using CLUSTALX2. A primer pair (RRV 2F and RRV 2R) were designed from selected conserved regions using the web application Primer 3 and the thermodynamic features of each primer was tested using mFOLD. A multitarget artificial positive control for routine diagnostics was also constructed to include the RRV primers. Reference primers targeting the plant gene Nad5 were also used for internal control. The sensitivity of the primer pair RRV2F/2R was assessed using plasmid DNA and found to be $1 \mathrm{fg}$. Twentyeight Rose spp. samples from 8 different states of the United States were screened using primers RRV2F/2R and endpoint RT-PCR. The expected amplification product is $108 \mathrm{bp}$. All collected samples tested positive, showing broad range detection of RRV among samples collected from different geographical locations. This endpoint RT-PCR method is sensitive, reliable and has potential to be used in routine diagnostics laboratories for disease management decisions.

Comparison of end point RT-PCR and RT-HDA using primer pairs for five viruses frequently infecting ornamental and nursery crops S. DOBHAL (1), M. Arif (1), J. Olson (2), F. M. Ochoa-Corona (1)

(1) National Institute for Microbial Forensics \& Food and Agricultural Biosecurity, Department of Entomology and Plant Pathology, Oklahoma State
University, Stillwater, OK, U.S.A.; (2) Department of Entomology and Plant Pathology, Oklahoma State University, Stillwater, OK, U.S.A.

Phytopathology 104(Suppl. 3):S3.34

Tobacco mosaic virus (TMV), Hosta virus X (HVX), Cucumber Mosaic Virus (CMV), Tomato spotted wilt virus (TSWV), and Impatiens necrotic spot virus (INSV) cause significant losses, reducing both quality and yield of ornamental crops. Specific primer pairs were designed to amplify regions from a conserved segment of the capsid protein gene of HVX and CMV; nucleocapsid protein gene of TSWV and INSV, and movement and capsid protein gene of TMV. Methods used were RT-PCR and RT-HDA (Helicase Dependent Amplification), which does not require a thermal cycler. The specificity and sensitivity of both methods were assessed using plasmid DNA and infected host tissue. For plasmid DNA, the sensitivity of RT-PCR and RT-HDA were similar ( $1 \mathrm{fg}$ ) if detecting TMV, HVX, and CMV. Whereas, RT-HDA was more sensitive (1fg) compared to RT-PCR (10 fg) if detecting TSWV and INSV. For infected host tissue, the sensitivity of RT-PCR was $1 \mathrm{fg}$ for HVX and CMV. Similarly, the sensitivity of RT-PCR was $10 \mathrm{fg}$ for TMV, TSWV and INSV. RT-HDA was found to be sensitive (1fg) than RT-PCR (10 fg) when detecting TMV and INSV. The sensitivity of RT-HDA was $10 \mathrm{fg}$ for CMV and $100 \mathrm{fg}$ each for HVX and TSWV. These five primer pairs were highly sensitive for detecting TMV, HVX, CMV, TSWV and INSV using these two DNA amplification methods. Either method will assist with the rapid detection of any of these viruses and will facilitate integrated pest management decisions for these plant viruses.

\section{Influence of sugar beet cultivar resistance to Cercospora leaf spot} threshold-reach and disease management

N. S. DOLOVAC (1), N. R. Trkulja (1), A. G. Milosavljevic (1), E. L. PfafDolovac (1)

(1) Institute for Plant Protection and Environment, Belgrade, Serbia Phytopathology 104(Suppl. 3):S3.34

Cercospora beticola, the causal agent of Cercospora leaf spot is the most serious disease of sugar beet worldwide. Use of resistant cultivar of sugar beet and fungicide application is the main mechanism of disease control. In the 2011, at two localities (Srem and South Banat), the disease incidence threshold (DI $=5 \%)$ is accompanied on two sensitive (Asketa and Solea) and two resistant cultivars (Marianka and Leopard) in order to make decision for first treatment of fungicides. Monitoring of disease symptoms was started after canopy closure growth stage $(\mathrm{BBCH} 39)$ and every two days was repeated. In all cultivars about 100 leaves were randomly selected by walking diagonally in a field. Procedure was repeated four times and average DI was calculated. After threshold was reached, three treatments with trifloxistrobincyproconazole fungicide were applied to resolve impact of cultivar resistance on disease management. At both localities threshold was reached first in Asketa and Solea cultivar 28th June, while in Leopard and Marianka one week later, 6th and 8th July at locality South Banat and Srem respectively. Most effective disease control was achieved by using trifloxistrobincyproconazole with Marianka cultivar and then Leopard, while in the fields with Asketa and Solea cultivars disease control was significantly lower. The results indicated great impact of sugar beet cultivar resistance to C. beticola on threshold-reach and disease management of Cercospora leaf spot.

Spatio-temporal spread of Grapevine leafroll disease in Washington vineyards

B. P. DONDA (1), R. Naidu (1)

(1) Washington State University, Prosser, WA, U.S.A.

Phytopathology 104(Suppl. 3):S3.34

Grapevine leafroll disease (GLD) is one of the major constraints for Washington's wine grape industry. We have been monitoring spatial and temporal spread of GLD in young vineyard blocks planted with virus-tested, own-rooted grapevine (Vitis vinifera L.) cultivars and located in close proximity to heavily infected old vineyard blocks. Symptomatic and asymptomatic grapevines, that are immediately adjacent to symptomatic plants, were tested by RT-PCR for the presence of Grapevine leafrollassociated virus 3 (GLRaV-3, genus Ampelovirus, family Closteroviridae). A comparison of the disease incidence obtained from each block in 2013 season with data obtained from previous seasons showed higher number of grapevines with GLD symptoms in each season compared to previous season, suggesting increased disease incidence with time in young plantings. The spatial distribution of symptomatic grapevines showed a disease gradient in which the highest percentage of symptomatic grapevines were located in rows closest to the heavily infected old blocks and the disease incidence declined in rows with increasing distance from old blocks. Furthermore, the spatial distribution of symptomatic grapevines showed an aggregated pattern, suggesting vine-to-vine spread within individual rows in young blocks. Cloning and sequencing a portion of the heat-shock protein-70 homolog of 
GLRaV-3 confirmed virus spread from neighboring blocks to young vineyards planted with virus-tested planting stock.

Mcg1, a NDT80/PhoG protein, controls completion of tumour maturation, teliospore development and meiosis in Ustilago maydis

C. DOYLE (1), H. K. Cheung (1), K. L. Spence (1), B. J. Saville (1)

(1) Trent University, Peterborough, ON, Canada

Phytopathology 104(Suppl. 3):S3.35

The model fungal plant pathogen, Ustilago maydis, requires growth in the host for sexual development and meiosis. Hence, meiotic initiation must be triggered by cues received from the plant during pathogenic development. To investigate this process, an ortholog of Saccharomyces cerevisiae meiotic control protein, Ndt80, which we termed Mcg1 (meiosis control gene 1) was identified in $U$. maydis. This is the only Ndt80/PhoG protein in U. maydis and it has orthologs with conserved synteny in other smut fungi. Infections with compatible $\Delta m c g 1$ strains were fully pathogenic, but teliospore development terminated early and teliospores germinated without completing meiosis. This suggests that Mcg1 is involved in the regulation of meiosis and teliospore formation. Microscopic observations in planta bear this out, with $\Delta m c g 1$ infections developing normally but producing fewer teliospores that do not fully mature. Complementation of the $\Delta m c g l$ mutants with wildtype $m c g l$ restores normal teliospore formation, and meiosis. Analysis of 1) strains created in which the Mcg1 protein is modified, 2) genes with altered transcript levels in $4 m c g l$ strains, and 3) motifs identified upstream of putative Mcg1 target genes, all suggest that Mcg1 acts as a transcription factor, which shares similar DNA binding properties to Ndt80. Together, these analyses identify components of a control pathway for in planta teliospore development in smut fungi.

Development of a PCR-RFLP based detection method for the oak pathogens Diplodia corticola and D. quercivora

T. DREADEN (1), J. Smith (1)

(1) University of Florida, Gainesville, FL, U.S.A.

Phytopathology 104(Suppl. 3):S3.35

The canker pathogens Diplodia corticola and D. quercivora were first reported affecting live oak (Quercus virginiana) in the United States in 2010. A rapid and cost effective method to identify isolates of these closely related fungal species was needed to facilitate survey and detection efforts. This was achieved by developing selective primers that generate a PCR product, from both taxa, followed by a restriction enzyme digestion with $M s e I$ and agarose gel electrophoresis to differentiate $D$. corticola from $D$. quercivora amplicons. The new method will allow for rapid and accurate diagnosis and monitoring for these potentially damaging pathogens of oak trees.

The biology of two canker-causing fungi on quaking aspen (Populus tremuloides)

M. DUDLEY (1), N. Tisserat (1), J. Negron (2)

(1) Colorado State University, Fort Collins, CO, U.S.A.; (2) USDA Forest Service, Fort Collins, CO, U.S.A.

Phytopathology 104(Suppl. 3):S3.35

We are investigating the relative abundance and pathogenicity of two species of fungi (Valsa sordida and V. notastroma) which cause Cytospora canker on quaking aspen (Populus tremuloides). Results from our previous survey of aspen health clearly indicated that Cytospora is the most common canker disease on Colorado aspen. Classification of this group of fungi has historically been very difficult, due to the great variability of morphological characteristics; nearly a dozen different Cytospora anamorphs have been described on aspen. Isolates were cultured from infected bark samples collected throughout Colorado, southern Wyoming, and Minnesota. To identify species and isolates within species, we are using genetic markers (SNPs) within the rDNA ITS, EF- $1 \alpha$, and $\beta$-tubulin genes. The two species appear to have similar distributions, and we have identified distinct isolates of each. We are also assessing the relative pathogenicity of the two Valsa species through several inoculation trials of young aspen trees. Preliminary analysis indicates that among drought-stressed trees, $V$. sordida is more pathogenic than $V$. notastroma, based on canker size. There were small but significant differences in overall canker size between isolates of each of the two species. Among the well watered control group of trees, no cankers formed. Finally, we are investigating a potential vector for Valsa, the aspen bark beetle (Trypophloeus populi), which appears to occur with Cytospora canker.

The effect of climatic events on the growth of quaking aspen (Populus tremuloides)

M. DUDLEY (1), N. Tisserat (1), J. Negron (2), W. Jacobi (1)

(1) Colorado State University, Fort Collins, CO, U.S.A.; (2) USDA Forest Service, Fort Collins, CO, U.S.A.

Phytopathology 104(Suppl. 3):S3.35
Widespread dieback of quaking aspen has been observed throughout Colorado and elsewhere since the late 1990s. In Colorado, in the years following the severe drought of 2002, stand mortality intensified, and surveys were initiated to investigate potential causal agents. We conducted health surveys of both healthy and dying aspen stands throughout CO and southern WY in 2009 and 2010. During that time, we collected increment cores from live, standing adult aspen in all healthy and damaged stands surveyed. Currently, we are investigating the influence of drought events on the growth of aspen in Colorado since 1909. We processed increment cores using standard dendrochrological techniques. All cores were cross-dated and annual rings were measured using an increnometer. Increment data was checked for errors (using COFECHA) and all non-climate related data trends were removed (using ARSTAN). A spatial drought index (obtained from the USFS) was used to derive yearly drought severity values for all plot (core) locations. Analysis indicates that stands located along the southern Front Range of Colorado responded strongest to drought episodes over the last century. Pearson correlations with drought data for the Pike and San Isabel national forests were $0.35(\mathrm{P}=0.004)$ and $0.25(\mathrm{P}=0.0113)$, respectively. Data from trees in the two northern and western forests (the White River and Medicine Bow-Routt N.F.) did not correlate well with drought data (correlations were below 0.10).

\section{Identification of Lasiodiplodia theobromae from novel sources in Nigeria and Mexico}

F. M. DUGAN (1), A. Osuagwu (2), E. A. Uyoh (2), E. Okpako (2), S. L. Lupien (1), T. Kisha (1)

(1) USDA ARS WRPIS, Pullman, WA, U.S.A.; (2) University of Calabar, Calabar, Nigeria

Phytopathology 104(Suppl. 3):S3.35

Lasiodiplodia theobromae is a pan-tropical plant pathogen, allied with several newly described, formerly cryptic species. In 2012, we consistently isolated from asymptomatic seeds of Tetrapleura tetraptera, a medicinal and culinary plant native to Nigeria, and in 2010 from fruit of Cocos nucifera, imported from Mexico and purchased at a local retail outlet, fungi conforming to the standard morphological description for L. theobromae. On the basis of DNA sequences of elongation factor 1 gene, two isolates from Tetrapleura were 98\% identical to CBS 289.56 (from sail-cloth in New Guinea), 99\% homologous to "ex-type" CBS 111530 (from Leucospermum sp. in Hawaii), and $100 \%$ homologous to ex-type CBS 164.96 (from unknown fruit in Papua New Guinea); and $98 \%$ or less to other Lasiodiplodia spp., including $L$. pseudotheobromae. The coconut isolate was $98 \%$ homologous with CBS 164.96, 99\% with CBS 111530 and CBS 287.47 (from Musa sapientum, locale unknown), and $100 \%$ homologous with CBS 289.56 ; and $97 \%$ or less to other Lasiodiplodia spp., (e.g., L. pseudotheobromae). Coconut and Tetrapleura isolates were $98 \%$ homologous to each other. CBS 111530 was referenced as "ex-type" in 2011 but without formal designation. CBS 164.96 was formally designated ex-type in 2013. The name L. theobromae is based on Botryodiplodia theobromae Patouillard (1892) from Theobroma cacao in Ecuador. We have not encountered prior reports of $L$. theobromae from coconut fruit in Mexico, nor from Tetrapleura.

Inactivation of $r s m D$ of the soft rotting Pectobacterium atrosepticum leads to overproduction of exoenzymes and hypervirulence

K. DUMENYO (1), C. M. Kersey (2), B. Hageman (1)

(1) Tennessee State University, Nashville, TN, U.S.A.; (2) Department of Biological, Physical, and Human Sciences, Freed Hardeman University, Henderson, TN, U.S.A.

Phytopathology 104(Suppl. 3):S3.35

Pectobacterium atrosepticum $(\mathrm{Pa})$, a member of soft rot Enterobacteriaceae is an economically important pathogen that causes soft rot of potato tubers and blackleg of stems in the field. Virulence is regulated by, among other things, post-transcriptional Rsm system made up of RNA-binding RsmA and the noncoding rsmB RNA. Studies with the homologous Csr system of $E$. coli suggest an additional member, (designated $r s m D$ ) could be involved in this regulation. The cosmid clone of $r s m D^{+}$DNA from Pa strain SCRI 1043 was isolated, inactivated and use to construct $\mathrm{RsmD}^{-}$strain. Compared to the parental $\mathrm{RsmD}^{+}$strain, the RsmD mutant overproduced pectate lyase (Pel), cellulase $(\mathrm{Cel})$ and protease (Prt) in both minimal and host extract-supplemented media and macerated more celery petioles. By contrast, the $\mathrm{RsmD}^{-}$strain produced less extracellular polygalacturonase (Peh) than the $\mathrm{RsmD}^{+}$parent. A wild type extra-chromosomal copy of $r s m D$ complemented the altered Pel, Prt, Cel and virulence phenotype of the mutant. Similarly, multicopy $r s m A$, encoding the post-transcriptional regular, suppressed, Pel, Prt, Cel and virulence. The extrachromosomal copy also suppressed the levels of these enzyme and virulence in the parent. These data suggest that $r s m D$ is a component of the post-transcriptional Rsm system in $\mathrm{Pa}$ and could function analogous to $\operatorname{crs} D$ of $E$. coli. 
Variation in host response to Septoria canker in a population of Populus nigra

K. L. DUNNELL (1), W. Berguson (2), B. McMahon (2), J. M. LeBoldus (1) (1) North Dakota State University, Fargo, ND, U.S.A.; (2) University of Minnesota Duluth, Duluth, MN, U.S.A.

Phytopathology 104(Suppl. 3):S3.36

Septoria musiva causes stem cankers on hybrid poplar impacting the sustainability of commercial poplar plantations across eastern and central North America. The objective of this study was to characterize the variability in Septoria canker resistance in Populus nigra, a parent species commonly used in hybrid poplar breeding programs. Fifty vegetatively propagated genotypes of $P$. nigra, from seven locations in Europe, were inoculated with six isolates of $S$. musiva. Three weeks after inoculation disease severity was estimated in two ways: the number of necrotic lesions (cankers) $\mathrm{cm}^{-1}$ and a disease severity rating (1-resistant to 5 -susceptible). The results indicated that location explained $72 \%$ and $26 \%$ of the variation, respectively, while clone nested within location explained $28 \%$ and $51 \%$ of the variation, respectively. A second experiment was conducted to determine the relationship between mortality and disease severity. Thirteen genotypes with a range of disease severities (1-5) were selected and inoculated with a bulk spore suspension of six isolates. Host response was evaluated after three weeks, as described above, and mortality was determined at 12 weeks. Results indicated a similar range of disease severities as the first experiment. A threshold of 0.1 cankers $\mathrm{cm}^{-1}$ resulted in $>80 \%$ mortality of each genotype. The variability of resistance to $S$. musiva indicates that it is possible to select resistant genotypes of $P$. nigra for hybrid poplar breeding programs.

Interaction of Bean pod mottle virus, Soybean mosaic virus and Reniform nematode on yields of soybean

L. T. DUONG (1), R. B. Sikkens (1), J. F. Murphy (1), K. S. Lawrence (1)

(1) Auburn University, Auburn, AL, U.S.A.

Phytopathology 104(Suppl. 3):S3.36

While Bean pod mottle virus (BPMV), Soybean mosaic virus (SMV) and Reniform nematode (Rotylenchulus reniformis) (RN) individually cause severe yield losses in soybean (Glycine max. L.), it is believed that a virus and nematode combination can further exacerbate the losses. An experiment combining both viruses with- and without $\mathrm{RN}$ on two conventional soybean varieties (Hutcheson and Hartwig) were conducted on microplots in Auburn, Alabama. Under RN-free conditions, SMV caused yield losses ranging from $32 \%$ to $41 \%$ on Hartwig and Hutcheson, respectively. Yield losses due to BPMV amounted to $31 \%$ and $57 \%$ for these two varieties under similar RNfree conditions. In our experiment, yield losses did not increase significantly due to the combination of one of the viruses with $\mathrm{RN}$, nor due to the virus combination of SMV+BPMV with RN, compared to the presence of the viruses without the RN. Exposure to RN alone, without any of the viruses present, decreased the yield for Hutcheson by $18 \%$, while in the case of Hartwig, a 13\% higher yield was observed on the RN infested plots compared to $\mathrm{RN}$-free conditions. Yields were more suppressed by the presence of the viruses than by the RN, whereas the presence of RN did not noticeably affect overall yield losses due to the viruses.

\section{Yellow rust resistant genes for winter wheat breeding in Kazakhstan}

Y. DUTBAYEV (1), M. Koishibayev (2), N. Sultanova (2)

(1) Kazakh National Agrarian Univ, Almaty, Kyrgyzstan; (2) Kazakh Research Institute of Pant Protection, Almaty, Kazakhstan

Phytopathology 104(Suppl. 3):S3.36

Yellow rust (Puccinia striiformis f.sp. tritici) occurs regularly in the piedmont zone of southern and south-eastern regions Kazakhstan. This disease also occurs in northern Kazakhstan, but less frequently. Outbreaks of yellow rust occurred in 1999, 2002, and 2009; low incidence of the disease was observed in 2000, 2003, 2006 and 2010; yellow rust did not develop in 2001, 2004, 2005, 2007, 2008, 2011, 2012 and 2013 in Kazakhstan. During the outbreaks of stripe rust, commercial cultivars of winter wheat grown in Kazakhstan, including Steklovydnaya 24, Eritrospermum 350, Zhetusu, Karlygash, Bogarnaya 56, Progress, Krasnovopadskaya 210, and Opax showed high susceptibility to $P$. striiformis. Cultivars Sapaly, Naz, Yegemendi, Tungush, Akdan, and Konditerskaya were found resistant to yellow rust. Analysis of resistance in winter wheat cultivars/lines showed presence of several resistant genes to $P$. striiformis, including Yr3, Yr3V, Yr4+, Yr6+, Yr10, Yr15, Yr27, YrN, YrARP, YrSP, and YrSD and with pyramids of genes of Yr6+Yr7, Yr8+Yr18, and Yr2+ Yr11+ Yr25. Lines with genes YrA, Yr1, Yr6, Yr9, Yr17, Yr18 and Yr28 were not resistant to P. striiformis. European cultivars with Yr2, Yr3N, Yr3V, Yr4, Yr9, Yr7+, YrSD, YrSu, and YrSP genes showed high resistance to pathotypes of $P$. striiformis in Kazakhstan.
A novel pathovar of Pseudomonas coronafaciens (pv. morscepanov.), responsible for yellow bud disease in onion (Allium cepa)

B. DUTTA (1), R. Gitaitis (1), T. Coutinho (2), D. Langston (1)

(1) University of Georgia, Tifton, GA, U.S.A.; (2) University of Pretoria, Pretoria, South Africa

Phytopathology 104(Suppl. 3):S3.36

Yellow bud (YB) is an emerging disease of onion has only been reported from Georgia. The casual organism is a gram-negative, ice-nucleating, rod-shaped, aerobic bacterium. The bacterium belongs to LOPAT group Ia as it produces levan, is negative for oxidase, potato rot, and arginine dihydrolase and produces a hypersensitive reaction in tobacco. Sequencing of $16 \mathrm{~S}$ rRNA and coronafacate ligase genes and MLSA analysis, based on sequences of gyrB, rpoD, and gltA, allocated the genus-species of YB strains as Pseudomonas coronafaciens. However, unlike P. coronafaciens, YB stains were nonfluorescent on King's medium B. Repetitive extragenic palindrome (REP)PCR analysis gave identical banding patterns for the onion strains as that of a type strain of $P$. coronafaciens (ATCC 19608). More than $90 \%$ DNArelatedness was observed for $\mathrm{YB}$ strains with the $P$. coronafaciens (type strain). Inoculation of YB strains and $P$. coronafaciens (type strain) onto rye, oat, Italian ryegrass and onion resulted in symptoms on onion only with YB strains but not with the type strain. Additionally, inoculation of type strain resulted in symptoms on rye, oat and Italian ryegrass whereas YB strains did produce symptoms on these plants. This confirmed that the YB strains were indeed a novel pathovar of $P$. coronafaciens and the proposed named for the organism is $P$. coronafaciens pv. morcepa nov.

Characterization of novel Cercospora beticola effector proteins

M. EBERT (1), T. Friesen (1), G. Secor (2), M. D. Bolton (1)

(1) USDA-ARS, Fargo, ND, U.S.A.; (2) North Dakota State University, Fargo, ND, U.S.A.

Phytopathology 104(Suppl. 3):S3.36

Cercospora Leaf Spot (CLS), caused by the hemibiotrophic fungus Cercospora beticola, is the most destructive foliar disease of sugar beet worldwide. During infection, plant pathogens secrete effectors that help establish disease. Although many effectors have been characterized in several pathosystems, no $C$. beticola effector proteins have been reported to date. To identify novel $C$. beticola effectors, we grew $C$. beticola in vitro under specific conditions in which infiltration of culture filtrate caused necrosis in sugar beet leaves within $24 \mathrm{~h}$. Treatment of culture filtrate with a mixture of proteases abolished necrosis-inducing activity, confirming that the $C$. beticola effector(s) responsible for necrosis was proteinaceous in nature. Active culture filtrates were partially purified using liquid chromatography (LC). A single (LC) fraction was repeatedly identified that caused necrosis upon infiltration into host tissue. MS/MS analysis of this fraction identified three effector protein candidates. Candidates exhibited classic effector characteristics, including secretion signal, high cysteine content, and low molecular weight (6 to $11 \mathrm{kDa}$ ). Candidate effector proteins are currently being produced in Pichia pastoris for further analysis. A detailed characterization of these putative effectors will be presented. Gaining an understanding of $C$. beticola effector biology will give new insight in $C$. beticola pathology and may lead to novel CLS control measures.

Resistance reaction of flax germplasm to fusarium wilt

V. P. EDIRISINGHE (1), H. R. Kutcher (1), H. Booker (1), K. Y. Rashid (2), S. Cloutier (3), F. M. You (2)

(1) University of Saskatchewan, Saskatoon, SK, Canada; (2) Agriculture \& Agri-Food Canada, Morden, MB, Canada; (3) Agriculture \& Agri-Food Canada, Ottawa, ON, Canada

Phytopathology 104(Suppl. 3):S3.36

The fusarium wilt pathogen, Fusarium oxysporum f. sp. lini (Fol), is able to survive in soil for long periods and cause considerable yield loss. Varietal resistance is the most effective method to control the disease and it is essential to identify resistant cultivars and genes. Disease reaction of a subset (160) of RIL lines developed from parent cultivars 'Aurore' (moderately resistant) and 'Oliver' (susceptible) was assessed under controlled environment conditions to two Fol isolates (isolate numbers 81 and 131). Disease severity (DS) was determined (grading scale, 0 to 9) and the area under the disease progress curve (AUDPC) was calculated. The population varied from resistant to highly susceptible; 5 to $14 \%$ of the RILs severely wilted (scores of 8 and 9) with isolates 131 and 81, respectively. Similarly, in wilt nurseries, at Saskatoon and Morden, evaluation of the full set of 200 RILs, the disease reaction varied from resistant to susceptible, with $21 \%$ and $33 \%$ of RILs severely wilted, respectively, at the green boll stage. These results indicate genetic variation among the RILs for DS and AUDPC. The results from the two locations were significantly different, although moderately correlated $\left(r^{2}=0.6127\right)$. Differences in environmental and experimental conditions (such 
as seeding date) at the two locations, as well as different $\mathrm{Fol}$ strains in the soil combined with the subjectivity of the grading system may explain the significant differences between locations.

\section{Influence of resistance and surface residue on incidence of epiphytic Clavibacter michiganensis subsp. nebraskensis and Goss's leaf blight of corn}

S. K. Eggenberger (1), F. W. Nutter (1), A. E. ROBERTSON (1)

(1) Iowa State University, Ames, IA, U.S.A.

Phytopathology 104(Suppl. 3):S3.37

The primary source of inoculum for epidemics of Goss's wilt and leaf blight of corn (GWLB) is Clavibacter michiganensis subsp. nebraskensis (Cmn)infested surface residue. Little is known about how surface residue and hybrid resistance contribute to the development of epiphytic $\mathrm{Cmn}$ populations, which can be detected on asymptomatic corn early in the growing season. In 2013, we monitored the incidence of $\mathrm{Cmn}$ on asymptomatic, green leaf tissue in a 2 X 2 factorial experiment with a susceptible and a resistant hybrid and two levels $(<5 \%,>50 \%)$ of $\mathrm{Cmn}$-infested surface residue. The incidence of plants with GWLB symptoms was also monitored. Incidence of epiphytic Cmn fluctuated through the season and declined after mid-August. Season-long incidence of epiphytic Cmn was highest in plots with high surface residue, regardless of hybrid, and lowest in plots with the resistant hybrid and low surface residue. Only two lesions were observed in the resistant hybrid, but in the susceptible hybrid, the mean incidence of GWLB at the end of the season was $16 \%$ and $43 \%$ in the low and high residue treatments, respectively. The studies show that Cmn-infested residue can serve as a source of inoculum for epiphytic Cmn. Since Cmn can persist as an epiphyte on both susceptible and resistant hybrids through the growing season, epiphytic $\mathrm{Cmn}$ may be undetected in fields but provide another source of inoculum for GWLB.

Disease severity assessment in epidemiological studies: Accuracy and reliability of visual estimates of Septoria leaf blotch (SLB) in winter wheat M. El Jarroudi (1), L. Kouadio (2), C. Mackels (1), B. Tychon (1), P. Delfosse (3), C. H. BOCK (4)

(1) Univ of Liege, Arlon, Belgium; (2) Agriculture and Agri-Food Canada Agriculture et Agroalimentaire Canada, Lethbridge, Alberta, Canada; (3) Centre de Recherche Public - Gabriel Lippmann, Belvaux, Luxembourg; (4) USDA-ARS-SEFTNRL, Byron, GA, U.S.A.

Phytopathology 104(Suppl. 3):S3.37

The accuracy and reliability of visual assessments of SLB severity by raters (i.e. one plant pathologist with extensive experience and three other raters trained prior to field observations using standard area diagrams and DISTRAIN) was determined by comparison with assumed actual values obtained by digital image analysis. Initially analyses were performed using SLB severity over the full $0-100 \%$ range; then, to explore error over short ranges of the $0-100 \%$ scale, the scale was divided into sequential $10 \%$ increments based on the actual values. Lin's concordance correlation (LCC) analysis demonstrated that all raters were accurate when compared over the whole severity range (LCC coefficient $\left(\rho_{c}\right)=0.92-0.99$ ). However, agreement between actual and visual SLB severities was less good when compared over the short intervals of the $10 \times 10 \%$ classes $\left(\rho_{c}=-0.12-0.99\right)$, demonstrating that agreement will vary depending on the actual disease range over which it is compared. Inter-rater reliability between each pair of raters over the full 0$100 \%$ range (correlation analysis $r=0.970-0.992, P<0.0001$ ), and inter-class correlation coefficient $(\rho \geq 0.927)$ were very high. This study provides new insight into using a full range of actual disease severity vs limited ranges to ensure a realistic measure of rater accuracy and reliability, in addition to contributing to the ongoing debate on the use of visual disease estimates based on the $0-100 \%$ ratio scale for epidemiological research.

The implications of eggplant as a host in late blight epidemiology in Egypt

S. M. El-Ganainy (1), Y. M. Ahmed (1), M. S. Soliman (1), D. E. L. Cooke (2), A. M. M. TOHAMY (1)

(1) Plant Pathology Research Institute, Giza, Egypt; (2) The James Hutton Institute, Dundee, United Kingdom

Phytopathology 104(Suppl. 3):S3.37

Late blight disease caused by the oomycete Phytophthora infestans is the most devastating disease threats potato and tomato crops worldwide. In Febrauary 2014 weather conditions were favorable to the development of late blight epidemic. Unexpectedly, late blight symptoms were noticed in scattered foci in an eggplant (Solanum melongena L.) field in northern Egypt. A typical "V"-shaped lesions were cultured on Phytophthora-selective medium. Pure cultures were obtained by single-hypha transfers and the isolates were identified as $P$. infestans according to their morphological and microscopic features. Pathogenicity test proved that eggplant isolate was virulent on potato
(Lady Rosetta cv.) and tomato (Super Strain B cv.). Mating type testing showed that eggplant isolate belonged to mating type A1. This is considered the first report of $P$. infestans on eggplant in Egypt. Currently, variation in aggressiveness' components between 3 isolates of potato, tomato and eggplant is compared. In addition, further work will be done to study: 1. Whether eggplant is a sorce of inoculum to potato and tomato crops or vice versa? 2. Is only one lineage able to infect eggplant or is it both 13_A2 and 23_A1?

Calcium plays a key role in mediating the $\boldsymbol{R}_{M c 1(b l b)}$ resistance response against Meloidogyne chitwoodi in potato

A. A. ELLING (1), L. J. Davies (1), C. R. Brown (2)

(1) Department of Plant Pathology, Washington State University, Pullman, WA, U.S.A.; (2) USDA-ARS, Prosser, WA, U.S.A.

Phytopathology 104(Suppl. 3):S3.37

Columbia root-knot nematode, Meloidogyne chitwoodi is an important pathogen in temperate agriculture, causing extensive damage to potato. An advanced breeding line has been developed in which a $M$. chitwoodi resistance (R) gene from the wild potato species, Solanum bulbocastanum was introgressed into $S$. tuberosum. Isolates of $M$. chitwoodi, avirulent and virulent against the $R_{M c 1(b l b)}$ gene were used to functionally characterize the $\mathrm{R}$ gene in potato. Histological observations of avirulent and virulent $M$. chitwoodi interactions indicated the occurrence of a hypersensitive response (HR). A fluorescent indicator was used to quantify the activity of reactive oxygen species (ROS) in $R_{M c l(b l b)}$ roots. A biphasic release of ROS was observed in response to avirulent $M$. chitwoodi. This confirmed the $R_{M c l(b l b)}$ mediated resistance response was HR-dependent. To gain an insight into the signal transduction pathways mediating HR, chemical inhibitors were utilized. The calcium channel inhibitor, $\mathrm{LaCl}_{3}$ caused a significant reduction $(-147.1 \%$ compared to controls) in electrolyte leakage, an indicator of cell death. This suggested a role for calcium in $R_{M c l(b l b)}$ mediated resistance. Labeling of calcium fluxes and quantitative real-time PCR analysis, provided further evidence for the importance of calcium. This study is the first characterization of an R gene against $M$. chitwoodi and the first evidence of calcium mediating resistance against plant-parasitic nematodes.

Detection of Fusarium oxysporum f. sp. phaseoli in common bean seeds using magnetic capture hybridization and quantitative PCR

M. L. ELLIS (1), M. Viana de Sousa (2), G. P. Munkvold (1)

(1) Iowa State University, Ames, IA, U.S.A.; (2) Universidade Federal de Lavras, Lavras, Brazil

Phytopathology 104(Suppl. 3):S3.37

Fusarium oxysporum f. sp. phaseoli (Fop) is the causal agent of Fusarium wilt of common bean (Phaseolus vulgaris), and is considered one of the most important pathogens of this crop worldwide. The pathogen can be disseminated by contaminated seeds; therefore accurate and sensitive seed health testing methods are needed to ensure seed quality. The objective of this research was to assess whether magnetic capture hybridization $(\mathrm{MCH})$ would improve upon the sensitivity of a quantitative PCR (qPCR) protocol developed for the detection of Fop from seed. The PCR primers, TaqMan probe, and hybridization capture probe were designed using the Fusarium transcription factor 1 gene ( $f t f 1)$. DNA from fungal mycelium and artificially and naturally infested seed was extracted using a chloroform-based DNA extraction protocol. DNA from seed was also extracted using the Qiagen DNeasy Plant Mini Kit. The MCH-qPCR assay resulted in at least a 100 -fold increase in detection sensitivity for Fop DNA, from $10 \mathrm{fg} / \mu \mathrm{L}$ to $0.1 \mathrm{fg} / \mu \mathrm{L}$. DNA extracted from seeds using the chloroform-based method was not detectable using qPCR alone; however the pathogen was detectable from both artificially and naturally infested seed using MCH-qPCR. DNA extracted from seeds using the Qiagen Kit could be detected using qPCR alone; future work is needed to determine if the MCH-qPCR assay can improve detection sensitivity of the pathogen from DNA extracted from seeds using the Qiagen Kit.

Chemical inducers of systemic acquired resistance in plants

M. H. EL-SHETEHY (1), C. Wang (2), S. Baby (1), K. Yu (1), A. Kachroo (1), P. Kachroo (1)

(1) University of Kentucky, Lexington, KY, U.S.A.; (2) College of Agronomy and Plant Protection, Key Lab of Integrated Crop Pest Management of Shandong Province, Qingdao Agricultural University, 700 Changcheng Road, Qingdao 266109, China, Qingdao, China

Phytopathology 104(Suppl. 3):S3.37

Systemic acquired resistance (SAR) is a form of resistance that protects against a broad-spectrum of pathogens. SAR involves the generation of mobile signal(s) at the site of primary infection, which moves to, and arms distal portions of a plant against subsequent secondary infections. A number of signals contributing to SAR have been isolated and characterized. These 
include salicylic acid (SA), the nine carbon dicarboxylic acid azelaic acid (AzA), the phosphorylated sugar glycerol-3-phosphate (G3P), and two lipid transfer-like proteins (LTPs) DIR1 (Defective in Induced Resistance) and AZI1 (AzA insensitive). Earlier we showed that a feedback loop involving AzA, G3P, DIR1, and AZI1 regulates SAR. Recent results show that free radicals nitric oxide (NO) and reactive oxygen species (ROS) act upstream of the AzA-G3P branch of SAR, which operates in parallel to SA derived signaling. A role for NO and ROS in SAR was established using biochemical, cellular, molecular and genetic approaches. The interrelationship amongst the various chemical inducers of SAR will be discussed. Methyl jasmonate induces systemic resistance in grape berries against
postharvest gray mould caused by Botrytis cinerea

D. ERRAMPALLI (1), A. Sharon (2), P. H. Goodwin (3)

(1) Agriculture and Agri-Food Canada, Vinelnad Station, ON, Canada; (2) Tel-Aviv University, Tel-Aviv, Israel; (3) University of Guelph, Guelph, ON, Canada

Phytopathology 104(Suppl. 3):S3.38

Botrytis cinerea, a necrotrophic pathogen causes gray mold in grapevine berries. Methyl jasmonate (MeJA) has signalling role in eliciting induced systemic resistance (ISR) against diseases. In this study, the effect of exogenous MeJA on the suppression of postharvest botrytis in green grape cultivars 'Thomson', 'Chardonnay' and 'Vidal.' and in red grape cultivars 'Merlot', 'Flame' and 'Cabernet Sauvignon' was tested. The grape bunches (15 grapes/bunch and three replicate treatments) were spray-treated with 1 $\mathrm{mM}$ of MeJA, air dried for 3 hours. Three days after the MeJA treatment, each of the grape berry in the bunch was wounded with a needle and inoculated with $1 \times 10^{4}$ spores of $B$. cinerea B 05.10 and incubated in the dark at $20^{\circ} \mathrm{C}$ and $85 \%$ RH. Control treatment did not receive MeJA. The lesion diameter was recorded at 7 and 14 days after inoculation. The elicitor, MeJA induced defense response by significantly suppressing the Botrytis bunch rot in all the grape cultivars. Defense response, expressed as $P A L$ gene, in grapevine berries towards $B$. cinerea, was studied. Maximum levels of induction of $P A L$ gene was observed at 48 hours post inoculation and a significantly lower level of $P A L$ gene was expressed in MeJA and B. cinerea infected grapevine berries, as compared to $B$. cinerea only infected berries.

Resistance to fungicides in Botrytis cinerea isolates from strawberry in Ontario

D. ERRAMPALLI (1)

(1) Agriculture and Agri-Food Canada, Vineland Station, ON, Canada

Phytopathology 104(Suppl. 3):S3.38

Chemical control is essential to prevent pre- and postharvest fruit gray mold caused by Botrytis cinerea Pers,:Fr. in strawberries. In a two-year study, 392 single-spore isolates of $B$. cinerea from 5 strawberry fields in Ontario were tested for sensitivity to the benzimidazole thiabendazole, the anilinopyrmidine cyprodinil and pyrimethanil, the phenylpyrrole fludioxonil and the dicarboximide iprodione, on PDA media amended with a discriminatory concentration of $5 \mathrm{ug} / \mathrm{ml}$ of each of the fungicides. Sensitivity determinations to the fungicides revealed a total of four phenotypes: All of the isolates were sensitive to fludioxonil; all were resistant to anilinopyramidines; and $70 \%$ of isolates were resistant to three out of four chemical groups. Detached fruit assays demonstrated that all isolates were pathogenic on strawberries. The genotyping of the 20 select isolates with BenA gene and BcOS1 gene showed that all benzimidazole resistant isolates carried the 198 Glu to Ala (E198A) mutation in BenA gene in beta-tubulin and all dicarboximide resistant isolates carried 365 Ile to Ser (I365S) mutation in BcOS1 gene that encodes histidine kinase. As expected, cross resistance was observed between two of the anilinopyramidine fungicides, cyprodinil and pyrimethanil. The absence of cross-resistance between fludioxonil and other fungicides tested indicates that multidrug resistance phenotypes do not confer resistance to fludioxonil.

Patterns of disease loss in soybean - exploration of the soybean yield loss database in the United States

P. D. ESKER (1), S. R. Koenning (2), J. A. Wrather (3), C. A. Bradley (4)

(1) University of Costa Rica, San Jose, Costa rica; (2) North Carolina State University, Raleigh, NC, U.S.A.; (3) University of Missouri, Portageville, MO, U.S.A.; (4) University of Illinois, Urbana, IL, U.S.A.

Phytopathology 104(Suppl. 3):S3.38

Since 1996, data have been collected regarding estimated losses due to major soybean diseases in the United States. Estimates have been made by extension specialists or other experts from the major soybean producing states. The data are essential to understanding the impact of these diseases on production and to the soybean industry in order to prioritize research and outreach efforts. What has not been examined in great deal has been if there are inherent patterns temporally or spatially in these data. Therefore, we have started to explore this database in more detail using a series of exploratory and empirical methods to ask specific questions, for example, "Has the discovery of soybean rust (Phakopsora pachyrhiizi) in the United States led to increases in losses due to other foliar diseases?" For this question, our results have indicated that "yes", diseases such as frogeye leaf spot (Cercospora sojina), bacterial blight (Pseudomonas savastanoi pv. glycinea), Septoria brown spot (Septoria glycines) and downy mildew (Peronospora manshurica) have increased. Furthermore, new information has enabled us to classify some diseases by region versus those that have been documented over a wider region. Lastly, we have integrated information from the USDA production statistics, such as yield and harvested and production areas, to further examine diseases by different groupings. Overall, the goal in these efforts is to identify potential areas for further collaboration.

Plant host and community diversity impact the dynamics of resource use by soil Streptomyces

A. ESSARIOUI (1), N. LEBLANC (1), H. C. Kistler (2), L. Kinkel (1)

(1) University of Minnesota, St. Paul, MN, U.S.A.; (2) USDA/University of Minnesota, St. Paul, MN, U.S.A.

Phytopathology 104(Suppl. 3):S3.38

Nutrient use overlap among coexisting Streptomyces is thought to be a predictor of pathogen inhibitory capacity of soil populations. Yet there is little information on the diversity or on the factors that affect nutrient use overlap among sympatric populations. We examined the effect of plant species and diversity on nutrient use by Streptomyces from the rhizosphere of Andropogon gerardii (Ag) and Lespedeza capitata (LC) in communities of 1 or 16 plant species. Growth on 95 substrates was assessed over $5 \mathrm{~d}$ using Biolog SF-P2 plates. Growth of $A g$ isolates was higher in monoculture than polyculture during the first $36 \mathrm{~h}$ post-inoculation. Between 48 and $120 \mathrm{~h}$ Streptomyces from 16-species plots grew significantly more than Streptomyces from monoculture. Niche overlap for all pairewise combinations among sympatric isolates was greater in monoculture than polyculture suggesting stronger niche specialization among isolates in polyculture. Growth of Streptomyces from $L c$ in 16-species was also significantly higher than 1-species. Streptomyces from polyculture used 93 nutrients whereas those from monoculture used only 73 . Streptomyces from $L c$ used carbon sources more efficiently than those from $\mathrm{Ag}$ regardless of plant diversity. These results suggest differential selection imposed by plant host and diversity on Streptomyces. Nutrient competition among Streptomyces in carbon-limited monocultures is likely to select for inhibitory phenotypes associated with pathogen suppression.

Genetic and phenotypic variation among Cephalosporium gramineum isolates collected in the Pacific Northwest United States

K. Esvelt Klos (1), J. G. Evans (2), T. D. MURRAY (3)

(1) USDA-ARS Small Grains Germplasm Research Unit, Aberdeen, ID, U.S.A.; (2) Oklahoma State University, Stillwater, OK, U.S.A.; (3) Washington State University, Pullman, WA, U.S.A.

Phytopathology 104(Suppl. 3):S3.38

Cephalosporium gramineum ( $\mathrm{Cg}$ ) causes Cephalosporium stripe, a chronic disease of winter wheat in the US states of Washington, Oregon and Idaho (PNW). In order to assess genetic variation and infer reproductive strategy, $270 \mathrm{Cg}$ isolates collected from the PNW were characterized for variation in cultural morphology and amplified fragment length polymorphism. Isolates differed in mycelial texture, color and growth rate on malt extract agar, and no shared multilocus genotypes were observed. Isolates collected prior to 2010 were more likely to have fine texture, darker color with warmer hue and grow at a slightly faster rate than others. Evidence of population differentiation among isolates collected before and after 2010 was marginally significant; however, principal component analysis of the genetic distance among isolates did not reveal evidence of population structure. Fields near Lewiston, ID and Oakesdale, WA were intensively sampled in 2010. Isolates from these two fields were similar culturally, though Lewiston had a greater proportion of coarse-textured isolates. Fourteen percent of genetic variation among isolates was explained by location of origin and there was evidence of population differentiation. The null hypothesis of random mating was rejected in both fields. Implications of the phenotypic and genotypic variation are unknown but consistent with a recent study that identified four haplotypes based on IGS variation in a world collection of $\mathrm{Cg}$ isolates.

Managing downy and powdery mildew in organically-grown cucurbit crops

K. L. EVERTS (1)

(1) University of Maryland College Park, Salisbury, MD, U.S.A.

Phytopathology 104(Suppl. 3):S3.38

Downy mildew (Pseudoperonospora cubensis) and powdery mildew (Podosphaera xanthii) are important diseases in organic cucurbit production. 
A trial was conducted to evaluate Champ (copper hydroxide) alone, or alternated with Serenade (Bacillus subtilis), Regalia (Reynoutria sachalinensis), Actinovate (Streptomyces lydicus), or Oxidate (hydrogen dioxide) for downy and powdery mildew on Cucumis sativus and C. melo. Fungicide program efficacy based on area under the downy mildew curve (AUDMC) depended on crop; disease was greater on C. sativus than on C. melo. All programs reduced AUDMC compared to no treatment on both crops. However on C. sativus, Champ alone or alternated with Serenade reduced AUDMC more than when alternated with Oxidate. Powdery mildew severity on the lower leaf surface of $C$. sativus was similar in all treatments. However on C. melo, Champ alternated with Regalia or Serenade reduced powdery mildew more than Champ alone. A second trial examined the efficacy of Champ alone or alternated with Serenade or Regalia on Cucurbita moschata or C. pepo. On C. moschata, all programs reduced downy mildew compared to no treatment, and there were no differences among programs. On C. pepo, Champ alone or alternated with Serenade reduced downy mildew compared to no treatment, but not when alternated with Regalia. Overall, Champ alone or alternated with Serenade was most effective on downy mildew and Champ alternated with Regalia was most effective on powdery mildew.

\section{Influence of plant stage on resistance to anthracnose in Andean lupin} (Lupinus mutabilis Sweet)

C. E. FALCONI (1), R. G. Visser (2), S. W. van Heusden (2)

(1) Universidad de las Fuerzas Armadas, Sangolqui, Pichincha, Ecuador; (2) Wageningen University, Wageningen, Netherlands

Phytopathology 104(Suppl. 3):S3.39

Anthracnose, caused by C. acutatum, is the most devastating fungal disease in Andean lupin (Lupinus mutabilis) in Ecuador and other lupins around the world. Bending of the main stem is the result of anthracnose infection. Because there are not Andean lupin anthracnose resistant varieties, a rapid method is required to investigate lupin-C. acutatum interactions. I-450 and I451 cultivars were inoculated on the meristematic section of the main stem either by spraying or pipetting on an artificial wound with one $C$. acutatum isolate. Although the two methods gave similar results, spraying the inoculum was the best method because it mimics natural pathogen infection. Plantpathogen interactions were determined in five different phenological stages $(2-3,4-5,6-7,8-9$, and 10-11 leaf stage) with three C. acutatum isolates. A scale (0-5) was used to assess disease. Anthracnose symptoms were greater in inoculated early seedling (2-3 leaf stage), decreasing significantly in early vegetative phase (6-7 leaf stage) and increasing again when the flower stage began (10-11 leaf stage). The present results suggest that anthracnose resistance of these two lupin cultivars is not equally expressed at all developmental stages. We recommend scoring at the most resistant stage (6-7 leaves plants, 1.5 month old) and confirm at the most susceptible stage in 1011 leaves plants ( 2.5 months old) to identify material with improved response.

\section{Effects of carbon amendments on pathogen-inhibitory soil Strepto-} mycetes

L. FELICE (1), R. Dill-Macky (1), L. L. Kinkel (1)

(1) University of Minnesota, St. Paul, MN, U.S.A.

Phytopathology 104(Suppl. 3):S3.39

Suppression of soil-borne diseases by indigenous soil bacteria is an appealing target of crop management. Composts and green manures have been used to enhance indigenous suppression, but effectiveness varies widely. We set out to test the hypotheses that amending soil with higher doses and single carbon sources would result in greater pathogen-suppressive capacity than amendments of lower doses and more complex carbon sources. Two soils were collected from the field and amended over nine months with three single carbon sources (glucose, fructose, malic acid) separately and in combination at two different dosages each. The density and proportion of Streptomycetes inhibitory against two common soil pathogens were measured using culturebased techniques. Inhibitor densities against Streptomyces scabies after amendments ranged from 3.4 to $5.6 \log \mathrm{CFU} / \mathrm{g}$ soil. The high-organic matter (OM) soil harbored higher densities of pathogen-inhibitors, but was less responsive to carbon amendments than the low-OM soil. In the low-OM soil, greater shifts in densities of pathogen-inhibitors occurred with lower dose amendments. These results suggest that carbon dose, soil physicochemical properties, and initial soil microbial community characteristics interact to determine the community response to carbon amendments and resulting pathogen suppression by soil Streptomyces.

Molecular mapping of $\mathrm{YrSP}$, a wheat gene for resistance to stripe rust J. Feng (1), M. Wang (1), X. CHEN (2)

(1) Washington State University, Pullman, WA, U.S.A.; (2) USDA ARS, Pullman, WA, U.S.A.

Phytopathology 104(Suppl. 3):S3.39
Stripe rust, caused by Puccinia striiformis f. sp. tritici (Pst), is an important disease of wheat worldwide. Resistance is the best approach for control the disease. $Y r S P$, a stripe rust resistance gene originally from wheat variety Spaldings Prolific, is used for differentiating $P$ st races, but its chromosomal location is not clear. To map $\mathrm{YrSP}$, its near-isogenic line was backcrossed to the recurrent parent, Avocet $\mathrm{S}$. Genetic analysis of the $\mathrm{F}_{1}, \mathrm{BC}_{1}, \mathrm{~F}_{2}$, and $\mathrm{F}_{2: 3}$ progeny confirmed the single dominant gene. A total of $182 \mathrm{~F}_{2}$ plants and their derived $\mathrm{F}_{2: 3}$ lines were phenotyped with an avirulent Pst race and genotyped with simple sequence repeat (SSR), single-nucleotide polymorphism (SNP), and sequence tagged site (STS) markers. A linkage map was constructed with $3 \mathrm{SSR}, 17 \mathrm{SNP}$, and 3 STS markers covering $27.6 \mathrm{cM}$. Markers $S 3$ and $d p 269$ were 0.4 and $1.8 \mathrm{cM}$ from $\mathrm{YrSP}$, respectively. The gene was mapped to the long arm of chromosome $2 \mathrm{~B}$ bin $2 \mathrm{BL}-7$ within $50 \%$ of the chromosomal arm to the centromere. Allelism tests determined that $Y r S P$ is at a locus different from $Y r 5, Y r 7, Y r 43, Y r 44$, and $Y r 53$, which are also located in $2 \mathrm{BL}$. The reaction data from tests with seven historical and currently predominant $P$ st races in the U.S. also demonstrated the differences of $Y r S P$ from all of the other genes. The result indicates that $Y r S P$ is valuable for differentiating $P s t$ races, studying the plant-pathogen interactions, and properly using together with other genes in breeding programs.

Molecular study of the sucrose non-fermenting protein kinase 1 gene in the canola blackleg pathogen Leptosphaeria maculans

J. FENG (1), H. Zhang (2), S. F. Hwang (1), S. E. Strelkov (3)

(1) Alberta Agriculture and Rural Development, Edmonton, AB, Canada; (2) The Institute of Vegetables and Flowers, Chinese Academy of Agricultural Sciences, Beijing, AB, China; (3) University of Alberta, Edmonton, AB, Canada

Phytopathology 104(Suppl. 3):S3.39

Leptosphaeria maculans is a fungal pathogen causing blackleg in canola. Its virulence has been attributed, among other factors, to the activity of hydrolytic cell wall degrading enzymes (CWDEs). Studies on the pathogenicity function of CWDEs in plant pathogenic fungi have been difficult because of the redundancy of the encoding genes. In microorganisms, many CWDE genes are repressed by glucose and derepressed by the function of the sucrose nonfermenting protein kinase 1 ( $S N F 1)$ gene. To address the molecular function of $S N F 1$ in $L$. maculans, $L m S N F 1$ was cloned and functionally characterized using a gene knockout strategy. Growth of the $L m S N F 1$ knockout strains was severely disturbed, as was their sporulation, spore germination and ability to attach to the plant surface. When inoculated on canola cotyledons, the LmSNF1 knockout strains did not cause any symptoms, indicating the loss of pathogenicity. The expression of 11 selected CWDE genes and a pathogenicity gene $(L o p B)$ was significantly down-regulated in the LmSNF1 knockout strains. In conclusion, knockout of $L m S N F 1$ prevented the fungus from properly derepressing the production of CWDEs, compromised the utilization of certain carbon sources, and impaired the fungal pathogenicity on canola.

Transcriptome analysis of spinach differential cultivars for downy mildew pathogens

C. FENG (1), B. Bluhm (1), J. Tipton (1)

(1) University of Arkansas, Fayetteville, AR, U.S.A.

Phytopathology 104(Suppl. 3):S3.39

Spinach downy mildew disease, caused by the obligate pathogen Peronospora farinosa f. sp. spinaciae $(P f s)$ is the most economically important disease of spinach. There are 14 races of $P f s$ that have been characterized, 11 of which have been identified in last 25 years. A set of spinach cultivars containing none, one or two known resistance loci, is used to differentiate races of Pfs. To compare the transcriptomes of seven differential cultivars and the wild species Spinacia turkestanica, RNA was extracted from one-month old leaves and sequenced with Illumina Hiseq-2000. There were 30 to $48 \mathrm{M}$ 100-bp paired-end reads obtained from these cultivars, which were assembled into 44 to $123 \mathrm{k}$ contigs with average lengths of 359 to $651 \mathrm{bp}$ and total lengths of 26 to $45 \mathrm{M}$ bp for each cultivar. The GC content in spinach was about $40 \%$. Spinach genes responsive to environmental stresses (such as heat, cold, salt, etc.) and related reproduction (such as sex determination, self-incompatibility, pollen specific protein, and fertility restorer) were identified. Hundreds of disease resistance genes containing nucleotide binding-site leucine rich repeat (NBS-LRR) domains as well as other resistance genes, including pathogenesis-related proteins, chitinase (classes I $\sim$ IV), virus resistance were also identified. Thousands of simple sequence repeats (SSRs) and single nucleotide polymorphisms (SNPs) were found in the RNA sequences and could be useful for spinach genetic and breeding research. 
A host-pathogen interaction paradigm: Is Leptosphaeria maculans's new races adopting rapidly to Canadian canola in western Canada?

D. FERNANDO (1), S. Liban (1), X. Zhang (1), G. Peng (2), D. Cross (3) (1) University of Manitoba, Winnipeg, MB, Canada; (2) Agriculture and AgriFood Canada, Saskatoon, Saskatoon, SK, Canada; (3) Agriculture and AgriFood Canada, Melfort, Melfort, SK, Canada

Phytopathology 104(Suppl. 3):S3.40

Blackleg caused by Leptosphaeria maculans is the most destructive disease affecting Canola (Brassica napus L.). Genetic resistance has proven to be an effective means of disease control in western Canada. However, this pathogen has appeared on cultivars rated as resistant. Information on genetic variability in the population is essential in developing an effective control strategy. Race structure was assessed on avirulence alleles AvrLm1, AvrLm2, AvrLm3, AvrLm4, AvrLm6, AvrLm7, AvrLm9, LepR1, LepR2, and AvrlmS. Overall, certain alleles were more prevalent in the pathogen population with AvrLm6 and Avrlm7 present in $>90 \%$ of isolates and AvrLm3, Avrlm9, and AvrLepR2 present in $<10 \%$ of isolates. The latter 3 alleles have changed to the virulent forms affecting the majority of the Canadian canola cultivars which carry the corresponding Rlm genes (i.e. $R \operatorname{lm} 3, \operatorname{Rlm} 2$ ). However, some loci differed greatly across geographic locations. For example, AvrLm2 ranged from $86 \%$ in Vegreville, Alberta to $38 \%$ in Plum Coulee, Manitoba. Selection pressure from different race-specific resistance genes in commercial canola cultivars (i.e. $R \operatorname{lm} 3$ ) is postulated as the most significant factor influencing the variation in the emergence of 'newer' virulence forms observed. The presentation will address some of the innovative measures undertaken by the researchers and industry to address this change in race structure and mitigate blackleg spread across the Prairie Provinces.

Identification of Botrytis spp. causing blossom blight in Japanese plums and nectarines in Chile

E. E. FERRADA (1), B. A. Latorre (1), G. A. Diaz (1), J. P. Zoffoli (1)

(1) Pontificia Universidad Catolica de Chile, Santiago, Chile

Phytopathology 104(Suppl. 3):S3.40

Blossom blight commonly affects nectarines (Prunus persicae var. nectarina) and Japanese plums (P. salicina) in Chile. In 2013, prevalence of 25 and 37\% were estimated in nectarines and Japanese plums, respectively. First symptoms consisted on a light brown necrosis of the petals followed by necrosis of the entire flower. Eventually, gray masses of conidia appeared on senescent blossoms. The aim of this study was identify the species of Botrytis associated to blossom blight. A total of 150 Botrytis isolates, with either a high or low sporulation, were obtained on PDA. These phenotypes were also expressed on King medium B (BK) and pea agar (PA). High sporulated isolates produced gray colonies and sclerotia on PDA, BK and PA while the low sporulated isolates produced whitish colonies and sclerotia were obtained only on PA after 14 days at $20^{\circ} \mathrm{C}$. Glyceraldehyde 3-phosphate dehydrogenase (G3PDH), heat-shock protein 60 (HSP60) and DNA-dependent RNA polymerase subunit II (RPB2) genes coding sequence alignments and the concatenated phylogenetic analysis revealed $B$. cinerea as the high sporulated phenotype while the low sporulated phenotype remained as a non-identified species (Botrytis sp.1). Isolates of B. cinerea $(\mathrm{n}=3)$ and Botrytis $\mathrm{sp} .1(\mathrm{n}=3)$ were pathogenic on flowers and fruits of Japanese plums. On the basis of the results obtained, $B$. cinerea and a new non-identified species of Botrytis were associated to blossom blight of Japanese plums and nectarines in Chile.

\section{Molecular strategies employed by pea cultivars during the interaction} with white mold Sclerotinia sclerotiorum

P. C. FERREIRA DOS SANTOS (1), X. Zhuang (1), C. Foster (1), A. Rojas (1), J. Wang (1), K. McPhee (2), T. E. Coram (3), M. Chilvers (1)

(1) Michigan State University, East Lansing, MI, U.S.A.; (2) North Dakota State University, Fargo, ND, U.S.A.; (3) Dow AgroSciences LLC, Indianapolis, IN, U.S.A.

Phytopathology 104(Suppl. 3):S3.40

The molecular aspects of the interactions between pea (Pisum sativum L.) and Sclerotinia sclerotiorum were investigated with RNAseq at 12, 24 and 48 hpi, of both susceptible (Lifter) and partially resistant (PI240515). The results showed that 2,528 (77\%) and 1,517 (63\%) genes from PI240515 and Lifter, respectively, were specifically expressed within each genotype in response to infection. PI240515 expressed more resistance genes, including those related to programmed cell death. Sclerotinia is able to induce apoptotic-like events in plants during disease development. We selected a few genes and evaluated their expression by qRT-PCR. Interestingly, at $24 \mathrm{hpi}$, PsHxk-1, PsPLD and PSBAKl (encoding a hexokinase, a phospholipase and a BR1-associated kinase, respectively) transcript levels were significantly $(\mathrm{P}<0.05)$ up-regulated in inoculated Lifter when compared with inoculated PI240515. Expression patterns between the two cultivars was particularly dissimilar at $24 \mathrm{hpi}$, with the most highly differentially expressed genes in PI240515 being involved in lignin and flavonoid production. Lignin content was determined by the acetyl bromide method and light microscopy. Additionally, expression patterns of PI240515 clustered with $\mathrm{H}_{2} \mathrm{O}_{2}$-treated Arabidopsis samples followed by MeJA-treated ones. These results hint that PI240515 plants are able to delay cell death, invest in PR gene expression, cell wall synthesis and possibly phytoalexin production as a way to fight off disease.

Dynamics of Pseudomonas savastanoi populations in galls and on leaves of olive in California

E. J. FICHTNER (1), G. Kasun (2), C. DeBuse (3), W. H. Krueger (4)

(1) UC ANR, Tulare, CA, U.S.A.; (2) Portland State University, Portland, OR, U.S.A.; (3) USDA ARS, Davis, CA, U.S.A.; (4) UC ANR, Orland, CA, U.S.A.

Phytopathology 104(Suppl. 3):S3.40

Management of olive knot caused by Pseudomonas savastanoi relies exclusively on copper applications and pruning/removal of symptomatic tissues. Epiphytic populations and bacteria residing in galls serve as primary inoculum. The pathogen population dynamics on California (CA) olives is unknown. To address survival of primary inoculum, specific objectives include: i) assessment of epiphytic pathogen population dynamics in three CA regions, and ii) characterization of pathogen survival in galls. Epiphytic populations were assessed repeatedly over two years on 5 flagged trees in each of 10 orchards ranging over $550 \mathrm{~km}$ of the Central Valley. Foliar samples were incubated in buffer and resulting bacterial suspensions were placed on selective medium. To produce galls of equal age, leaf scars were inoculated in April 2011, and tissues were excised and sampled for pathogen populations over time. Populations in galls declined over $90 \%$ during the first summer survival period, suggesting that galls are mainly infective the first winter and spring after symptom development. Winter epiphytic populations were generally lower in the southern San Joaquin Valley than either the southern or northern Sacramento Valley; summer populations were generally undetectable across all three regions. Despite copper use, a single orchard exhibited a postharvest epiphytic population burst in 2013, suggesting the potential lack of sensitivity to the applied bactericide.

\section{Unraveling stem rust resistance in Brachypodium distachyon}

M. FIGUEROA (1), C. Castell-Miller (1), S. Gordon (2), D. F. Garvin (3), S. Filichkin (4), S. Alderman (5), J. Vogel (2), T. Mockler (6), W. Pfender (5)

(1) University of Minnesota, St. Paul, MN, U.S.A.; (2) USDA, ARS, PWA, WRRC-GGD, Albany, CA, U.S.A.; (3) USDA ARS, St. Paul, MN, U.S.A.; (4) Oregon State University, Corvallis, OR, U.S.A.; (5) USDA ARS Forage Seed and Cereal Research, Corvallis, OR, U.S.A.; (6) Donald Danforth Plant Science Center, St. Louis, MO, U.S.A.

Phytopathology 104(Suppl. 3):S3.40

Stem rust is a devastating pathogen of wheat and barley. Advancing our understanding of stem rust disease resistance is critical to develop strategies to prevent agronomic losses and enhance grain production. Brachypodium distachyon has been developed as an experimental model to study cereals and grasses. The natural genetic variation of $B$. distachyon and its genotypedependent resistance against stem rust support the potential of this species to provide durable disease resistance. We developed an experimental framework to identify genes in $B$. distachyon that may play a role in the molecular recognition and resistance to three different stem rust isolates: $P$. graminis $\mathrm{f}$. sp. tritici (Pg-tr), P. graminis f. sp. lolii (Pg-lo) and P. graminis f. sp. phleipratensis (Pg-pp). For each of these isolates, we analyzed gene expression profiles during two distinctive stages of infection, appressorium formation (12 hpi, hours post-inoculation), and fungal penetration (18 hpi). Based on changes of mRNA levels and predicted gene function we prioritized a list of genes for downstream functional characterization. Cross-reference of our list of genes of interest with a collection of $B$. distachyon T-DNA insertional mutants allowed screening of 50 mutants which represent genes with possible receptor, transporter or antifungal functions. Here, we report the molecular activities associated with these interactions and disease susceptibility of several $B$. distachyon mutants of interest.

Gene expression, proteome analysis, and validation of effectors associated with psyllid-mediated Liberibacter transmission

T. W. FISHER (1), E. V. Sheveleva (1), J. K. Brown (1)

(1) University of Arizona, Tucson, AZ, U.S.A.

Phytopathology 104(Suppl. 3):S3.40

$\mathrm{Ca}$. Liberibacter asiaticus and solanacearum are putative fastidious, bacterial causal agents of Huanglongbing and Zebra chip, important diseases of citrus and solanaceous crops, respectively, and transmitted by psyllids in a propagative, circulative manner. Elucidation and functional characterization of gene products specifically involved in $\mathrm{Ca}$. Liberibacter primary infection 
and establishment, and acquisition processes in the psyllid vectors are largely incomplete. The 110,937 and 83,231 Asian citrus psyllid (ACP) Diaphorina citri Kuwayama and potato psyllid (PoP) Bactericera cockerelli Sulc midgut and salivary gland transcripts, respectively, were used to identify psyllid proteins obtained by nano-liquid chromatography with tandem mass spectrometry (LC-MS/MS) analysis of excised adult midguts and salivary glands, producing approximately 500 unique proteins. In silico profiling of $\mathrm{ACP}$ and PoP midguts and salivary glands identified transcripts and proteins with altered expression in response to $\mathrm{Ca}$. Liberibacter infection and tissue specificity in psyllids. The results implicated transcripts involved in immune response, endocytosis, and cytoskeleton assembly that feasibly could serve as effectors of infection, invasion, propagation, and acquisition to facilitate psyllid-mediated $\mathrm{Ca}$. Liberibacter transmission. Selected transcriptLiberibacter interactions were subjected to validation using RNA-interference, qPCR, pull down assays, and confocal immuno-localization.

Functional characterization of the holin and endolysin of 'Candidatus Liberibacter asiaticus' bacteriophage $\mathrm{SC} 1$ and validation of a late gene promoter

L. A. FLEITES (1), S. Zhang (2), M. Jain (1), D. W. Gabriel (1)

(1) University of Florida, Gainesville, FL, U.S.A.; (2) Integrated Plant Genetics, Gainesville, FL, U.S.A.

Phytopathology 104(Suppl. 3):S3.41

Cell lysis at the end of the bacteriophage lytic cycle is generally achieved by two proteins: the holin, an inner membrane permeabilizing protein with alpha helical structure, and the endolysin, a muralytic enzyme that traverses the holes created by holins and degrades the cell wall. We previously identified several late genes by RT-qPCR of a prophage of $\mathrm{Ca}$. Liberibacter asiaticus (causes citrus Huanglongbing) that were highly expressed in periwinkle (in which phage particles form), but not citrus (where phage have not been seen). To functionally confirm lytic cycle genes, we cloned and expressed the annotated prophage holin (SC1_gp110) and endolysin (SC1_gp035), as well as a gene outside of the prophage region annotated as an endolysin (CLIBASIA_04790). Functional expression of SC1_gp110 in pET27b in Escherichia coli revealed bacteriostatic activity characteristic of holins. Expression of SC1_gp035, but not CLIBASIA_04790, in pET27b led to lysis of E. coli, confirming SC1 gp035 as an active endolysin. An SC1 gp110 holin promoter region ("hPro") was fused with a GUS and a lacZ alpha reporter, forming hPro::GUS and hPro::lacZ, respectively. E. coli colonies carrying $\mathrm{hPro:}: l a c Z$ were white on $\mathrm{X}$-gal media, indicating a lack of promoter recognition, but Liberibacter crescens colonies carrying hPro::GUS constructs were blue, indicating strong promoter activity. Screening of potential transcription factors for trans-activation of the holin promoter is in progress.

\section{Characterization of Sec-Translocon dependent extracytoplasmic proteins} of Candidatus Liberibacter asiaticus

S. FNU (1), N. Wang (1)

(1) University of Florida, Lake Alfred, FL, U.S.A

Phytopathology 104(Suppl. 3):S3.41

Candidatus Liberibacter asiaticus (Las) is the causative agent of Huanglongbing, a destructive disease of citrus globally; causing extensive loses in the citrus industry. The molecular basis of the interaction of Las with its hosts has not yet been established, owing to the inability to culture Las. Interestingly, Las contains a complete Sec translocation apparatus, which represents a mechanism for the secretion of Las proteins, including putative virulence factors, into the extracytoplasmic milieu. Our goal was to characterize the Sec-translocon dependent extracytoplasmic proteins in Las in context of the host cell infection. A total of 150 Las proteins were predicted to contain signal peptides targeting them out of the cytoplasm via the Sectranslocon. For experimental validation of these putative signal peptides, Escherichia coli based alkaline phosphatase gene fusion assays were carried out. From the experimental analysis, out of the 132 tested proteins, $89(68 \%)$ showed positive result for the phoA assay, suggesting that their cognate proteins are translocated outside the cytoplasm via the Las Sec system. Additionally, we investigated the Sec-translocon dependent extracytoplasmic proteins of Liberibacter crescens. Functions of selected extracytoplasmic proteins are being studied using Sinorhizobium meliloti as a model. The putative function of these Sec-dependent extracytoplasmic proteins will be discussed.

Effect of temperature and wetness duration on anthracnose fruit rot development on different strawberry cultivars

B. B. FORCELINI (1), N. Peres (2)

(1) University of Florida, Wimauma, FL, U.S.A.; (2) University of Florida, Gulf Coast Research and Education Center, Wimauma, FL, U.S.A.

Phytopathology 104(Suppl. 3):S3.41
Florida is the largest producer of winter strawberries in the world Anthracnose Fruit Rot (AFR), caused by Colletotrichum acutatum, can greatly affect production if not controlled. The use of fungicides in addition to cultural practices such as the use of less susceptible cultivars are important tools for AFR control. In addition, disease forecasting models for AFR based on temperature and Wetness Duration (WD) have been developed to time and reduce fungicide applications on strawberry. The objective was to evaluate the effects of temperature and WD on the development of AFR on strawberry cultivars with different levels of susceptibility to the disease. Immature strawberry fruit of the cultivars Camarosa (highly susceptible) and Strawberry Festival (moderately susceptible) were inoculated with a $10^{6}$ conidia $/ \mathrm{ml}$ suspension and incubated under wetness periods ranging from 0.5 to 48 hours and temperatures 15,20 and $25^{\circ} \mathrm{C}$. Fruit were then dried and moved to $25^{\circ} \mathrm{C}$ for nine days and then evaluated for disease incidence. Disease incidence increased non-linearly with increasing temperature and WD for both cultivars and disease development curves at 15,20 , and $25^{\circ} \mathrm{C}$ and from 0 to $48 \mathrm{~h}$ of WD were similar. Disease symptoms start to develop on both cultivars at $15^{\circ} \mathrm{C}$ and with 3 hours of WD. Optimum temperature and wetness duration for both cultivars were $20^{\circ} \mathrm{C}$ and 48 hours, respectively. However, overall disease incidence was higher for 'Camarosa' than for 'Strawberry Festival'.

Virulence phenotypes and genetic differentiation of Puccinia triticina, the wheat leaf rust pathogen, in Ethiopia

A. FOX (1), K. Xiao (2), M. Acevedo (3), J. Kolmer (2)

(1) USDA ARS, St. Paul, MN, U.S.A.; (2) USDA, St. Paul, MN, U.S.A.; (3) North Dakota State University, Fargo, ND, U.S.A

Phytopathology 104(Suppl. 3):S3.41

Ethiopia has a unique history of wheat cultivation since tetraploid emmer and durum wheats have been grown for millennia and cultivation of common hexaploid wheats have increased in recent years. The objective of this research was to characterize the Puccinia triticina population in Ethiopia for virulence and molecular genotypes. A total of 180 single uredinial isolates were derived from 108 samples collected from common wheat, durum wheat, and cultivated emmer in Debre Zeit and surrounding areas from 2011-2013. The isolates were characterized for virulence phenotype on 20 near isogenic Thatcher differential wheat lines and for molecular genotypes with 10 simple sequence repeat (SSR) markers. Nearly $60 \%$ of the total isolates were phenotypes MCDSS or MBDSS, with virulence to common wheat. Isolates with BBBQJ phenotype that were virulent to durum wheat were $15 \%$ of the isolates and nearly $20 \%$ of the isolates were avirulent to Thatcher but had virulence to durum wheats. Virulence phenotypes of isolates were highly correlated with host of origin. Clustering of SSR genotypes using twodimensional principal coordinates placed the M-DSS, BBBQJ and the Thatcher avirulent isolates into three discrete groups with high levels of $F_{S T}$ and $R_{S T}$ differentiation. The Thatcher avirulent isolates found on durum wheat are unique to Ethiopia and may reflect the regions history as a center of diversity for tetraploid wheats.

\section{Evolution and spread of azole-insensitive Zymoseptoria tritici field isolates} in the UK

B. A. FRAAIJE (1), S. Atkins (1), J. Luo (1), P. Diez de la Fuente (1), N. J. Hawkins (1)

(1) Rothamsted Research, Harpenden, United Kingdom Phytopathology 104(Suppl. 3):S3.41

Azole fungicides have been used for more than three decades in the UK to control Septoria leaf blotch caused by the fungus Zymoseptoria tritici. Research has shown that the efficacy of some azoles (e.g. tebuconazole and triadimenol) can be compromised by the evolution and spread of insensitive strains carrying combinations of amino acid alterations (amino acid substitutions and a deletion) in the target protein sterol 14 $\alpha$-demethylase (CYP51). A stepwise accumulation of mutations has been observed since the early 1990s. Since 2012, CYP51 variants harbouring up to nine amino acid alterations can be found in the least sensitive strains. In addition, a $120 \mathrm{bp}$ insertion in the CYP51 promoter linked with CYP51 overexpression has been found since 2009 in some strains representing two different CYP51 variants. In vitro fungicide sensitivity testing of different CYP51 variants $(>60)$ shows significant shifts in azole efficacy for all compounds tested (i.e. epoxiconazole, prothioconazole, prochloraz and tebuconazole). Here we present the genotype-to-phenotype relationships for these strains and discuss the practical implications.

Use of loop-mediated isothermal amplification assays to detect azoleinsensitive CYP51-overexpressing strains of Zymoseptoria tritici

B. A. FRAAIJE (1)

(1) Rothamsted Research, Harpenden, United Kingdom

Phytopathology 104(Suppl. 3):S3.41 
Septoria leaf blotch is currently controlled by programmed applications of multisite inhibitors (e.g. chlorothalonil), azoles (e.g. epoxiconazole and prothioconazole) and a new generation of Succinate Dehydrogenase Inhibitors (e.g. bixafen, isopyrazam and fluxapyroxad). Azole fungicides have been used for three decades but their efficacy has eroded over time due to the evolution of azole insensitive strains carrying alterations in the sterol $14 \alpha$-demethylase (CYP51) target protein. Because of the importance of azoles as a mixing partner of SDHIs continued monitoring of azole sensitivity shifts is paramount. Recently, we have reported a new mechanism, a 120 bp insertion in the CYP51 promoter which is linked with 10- to 40 -fold CYP51 overexpression. Other isolates carry promoter variants based on a larger insert of $868 \mathrm{bp}$ but the impact of this insert on the regulation of CYP51 expression remains unclear. Here we present the development and application of loop-mediated isothermal amplification (LAMP) assays for rapid, on the spot detection of CYP51 promoter inserts in Zymoseptoria tritici (Mycosphaerella graminicola) isolates carrying CYP51 variants [L50S, S188N, I381V, $\Delta$ Y459/G460 \& N513K] and [L50S, S188N, A379G, I381V, $\Delta$ Y459/G460 \& N513K].

Pathogenicity and control of Boeremia exigua var. exigua causing agent of Black root rot of industrial chicory (Cichorium intybus L. var. sativum)

R. A. FRANCE (1), D. E. Grinbergs (1)

(1) INIA, Chillan, Chile

Phytopathology 104(Suppl. 3):S3.42

The main disease of industrial chicory in Chile is the Black root rot, which may cause yield losses up to $31 \%$ and industrial problems during the fructan extraction. The disease is difficult to control due to the pathogen is soil borne and develops late in the season, when the crop is fully covering the ground and the main root is deep into the soil. The objective was to screen the pathogenicity of different isolates of B. exigua var. exigua, study the effect of root injury and fungicides or resistance inducer in disease control. Pathogenicity test was performed in chicory root disc, inoculated with 20 isolates. Differences were observed among isolates $(\mathrm{P}<0.05)$, from 0 to $70 \%$ of root rot. Later, the most pathogenic isolate was used for whole root inoculation, with or without injury on the crown. In both cases the pathogen was able to colonize the root; however the disease progression was greater $(50 \%)$ in the injured ones. Fungicide test was first accomplished in Petri plates, using six concentrations of boscalid, boscalid + pyraclostrobin, pyraclostrobin + methylthiophanate, cyprodinyl + fludioxonil, azoxystrobin + cyproconazole and phosphite. Later the most effective products were applied to entire roots under humid chamber and potted plants. The results showed that phosphite was the most consistent treatment to control the disease and a good option for foliar application, when the crop is fully developed and the fungus start its pathogenicity process.

Characterization of virulence of different pathogenicity groups of Leptosphaeria maculans on Brassica napus $\mathbf{L}$.

J. FRANCESCHI (1), L. del Rio (1)

(1) North Dakota State University, Fargo, ND, U.S.A.

Phytopathology 104(Suppl. 3):S3.42

Leptosphaeria maculans is one of the most destructive pathogens of canola (Brassica napus). This fungus produces strains with different virulence profiles (pathogenicity groups, PG) as determined using cultivars 'Westar, Quinta and Glacier. However, little information is available on other traits that may characterize these groups. The objective of this study is to characterize the aggressiveness of $L$. maculans isolates belonging to PG 2, 3, 4, and T. Individual spore suspensions $\left(10^{7}\right.$ spores $\left.\mathrm{ml}^{-1}\right)$ were obtained from $7,10,14$, or 21 days-old colonies from several isolates belonging to each of these PGs. Spores were inoculated on the cotyledon leaves of seedlings from cultivar Westar. The study was conducted using a completely randomized design with six replications in greenhouse conditions. Disease reaction was recorded 4, 7, 11 and 15 days post inoculation and the area under the disease progress curve was calculated. Results suggest isolates vary in aggressiveness within each PG although PG-3 and 4 may be more aggressive than PG-2 and PG-T. Spores produced by older colonies had reduced aggressiveness in all PGs. These studies will improve our understanding of this pathogen and will allow us to identify the best isolates to be used to select resistant materials.

\section{Fusarium as a seed pathogen on Bromus tectorum}

J. FRANKE (1), B. Geary (2), S. E. Meyer (3), S. Saunders (2), T. Poh (2)

(1) Brigham Young University, West Jordan, UT, U.S.A.; (2) Brigham Young University, Provo, UT, U.S.A.; (3) USDA, Provo, UT, U.S.A.

Phytopathology 104(Suppl. 3):S3.42

Cheatgrass (Bromus tectorum) has become known as one of the most prominent invaders of the Western United States. Each year this invasive weed displaces millions of native plants creating a vast number of monocultures across the Great Basin. These monocultures sporadically exhibited stand failure, which has become known as a "dieoff". These dieoffs were thought to be caused by pink snow mold, which was known as Fusarium nivale, but has been reclassified as Microdochium nivalis. Recently, the genus Fusarium has become a reoccurring theme when studying these die-offs. Strains closely related to Fusarium tricinctum have been isolated multiple times from field die-off locations and are believed to play a major role in these die-offs. Isolated Fusarium strains were inoculated on to non-dormant seeds and kept at $-1.5 \mathrm{MPa}$ for 7 days at $25 \mathrm{C}$ in order to mimic the early-autumn environment of the Great Basin. When seeds were transferred to water to allow for germination, a white tuft of fungal mycelium was observed at the radical end of seeds that did not germinate. Approximately 40 to $80 \%$ of the non-dormant seeds subjected to water stress were killed. Samples were taken throughout the experiment and viewed using scanning electron microscopy. Results suggest that Fusarium has a significant role in causing cheatgrass dieoffs.

Evaluating the efficacy of fungicide treatments for blackleg (Leptosphaeria maculans) management in canola

M. C. FRASER (1), S. F. F. Hwang (2), G. D. Turnbull (2), H. U. Ahmed (2), W. Barton (3), S. E. Strelkov (1)

(1) University of Alberta, Edmonton, AB, Canada; (2) Crop Diversification Centre North, Edmonton, AB, Canada; (3) BASF Canada, Mississauga, ON, Canada

Phytopathology 104(Suppl. 3):S3.42

Blackleg of canola, caused by Leptosphaeria maculans, is associated with seedling death and stem cankers that reduce the yield and quality of this crop. The purpose of this study was to evaluate the performance of various fungicides in managing blackleg in a susceptible ('Westar') and moderately resistant ('73-15') cultivar. The study was conducted as greenhouse and field trials in 2012 and 2013 in Edmonton, Canada. Several fungicidal seed treatments were assessed, including Prosper FX (carbathiin, trifloxystrobin, and metalaxyl) and an experimental product formulation (pyraclostrobin and fluxapyroxad) applied at half and full rates. Two foliar fungicides also were applied in combination with the experimental formulation, and included Priaxor (pyraclostrobin and fluxapyroxad) and Tilt $250 \mathrm{EC}$ (propiconazole). In the field trials, Priaxor reduced stem canker severity by an average of $58 \%$ in '73-15' and by $67 \%$ in 'Westar', relative to a non-treated control. In 2013, Priaxor and Tilt increased yields in 'Westar' by $24 \%$ and $9 \%$, respectively. In one greenhouse trial, all treatments reduced stem canker severity from 58 $83 \%$ in ' $73-15$ ' and from $64-79 \%$ in 'Westar'. There were no significant differences in yield, except that 'Westar' had a 95\% greater yield following Priaxor treatment. The results of this study suggest a reduction in blackleg with the use of seed treatments in combination with foliar fungicides, especially when cropping a susceptible cultivar.

Evaluation of Solanum sisymbriifolium as a potential host for Colletotrichum coccodes and Verticillium dahliae

Z. A. FREDERICK (1), T. F. Cummings (1), D. A. Johnson (1)

(1) Washington State University, Pullman, WA, U.S.A.

Phytopathology 104(Suppl. 3):S3.42

The emphasis of Plant Pathology on disease management in agricultural systems has resulted in many positive outcomes for plant health. This success can be enhanced by understanding of how pathogens interact with their plant hosts in natural systems. One example is a wild relative of potatoes, Solanum sisymbriifolium, which can stimulate egg hatch of the Potato Cyst Nematode, Globodera pallida and Globodera rostochiensis, but does not support nematode reproduction. However the ability for S. sisymbriifolium to be a host for various pathogenic fungi of potatoes, such as Verticillium dahliae and Colletotrichum coccodes, has not been fully investigated. Given that $V$. dahliae and $C$. coccodes persist in soils through crop rotational cycles, the knowledge gained from this study would add to the continued success of crop rotations. Greenhouse trials were established to detect $V$. dahliae and $C$. coccodes from the stems of $S$. sisymbriifolium and three potato cultivars of varying susceptibility to $V$. dahliae. It was determined that $V$. dahliae genotypes that are aggressive on potato infected and reproduced in 50 percent of sampled $S$. sisymbriifolium stem tissues. $V$. dahliae genotypes that are aggressive on mint, along with all $C$. coccodes genotypes, were detected from 13 percent of sampled $S$. sisymbriifolium stems. Hence, utilizing $S$. sisymbriifolium in rotation just prior to potatoes would increase risks associated with the propagation of aggressive $V$. dahliae genotypes.

Effect of temperature on growth and sporulation of US-22, -23, and -24 Phytophthora infestans and implications for late blight epidemiology

K. FROST (1), A. Gevens (1), A. Seidl Johnson (1), D. Rouse (1)

(1) University of Wisconsin, Madison, WI, U.S.A.

Phytopathology 104(Suppl. 3):S3.42 
The population of Phytophthora infestans (Mont.) deBary, causal agent of late blight, continues to evolve with unique clonal lineages arising which differ in fitness and pathogenicity. For isolates of 3 recently identified clonal lineages of $P$. infestans, US-22, -23 , and -24 , sporulation rates were determined on potato and tomato foliage and the effect of temperature on lesion growth rate on tomato was measured. Lesion growth rates for US-22, -23 , and -24 isolates were $6.3,5.9$, and 4.0 , respectively. US-23 isolates had the highest sporulation rates (sporangia $\mathrm{x}$ meter $^{-2} \times$ day $^{-1} \times 10^{8}$ ) on potato (4.4) and tomato (3.6) leaves, US-22 readily infected both hosts, but sporulation by isolates of this lineage was overall less, and US-24 isolates, while having a high sporulation rate on potato (4.0), had the lowest overall sporulation rate on tomato (1.7). When parameters in the LATEBLIGHT model were modified to reflect the experimentally determined lesion growth rates and sporulation rates of the isolates, simulated epidemics for US-23 isolates using WI weather data (2009-2012) consistently progressed at a higher rate and were more severe than the simulated epidemics of the isolates of the US-22, US-24, or EC-1 clonal lineages. These results suggest that isolates of $P$. infestans US-23 may have the greatest fitness among currently prevalent lineages and may be the most likely lineage to persist in the population.

\section{New detection tools for grapevine trunk pathogens in apparently healthy} California vineyards

P. T. FUJIYOSHI (1), S. Schwebs (2), T. Diaz (2), R. Travadon (3), D. P. Lawrence (3), K. Baumgartner (1)

(1) USDA ARS, Davis, CA, U.S.A.; (2) Coastal Viticultural Consultants, Inc., Santa Rosa, CA, U.S.A.; (3) UC Davis, Davis, CA, U.S.A.

Phytopathology 104(Suppl. 3):S3.43

Symptoms of grapevine trunk diseases appear several years after infection, at which point the practices that protect pruning wounds are far less effective. To detect trunk pathogens in vineyards we designed PCR primers specific to three aggressive and widespread trunk pathogens (Eutypa lata, Neofusicoccum parvum, Phaeomoniella chlamydospora). Spores were trapped in three newly established (3-5 years, vines asymptomatic) and three mature (13-15 years; vines symptomatic) vineyards each in Napa and San Joaquin Counties. Spore traps compared at each site were microscope slides and Rotorod ${ }^{\circledR}$ traps, both coated with petroleum jelly. Trap catches were eluted for culture- and DNAbased detection. To optimize culture-based recovery of the slower-growing pathogens we used semi-selective medium or passed spore suspensions through $10-\mu \mathrm{m}$ filters. Culture-based recovery detected E. lata from 11 of 12 sites, Botryosphaeriaceous fungi such as $N$. parvum from nine sites, and $P$. chlamydospora from no sites. DNA-based detection revealed N. parvum from slides in the majority of sites, E. lata in no sites, and P. chlamydospora in two of the Napa sites. Rotorod ${ }^{\circledR}$ traps yielded fewer pathogens in culture than microscope slides but had the same species predominating. No PCR products resulted from Rotorod ${ }^{\circledR}$ traps. Vineyard age had no effect on detection. Early detection of trunk pathogens via spore traps may alert growers of the need for preventative practices in newly established vineyards.

\section{Development of a molecular marker to detect the Potato virus $\boldsymbol{Y}$ resistance} gene $\boldsymbol{R} \boldsymbol{y}_{\text {chc }}$ in diploid populations of potato

A. C. FULlADOLSA (1), S. H. Jansky (2), D. A. Halterman (3), A. O. Charkowski (1)

(1) University of Wisconsin-Madison, Madison, WI, U.S.A.; (2) University of Wisconsin-Madison/USDA-ARS, Madison, WI, U.S.A.; (3) USDA-ARS, Madison, WI, U.S.A.

Phytopathology 104(Suppl. 3):S3.43

The wild relatives of cultivated potato (Solanum tuberosum L.) are a valuable reservoir of useful traits, including plant resistance to biotic and abiotic stresses. The major disease problem in seed potato production and certification is Potato virus $Y$ (PVY), a rapidly evolving and widespread aphid-transmitted potyvirus, for which resistance has been identified but narrowly used in breeding programs in North America. The gene $R y_{c h c}$ confers extreme resistance to PVY and was found in accessions of the diploid species S. chacoense. To use this source of resistance in marker-assisted breeding, we developed diploid populations of potato segregating for the resistance phenotype by crossing a $S$. chacoense plant, heterozygous for $R y_{c h c}$, with a diploid, susceptible $S$. berthaultii plant. $175 \mathrm{~F} 1$ progeny were genotyped using the SolCAP 8303 SNP Infinium array and five single nucleotide polymorphisms (SNPs) on chromosome IX correlated with the resistance phenotype. Additionally, we sequenced the genome of a $S$. chacoense plant, homozygous for $R y_{c h c}$, from which a useful breeding clone and several populations have been developed, and are searching for polymorphisms to develop a tightly linked molecular marker by using published mapping data and the identified SNPs. The improved tools to use $R y_{\text {chc }}$ as a source of resistance will equip potato breeders for effective selection of resistant potato varieties that will reduce the incidence of PVY.
Response of Fusarium thapsinum to sorghum brown midrib lines and to phenolic metabolites

D. L. FUNNELL-HARRIS (1), S. E. Sattler (1)

(1) USDA ARS, Lincoln, NE, U.S.A.

Phytopathology 104(Suppl. 3):S3.43

Sorghum lines were bred for reduced lignin for cellulosic bioenergy uses, through the incorporation of brown midrib (bmr) bmr6 and/or 12 into two genetic backgrounds, either as single or double mutant lines. When these lines were assessed for resistance to $F$. thapsinum stalk rot, a cause of lodging, they were as resistant to $F$. thapsinum as near-isogenic wild-type lines. Peduncles of newly identified $b m r$ lines from an ethyl-methanesulfonate-mutagenized population, inoculated with $F$. thapsinum, were as resistant as the wild-type line, BTx623. One $b m r$ line (1107) had significantly smaller mean lesion lengths than BTx623, suggesting a mutation is associated with reduced susceptibility. Growing F. thapsinum on medium with ferulic, vanillic, sinapic, syringic and caffeic acids, phenolic compounds derived from the lignin pathway and elevated in different $\mathrm{bmr}$ lines, indicated that $F$. thapsinum was tolerant to these compounds. Eight sorghum fungi were also tested for effects on growth by the presence of these compounds and ferulic acid inhibited these fungi. Most of the phenolics inhibited $F$. verticillioides and $F$. proliferatum. Accumulation of phenolic metabolites in $\mathrm{bmr}$ plants may inhibit growth of some sorghum pathogens, while other factors, such as aromatic phytoalexins or salicylic acid, may be involved in resistance to $F$. thapsinum.

Characterization of fluorescent Pseudomonas spp. associated with roots and soil of two sorghum genotypes

D. L. FUNNELL-HARRIS (1), S. E. Sattler (1)

(1) USDA-ARS, Lincoln, NE, U.S.A.

Phytopathology 104(Suppl. 3):S3.43

Sorghum, useful for bioenergy feedstock, animal feed, and food, requires economical methods for disease prevention and control. Fluorescent Pseudomonas spp. were isolated from sorghum roots and adherent soil to identify isolates that inhibited sorghum fungal pathogens. Pseudomonads were collected from seedlings of sorghum cultivars RTx433 and Redlan and wheat cultivar Lewjain, grown in two soils. Lewjain supports growth of producers of the antibiotic, 2,4-diacetylphloroglucinol (2,4-DAPG). Isolates from all three plants were assessed for hydrogen cyanide $(\mathrm{HCN})$ and extracellular protease production, and for a 2,4-DAPG gene, phlD. Both soil and plant type affected $\mathrm{HCN}$ - and protease-production, but $p h l D$ was unaffected. $p h l D^{+}$isolates were chosen to determine phlD genotypes and to conduct in vitro inhibition assays against sorghum pathogens. Most isolates were genotype D, previously associated with superior root colonization. phlD ${ }^{+}$sorghum isolates were cocultured with five pathogens. One isolate from each sorghum line inhibited all five pathogens but more Redlan isolates were inhibitory to the virulent pathogen, Fusarium thapsinum, than RTx433 isolates. Nearly all inhibitory isolates from both cultivars were from one soil. This is consistent with previous observations from sorghum field studies: soil type played a significant role in determining characteristics of fluorescent pseudomonads, but sorghum genotype also had a considerable effect.

Detecting the grapevine wood-canker pathogen Neofusicoccum parvum (Botryosphaeria dieback) based on host markers of infection

E. R. GALARNEAU (1), S. Czemmel (2), R. Travadon (3), G. Cramer (2), A.

J. McElrone (1), K. Baumgartner (1)

(1) USDA-ARS CPGRU Davis, Davis, CA, U.S.A.; (2) University of NevadaReno, Reno, NV, U.S.A.; (3) University of California-Davis, Davis, CA, U.S.A.

Phytopathology 104(Suppl. 3):S3.43

Botryosphaeria dieback (causal fungus Neofusicoccum parvum) is a woodcanker disease that limits vineyard productivity. Early detection is hampered by the internal nature of the canker and absence of leaf symptoms. A detection tool for the early stage of infection, and from leaves instead of wood, would inform control practices. We defined the early stage of infection of inoculated vs. non-inoculated (both wounded and non-wounded) plants, based on light microscopic investigation of: 1) fungal colonization at and beyond the inoculation site, and 2) plant anatomical responses to wood colonization. Then we identified differentially-expressed genes from newly-expanding leaves that were specific to the early stage of infection, based on RNA-seq. Fungal colonization occurred in the xylem, mostly the fibers, and was restricted to the inoculation site until 2 months post-inoculation (MPI). Also at 2 MPI, a significantly higher percentage of xylem vessels fully-occluded by gels and xylem tissue with lower starch content were quantified in inoculated plants, but not in wounded or non-wounded plants. RNA-seq analysis of leaves identified 2,340 differentially-expressed candidate genes. qPCR analysis showed genes that were significantly induced and maintained at high levels in the early stages of infection were associated with plant defense responses, 
such as: 1) altered gene regulation, 2) cell wall modification, and 3) adaptation of amino acid biosynthesis.

Field and laboratory evaluation of resistance to boxwood blight in Buxus cultivars

M. GANCI (1), K. Ivors (1), D. M. Benson (1)

(1) North Carolina State University, Raleigh, NC, U.S.A.

Phytopathology 104(Suppl. 3):S3.44

Boxwood blight is caused by the fungal pathogen Calonectria pseudonaviculata $(=$ Cylindrocladium pseudonaviculatum $=$ C. . buxicola $)$. During 2012 and 2013, a wide range of resistance was observed when Buxus cultivars were inoculated on a container pad, suggesting that some cultivars expressed quantitative resistance to boxwood blight. An experiment was conducted in a growth chamber to evaluate disease severity and quantify the components of resistance. The objective was to compare boxwood blight resistance of intact plants between lab and field tests. Eight cultivars that expressed varying levels of field resistance were spray inoculated with a water suspension of 10,000 conidia $/ \mathrm{ml}$ and incubated at $20^{\circ} \mathrm{C}$. Disease severity (DS), incubation period, latent period, lesion area, and spore production were evaluated. Significant $(P$ $<0.0001)$ differences in DS ratings were observed among the cultivars tested. For instance, $B$. sinica var insularis 'Nana' was resistant (7\% DS) while $B$. sempervirens 'American' was highly susceptible ( $89 \%$ DS), confirming results with inoculations in the field ('Nana' $0.02 \%$ DS, 'American' $42 \%$ DS; $P<0.0001)$. There was significant $(P=0.0114)$ correlation between lab and field DS ratings with a Spearman's rank correlation coefficient of 0.83 . Further analysis of the components of partial resistance of Buxus cultivars to boxwood blight can reveal additional lab phenotypic assays that correlate with results from field studies.

\section{Development of control method for strawberry bacterial angular spot} disease

G. GANG (1), H. Cho (1), J. Kim (2), Y. Kwak (3)

(1) Division of Applied Life Science, GNU, Jinju, South Korea; (2) GNU, Jinju, Korea; (3) Gyeongsang National University, Jinju, South Korea Phytopathology 104(Suppl. 3):S3.44

In Korea, Strawberry bacterial angular leaf spot disease, caused by Xanthomonas fragariae, was reported in 2010 and the disease listed as a quarantine pest (A2 list). However, no strawberry angular leaf spot disease control methods are available. Therefore, in this study we selected eight bactericides (Validamycin-A, Oxolinic acid, Kasugamycin, Streptomycin, Streptomycin/Validamycin-A, Oxolinic acid/Streptomycin, Copper oxychloride/Kasugamycin and Polyoxin D/Kasugamycin) with mode-of-action of the bactericides. The $\mathrm{EC}_{50}$ for each bactericide was calculated with a standard pathogen strain (Validamycin-A $19.269 \mathrm{ug} / \mathrm{mL}$, Oxolinic acid $28.909 \mathrm{ug} / \mathrm{mL}$, Kasugamycin $36.081 \mathrm{ug} / \mathrm{mL}$, Streptomycin $64.954 \mathrm{ug} / \mathrm{mL}$, Streptomycin/Validamycin-A 36.458, Oxolinic acid/Streptomycin $46.535 \mathrm{ug} / \mathrm{mL}$, Copper oxychloride/Kasugamycin $45.130 \mathrm{ug} / \mathrm{mL}$ and Polyoxin D/Kasugamycin $36.414 \mathrm{ug} / \mathrm{mL}$ ). To field test, we applied the bactericides in June and October as nursery stage and cultivation stage, respectively. In nursery stage with high temperature condition, Oxolinic acid (97.2\%), Kasugamycin $(93.2 \%)$ and Validamycin (87.7\%) showed significant control effect. However, in cultivation stage with low temperature, only Validamycin (93.3\%) showed reliable control effect against the disease. The results suggested that different bactericide treatments during nursery and cultivation stages are required to maximization effective control of the strawberry angular leaf spot disease.

Soil microbial networks in organic and conventional tomato: Comparing long-term and transient communities by soil RNA

K. A. GARRETT (1), L. Gomez-Montano (1), A. Jumpponen (1), M. Kennelly (1)

(1) Kansas State University, Manhattan, KS, U.S.A.

Phytopathology 104(Suppl. 3):S3.44

Soil microbes are fundamental for the productivity of agricultural systems and may be affected by fertility management. We evaluated total and active microbial community responses in a soil fertility field experiment after three cycles of a two-year tomato and Chinese cabbage rotation with low and high levels of organic or conventional fertility. Low fertility treatments in both systems received nutrients only through a cover crop (buckwheat). The conventional high fertility treatment used potassium nitrate, calcium nitrate, and inorganic pre-plant fertilizer, and the organic high fertility treatment included fish hydrolysate and compost pre-plant fertilizer. We compared total resident fungal and bacterial communities using PCR-amplified DNA and the actively metabolizing communities using reverse-transcribed, PCR-amplified cDNA. Data suggest that the high-fertility organic management increases bacterial diversity. We recovered bacterial genera with important agro- ecological roles, including Nitrospira, Rhizobium and Meiothermus. Networks of microbial associations showed co-occurrence of fungal and bacterial taxa that were actively metabolizing in the soil samples. Preliminary results showed higher fungal diversity in cDNA than in DNA samples and that cDNA and DNA provide different community profiles. For example, frequencies of Glomus, Cladosporium, Guehomyces, and Alternaria differed between the cDNA and DNA-derived communities.

Screening and characterization of hormetic responses to Thiophanatemethyl in multiple Sclerotinia homeocarpa strains

C. D. GARZON (1), S. Pradhan (1), V. F. Marcillo (2), N. R. Walker (1), L. Miller (3)

(1) Oklahoma State University, Stillwater, OK, U.S.A.; (2) Universidad de las Fuerzas Armadas ESPE, Sangolqui, Ecuador; (3) University of Missouri, Columbia, MO, U.S.A.

Phytopathology 104(Suppl. 3):S3.44

Hormetic dose-responses to stressors are characterized by low-dose stimulation and high-dose inhibition. Although the mechanisms that underlie hormesis remain unclear, validation of this phenomenon may have significant implications for management of plant pathogens. Intensive use of fungicides for management of plant diseases can result in exposure of resistant isolates to subinhibitory doses. Previous studies have demonstrated that radial growth of selected fungal and oomycete strains was stimulated by low doses of fungicides. In this study, the dose-responses of a population of 29 isolates of Sclerotinia homeocarpa, the causal agent of dollar spot, were evaluated to detect and estimate the hormetic effect of the fungicide thiopahanate-methyl on mycelial growth. Ten treatments were evaluated, including 9 fungicide concentrations and a fungicide-free control, with three repetitions per treatment. Radial growth and total mycelium area were measured and compared among treatments using a Brain-Cousens non-linear regression model, and dose-response parameters were determined. Sixteen isolates displayed hormetic responses with up to $16 \%$ stimulation. Future research will assess the effect of fungicide hormesis on virulence, and stimulatory responses will be characterized by differential gene expression.

Multilocus phylogeny of the Pythium irregulare complex

C. D. GARZON (1), P. A. Garrido (1), J. E. Weiland (2), F. J. Flores (1), M. Herrero (3), G. W. Moorman (4), M. L. Daughtrey (5), A. Levesque (6)

(1) Oklahoma State University, Stillwater, OK, U.S.A.; (2) USDA ARS, Corvallis, OR, U.S.A.; (3) Bioforsk, As, Norway; (4) Pennsylvania State University, University Park, PA, U.S.A.; (5) Cornell University, Riverhead, NY, U.S.A.; (6) Agriculture and Agri-Food Canada, Ottawa, Canada Phytopathology 104(Suppl. 3):S3.44

Pythium irregulare is a prevalent pathogenic species of numerous plant hosts worldwide. Within $P$. irregulare sensu lato exist high levels of genetic diversity. Two cryptic species, $P$. irregulare sensu stricto (s.s.), and $P$. cryptoirregulare have been described. However, additional groups have been tentatively identified within the $P$. irregulare complex. Therefore, further analyses for resolving the phylogenetic status of this group of oomycetes are needed. Ninety six isolates from various geographical origins were collected and compared based on DNA sequence data of $\beta$-tubulin (Btub), heat shock protein 90 (HSP90), cytochrome oxidase subunit I, including the cox I-II spacer (coxI-II) and the internal transcribed spacer regions of the ribosomal DNA including the $5.8 \mathrm{~S}$ unit (ITS). To further assess the genetic diversity of this complex and define species boundaries, a subset of samples were analyzed using SSR and ISSR markers. Analyses of DNA sequence data and DNA fingerprinting revealed four discrete clades within the complex, supporting the species status of $P$. irregulare s.s. and $P$. cryptoirregulare, and of two additional groups, previously reported as P. 'vipa' I and P. 'vipa' II. Phylogenetic relationships of these species and other members of Pythium clade $\mathrm{F}$ will be discussed.

Population dynamics of Pythium species in floricultural greenhouses in Long Island, New York

C. D. GARZON (1), L. Sain (1), P. A. Garrido (1), I. Sanchez (2), G. Garcés (2), P. Espinosa (2), A. Medina (2), F. Proaño (2), F. Cornejo (3), M. L. Daughtrey (4)

(1) Oklahoma State University, Stillwater, OK, U.S.A.; (2) Universidad de las Fuerzas Armadas ESPE, Sangolqui, Ecuador; (3) Pontificia Universidad Catolica del Ecuador, Quito, Ecuador; (4) Cornell University, Riverhead, NY, U.S.A.

Phytopathology 104(Suppl. 3):S3.44

Pythium species are ubiquitous pathogens that cause root rots, black leg of cuttings, and damping-off of seedlings in ornamentals. Pythium inoculum can be present in plant materials, water, and soilless potting mixes used in floricultural operations. Epidemiological studies of Pythium diseases in 
ornamentals are challenging because of the diversity of crops involved, the multitude of potential sources of inoculum, and the difficulty of species identification. Overlapping morphological characteristics and the existence of cryptic species make species identification an arduous process, even for experienced diagnosticians. This study identified species and characterized Pythium populations fluctuations over several years in floricultural greenhouses from Long Island, New York. Isolates were collected yearly from 2008 to 2013 from diverse ornamental plant hosts. Species morphological identifications were verified by DNA sequencing of the ITS region, including the 5.8S rDNA. DNA sequence analysis of 428 Pythium isolates identified 22 species. The most abundant species were $P$. irregulare sensu lato $(\mathrm{n}=296), P$. segnitium $(\mathrm{n}=57)$, and $P$. ultimum s.l. $(\mathrm{n}=17)$. Species diversity fluctuated over time at each location. Although the Pythium species recovered from the floricultural operations studied were diverse and dynamic, species of the $P$. irregulare complex were the most prevalent.

Foliar fungicides for the control of leaf diseases and plant health in spring wheat

P. GAUTAM (1)

(1) North Dakota State University, Langdon, ND, U.S.A.

Phytopathology 104(Suppl. 3):S3.45

An experiment, randomized complete block design with four replications, was established during summer 2013 at the North Dakota State University Langdon Research Extension Center, Langdon, ND to evaluate efficacy of foliar fungicides for the control of leaf diseases and its impact on plant health and yield of spring wheat. Trial was naturally infected without artificial inoculation. Fungicides were applied at the water volume of $10 \mathrm{GPA}$ at Feekes growth stage 3 . Leaf disease severity was rated on the day of treatment application and subsequently on 14, 28 and 42 Days after treatment (DAT). Plots were harvested at maturity with a small plot combine and the yield and test weight determined. Data on $0,14,28$ and 42 DAT severity were $\log$ transformed to achieve homoscedasticity. 28 and 42 DAT incidences were squared root transformed. Leaf disease levels were statistically similar in all plots before the application of treatments (0 DAT). Numerically all fungicides resulted in reduced disease incidence and severity $28 \mathrm{DAT}$. Overall, Priaxor at $2 \mathrm{oz} / \mathrm{A}$ and Tilt $2 \mathrm{oz} / \mathrm{A}$ were the most efficacious in terms of reducing leaf disease incidence and severity compared to the non-treated. However, the reduction of leaf diseases was not reflected in terms of yield. None of the fungicide resulted in significantly higher or lower test weight than nontreated.

Participatory evaluation of potato varieties on organic farms: Opportunities for education and research

R. K. GENGER (1), D. I. Rouse (1), R. L. Groves (1), S. H. Jansky (2), A. O. Charkowski (1)

(1) University of Wisconsin-Madison, Madison, WI, U.S.A.; (2) University of Wisconsin-Madison/USDA-ARS, Madison, WI, U.S.A.

Phytopathology 104(Suppl. 3):S3.45

On-farm variety evaluation has the potential to provide farmers with information relevant to their farming environment and methods, enabling better informed variety choices. This farmer-researcher partnership also provides opportunities for researchers to understand the realities of the farm operation, to provide information to farmers on disease and pest issues that impact farm productivity and sustainability, and to design research programs with relevance to on-farm pest and disease problems. From 2010 to 2012, we evaluated commercially available potato varieties on organic farms throughout Wisconsin, and on organically managed land at the West Madison Agricultural Research Station. In 2013, 24 heirloom potato varieties were evaluated, in comparison to the best-performing varieties from previous trials, at 25 organic sites. Varieties were evaluated for early vigor, vine size, insect and disease damage, total and marketable yield, and tuber defects. Tuber defect diseases were a significant cause of loss, with between 24 and $38 \%$ culled. Common scab (Streptomyces scabies), silver scurf (Helminthosporium solani), and black scurf (Rhizoctonia solani) were the most common causes of post-harvest losses. Several heirloom varieties with potential for drought tolerance, early blight resistance, and potato leafhopper resistance were identified.

Susceptibility of crown buds to Podosphaera macularis and its association with perennation of the hop powdery mildew fungus

D. H. GENT (1), G. Grove (2), M. E. Nelson (2), C. Probst (2), M. Twomey (3), S. Wolfenbarger (3)

(1) USDA ARS NFSPRC, Corvallis, OR, U.S.A.; (2) Washington State University Irrigated Agriculture Research and Extension Center, Prosser, WA, U.S.A.; (3) Oregon State University Department of Botany and Plant Pathology, Corvallis, OR, U.S.A.

Phytopathology 104(Suppl. 3):S3.45
The hop powdery mildew fungus, Podosphaera macularis, overwinters in association with host tissue by means of bud perennation, leading to development of flag shoots, and apart from the host as ascocarps. However, the ascigerious stage is not known to exist in the Pacific Northwest, thus flag shoots are the only source of primary inoculum. The phenology of bud development, their susceptibility to powdery mildew, and dynamics of flag shoot emergence in the following season were assessed to clarify the overwintering process of $P$. macularis. During three years of study, small surface crown buds were present by mid July, and both the number and size of buds increased linearly over time. Buds of varying sizes were susceptible to powdery mildew during summer. However, bud susceptibility decreased markedly after September. Flag shoots emerged over a three-month period spanning late winter and spring in sync with shoot emergence. The number of flag shoots produced in spring was associated with timing of inoculation in the previous year. A small proportion of flag shoots emerged following chemical desiccation of shoots, a cultural practice intended to reduce inoculum of $P$. macularis. In separate field studies, delaying spring pruning significantly reduced development of powdery mildew on leaves without negatively impacting yield. These findings have important implications for strategies to reduce overwintering survival of $P$. macularis and development of powdery mildew.

Endophytic microbes of Brachiaria grasses from the native home (East Africa) and their roles in host fitness

S. GHIMIRE (1), J. Njuguna (1), L. Kago (1), C. Mutai (1), M. Ahonsi (1)

(1) International Livestock Research Institute, Nairobi, Kenya

Phytopathology 104(Suppl. 3):S3.45

Brachiaria grasses are native to East and Central Africa and form important components of Savannah grassland ecosystem. These grasses were introduced into the humid tropical regions of South America, Southeast Asia, and northern Australia where they have transformed grassland landscape and animal production systems. However, the potential of these important grasses in their native home remains largely unexplored. To address this issue, a study has been initiated at Biosciences eastern and central Africa - International Livestock Research Institute Hub (BecA-ILRI Hub) to explore natural diversity of Brachiaria grasses, evaluate improved Brachiaria grass cultivars and investigate endophytic microbes associated with these native grasses. The overall objective is to develop Brachiaria varieties that are better adapted to semi-arid environments of sub-Saharan Africa where soils are mainly less fertile, low in $\mathrm{pH}$ and high in aluminium toxicity. Specifically, the study component reported here presents assemblages of Brachiaria endophytes in their native home and elucidation of the multiple benefits the organisms provide to the host, such as plant growth promotion, nutrient utilization, protection against pest and pathogens and tolerance to drought and other abiotic stresses.

\section{Virus incidence in Texas peach orchards}

M. Giesbrecht (1), K. ONG (1)

(1) Texas A\&M AgriLife Extension Service, College Station, TX, U.S.A. Phytopathology 104(Suppl. 3):S3.45

During the spring and summer of 2013, five commercial peach orchards and one peach breeding orchard in six counties in Texas were tested for the presence of three different stone fruit viruses. A total of 877 foliar peach and plum samples were tested for plum pox virus (PPV) and 717 samples were tested for prune dwarf virus (PDV) and $<$ Prunus $>$ necrotic ringspot virus (PNRSV). Of the plum pox virus samples, 479 were collected from Texas A\&M breeding plots and 398 were collected from the commercial orchards. Each sample was tested by ELISA using Agdia ELISA kits and following USDA APHIS PPQ and National Plant Diagnostic Network protocols. All 877 samples tested negative for all five strains of plum pox virus. Of the 717 samples tested for the two other viruses, again 479 of the samples originated from Texas A\&M breeding stock and the remaining 238 were collected from the commercial orchards. Samples were tested with Agdia ELISA kits following kit instructions. These results demonstrated all Texas A\&M breeding stocks to be free of these viruses but commercial orchard testing uncovered two positive samples for PDV from two different counties and eleven positive samples for PNRSV from three different counties. In 2014, we plan to continue sampling Texas peach orchards for these three viruses as well as for the phytoplasma disease European stone fruit yellows.

Propagation of Xanthomonas fragariae in strawberry nursery stock: Epidemiological analysis using both experimental and modeling approaches

C. GIGOT (1), N. McRoberts (1), W. Turechek (2)

(1) University of California, Davis, CA, U.S.A.; (2) Horticulture Researsh Laboratory, USDA-ARS, Fort Pierce, FL, U.S.A.

Phytopathology 104(Suppl. 3):S3.45 
As a quarantine organism, the bacterium Xanthomonas fragariae - the cause of strawberry Angular Leaf Spot (ALS) - is a concern for U.S. nursery growers wishing to export plants. In addition, under conducive environmental conditions, ALS can cause high yield losses in fruit production. Current knowledge of ALS, especially its epidemiology, is incomplete. So, in collaboration with California nurseries, we are (i) analyzing disease spatial patterns under field conditions, and (ii) identifying and characterizing the potential entrance points of the bacterium in the multi-year strawberry growing process. The study of spatial patterns allows us to disentangle and rank pathogen dispersal mechanisms, a necessary step to optimize ALS control. For example, there are infections due to overhead irrigation (anisotropic patterns under still air conditions), mechanical and anthropic interventions (isotropic patterns linked to crop rows), and infections coming from outside the plots. No totally effective chemical or genetic control exists for this disease. ALS management is almost completely dependent on pathogen exclusion and physical removal of symptomatic plants. However infected hosts may be asymptomatic during long periods. To design healthier production systems, it is essential to know where and when the bacterium is most likely to contaminate $X$. fragariae-free plants, and the pathogen basic reproduction number in different variants of the production process.

Using wheat cultivar mixtures as a control method against septoria tritici blotch: Experimental and modeling approaches

C. GIGOT (1), S. Saint-Jean (2), L. Huber (3), C. de Vallavieille-Pope (4)

(1) University of California, Davis, CA, U.S.A.; (2) AgroParisTech-INRA, UMR1091 Environnement et Grandes Cultures, Thiverval-Grignon, France; (3) INRA-AgroParisTech, UMR1091 Environnement et Grandes Cultures, Thiverval-Grignon, France; (4) INRA, UR1290 BIOGER-CPP, ThivervalGrignon, France

Phytopathology 104(Suppl. 3):S3.46

Septoria tritici blotch (STB), caused by Zymoseptoria tritici, is a worldwide wheat disease of concern that may lead to major yield-damaging epidemics. Because STB is rain-splash dispersed at short distances during the host growing season, cultivar mixtures are expected to provide a rather low efficiency in disease reduction. However, quantitative data are still lacking to specify what it actually is. We used both experimental and modeling approaches in order to figure out how mixing cultivars can decrease STB severity. From 2008 to 2012, field experiments were conducted on binary wheat mixtures, with a $1: 3$ moderately susceptible:moderately resistant ratio. The number of sporulating lesions, and the diseased and green leaf areas were assessed on a weekly basis. All the results showed consistent reductions of STB symptoms on the more susceptible cultivar (ca. $40 \%$ less diseased area over the five years), without significantly affecting the more resistant cultivar, comparatively to their respective pure stands. In parallel, a biophysical model was developed to extend our experimental findings. It relied on the combination of virtual 3D wheat-like canopies with heterogeneous host resistance levels, and mechanical description of trajectories of pathogenbearing splash droplets. Using this modeling approach, we pointed out the necessity to consider the accurate cultivar resistance levels in order to find the optimal cultivar ratio, maximizing the mixture efficiency.

Bull's-eye: Tissue processing improvements for isolating high quality RNA from laser microdissected pathogen infected cells and tissues I. J. GIRARD (1), D. Fernando (1), M. G. Becker (1), M. F. Belmonte (1)

(1) University of Manitoba, Winnipeg, MB, Canada

Phytopathology 104(Suppl. 3):S3.46

Canola is a Canada's number one oilseed crop, however damage from fungal attacks by the pathogen Leptosphaeria maculans (blackleg) results in millions of dollars in lost productivity every year. The severity of each host-pathogen interaction is governed by complex regulation of transcriptional circuitry that mediates the activation of plant defenses. These regulators are specified in cells at the site of the infection and over time to neighboring cells as the plant responds to the attack. The first step in revealing these processes with a genomics approach is contingent on the isolation of high quality RNA from cells located at and distal to the site of infection, allowing accurate profiles of gene activities for downstream analyses. Laser microdissection is the only method capable of isolating individual cells and tissues from the site of the infection with such a high degree of precision. Tissues of interest are fixed and embedded in paraffin wax to preserve cell structure and RNA, then sectioned for visualization of target cells and microdissected using a Leica LMD 7000 microscope. Optimization of each step of the process is required to overcome RNA degradation from exposure to harsh chemicals like xylenes used in tissue clearing and high temperatures used for wax infiltration. We present quantitative data based on careful optimization of each step in the tissue processing protocol and discuss implications of RNA quality to next generation sequencing strategies.
Distinguishing resistant from tolerant host-pathogen interactions between Fragaria vesca and Verticillium dahliae

G. GLEESON (1), M. Wallhead (1), K. Broders (1)

(1) University of New Hampshire, Durham, NH, U.S.A.

Phytopathology 104(Suppl. 3):S3.46

There is considerable variation in Verticillium wilt resistance between different cultivars of strawberry. However, little is known about the mechanisms responsible for these variable resistance or tolerance reactions. Current methods of categorizing susceptible and resistant cultivars of strawberry based on severity of disease symptoms, although cheap and efficient, lack the resolution to describe the true nature of the host-pathogen interactions. The objective of this study was to use confocal microscopy, hyperspectral imaging, and plating assays, to more precisely and accurately describe host-pathogen interactions. Progeny of a cross between a resistant and a susceptible parent of Fragaria vesca were inoculated with a transgenic strain of Verticillium dahliae expressing the Green Fluorescent Protein (GFP) in greenhouse trials and scored for resistence/susceptibility by visual rating, tracking the infection by GFP, and recording the spectral reflectance of plant leaves. Progeny from the susceptible $\times$ resistant parent cross of the wild strawberry, Fragaria vesca, showed variable levels of colonization by $V$. dahliae as measured by confocal microscopy, while demonstrating little to no difference in visual disease symptom severity. Distinguishing tolerant from resistant host-pathogen interactions will allow for more effective disease management of vascular wilt pathogens by minimizing cultivars that harbor the pathogen.

Food fight: Fungal foe frustration (Fusarium verticillioides vs. the world of xenobiotics)

S. E. GOLD (1), M. Gao (2), N. Crenshaw (3), M. Rath (2), S. Gao (2), A. E. Glenn (3)

(1) USDA ARS, Athens, GA, U.S.A.; (2) Plant Pathology, University of Georgia, Athens, GA, U.S.A.; (3) USDA-ARS Toxicology and Mycotoxin Research Unit, Athens, GA, U.S.A.

Phytopathology 104(Suppl. 3):S3.46

Fusarium verticillioides infects maize and produces the fumonisin mycotoxins. The genome of the fungus encodes approximately 30 proteins containing beta-lactamase domains that are roughly evenly split between two families, metallo beta-lactamases and cephalosporinases. In bacteria betalactamases are well known to confer resistance to antibiotics, including penicillin. However, their roles in fungal biology are cryptic. In $F$. verticillioides the function of only one of these genes (FVEG_08291) has been demonstrated. This gene is critical for resistance to the maize benzoxazolinone phytoanticipins. The enzyme hydrolyzes 2-benzoxazolinone (BOA), which contains a gamma-lactam moiety, to produce the intermediate 2-aminophenol in the detoxification pathway. We hypothesize that many of the $F$. verticillioides beta-lactamases function similarly to FVEG_08291 to detoxify environmental xenobiotics. To test this hypothesis, we are engaged in a long-term project to delete each gene within the two beta-lactamase families. This is being accomplished primarily through undergraduate student research projects. We plan to test the mutant collection for increased sensitivity to various lactam containing compounds, particularly those known from organisms with overlapping niche space with $F$. verticillioides, such as the maize endophyte Acremonium zeae and its lactam containing antimicrobial metabolites, pyrrocidines A and B. This presentation will provide a snap shot on our progress.

\section{Pathogenicity of Fusarium proliferatum on common bean in Costa Rica}

J. P. González Venegas (1), P. ESKER (1), A. Murillo Williams (1)

(1) Universidad de Costa Rica, San Pedro Montes de Oca, Costa rica

Phytopathology 104(Suppl. 3):S3.46

Fusarium proliferatum $(\mathrm{Fp})$ is a plant pathogen that is widely distributed around the world and has been identified as the causal agent of numerous diseases on several crops. In Costa Rica, Fp has not been reported as a pathogen of common bean (Phaseolus vulgaris), a widely consumed grain in the majority of countries in Latin America. Furthermore, it is known that this pathogen can produce fumonisins, increasing the potential risk to consumers. To determine if isolates of $\mathrm{Fp}$ from rice can be pathogenic on common bean, a series of studies were conducted. Two bean varieties, Guaymí and Cabécar, and 10 isolates of $\mathrm{Fp}$ were tested. Overall, a low degree of pathogenicity was observed on both bean varieties, with primary damage to the base of the stem and the main root of seedlings. Nonetheless, some isolates were found to cause greater damage, and differences in isolate pathogenicity were noted depending on the bean variety. In growth chamber studies to examine the effect of temperature, the highest diseases severity was observed at $35^{\circ} \mathrm{C}$ for the most pathogenic isolate. Overall, across experiments the variety Cabécar was found to be more affected by Fp. Lastly, it was noted that fumonisins 
could be produced on both varieties, with average concentrations of $6.6 \mathrm{mg}$ $\mathrm{kg}^{-1}$ (Guaymí) and $6.1 \mathrm{mg} \mathrm{kg}^{-1}$ (Cabecar), respectively. These results suggest that $\mathrm{Fp}$ can infect bean seedlings and has the ability to produce fumonisins when growing on bean seeds as substrate.

Effects of temperature and moisture on infection of wheat by Puccinia triticina under field conditions in Kansas

B. S. GRABOW (1), E. D. De Wolf (1)

(1) Kansas State University, Manhattan, KS, U.S.A.

Phytopathology 104(Suppl. 3):S3.47

Leaf rust (Puccinia triticina) is an economically important wheat disease in Kansas. In 2005, 2007, and 2008 Kansas has experienced 2, 14, and 4.7 percent statewide yield losses respectively. A bioassay was used to identify weather conditions that were favorable for the infection of a $P$. triticina isolate in an outdoor field environment. The bioassay included inoculating potted seedlings with $P$. triticina and exposing the plants to an outdoor environment overnight (16 hours). During the nightly exposure period, two pots were misted with distilled water and then covered with a plastic bag to retain moisture, and two pots were exposed to ambient conditions. Temperature, relative humidity, leaf wetness, and precipitation were collected on-site. Following exposure, the plants were then placed in a growth chamber for 13 days at $20^{\circ} \mathrm{C}$ and evaluated for disease severity. The bioassay was repeated on 126 nights representing both fall and spring growing seasons for winter wheat in Kansas. The misted treatment plants were used to determine the temperature range for infection if moisture was not limiting. Infections occurred at a wide range of temperatures $\left(5-25^{\circ} \mathrm{C}\right)$, and were most frequent when more than 6 hours of leaf wetness occurred during exposure. Future research will use these results to determine weather conditions that influence epidemics and to develop prediction models for leaf rust in Kansas.

\section{Variation in the reaction of hard red winter wheat cultivars to common} root rot and spot blotch

N. GRAF GRACHET (1), R. M. Hunger (1), M. E. Payton (1), J. Edwards (1)

(1) Oklahoma State University, Stillwater, OK, U.S.A.

Phytopathology 104(Suppl. 3):S3.47

The incidence of residue-borne wheat diseases has increased in Oklahoma due to adoption of no-till cultivation. This includes common root rot (CRR) and the foliar disease spot botch (SB), both caused by Bipolaris sorokiniana. Screening for reaction to CRR and SB currently is not conducted, so the goal was to determine if useful levels of resistance to these diseases exist in hard red winter wheat (HRWW) cultivars. For SB, seedling foliage of 14 cultivars was sprayed with a spore solution $\left(10^{5}\right.$ and $10^{6}$ spores $\left./ \mathrm{mL}\right)$. Disease was visually assessed by rating percent infected leaf area and by using Assess quantification software (Lamari, 2008, APS Press). For CRR, seeds of 3 cultivars were inoculated with a spore solution $\left(10^{5}\right.$ and $10^{6}$ spores $\left./ \mathrm{mL}\right)$ and incubated at $25^{\circ} \mathrm{C}$ in the dark between wet filter paper. Root system reduction (area and length) was determined using Assess. For SB, a high correlation $\left(\mathrm{R}^{2}\right.$ $=0.91$ ) was observed between visual rating and Assess. Average severity was $30 \%$ across all cultivars; Billings, Ruby Lee and Garrison showed the best resistance $(12 \%)$. For CRR, root length and root area were highly correlated (average $\mathrm{R}^{2}=0.86$ ), but root length provided greater differentiation between varieties. Percent root length reductions were 12, 22, and 41 for Duster, Endurance, and Iba, respectively. These results indicate useful levels of resistance to $\mathrm{SB}$ and CRR may be available in HRWW varieties.

Excess bicarbonate in soil and irrigation water increases fibrous root loss and decline of Huanglongbing-affected citrus trees in Florida

J. H. GRAHAM (1), K. M. Gerberich (1), D. B. Bright (1), E. G. Johnson (1) (1) University of Florida, Citrus Research and Education Center, Lake Alfred, FL, U.S.A.

Phytopathology 104(Suppl. 3):S3.47

An extensive survey of HLB-affected groves indicated that greater decline in fibrous root health and greater expression of HLB symptoms is observed where irrigation water is high in bicarbonates $(>100 \mathrm{ppm})$ and/or soil $\mathrm{pH}>$ 6.5. Affected orchards employ micro-sprinkler irrigation that concentrates fibrous roots in the wetted zone and soils often have a history of excessive dolomite liming to manage high residual copper. Affected orchards have offcolor foliage, thin canopies due to excessive leaf drop, twig dieback and more severe HLB symptoms in leaves and fruit. HLB symptom expression of trees on different rootstocks is ranked Swingle citrumelo $>$ Carrizo citrange $>$ sour orange $>$ Cleopatra mandarin which follows rootstock intolerance of bicarbonate. To confirm the relationship between HLB decline and bicarbonate stress, fibrous roots of 8-15 yr old Valencia orange trees on Swingle or Carrizo rootstock were sampled in 37 orchards with varying soil $\mathrm{pH}$ and irrigation water quality. Lower root density was directly related to irrigation water $\mathrm{pH}$ and to soil $\mathrm{pH}>6.2$. Fruit production over three seasons
(2009-2012) during which HLB incidence was accelerating revealed that orchards under high bicarbonate stress declined $20 \%$ in yield compared to orchards with low bicarbonate stress with a $6 \%$ increase in production. Yield loss was correlated with reduced fibrous root density.

Black root rot of industrial chicory (Cichorium intybus $\mathrm{L}$. var. sativum) in Chile caused by Boeremia exigua var. exigua

D. E. GRINBERGS (1), R. A. France (1)

(1) INIA, Chillan, Chile

Phytopathology 104(Suppl. 3):S3.47

The industrial chicory is cultivated for fructan extraction from their roots, food which promotes human health. In Chile the crop was introduced in 2006 and from the beginning showed an undescribed disease, causing significant damages to the root and yield losses up to $31 \%$. Besides the industrial problems in fructan extraction. The objectives were to describe and identify the causing agent and to assess the incidence, severity and pathogenicity. This investigation was conducted between 2009 and 2013. The symptoms on the crown and upper taproot started as small, dark, firm, sunken lesions, and then turned from brownish to black. Root cavities lacked fungal signs and covered the entire root up to $20 \%$ of the analyzed plants at harvest. In 2009 the disease incidence occurred on $39 \%$ of the sampled plants. From the infected roots, a fungus was consistently isolated on acidified PDA and water agar. The pathogenicity tests showed that the pathogen caused rot on detached roots, entire roots, potted plants and plants growing on inoculated soil. The fungus was reisolated from the symptomatic tissues, confirming Koch's postulates. On the basis of its morphological characteristics on oatmeal agar, the presence of metabolite ' $E$ ' on malt agar and the pathogenicity tests, the isolates were identified as Boeremia exigua var. exigua. To our knowledge this is the first report of B. exigua var. exigua in industrial chicory in Chile.

Red stripe caused by Acidovirax avenae subsp. avenae in Louisiana sugarcane

M. P. GRISHAM (1), R. M. Johnson (1)

(1) USDA ARS Sugarcane Research Unit, Houma, LA, U.S.A. Phytopathology 104(Suppl. 3):S3.47

Red stripe of sugarcane caused by Acidovirax avenae subsp. avenae is considered to be of minor importance because, most often when found, only the mild leaf stripe symptom is observed. In 2010, both leaf stripe and the more severe top rot symptom were observed in commercial sugarcane fields in Louisiana. An experiment was established in two naturally infected fields of cultivar HoCP 00-950. One, a plant-cane field, was divided into 113 plots; the other, a first-ratoon field, into 84 plots. A negative correlation was found between disease incidence and $\mathrm{kg}$ sucrose yield per hectare with $\mathrm{kg}$ sucrose per tonne of cane the component most affecting yield loss. In a nitrogen fertility rate experiment, incidence of red stripe was higher among plots with heavier, clay soils than among those with lighter, more silty soils, and among plots with added nitrogen compared to the plots with no added nitrogen. In another experiment, the effect of planting a new crop with stalks from a field with red stripe was tested. Treatments included planting plots with 1) stalks exhibiting top rot, 2) stalks exhibiting leaf stripe, 3) a mixture of stalks exhibiting both symptom types and nonsymptomatic stalks, and 4) nonsymptomatic stalks only. Stalk stand counts and yield (kg sucrose per hectare) were reduced in the three treatments planted with symptomatic stalks compared to the one planted with nonsymptomatic stalks. Incidence of red stripe was very low in the new crop and first-ratoon crop.

Quantification of enzymes elicited by Sarocladium oryzae in relation to suppression of leaf blast severity

R. A. Guimarães (1), V. L. SILVA-LOBO (2), M. C. C. Filippi (2), M. M. Barros (2), A. S. Prabhu (2)

(1) Universidade Federal de Goiás, Goiânia, Brazil; (2) Embrapa Rice and Beans, Santo Antonio De Goias, Brazil

Phytopathology 104(Suppl. 3):S3.47

Magnaporthe oryzae is the casual agent of rice blast and a major rice disease in Brazil. This fungus is responsible for significant yield losses, and the use of cultivar resistance and chemical control were not adequate for suppressing the disease. Isolates of Sarocladium oryzae showed, in vitro, antagonism against $M$. oryzae in paired culture test on PDA. The production of cerulenin, a secondary metabolite produced by $S$. oryzae, quantified by thin layer chromatography (TLC) was variable in different isolates, being greater in So 03. Rice plants of $\mathrm{cv}$. BRS Primavera were sprayed with conidial suspension (CS) and culture filtrate (CF) 48 hours before the inoculation with M. oryzae. The SC reduced leaf blast severity by $68.8 \%$ and $\mathrm{CF}$ by $75.5 \%$. The enzymatic quantification showed greater activity in relation to water control in chitinase (EC 3.2.1.14) and $\beta$ 1,3-Glucanase (EC 3.2.1.39) 3 and 24 hours after inoculation with M. oryzae (H.A.I.Mo), respectively, in SC and CF Peroxidase 
(EC 1.11.1.7), Phenylalanine ammonia-lyase (EC 4.3.1.5) e Lipoxygenase (EC1.12.11.12) exhibited oscillations in time and treatments. Salicylic acid had the strongest activity with peaks 3, 24 and 48 H.A.I.Mo. Spraying plants with $\mathrm{SC}$ and $\mathrm{CF}$ reduced leaf blast severity and possibly play a role in plant defense by biochemical activation processes that are expressed by enzymatic activity.

Increased $\mathrm{CO}_{2}$ and temperature effects on Fusarium oxysporum f. sp. lactucae on lettuce plants grown under simulated environmental conditions

M. L. GULLinO (1), I. Ferrocino (2), W. Chitarra (3), M. Pugliese (4), G. Gilardi (3), A. Garibaldi (3)

(1) University of Torino, Grugliasco Torino, Italy; (2) Agroinnova, Disafa, University of Torino, Grugliasco (TO), Italy; (3) Agroinnova, University of Torino, Grugliasco (TO), Italy; (4) Disafa, Agroinnova, University of Torino, Grugliasco (TO), Italy

Phytopathology 104(Suppl. 3):S3.48

The pathosystem lettuce (Lactuca sativa) - Fusarium wil (Fusarium oxysporum f. sp. lactuace) was chosen as model to assess the potential impact of increased $\mathrm{CO}_{2}$ and temperature on disease under controlled environment. Potted plants were grown in phytotrons under 4 different simulated climatic conditions: (1) standard temperature (ranging from $18^{\circ}$ to $22^{\circ} \mathrm{C}$ ) and standard $\mathrm{CO}_{2}$ concentration $(450 \mathrm{ppm}) ;(2)$ standard temperature and elevated $\mathrm{CO}_{2}$ concentration ( $800 \mathrm{ppm}$ ); (3) elevated temperature (ranging from $22^{\circ}$ to $26^{\circ} \mathrm{C}$, $4^{\circ} \mathrm{C}$ higher than standard) and standard $\mathrm{CO}_{2}$ concentration; (4) elevated temperature and $\mathrm{CO}_{2}$ concentration. Each pot was inoculated with a talc containing chlamydospores of Fusarium oxysporum f. sp. lactuace to reach a final concentration of $1 \times 10^{4} \mathrm{CFU} \mathrm{g} 1^{-1}$ of substrate. Non infested pots were used as control. Disease severity and physiological parameters (chlorophyll content, fluorescence) were assessed every 7 days. At the same time substrate samples were collected and plate counts, enzymatic assays, and PCR-DGGE analyses were carried out on to evaluate the effect of climate change on the microbial population. Disease severity and Fusarium spp. population were positively influenced by higher temperatures but not by increasing $\mathrm{CO}_{2}$ concentration. Considering the future environmental conditions and the expected rising of global temperature, we can assume that this could increase the severity of Fusarium wilt of lettuce and probably of other Fusarium wilts.

Effect of light on the fumonisin production of members of the Gibberella fujikuroi species complex isolated from rice

M. L. GULLINO (1), D. Spadaro (2), S. Matic (3), A. Garibaldi (3)

(1) University of Torino, Grugliasco (Torino), Italy; (2) Disafa, Agroinnova, University of Torino, Grugliasco (TO), Italy; (3) Agroinnova, University of Torino, Grugliasco (TO), Italy

Phytopathology 104(Suppl. 3):S3.48

Some Fusarium species belonging to the Gibberella fujikuroi species complex and associated to bakanae disease of rice were investigated for their ability to produce fumonisins $\left(\mathrm{FB}_{1}\right.$ and $\left.\mathrm{FB}_{2}\right)$ under different light conditions, and for pathogenicity. Highly stimulating conditions for fumonisin production compared to darkness were yellow and green light in F. verticillioides strains; white and blue light, and light/dark alternation in F. fujikuroi and $F$. proliferatum strains. All light conditions influenced positively the fumonisin production with respect to the dark. Expression of the FUMI gene, which is necessary for initiation of fumonisin production, was in accordance with the fumonisin biosynthetic profile. High and low fumonisin-producing $F$. fujikuroi strains showed typical symptoms of bakanae disease, abundant fumonisin-producing $F$. verticillioides strains caused chlorosis and stunting of rice plants, while fumonisin-producing $F$. proliferatum strains were asymptomatic on rice. F. fujikuroi might be an abundant fumonisin producer with levels comparable to that of $F$. verticillioides and $F$. proliferatum, highlighting the need of deeper mycotoxicological analyses on rice isolates of $F$. fujikuroi. Our results showed for the first time the influence of light on fumonisin production in isolates of $F$. fujikuroi, $F$. proliferatum, and $F$. verticillioides from rice.

Control of downy mildew of basil with fungicides and resistance inducer products under greenhouse conditions

M. L. GULLINO (1), G. Gilardi (2), A. Garibaldi (2)

(1) University of Torino, Grugliasco Torino, Italy; (2) Agroinnova, University of Torino, Grugliasco (TO), Italy

Phytopathology 104(Suppl. 3):S3.48

Downy mildew of basil, incited by Peronospora belbahrii, causes particularly severe losses in several countries. The management of downy mildew of basil is complicated by the very limited availability of chemicals on this crop and by the absence of basil varieties resistant to the pathogen. Trials were carried out under greenhouse conditions to evaluate a number of compounds, known for their capability of inducing resistance compared with systemic fungicides and copper salts. Treatments were applied alone and in rotation as foliar spray on basil plants 16-21 days after sowing, by using a dosage in according to manufacturer's instructions. In the presence of a disease incidence ranging from $66.8 \%$ to $88.8 \%$, metalaxyl-M + copper hydroxide, azoxystrobin, mandipropamid, the based-phosphite fertilizer were the most effective in disease reduction compared with the untreated control. The prohexadione-Ca and mustard oil were only partially reduced disease incidence and severity (43\% - 69\% and from 33\% - 70\%, respectively) compared with the untreated control, while B. subtilis QST 713 and thyme oil extract were not effective in two out of three trials. Effective control was obtained either using rotation of fungicides with compounds that can induce resistance as well as by using rotation with different resistance-inducing compounds on their own.

Functional conservation and divergence of cAMP-PKA signaling pathway in cereal pathogenic Fusarium graminearum and $F$. verticillioides

L. GUO (1), X. Zhou (2), A. Breakspear (3), G. Zhao (1), L. Gao (1), J. R. Xu (2), H. C. Kistler (3), L. J. Ma (1)

(1) University of Massachusetts Amherst, Amherst, MA, U.S.A.; (2) Purdue University, West Lafayette, IN, U.S.A.; (3) USDA-ARS Cereal Disease Lab, St. Paul, MN, U.S.A.

Phytopathology 104(Suppl. 3):S3.48

Fusarium graminearum $(F g)$ and $F$. verticillioides $(F v)$ are two pathogenic ascomycetes that cause cereal crop diseases and produce mycotoxins, threatening global food safety and human health. Molecular mechanisms for pathogenesis are crucial for disease control but not well understood. Genetic analysis of $F g$ and $F v$ cAMP-PKA pathway showed this pathway was required for normal pathogenesis in both species but also played speciesspecific roles in secondary metabolism. To understand gene regulatory networks and differences of $F g$ and $F v$ cAMP-PKA pathway, we compared transcriptomes of FAC1 and CPK1 mutant. Overall this essential pathway is highly conserved between the two closely related species. The conservation signals enable identification of unique components of cAMP signaling pathway for $F g$ and $F v$. Based on functional enrichment of differentially expressed genes, conserved pathways control essential functions such as metabolism, cell cycle and protein synthesis. Diverged pathways play more species-specific roles such as isoprenoid and aurofusarin biosynthesis in $\mathrm{Fg}$ but polyketide synthesis, $\mathrm{pH}$ response and macroconidiation in $F v$, suggesting that although much conserved in $F g$ and $F v$, cAMP-PKA pathway has diverged to perform species-specific functions during speciation. This result, in strong agreement with the mutant phenotypes, dissects cAMP-PKA pathway in the two pathogenic fungi and provides a powerful approach to correlate genotypes with fungal physiological data.

East meets west, the story of Geosmithia morbida in the United States D. HADZIABDIC (1), K. A. Nix (1), L. M. Vito (1), P. R. Merten (2), M. T. Windham (1), P. L. Lambdin (1), J. F. Grant (1), G. J. Wiggins (1), J. W. Pscheidt (3), P. A. Wadl (1), W. Cranshaw (4), R. N. Trigiano (1)

(1) University of Tennessee, Knoxville, TN, U.S.A.; (2) USDA Forest Service, Forest Health Protection, Asheville, NC, U.S.A.; (3) Oregon State University, Corvallis, OR, U.S.A.; (4) Colorado State University, Fort Collins, CO, U.S.A.

Phytopathology 104(Suppl. 3):S3.48

The fungus Geosmithia morbida and walnut twig beetle, Pityophthorus juglandis, have been associated with a disease complex of black walnut (Juglans nigra) known as thousand cankers disease (TCD). Disease symptoms include branch dieback, canopy loss, and tree mortality. During the current epidemic, TCD has expanded from the western to the native eastern range of black walnut and has recently been discovered in northwestern Italy. As a result of pathogen movement and possible global distribution, there is a critical need to understand genetic diversity and population structure of $G$. morbida. We investigated the population structure of 135 isolates of $G$. morbida from eastern (58) and western (77) United States using eight microsatellite loci. There is high genetic diversity among seven G. morbida subpopulations with evidence of gene flow and a significant correlation between geographic and genetic distance. The fungus exhibited 131 unique haplotypes. Analysis of molecular variance (AMOVA) indicated that most of the genetic variation was attributed to individual variation $(88 \%)$ rather than divergence across sampling localities $(18 \%)\left(\Phi_{S T}=0.28, P<0.001\right)$. Bayesian clustering analyses identified two distinct genetic clusters (delta $K=2$ ), with the highest admixture found in Oregon and Colorado subpopulations. Understanding the genetic composition and demography of this hostpathogen-insect complex can provide insight into future predictions of TCD epidemics. 
Biomass yield and rust intensity on ornamental switchgrass is impacted by fungicide selection and application interval

A. K. HAGAN (1), K. L. Bowen (1)

(1) Auburn University, Auburn, AL, U.S.A.

Phytopathology 104(Suppl. 3):S3.49

'Cloud 9'ornamental switchgrass was transplanted from 2 in. pots in 2011 at the Brewton Agricultural Research Unit. Plants were drip irrigated as needed. An application of $400 \mathrm{lb} / \mathrm{A}$ of 5-10-15 fertilizer was made each year in March. A factorial design arranged in a split plot of five single plant replications with fungicides as the main plot and application interval as the split plot was used. Daconil Ultrex, Heritage 50WDG, and Eagle 20EW were applied from June to September at 1-, 2-, 3- and 4-wk intervals in 2011, 2012, and 2013. Rust intensity was visually rated monthly during the study period using a 1 to 10 scale. In December, plant tops were cut and weighed. To determine dry biomass yield, shoot samples were weighed wet, oven dried, and weighed dry. Since the fungicide $\times$ application interval for rust intensity and biomass yield are not significant, data are pooled. While all fungicides reduced rust intensity compared with the non-treated control, Eagle 20EW gave better control than Heritage 50WDG in two of three yr, while Daconil Ultrex was least efficacious. Rust intensified as application interval went from 1 to $4 \mathrm{wk}$. Eagle 20EW, Heritage 50WDG, and Daconil Ultrex boosted biomass yield from 15 to $40 \%$ compared with the non-treated control in three, two, and one of the three study yrs, respectively. Biomass yield was higher in two of three yr for fungicides applied weekly than at longer intervals and was lowest for the nontreated control.

Yield parameters and disease activity on biomass, forage, and sweet sorghum varieties in Alabama

A. K. HAGAN (1), K. L. Bowen (1)

(1) Auburn University, Auburn, AL, U.S.A.

Phytopathology 104(Suppl. 3):S3.49

Disease reaction, aboveground dry matter (ADM), ${ }^{\circ} \mathrm{Bx}$, and total fermentable sugar yield (TFSY) of commercial sweet and forage sorghums along with hybrid sweet and biomass sorghums were assessed at three locations in Alabama. All varieties were sown between May 1 and May 15. Disease severity was periodically assessed on a 1 to 9 scale thru harvest, which consisted of cutting stalks $5 \mathrm{~cm}$ above the soil line in $1.5 \mathrm{~m}$ length of row at soft dough. Stalks were weighed and milled after deheading to calculate ${ }^{\circ} \mathrm{Bx}$ and TFSY. Stalk samples were weighed wet, dried, then weighed to calculate ADM yield. Anthracnose was the primary disease at all sites, while rough leaf spot, gray leaf spot, zonate leaf spot, and rust caused minor damage. At a Central AL site, anthracnose, which was first noted on SS405 forage sorghum leaves at 40 DAP, caused nearly $70 \%$ and $90 \%$ leaf area necrosis at 60 DAP and 120 DAP, respectively. While anthracnose development was slower on the sweet sorghum varieties, their final anthracnose ratings nearly matched those of SS405 at one of three sites but were low at the other two. Similarly high ADM yields were often seen for the M81-E and Topper 76-6 sweet sorghums and hybrid sweet and biomass varieties. Hybrid sweet sorghum variety ${ }^{\circ} \mathrm{Bx}$ and TFSY matched or exceeded those of M81-E and Topper 76-6 and were higher compared to Dale sweet sorghum. While two hybrid sweet sorghum varieties had similar anthracnose ratings to M81-E, one proved highly resistant.

The stem nematodes of Ditylenchus weischeri and $D$. dipsaci can be reared on carrot disks but not on fungal cultures

A. HAJIHASSANI (1), M. Tenuta (1)

(1) University of Manitoba, Winnipeg, MB, Canada

Phytopathology 104(Suppl. 3):S3.49

Monoxenic cultures can provide sufficient amount of nematode inoculum in studying plant-nematode associations. Among species of Ditylenchus, some have been reared on callus of alfalfa, chicory or carrot as well as fungal cultures. A population of $D$. weischeri from creeping thistle in Manitoba and D. dipsaci from garlic in Ontario were examined for potential to be reared on carrot disks and fungal cultures. The nematodes were isolated from plant tissues, placed in streptomycin sulphate $\left(4,000 \mathrm{mg} \mathrm{L}^{-1}\right)$ and repeatedly rinsed in sterile distilled water. Eighty individuals (male, female and J4) of each species were then transferred to plates containing either surface sterilized carrot disks without nutrient agar or the fungi, Fusarium solani, Botrytis cinerea, Rhizoctonia solani, Verticillium dahliae, Sclerotinia sclerotiorum, Colletotrichum gloeosporioides, and an unidentified species from creeping thistle, cultured on PDA. The number of nematodes recovered from carrot disks varied significantly for the two species. The number of $D$. weischeri increased 51 times, however, the number of $D$. dipsaci increased more, being 238 times. $D$. weischeri and $D$. dipsaci couldn't be reared on any of the fungal isolates examined. The results indicate feeding behaviour of $D$. dipsaci and $D$. weischeri is different from other Ditylenchus species capable of rearing on fungi. Rearing both nematode species on carrot disks will provide large numbers of individuals for plant host range studies.

Greenhouse reproduction fitness of the stem nematodes, Ditylenchus weischeri and $D$. dipsaci on pulse and non-pulse crops

A. HAJIHASSANI (1), M. Tenuta (1), R. Gulden (2)

(1) Department of Soil Science, University of Manitoba, Winnipeg, MB, Canada; (2) Department of Plant Science, University of Manitoba, Winnipeg, MB, Canada

Phytopathology 104(Suppl. 3):S3.49

Two series of greenhouse trials were conducted to evaluate the host range of Ditylenchus weischeri, a newly described stem nematode species on creeping thistle (Cirsium arvense), and D. dipsaci, a quarantine parasitic species of many crops. Pulse crops included five yellow pea varieties, a pinto, a kidney and a navy bean, a desi and a kabuli chickpea, and a large green lentil variety. Non-pulses examined were spring wheat (var. AC Barrie), canola (var. Invigor), creeping thistle and garlic. Trials were conducted in a completely randomized design with nine replications. Each replicate unit a potted plant inoculated with $100 \mathrm{~J} 4$ stage individuals of either $D$. weischeri or D. dipsaci in $15 \mu \mathrm{l}$ of $1.5 \%$ carboxymethyl cellulose, applied to the axils of the first true leaves and then grown for eight weeks. Plants inoculated with water alone were as controls. Both $D$. weischeri and $D$. dipsaci had no significant impact on the aboveground biomass weight of all crops tested. The yellow pea varieties (range $R_{f}=2.1$ to 6.9 ), bean (1.4 to 2.4), chickpea (1.1 and 1.5), and garlic (6.2) were hosts for $D$. dipsaci but lentil, spring wheat, canola and creeping thistle were not. The $R_{f}$ for $D$. weischeri on yellow pea varieties ranged from 0.9 to 1.7. Creeping thistle was a very good host for $D$. weischeri with an $R_{f}$ of 5.7. All other crops examined were not hosts for D. weischeri. This study confirmed the low potential of $D$. weischeri to reproduce on pulse crops compared to D. dipsaci.

Context dependency of mutation in P3 of Soybean mosaic virus required for gain of virulence on Rsv4-genotype soybeans

M. R. HAJIMORAD (1), Y. Wang (1), B. Khatabi (1)

(1) University of Tennessee, Department of Entomology \& Plant pathology, Knoxville, TN, U.S.A.

Phytopathology 104(Suppl. 3):S3.49

Rsv4 in soybean recognizes P3 of Soybean mosaic virus (SMV) and confers broad resistance against SMV. It has previously been reported that amino acid substitutions at polyprotein positions 1033 (Q to $\mathrm{K}$ ) or 1054 ( $\mathrm{G}$ to $\mathrm{R}$ ), both residing on SMV P3, converts avirulent isolates belonging to $\mathrm{G} 2$ strain to virulent. In other hand, the virulence determinant of another unassigned SMV isolate to a specific strain was reported at position 1053 where $\mathrm{S}$ to $\mathrm{N}$ substitution resulted in virulence. In contrast, virulence determinants of an isolate belonging to $\mathrm{G} 7$ strain has been reported to HC-Pro, P3 and CI. In this study, virulence impact of amino acid substitutions at corresponding positions of SMV-G7 and SMV-G7d (two isolates belonging to G7 strain) were examined. Additionally, S1053N substitution in P3 of SMV-N, an isolate belonging to $\mathrm{G} 2$ strain, was also evaluated. The results confirm that virulence determinant(s) of SMV on Rsv4-genotype soybeans solely reside on P3. Furthermore, data suggest that i) amino acid substitution at corresponding position 1053 of none of these viruses play a role in virulence, ii) amino acid substitution at polyprotein position corresponding to 1054 of SMV-G7 and SMV-G7d do not confer virulence, and iii) genetic composition of P3 plays a crucial role in virulence of SMV on Rsv4-genotype soybeans.

Molecular quantification of Colletotrichum lindemuthianum in dry edible pinto beans

J. HALVORSON (1), K. Simons (1), R. Lamppa (1), J. S. Pasche (1)

(1) North Dakota State University, Fargo, ND, U.S.A.

Phytopathology 104(Suppl. 3):S3.49

Dry bean anthracnose, caused by Colletotrichum lindemuthianum, is a damaging disease of dry bean (Phaseolus vulgaris L.) that can cause large yield reductions and reduced seed quality. The introduction of $C$. lindemuthianum into a field most commonly occurs through planting infected seed; therefore, the most important management practice is the use of certified seed. Early infections caused by seed to seedling transmission can be devastating and have been proven to be the most important factor in disease development. Seed to seedling transmission has been documented to be as high as $15 \%$ in symptomless seed and increases with increasing symptom severity. Anthracnose of dry bean traditionally has been diagnosed by visualizing the pathogen on infected tissues; however, this method may not be sensitive enough to detect symptomless seed infections. A real-time PCR assay was developed for the quantification of $C$. lindemuthianum in infected host tissue, regardless of the presence of disease symptoms. Primer pair $\mathrm{ClF} 1527 / \mathrm{ClR} 1609$ was developed from the single copy nitrate reductase gene 
and was able to detect less than $5 \mathrm{pg} / \mu \mathrm{l}$ of pathogen DNA with an $\mathrm{R}^{2}$ value of 0.992 and efficiency of $100.1 \%$ in a $10 \mu 1$ reaction. No product was amplified from DNA of other dry bean pathogens or Colletotrichum species. The assay successfully detected C. lindemuthianum in $46 \%$ of symptomless seeds produced from infected pods compared to a $4 \%$ detection rate using traditional fungal growth methods.

An RNA-sequencing analysis implicates the presence of multiple cell-tocell signaling pathways in the rice pathogenic bacterium Burkholderia glumae

J. H. HAM (1), R. Chen (1)

(1) Dept. Plant Pathology and Crop Physiology, Louisiana State University Agric Ctr, Baton Rouge, LA, U.S.A.

Phytopathology 104(Suppl. 3):S3.50

Burkholderia glumae is the Gram-negative bacterium causing bacterial panicle blight of rice. Major virulence factors of this pathogen, including toxoflavin (a phytotoxin), lipase and flagella, are known to be controlled by the quorum-sensing (QS) system composed of the LuxI and LuxR homologs, TofI and TofR. Our recent study with $B$. glumae $336 \mathrm{gr}-1$, a virulence strain isolated from a Louisiana rice field, revealed that toxoflavin production can be produced without $t o f I$ and/or $t o f R$ on a solid medium, implying the presence of unknown cell-to-cell signaling pathways independent of tofI and/or tofR. RNA sequencing and transcriptome analysis with B. glumae QS mutants revealed that genes predicted to function for the synthesis of BDSF (a fatty acid-type QS signaling molecule) and cyclic di-GMP (a key intracellular signaling molecule) as well as for bacterial programmed cell death were expressed in the dependence of the tofI/tofR QS system. In this study, it was also found that many genes involved in signal perception, transduction and transport were upregulated specifically in the cells grown on a solid medium, suggesting that alternative cell-to-cell signaling pathways are activated in bacterial cells grown in a sessile form.

Taxonomy and DNA sequence variation of the rust fungi infecting four prairie grass species

S. HAMBLETON (1), K. Temple (1), M. Liu (1)

(1) Agriculture and Agri-Food Canada, Ottawa, ON, Canada

Phytopathology 104(Suppl. 3):S3.50

Native, warm season, perennial prairie grasses offer advantages as biomass or bioenergy crops because of their high productivity with low agricultural inputs. All are susceptible to rust fungi but few modern systematic studies address these species. In late fall 2012, rust-infected leaves were collected from four grass hosts being evaluated at sites in Ontario for their potential as biofuels. Identification of the pathogen was expected to be straightforward. Teliospore morphology suggested one species was involved and multiple recent reports link Puccinia emaculata to one of the hosts, but ITS sequencing indicated some DNA extracts were mixed. Single pustule DNA extractions and PCR-based cloning were used to obtain clean DNA sequences, yielding multiple genotypes from some specimens. BLAST searches suggested an affinity to $P$. andropogonis, a complex of at least 5 taxa, or $P$. emaculata. To build a broader reference data set, herbarium specimens were sampled for additional representatives and related species. Bayesian analyses highlighted four groups (taxa) distinct from other clades. One included several specimens of $P$. emaculata, including one from Sorghastrum, while the other three represented as yet unidentified taxa. A comprehensive study of rusts reported from prairie grass hosts is needed to ensure future diagnostic assays are designed to target all species that could affect yield in biofuel crops.

\section{Advances toward DNA-based identification and differentiation of North} American Heterobasidion spp.

C. HAMMETT (1), S. F. Shamoun (2), I. Kassatenko (2), X. Li (3)

(1) UBC Forestry Sciences and Conservation, Vancouver, BC, Canada; (2) Canadian Forestry Service, Victoria, BC, Canada; (3) Canadian Food Inspection Agency, Charlottetown, PE, Canada

Phytopathology 104(Suppl. 3):S3.50

Heterobasidion annosum sensu lato is a species complex of pathogenic whiterot wood decay fungi which cause root and butt rot in conifer and hardwood stands across the Northern hemisphere. In North America there are two species: H. irregulare often found on Pinus and Picea species in eastern and central North America, and $H$. occidentale often found on several conifer and broad-leaf species in western North America. H. irregulare is of great interest as it tends to be more necrotrophic, virulent, and invasive then $H$. occidentale which behaves more as a saprophyte and agent of wood decay. The overlapping distribution, shared host range, similar disease symptoms and fruiting bodies have made discrimination between these two species difficult. We developed two sets of primers that bind specifically to conserved alleles in glyceraldehyde 3-phosphate dehydrogenase and elongation factor $1 \alpha$ genes for
H. irregulare and $H$. occidentale, respectively. The method is sensitive enough to detect either species from infected wood. New distribution maps of the species in North America, including the detection of $H$. irregulare for the first time on ponderosa pine in British Columbia as well as on western red cedar in Washington state, where it was previously thought to be $H$. occidentale. This method would serve as a valuable tool in detecting the pathogen in trade and forest stands.

Mutagenic strain development of Trichoderma harzianum to enhance antagonism against soil borne plant pathogens and its commercialization A. HANNAN (1), M. J. Saleem (2), S. T. Sahi (1), K. Riaz (1)

(1) University of Agriculture Faisalabad, Faisalabad, Pakistan; (2) University of the Punjab Lahore Pakistan, Lahore, Pakistan

Phytopathology 104(Suppl. 3):S3.50

Trichoderma harzianum has antagonistic potential against numerous soil borne plant pathogens. Present study was an effort to commercialize hyperactive mutants of $T$. harzianum. For this purpose 28 strains of $T$. harzianum were purified and screened for antagonistic efficiency against Fusarium oxysporum, Alternaria alternata, Macrophomina phaseolina and Rhizoctonia solani. The data revealed that strains $\left(\mathrm{Th}_{\mathrm{S} 3}, \mathrm{Th}_{\mathrm{S} 6}\right.$ and $\left.\mathrm{Th}_{\mathrm{S} 17}\right)$ isolated from rhizosphere of vegetable crops showed higher percentage of reduction in colony diameter over control (36.5, 38.0 and $37.5 \%$ respectively). The efficient strains were mutated through ultra violet irradiations and chemicals. The mutants survived on minimal inhibitory concentration of benomyl (mutant detector) were re-evaluated, where efficient individuals $(48.5,52.5$ and 52\%) were retained for genetic characterization. Band expression of mutants and parental strains exhibited isogenic variant of parental strains and their outperformance could be attributed to modified genetic make-up. Moreover these hyperactive mutants were characterized for conidial yield on cheaper substrates under solid state fermentation. The conidial count was higher on rice bran supplemented with chick pea flour $(2.5$ $\times 10^{8} \mathrm{cfu} / \mathrm{g}$ after 12 days of incubation at $27^{\circ} \mathrm{C}$. These findings explored a cost effective technology for production of hyperactive mutants of $T$. harzianum at pilot scale.

Storage rot in sugar beet: Variable response over time and with different host germplasm

L. E. HANSON (1), J. McGrath (1), T. R. Goodwill (1), M. Shaaban (2), R. M. Beaudry (2)

(1) USDA ARS, East Lansing, MI, U.S.A.; (2) Michigan State University, East Lansing, MI, U.S.A.

Phytopathology 104(Suppl. 3):S3.50

Sugar beet (Beta vulgaris) is commonly stored in outdoor piles prior to processing for food and animal feed. While in storage the crop is subject to multiple post-harvest rots. In the Michigan growing region, little loss due to storage rots is observed until beets have been in storage for several months. A recommendation to screen for resistance when beets had been stored for between 80 and 120 days was published 1970s, but there was little information about why this was recommended. Using USDA germplasm and recombinant inbred lines (RILs) of sugar beet, materials were screened for their response to some fungal storage rots and for changes in response with varying length of storage. Significant differences $(\mathrm{P}<0.05)$ were found in the amount of rot caused by pathogens in beets after different storage duration and in the response to various fungal pathogens. A poor correlation between reduced damage by different pathogenic genera suggests independent genetic control of susceptibility. Germplasm was identified that shows reduced damage with storage rot pathogens or a delay in development of extensive rot with several of the major storage rot pathogens. There is the potential to develop more resistant material to improve long term storage.

Pathogen and pest responses to forest management in the southern Sierra Nevada

A. E. HAWKINS (1), D. M. Rizzo (1)

(1) University of California, Davis, CA, U.S.A.

Phytopathology 104(Suppl. 3):S3.50

Forest pathogens and pests accelerate tree mortality, increase stand structural heterogeneity, and alter tree community composition. At mid-elevations in the Sierra Nevada white fir, red fir, sugar pine, Jeffrey pine and incense cedar coexist and host a variety of pathogens and pests. Various management strategies commonly applied to Sierran forests for conservation, ecological restoration, and fire prevention impact disease dynamics. In the Teakettle Experimental Forest (Sierra National Forest, $\sim 80 \mathrm{~km}$ east of Fresno, CA) six treatments were applied to 18, 4-hectare plots in 2000 and 2001. Treatments consisted of two burning (burn, no burn) and three thinning treatments (overstory thinning, understory thinning, no thinning) with three replicates per treatment. Pathogen and pest data were collected for all trees (over 30,000) in 
each plot pre- and post-treatment. I am collecting pathogen and pest data on all trees and stumps in each plot to investigate pathogen and pest responses a decade after treatments. Although mortality has decreased since last survey, specific pathogen incidences have increased in stumps and saplings. Mortality was proportional to the abundance of each tree species. Increases in Heterobasidion occidentale incidences may result in future increases in mortality.

\section{Selecting Minnesota American elms for resistance to Dutch elm disease}

B. W. HELD (1), R. A. Blanchette (1), C. P. Giblin (2)

(1) University of Minnesota, Department of Plant Pathology, St. Paul, MN, U.S.A.; (2) University of Minnesota, Department of Forest Resources, St. Paul, MN, U.S.A.

Phytopathology 104(Suppl. 3):S3.51

Dutch elm disease (DED) was first introduced to the United States in the 1920 's and over the past decades it has devastated the native American elm population. Like most other introduced diseases, the DED pathogen, Ophiostoma ulmi, encountered little resistance in its host which led to unabated disease spread across the United States where elms were planted. Despite the tremendous losses, the American elm is still an iconic species because of its elegant form and its suitability for the urban environment. As the disease has progressed through the landscape, some trees have survived in areas with high disease pressure. Survivor elms in Minnesota were identified, clonally propagated and challenged with Ophiostoma novo-ulmi to determine levels of resistance. Results indicate that wild type selections die quickly but resistant selections show reduced symptoms but recover and live, in both greenhouse and field trials. Recently it was discovered that elms in the U.S. are a polyploid complex and not mainly tetraploid as previously thought. Ploidy of DED-resistant Minnesota elms will also be presented. This research to identify and test putative resistance of selected elms is an effort to bring disease-resistant, cold hardy, and aesthetically pleasing native elms back into Minnesota landscapes.

Global crop connectivity as a risk factor for pathogen and pest invasion: The case of banana, cassava, potato, and sweetpotato

J. F. HERNANDEZ NOPSA (1), Y. Xing (1), J. Andrade-Piedra (2), F. Beed (3), G. Bloome (4), M. Carvajal Yepes (5), G. Forbes (6), J. Kreuze (7), J. Kroschel (8), J. Legg (3), M. Parker (9), E. Schulte-Geldermann (9), K. A. Garrett (1)

(1) Kansas State University, Manhattan, KS, U.S.A.; (2) International Potato Center (CIP), Quito, Ecuador; (3) International Institute of Tropical Agriculture (IITA), Dar es Salaam, Tanzania; (4) Bioversity, Kampala, Uganda; (5) International Center for Tropical Agriculture (CIAT), Cali, Colombia; (6) International Potato Center (CIP), Beijing, China; (7) International Potato Center (CIP), Lima, Peru; (8) International Potato Center / Global Crop Diversity Trust, Filderstadt, Germany; (9) International Potato Center (CIP), Nairobi, Kenya

Phytopathology 104(Suppl. 3):S3.51

The geographic connectedness of croplands is an important risk factor for the invasion of crop-specific pathogens. Understanding the structure of these networks supports sampling and mitigation strategies. We evaluated global networks of the vegetatively-propagated crops banana/plantain, cassava, potato, and sweetpotato, because of their major role as a source of energy in the tropics, and their importance to smallholder farmers in developing countries. Diseases transmitted through vegetative propagation are notoriously damaging. We analyzed the structure of the crop networks, where the existence of a link between geographic location pairs was determined using a gravity model, as a function of the distance between the pair of locations and the product of the harvested crop area in the two locations. Networks within continents were evaluated using metrics such as average path length (APL: mean shortest path length between pairs of linked nodes), transitivity (T: probability that the adjacent nodes of a node are connected), and the distribution of node degree (sum of link weights at a node). E.g., the highest connectivity for cassava in these networks was in Asia $(\mathrm{APL}=1.87, \mathrm{~T}=0.41$ ) and the highest connectivity for sweetpotato was in Africa (APL $=1.40, \mathrm{~T}=$ 0.72). Identifying the highly-linked hub locations and bridge locations supports prioritizing surveillance for new invasive pests and diseases, and tracing intra-region movement of diseases.

Networks of stored wheat: Towards improving sampling and management strategies in the United States and Australia

J. F. HERNANDEZ NOPSA (1), G. Daglish (2), D. Hagstrum (3), J. Leslie (4), T. Phillips (3), C. Scoglio (5), S. Thomas-Sharma (4), G. Walter (6), K. Garrett (7)

(1) Kansas State University and Plant Biosecurity Cooperative Research Centre (CRC), Canberra, Australia, Manhattan, KS, U.S.A.; (2) Department of
Agriculture, Fisheries and Forestry, Agri-Science Queensland, Australia, and Plant Biosecurity Cooperative Research Centre (CRC), Canberra, Australia, Brisbane, Australia; (3) Department of Entomology, Kansas State University, Manhattan, KS, U.S.A.; (4) Department of Plant Pathology, Kansas State University, Manhattan, KS, U.S.A.; (5) Department of Electrical and Computer Engineering, Kansas State University, Manhattan, KS, U.S.A.; (6) School of Biological Sciences at the University of Queensland, Australia, Brisbane, Australia; (7) Department of Plant Pathology, Kansas State University, and Plant Biosecurity Cooperative Research Centre (CRC), Canberra, Australia, Manhattan, KS, U.S.A.

Phytopathology 104(Suppl. 3):S3.51

Wheat is the second most important staple worldwide. Insect, fungal, and mycotoxin contamination affect wheat grain quality during storage, and farmers and storage companies suffer economic losses due to mycotoxin contamination, trade limitation, and pesticide resistant insect populations. Stored grain moves from fields to storage structures (silos, elevators, depots), among storage structures, and to a final destination in the milling industry by train, truck, and barges. The network of stored grain movement may facilitate the dispersal of fungi, insect, and other contaminants among the nodes. We developed network models with 37 nodes (states) for the United States and 41 nodes (sites) for Australia (AU). Metrics such as average shortest path (2.1 and 6.7) and transitivity ( 0.38 and 0.35$)$ were obtained for the US and AU, respectively. Analysis of shortest paths, betweenness centrality, and node degree highlighted the importance of KS, IL, and ID in the US and the Toowoomba, Natcha, and Malu in AU. A striking difference between the countries was that highly connected nodes were in the central US but in coastal AU, suggesting different optimal sampling and mitigation strategies for the two systems. Developing multilayer and interconnected network models of a) grain transportation, b) movement of fungi and insects, and c) management communication may be an important next step for understanding the risk of subpopulations of pesticide-resistant insects and mycotoxins.

Foliar use of Oso 5\% SC (polyoxin D zinc salt) from Certis USA in fungicide programs to control diseases of cucurbits in the US

B. HIGHLAND (1), M. Dimock (2), S. Ockey (3)

(1) Certis USA, Nokomis, FL, U.S.A.; (2) Certis USA, Columbia, MD, U.S.A.; (3) Certis USA, Yakima, WA, U.S.A.

Phytopathology 104(Suppl. 3):S3.51

Foliar use of Oso $5 \% \mathrm{SC}$ (polyoxin D zinc salt) from Certis USA in fungicide programs to control diseases of cucurbits in the US. Oso 5\% SC (trade name Tavano $5 \% \mathrm{SC}$ for berries) is a broad-spectrum, high potency, preventative fungicide for control or suppression of fungal plant diseases, and is labelled for use in a variety of vegetables and other crops. Oso $5 \% \mathrm{SC}$ can be mixed with insecticides, fungicides, surfactants or fertilizers, and is active on various cucurbit diseases, including powdery mildew, downy mildew, anthracnose, southern blight, and gummy stem blight. Oso $5 \% \mathrm{SC}$ is a unique fungicide with a unique mode of action (FRAC code 19), and works by chitin synthase inhibition, inhibiting fungal cell wall formation. It is exempt from tolerance, and has a $4 \mathrm{hr}$. re-entry interval, and 0 days to harvest. Results from field trials show that Oso $5 \% \mathrm{SC}$ when used in a fungicide program in cucurbits increases yield, reduces disease infection, and improves size and grade of fruit.

Graft and psyllid transmissions indicate resistance to 'Candidatus Liberibacter asiaticus' is expressed in the citrus relative orange jasmine

M. E. HILF (1), D. G. Hall (1)

(1) USDA ARS USHRL, Fort Pierce, FL, U.S.A.

Phytopathology 104(Suppl. 3):S3.51

Huanglongbing (aka citrus greening) is a systemic bacterial disease of citrus associated with infection by the phloem-colonizing bacteria ' $\mathrm{Ca}$. Liberibacter asiaticus', (CLas) which is transmitted to non-infected trees by the Asian citrus psyllid (Diaphorina citri, Kuwayama) or by grafting with infected budwood. The rutaceous flowering ornamental plant, Murraya exotica L., commonly called orange jasmine (aka orange jessamine) is a preferred host for $D$. citri and is a popular landscape ornamental. Experiments were done to evaluate its susceptibility to infection with CLas by psyllid and graft transmission. Transmission experiments with $D$. citri and $M$. exotica seedlings conducted in fall 2012 and summer 2013 yielded 1/16 and 5/80 infected seedlings respectively. Grafting experiments done to transmit CLas from citrus to $M$. exotica showed a significant difference in the survival of budwood which was dependent on the budwood source, with survival of $80 \%$ of budwood from non-infected trees and of only $32 \%$ of budwood from CLasinfected trees. The psyllid transmission data suggest $M$. exotica seedling populations are heterozygous for susceptibility to infection and that these results are compatible with a model where resistance is a dominant trait controlled by two genes. The results from grafting experiments suggest this is 
an active resistance to infection and that resistant $M$. exotica seedlings can be identified by grafting with budwood from infected citrus trees.

\section{Identification and characterization of genes regulating conidiogenesis in} the maize pathogen Fusarium graminearum

R. L. HIRSCH (1), A. N. Thompkins (1), S. L. Atkinson (1), J. E. Flaherty (1)

(1) Coker College, Hartsville, SC, U.S.A.

Phytopathology 104(Suppl. 3):S3.52

Fusarium graminearum is an agronomically important plant pathogen that causes major yield-limiting diseases like head blight of wheat and ear rot of maize. F. graminearum persists in the soil in the absence of a host and reproduces asexually through the production of conidia to initiate repeating cycles of infection. Despite the importance of environmental stress tolerance and conidiation in the disease cycle, the underlying regulatory mechanisms are poorly understood. In order to identify putative regulatory genes, a 5000strain collection of random insertional mutants was assayed for variations in conidiation and growth during exposure to osmotic stress. Several strains expressing phenotypic abnormalities were selected for characterization, and isolate re-sequencing identified genomic lesions in three of the random insertional mutant strains. The genes were deleted by split-marker recombination from the wild-type strain, and targeted gene deletion mutants were assayed for defects in growth and development. The results from the screen identified genes involved in the overproduction of conidia in a variety of metabolic substrates, and both tolerance and sensitivity to osmotic stress during growth in axenic culture. The identification and functional characterization of novel genes discovered during this study has informed the models describing crucial developmental processes that are required for the survival and dissemination of an important plant pathogen.

VirFind: An online bioinformatics tool for plant virus detection and discovery

T. HO (1), I. E. Tzanetakis (1)

(1) University of Arkansas, Fayetteville, AR, U.S.A

Phytopathology 104(Suppl. 3):S3.52

Until today there is no publicly available bioinformatics tool for plant virus discovery. We have developed an online package, VirFind, with the purpose to facilitate virologists with virus detection and discovery using next generation sequencing. This is an automated bioinformatics pipeline that can accept input files in 454, Illumina or fasta formats, and filter out host reads, virus reads with their corresponding Blastn and Blastx reports, and perform conserved domain search for reads of unknown origin. Using VirFind to process over 30 plant samples sequenced with 454 or Illumina platforms, we have detected various viruses and extended genome sequences of Elderberry latent virus (Carmovirus, Tombusviridae) in elderberry, Mint vein bandingassociated virus (unassigned genus, Closteroviridae) in mint, and Peach rosette mosaic virus (Nepovirus, Secoviridae) in grape. We also discovered seven novel virus species including a trichovirus and a waikavirus in blackcurrant, and five carlaviruses in elderberry. Furthermore, VirFind was independently and successfully tested by four external users with seven different dsRNA, siRNA or total RNA datasets from plants or insects, demonstrating that the tool can be used as a universal virus discovery package. VirFind is now open on the internet with a front-end graphical interface for public use.

Spatial and temporal distribution of fungicides applied to creeping bentgrass

K. R. HOCKEMEYER (1), R. X. Latin (1)

(1) Purdue University, West Lafayette, IN, U.S.A.

Phytopathology 104(Suppl. 3):S3.52

Turf managers often rely on fungicides to limit damage caused by root diseases. Since fungicides do not move basipetally, they can only be effective if fungitoxic concentrations can be delivered to the rhizosphere. This research focused on the distribution of modern fungicides in verdure, thatch, sand, and roots of creeping bentgrass maintained as a putting green. Fungicides (azoxystrobin, propiconazole, pyraclostrobin, thiophanate-methyl) were applied to replicate field plots in a water volume of $815 \mathrm{~L} \mathrm{ha}^{-1}$. Plots were sampled over time $(0,3,7,10,14,17,21$ days after application) by extracting cores measuring $2 \mathrm{~cm}$ diameter by $3.8 \mathrm{~cm}$ deep. Cores were separated into verdure/thatch, sand, and roots before quantitative determination (liquid chromatography, triple quadrupole mass spectrometry) of fungicide residues over time. Fungicide residues in verdure/thatch declined steadily over time and support previously reported results describing fungicide depletion. Fungicides were detected in roots and sand within 5 hours post application, although at very low (1-15 ppm) concentrations. Residues in roots and sand remained at low levels throughout the course of the experiment. Fungicides differed with respect to amounts recovered in each section. These results describe fungicide depletion in the rhizosphere over time.

\section{A bio-control of Cenchrus ciliare by secondary metabolites of fungal} pathogens

A. HOLLINGSHEAD (1), B. Geary (1), S. Meyer (2), M. Masai (1)

(1) Brigham Young University, Provo, UT, U.S.A.; (2) USDA, Provo, UT, U.S.A.

Phytopathology 104(Suppl. 3):S3.52

Cenchrus ciliare (Buffelgrass) is a drought tolerant bunchgrass invasive to regions of the southwestern US. Due to its presence, wildfires are more frequent and biodiversity is negatively impacted. Magnaporthe grisea is a natural pathogen of $C$. ciliare and may produce secondary metabolites that control it without damaging native grasses. Magnaporthe grisea has been isolated from C. ciliare lesions, along with Cochlioblus australiensis and other fungi. Light microscopy and DNA sequencing were used to identify the fungi. Isolates of M. grisea and C. australiensis were grown in liquid medium, filtered, freeze dried, and the metabolites extracted using ethyl acetate. The extract was fractionated based on polarity by a column of silica gel. Bioassays used a $15 \mu 1$ drop of extract from each fraction applied to leaf surfaces punctured by a hypodermic needle. Extract was tested on Triticum sp., Solanum lycopersicum, C. ciliare, and two native desert grasses (Digitaria californica and Heteropogon contortus). After 4 days, lesion size was measured with an electronic caliper. Cenchrus ciliare leaves were the most sensitive to fractions 1,2 , and 3 from the $C$. australiensis extract, average lesion size was over $1 \mathrm{~mm}$. Fractions 5, 6, 8, 12, and 13 from M. grisea were active on $C$. ciliare with lesions averaging $0.78,0.94,1.07,1.29$, and 1.23 $\mathrm{mm}$, respectively. Triticum and D. californica showed minimal lesions from these extract fractions.

Comparison of soil bacterial communities from fields treated with Anaerobic Soil Disinfestation located on Florida's east to west coast J. C. HONG (1), K. J. Martin (2), N. Kokalis-Burelle (1), D. Butler (3), E. N. Rosskopf (1)

(1) USDA-ARS, Fort Pierce, FL, U.S.A.; (2) William Paterson University, Wayne, NJ, U.S.A.; (3) University of Tennessee, Knoxville, TN, U.S.A. Phytopathology 104(Suppl. 3):S3.52

Since the phase out of methyl bromide, growers are in need of alternative methods for controlling soil-borne pathogens and weeds. Anaerobic soil disinfestation (ASD) has been used in the U.S., Japan, and the Netherlands for suppressing a variety of soil-born pests for a diversity of crops. Application of ASD in Florida utilizes the principles of soil solarization, incorporating organic amendments, including composted poultry litter (CPL), and soil saturation to create favorable conditions for anaerobic and facultative anaerobic bacteria. CPL provides a source of nitrogen, maintains soil moisture, and may affect the microbial community. Growers are hesitant to adopt ASD due to the potential contamination of Salmonella. For this study, 5 fields on the east and west coasts of Florida were treated with ASD. Soil samples were taken every 2-3 days. The $\mathrm{pH}$ was taken and DNA was extracted from each soil sample. Length heterogeneity pcr using V2 and V3 primers was performed on each sample to determine the bacterial communities. Metagenomic sequencing was performed on the pre and post treatment samples from each field. Bacterial communities were compared from each field to determine which populations were important in ASD. Soil and fruit samples were also tested for Salmonella by enriching the samples, extracting the DNA, and running pcr with Salmonella specific primers.

Evaluation of oxathiapiprolin for management of downy mildew on sunflower

R. HUMANN (1), K. Johnson (2), T. Gulya (3), S. Meyer (1), J. Jordahl (1), A. Friskop (1), M. Wunsch (4), S. Markell (1)

(1) North Dakota State University, Fargo, ND, U.S.A.; (2) DuPont Field Development, Grand Forks, ND, U.S.A.; (3) USDA ARS, Fargo, ND, U.S.A.; (4) NDSU Carrington Research Extension Center, Carrington, ND, U.S.A. Phytopathology 104(Suppl. 3):S3.52

Downy mildew of sunflower is a yield-limiting disease caused by Plasmopara halstedii. Two tools are commonly used to manage the disease: fungicidal seed treatments and genetic resistance. The FRAC 4 fungicides, metalaxyl and mefenoxam, were effective until resistant $P$. halstedii isolates were identified in the late 1990s. Currently, only one seed care package containing the FRAC 11 fungicide azoxystrobin as the primary active ingredient, is marketed for use on sunflowers. The objective of this study was to evaluate the experimental fungicide oxathiapiprolin for management of downy mildew. Oxathiapiprolin has not yet been assigned to a FRAC group. Eight field research trials were conducted throughout 2012 and 2013, artificially inoculated with $P$. halstedii zoosporangia, and irrigated after inoculation. Incidence levels were 
determined by recording systemically infected plants four times throughout the growing season. In the six trials with measureable disease pressure, all treatments that contained oxathiapiprolin had significantly lower disease incidence than the non-treated checks. Similarly, all treatments containing oxathiapiprolin had disease incidence levels the same or lower than azoxystrobin treatments. Results indicate oxathiapiprolin could provide another management option for downy mildew.

\section{First report of Fusarium proliferatum causing root rot disease in soybean} (Glycine max) in Canada

S. F. Hwang (1), K. F. CHANG (1), H. U. Ahmed (1), Q. Zhou (1), S. E. Strelkov (2), R. L. Conner (3), D. McLaren (4), B. D. Gossen (5), G. D. Turnbull (1)

(1) Alberta Agriculture and Rural Development, Edmonton, AB, Canada; (2) University of Alberta, Edmonton, AB, Canada; (3) Agriculture and Agri-Food Canada, Morden, MB, Canada; (4) Agriculture Canada, Brandon, MB, Canada; (5) Agriculture and Agri-Food Canada, Saskatoon, SK, Canada Phytopathology 104(Suppl. 3):S3.53

Soybean plants showing symptoms of root rot were collected from fields in western Canada to determine the etiology of the disease. Four Fusarium spp. including $F$. avenaceum, $F$. culmorum, $F$. oxysporum and $F$. proliferatum were identified based on cultural and morphological characteristics, and all isolates of these species were found to be pathogenic on soybean. The identity of $F$. proliferatum was confirmed by PCR analysis with the $F$. proliferatumspecific primer set CLPRO1/CLPRO2. Amplicons of the target fragments (partial calmodulin (cld) gene, $526 \mathrm{bp}$ ) were obtained only from DNA of $F$. proliferatum. The amplicon was sequenced and shared $100 \%$ identity with cld gene sequences of $F$. proliferatum available in GenBank. Fusarium proliferatum was the most virulent of the four Fusarium species identified, causing the greatest root rot severity and reduction of seedling emergence. Fusarium avenaceum was the second most virulent and caused a greater reduction in seedling numbers than $F$. culmorum or $F$. oxysporum. $F$. proliferatum inoculum density showed strong log linear negative relationships with seedling emergence, shoot dry weight, seed yield, and a log linear positive relationship with root rot disease severity. To our knowledge, this is the first report of $F$. proliferatum causing soybean root rot in Canada.

Draft genome sequence and partial annotation of Lonsdalea quercina subsp. quercina type strain and field isolates from Colorado USA

J. R. IBARRA CABALLERO (1), M. M. Zerillo (1), J. Snelling (2), W. Cranshaw (1), C. Boucher (1), N. A. Tisserat (1)

(1) Colorado State University, Fort Collins, CO, U.S.A.; (2) Oregon State University, Corvallis, OR, U.S.A.

Phytopathology 104(Suppl. 3):S3.53

Lonsdalea quercina (formerly Erwinia quercina and Brenneria quercina) is associated with bacterial gummosis and twig necrosis of several oak especies (Quercus spp.). We recovered two isolates from symtomatic pin oak $(Q$. palustris) and northern red oak (Q. rubra) that exhibited small cankers and gummosis at Kermes scale (Allokermes galliformis) feeding sites. We sequenced the genomes of these two isolates, as well as the type strain ATCC 29281 originally isolated from California live oak (Q. agrifolia) in California USA, using Illumina Hi_Seq 2000 system, and them we assembled them using Mira assembler. The resulting assemblies were similar to each other, having a total length of around $3.85 \mathrm{Mbp}$ and a GC content of 55.1-55.6\%. A total of about 3,400 coding sequences representing more than 470 gene categories were identified using the RAST server. The two field isolates were very similar, showing less similarity to the type strain; however, a multi-locus phylogenetic tree based on the Bayesian inference grouped the isolates in a single cluster, distinct from L.q subsp. iberica and L.q. subsp. britanica.

Deep sequencing reveals Cotton leaf curl Gezira helper virus and sub-viral DNAs in weed, ornamental, and cultivated crops throughout Arabia A. IDRIS (1), M. A. Al-Saleh (2), J. K. Brown (3)

(1) King Abdullah University of Science and Technology, Thuwal, Saudi Arabia; (2) King Saud University, Riyadh, Saudi Arabia; (3) University of Arizona, School of Plant Sciences, Tucson, AZ, U.S.A.

Phytopathology 104(Suppl. 3):S3.53

The whitefly-transmitted, monopartite Cotton leaf curl Gezira virus $(\mathrm{CLCuGeV})$, is a monopartite begomovirus that causes vein-thickening and leaf curl symptoms. Leaves of plants with symptoms reminiscent of $\mathrm{CLCuGeV}$ infection were collected from hollyhock, Malva spp., and okra plants throughout Arabia. Total DNA was isolated from leaves and used for circular, ssDNA virus enrichment by rolling circle amplification (RCA). RCA products were subjected to Next-Generation Sequencing (NGS) using the Illumina platform. Short reads were assembled, and single nucleotide polymorphisms were identified, for the resultant begomovirus $(\sim 2.8 \mathrm{~kb})$ and satellite $(\sim 1.4 \mathrm{~kb})$ molecules. Also, the RCA products were digested with restriction enzymes to linearize and enable cloning and sequencing of viral components and satellites. BLASTn analysis of NGS- and RCA-derived products indicated that the begomoviral genomes and satDNAs were most closely related to $\mathrm{CLCuGeV}$, endemic to the Nile Basin, and to close relatives in Jordan and Pakistan thought recently introduced. Several betasatellites, together with $\mathrm{CLCuGeV}$, co-infected three host plant species from four locations. In one location, only defective betasatellites were detected. Nucleotide (nt) sequence comparisons revealed two types of alphasatellites, at $88 \%$ and $64 \%$ shared nt identity, respectively, with Cotton leaf curl Gezira alphasatellite (DNA-1) and Ageratum yellow vein Singapore alphasatellite (DNA-2) from Oman and Singapore.

Proposal to establish a public DNA repository for plant pathogenic fungi P. INDERBITZIN (1), K. V. Subbarao (2)

(1) University of California, Davis, CA, U.S.A.; (2) University of California, Salinas, CA, U.S.A.

Phytopathology 104(Suppl. 3):S3.53

We propose the establishment of a public DNA repository for the long-term preservation of DNA samples from studies in plant pathology. The goal of the repository is to preserve fungal biodiversity, contribute to the continuity of science and improve disease management. Preservation of the DNA from strains used in experiments guarantees that identification of species and other taxa can be assessed retroactively. This is particularly useful in the case of ambiguous species boundaries and following taxonomic changes, which can confuse our knowledge of the biology of individual species. The stored DNA is also available for future genome sequencing, which in conjunction with existing knowledge about the species, will further our understanding of fungal biology, and contribute to improvement of disease management strategies. Establishing a DNA repository is much easier than a new culture collection, which APS has been exploring. DNA storage requires little maintenance and no APHIS permits, and even large numbers of samples can be stored relatively cheaply. The main disadvantage of DNA over cultures is that DNA cannot be used in biological experiments. But in the future, it is likely that living organisms will be routinely recreated from DNA, as was already demonstrated in bacteria. To illustrate the advantages of a DNA repository for plant pathology, we provide practical examples from Verticillium, the causal agents of Verticillium wilt.

Stability of boscalid resistance and fluopyram sensitivity of Corynespora cassiicola strains in commercial cucumber greenhouses

H. ISHII (1)

(1) Natl Inst for Agro-Environmental Sciences, Tsukuba, Japan

Phytopathology 104(Suppl. 3):S3.53

Corynespora cassiicola, the cause of Corynespora leaf spot disease, is one of the most important pathogens on cucumber in Japan. This fungus easily developed resistance to various fungicides such as benzimidazole, dicarboximide, QoI, and SDHI fungicides. Spray applications of SDHI fungicides were stopped and alternative fungicides have been used in the commercial greenhouse tested in this study as boscalid-resistant strains of $C$. cassiicola were found to be widely distributed in the area. In order to know the stability of boscalid resistance after withdrawal of SDHI fungicide applications, infected cucumber leaves were sampled every year between 2010 and 2013, single conidium isolates obtained, and mycelial growth tests conducted on fungicide-amended YBA agar medium to examine sensitivity. All of the 21 isolates collected from the greenhouse in 2013, three years after the withdrawal, still showed very high resistance to boscalid indicating that this type of resistant strains were stable in a greenhouse in the absence of selection pressure by SDHI fungicides. In contrast, these isolates all exhibited sensitivity to fluopyram which further confirmed the lack of cross resistance in very highly boscalid-resistant isolates to fluopyram as reported before.

FvSNF1, the protein kinase gene in Fusarium virguliforme, is required for cell wall-degrading enzyme expression and virulence on soybean

K. T. ISLAM (1), J. Bond (1), A. M. Fakhoury (1)

(1) Southern Illinois University, Carbondale, IL, U.S.A.

Phytopathology 104(Suppl. 3):S3.53

Fusarium virguliforme is a soil-borne pathogen that causes Sudden Death Syndrome (SDS). SDS is one of the most devastating diseases of soybean resulting in significant economic losses in most of the soybean producing regions of the world. Despite the importance of SDS, a clear understanding of fungal genetic factors that affect the development of the disease is still lacking. The aggressiveness of $F$. virguliforme on infected soybean plants is believed to require the activity of cell wall-degrading enzymes (CWDE). The production of these CWDEs in phytopathogenic fungi is under catabolic 
repression. In Saccharomyces cerevisiae, catabolic repression is regulated by SNF1 (sucrose non-fermenting 1). To investigate the role of cell walldegrading enzymes as determinants of $F$. virguliforme aggressiveness, the $F$. virguliforme $S N F 1$ homologue $F V S N F 1$ was targeted for disruption. The resulting $F v \Delta s n f l$ mutant showed significantly reduced expression of several CWDE genes. Interestingly, disruption of FvSNF1 in $F$. virguiliforme abolishes galactose utilization and causes poor growth on xylan, arabinose and sucrose. However, the mutation did not seem to affect the ability of the fungus to grow on glucose, fructose, maltose, or xylose as the main source of carbon. More importantly, in greenhouse experiments, the $F v \Delta s n f 1$ mutant was severely impaired in its ability to cause SDS on challenged soybean plants.

\section{Genetic characterization of Aspergillus flavus soil populations in Kenya} using simple sequence repeat (SSR) markers

M. S. ISLAM (1), K. A. Callicott (1), K. T. Meyers (2), R. Bandyopadhyay (3), C. Mutegi (4), P. J. Cotty (1)

(1) USDA-ARS, Tucson, AZ, U.S.A.; (2) School of Plant Sciences, The University of Arizona, Tucson, AZ, U.S.A.; (3) International Institute of Tropical Agriculture, Ibadan, Nigeria; (4) International Institute of Tropical Agriculture, Nairobi, Kenya

Phytopathology 104(Suppl. 3):S3.54

Human populations in Kenya are frequently exposed to dangerous levels of aflatoxins. Biocontrol products based on atoxigenic isolates of Aspergillus flavus are the most effective management tools for preventing aflatoxin contamination. Aflasafe KE01, containing four atoxigenic isolates, is under development for prevention of aflatoxin contamination in Kenya. The four isolates in KE01 were originally isolated from maize produced in Kenya, and each belongs to a distinct vegetative compatibility group with characteristic simple sequence repeat (SSR) haplotypes. However, natural distributions of these isolates beyond grain produced in 2004 to 2006 have not been studied. Prior to initial treatment of farmer's fields with KE01 in 2012, soil samples were collected from 287 farms in 13 districts located in 5 Kenya counties: Embu (68 farms / 2 districts), Kitui (47/3), Machakos (65/4), Makueni (67/3) and Tana River (40/1). Isolates (1070) recovered from these soils were characterized with 17 SSR markers with 8 to 62 alleles. A total of 892 haplotypes (out of a possible $4.7 \times 10^{23}$ combinations under random assortment) were detected; 97 of these were displayed by two or more isolates ( 2 to 16). Most haplotypes detected multiple times were geographically restricted, but $27 \%$ occurred in two or more of the five districts. Haplotypes of three of the four KE01 isolates were detected in the farm soils, with one KE01 haplotype being found in the most samples (2.1\%) and districts (5).

A rapid foliar assay for screening butternut trees for resistance to butternut canker disease

J. J. JACOBS (1), M. E. Ostry (2), M. J. Moore (2), C. H. Michler (3), K. E. Woeste (3)

(1) USDA Forest Service, Region 3 Forest Health Protection, Albuquerque, NM, U.S.A.; (2) USDA Forest Service, Northern Research Station, St. Paul, MN, U.S.A.; (3) USDA Forest Service, Hardwood Tree Improvement and Regeneration Center, Purdue University, Dept. Forestry and Natural Resources, West Lafayette, IN, U.S.A.

Phytopathology 104(Suppl. 3):S3.54

Butternut canker disease caused by the fungus Ophiognomonia clavigignentijuglandacearum $(O c-j)$ has decimated many populations of butternut (Juglans cinerea) and is one of the main impediments to restoration of the species throughout its native range. Small elliptical cankers form at wound sites and natural openings on all woody tissues, coalesce and kill branches and trees of all ages. The search for resistance to butternut canker disease has been complicated by inefficient techniques to identify resistant individuals within populations. An efficient and reliable screening methodology is essential if the goals of a single tree selection conservation breeding program are to be met. Recent evidence that $O c-j$ can infect foliar tissue under natural conditions may allow for the development of a reliable foliar inoculation protocol that would provide a rapid technique for screening collected germplasm using seedlings in contrast to the current methodology of inoculating larger (older) stems of selected trees. We report the development of a foliar inoculation protocol using unwounded leaflets from 1-2 year old trees grown in the greenhouse that correlates well with stem lesions produced by traditional inoculation techniques. This modified resistance screening technique utilizes a conidial suspension to produce necrotic lesions in a moist chamber reducing the evaluation time to less than two weeks compared with stem inoculation procedures lasting one to many years.

Use of Bacillus mycoides isolate $J$ induced resistance in IPM programs B. J. JACOBSEN (1), S. C. Ockey (2), H. B. Highland (3), M. B. Dimock (4)
(1) Montana State University, Bozeman, MT, U.S.A.; (2) Certis USA, Yakima, WA, U.S.A.; (3) Certis USA, Nokomis, FL, U.S.A.; (4) Certis USA, Columbia, MD, U.S.A.

Phytopathology 104(Suppl. 3):S3.54

Bacillus mycoides isolate $\mathrm{J}(\mathrm{BmJ})$ induced resistance provides control of bacterial, fungal, oomycete and viral pathogens on a wide range of crop plants. BmJ induced resistance is signaled through the NPR -1 gene and involves several PR proteins and ethylene. Optimal systemic resistance induction occurs 3-5 days after application and provides disease control for 14-21 days depending on the plant induced. $\mathrm{BmJ}$ is compatible with a wide range of pesticides including; triazole, EDBC, and QoI class fungicides and a wide range of insecticides. Disease control equal to commercial standards has been achieved with $\mathrm{BmJ}$ alone or in combination with low rates of fungicides or in alternating programs where $\mathrm{BmJ}$ replaces half the fungicide used in fungicide alone programs. BmJ has also been demonstrated to be of benefit to triazole and QoI fungicide resistance management programs in Cercospora leaf spot of sugarbeet control programs. BmJ used in combination with insecticides and roquing has produced $>50 \%$ control of potato $\mathrm{PVY}$ in multiple years in the field. Data will be presented for disease control of cucurbit crops, tomato, pepper, potato, pecans, spinach, lettuce and sugarbeet with comparisons to commercial pesticide standards. Control of postharvest diseases using preharvest application will be discussed. $\mathrm{BmJ}$ is licensed to CERTIS USA by Montana State University. Registration materials have been submitted to the USEPA and OMRI certification is expected.

Evaluation of residence, sporulation and efficacy of two formulations of the biocontrol Aspergillus flavus AF36 in commercial cotton fields in Arizona

R. JAIME (1), M. Foley (2), G. Barker (1), L. Liesner (2), L. Antilla (2), R. Bandyopadhyay (3), P. J. Cotty (4)

(1) University of Arizona, Tucson, AZ, U.S.A.; (2) Arizona Cotton Research and Protection Council, Phoenix, AZ, U.S.A.; (3) International Institute of Tropical Agriculture (IITA), Ibadan, Nigeria; (4) USDA ARS, School of Plant Sciences, University of Arizona, Tucson, AZ, U.S.A.

Phytopathology 104(Suppl. 3):S3.54

Aflatoxins are toxic, carcinogenic metabolites produce by Aspergillus fungi that reduce crop value. Competitive displacement by atoxigenic strains of $A$. flavus is the only method proven to reliably reduce aflatoxins in crops. Commercial atoxigenic biocontrol products use grain as both carrier and source of nutrients for reproduction and dispersal of the atoxigenic biocontrol agent. The first registered atoxigenic has been produced by inoculating steam sterilized wheat grain with the atoxigenic strain $\mathrm{AF} 36$, a slow and expensive process. A new formulation using dry roasted sorghum grain coated with a spore suspension of AF36 is less expensive and faster to produce. Performances of the two formulations were compared under commercial cotton production in eight fields in each of two seasons. Plots $(\sim 4$ ha) were separated by at least $100 \mathrm{~m}$ and harvested individually with a spindle picker. Roasted sorghum and steam sterilized wheat produced similar quantities of spores per gram. However, persistence differed between the formulations with more sporulating grains for sorghum than wheat after various periods in both seasons. The AF36 VCG was significantly higher in cottonseed and soil after harvest in both sorghum and wheat treatments compared to controls in 2012, but in 2013 only sorghum was higher. Increases in AF36 were associated with both decreased incidences of the $\mathrm{S}$ strain of A. flavus and reductions in crop aflatoxin content.

Characterization of dry bean transcriptome in response to soybean cyst nematode infection

S. JAIN (1), K. Chittem (1), J. Richards (1), R. Brueggeman (1), B. Nelson (1) (1) Department of Plant Pathology, North Dakota State University, Fargo, ND, U.S.A.

Phytopathology 104(Suppl. 3):S3.54

To understand changes in the transcriptome of Phaseolus vulgaris roots during infection by soybean cyst nematode (SCN; Heterodera glycines, HG type 0), gene expression profiling was conducted using next generation RNA sequencing on the Ion Torrent PGM $^{\mathrm{TM}}$ platform. Two pinto bean genotypes, PI 533561 and GTS-900, resistant and susceptible to SCN infection, respectively, were used as RNA sources eight days after inoculation. Total reads generated ranged between $\sim 3.5$ and 5.7 million per library. The dry bean transcript dataset (ftp://ftp.jgi-psf.org/pub/compgen/phytozome/v9.0/ Pvulgaris/) was used as a reference for mapping reads using bowtie2, and the differential expression was estimated using DESeq. Approximately $58 \%$ and $24 \%$ of the filtered reads from infected and non-infected roots, respectively, of PI 533561 were uniquely mapped to the reference data. In GTS-900, 27\% and $18 \%$ of the filtered reads from infected and non-infected roots, respectively, aligned uniquely to the reference data. In the infected roots of PI 533561, 430 
genes were up-regulated and 286 genes were down-regulated in comparison to non-infected roots. In contrast, 118 genes were expressed at greater levels and 485 genes were expressed at lower levels in SCN-infected roots of GTS-900 than in non-infected roots. This is the first report describing gene/transcripts involved in SCN-dry bean interaction and the results have important implications for further characterization of $\mathrm{SCN}$ resistance genes.

Regulation of a $\mathrm{Ca}$. Liberibacter asiaticus prophage late gene promoter by extracts of citrus and periwinkle leaves, and Asian citrus psyllid

M. JAIN (1), L. A. Fleites (1), D. W. Gabriel (1)

(1) University of Florida, Gainesville, FL, U.S.A.

Phytopathology 104(Suppl. 3):S3.55

Two prophage genomes ( $\mathrm{SC} 1$ and $\mathrm{SC} 2)$ have previously been described in the Ca. Liberibacter asiaticus (Las, causal agent of citrus Huanglongbing disease) genome. SC1 becomes replicative, and its lytic cycle genes activated, when Las has infected plants, but not when infecting the psyllid host. Among the SC1 late genes is SC1_gp110, annotated as a holin. The SC1_gp110 gene was recently found to be both functional in E. coli and strongly activated in planta; and its promoter was cloned into a wide host range shuttle vector pUFR071. The lacZ promoter of pUFR071 was replaced with the SC1 gp110 promoter and fused with promoter-less GUS (beta-glucuronidase) coding region to yield a late gene reporter plasmid, pLF057. The GUS coding region was also cloned downstream of the lac $Z$ promoter in pUFR071 to yield the constitutively GUS $^{+}$control vector pLF058. Both pLF057 and pLF058 were transformed into Liberibacter crescens strain BT-1; and exhibited strong GUS activity (blue color) using X-glucuronide. Cell-free extracts of citrus, eggplant, tobacco and periwinkle leaves, as well as cell-free psyllid extracts all inhibited GUS activity in a dose-dependent manner. Notably, inhibition of GUS activity was not observed following heat inactivation of the leaf or psyllid extracts. Attempts to identify possible late gene promoter element repressor/activators in $L$. crescens, as well as potential plant or psyllid factors affecting the pLF057 reporter, are in progress.

Evaluation of a metagenomics approach for the early detection of Salmonella in cilantro

K. G. JARVIS (1), C. J. Grim (2), J. J. Beaubrun (1), L. Ewing (1), K. Dudley (1), A. Ottesen (1), C. Wang (3), J. Pettengill (3), E. Brown (3), D. Hanes (1)

(1) FDA, Laurel, MD, U.S.A.; (2) Oak Ridge Institute for Science and Technology, Oak Ridge, TN, U.S.A.; (3) FDA, College Park, MD, U.S.A. Phytopathology 104(Suppl. 3):S3.55

Surveillance activities for Salmonella in leafy greens rely on culture-based methods, which include non-selective and selective enrichment. Fifty-nine cilantro samples from five different states were analyzed for the presence of Salmonella following the FDA BAM method. Total nucleic acids were extracted from cilantro samples prior to and following a non-selective enrichment and metagenomic sequencing was performed. The results were analyzed using QIIME, BLAST and a Kmer (25-mer) algorithm. Qiime analysis of $16 \mathrm{~s}$ RNA genes showed that the microbial population of unenriched samples was highly variable. However, upon enrichment in $\mathrm{mBPW}$, the alpha diversity decreased dramatically and resulted in a dramatic increase in abundance of the Firmicutes, often to as high as $98 \%$ of the total population. Blast analysis of Salmonella culture positive cilantro samples matched $0.47 \%$ of pre-enriched and $15.07 \%$ of the $24 \mathrm{hr}$-enriched WGS sequence reads to $S$. enterica Newport SL317. Kmer analysis with an enteric pathogen panel matched 0.11 and $2.55 \%$ of sequence reads from the preenriched and 24hr-enriched samples respectively. Primary non-selective enrichment is viewed as an important first step in resuscitation and recovery of injured Gram-negative bacteria, including pathogenic enteric organisms. These results indicate that counter-selection of Gram-positives, particularly the Firmicutes, may increase sensitivity, and potentially decrease assay time.

Isolation and characterization of Pythium litorale from irrigation ponds used for vegetable production

P. Ji (1), V. Parkunan (1), M. D. Purvis (1), A. PETKAR (1)

(1) University of Georgia, Tifton, GA, U.S.A.

Phytopathology 104(Suppl. 3):S3.55

Irrigation water can harbor propagules of pathogenic oomycetes that may be a primary source of disease outbreak in crop plants. Irrigation ponds associated with vegetable production in southern Georgia, USA, were assessed in this study. Camellia and rhododendron leaves were used as baits for recovery of oomycetes that were further identified by morphological characteristics and analysis of the internal transcribed spacer rDNA regions. Pythium litorale was frequently isolated from all irrigation ponds sampled. Assessment of growth rates of $P$. litorale isolates at 5 to $45^{\circ} \mathrm{C}$ indicated that the optimum and maximum temperatures were 30 and $40^{\circ} \mathrm{C}$, respectively. It appeared that these isolates were more tolerant to higher temperatures than previously described
$P$. litorale isolates from Europe that had a maximum growth temperature of $35^{\circ} \mathrm{C}$. Pathogenicity assay with the $P$. litorale isolates showed that they caused fruit rot and seedling damping-off of yellow squash. Pythium litorale was first described as a new species in 2006 and the present study is the first report indicating it is pathogenic on vegetable crops. This study provides useful information for a more comprehensive understanding of the nature of $P$. litorale and its potential impact on vegetable production.

Purification, storage, and pathogenicity assay of rice false smut fungus under controlled environmental conditions

Y. JIA (1), R. El-Shafey (1), Z. Zhang (1)

(1) USDA ARS Dale Bumpers National Rice Research Center, Stuttgart, AR, U.S.A.

Phytopathology 104(Suppl. 3):S3.55

Rice false smut, caused by Ustilaginoidea virens, is one of the most serious diseases that affect grain yield and quality. In the present study, a method to purify, store, and evaluate pathogenicity of $U$. virens under controlled environmental conditions was developed. Yellow chlamydospores were collected from fresh smut balls, striped onto a potato sucrose agar (PSA) plate, and incubated for seven days at room temperature. Single chlamydospores were visually identified and transferred to a $1.8 \mathrm{~mL}$ cryovial with $0.9 \mathrm{~mL}$ PSA and allowed to grow for seven days at room temperature. An equal volume of $60 \%$ glycerol was added to the cryovial for permanent storage at $-80^{\circ} \mathrm{C}$. For the pathogenicity assay, a section approximately $2-3 \mathrm{~mm}$ was removed from the cryovial and grown on a PSA plate for 7-10 days. Then a piece of agar with fungus was extracted from the plate, placed in liquid wheat bran media, and incubated at room temperature for 7 days with gentle shaking. Spores were filtered through four layers of cheese cloth and were re-suspended in sterilized distilled $\mathrm{H}_{2} \mathrm{O}$. Rice plants at the booting stage were inoculated by injecting the spore suspension at the tip of the rice sheath via a syringe. After inoculation, plants were maintained in a plastic growth chamber with high humidity for 7 days and then moved to a greenhouse for an additional two weeks. Typical smut symptoms ranging from silver white to yellow to darkgreen on the infected grains were observed.

Fungicide sensitivity testing of Cercospora beticola from sugarbeet in the Great Lakes Region

Q. JIANG (1), W. W. Kirk (1), N. Rosenzweig (1), P. M. Somohano (1), L. E. Hanson (2)

(1) Michigan State University, East Lansing, MI, U.S.A.; (2) USDA-ARS, East Lansing, MI, U.S.A.

Phytopathology 104(Suppl. 3):S3.55

Cercospora leaf spot (CLS), caused by Cercospora beticola (Sacc.), is the most serious foliar disease of sugarbeet (Beta vulgaris L.) worldwide. CLS is problematic in most sugarbeet growing regions and can cause significant economic losses. Among all the management strategies, timely and effective fungicide application is one of the most effective management tools. However, resistance has been reported in populations of $C$. beticola to several classes of fungicides including benzimidazoles, organotins and demethylation inhibitors (DMI). In 2011, quinine outside inhibitor (QoI)-tolerance was identified in Michigan, and later in other areas. In 2012, a spiral gradient method was used to estimate the fungicide concentration that resulted in a $50 \%$ inhibition of spore germination or mycelial growth $\left(\mathrm{EC}_{50}\right)$ to examine sensitivity to QoIs and benzimidazoles, respectively. Nearly $90 \%$ of isolates tested were resistant to thiophanate-methyl, indicating an increase from about $50 \%$ observed from 2002-2011. Out of 49 isolates tested for resistance to pyraclostrobin, $71 \%$ had $\mathrm{EC}_{50}$ values greater than $64 \mathrm{ppm}$. A similar ratio was found using sequencing of PCR products with 25 out of 32 having $100 \%$ identity with a sequenced QoI-resistant $C$. beticola. Testing isolates for resistance has continued in 2013. The majority of the isolates were sensitive to organotin and DMI in both years. Additional tests of fungicide sensitivity using PCR-RFLP analysis are ongoing.

Compounds from zoospore exudate serve as a signal to promote zoosporic germination and infection of Phytophthora erythroseptica

H. JIANG (1), J. Hao (1), K. D. Bishop (1)

(1) University of Maine, Orono, ME, U.S.A.

Phytopathology 104(Suppl. 3):S3.55

Phytophthora erythroseptica causes pink rot of potato. It produces flagellate zoospores that serve as inocula for host infection. Furthermore, the zoosporic behavior is density dependent. Zoospores at $10^{4}$ zoospores $/ \mathrm{ml}$ germinated and its suspension successfully caused necrotic lesions on potato tuber slices, but it was not observed at concentration of 100 zoospores $/ \mathrm{ml}$. In order to determine whether this behavior is regulated by extracellular compounds in zoospore exudates (ZE), effects of $\mathrm{ZE}$ on zoosporic germination of $P$. erythroseptica were examined under laboratory conditions. ZE was obtained 
from filtrated zoospore suspension $\left(10^{4}\right.$ zoospores $\left./ \mathrm{ml}\right)$. Freshly produced zoospores at different concentrations were treated either with or without $\mathrm{ZE}$, then incubated on a depression-well slides at $22^{\circ} \mathrm{C}$ for four hours, zoosporic germination was examined using a microscope. The results showed that zoospores only germinated when the concentration was at or above $10^{4}$ zoospores $/ \mathrm{ml}$. However, when treated with $\mathrm{ZE}$, germination occurred at 100 zoospores $/ \mathrm{ml}$. Similarly, $10 \mathrm{ul}$ of ZE-treated zoospores at 100 zoospores $/ \mathrm{ml}$ caused potato tuber infection, when inoculated on the eye of potato. The activity of ZE was not affected by high temperature $\left(100^{\circ} \mathrm{C}\right.$ for $\left.30 \mathrm{~min}\right)$, proteinase $\mathrm{K}$ and catalase. To determine the active compounds related to this activity, nuclear magnetic resonance, gas chromatography/mass spectrometry were performed. The results are under analyses.

Use of custom GoldenGate SNP assay to analyze global populations of Puccinia graminis f. sp. tritici

J. L. JOHNSON (1), L. J. Szabo (1), Y. Jin (1), P. Olivera (1), M. Newcomb (1)

(1) USDA-ARS, St. Paul, MN, U.S.A.

Phytopathology 104(Suppl. 3):S3.56

As the causative agent of wheat stem rust, the fungus Puccinia graminis $f . s p$. tritici (Pgt) has caused devastating crop losses worldwide, throughout the centuries into the present day. A custom Illumina GoldenGate Assay of 1536 SNP markers was constructed based on sequence analysis of Pgt. The array was designed using data from whole genome sequences of five Pgt strains. Markers, spaced on average every $27 \mathrm{~kb}$, provide coverage over $50 \%$ of the sequenced reads in the assembled reference genome. Almost 1300 of the SNP markers were chosen to lie within predicted genes, and selections were made so as not to bias the marker set for or against any of the sequenced genomes. Early experiments were run using reference DNA as a positive control and DNA from other members of the Puccinia genus, and other fungi as a negative control. Markers were found overall to be specific for Pgt. The performance of the SNP assay does not vary whether purified spores or infected plant material is used as the source for DNA isolation. To date, over 160 North American and 225 global Pgt isolates have been analyzed on a core set of 1113 SNP markers. Results show that around 30 genetic clusters can be assigned, and that in many cases these correlate to phenotypic race groups. We have created a robust assay that can be used in global diagnostics of Pgt samples.

\section{Therapeutic SAR-inducing branch paints aid the restoration of pear and} apple tree health after fire blight infection

K. B. JOHNSON (1), T. N. Temple (1)

(1) Oregon State University, Corvallis, OR, U.S.A.

Phytopathology 104(Suppl. 3):S3.56

Fire blight canker expansion in young pear and apple trees can be severely damaging in spite of therapeutic pruning to remove infections from trees. Disease symptoms commonly reoccur ('re-ignite') due to residual inoculum inside the tree. In the greenhouse, the systemic acquired resistance-inducing material, acibenzolar-S-methyl (ASM), applied as a pot drench or foliar spray suppressed fire blight canker expansion in young trees, but in the orchard similar rates of ASM by drench or spray were ineffective. In contrast, branch paints of ASM mixed with a silicone surfactant and applied locally to symptomless tissue below the pruning cut suppressed disease reoccurrence. In the greenhouse, trunk paints of ASM suppressed canker expansion in pear and apple and compared to sprays and drenches, showed a strong relative induction of pathogenesis-related protein genes, an indicator of SAR expression in the host. In orchards, paints of ASM applied to symptomless tissue after pruning significantly reduced the end of season 'yield' of blighted branches that developed from re-ignition. For example, for diseased Bosc pear trees pruned $15 \mathrm{~cm}$ below the proximal edge of expanding cankers, ASM (15 g a.i./L) painted onto $30-40 \mathrm{~cm}$ of branch tissue immediately below pruning cuts reduced re-ignited blight by $60 \%$ compared to pruning alone. Induction of systemic acquired resistance could prove practical as an aid to cutting blight in young pear and apple trees.

Two phases of root loss caused by citrus huanglongbing are independent of root growth

E. G. JOHNSON (1), K. M. Gerberich (1), J. Wu (2), J. H. Graham (1)

(1) University of Florida, Citrus Research and Education Center, Lake Alfred, FL, U.S.A.; (2) University of Florida, Lake Alfred, FL, U.S.A.

Phytopathology 104(Suppl. 3):S3.56

Huanglongbing (HLB) is a devastating disease of citrus, caused by phloem limited Candidatus Liberibacter spp. Early symptoms of disease include fibrous root loss and blotchy mottle on leaves followed by yield declines, leaf drop, and dieback. Early root loss was previously described as a $30-50 \%$ reduction in root density that begins before foliar symptoms develop. Early root loss is dependent on local bacterial infection and occurs before carbohydrate starvation caused by phloem plugging in the canopy. Continued sampling has identified a second phase of root loss (70-80\%) that begins at the early stages of canopy thinning from leaf drop. During this phase carbohydrate supply to the roots is severely limited by canopy disease. Surprisingly, in both phases of root loss, root growth was not significantly different from presumed healthy trees through all root flushes sampled to date. This suggests that root density reductions result from the shortened lifespan of fibrous roots. In the absence of anatomical effects of infection on roots, such as phloem-plugging, the cause of this shortened lifespan is still under investigation.

Analyzing the role of constitutive XEGIP expression on limiting potato late blight, caused by Phytophthora infestans

R. JONES (1)

(1) USDA ARS, Beltsville, MD, U.S.A.

Phytopathology 104(Suppl. 3):S3.56

The late blight pathogen Phytophthora infestans causes a rapid collapse of infected leaf tissue, presumably due to secretion of plant cell wall modifying enzymes, principally pectinases and endoglucanases. Inhibition of these enzymes may be useful in limiting leaf damage. The cultivated potato genome contains only one known endoglucanase inhibitor, a protein known as XEGIP (xyloglucan-specific endoglucanase inhibitor protein), but the genome harbors at least nine copies of this enzyme inhibitor. To test the ability of one XEGIP (AY321357) to reduce late blight tissue damage, transgenic, 35S driven XEGIP overexpressing potato (cv Kennebec) plants were generated. While there was no change in tissue damage after infection by $P$. infestans US11, there were dramatic changes in the plant architecture. The XEGIP transgenic plants average a 50 percent reduction in petiole length, thickened leaves with 50 percent higher chlorophyll, greatly reduced leaf trichome numbers, slow root formation, and spindle shaped tubers. These results suggest that at least one XEGIP influences plant encoded cell wall modifying enzymes that may be involved in plant cell wall expansion. The role of XEGIP in plant disease interactions may be more important for pathogens other than P. infestans.

Soybean cultivars and fungicide responses to frogeye leaf spot - ten years of field data

W. J. JORDAN (1), H. M. Kelly (1), M. A. Newman (1)

(1) University of Tennessee, Jackson, TN, U.S.A.

Phytopathology 104(Suppl. 3):S3.56

Frogeye leaf spot (FLS) caused by Cercospora sojina Hara can be a devastating foliar disease in many part of the United States. Reductions in yields of $20-40 \%$ are not uncommon under environmental conditions that promote growth and sporulation of the fungus. Fungicide spray regimes and cultivar selection have been used to combat the impact of the disease by many producers. Over 600 different commercially available cultivars were evaluated in Milan, TN from 2003 through 2013. Cultivars tested included maturity groups III, IV, and V. All plots were sprayed at the R3 growth stage with a commercially available fungicide, and were visually rating for FLS lesions at the R5-R6 growth stage. Yield was collected for each of the sprayed and unsprayed portions of the plots. Fungicide application significantly reduced FLS severity and increased yield across all cultivars and years. Fungicide application reduced FLS severity proportionately to the susceptible of the cultivars (i.e. the greater the susceptibility the greater the reduction in disease with a fungicide application). Average yield saved with a fungicide application also increased as FLS susceptibility increased. Economic analysis on the profitability of a fungicide application based on cultivar selection will be discussed. Results based on this research support cultivar selection and appropriate fungicide applications are critical in minimizing the loss of yield due to FLS.

Controlling cherry leaf spot disease in ornamental cherries in midTennessee

J. O. JOSHUA (1), M. Mmbaga (2)

(1) TN State College of Agric Human \& Natural Sci, Nashville, TN, U.S.A.; (2) TN State University School of Agric \& Con Sci, Nashville, TN, U.S.A.

Phytopathology 104(Suppl. 3):S3.56

Cherry Leaf spot (CLS) disease caused by Blumeriella jaapii has become a significant constraint in nursery production of ornamental cherries in the southeastern United States and controlling this disease has been challenging and ineffective. Disease management based on host resistance and weatherbased fungicide applications have been recommended for fruiting cherries primarily grown in cooler northern and northwest regions of the USA. This study was conducted to provide guidance to ornamental cherry growers in the warmer regions. Six commercial cultivars were evaluated for host resistance; 
only two cultivars, 'Kwanzan' and 'Akebono' displayed moderate resistance to CLS in commercial nursery fields while four cultivars, 'Snowgoose', 'Yoshino', 'Okame' and 'Autamnalis' were highly susceptible. Fungicide applications guided by availability of primary inoculum in the local area showed that rotations of captan (Captan ${ }^{\mathrm{TM}}$ ), tebuconazole (Orius $20 \mathrm{Q}^{\mathrm{TM}}$ ) and trifloxystrobin (Flint $50 \mathrm{WG}^{\mathrm{TM}}$ or compass ${ }^{\mathrm{TM}}$ ), captan with chlorothalonil and acibenzolar-S-methyl (Daconil Action ${ }^{\mathrm{TM}}$ ) were most effective in controlling CLS. A neem plant extract (Triact $70^{\mathrm{TM}}$ ) and a bacterial biological control isolate were also effective in controlling CLS; copper sulphate with hydrated lime (Bordeaux ${ }^{\mathrm{TM}}$ ) was not effective when used alone or in rotations with other fungicides and it caused phytotoxicity.

Effects of gene silencing suppressors on Potato virus $X(\mathrm{PVX})$ and Potato leafroll virus (PLRV) infection in two Nicotiana species

H. JU (1), P. Phat (1), G. Youn (1)

(1) Chonbuk Natl University, Jeonju-city Chonbuk, South Korea

Phytopathology 104(Suppl. 3):S3.57

Suppressor $\mathrm{p} 19$ of Tomato bushy stunt virus and $2 b$ of Cucumber mosaic virus have been suppressed plant silencing-derived defence mechanisms resulting in the efficient of transgene expression. Here we report the effect of gene silencing suppressors on transient expression in transgenic Nicotiana benthamiana and $N$. tabacum on symptom exhibition, viral RNA accumulation, and systemic spread of PVX and PLRV infection. Suppressor p19 serve as the most efficient on transient expression of green fluorescent protein (GFP) in non-transformed $N$. benthamiana. For $N$. benthamiana, the time of PVX and PLRV symptom exhibition in systemic leaves of transgenic plants expressing $\mathrm{p} 19$ and $2 \mathrm{~b}$ were $2-4$ days more rapid than non-transformed plants. In addition, PVX symptoms were exhibited in transgenic plants of $N$. tabacum expressing p19 and $2 \mathrm{~b}$ at 12 days post inoculation (dpi), but not in non-transformed plants, indicating that suppressor p19 and $2 \mathrm{~b}$ induced the PVX accumulation led to the exhibition of systemic symptom. Suppressor p19 and $2 \mathrm{~b}$ also enhanced systemic infection of PLRV. At higher temperature, up to $32^{\circ} \mathrm{C}$, PVX infection was restricted to both the inoculated leaves and systemically infected leaves of $N$. tabacum plants, indicating that the susceptibility of PVX infection in $N$. tabacum was temperature sensitive. It was clear that the application of $\mathrm{p} 19$ and $2 \mathrm{~b}$ are useful to enhance transgene expression and induce PVX and PLRV symptoms and accumulation.

Characterization of virus-derived siRNAs from RBSDV-resistant and susceptible rice genotypes by deep sequencing

Z. Y. JUN (1), L. Ying (2)

(1) Institute of Plant Protection, Jiangsu Academy of Agricultural Sciences, Nanjing, China; (2) Jiangsu Academy of Agricultural Sciences, Nanjing, China

Phytopathology 104(Suppl. 3):S3.57

Rice black-streaked dwarf virus (RBSDV), a member of the genus Fijivirus in the family Reoviridae, is transmitted by the small brown planthopper (SBPH). It severely affects rice and maize production in China in recent years. Small RNA-mediated gene silencing plays evolutionarily conserved roles in gene regulation and defense against invasive nucleic acids. Virus-derived small interfering RNAs (vsiRNAs) produced from their insect vectors are well described but those from their hosts are less characterized. Through deep sequencing, we examined vsiRNAs from infected leaves and shoots of both RBSDV-resistant and susceptible rice genotypes. More vsiRNAs were derived from the positive strands and mostly were 21-22nt long. Further analysis showed that vsiRNAs were generated from hotspots on 10 different segments of viral double-stranded genomic RNA (S1-S10), in which S2 occupied the most. VsiRNAs from S1, S5 and S7 were mostly mapped to the 5' terminal regions, which were opposite to the 3' terminal regions in S6 and S8 segments. These hotspots were not associated with the predicted RNA secondary structures. Some vsiRNAs hotspots were different between two genotypes, and identification of them would be useful for efficient RNAi target designing, and the further resistant mechanism research.

\section{Diversity of Aspergillus species causing aflatoxin contamination of maize and groundnuts in Zambia}

P. W. KACHAPUlulA (1), R. Bandyopadhyay (2), J. Akello (3), M. Mukanga (4), P. J. Cotty (5)

(1) University of Arizona, Tucson, AZ, U.S.A.; (2) IITA Nigeria, IBADAN, Nigeria; (3) IITA ZAMBIA, LUSAKA, Zambia; (4) Zambia Agricultural Research Institute, LUSAKA, Zambia; (5) University of Arizona/USDAARS, Tucson, AZ, U.S.A.

Phytopathology 104(Suppl. 3):S3.57

Aflatoxins are carcinogenic, immunosuppressive toxins that frequently contaminate maize and groundnut, important staples in Zambia. Aspergillus flavus, A. parasiticus and two unnamed taxa have been implicated as causal agents of aflatoxin contamination in Africa. Improved understanding of contamination etiology in Zambia may facilitate aflatoxin management. Maize, groundnut and soil from the three agroecologies of Zambia were examined from 2012 to 2013. Soils (125) and crops (188) were assayed for Aspergillus section Flavi; aflatoxins were quantified with lateral flow immunochromatographic assays. Many fungal communities were dominated by isolates morphologically assignable to $A$. parasiticus. Multi-gene phylogenetic analyses with partial gene sequences for nitrate reductase (niaD, $1.8 \mathrm{~kb})$ and the aflatoxin pathway transcription factor $(a f l R, 2.1 \mathrm{~kb})$ resolved 200 isolates with $A$. parasiticus morphology from across Zambia into five well supported lineages. All crop samples tested had at least 2 ppb total aflatoxin, with $8 \%$ and $4.7 \%$ of the samples above $10 \mathrm{ppb}$ and $100 \mathrm{ppb}$ respectively. Fungal community structures in soil and crops, differed. Soil communities were dominated by $A$. parasiticus $(68 \%)$ while the $A$. flavus Lstrain morphotype was highest in maize $(61 \%)$ and fungi with S-strain morphology on groundnut $(51 \%)$. Characteristics of the identified lineages and factors that underlie community structure within Aspergillus section Flavi are under investigation.

Evolution and prevention of late blight caused by Phytophthora infestans genotype US-23

M. Kalischuk (1), L. KAWCHUK (2), R. Peters (3), K. Al-Maghrabi (4), F. Daayf (5), M. Harding (6)

(1) Lethbridge College, Lethbridge, AB, Canada; (2) AAFC, Lethbridge, AB, Canada; (3) AAFC, Charlottetown, PE, Canada; (4) AAF, Wicklow, NB, Canada; (5) University of Manitoba, Winnipeg, MB, Canada; (6) AARD, Brooks, AB, Canada

Phytopathology 104(Suppl. 3):S3.57

Late blight, caused by Phytophthora infestans, is historically infamous for the Irish Potato Famine and remains a devastating worldwide disease of potatoes and tomatoes. In recent years, there has been a resurgence of late blight in Canada and the United States, causing considerable disease losses. As part of a national late blight survey conducted in Canada during 2013 over 100 isolates of $P$. infestans were collected from the major potato growing areas and determined that the US-23 genotype dominated pathogen populations in most provinces. Many isolates of the $P$. infestans US-23 genotype had developed resistance to the systemic fungicide metalaxyl-m and that garden tomatoes were contributing to the prevalence of this strain of the late blight pathogen. Displacement of a $P$. infestans genotype within a province was observed within a single season and disease prevention strategies should include actions that reduce inoculum initiating from tomatoes, which could spread to potatoes and other economically important plants. Clonal propagation also contributed to the $P$. infestans US-8, US-11, and US-24 genotypes becoming established in isolated areas of Canada. In some areas, both A1 and A2 mating types of the pathogen were found in the same production region, potentially increasing the opportunity for sexual recombination within the $P$. infestans pathogen populations.

Application of the $\mathbf{R}$ package poppr for analysis of population genetic data

Z. N. KAMVAR (1), J. F. Tabima (1), N. J. Grünwald (2)

(1) Oregon State University, Corvallis, OR, U.S.A.; (2) USDA-ARS, Corvallis, OR, U.S.A.

Phytopathology 104(Suppl. 3):S3.57

Plant pathogen populations are often clonal or partially clonal, making analysis difficult. Many analysis methods assume panmixia and cannot be used for clonal or partially clonal populations. We thus created the $\mathrm{R}$ package poppr for conducting analysis of these populations commonly observed in plant pathology. Poppr provides a set of tools for repeatable analysis using hierarchically sampled populations. Clone censoring data is readily implemented for any level of a population hierarchy. To test for clonal reproduction we included analysis of linkage disequilibrium using the index of association combined with significance tests based on permutation. Several genetic distances, such as Nei's, Roger's and Bruvo's distance for microsatellites, are also implemented with the ability to produce dendrograms with bootstrap support or minimum spanning networks. With other packages in R, poppr further allows analyses of populations including richness, diversity, and evenness among multilocus genotypes. Poppr can analyze dominant and co-dominant marker data resulting from AFLP, SSR, SNP or similar kinds of data. We have made use of the functions in poppr for visualization of multilocus genotypes at http://phytophthora-id.org and have applied this package for analyses of Phytophthora plurivora populations. We continue to develop poppr and are currently working on tools for analysis of dense single nucleotide polymorphism data resulting from genotyping-bysequencing. 
Mapping quantitative resistance loci for bacterial leaf streak disease in hard red spring wheat using an identity by descent mapping approach Y. R. KANDEL (1), L. E. Osborne (2), J. L. Gonzalez-Hernandez (3), K. D. Glover (3)

(1) Iowa State University, Brookings, SD, U.S.A.; (2) Pioneer Hi-Bred International, Brookings, SD, U.S.A.; (3) South Dakota State University, Brookings, SD, U.S.A.

Phytopathology 104(Suppl. 3):S3.58

Bacterial leaf streak ((BLS; caused by Xanthomonas campestris pv. translucens) has become a serious disease of wheat in the Midwest USA. This study was conducted to map quantitative trait loci (QTL) for BLS resistance in spring wheat using an identity by descent (IBD) mapping approach. The mapping population consisted of 825 individuals representing 60 families. Resistance phenotyping was carried out in greenhouse and field research plots. Quantitative trait loci analysis was performed using both linkage and association-based approaches. Two genomic regions near the simple sequence repeat (SSR) markers Xwmc522 and Xbarc134, on chromosomes 2A and 6B, respectively, were significantly associated with resistance in both greenhouse and field evaluations. The markers Xwmc291 and Xbarc3 were also significantly associated with resistance from greenhouse evaluations, and Xgwm550 was associated from field observations. Variance explained by the significant markers ranged from 0.56 to $29.56 \%$, Xwmc522 was most associated with resistance, and narrow-sense heritability for resistance was 0.28 . This study demonstrates that the IBD mapping technique is a viable alternative to traditional bi-parental mapping for detecting markers associated with complex traits like partial disease resistance in breeding populations. This information will be useful for marker-assisted selection and will ultimately accelerate the development of cultivars with enhanced from breeding programs.

Effect of glyphosate application on sudden death syndrome of soybean under different field conditions

Y. R. KANDEL (1), C. A. Bradley (2), K. A. Wise (3), M. Chilvers (4), A. Tenuta (5), V. M. Davis (6), P. Esker (6), D. L. Smith (6), D. Mueller (1) (1) Iowa State University, Ames, IA, U.S.A.; (2) University of Illinois, Urbana, IL, U.S.A.; (3) Purdue University, West Lafayette, IN, U.S.A.; (4) Michigan State University, East Lansing, MI, U.S.A.; (5) Ontario Ministry of Agriculture and Food, Guelph, ON, Canada; (6) University of WisconsinMadison, Madison, WI, U.S.A.

Phytopathology 104(Suppl. 3):S3.58

Sudden death syndrome (SDS), caused by Fusarium virguliforme, is an important yield limiting disease of soybean. Glyphosate has been used for many years to control weeds in glyphosate-tolerant soybean. The effect of glyphosate application on SDS is not clearly understood. The objective of this study was to examine how glyphosate use affected SDS severity under field conditions. Fifteen field experiments were conducted in IA, IL, IN, MI, WI, and Ontario, CAN during 2011, 2012, and 2013. Six treatment combinations of non-glyphosate herbicides and glyphosate including pre- and postemergence, single and multiple applications were compared. Disease severity was significantly different $(P<0.05)$ across the location-years. Mean foliar disease index (FDX) ranged from 0 to $65 \%$ across field trials. Highest disease was noted in irrigated plots. There were no statistically significant effects of herbicide treatments or interactions $(P>0.05)$ on disease severity. The mean FDX among the treatments ranged from 10 to $14 \%$. These results indicate that the environment, particularly soil moisture, played a significant role on SDS development. Glyphosate-treated plots did not have more SDS than plots with other herbicides. Glyphosate-treated plots tended to yield more than plots treated with other herbicides. This fifteen location-year study demonstrated that glyphosate application does not increase SDS severity or adversely affect soybean yield under field conditions.

Effect of growth media composition on natural competence of Xylella fastidiosa

P. P. KANDEL (1), L. De La Fuente (1)

(1) Auburn University, Auburn, AL, U.S.A.

Phytopathology 104(Suppl. 3):S3.58

Xylella fastidiosa (Xf), a gram-negative bacterial plant pathogen that causes economically important diseases on crops such as grapes, citrus, and almond, has recently been described as naturally competent. Natural competence, a phenomenon in which bacterial cells take up free DNA from the environment and recombine it into their genomes, could be the explanation behind the appearance of new and virulent strains of Xf. It has been reported in several other bacterial species that nutrient composition of the growth environment/medium greatly influences natural competence and hence, can govern the rate of bacterial evolution. Using Xf strains and plasmids tagged with marker genes (GFP, antibiotic resistance), combinations of recipient $\mathrm{Xf}$ and plasmids or dead donor $\mathrm{Xf}$ cells were cultured together to asses $\mathrm{Xf}$ competency in media differing in the composition of minerals $(\mathrm{Ca}, \mathrm{Mg}, \mathrm{Zn})$, amino acids, and carbon sources and also in grape sap collected from different cultivars. The experiments were conducted on plates, in liquid batch cultures, and in micro-fluidic chambers (MCs) that mimic xylem vessels Xf naturally inhabits. Recombinants that contain the marker genes from the plasmids or dead donor cells were recovered using selective media. We found that media composition greatly influences natural competence in $\mathrm{Xf}$, and grape sap also supports competence in MCs, suggesting the occurrence of this process in nature.

Fungal pathogens associated with mature watermelon wilt symptoms in Korea

H. J. KANG (1), Y. S. Kim (2), B. Han (2), T. Kim (2), J. Noh (2), Y. Kim (3), C. U. Han (2), S. Y. Nam (2)

(1) Watermelon Research Institute, CBARES, Eumseong-gun Chungcheongbuk-do, South Korea; (2) Watermelon Research Institute, CBARES, Eumseong-gun, South Korea; (3) Enviroment-friendly Agriculture Research Division, CBARES, Cheongwon-gun, South Korea Phytopathology 104(Suppl. 3):S3.58

The majority of watermelons in Korea are grown under greenhouse conditions. To avoid plant diseases caused by consecutive cropping in the same location and to increase yield, almost all planted watermelons are grafted on wilt-resistant stocks. However, near harvest, various types of wilt symptoms (including sudden wilt or gradual wilt) accompanied by yellowing, vine decline, and leaf necrosis are observed. To determine the factors associated with wilt symptoms, we conducted field surveys and isolated fungal pathogens associated with the symptoms. Rhizonctonia spp. were the major pathogens isolated from wilted plants showing yellowing, leaf necrosis, and root rot. From the plants showing sudden wilt symptoms as well as healthy plants, Fusarium oxysporum was not isolated. However, pathogenic strains of $F$. oxysporum were isolated from one farm, in which the Fusarium wilt-resistant root stocks ("Ojakkyo" cultivar originated from Citrullus vulgaris, wild watermelon) had been used for more than 20 years. These results suggest that long-term use of the same resistant stock cultivar could cause an outbreak of Fusarium wilt under greenhouse conditions. In addition, this is the first report of Fusarium wilt on grafted watermelon in Korea.

Identification of two 6-methoxyflavonoid phytoanticipins from Prunus maackii

L. J. KANISZEWSKI (1), R. Hammerschmidt (1)

(1) Michigan State University, East Lansing, MI, U.S.A

Phytopathology 104(Suppl. 3):S3.58

Phytoanticipins and structural barriers such as periderms provide constitutive defenses against pathogen invasion. As part of a search for resistance in Prunus spp against Armillaria ostoyae, strong antifungal activity against $A$. ostoyae was detected when periderm from Prunus maackii was incorporated into culture media. Similar antifungal activity was not detected in periderm from other Prunus spp. Using thin layer chromatography bioassays, two major antifungal compounds were detected in acetone extracts of $P$. maackii periderm. These compounds were either not detected or detected at very low concentrations in other Prunus spp. tested. High performance liquid chromatography coupled with UV or mass spectroscopy identified the two compounds as flavonoids. The structures of the two compounds were confirmed as 3,5,7-trihydroxy-6-methoxyflavone and 3,5,7-trihydroxy-6methoxyflavanone by X-ray crystallography. The antifungal spectrum of activity of $P$. maackii periderm will also be described.

Identification of quantitative trait loci associated with resistance to Pyrenophora tritici-repentis in a doubled haploid tetraploid wheat population

G. KARIYAWASAM (1), S. S. Xu (2), J. D. Faris (2), J. B. Rasmussen (1), Z. Liu (3)

(1) Department of Plant Pathology, North Dakota State University, Fargo, ND, U.S.A.; (2) Cereal Crops Research Unit, Northern Crop Science Laboratory, USDA-ARS, Fargo, ND, U.S.A.; (3) North Dakota State University, Fargo, ND, U.S.A.

Phytopathology 104(Suppl. 3):S3.58

Tan spot of wheat, caused by Pyrenophora tritici-repentis, is an important disease of common and durum wheat across the world. The disease not only causes significant yield losses, but also can diminish grain quality. In particular, red smudge caused by fungal infection of wheat kernels can lead to significant downgrading of durum wheat in the market. Fewer studies have been conducted to determine the chromosomal locations of tan spot resistance genes in tetraploid wheat compared to hexaploid wheat. Previously, a doubled haploid population consisting of 146 lines derived from durum cultivar 
Lebsock and Triticum turgidum subsp. carthlicum accession PI 94749 was used to identify QTL associated with resistance to races 1 and 2. In this work, we evaluated this population for the reaction to race 3 (isolate 331-9), race 5 (isolate DW5) and a newly identified race (isolate Ark CroB10). QTL significantly associated with tan spot resistance were identified on chromosomes 3A, 4A, 5A, 5B, 7A and 7B, respectively. The 3A QTL, which was previously shown to be associated with resistance to races 1 and 2 , was associated with all three isolates in this work and explained from $10-14 \%$ of the disease variation. The other QTL were associated with resistance to one or two isolates. Lebsock contributes the resistance effects of all but the 7B and $5 \mathrm{~A}$ QTL. This work further indicates that both race specific and racenonspecific resistance are presented in wheat tan spot system.

Using effectors of Phytophthora infestans to teach pathogenesis: Our attempt to provide a more comprehensive education

V. KARTANOS (1), D. A. Halterman (2), M. Hayslett (1), D. Rouse (1)

(1) University of Wisconsin-Madison, Madison, WI, U.S.A.; (2) USDA-ARS, Madison, WI, U.S.A.

Phytopathology 104(Suppl. 3):S3.59

The topic of pathogenesis mechanisms (R/avirulence genes, effectors, and hypersensitive response) has proved challenging for students in our introductory plant pathology course. An apparent gap exists in the curriculum between this introductory course and higher level plant-microbe interaction courses. To address this, lectures were altered to provide a better introduction to pathogenesis and to reflect recent research. Also, a novel laboratory exercise was developed for students to use the terms and concepts. Students infiltrated leaves of transgenic tobacco, expressing the potato RB resistance gene, with Agrobacterium tumefaciens expressing the effectors IPI-O1 and IPI-O4 from Phytophthora infestans. They observed the interaction between the effectors and the R-gene product by examining the plants for hypersensitive cell death. To assess these new additions, a pre-quiz was administered prior to the laboratory activity and around the same time the companion lectures began. The same or similar questions were asked on a quiz after the lab and on the final exam. Of two questions that were given verbatim on the pre-quiz and the lab quiz, the score improved for both $(\mathrm{p}<0.05)$. When all exam questions on these topics were compared to the total pre-quiz scores, the average score on the exam questions was $7.28 \%$ higher (p $=0.05)$. We conclude that these changes to the lecture and the lab improved student understanding of the molecular mechanisms involved in pathogenesis.

Reduction of pathogen populations at grapevine wound sites is associated with the mechanism of biological control of crown gall by strain ARK-1 A. KAWAGUCHI (1)

(1) Okayama Pref Tech Ctr for Agric, For, Fish, Akaiwa, Japan

Phytopathology 104(Suppl. 3):S3.59

A nonpathogenic strain of Agrobacterium (=Rhizobium) vitis, ARK-1, limits the development crown gall of grapevine. Co-inoculation with ARK-1 and the tumorigenic strain VAT07-1 at a 1:1 cell ratio result in a higher population of ARK-1 than of VAT07-1 in shoots without tumors, but a significantly lower population of ARK-1 than VAT07-1 in grapevine shoots with tumors. This indicates that ARK-1 reduced the pathogen population at the wound site through biological control. Although ARK-1 produced a zone of inhibition against other tumorigenic Agrobacterium spp. in vitro assays, the antibiosis depended on the culture medium. ARK-1 did not inhibit the growth of tumorigenic A. vitis strain 9-3-4 in the antibiosis assay, but suppressed tumor formation on grapevine shoots caused by 9-3-4, suggesting that antibiosis by ARK-1 is not the main mechanism of biological control. Moreover, in grapevine stems inoculated with ARK-1 by needle-prick method, expression of defense-related gene such as PR-1 was induced in stem tissues.

Distribution, genetic diversity, and impact of fungicide resistant Cercospora sojina, the cause of frogeye leaf spot in soybean, in Tennessee H. KELLY (1), K. Lamour (2), W. J. Jordan (1), A. Cochran (1)

(1) University of Tennessee, Jackson, TN, U.S.A.; (2) University of Tennessee, Knoxville, TN, U.S.A.

Phytopathology 104(Suppl. 3):S3.59

Frogeye leaf spot (FLS) of soybean, caused by Cercospora sojina $\mathrm{K}$. Hara, is a common foliar disease in the southern USA and parts of the midwestern and northern USA. Yield losses ranging from $20-60 \%$ are not uncommon in years where weather conditions are favorable for fungal growth and cultivars lack genetic resistance. Management strategies to control FLS include planting resistant varieties and applying foliar fungicides. In 2010, the first C. sojina isolate with resistance to quinone outside inhibitor (QoI) fungicides was reported in Tennessee. Since that time C. sojina isolates with resistance to QoI fungicides have been confirmed in 10 states, across 67 counties/parishes in the US. C. sojina field studies have been conducted across Tennessee to investigate genetic diversity, disease severity, response to fungicides, and impact on yield. The results of these studies and the impact of QoI resistant $C$. sojina isolates will be discussed.

Developing a DNA-based toolkit for detection and identification of wheat rust pathogens

P. Kesanakurti (1), S. HAMBLETON (1)

(1) Agriculture and Agri-Food Canada, Ottawa, ON, Canada

Phytopathology 104(Suppl. 3):S3.59

Wheat is the primary host for the three rust fungi, Puccinia graminis (Pgt), P. triticina $(\mathrm{Pt})$ and $P$. striiformis (Pst). It is common to find plants infected with more than one species, making disease diagnosis difficult in early stages of rust development. Effector genes assumed to play a major role in disease mechanisms offer the potential to design specific markers for diagnostics. Two recent publications identified more than 1600 putative effector genes in Pgt, Pt and Pst in silico. Analyses of publicly available genomes were used to select candidates which were single copy, species-specific and variable at a sub-specific level. A total of 30,67 and 26 primer pairs were designed and tested on pure DNA extracts of 8,25 and 9 isolates respectively for Pgt, Pt and Pst. Further testing on non-target species identified $3(\mathrm{Pgt}), 24(\mathrm{Pt})$ and 13 (Pst) species-specific primer pairs. Two Allele Specific Oligos (ASOs) designed for Pgt differentiated two groups of isolates corresponding to provenance from Africa versus North America. Combinations of primer sets were used to confidently identify the targets in a mixed DNA sample containing all three species, without DNA sequencing. Their use in conjunction with assays diagnostic for all three species guarded against false negative results. The observed variation in PCR amplicon sizes allows for development of a multiplex assay, eliminating the need for laborious cloning and costly probes for identification.

A new record of Meloidogyne partityla and an unknown species of Punctodera from Arkansas

C. KHANAL (1), W. Ye (2), R. T. Robbins (1)

(1) University of Arkansas, Fayetteville, AR, U.S.A.; (2) Nematode Assay Section, Agronomic Division, North Carolina Department of Agriculture \& Consumer Services, Raleigh, NC, U.S.A.

Phytopathology 104(Suppl. 3):S3.59

Soil and root samples of pecan (Carya illinoinensis) were collected from a pecan orchard near Paris, Logan County, Arkansas. Soil was processed using sugar floatation method to determine the presence of nematodes. Extracted nematodes were tentatively identified to genus using a stereo microscope. Root-knot nematode J2's as well as cysts were obtained from the soil. Rootknot females with egg masses were obtained from pecan roots. These nematodes were identified using morphological and molecular diagnostics. For root-knot nematode, polymerase chain reaction (PCR) was performed to amplify the region between COII and 16S rRNA of mitochondrial gene. PCR product was purified and sent for sequencing to DNA Sequencing Core Facility, Little Rock, Arkansas. Sequences obtained were compared with nonredundant nucleotide sequences in Genbank through NCBI BLAST. The Blast result showed highest identity with Meloidogyne partityla for the root-knot nematode. This is the first report of M. partityla in Arkansas which was previously reported from Georgia, New Mexico, Florida, Texas, Arizona and Oklahoma. This nematode is posing a threat to the pecan industry. 18S, ITS and $28 \mathrm{~S}$ regions of Ribosomal gene was amplified to identify the cyst nematode. Sequencing results show similarity to Punctodera, but not an exact match. Phylogenetic, morphological and morphometric studies of both the nematodes were done. Males of neither were found.

Endornaviruses in common bean breeding lines, cultivars, landraces, and wild genotypes from the two main centers of domestication

S. Khankhum (1), R. A. VALVERDE (1)

(1) Louisiana State University Agricultural Center, Baton Rouge, LA, U.S.A. Phytopathology 104(Suppl. 3):S3.59

Plant endornaviruses are persistent viruses that do not cause visible symptoms and have been reported in several economically important plant species. Two endornaviruses, Phaseolus vulgaris endornavirus 1 (PvEV-1) and Phaseolus vulgaris endornavirus 2 (PvEV-2) with RNA genomes of 14 and $15 \mathrm{~kb}$ respectively have been reported in common bean (Phaseolus vulgaris) cultivars. These endornaviruses, lack coat protein, and are transmitted only through the gametes. We investigated the occurrence of these viruses in breeding lines, cultivars, landraces, and wild genotypes of $P$. vulgaris from the two centers of domestication: Mesoamerica and the Andes. Genotypes were tested for the presence of viral double-stranded RNA by gel electrophoresis and RT-PCR using virus-specific primers. Amplicons from PCR reactions using selected samples representing the various genotypes were sequenced. In general, the two endornaviruses were detected in most of the 
common bean genotypes of Mesoamerican origin but not in those of Andean origin. Viruses were more frequent in modern cultivars and breeding lines than in landraces and wild genotypes. Sequence of PCR products confirmed that endornaviruses from wild $P$. vulgaris are closely related to those of landraces, breeding lines, and modern cultivars. These results suggest that these viruses may have a beneficial effect to Mesoamerican common beans.

Influence of cytoplasmic genomes on plant defense response

P. KIANIAN (1), F. Ghavami (2), R. Burciaga (3), S. Meinhardt (4), M. Acevedo (4), S. Kianian (5)

(1) University of Minnesota, St. Paul, MN, U.S.A.; (2) BioDiagnostics, River Falls, WI, U.S.A.; (3) ARS-USDA, Byron, GA, U.S.A.; (4) North Dakota State University, Fargo, ND, U.S.A.; (5) ARS-USDA, St. Paul, MN, U.S.A. Phytopathology 104(Suppl. 3):S3.60

Alloplasmic lines can be produced by replacing the nucleus of one species in the cytoplasm of a closely related species through repeated backcrossing. Wheat has an extensive collection of alloplasmic lines many with over 10 backcrosses ( $>99 \%$ of the nuclear genome is replaced) and some with over 20 backcrosses to the parental nuclear donor species. Studies indicate that these cytoplasmic substitutions can change plant fertility, heading dates, and biomass. This study was conducted to investigate the response of wheat alloplasmic lines in relation to the parental control to fungal pathogens Puccinia triticina (leaf rust) and Pyrenophora tritici-repentis (tan spot). Results indicate that $24 \%$ and $9.4 \%$ of the lines tested had significantly increased resistance to tan spot isolates BR15 (race eight) and Pti2 (race one), respectively. The alloplasmic lines showing increased resistance to tan spot and derived from paternal donor cultivar 'Chris', which is susceptible to a number of leaf rust isolates, were further tested with the bulk mixture of four leaf rust isolates THBL, MCPL, MFPS, and TDBG. Two of the eight lines tested showed significantly increased resistance with the line carrying Aegilops heldreichii cytoplasm exhibiting a highly resistant response. These studies indicate that cytoplasmic component of the cell contribute to plant's ability to respond to pathogen attack and further detailed analysis of this interaction is warranted.

New resistance in old places: Resistance to the Ug99 race group of Puccinia graminis f. sp. tritici in intergeneric hybrids and historic germplasm

J. KIELSMEIER-COOK (1), T. Danilova (2), B. Friebe (2), M. N. Rouse (3)

(1) University of Minnesota, St. Paul, MN, U.S.A.; (2) Kansas State University, Manhattan, KS, U.S.A.; (3) USDA-ARS/University of Minnesota, St. Paul, MN, U.S.A.

Phytopathology 104(Suppl. 3):S3.60

New races of the stem rust pathogen, Puccinia graminis f. sp. tritici (Pgt), threaten global wheat production. Rapid evolution of Pgt requires continuous effort to deploy new resistance. The bottleneck for new Pgt resistance is not a lack thereof but a dearth of cultivars with new resistance. In order to rapidly introgress new resistance into modern cultivars, we crossed historic wheat lines identified as resistant to Ug99 with modern cultivars. Phenotype segregation of one population indicated two Ug99 resistance genes: $\mathrm{Sr} 24$ and an uncharacterized gene. Top-cross $F_{1}$ plants were screened with markers to simultaneously combine desirable agronomic and disease resistance loci including Glu-A1/Glu-D1, Rht-B1/Rht-D1, Lr34, and Sr24. Doubled haploid progeny of the selected plants were screened with Pgt race TTKST (Ug99 + $\mathrm{Sr} 24$ virulence) to resolve genomic location of the uncharacterized gene. We also screened the W.J. Sando collection of wheat-intergeneric hybrids with Pgt race TTKSK (Ug99) and variants. Four lines were resistant at the seedling stage and cytologically similar to wheat. GISH revealed each line possessed 1 to 2 pairs of Thinopyrum ponticum chromosomes. We used homoeologspecific FISH cDNA probes to identify individual wheat and alien chromosomes. By combining germplasm screening, mapping, and markerassisted pre-breeding into a single workflow we rapidly incorporated new resistance into modern breeding germplasm.

Isolation and formulation of antagonistic bacteria for biological control of root rot on ginseng

Y. S. KIM (1), Y. H. Jeon (1)

(1) Andong National University, Andong, South Korea

Phytopathology 104(Suppl. 3):S3.60

Ginseng (Panax ginseng) is one of the most widely cultivated as herbal medicine in Korea. Ginseng should be grown in the same field soil for several years and root condition of ginseng is getting worse and worse. This cultivation practice makes ginseng vulnerable to attacks by a variety of soilborne pathogens including fungi, bacteria, and nematode. Fungi are the major pathogens causing root diseases on ginseng, among which Cylindrocarpon destructans (Zins.) Sholten (teleomorph: Nectria radicicola Gerlach \& L.
Nilsson) is one of the most important root-rot causing pathogen and the main cause of replanting problems in ginseng cultivation. This study aimed to develop a biocontrol system for root rot caused by $C$. destructans on ginseng. One hundred eighty-five bacteria were isolated from ginseng roots and various soils samples were used screening for their antifungal activity against the fungal pathogen. Three isolated bacteria were showed inhibition of hyphal growth and two isolated bacteria were impeded chlamydospore formation of C. destructans. All of these results suggest that the bacterial isolates have good potential as a microbial agent for the biocontrol on ginseng root rot by C. destructans. This research will be shed light on development of ecofriendly control strategy of $C$. destructans to reducing ginseng replant disease.

Screening and identification of wide-spectrum antifungal bacteria isolated from aerated compost tea

M. J. KIM (1), C. K. Shim (1), Y. K. Kim (1), H. J. Jee (1), S. J. Hong (1), J. H. Park (1), E. J. Han (1), M. Lee (1), J. C. Yun (1)

(1) National Academy of Agricultural Science, Suwon, South Korea Phytopathology 104(Suppl. 3):S3.60

A total of 100 bacterial isolates were isolated from aerated compost tea and screened for antagonism against six plant pathogenic fungi, Botrytis cinerea, Colletotrichum gloeosporioides, Fusarium oxysporum, Phytophthora capsici, Rhizoctonia solani, and Sclerothium sclerotinia and used to control major plant pathogens. $83 \%$ isolates showed antagonistic activities against three of six fungal pathogens with varying degrees. Among them, 26 of 100 isolates showed significantly inhibited the mycelial growth of six major plant pathogens and also formed siderophore and indole acetic acid, and had nitrogen fixation activity in vitro. Through its biochemical, physiological, and $16 \mathrm{~S}$ rDNA characteristics, the selected bacteria were identified as Bacillus amyloliquefaciens, Bacillus pumilus, Paenibacillus terrae, and Sphingobacetrium composti. Therefore we think the candidates antifungal isolate can be a promising biocontrol agents for controlling of plant disease in organic vegetable cultivation.

Site-directed mutagenesis and immunolocalization of SnTox1, a necrotrophic effector produced by the wheat pathogen Parastagonospora nodorum

Y. M. KIM (1), Z. Liu (1), J. D. Faris (2), T. L. Friesen (2)

(1) North Dakota State University, Fargo, ND, U.S.A.; (2) USDA-ARS, Northern Crop Science Laboratory, Cereal Crops Research Unit, Fargo, ND, U.S.A.

Phytopathology 104(Suppl. 3):S3.60

SnTox 1 is a necrotrophic effector (NE) produced by the destructive wheat pathogen, Parastagonospora nodorum (syn. Stagonospora nodorum). SnTox1 induces necrosis on wheat genotypes carrying Snn1. After secretion with the cleavage of a 17 amino acid (aa) signal peptide, the mature SnTox1 is 100 aa and contains 16 cysteine residues with multiple putative protein motifs including chitin-binding domains (CBDs) and a lysine-rich region. To further investigate the mode of action of SnTox1, site-directed mutagenesis was conducted. While mutations at CBDs did not affect SnTox1 induced necrosis, necrosis was eliminated when cysteine residues were substituted or deleted. When mutations were produced at a casein kinase II phosphorylation site, a slight reduction in necrosis was observed. However, elimination of necrosis was observed in preliminary experiments when all six lysine residues in a predicted helical region were substituted. We demonstrated that SnTox1 binds to the cell wall in fungal mycelium, which supports the chitin-binding function of SnTox1. In addition, immunolocalization of SnTox1 by surface application revealed that SnTox1 remains on the leaf surface while necrosis develops in the mesophyll of the leaf as time progresses, indicating cell to cell signaling. Our results suggest that cysteine residues in SnTox1 are important in maintaining structural integrity of the NE, and that the lysine-rich region plays a critical role in SnTox1-induced necrosis signaling.

Phytotoxin solanapyrone A produced by Ascochyta rabiei and Alternaria solani is nonessential for pathogenicity, but likely plays ecological roles W. KIM (1), C. M. Park (1), J. J. Park (1), D. R. Gang (1), M. Xian (1), F. M. Dugan (1), T. L. Peever (1), W. Chen (1)

(1) Washington State University, Pullman, WA, U.S.A. Phytopathology 104(Suppl. 3):S3.60

Ascochyta rabiei and Alternaria solani, causal agents of chickpea and potato blights respectively, produce the same phytotoxin solanapyrone A (SolA). The toxicity of SolA to plants has been documented, but its role in pathogenicity has not been investigated. In this study, we generated solanapyrone-deficient mutants of both A. rabiei and Al. solani using targeted mutagenesis. The mutants completely lost production of SolA. Pathogenicity tests showed that SolA-deficient mutants are equally pathogenic as the wild type strains suggesting SolA is nonessential for disease development. Concordantly, SolA 
was not detected during its parasitic stage. The expression of SolA genes culminated during sporulation and was concentrated around the spore-bearing structure at maturity. In competition assays, the solanapyrone-deficient mutants did not inhibit the growth of several saprobic fungi isolated from chickpea debris, whereas wild-type strains effectively kept the potential competitors away from their colony boundaries. These results suggest that the two fungi utilize SolA to protect their vegetative and reproductive propagules from other microorganisms during saprobic life stage, thereby being successful in spreads of the diseases in the next growing season.

\section{Phytophthora species associated with Abies root rot and their distribution in Pennsylvania}

S. H. KIM (1), S. Kang (2), T. N. Olson (1), E. Nikolaeva (1)

(1) Pennsylvania Department of Agriculture, Harrisburg, PA, U.S.A.; (2) Phytophthora Database, Pennsylvania State University, State College, PA, U.S.A.

Phytopathology 104(Suppl. 3):S3.61

Phytophthora root rot is a serious threat to Christmas tree production in PA. Plant inspectors of the PA Department of Agriculture (PDA) have collected Abies spp. showing root rot symptoms, mostly from nurseries, while providing inspection and certification services over the last 28 years (1986-2013). Among 300 Abies samples displaying Phytophthora root rot symptoms, collected from 47 Counties, 229 (from 45 Counties) turned out to be Phytophthora positives. Fraser fir, Abies fraseri, seems to be the most vulnerable host with $74.7 \%$ of the positives (37 Counties) corresponding to this host. White fir (A. concolor) was the next most common host $(8.7 \%$ from 12 Counties). The remaining samples corresponded to Balsam (A. balsamea), Canaan (A. intermedii), Noble (A. proera), Grand (A. grandis), and Korean $(A$. koreana) firs. Phytophthora isolates cultured from the 229 samples using PARP medium belonged to eight species, based on both morphological characteristics and ITS sequences, and were stored in PDA culture collection. $P$. cryptogea, $P$. cactorum, $P$. drechsleri and $P$. citricola were associated with $83 \%$ of the samples, and the rest was associated with $P$. cinnamomi, $P$. medicaginis $\times$ cryptogea, $P$. citrophthora, and $P$. cambivora. A significant portion of Fraser fir samples displaying Phytophthora root rot symptoms (40.5\%) came from three counties, in which P. cryptogea, P. drechsleri, $P$. cactorum, and P. citricola were associated with $93.3 \%$ of the samples.

Underestimation of Fusarium mycotoxin contamination due to "masked" mycotoxins

H. Klink (1), J. A. Verreet (1), T. BIRR (1)

(1) University of Kiel, Kiel, Germany

Phytopathology 104(Suppl. 3):S3.61

The so-called "masked" mycotoxins are of particular importance with regard to mycotoxin safety limits. Wheat as well as corn are able to conjugate deoxynivalenol (DON) with glucose (or "mask" it) to the non-phytotoxic DON-3-glucoside (D3G). A few studies have demonstrated that lactobacilla in the mammalian gut deglycosylate D3G to DON and glucose ("unmasking"), whereby the toxicological risk is increased by "masked" DON. Standard analysis methods do not take into account "masked" DON, resulting in an underestimation of the actual DON exposure. DON and D3G contents of wheat (3 varieties, 2012 and 2013) and corn (4 varieties, 2011-2013) were determined in Germany in the course of an over-regional monitoring. In wheat, D3G could be detected in all 3 varieties. In the highly susceptible wheat cultivar Ritmo, $10 \%$ of the total DON was detected in the glycosylated form, in the moderately susceptible variety Inspiration $13 \%$, and in the tolerant variety Dekan $24 \%$. By taking into account D3G in addition to nonglycosylated DON, the EU's maximum DON amount of $1250 \mu \mathrm{g} \mathrm{kg}^{-1}$ dry grain mass was exceeded significantly more often than in sole consideration of DON without D3G. In corn, D3G also was detected in all varieties tested. A "realistic" view of Fusarium toxin contamination requires therefore that the "masked" mycotoxins (especially D3G) are considered in addition to the unmasked toxins.

Effects of chemical class and physical properties on the translaminar activity of fungicides

C. J. R. KLITTICH (1), S. L. Ray (1)

(1) Dow AgroSciences LLC, Indianapolis, IN, U.S.A.

Phytopathology 104(Suppl. 3):S3.61

The translaminar activity of 61 fungicides in three classes was examined using cucumber powdery mildew as a model system. Application rates were normalized to foliar $\mathrm{LC}_{50}$ and formulation was identical for all compounds. Lipophilicity and water solubility were the most predictive parameters in multiple and logistic regression models. Foliar $\mathrm{LC}_{50}$ was a key third parameter for predicting size of the control zone. Triazole and strobilurin fungicides fell into four groups: moderate translaminar movement/small zone of control (pyraclostrobin, fluoxastrobin); moderate translaminar movement/large zone of control (propiconazole, epoxiconazole, tebuconazole); strong translaminar movement/large zone of control (picoxystrobin, fenbuconazole, myclobutanil, prothioconazole); very strong translaminar movement/large zone of control (cyproconazole, azoxystrobin). Although triazoles as a class produced significantly larger control zones than the other fungicide classes, individual strobilurins can have very strong translaminar movement. When triazole and strobilurin fungicides were compared for lipophilicity and water solubility they were distributed across the range of physical properties with no retention of the translaminar group clustering. Although lipophilicity and water solubility were the most predictive factors in our models, other (unknown) physical properties must also affect the translaminar redistribution of strobilurins and triazoles.

Detection of the downy mildew pathogens of spinach (Peronospora effusa) and beet $(P$. schachtii) using spore traps and quantitative PCR assays S. J. KLOSTERMAN (1), A. Anchieta (1), N. McRoberts (2), S. Koike (3), K. V. Subbarao (4), H. Voglmayr (5), Y. J. Choi (6), M. Thines (7), F. N. Martin (1)

(1) USDA ARS, Salinas, CA, U.S.A.; (2) University of California, Davis, CA, U.S.A.; (3) University of California Cooperative Extension - Monterey County, Salinas, CA, U.S.A.; (4) University of California, Salinas, CA, U.S.A.; (5) University of Vienna, Vienna, Austria; (6) Goethe University and Biodiversity and Climate Research Center, Frankfurt, Germany; (7) Biodiversity and Climate Research Center, Frankfurt, Germany

Phytopathology 104(Suppl. 3):S3.61

Downy mildew of spinach, caused by Peronospora effusa, is a disease constraint on spinach production worldwide. The aim of this study was to develop a real-time quantitative PCR assay for detection of airborne inoculum of $P$. effusa in California. This type of assay may, in combination with disease-conducive weather forecasting, be helpful to time fungicide applications for disease management. Among oomycete ribosomal DNA (rDNA) sequences examined, the highest nucleotide sequence identity was observed between rDNA sequences of $P$. effusa and Peronospora schachtii, the cause of downy mildew on Beta vulgaris (beet and Swiss chard). Single nucleotide polymorphisms (SNPs) were detected between $P$. effusa and $P$. schachtii $18 \mathrm{~S}$ rDNA regions for design of $P$. effusa- and $P$. schachtii-specific TaqMan probes and reverse primers. An allele-specific probe and primer amplification method was applied to determine the frequency of both $P$. effusa and $P$. schachtii rDNA target sequences in pooled DNA samples, enabling quantification of rDNA of $P$. effusa from impaction spore trap samples. The rDNA copy numbers of $P$. effusa were on average 3400-fold higher from trap samples collected near an infected spinach field than at sites remote from such fields.

Diversity of foliar Phytophthora species on rhododendron in Oregon nurseries

B. J. KNAUS (1), V. J. Fieland (2), N. J. Grunwald (1)

(1) USDA-ARS, Corvallis, OR, U.S.A.; (2) Oregon State University, Corvallis, OR, U.S.A.

Phytopathology 104(Suppl. 3):S3.61

The genus Phytophthora is considered to contain some of the most important plant pathogens affecting nursery crops. Given the recent emergence of the sudden oak death pathogen $P$. ramorum, particularly in association with Rhododendron, it has become prudent to characterize Phytophthora communities in nursery environments. Many taxa may present syptoms similar to regulated taxa and may vary in pathogenicity from regulated taxa, making determination important. We present a survey of Phytophthora taxa observed from seven nurseries in the U.S. state of Oregon. Incidence and diversity of Phytophthora communities differed significantly among nurseries and among seasons. The taxa $P$. syringae and $P$. plurivora were widespread and detected at most of the nurseries sampled. Nine other taxa were also detected but were found either in a single nursery or were shared among only a few nurseries. Characterization of the Phytophthora communities present in nurseries is an important step towards understanding the ecology of these organisms as well as an aid to nursery managers in determining what risks may be present when symptomatic plants are observed. This study builds on an increasing literature which characterize Phytophthora community structure in nurseries.

Inheritance of powdery mildew disease resistance in Cornus florida

S. KODATI (1), L. Parikh (1), M. T. Mmbaga (1)

(1) TN State College of Agric Human \& Natural Sci, Nashville, TN, U.S.A. Phytopathology 104(Suppl. 3):S3.61

Efforts to breed for powdery mildew (Erysiphe pulchra) resistance in flowering dogwoods (Cornus florida L.) have been undertaken, but only a few 
cultivars have displayed powdery mildew resistance al beit moderate. Furthermore, the identified resistance has not been consistent over time and locations and fungicide applications become necessary when environmental condition are highly favorable to powdery mildew. The objective of this research was to identify the inheritance pattern and gene interactions for powdery mildew resistance in $C$. florida. Hand pollinations were used to make 8 different controlled crosses between 'Cherokee Brave', 'Cherokee Princess' and selections R14 and MI9. The progeny seedlings were maintained in greenhouse environment, exposed to powdery mildew and rated for powdery mildew disease reaction. Progeny segregation was grouped into different disease reaction categories based on their disease severity. The results were analyzed and their ratios were calculated for gene interaction and identification of gene flow.

\section{Etiology and management of stem rot diseases of Stevia}

A. KOEHLER (1), H. D. Shew (1)

(1) North Carolina State University, Raleigh, NC, U.S.A.

Phytopathology 104(Suppl. 3):S3.62

Stevia (Stevia rebaundia) is an herbaceous perennial that is an emerging crop in the US. Stevia is typically grown for 3-5 years and is harvested twice per growing season. Stevia leaves contain multiple glycosides that are extracted for use as a nonnutritive sweetener which was approved as a food additive by the USDA in 2008. Stevia plantings in North Carolina began in 2011 and are expanding rapidly in the state. Wilting and death of plants in first and second year commercial plantings were observed in NC in 2012 and 2013. Sclerotium rolfsii and Sclerotinia sclerotiorum were observed on diseased plants and Koch's postulates were performed to verify these organisms as pathogens of Stevia. The biology of these pathogens on Stevia is not yet clearly understood. Initial work is underway to screen Stevia germplasm for resistance to these two stem rot pathogens as well as other potential pathogens that are endemic in Stevia production areas. The goal of this work is to better understand when and where infection is occurring and if any of the genotypes currently available have usable levels of partial resistance. There are currently no products labeled for use on Stevia, which is a limiting factor to the commercial production of Stevia. Fungicide and biocontrol field trials will be conducted to determine optimal application rates and timing.

Isolation of Phytophthora megakarya informed by developmental expression of disease lesion pattern on cocoa (Theobroma cacao) pod

O. O. KOLAWOLE (1), A. A. Ogunjobi (2), E. M. Goss (3)

(1) University of Florida; University of Ibadan, Nigeria; Cocoa Research Institute of Nigeria, Gainesville, FL, U.S.A.; (2) University of Ibadan, Ibadan, Nigeria; (3) University of Florida, Gainesville, FL, U.S.A.

Phytopathology 104(Suppl. 3):S3.62

Phytophthora pod rot (black pod disease) is a major constraint to cocoa production in Africa, particularly endemic to Central and West Africa cocoaproducing countries. This disease is caused predominantly by Phytophthora megakarya, a devastating, invasive, aggressive and highly virulent pathogen, which frequently causes loss of cocoa pods up to $60-100 \%$ especially if left uncontrolled. This pathogen can be easily confused with other species of Phytophthora which also cause black pod disease. Phytophthora species have traditionally been delineated by their morphology, cytology and biochemistry, however, there are complications in using phenotypic taxonomic characters, due to overlapping characters between species and variation among isolates of the same species. Nonetheless, it is believed that black pod disease caused by P. megakarya can be distinguished from other Phytophthora using the irregular edge lesions it produces on cocoa pods. We obtained 379 Phytophthora isolates from the interphase of developing irregular edge lesion on cocoa pods from six states across two cocoa-growing agro-ecological zones in Nigeria. P. megakarya can be distinguished from P. palmivora and other Phytophthora species by PCR amplification of the ITS region followed by restriction analysis with $A l u$ I. PCR-RFLP of the 379 isolates showed that they were all $P$. megakarya, confirming that this species produces a characteristic lesion morphology.

Global population genetics of the wheat leaf rust fungus Puccinia triticina J. KOLMER (1), M. Ordonez (2)

(1) USDA ARS, St. Paul, MN, U.S.A.; (2) Pontifical University Catholica, Quito, Ecuador

Phytopathology 104(Suppl. 3):S3.62

Collections of Puccinia triticina were obtained from North America, South America, Central Asia, Europe, the Middle East, Russia and China to examine the genetic diversity within each continental region and genetic relationship between regions. Approximately 100 single uredinial isolates from each region were tested for virulence phenotype to 20 near-isogenic Thatcher wheat lines and for molecular genotype with 23 simple sequence repeat (SSR) primers. All continental populations had significantly higher than expected SSR allele heterozygosity and had very high $(>3.00)$ index of association $\left(\mathrm{I}_{\mathrm{A}}\right)$ values, indicating clonal reproduction worldwide. Genetic differentiation for SSR genotype groups varied from 8 groups in Europe to 2 groups in Russia. In all regions $R_{S T}$ differentiation was greater than $F_{S T}$ indicating the likely importance of SSR mutation in generating variation. Within all populations $F_{S T}$ and $R_{S T}$ differentiation based on geographic regions was lower than differentiation based on SSR genotype groups, indicating the migration of SSR genotypes within continental regions. In all populations SSR genotypes were significantly correlated from 0.55 to 0.42 with virulence phenotypes. Grouping based on $F_{S T}$ and $R_{S T}$ in neighbor joining dendrograms of the worldwide SSR genotype groups had low correlation, however both measures separated groups of SSR genotypes that originated from durum wheat from collections from common wheat.

Can antagonistic fungi reduce the growth of Cryphonectria parasitica in dual culture?

M. KOLP (1), A. M. Jarosz (1)

(1) Michigan State University, East Lansing, MI, U.S.A.

Phytopathology 104(Suppl. 3):S3.62

Cryphonectria parasitica is the introduced fungus causing chestnut blight on American chestnut (Castanea dentata). The blight epidemic has decimated chestnuts throughout its native range. This necrotrophic fungus enters the host through wounds in the outer bark and rapidly kills host cells. Once infected, the cambium and inner bark become necrotic resulting in a canker that can expand and girdle a stem. However, we have observed secondary saprotrophic fungi that colonize the center of older blight cankers. These fungi were collected from natural cankers in chestnut stands in Michigan and Wisconsin and identified as Trichoderma spp. and Penicillium spp., which are noted in the literature to be competitive soil saprotrophs. To begin evaluating the potential of these secondary fungi as biological control agents, a dual-culture plating experiment was initiated to compare in vitro growth responses of two different strains of Cr. parasitica separately against nine isolates of secondary fungi. When plated against each of three strains of Trichoderma spp., the two strains of Cr. parasitica used in this study (EP 155 and LE 221) grew significantly less compared to the no competitor control. Penicillium spp. did not significantly change the growth of either strain of $\mathrm{Cr}$. parasitica nor did the other four taxa of secondary fungi. Future research will investigate the ability of Trichoderma spp. to control canker expansion on excised and living chestnut tissue.

A proposed generic research protocol for phytosanitary treatments of regulated plant pathogens in seed

D. A. KOMM (1)

(1) USDA, Raleigh, NC, U.S.A.

Phytopathology 104(Suppl. 3):S3.62

Phytosanitary treatments are used to prevent the introduction of regulated pests into the United States. United States Department of Agriculture (USDA), Animal and Plant Health Inspection Service (APHIS), Plant Protection Quarantine (PPQ) has a documented generic research protocol for phytosanitary treatments for insects, but not for plant pathogens. Recent interceptions of Diaporthe viticola on spinach seed and Cucumber Green Mottle Mosaic Virus on melon required phytosanitary treatments for the associated plant pathogens. In response, PPQ developed a generic research protocol for phytosanitary treatments of plant pathogens in seed. Phytosanitary treatments must be $100 \%$ effective in killing and/or inactivating the plant pathogen. The protocol must be detailed about all aspects of the research. Small scale testing or preliminary tests must be done to determine the best treatment options. Multiple doses and time/temperature combinations are useful to determine the optimum treatment. Each treatment must have a minimum of 4 replications with equivalent of 100 infected seeds per replication. A confirmatory test is conducted following the small scale testing. The sample size for the confirmatory testing will be mutually agreed upon by APHIS and researcher. The resulting treatment will provide quarantine protection for United States agriculture.

Population structure and fungicide resistance profile of Botrytis spp. causing damping-off disease in stone and pome fruit rootstock seedlings S. KONSTANTINOU (1), G. Sarmis (1), G. S. Karaoglanidis (2)

(1) Aristotelian Univ of Thessaloniki, Faculty of Agriculture, Plant Pathology Laboratory, Thessaloniki, Greece; (2) Aristotelian Univ of Thessaloniki, Thessaloniki, Greece

Phytopathology 104(Suppl. 3):S3.62

Botrytis spp. is the main causal agent of damping-off in micro-propagated pome and stone fruit rootstock seedlings in greenhouse nurseries. The study was conducted to investigate the fungal population structure and fungicide 
resistance profile. Population structure was investigated in 134 isolates, using 2 molecular markers, the presence of transposable elements (TEs, Boty and Flipper) and the presence of a $21 \mathrm{bp}$ insertion in the $m r r l$ gene, encoding a transcription factor, that distinct B. cinerea sensu stricto, from Botrytis Group $\mathrm{S}$, a novel clade within Botrytis populations. Results showed that transposa isolates (both TEs present) were predominant (83.6\%), while Boty, Flipper or vacuma (both TEs absent) isolates were found at frequencies of $11.9,1.5$ and $3 \%$, respectively. Similarly, was found that $B$. cinerea sensu stricto was predominant in the population $(69.7 \%)$. However a significant proportion of the isolates were identified as Botrytis group S (29.6\%), while 1 isolate was identified as B. pseudocinerea. Measurements of fungicide sensitivity revealed the presence of high frequencies of multiple resistance to more than 2 different fungicide classes, mostly associated with target site mutations. The higher frequencies of resistance were observed for QoIs, SDHIs and benzimidazoles with values of $89.8,73$ and $64.6 \%$, respectively, while frequencies of resistance to anilinopyrimidines, hydroxyanilides, dicarboximides and phenylpyrroles were lower.

\section{Identification, characterization and mycotoxigenic ability of Alternaria} spp. causing core rot of apple fruit

S. KONSTANTINOU (1), P. Ntasiou (1), C. Myresiotis (2), E. Mourkidou (2), G. S. Karaoglanidis (3)

(1) Aristotelian Univ of Thessaloniki, Faculty of Agriculture, Plant Pathology Laboratory, Thessaloniki, Greece; (2) Aristotelian Univ of Thessaloniki, Faculty of Agriculture, Laboratory of Pesticide Science, Thessaloniki, Greece; (3) Arisotelian Univ of Thessaloniki, Thessaloniki, Greece

Phytopathology 104(Suppl. 3):S3.63

Alternaria core rot is a major postharvest disease of apple fruit in Greece. Seventy five Alternaria spp. isolates obtained from fruit showing core rot symptoms were identified as either A. tenussima or A. arborescens at frequencies of 89.3 and $11.7 \%$, respectively, based on ITS sequence. Moreover, phylogenetic analysis based on the sequence of two additional genes, Alta 1 and endoPG supported the distinction of the isolates into two separate clades. Artificial inoculations of fruit (cv. Fuji) and incubation at two different temperatures $\left(0\right.$ and $\left.23^{\circ} \mathrm{C}\right)$ showed that $A$. tenussima was more aggressive compared to $A$. arborescens. In addition, in 30 isolates of both species was measured the production of 3 mycotoxins, alternariol (AOH), alternariol monomethyl ether (AME) and tentoxin (TEN). Measurement was conducted both in vitro, on artificial nutrient medium and in vivo on artificially inoculated apple fruit, using a high performance liquid chromatography with diode array detector (HPLC-DAD). The results showed that most of the isolates of both species were producing all the 3 metabolites both in vivo and in vitro. On apple fruit $A$. tenuissima isolates were producing more $\mathrm{AOH}$ than $A$. arborescens isolates, while the latter were producing more TEN compared to the former. Such results indicate that Alternaria core rot of apple fruit represents a major threat not only due to quantitative yield losses but also for qualitative deterioration of apple by-products.

\section{UV-C treatment to induce disease resistance for long-term storage of} carrots

N. KOUASSI (1), R. Corcuff (1), R. Tweddell (2), D. Michaud (2), J. Arul (1) (1) Department of Food Science and Nutrition and Horticultural Research Centre, Laval University, Quebec, QC, Canada; (2) Department of Plant Biology and Horticultural Research Centre, Laval University, Quebec, QC, Canada

Phytopathology 104(Suppl. 3):S3.63

Postharvest diseases cause serious storage losses and early termination of the storable life of fresh carrots. UV-C was applied to induce disease resistance in carrots and increase their storage life. The hormetic (optimum) UV-C dose, delay after harvest before treatment, storage temperature, and carrots longterm storability of the treated carrots were evaluated by resistance to Botrytis cinerea, Sclerotinia sclerotiorum, and Rhizoctonia carotae, the accumulation of the phytoalexin 6-methoxymellein (6-MM), the induction of defense proteins PR-2 ( $\beta$-1,3-glucanases) and PR-3 (chitinases), severity of natural infection, and senescence (sprouting and rooting). The hormetic UV-C dose for induction of resistance was about $5.4 \mathrm{~kJ} . \mathrm{m}^{-2}$, and the resistance strongly related to the accumulation of 6-MM. Carrots treated immediately after harvest developed and maintained stronger defense against diseases. The storage temperature significantly influenced the resistance to pathogens and the accumulation as well as the degradation of 6-MM, and the proteins PR-2 and PR-3. The long-term storage studies showed that the UV-C treatment could improve carrot shelf life, as seen by reduced natural infection, lateral roots and sprouts, and maintenance of inhibitory level of 6-MM. It was concluded that UV-C treatment could be a beneficial technology and/or be a key part of an integrated diseases management for long-term storage of carrots.
Pre-inoculation heat stress increases resistance to Phytophthora root rot in warm climate but not cold climate populations of Rhododendron minus S. KREBS (1), J. Wang (1), P. Bonello (2), J. Blakeslee (3)

(1) The Holden Arboretum, Kirtland, OH, U.S.A.; (2) The Ohio State University, Columbus, OH, U.S.A.; (3) The Ohio State University - OARDC, Wooster, OH, U.S.A.

Phytopathology 104(Suppl. 3):S3.63

The ability to tolerate disease under high temperature stress may be an important component of warm climate adaptation in plants. To test this concept, we measured the responses of two native Rhododendron minus populations to inoculation with Phytophthora cinnamomi following root heat stress. Pot-grown seedlings from a northern high-elevation population $(\mathrm{NH})$ and a southern low-elevation population (SL) were placed in a water bath to bring the root zone to target temperatures of $26^{\circ}, 34^{\circ}, 42^{\circ}$, or $50^{\circ} \mathrm{C}(1$ hour to, 1 hour at target). After cooling, seedlings were inoculated with $P$. cinnamomi and mortality was tracked over time. All seedlings in uninoculated control pots survived the highest heat treatments. Staining with TTC revealed no significant difference in root viability between the $26^{\circ}$ and $42^{\circ} \mathrm{C}$ treatments, but a significant $(52 \%)$ reduction in viability occurred at $50^{\circ} \mathrm{C}$. Paired $t$ tests of areas under the disease progress curves for the two populations $\left(\mathrm{AUC}_{\mathrm{NH}}\right.$ and $\mathrm{AUC}_{\mathrm{SL}}$ ) resulted in no significant difference at $26^{\circ} \mathrm{C}$ and $50^{\circ} \mathrm{C}$ root treatment levels, a $13 \%$ lower $\mathrm{AUC}_{\mathrm{SL}}$ in the $34^{\circ} \mathrm{C}$ treatment relative to $\mathrm{AUC}_{\mathrm{NH}}$ $(p=.035)$, and a $19 \%$ reduction in $\mathrm{AUC}_{\mathrm{SL}}$ relative to $\mathrm{AUC}_{\mathrm{NH}}$ after root exposure to $42^{\circ} \mathrm{C}(p=.003)$. At non-injurious stress temperatures $\left(\leq 42^{\circ} \mathrm{C}\right)$, these data suggest that warm climate-adapted $R$. minus populations are less predisposed to disease at high temperatures because of a heat-inducible defense response that is not present in populations from colder climates.

Grapevine red blotch-associated virus is widespread in United States vineyards

B. Krenz (1), J. R. Thompson (1), H. L. McLane (1), M. FUCHS (2), K. L. Perry (1)

(1) Cornell University, Ithaca, NY, U.S.A.; (2) Cornell University, Geneva, NY, U.S.A.

Phytopathology 104(Suppl. 3):S3.63

Grapevine red blotch-associated virus $(\mathrm{GRBaV})$ is highly correlated with red blotch, a recently recognized disease of grapevines. This virus is tentatively assigned to the family Geminiviridae with limited significant affinities to members of recognized genera and novel plant-infecting gemini-like viruses. To determine the distribution and genetic variability of GRBaV isolates, more than one hundred infected grapevine samples were collected in or received from vineyards in eight states (AR, CA, MD, NJ, NY, OR, PA and VA), demonstrating a widespread occurrence of the virus in the United States. Phylogenetic analyses of a viral replication-associated protein (Rep) gene fragment from 42 isolates of GRBaV demonstrated two distinct clades, with clade 1 showing the greatest variability and clade 2 grouping the majority of isolates. The full-length genome of six GRBaV isolates was sequenced and phylogenetic analyses of 14 whole genomes recapitulated phylogenetic relationships seen for the Rep gene. A comparison of GRBaV genomes indicated recombination events underlying some of the variation seen among isolates within clade 1 . These results shed light on the distribution and genetic variability of the newly identified GRBaV.

A multi-year search for Candidatus Liberibacter spp. in orange jasmine plants in Texas by field surveys and multi-loci PCR assays

M. KUNTA (1), W. Li (2), J. V. da Graça (1), L. Levy (3), C. de la Garza (1), C. C. Parra (1), M. Gonzalez (1), S. Chavez (1), E. S. Louzada (1), M. K. Nakhla (2), M. Sétamou (1)

(1) Texas A\&M University Kingsville, Weslaco, TX, U.S.A.; (2) USDA APHIS PPQ CPHST Beltsville Laboratory, Beltsville, MD, U.S.A.; (3) USDA APHIS PPQ CPHST, Riverdale, MD, U.S.A.

Phytopathology 104(Suppl. 3):S3.63

Citrus species found adjacent to orange jasmine trees are the preferred host plants for psyllids and can act as reservoirs for Liberibacters in dooryards of the Lower Rio Grande Valley. The primers and probe based on 16S rRNA were used to identify Liberibacters by conventional (cPCR) and TaqMan quantitative PCR (qPCR). During 2009-2010, out of 440 orange jasmine leaf samples collected from 55 plants and psyllids in the vicinity of these plants, 44 orange jasmine samples showed high threshold cycle $(\mathrm{Ct})$ values 33-37 while pysllids did not produce any high $\mathrm{Ct}$ values in the quantitative PCR (qPCR). Suspect samples were recollected in March, 2011 from these plants along with ACP adults and nymphs for DNA extraction. New TaqMan primer/probe sets based on other Candidatus Liberibacter asiaticus (CLas) genes were used to verify the $16 \mathrm{~S}$ qPCR results. From 16 citrus plants, 90 orange jasmine and $22 \mathrm{ACP}$ samples tested, 3 orange jasmine samples yielded high qPCR Ct values (1 using 16S rRNA gene and 2 for cold shock protein 
gene) in multi-loci qPCR assays using 7 primer/probe combinations that amplify different Las genes. Subsequent multi-loci PCR assays on leaf samples collected from 44 orange jasmine plants in different months of 2011/ 2012 and leaf and root samples from 37 orange jasmine and 10 citrus plants collected in 2014 did not produce any high Ct values. All samples were symptomless and tested negative in cPCR and nested PCR reactions.

Citrus Huanglongbing incidence, spread, and current situation in Texas M. KUNTA (1), M. Sétamou (1), E. S. Louzada (1), J. V. da Graça (1), D. W. Bartels (1)

(1) Texas A\&M University-Kingsville, Weslaco, TX, U.S.A.

Phytopathology 104(Suppl. 3):S3.64

After 6 years of extensive statewide surveys, the occurrence of Huanglongbing (HLB) in Texas citrus was confirmed in 2012. An extremely low percentage $(0.03 \%)$ of psyllid samples collected in 2011 showed threshold cycle values less than 32 and 1 year later, the first detection of HLBinfected sweet orange (cultivar 'Valencia') tree was made. From the initial CLas detection in psyllids in 2011, there has been a substantial increase in the percentage of positive psyllids detected through time, with a $0.33 \%$ of psyllids tested $(\mathrm{n}=11075)$ in 2013 . The percentage of CLas-positive leaf tissue increased from $0.61 \%(\mathrm{n}=7680)$ in 2012 to $1.03 \%(\mathrm{n}=8320)$ in 2013 . As reported in several previous studies, detection of CLas in psyllids was at least one year earlier before its detection in citrus tissue sample. A positive and significant relationship was obtained between the yearly percentage of CLaspositive leaf tissue samples $(\mathrm{Y})$ and the percentage of CLas-positive psyllids (X) $\left(\mathrm{Y}=-0.0302+3.21 \mathrm{X}\right.$ with adjusted- $\left.R^{2}=0.99\right)$, for the period of 2009 2013. It shows that an increase of $3.21 \%$ CLas positive pyllids lead to $1 \%$ increase in CLas positive leaf tissue samples. Proactive psyllid management in commercial groves and residential trees, removal of infected trees, and quarantine regulations on movement of citrus plants along with environmental factors may be slowing the spread of the disease in Texas compared to other citrus producing areas.

Oversummering of 'Candidatus Liberibacter solanacearum' in silverleaf nightshade, Solanum elaeagnifolium, in the lower Rio Grande Valley of Texas

M. KUNTA (1), J. Thinakaran (2), E. Pierson (3), D. Henne (2)

(1) Texas A\&M University Kingsville, Weslaco, TX, U.S.A.; (2) Texas AgriLife Research, Weslaco, TX, U.S.A.; (3) Texas A\&M University, College Station, TX, U.S.A

Phytopathology 104(Suppl. 3):S3.64

Certain cultivated and wild solanaceous plant species are significant in the epidemiology of zebra chip (ZC) disease of potatoes and are alternative hosts for the potato psyllid, Bactericera cockerelli, and its endosymbiotic bacterial pathogen, 'Candidatus Liberibacter solanacearum' (Lso). Therefore, experiments were conducted under controlled conditions to study transmission of Lso from infected potato to uninfected silverleaf nightshade (SLN), and vice versa. Lso-free $B$. cockerelli reared on Lso-infected SLN plants became infective, and transmitted Lso to potato plants. Detection of Lso in SLN was inconsistent, due to uneven distribution and variation in Lso titer, as reported for other solanaceous plants. Both vector and pathogen reportedly do not tolerate temperatures above $35^{\circ} \mathrm{C}$. Lso-infected SLN plants were planted outdoors to determine if Lso persists in these plants after exposure to temperatures routinely $>35^{\circ} \mathrm{C}$, and possibly serve as primary sources of Lso inoculum during the following winter potato growing season. Under field conditions, it was observed that SLN plants are shrubby, producing daughter plants via stolons, with subsequent death of the mother plant. Aerial parts of Lso-infected field planted SLN did not test positive for Lso by the following autumn. A search is in progress for Lso in SLN roots and stolons, and acquisition studies with $B$. cockerelli are also being conducted to verify Lso presence/absence in field planted SLN.

Management of bacterial wilt of tomato with a combination of grafting and a systemic acquired resistance inducer

S. KUNWAR (1), M. L. Paret (2), J. B. Jones (1), L. Ritchie (2), J. H. Freeman (2), S. M. Olson (2)

(1) Plant Pathology Department, University of Florida, Gainesville, FL, U.S.A.; (2) NFREC, University of Florida, Quincy, FL, U.S.A.

Phytopathology 104(Suppl. 3):S3.64

Field trials and greenhouse studies were conducted in Florida from 2012-2013 to determine the integrated effect of grafting and application of acibenzolar-Smethyl (ASM), a systemic acquired resistance (SAR) inducer, for control of bacterial wilt disease of tomato caused by Ralstonia solanacearum. In greenhouse experiments, ASM $(50 \mathrm{mg} / \mathrm{l})$ was applied twice either as foliar spray or as soil drench before inoculation. Soil application of ASM significantly reduced disease severity on a susceptible tomato cultivar (BHN
$602)$ as compared to foliar treated or the untreated control $(\mathrm{P} \leq 0.05)$. Similarly, in the field, a single drip application of ASM $(50 \mathrm{mg} / \mathrm{l})$ before transplanting and four weekly drip applications of ASM $(0.5 \mathrm{oz} / \mathrm{A})$ after transplanting significantly reduced disease incidence in BHN $602(\mathrm{P}=0.0045)$, but did not significantly improve yield $(\mathrm{P}=0.0057)$ relative to the untreated control. Grafting (BHN 998 rootstock and BHN 602 scion), alone or in combination with drip application of ASM $(0.5 \mathrm{oz} / \mathrm{A})$, provided significantly better disease control and yield relative to the untreated control. In contrast, foliar ASM application combined with grafting provided a marginal increase in disease incidence and significantly reduced yield in one of the field trials as compared to the untreated grafted control $(\mathrm{P}<0.0001)$. The combination of grafting and ASM application via drip, however, was statistically equivalent to grafting in controlling the disease and improving yield.

Resistance to Fusarium virguliforme in soybean cultivars Minnsoy, Noir, and recombinant inbred lines derived from a cross of Minnsoy $\mathbf{x}$ Noir

J. E. KURLE (1), M. V. Milenovic (2), G. M. Anderson (1)

(1) Department of Plant Pathology, University of Minnesota, St. Paul, MN, U.S.A.; (2) Department of Agronomy and Plant Genetics, University of Minnesota, St. Paul, MN, U.S.A.

Phytopathology 104(Suppl. 3):S3.64

Sudden death syndrome (SDS) caused by Fusarium virguliforme $(F v)$ is a major yield limiting disease of soybean. Infection during germination is followed by root rot and foliar symptoms. Resistance screening results are affected by environmental conditions, symptoms assessed for the resistance rating, and inoculation method. The objectives of this study are 1) to characterize resistance in soybean lines differing in susceptibility to SDS and 2 ) to determine if symptom ratings obtained using different evaluation methods are correlated. 'Minnsoy' and 'Noir1', sixteen recombinant inbred lines, and check varieties, AG2107, McCall, and MN1606SP, were included in the study. Treatments were an uninoculated control, inoculation with infested sorghum, and immersion in $\mathrm{Fv}$ toxin. Foliar symptoms were evaluated 32 days after planting (DAP), root rot and root development was evaluated 36 DAP, and reproductive stage foliar ratings from field experiments were included for comparison. Foliar symptom severity ranged from 4.3 (Minnsoy) to 1.0 (Noir and 3 other cultivars). Root rot severity ranged from 8.7 to 2.5. Cultivar ranking differed when differing disease assessment methods were compared. Foliar and root rot ratings correlated poorly. Severe root rot was associated with reduced foliar symptoms in some cultivars. The results indicate that multiple mechanisms provide resistance to SDS and that multiple screening methods are necessary to phenotype resistance to SDS.

Genetic diversity of Agrobacterium vitis from diverse geographic origins based on RAPD and rec $A$ gene sequence analysis

N. KUZMANOVIC (1), E. Biondi (2), A. Bertaccini (2), A. Obradovic (1)

(1) University of Belgrade, Faculty of Agriculture, Belgrade, Serbia; (2) Università di Bologna, Facoltà di Agraria, Bologna, Italy

Funded by the national III46008 and EU AREA No 316004 projects. Phytopathology 104(Suppl. 3):S3.64

Agrobacterium vitis is a primary causal agent of grapevine crown gall worldwide. Major mode of the pathogen long distance dispersal is via grapevine propagating material. The aim of this study was to assess genetic diversity and phylogenetic relatedness of 62 tumorigenic strains of $A$. vitis originating from eight European countries (58 strains), North America (2 strains), Australia (1 strain) and North Africa (1 strain). Initial evaluation of genetic diversity among strains was determined by RAPD using three primers: A9, A10 and R13. Genetic profiles were transformed into a binary matrix used for phylogenetic analysis and construction of the dendrogram. The strains were differentiated into 12 genetic groups at $80 \%$ similarity level. A total of 27 representative strains, at least one per each RAPD group, were selected for amplification and sequencing of $\mathrm{rec} A$ housekeeping gene using primers recAF1/recAR1 designed to amplify 615 bp DNA fragment. The sequence similarities among the strains were $92.1-100 \%$. Four clusters were identified in the maximum likelihood phylogenetic tree. The correlation between the genetic groups and geographical origin of the strains was not determined. Genotypic relatedness of the strains belonging to a particular phylogenetic group indicates that they may have common origin and were probably distributed worldwide following the movement of infected grapevine planting material.

Validation of an ascospore prediction model for circular leaf spot disease caused by Mycosphaerella nawae of persimmon

J. H. KWON (1), O. Choi (2), J. J. Park (2), H. S. Lee (1), B. K. Chung (1), T. S. Kim (1), S. D. Lee (1), H. Y. Shin (1), Y. S. Kwak (2), J. Kim (2)

(1) Gyeongsangnam-do Agricultural Research and Extention Services, Jinju, South Korea; (2) Gyeongsang National University, Jinju, South Korea

Phytopathology 104(Suppl. 3):S3.64 
Circular leaf spot (CLS) disease caused by Mycosphaerella nawae continues to cause fruit yield losses of persimmon. Ascospoores are the primary inocula for the outbreaks of CLS. We describe a computer model used cumulative degree days to simulate ascospore release data. We investigated the seasonal pattern of ascospore release of CLS and the association with day degrees affecting spore release based on spore trapping and fungal isolation. Ascospore release data of CLS were recorded from two regions for three persimmon-growing seasons (2010-2012). The ascospores of CLS start to release from the first week of May and released until the end of July, and maximum release of spores was observed in the late of June. Cumulative degree days were used to simulate ascospore release. The regression parameters were used to calculate the cumulative degree days at which $50 \%$ release occurred. The overall mean value of cumulative degree days from 01 May to $50 \%$ ascospore release was $1174^{\circ} \mathrm{C}$, which was not significantly different from the simulated value of $1,144^{\circ} \mathrm{C}$. The difference of mean value between observed and simulated ascospore release percentage was not significantly different from zero, indicating that the computer model was not biased towards over- or under-prediction. Our model is useful in estimating relative inoculum levels, and the starting, peak and end of ascospore release, thus determine to time fungicides.

Comparative analysis of Populus trichocarpa resistance to Melampsora spp. leaf rust disease

J. LA MANTIA (1), J. Klapste (1), Y. A. El-Kassaby (1), S. D. Mansfield (1), C. J. Douglas (1), R. C. Hamelin (1)

(1) University of British Columbia, Vancouver, BC, Canada

Phytopathology 104(Suppl. 3):S3.65

Populus trichocarpa is a native natural resource to the Pacific Northwest; providing fast-growing woody biomass for utilization in wood and paper industries and biofuels production. Poplars co-evolve with native Melampsora spp; however, hybrid poplar breeding and international trade has introduced an exotic rust species to North America, Melampsora larici-populina. Moreover, a new hybrid rust; M. $\times$ columbiana, has enhanced pathogenicity and has become the principal leaf rust in the Pacific Northwest. In the presented study, 407 and 397 P. trichocarpa genotypes were screened for resistance to M. larici-populina and $M$. $\times$ columbiana in independent trials of inoculated leaf disks, respectively. A total of 29,355 SNPs in 3,543 genes were used for association analysis to compare host resistance mechanisms. Resistance to each species displayed a continuous range from complete resistance to upwards of 40 pustules per leaf disk at 14 days post inoculation. Final number of pustules and area under the disease progress curve were significantly correlated amongst 378 common poplar genotypes $\mathrm{R}^{2}=16.0 \%$ at $p<0.01$ and $\mathrm{R}^{2}=12.0 \%$ at $p<0.05$, respectively. A total of 13 SNPs were significant at $P<3.30 \times 10^{-5}$. Associated SNPs were located in genes regulating ROS, auxin signaling, and anthocyanidin biosynthesis revealing the first non-R gene resistance loci. No SNP was associated in both analyses, but more in-depth SNP coverage may results in non-species specific rust resistance.

Effect of biorational fungicides on in vitro germination of Monilinia fructicola conidia

N. LALANCETTE (1), K. A. McFarland (1), J. Gager (1)

(1) Rutgers University, Bridgeton, NJ, U.S.A.

Phytopathology 104(Suppl. 3):S3.65

Basic efficacy information is lacking for many biorational fungicides. The objective of this study was to quantitatively assess inhibition of $M$. fructicola spore germination by three biofungicides currently registered on peach: potassium bicarbonate (Kaligreen), Bacillus subtilis (Serenade MAX), and hydrophobic extract of neem oil (Trilogy). Shaker flasks containing an aqueous medium of $0.025 \mathrm{M}$ potassium phosphate buffer plus $0.1 \%$ sucrose and $0.1 \%$ yeast extract were amended with each biofungicide at seven different concentrations in a logarithmic series. Non-amended buffer-substrate medium served as a control. After adding conidia, flasks were incubated for 6 $\mathrm{h}$ with gentle agitation at $25^{\circ} \mathrm{C}$. The dependent response variable was $\%$ relative spore germination $=\%$ germinated $($ amended) $/ \%$ germinated (control). Examination of the linear response - log dose curves revealed that all three biofungicides were capable of inhibiting germination. To quantitatively evaluate efficacy, the logistic decline model was fit to each response-dose curve using nonlinear regression analysis. The effective concentration at which germination is inhibited by $50 \%\left(\mathrm{EC}_{50}\right)$ was estimated as $0.0199,10.43$, and $0.6015 \mathrm{~g}$ a.i./L for potassium bicarbonate, $B$. subtilis, and neem oil, respectively. These results indicate that potassium bicarbonate has the greatest intrinsic efficacy with respect to inhibition of conidia germination. The findings will be discussed within the context of field application.
Bremia lactucae infection efficiency under field conditions is modulated by leaf wetness duration and temperature

M. LAMINE FALL (1), C. Beaulieu (1), H. Van Der Heyden (2), O. Carisse (3) (1) University of Sherbrooke, Sherbrooke, QC, Canada; (2) Compagnie de recherche Phytodata Inc, Sherrington, QC, Canada; (3) Agric \& Agri-Food Canada, St-Jean-sur-Richelieu, QC, Canada

Phytopathology 104(Suppl. 3):S3.65

More than 80 percent of the Canadian lettuce production is located in the province of Quebec. Most of the knowledge on epidemiology of downy mildew (Bremia lactucae) is derived from controlled conditions experiments or field experiments conducted in subtropical climate and cannot readily be applied to the Quebec lettuce production. The influence of temperature and leaf wetness duration on B. lactucae infection efficiency (IE) was studied under both field and growth chambers conditions in 2003, 2004, 2012 and 2013. The IE was defined as the ratio of number of lesions per leaf to airborne conidia concentration (ACC). ACC of (B. lactucae was measured with rotating-arms samplers three times per week while 72 traps plants per sampling day were randomly exposed in lettuce field. Under controlled environment, an ACC of 1 conidia $\mathrm{m}^{-3}$ was sufficient to cause 1 lesion per leaf and the IE ranged from 0.25 to 1.00 . Under field condition, an ACC of 10-14 conidia $\mathrm{m}^{-3}$ was required to cause 1 lesion per leaf and IE ranged from 0.02 to 0.10 except in 2003. The IE increased with increasing leaf wetness duration, whereas it decreased as the temperature increased. Also, below temperature of $20^{\circ} \mathrm{C}$, leaf wetness duration of $2 \mathrm{~h}$ was sufficient for infection. Therefore, under the Quebec lettuce production conditions, a leaf wetness period of 2-3h and an ACC of 10-14 conidia $\mathrm{m}^{-3}$ can be used as risk indicators and to facilitate disease management decisions.

Simultaneous detection and differentiation of three sweet potato potyviruses by a multiplex TaqMan real-time RT-PCR

P. LAN (1), F. Li (2), J. Abad (3), L. Pu (1), R. Li (1)

(1) USDA-ARS, Beltsville, MD, U.S.A.; (2) Yunnan Agricultural University, Kunming, China; (3) USDA-APHIS, Beltsville, MD, U.S.A.

Phytopathology 104(Suppl. 3):S3.65

Sweet Potato Viral Disease (SPVD) is the most devastating disease of sweet potato worldwide. The disease is caused by mix infection of Sweet potato chlorotic stunt virus, a member of the genus Crinivirus in the family Closteroviridae, and several viruses including Sweet potato latent virus (SPLV), Sweet potato virus G (SPVG) and Sweet potato mild mottle virus (SPMMV) in the family Potyviridae. In this study, a one-tube reverse transcription (RT) TaqMan real-time PCR was developed for the simultaneous detection and differentiation of SPLV, SPVG and SPMMV. Amplification and detection of a fluorogenic cytochrome oxidase gene (COX) was included as an internal control. Sensitivities of the multiplex assay were $10^{-7}$ for each of the target viruses and for the COX mRNA, similar to those of simplex assays. The mulitplex assay was evaluated by testing 74 field samples collected from China. The multiplex assays detected SPVG and SPLV in 63 and 6 samples, respectively. SPLV was existed as co-infection, and SPMMV was not detected in any of the samples. The results agreed with those of conventional simplex RT-PCR, indicating the multiplex assay was sensitive. Sequence analysis of selected samples showed the multiplex assay was specific. The multiplex assay described here offers a valuable tool for the differential detection of the causal viruses of SPVD. It will be useful for rapid and reliable identification of these viruses in quarantine, certificate programs and virus surveys.

Greenhouse evaluation of inoculation methods and commercial cotton cultivars in the presence of verticillium wilt

C. J. LAND (1), K. Lawrence (1)

(1) Auburn University, Auburn, AL, U.S.A.

Phytopathology 104(Suppl. 3):S3.65

Verticillium Wilt is caused by Verticillium dahliae, which colonizes the vascular cylinder of cotton and causes defoliation, stunting, and yield loss. Objectives were to optimize efficiency of infection and identify tolerant cotton varieties in greenhouse screens. Inoculation methods examined at planting included: 1) 3.5 grams of Verticillium dahliae microsclerotia; 2) $10 \% \mathrm{vol} / \mathrm{vol}$ V. dahliae infested oat seeds; 3) $10^{7}$ spore drench; 4) stem injection of 25,000 conidia at 4WAP; and 5) an untreated control. All treatments were planted with a susceptible cotton variety Phytogen $375 \mathrm{WRF}$. Cotton varieties and lines were evaluated in the greenhouse as well. Tests were statistically analyzed by Means/Anova and means compared using Tukey-Kramer HSD $(\alpha$ $=.1)$. Inoculation methods indicated stem injection was most effective ( $\mathrm{P} \leq$ 0.10 ) at reducing plant biomass by $41 \%$ compared to the control. The stem injection was the only inoculation technique that produced visual foliar symptoms and necrosis in the root system indicating the pathogen's movement downward in the plant. Stoneville 4946 GLB2 supported the 
greatest shoot weight $(\mathrm{P} \leq 0.10)$ at 17.5 gm producing a plant that was $57 \%$ larger than PHY 375 WRF; however root development was similar to other varieties. Phytogen $375 \mathrm{WRF}$ displayed the opposite growth pattern with the lowest shoot weight of 7.4 grams and root weight of which was $(P \leq 0.10)$ greater compared to other varieties tested.

Geographical assessment of genetic diversity within Clavibacter michiganensis subsp. nebraskensis using BOX-PCR and AFLP techniques C. B. Langemeier (1), S. A. SCHLUND (2), I. V. Agarkova (2), T. A. Jackson-Ziems (2), G. R. Kruger (3)

(1) University of Nebraska-Lincoln, Department of Plant Pathology; Hoegemeyer Seed, Hooper, NE, U.S.A.; (2) University of Nebraska-Lincoln, Department of Plant Pathology, Lincoln, NE, U.S.A.; (3) University of Nebraska-Lincoln, Department of Agronomy and Horticulture, North Platte, NE, U.S.A.

Phytopathology 104(Suppl. 3):S3.66

Goss's wilt and leaf blight of corn is a bacterial disease, caused by Clavibacter michiganensis subsp. nebraskensis $(C M N)$, that has been increasing in incidence since the early 2000's. The objective of this research was to determine if the rapid expansion of Goss's wilt was due to alterations in the genome of the bacterium. The population structure of $C M N$ strains $(\mathrm{n}=$ 466) isolated in 2010-2011 from eight Midwest states was determined using an amplified fragment length polymorphism (AFLP) analysis and reppolymerase chain reaction (rep-PCR). Analysis of the data sets of AFLP and BOX-PCR fingerprints revealed three groups: two major groups (A and B) and a minor group $(\mathrm{C})$. Group $\mathrm{C}$ was very heterogeneous and was comprised of several subgroups. Rep-PCR had an overall mean similarity cutoff at $92.5 \%$ with $82 \%$ of the tested isolates in group A, $16 \%$ in group B, and $2 \%$ in group $\mathrm{C}$ comprised of seven sub-groups. The mean similarity cutoff for AFLP was $87 \%$ and results showed that group A consisted of $80 \%$ of the isolates, group B had $10 \%$, and $10 \%$ in group C that had 22 sub-groups. The clustering of strains was not correlated to strain geographical origin.

Tracking Raspberry bushy dwarf virus from pollen to systemic infection reveals RNA1 replicates in a resistant cultivar in the absence of RNA2

K. K. LANNING (1), P. P. Moore (1), R. R. Martin (2)

(1) Washington State University, Puyallup, WA, U.S.A.; (2) USDA-ARS Horticulture Crops Research Unit, Corvallis, OR, U.S.A.

Phytopathology 104(Suppl. 3):S3.66

Raspberry bushy dwarf virus (RBDV) is the most prevalent virus of Rubus species worldwide. It's a pollen-borne virus that often causes drupelet abortion resulting in significant quality and yield reductions. Little has been done on the movement of RBDV from infected pollen to the stigma and subsequent systemic movement through the pollinated plant. To address this, a pollination experiment was designed in which 'Willamette' (R) and 'Meeker' (S) plants were placed between RBDV-infected plants in a screenhouse. Bumblebees were introduced at the time of bloom. Receptacle, pedicle, fruiting lateral and floricane tissue was destructively sampled and analyzed for RBDV using real-time RT-PCR with RBDV-RdRp specific primers and probe. RBDV was detected in all tissue types in both 'Willamette' and 'Meeker' plants three weeks post-bloom. Grafting from RBDV-positive 'Willamette' plants confirmed that the virus was graft transmissible. Further analysis using RBDV-CP specific primers in RT-PCR resulted in amplicons of the correct size amplified consistently from 'Meeker' samples, while only two 'Willamette' samples yielded weak bands. Previously reported to be resistant to RBDV based on repeated negative ELISA tests, these results suggests RBDV RNA1 is able to replicate and move systemically in 'Willamette'. Further work is underway to determine if RBDV is present in pollen of 'Willamette', and if present does it transfer horizontally during pollination.

Genotype rotation as strategy for reducing the impact of leaf anthracnose in sorghum

F. E. LANZA (1), L. V. Cota (1), D. D. Silva (1), R. V. Costa (1), C. R. Casela (1), A. G. Souza (1)

(1) EMBRAPA CNPMS, Sete Lagoas, Brazil

Phytopathology 104(Suppl. 3):S3.66

Anthracnose, caused by Colletotrichum sublineolum, is the most important disease of sorghum (Sorghum bicolor) in Brazil. The sorghum anthracnose can cause losses up to $50 \%$ of the production in Brasil. The disease control is basically made by host resistance but due the high variability of the pathogen more knowledge is necessary to use with efficiency the genetic control on this pathossystem. Therefore, the aim of this study was to evaluate the genotype rotation towards a sustainable management strategy for the foliar anthracnose disease in sorghum. Three grain sorghum hybrids, being one susceptible (BR304) and two resistant to anthracnose (1G150 and DAS740) were evaluated. The experiments were conducted following the treatments: icontinuous planting of each hybrid; ii- rotation plating of BR304 with 1G150; iii- rotation plating of BR304 with DAS740; iv- rotation plating of DAS740 with $1 \mathrm{G} 150$. The experiment was carried out during seven growing seasons from 2006 to 2011 . We evaluated the severity of the disease at $69,83,96$, and 110 days after planting. The disease severity in the susceptible genotype BR304 decreased to $42 \%$ and $37 \%$ in rotation with the resistant genotypes 1G150 and DAS740, respectively. Grain yields increase of the BR304 in rotation with $1 \mathrm{G} 150$ and DAS740 were $14 \%$ and $39 \%$, respectively. The rotation of sorghum genotypes can be considered a management strategy for reducing the impact of anthracnose and to keep the host resistance in sorghum.

Characterization of Pythium spp. frequently found in recycled irrigation water

C. E. LANZE (1), G. W. Moorman (2)

(1) The Pennsylvania State University, State College, PA, U.S.A.; (2) The Pennsylvania State University, University Park, PA, U.S.A.

Phytopathology 104(Suppl. 3):S3.66

While many commercial greenhouse growers recycle irrigation water for the financial and environmental benefits of reusing water and fertilizer, they may be faced with plant disease after recycling water-borne pathogens, especially Pythium spp. The objective of this work is to identify and characterize the Pythium species that were isolated from recycled irrigation water tanks of two commercial greenhouses in Pennsylvania, as well as determine the role of their interactions in disease. Most of the species isolated from the tanks are of unknown identities. Several of these species can cause geranium damping-off in lab tests and some of them were determined to be resistant to mefenoxam. To test the interactions of these Pythium spp. on roots of more mature plants, geraniums were co-inoculated with the unknown species and with known pathogens, Pythium aphanidermatum, Pythium irregulare, and Pythium cryproirregulare. In future work, the species that cause root rot or change disease progressions will be characterized genetically and morphologically. The effect of mefenoxam on co-inoculated geraniums will be examined.

Cumulative and residual effects of potato cropping system management strategies on soilborne diseases and soil microbial communities over time R. P. LARKIN (1), J. M. Halloran (1)

(1) USDA ARS, Orono, ME, U.S.A.

Phytopathology 104(Suppl. 3):S3.66

In field trials established in 2004, different 3-yr potato cropping systems focused on specific management goals of soil conservation (SC), soil improvement (SI), and disease-suppression (DS) were evaluated and compared to a 2-yr standard rotation (SQ) and a non-rotation control (PP) for their effects on soilborne diseases, tuber yield, and soil microbial community characteristics under both rainfed and irrigated conditions. Systems were actively managed through 2010 , with potato crops planted in subsequent years (2011-12) to examine residual effects. All rotations reduced disease and increased yield over baseline levels after one rotation cycle, but diseases increased overall after two rotation cycles, then stabilized. The DS system, which utilized Brassica and other disease-suppressive rotation crops, maintained lower soilborne disease levels than all other rotations, as well as high yields, throughout the study. The SI system, characterized by yearly compost amendments, and irrigation, both resulted in higher yields, but also higher levels of black scurf and common scab. Cropping system and irrigation effects were still significant even after systems were no longer maintained. Soil microbial community data, exemplified by FAME profiles, showed significant changes associated with cropping system, and differences among systems increased over time. Overall, cropping system strategy had significant and lasting effects on soil microbiology and soilborne diseases.

\section{Actnidia sp. susceptibility to Phytophthora}

K. Lawrence (1), J. LUANGKHOT (1)

(1) Auburn University, Auburn, AL, U.S.A.

Phytopathology 104(Suppl. 3):S3.66

Recently it was observed that Phytophthora killed several kiwifruit vines on a research plot in central Alabama. The Phytophthora was baited out of the soil using Eucalyptus sp., Camellia japonica, and Camellia sasanqua species of susceptible leaf disks. Leaf disks were sampled at 24, 48, and 72 hours, aseptically plated on to PARPH media and allowed to grow for $5-7$ days in the dark at 25-C. Isolation frequency was statistically analyzed using SAS 9.1. Leaf disks from Eucalyptus sp. were the most efficient at extracting Phytophthora with means of $19.5 \%$ and $17 \%$ at 48 and 72 hour sampling periods, respectively. Camellia japonica was statistically similar recovering Phytophthora at 11 and $19 \%$ at 24 and 48 hours, respectively. Isolation frequency of Phytophthora found was $13.3 \%$ of Eucalyptus, $11.5 \%$ from $C$. japonica, and $6.6 \%$ from the C. sasanqua. Thus Eucalyptus and C. japonica 
are equally effectivebaits. The three sampling time periods observed indicated 48 hours and 72 hours were similar, recovering 13.7 and $10.7 \%$ of Phytophthora, respectively. Eucalyptus and C. japonica are good baits for isolating Phytophthora from samples in 48 to 72 hours sampling periods of the soil mixtures. The Phytophthora isolates will be identified to species and employed in kiwifruit variety trials to determine possible IPM management options and variety selections.

\section{Diversity of Diaporthe species causing wood-canker diseases of fruit and} nuts crops in northern California

D. LAWRENCE (1), R. Travadon (1), K. Baumgartner (2)

(1) University of California-Davis, Davis, CA, U.S.A.; (2) USDA-ARS, Davis, CA, U.S.A.

Phytopathology 104(Suppl. 3):S3.67

Diaporthe species cause many plant diseases worldwide including dieback and the formation of cankers in the permanent woody structure of economically important crops such as apricots, almonds, and grape. This study aimed at revealing potential inoculum sources of Diaporthe species associated with Phomopsis dieback of grape in California, where cultivars of table grape are among the most susceptible. Diaporthe isolates were collected from wood cankers in vineyards, orchards (almond, apricot, pear), and from the wild host willow in riparian areas surrounding these cultivated crops. Morphological characterization based on colony growth rates (ranging from $12-80 \mathrm{~mm}$ after 8 days) and alpha conidia sizes (ranging from 5.6-11.2 $\times 1.9$ $4.4 \mu \mathrm{m})$ identified seven morphological groups. Nine species (D. ambigua, $D$. ampelina, D. australafricana, D. chamaeropis, D. eres, D. foeniculina, D. nobilis, D. novem, and Diaporthe sp. nov.) were revealed based on phylogenetic analysis of three loci (ITS, translation elongation factor 1-alpha, calmodulin). This includes the first report of $D$. australafricana, $D$. chamaeropis, D. nobilis, and D. novem in California vineyards, with $D$. chamaeropis reported on grape for the first time as well as the circumscription of a novel Diaporthe sp. isolated from willow. Pathogenicity testing on grape is currently underway to elucidate the role of all nine identified Diaporthe species in the etiology of Phomopsis dieback.

Fusarium community structure and carbon metabolism phenotypes respond to grassland plant community richness and plant host

N. LEBLANC (1), A. Essarioui (1), L. L. Kinkel (1), H. C. Kistler (2)

(1) University of Minnesota, St. Paul, MN, U.S.A.; (2) USDA ARS Cereal Disease Laboratory/ University of Minnesota, St. Paul, MN, U.S.A. Phytopathology 104(Suppl. 3):S3.67

Fungi in the genus Fusarium are well-studied plant pathogens, but their diversity and abundance in soil suggests they play additional important functional roles in ecosystem processes. This work characterized Fusarium diversity and community structure, as well as individual strain carbon utilization phenotypes in response to grassland plant diversity and species. The legume Lespedeza capitata and the C4 grass Andropogon gerardii were grown in monoculture and in 16-species native grassland plant communities established in 1994 within the University of Minnesota Cedar Creek Ecosystem Science Reserve. Soil sampled from the base of the plants was used to extract DNA and to isolate Fusarium strains using semi-selective media. The RPB2 locus was amplified from the DNA and sequenced. Biolog SFP-2 plates were used to determine growth dynamics of each isolate on 95 different carbon substrates over 5 days. Fusarium community diversity was greater in polyculture vs. monoculture plant communities. Fusarium communities differed between the two plant hosts in monoculture but not in polyculture. Fusarium isolates from $L$. capitata utilized the most substrates, while isolates from $A$. gerardii grew most efficiently. These results show that plant community diversity and plant identity strongly influence Fusarium community structure, diversity, and function (carbon utilization phenotypes) of these common soil borne fungi, showing a strong link between above and belowground dynamics.

Trichoderma promoted growth and development of Jamaica scotch bonnet seedlings in the greenhouse

L. LEE (1), C. Cotton (1), F. Hashem (1), B. Bailey (2)

(1) University of Maryland Eastern Shore, Princess Anne, MD, U.S.A.; (2) Sustainable Perennial Crops Lab, USDA-ARS, Beltsville, MD, U.S.A. Phytopathology 104(Suppl. 3):S3.67

Jamaica Scotch Bonnet peppers could become a potential niche market for small farmers on the Delmarva Peninsula. Trichoderma are plant symbionts that may be used as seed treatment to control disease and enhance seedling vigor. This study was conducted to examine the persistence of Trichoderma on growth and development of hot pepper seedlings. Seeds were sown in sterile promix amended with three treatments, Trichoderma harzianum (T22), Trichoderma virens (GL13), and a control. Each fungal strain was applied to sterlie promix at a rate of $0.1 \%(\mathrm{v} / \mathrm{v})$. Seeds were sown into plug trays and placed into the growth chamber for ten days at $29^{\circ} \mathrm{C}$ and then transferred to the greenhouse. Microbial samples derived from the promix showed an increase in Trichoderma population from $0 \mathrm{CFU} \mathrm{g}^{-1}$ (Day 0) to 6.31 and 6.63 $\log _{10} \mathrm{CFU} \mathrm{g} \mathrm{g}^{-1}$ (Day 21) for T22 and GL 13, respectively. Trichoderma population sampled from the rhizosphere at the end of day 56 post-inoculation were 6.33 and $6.39 \log _{10} \mathrm{CFU} \mathrm{g} \mathrm{g}^{-1}$ for T22 and GL 13, respectively. Days to seedling emergence above the soil line was significantly higher in the treated seeds when compared to untreated ones. Seedling biomass and root colonization were also significantly higher on Trichoderma treated seedlings than the untreated. The positive benefits of Trichoderma colonization on hot pepper seedlings shown in this study indicate that critical seedling characteristics can be improved and thus influence seedling vigor and uniformity.

Interactions between fungi and plants through volatile signaling: The effects of volatile organic compounds (VOCs) emitted by Trichoderma on plants

S. LEE (1), R. Hung (1), J. W. Bennett (1)

(1) Rutgers, The State University of New Jersey, New Brunswick, NJ, U.S.A. Phytopathology 104(Suppl. 3):S3.67

Members of one of the most frequently isolated genera of free-living soil fungi, Trichoderma, are well studied for their ability to reduce plant disease, promote plant growth and productivity. Previously, we demonstrated that $T$. viride emitted VOCs induced growth promotion in Arabidopsis thaliana. The purpose of this study was to examine and evaluate the effects of VOCs from several species of Trichoderma (20 strains total) including: T. aggressivum, $T$. asperellum, T. atroviride, T. brevicompactum, T. harzianum, T. longibrachiatum, T. pseudokoningii, T. virens and T. viride on Arabidopsis and Lycopersicum. Plants and fungi were grown together while physically separated, allowing only the gas exchanges to occur. Plants exposed to VOCs of several species of Trichoderma exhibited growth promotion and developmental changes including larger leaf size, increased shoot weight, increased lateral root branching, and increased total chlorophyll concentration. Interestingly, different strains of $T$. asperellum induced varied responses; certain strains significantly inhibited plant growth. Real-Time qRT-PCR data showed that several auxin-related genes encoding efflux carrier, IAA, and SAUR-like proteins in A. thaliana were affected by the exposure to Trichoderma VOCs. Using GCMS, we have identified over 100 unique compounds emitted by Trichoderma. A study of the differential responses of Arabidopsis to individual fungal VOCs is currently underway.

Integration host factors are required for sigma 54-dependent $h r p L$ gene expression and virulence in Erwinia amylovora

J. H. LEE (1), V. Ancona (2), Y. Zhao (2)

(1) University of Illinois at Urban-Champaign, Urbana, IL, U.S.A.; (2) University of Illinois, Urbana, IL, U.S.A.

Phytopathology 104(Suppl. 3):S3.67

Erwinia amylovora is a necrogenic bacterium, whose virulence is dependent upon a functional hrp-type III secretion system (T3SS). We have recently reported that $h r p L$, encoding the master regulator of T3SS, is under control of sigma $54(\mathrm{RpoN})$, its modulation protein $\mathrm{YhbH}$ and sigma 54-enhancer binding protein HrpS. In this study, we investigated the role of integration host factors (IHFs) in regulating RpoN-dependent $h r p L$ and T3SS gene expression. IHFs are nucleoid-associated proteins and consist of two subunits, i. e. IHF $\alpha$ and IHF $\beta$. IHF $\alpha$ and IHF $\beta$ usually form heterodimers, which could regulate gene expression by influencing nucleoid structure and DNA bending. Two single mutants (ihfA and $i h f B$ ) were generated and characterized in $E$. amylovora. Our results showed that both ihfA and ihfB mutants failed to colonize and produce necrotic lesions on immature pear fruits. Bacterial growth of both mutants in pear fruits was greatly reduced and expression of $h r p L, d s p E, h r p A$ and $h r p N$ was also significantly down-regulated as compared to wild type (WT) strain. In addition, both ihfA and ihfB mutants exhibited slower growth in rich medium as compared to WT strain and were hypermotile on motility plates. Furthermore, both IHFs positively regulated expression of small non-coding regulatory RNA $\operatorname{csr} B$, which also negatively regulates motility. These results indicate that IHFs are required for RpoNdependent $h r p L$ gene expression and virulence in E. amylovora.

Identification and characterization of unique genes encoding nonribosomal peptide synthetases in the cereal fungal pathogen Bipolaris sorokiniana

Y. LENG (1), S. Zhong (1)

(1) North Dakota State University, Fargo, ND, U.S.A.

Phytopathology 104(Suppl. 3):S3.67

Bipolaris sorokiniana is the causal agent of spot blotch, common root rot and kernel blight in barley and wheat. Previous studies showed that deletion of the 
gene for Sfp-type 4'-phosphopantetheinyl transferase (PPT) in B. sorokiniana led to the mutants becoming non-pathogenic on barley plants, suggesting that PPT-activated polyketide synthases (PKSs) or non-ribosomal peptide synthethases (NRPSs) are required for fungal pathogenicity or virulence, presumably by biosynthesis of secondary metabolic toxins. To elucidate the mechanisms underlying pathogenicity and virulence of $B$. sorokiniana, we analyzed genome sequences of four isolates (ND90Pr, ND85F, ND07-47-1 and ND4008) of the fungus, each representing one of the four pathotypes identified so far based on their different virulence pattern on three barley differentials (Bowman, ND 5883 and ND B112). A total of 25, 26, 30 and 28 candidate genes for NRPSs were identified from each of the fungal genomes, respectively. Phylogenetic analyses of the adenylation domains among candidate NRPSs identified two, one, four and two unique candidate genes for NRPSs in ND90Pr, ND4008, ND07-47-1 and ND85F, respectively. The two unique NRPS-coding genes in ND90Pr have been demonstrated to be required for high virulence on barley cv. Bowman. The functions of NRPS-coding genes unique to other fungal isolates are being characterized by the gene knockout approach and the results will be presented.

Time-course of Zebra Chip symptoms in potato tubers and correlation between sprouting and bacterial titer in seed potatoes

J. G. LEVY (1), D. Scheuring (1), J. Koym (2), A. Ravindran (1), D. Henne (3), E. A. Pierson (1), C. J. Miller (1)

(1) Texas A\&M University, College Station, TX, U.S.A.; (2) Texas AgriLife Extension Service, Lubbock, TX, U.S.A.; (3) Texas A\&M University, Weslaco, TX, U.S.A.

Phytopathology 104(Suppl. 3):S3.68

Zebra Chip (ZC) disease is a major problem for potato producers causing reductions in both tuber yield and quality. This study aimed to characterize symptom development in the tubers of two potato cultivars that according to field testing vary in susceptibility to ZC. Experiments were conducted in caged field plots to control insect pressure. At 61 days after planting, all plants were infested with five bacterialiferous insects, e.g. carrying "Candidatus Liberibacter solanacearum" (Lso), the causative agent of ZC. At 75 days after planting ( 2 weeks after infection) single plants of each variety were harvested every other day and tuber yield and chip quality (e.g. presence/absence of discoloration symptomatic of $\mathrm{ZC}$ ) were evaluated. The tubers were stored at room temperature and scored for sprouting after $\sim 20$ weeks. The progression of ZC symptom development based on chip appearance was less rapid and less severe for the cultivar previously identified as being somewhat resistant in field trials. Sprouting occurred in a number of tubers from infested plants that also tested positive for the presence of Lso and were evaluated to determine whether sprouting is related to bacterial haplotype, titer, or both.

Student recruitment in plant pathology: Filling the gaps to connect to prospective students

M. M. LEWANDOWSKI (1), S. D. Williams (1), T. K. Mitchell (1), A. E. Dorrance (2), T. L. Niblack (1), D. N. Alvis (1), R. D. Capouya (1), K. S. McGlone (1), B. K. Mowery (1), B. D. Weaver (1)

(1) Ohio State University, Columbus, OH, U.S.A.; (2) Ohio State University, Wooster, OH, U.S.A.

Phytopathology 104(Suppl. 3):S3.68

Raising awareness and recruiting students to plant pathology are key to our educational mission. To guide communications and student recruitment strategies for the Department of Plant Pathology at The Ohio State University, we evaluated feedback from focus groups, surveys, student discussions and website analytics. Additional input was incorporated from academic and communications units in the university's College of Food, Agricultural, and Environmental Sciences. Common questions from students centered on careers, course requirements and financial matters (funding, scholarships). To address these topics, we focused on developing and retooling content for the department website (plantpath.osu.edu) and the Master in Plant Health Management website (mphm.osu.edu). We are working on ways to provide deeper insight into various careers, because students often express unfamiliarity about the day-to-day activities of plant pathologists. We also surveyed students to learn how they obtain information about courses to help us target publicity for a new course, Mushrooms, Molds and Mankind. In addition, we are exploring how social media (Twitter, Facebook, Pinterest) can connect us with audiences who share overlapping interests in areas such as science education, mushroom foraging, gardening, and arts and crafts.

Relationship between efficacy of biocontrol of aflatoxin in maize and genetic structure of native Aspergillus flavus

M. H. LEWIS (1), I. Carbone (1), G. A. Payne (1), K. L. Bowen (2), A. K. Hagan (2), R. Kemerait (3), R. Heiniger (1), P. Ojiambo (1)
(1) North Carolina State University, Raleigh, NC, U.S.A.; (2) Auburn University, Auburn, AL, U.S.A.; (3) University of Georgia, Tifton, GA, U.S.A.

Phytopathology 104(Suppl. 3):S3.68

Aflatoxin contamination occurs when toxigenic strains of Aspergillus flavus infect maize kernels. Biocontrol using atoxigenic strains of $A$. flavus has the greatest potential to control aflatoxin contamination. However, the impact of the genetic structure of native populations of Aspergillus section Flavi on biocontrol efficacy is not fully understood. Our hypothesis is that use of a biocontrol strain that is genetically similar to the native soil population of $A$. flavus will increase biocontrol effectiveness. Field trials were conducted in Alabama, Georgia, and North Carolina in 2012 and 2013. Biocontrol products AF36 ${ }^{\circledR}$ and AflaGuard ${ }^{\circledR}$ were applied and soil samples were collected prior to and 1 week after biocontrol application and at harvest. A. flavus $\mathrm{L}$-strains and A. parasiticus were recovered from fields in NC and GA while A. parasiticus and A. flavus L- and S-strains were present in AL. About $10 \%, 36 \%$, and $48 \%$ of isolates in GA, AL, and $\mathrm{NC}$, respectively, were of the AflaGuard lineage, while only $4 \%$ of the isolates in all three states belonged to the AF36 lineage. In $\mathrm{NC}$, aflatoxin contamination was lower in AflaGuard treated plot grain than in AF36 treated plot grain. While AF36 was not applied in AL, aflatoxin contamination was lower in AflaGuard treated plots than in untreated controls. These results seem to suggest that matching the biocontrol product to the genetic structure of the native soil populations of A. flavus will increase biocontrol efficacy.

Discovery of a new genotype of Squash mosaic virus through deep sequencing of small RNAs and development of a qRT-PCR for broad spectrum detection

R. LI (1), S. Gao (2), S. Berendsen (3), Z. Fei (4), K. S. Ling (1)

(1) USDA-Agricultural Research Service, U.S. Vegetable Laboratory, Charleston, SC, U.S.A.; (2) Boyce Thompson Institute for Plant Research, Cornell University, Ithaca, NY, U.S.A.; (3) Rijk Zwaan Breeding B.V., De Lier, Netherlands; (4) Boyce Thompson Institute for Plant Research, USDAARS, Robert W. Holley Center for Agriculture and Health, Cornell University, Ithaca, NY, U.S.A.

Phytopathology 104(Suppl. 3):S3.68

Squash mosaic virus (SqMV), a seed-borne virus belonging to the genus Commovirus in the family Comoviridae, could cause a serious yield loss on cucurbit crops worldwide. SqMV has a bipartite single-stranded RNA genome (RNA-1 and RNA-2) encapsidated separately with two capsid proteins. Both RNA molecules contain a genome-linked viral protein $(\mathrm{Vpg})$ and a poly (A) tail. At present, two serotypes (genotypes) of SqMV with only $87 \%$ sequence identity between them are recognized and a qRT-PCR has been developed to detect SqMV isolates belonging to both genotypes. However, a novel SqMV isolate collected on squash in 2010 in Spain was not detectable by this qRTPCR, which prompted us to characterize its genome sequence. The genome sequences of both RNA molecules were obtained using small RNA deep sequencing and assembly technology and validated with Sanger sequencing of RT-PCR products. Both RNA genomes generated approximately $87 \%$ sequence identity to isolates in two known genotypes, suggesting the need to create a third genotype for SqMV. To improve primer and probe design for broad spectrum detection in qRT-PCR to all known SqMV genotypes, a conserved sequence in the 3'-terminal region of RNA-2 was identified through a multiple sequence alignment. This new qRT-PCR was shown to allow sensitive and reliable detection of various SqMV isolates in seed and plant tissues.

Assessing the population genetic structure of Didymella bryoniae to better understand the epidemiology of gummy stem blight of cucurbits

H. X. LI (1), M. Brewer (1)

(1) University of Georgia, Athens, GA, U.S.A.

Phytopathology 104(Suppl. 3):S3.68

Knowledge of the sources of inoculum for epidemics is essential for effective plant disease management. Gummy stem blight, caused by Didymella bryoniae, is the most destructive foliar disease of cucurbits in the southeastern USA. Our objectives were to assess genetic diversity within and among fields, identify the major sources of inoculum, and infer mechanisms of dispersal by determining the geographic, temporal, and fine-scale population structure of D. bryoniae. During epidemics in 2012 and 2013, 448 isolates from watermelon were collected from four locations: Colleton Co., SC; Cook Co. and Tift Co., GA; and Suwannee Co., FL. In 2013, the within-field sampling location of all isolates in the two Georgia fields was recorded. All isolates were genotyped with 16 microsatellite loci. Although clonal genotypes were common and widespread, genotypic diversity was high within each field. Among fields, population differentiation was low, but significant with only a single exception; however, differentiation was not correlated with geographic 
distance. Significant differentiation was also detected at each site between years. Overall, this suggests that non-local sources of inoculum, such as infected seed or transplant seedlings, contribute to gummy stem blight epidemics. Additionally, there was no spatial aggregation of clones or correlation of genetic distance with geographic distance within fields indicating widespread dispersal within fields.

\section{Development of soybean with novel sources of resistance to Phomopsis} seed decay

S. LI (1), P. Chen (2), D. Walker (3), J. Rupe (2)

(1) USDA ARS, Crop Genetics Research Unit, Stoneville, MS, U.S.A.; (2)

University of Arkansas, Fayetteville, AR, U.S.A.; (3) USDA-ARS, Soybean/Maize Germplasm, Pathology and Genetics Research Unit and University of Illinois, Urbana, IL, U.S.A.

Phytopathology 104(Suppl. 3):S3.69

Phomopsis seed decay (PSD) is an important soybean disease that results in poor seed quality in most soybean production areas of the United States. PSD is caused primarily by the fungal pathogen Phomopsis longicolla. In 2009, due to the prevalence of hot and humid environments from pod fill to harvest, PSD caused significant yield losses in 16 southern states. Planting PSDresistant soybean cultivars would be the most economical and environmentally friendly means of protecting soybean crops from PSD, but few resistant cultivars are currently available. In the past eight years, USDA and university scientists have collaborated to screen approximately 1,675 of soybean germplasm accessions, breeding lines, and cultivars for resistance to PSD, and have identified novel sources of resistance. Our current research is focused on development of soybean lines with resistance. In 2013, 17 new cross combinations were made to incorporate resistance genes into high yielding cultivars or breeding lines, and five other crosses were made to develop mapping populations in Arkansas. Crosses were also made in Illinois between elite Midwestern lines and five resistant USDA germplasm accessions to develop breeding lines and potential mapping populations. Analysis of these populations will help us understand the genetics of PSD resistance, identify molecular markers for selection of PSD resistance, and develop high yielding lines with PSD resistance.

Biofilm formation in Xanthomonas citri subsp. citri and potential of biofilm inhibitors to control citrus canker disease

J. LI (1), N. Wang (1)

(1) University of Florida, Lake Alfred, FL, U.S.A.

Phytopathology 104(Suppl. 3):S3.69

Xanthomonas citri subsp. citri (Xcc) causes citrus canker, a destructive disease of citrus worldwide. Xcc forms a biofilm on host plant surfaces; however, its role in the infection process of Xcc is largely unknown. Here, we provided evidence showing that biofilm formation is an important infectionpromoting factor in Xcc. Forming a biofilm is essential for Xcc survival on citrus leaves and successful infection. Mutant strains of Xcc containing individual mutated genes were dramatically reduced in ability to form a biofilm, tolerate environmental stresses, and induce canker symptoms. These results led to the identification of genetic determinants for biofilm formation in Xcc and the discovery of small molecules inhibiting biofilm formation to reduce Xcc infection. A total of 92 genes including 33 novel genes were discovered to be associated with biofilm formation in Xcc. D-leucine and 3indolylacetonitrile (IAN) were found to prevent biofilm formation by Xcc on abiotic surfaces and host leaves. qRT-PCR analysis revealed that IAN repressed expression of chemotaxis/motility related genes in Xcc. Treatment of cells with D-leucine or IAN reduced copper resistance by the pathogen, and combinations of D-leucine and IAN with copper were more effective than copper alone in reducing canker symptoms and pathogen populations on grapefruit leaves. These findings demonstrate the potential of small molecule biofilm inhibitors for managing citrus canker disease.

Identification, detection and quantification of Pythium species caused root rot of calla lily in California

W. LI (1), S. Dangi (1), B. Hanson (2), J. Gerik (1)

(1) Parlier, Parlier, CA, U.S.A.; (2) UC Davis, Davis, CA, U.S.A.

Phytopathology 104(Suppl. 3):S3.69

In Monterey and Santa Cruz countries of California, hybrid calla lilies are highly limited by soilborne pathogens, particularly Pythium species. Fifty samples were collected form heavily infected calla lily roots from at least six field sets respectively in 2010and 2013. Pathogens were isolated from the roots and pathogenicity tests were conducted in the greenhouse. To identify the pathogenic species of Pythium, PCR-RFLP was performed to amplify a portion of the mitochondrial encoded cytochrome oxidase II gene (cox II) and the spacer region between coxI and coxII flowing enzyme digestion. Based on digestion patterns, PCR products were selected for sequencing. Twenty eight of thirty five in 2010 and fourteen of fifteen in 2013 were high hit to the sequence of $P$. sylvaticum. Only one isolate in 2013 showed sequence similar to $P$. ultimum. Corresponding to pathogenicity test, isolates similar to $P$. sylvaticum caused serious diseases and $P$. ultimum caused the mild disease. Morphological characterizations of pathogenic species of Pythium were also observed. Based on the sequences of Pythium species, we designed real time PCR (qPCR) primers which can be specifically used to detect and quantify pathogenic Pythium species in soil. Knowing the pathogen population in soil will help growers make decisions on field selection and soil fumigation before planting calla lily.

Characterization of foliar pathogens infecting perennial rye grass in the northeastern U.S.

F. LICHTNER (1), K. Broders (1)

(1) University of New Hampshire, Durham, NH, U.S.A.

Phytopathology 104(Suppl. 3):S3.69

Perennial rye grass (Lolium perenne) is one of the most common grass species in fertilized grasslands under temperate climates and deserves diligent pest management. Even with the importance of $L$. perenne as a forage crop in the northeast there remains limited knowledge regarding the fungal pathogens associated with this crop as well as their distribution in the northeast. The main objective of this project was to characterize the fungal pathogens associated with $L$. perenne in the northeastern U. S. Isolates were recovered from symptomatic lesions on $L$. perenne across a range of locations from Pennsylvania to Maine over two growing seasons. In total, 65 isolates were recovered from 4 locations across the northeast. Isolates were identified based on morphological features as well as by sequencing the ITS region and comparing sequence data to known species in GenBank. To date ten species of fungi have been identified, including species of Colletotrichum, Drechsler, Fusarium, and Puccinia. Koch's postulates are currently being conducted to determine pathogenicity of all isolates recovered. This work represents one of the first assessments of the pathogen diversity associated with foliar disease symptoms of perennial ryegrass in the northeastern U. S. Future research will focus on determining the distribution and frequency of each of these species as well as evaluating host resistance in $L$. perenne in order to develop more efficient disease management strategies.

Double-stranded RNA-binding protein 4 is required for resistance signaling against viral and bacterial pathogens

G. H. LIM (1), S. Zhu (1), K. Yu (1), A. Kachroo (1), P. Kachroo (1)

(1) Department of Plant Pathology, University of Kentucky, Lexington, KY, U.S.A.

Phytopathology 104(Suppl. 3):S3.69

Species-specific immunity is induced when an effector protein from a specific pathogen strain is perceived by a cognate resistance $(\mathrm{R})$ protein in the plant. In Arabidopsis, the R protein HRT, which confers resistance to turnip crinkle virus (TCV) $(1,2)$, is activated upon recognition of the TCV coat-protein (CP), a potent suppressor of host RNA silencing. Recognition by HRT does not require RNA silencing suppressor function of $\mathrm{CP}$ and is not associated with the accumulation of TCV-specific small-RNA. However, several components of the host RNA silencing pathway participate in HRT-mediated defense against TCV. For example, the double stranded RNA binding protein (DRB) 4 interacts with the plasma membrane localized HRT, and is required for its stability (3). Intriguingly, TCV infection promotes the cytosolic accumulation of the otherwise primarily nuclear DRB4, and this in turn inhibits HRT-DRB4 interaction. These data together with differential localization of DRB4 in plants inoculated with avirulent and virulent viruses, suggests that sub-cellular compartmentalization of DRB4 plays an important role in activation of HRT. (1) PNAS, 2010; 107:13538-13543 (2) PLoS Pathog, 2011, 7: e1002318 (3) Cell Reports, 2013; 4:1-17.

Plant ferredoxin-like protein enhances disease resistance against bacterial soft rot through intensifying PAMP-triggered immunity

Y. H. LIN (1), Y. H. Su (2), C. Y. Hong (2)

(1) National Pingtung University of Science and Technology, Pingtung, Taiwan; (2) Department of Plant Medicine, National Pingtung University of Science and Technology, Pingtung, Taiwan

Phytopathology 104(Suppl. 3):S3.69

Application of a transgene such as plant ferredoxin-like protein (PFLP) is a possible strategy to protect plants against bacterial diseases. Our previous reports demonstrate that transgenic plants express extracellular PFLP (ESF plants) are more resistant against bacterial pathogens. This protein intensifies hypersensitive response (HR) in plants while a pathogen associated molecular pattern (PAMP), harpin, was infiltrated. Thus, we attempted to determine whether PFLP participates in intensifying PAMP-triggered immunity to enhance disease resistance. Firstly, we showed that ESF plants were resistant 
against bacterial soft rot caused by Pectobacterium carotovorum subsp. carotovorum. Then, the $f l i C$ which encoded flagellin from Pcc was cloned and expressed. In Col-0 plants, the ROS generation and HR ratio were induced by the treatment with both PFLP and FliC, which did not occur as a result of treatment with PFLP or FliC alone. In ESF plants, FliC also elicited more callose deposition. Moreover, we demonstrated that the PFLP-intensified ROS generation and $\mathrm{HR}$ were related to $\mathrm{Ca}^{2+}$ influx and activation of NADPH oxidase. We concluded that the PFLP-intensified disease resistance is associated with the intensification of PAMP-triggered immunity.

\section{The phenotypic and genetic diversities of the ordinary strain of Potato virus $\mathrm{Y}\left(\mathrm{PVY}^{\mathrm{O}}\right)$}

Y. H. LIN (1), K. Green (2), A. V. Karasev (2), S. M. Gray (3)

(1) Cornell University, Ithaca, NY, U.S.A.; (2) University of Idaho, Moscow, ID, U.S.A.; (3) USDA ARS and Cornell University, Ithaca, NY, U.S.A.

Phytopathology 104(Suppl. 3):S3.70

Potato virus $\mathrm{Y}(\mathrm{PVY})$ is classified into several strain groups based on genome organization and various phenotypic characteristics. Isolates of the ordinary strain of PVY, PVY ${ }^{\circ}$, all have highly conserved genome sequences and genome organization, but can be divided into variant groups based on serology and symptom type and severity in potato. Phenotypic variation was determined in 37 fully sequenced isolates of $\mathrm{PVY}^{\mathrm{O}}$ and $\mathrm{PVY}^{\mathrm{O}}-\mathrm{O} 5$, a serological variant, in an attempt to identify sequences correlated with phenotype. Eight of $21 \mathrm{PVY}^{\mathrm{O}}$ and 10 of $16 \mathrm{PVY}^{\mathrm{O}}-\mathrm{O} 5$ isolates induced severe foliar necrosis, leaf drop and plant death in Yukon Gold. The remaining 19 isolates induced mosaic and mild necrotic foliar symptoms. Twelve of 21 $\mathrm{PVY}^{\mathrm{O}}$ and 15 of $16 \mathrm{PVY}^{\mathrm{O}}-\mathrm{O} 5$ isolates induced mild to severe tuber necrosis on Yukon Gold. None of the phenotypic characteristics correlate with phylogenetic groupings of the virus isolates generated using nucleotide or deduced amino acid sequences of the entire genome or individual cistrons. Analysis of 38 nonsynonomous amino acid substitutions across the genomes of the 37 isolates found that no single substitution or linear arrangement of changes could be associated with symptom type or severity. While foliar veinal necrosis in tobacco is a phenotype that has been attributed to 2-3 specific amino acid positions in the HC-pro cistron, it appears that simple linear genome sequence changes are not responsible for foliar or tuber necrosis in potato.

Transmission efficiency of different Potato virus $Y$ strains affecting potato from single and mixed infections

Y. H. LIN (1), J. T. Ingram (2), S. M. Gray (3)

(1) Cornell University, Ithaca, NY, U.S.A.; (2) USDA, ARS, Ithaca, NY, U.S.A.; (3) USDA, ARS and Cornell University, Ithaca, NY, U.S.A.

Phytopathology 104(Suppl. 3):S3.70

Potato virus $Y$ (PVY) is transmitted by numerous species of colonizing and transient aphid species; the Green peach aphid (GPA) is, by far, the most efficient vector. Differences in transmission efficiency may be one factor driving the change in PVY strain predominance from the ordinary strain, $\mathrm{PVY}^{\mathrm{O}}$, to the recombinant strains, $\mathrm{PVY}^{\mathrm{NO}}$ and $\mathrm{PVY}^{\mathrm{NTN}}$. GPA acquired virus from potato plants (cv Goldrush and NY129) infected with single (PVY $\left.\mathrm{PVY}^{\mathrm{NO}}, \mathrm{PVY}^{\mathrm{NTN}}\right)$, double $\left(\mathrm{PVY}^{\mathrm{O}}+\mathrm{PVY}^{\mathrm{NO}}, \mathrm{PVY}^{\mathrm{O}}+\mathrm{PVY}^{\mathrm{NTN}}, \mathrm{PVY}^{\mathrm{NO}}+\mathrm{PVY}^{\mathrm{NTN}}\right)$, or triple $\left(\mathrm{PVY}^{\mathrm{O}}+\mathrm{PVY}^{\mathrm{NO}}+\mathrm{PVY}^{\mathrm{NTN}}\right)$ strain mixtures. Single aphids were transferred to potato seedlings to determine transmission efficiency. Multiplex RT-PCR, able to differentiate PVY ${ }^{\mathrm{O}}, \mathrm{PVY}^{\mathrm{NO}}$, and PVY ${ }^{\mathrm{NTN}}$, confirmed the virus strains in source tissues and those transmitted to the recipient plants. $\mathrm{PVY}^{\mathrm{NTN}}$ was transmitted more efficiently $(30 \%)$ than $\mathrm{PVY}^{\mathrm{O}}$ or $\mathrm{PVY}^{\mathrm{NO}}(19 \%$ and $16 \%$, respectively) when acquired from single strain infected Goldrush plants. Transmission from NY129 was similar for PVY ${ }^{\mathrm{NTN}}$ and $\mathrm{PVY}^{\mathrm{O}}$. If $\mathrm{PVY}^{\mathrm{O}}$ was mixed with $\mathrm{PVY} \mathrm{NTN}^{\mathrm{NT}}$ and/or $\mathrm{PVY}^{\mathrm{NO}}$ in the source tissues, the transmission efficiency of $\mathrm{PVY}^{\mathrm{NTN}}$ or $\mathrm{PVY}^{\mathrm{NO}}$ was significantly reduced. Mixing PVY ${ }^{\mathrm{NTN}}$ and $\mathrm{PVY} \mathrm{NO}^{\mathrm{NO}}$ in the source tissue decreased the transmission efficiency of $\mathrm{PVY}^{\mathrm{NO}}$. Differences in aphid transmission efficiency may partially explain a rise in $\mathrm{PVY}^{\mathrm{NTN}}$, but not $\mathrm{PVY}^{\mathrm{NO}}$ incidence. Results from mixed infections indicate synergism or antagonism among strains likely affect virus titer and distribution.

Using wild relatives of potato to illustrate genetic control against the pathogenic bacteria Pectobacterium carotovorum

J. LIND (1)

(1) University of Wisconsin-Madison, Madison, WI, U.S.A.

Phytopathology 104(Suppl. 3):S3.70

Bacterial soft rot caused by Pectobacterium is one of the most important bacterial diseases of potato and other stored vegetables. Although soft rot resistance is present in wild species, it has not been effectively introduced into cultivated potato due to difficulty of incorporating traits into potato, which is an out-crossing tetraploid. Since potato is grown by vegetative propagation, seed production is also complicated and inefficient compared to other types of vegetables. As a result, little progress has been made in many aspects of potato breeding over the past century. One objective of this research is to characterize soft rot resistance in wild potato species and to generate resistant and susceptible inbred diploid potato lines to aid in characterization of these resistance genes. A second objective is to determine if Solanum microdontum, $S$. violaceimarmoratum, and $S$. chacoense accessions that are resistant to Pectobacterium carotovorum subsp. carotovorum are also resistant to other Pectobacterium species such as $P$. carotovorum subsp. Brasilense, $P$. atrosepticum and $P$. wasabiae. The resistance of the inbred lines and a comparison of diploid and inbred diploid potato lines with cultivated tetraploid potato lines will be assessed with plants grown under greenhouse and field conditions. The long-term outcomes of this project are the availability of soft rot resistant potato lines and a contribution to simplifying and sustaining potato breeding and production.

\section{At what age sugar beet is resistant to Rhizoctonia solani}

\section{Y. LIU (1), M. F. Khan (2)}

(1) North Dakota State University, Fargo, ND, U.S.A.; (2) North Dakota State University and University of Minnesota, Fargo, ND, U.S.A.

Phytopathology 104(Suppl. 3):S3.70

Rhizoctonia crown and root rot of sugar beet (Beta vulgaris L.) caused by Rhizoctonia solani, has become the most important problem for growers in Minnesota and North Dakota. The use of sugar beet cultivars resistant to $R$. solani is an important strategy for disease management, especially when combined with timely fungicide application during the growing season. The objective of this greenhouse study was to determine at what age sugar beet expresses resistance to $R$. solani. Three sugar beet cultivars that were susceptible, moderately resistant, and resistant to $R$. solani were inoculated with $R$. solani-infested barley grain at 0 to 10 weeks after planting. Twentyeight days after inoculation, plants were pulled, washed and roots evaluated for disease severity. All cultivars were susceptible to $R$. solani when inoculated at planting to three weeks after planting. In the susceptible cultivar, disease severity became significantly lower in seven week old plants, whereas disease severity became significantly lower in four week old plants of the moderately resistant and resistant cultivar. This study indicated that sugar beet plants, irrespective of their level of resistance to $R$. solani, are vulnerable to infection up to three weeks after planting. Additional protection in the form of seed treatment or fungicide application may be required to protect vulnerable sugar beet when planted in fields with a history of $R$. solani under favorable environmental conditions.

Occurrence of bacterial spot (Xanthomonas cucurbitae) in pumpkin and squash fields in the North Central Region

Q. LIU (1), M. Babadoost (1)

(1) University of Illinois, Urbana, IL, U.S.A.

Phytopathology 104(Suppl. 3):S3.70

Bacterial spot, caused by Xanthomonas cucurbitae, has become one of the most important diseases of pumpkin and squash in the North Central Region. During 2012 and 2013, field surveys were conducted in Illinois, Iowa, Indiana, Kansas, Michigan, Missouri, Nebraska, Ohio and Wisconsin to assess incidence and severity of the bacterial spot in pumpkin and winter squash fields. During four weeks of the harvest, 132 and 126 fields in 2012 and 2013, respectively, were visited. In each field, 60 fruit in 12 locations (5 fruit per location) were examined in an M-shaped walking path. Bacterial spot was observed in jack-o-lantern pumpkin, processing pumpkin, and squash fields. X. cucurbitae-infected fruit were observed in $117(89 \%)$ and $113(90 \%)$ of the fields visited, with overall 23 and 19\% fruit infected, in 2012 and 2013, respectively. Severity of fruit infection (percent surface area of the fruit affected) ranged from 1 to $20 \%$, with an average of $1.34 \%$ in 2012 and $1.10 \%$ in 2013. Symptomatic fruit were collected from all of the fields with bacterial spot and examined for the presence of $X$. cucurbitae. X. cucurbitate was identified based on yellow, mucoid colonies on YDC medium, PCR amplification using primers RST2/3 and RST9/10, and pathogenicity test on pumpkin plants in a greenhouse. In addition to X. cucurbitate, Pseudomonas and Bacillus spp. were isolated from symptomatic fruit. Interactions between $X$, cucurbitae and other isolated bacterial are being investigated.

Characterization of resistance genes to rice blast fungus Magnaporthe oryzae in a "green revolution" rice variety

Y. LIU (1), Y. Jia (1), X. Qi (2), K. M. Olsen (2), A. Caicedo (3)

(1) USDA-Agricultural Research Service Dale Bumpers National Rice Research Center, Stuttgart, AR, U.S.A.; (2) Washington University in St. Louis, Biology Department, St. Louis, MO, U.S.A.; (3) University of Massachusetts Amherst, Biology Department, Amherst, MA, U.S.A.

Phytopathology 104(Suppl. 3):S3.70 
The indica variety Dee Geo Woo Gen (DGWG) provides the semi-dwarf gene $(S D 1)$ playing an important role in the rice green revolution. In the present study, resistance $(R)$ genes to the US race (isolate) IB54 (isolate unnamed) of Magnaporthe oryzae, the causal agent of rice blast disease, was investigated in DGWG. Two partial resistant quantitative trait loci (QTLs), $q B R 1.1$ and $q B R 6.1$, and one major resistant QTL, $q B R 11.1$, were identified using a mapping population using DGWG. Genetic locations of both $q B R 6.1$ and qBR11.1 were confirmed with another mapping population involving DGWG. The total phenotypic variation of three resistance QTLs was $51.09 \%$, with each ranging from $1.12 \%$ to $47.62 \%$. Each of the three QTLs was located in a small genomic region. The major QTL, $q B R 11.1$, was mapped at a $129 \mathrm{~kb}$ region on the chromosome 11 nearby nine known blast $R$ genes. Within this $129 \mathrm{~kb}$ of $q B R 11.1$, three putative nucleotide-binding site (NBS) and leucine rich repeats (LRR) disease resistance proteins and three WRKY transcription factors WRKY61, WRKY63 and WRKY41 were identified, and considered as the candidate genes of $q B R 11.1$, tentatively designated as Pi66(t). Identification of blast $R$ genes in DGWG should help continued deployment of useful $R$ genes for improving crop productivity and resistance to rice blast disease.

Insights into molecular mechanism of blast resistance in weedy rice Y. LIU (1), Y. Jia (1), X. Qi (2), K. M. Olsen (2), A. Caicedo (3), D. R. Gealy (1) (1) USDA ARS Dale Bumpers National Rice Research Center, Stuttgart, AR, U.S.A.; (2) Washington University in St. Louis, Biology department, St. Louis, MO, U.S.A.; (3) University of Massachusetts Amherst, Biology Department, Amherst, MA, U.S.A.

Phytopathology 104(Suppl. 3):S3.71

Weedy rice is a serious pest in direct-seeded rice fields in the U.S. and worldwide. Under situable conditions weedy rice can reduce crop yield up to $70 \%$. However, weedy rice may carry novel blast resistance genes. In the present study, blast resistance genes were investigated with two recombinant inbred line (RIL) populations consisting of 175 and 224 individuals (S and B), respectively, derived from the crosses of two U.S. weedy rice ecotypes, the straw hull type PI653435 (1135-01) and the black hull type PI653419 (19969), with a closely related indica rice variety Dee Geo Woo Gen (DGWG) from China. Genotypes of all individuals in the $\mathrm{F}_{5}$ generation were determined using the Genotype by Sequencing method. Two high resolution genetic maps with 6179 SNPs in the S population and 14382 SNPs in the B population were constructed. Disease reactions of three parental lines were evaluated with 14 U.S. races/isolates in a greenhouse. The two weedy rice ecotypes showed partial resistance to several U.S. isolates. Disease reactions of all individuals of both populations to the selected isolates, IC17 (ZN57), IE1(ZN13), IE1K (TM2), ID1 (ZN42) and IB33 (FL9, a laboratory strain) were determined in a greenhouse. Resistance genes to blast were mapped using R/qtl software. Candidate resistance genes were identified from genomic regions delimited by SNPs markers. Insights into the molecular mechanism of blast resistance in weedy rice will be presented.

\section{Antagonism of black rot Xanthomonas campestris pv. campestris in cabbage by mixtures of plant growth-promoting rhizobacteria (PGPR) strains}

K. LIU (1), C. Garrett (1), J. Kloepper (1)

(1) Auburn University, Auburn, AL, U.S.A.

Phytopathology 104(Suppl. 3):S3.71

Black rot, caused by Xanthomonas campestris p $v$. campestris, is the most important and potentially destructive disease in cabbage. The objective of this study was to screen individual PGPR strains and then form mixtures of PGPR strains for their capacity to control black rot via antibiosis. Tests were conducted in vitro, in the greenhouse, and in the field. Twenty-four PGPR strains of Bacillus spp. strains were tested in vitro, and eight strains (AP136, 201, 213, 218, 219, 295 and 305) were chosen greenhouse tests with single strains. Seven individual PGPR strains caused significantly antibiosis ability, and the best four strains (AP218, 219, 295 and 305) were evaluated together as a mixture (mixiture-1), and together with strain AP213 (mixture-2). The two mixtures and four individual PGPR strains were then tested three times in the greenhouse and one time in the field. All the treatments resulted in significant disease suppression, with mixture-2 and AP218 causing the most consistent disease reduction in two of three tests. In the field, treatments of AP219, 305, mixture-1 and mixture-2 resulted in significantly lower disease incidence and disease severity and showed higher yield, compared to the disease control. In conclusion, mixtures of selected PGPR demonstrated enhanced consistency of biocontrol of cabbage black rot.

Selecting individual plant growth-promoting rhizobacteria for biological control of multiple plant diseases in growth chambers

K. LIU (1), J. W. Kloepper (1), C. H. Hu (1), J. A. McInroy (1)

(1) Auburn University, Auburn, AL, U.S.A.

Phytopathology 104(Suppl. 3):S3.71
A study was designed to screen individual PGPR strains for providing broadspectrum disease suppression. Fifteen plant growth-promoting rhizobacteria (PGPR) strains with antibiosis ability to nine different pathogens in vitro were tested for biological control of multiple plant diseases in the growth chamber. The specific diseases and hosts test in this study included black spot of tomato caused by Xanthomonas campestris pv. vesicatoria (XCV), black speck of tomato caused by Pseudomonas syringae pv. tomato (PST), damping off of pepper caused by Rhizoctonia solani, and damping off of cucumber caused by Pythium ultimum. No individual PGPR strains significantly decreased all four diseases. All the tested individual PGPR strains except AP136 significantly reduced the severity of the two foliar bacterial diseases (XCV and PST) compared to the non-bacterized control. Only treatment AP199 significantly reduced the disease incidence of $P$. ultimum. There was no significant disease reduction of two fungal damping off diseases ( $R$. solani and P. ultimum). In summary, of the fifteen tested strains, the incidence or severity of three of the tested pathogens was reduced by one strain, and two of the tested pathogens by fourteen strains.

Understanding the basis of subspecies differences among Erwinia tracheiphila strains

Q. LIU (1), M. L. Gleason (1), G. A. Beattie (1)

(1) Iowa State University, Ames, IA, U.S.A

Phytopathology 104(Suppl. 3):S3.71

Cucurbit bacterial wilt, caused by Erwinia tracheiphila, is a devastating disease of cucurbit crops in United States. Previous work showed significant variation among 69 strains isolated from Cucumis and Cucurbita hosts based on fingerprinting profiles and host preference, with symptoms occurring faster on seedlings of the host genus of origin. The objective of this study was to identify genes influencing subspecies differences among $E$. tracheiphila strains. Phenotypic assays indicated the strains from Cucumis generally grew faster in media than those from Cucurbita, whereas strains of these two subspecies did not differ in motility. We identified two candidate target genes influencing subspecies differences in E. tracehiphila, orfB and $d s p E$, which encode, respectively, an ortholog of Eop1, a host range-limiting factor in $E$. amylovora, and DspE/WtsE, a critical pathogenicity factor in E. amylovora and Pantoea stewartii. An alignment of pathogenicity islands from draft genome sequences of strains representing the subspecies of E. tracheiphila (2 Cucumis and 2 Cucurbita strains) identified differences that correlated with the subspecies, including 5 and 14 polymorphisms within orfB and DspE proteins, respectively, with 7 polymorphisms in DspE correlated with reported functional domains. We are evaluating the strength of this correlation with more strains of each E. tracheiphila subspecies.

Identification of tan spot resistance loci in cultivated emmer wheat by association mapping

Z. LIU (1), Q. Sun (2), S. S. Xu (3), S. Chao (3), J. D. Faris (3), M. Mergoum (2) (1) North Dakota State University, Fargo, ND, U.S.A.; (2) Department of Plant Science, North Dakota State University, Fargo, ND, U.S.A.; (3) Cereal Crops Research Unit, Northern Crop Science Laboratory, USDA-ARS, Fargo, ND, U.S.A.

Phytopathology 104(Suppl. 3):S3.71

Tan spot, caused by Pyrenophora tritici-repentis (Ptr), is a major disease of both common and durum wheat around the world. Tan spot resistant sources of adapted wheat germplasm are limited. Cultivated emmer wheat (Triticum turgidum L. ssp. dicoccum), one of the earliest forms of cultivated wheat, contains many useful genes for resistance to fungal diseases. In this work, we evaluated a set of geographically diverse cultivated emmer wheat accessions for the reaction to four different $P t r$ races to identify sources of resistance and tan spot resistance QTL using association analysis. Among 180 lines evaluated, 19, 28, 11 and 24 were resistant to races 1 (isolate Pti2), 2 (isolate 86-124), 3 (isolate 331-9), and 5 (isolate DW5), respectively. Four lines, were highly resistant to all four races. Using association mapping with a total of 3230 SNPs (MAF $>=0.05)$ and a linear mixed-effect model, we identified a total of 168, 26, 158, 72 SNP markers that were significantly associated with resistance to races $1,2,3$, and 5 , respectively, with an FDR-adjusted $p$ value $<$ 0.05. Putative QTLs were identified on chromosomes $1 \mathrm{~B}, 2 \mathrm{~A}, 6 \mathrm{~B}$ and $7 \mathrm{~B}$ for Pti2; 5B and 7A for 86-124; 5A, 5B and 7B for 331-9; and 1B, 2A, 4A, and 7B for DW5. Some of these loci correspond to previously known tan spot resistance QTL, while others are novel. This work provides important tools for genetic analysis and breeding for resistance to tan spot of wheat.

Differential effects of compost type on strawberry plant health and productivity

M. LLOYD (1), T. R. Gordon (1)

(1) University of California, Davis, CA, U.S.A.

Phytopathology 104(Suppl. 3):S3.71 
Management of soilborne pathogens affecting strawberry production has long relied on pre-plant fumigation to maintain pathogen inoculum below damaging levels, but tightening restrictions on fumigants demands alternative management approaches. Compost is commonly used by strawberry growers in California, but little is known about how composts derived from different source materials differ in their effects on plant health and productivity. To redress this deficiency, four commercially available composts were evaluated: steer manure-based, spent mushroom compost, yard trimmings and vermicompost. Each type of compost was characterized by nutrient composition, microbial activity as measured by fluorescein diacetate hydrolysis, and microbial community profile. In addition, five field trials have been established to assess effects of the four composts on plant growth and yield throughout the season. Early season measurements show significant effects of compost type on plant canopy diameters. In controlled studies, some compost treatments had significant effects on root growth and frequency of infection by Verticillium dahliae, the cause of Verticillium wilt. These results indicate that composts may have beneficial effects on strawberry production and that these benefits can be dependent on the type of compost that is used.

Climate favorability and yield losses caused by angular leaf spot of common bean in different Brazilian regions

M. LOBO (1), F. Yoshida (2), J. T. Aguiar (2), D. S. d'Afonseca (3)

(1) Embrapa Rice and Beans, Santo Antonio De Goias, Brazil; (2) Graduate Program on Agronomy, Universidade Federal de Goiás, Goiania, Brazil; (3) Informatics Institute, Universidade Federal de Goiás, Goiânia, Brazil Phytopathology 104(Suppl. 3):S3.72

The objective of this study was to estimate the impact of the rainfall and El Niño-Southern Oscillation (ENSO) phenomenon on angular leaf spot (Pseudocercospora griseola) severity in common bean (Phaseolus vulgaris), in different Brazilian planting areas, from 2001 to 2010. Disease severity and yield data were collected from different field trials carried out in the rainy planting season (Nov-Feb) or in the "dry season" (Feb-May). Field experiments were always carried out under a randomized block design with three replications and plots of $8 \mathrm{~m}^{2}$. Field data was compared to climatic variables estimated by the Brazilian National Institute of Meteorology (INMET) and NASA Prediction of Worldwide Energy Resource (POWER) and statistically analyzed with ANOVA and principal component analysis. There were differences for cultivars, planting season and cropping year. Disease severity and common bean yield varied annually among cities, indicating that the geographical local was conducive or limiting for disease development. The principal component analysis, in turn, revealed that rainfall and ENSO influences statistically disease severity and crop yield according to the crop area localization in the different Brazilian states. These results provide new information on the impacts of the disease at a regional scale, supports the development of a predictive model of angular leaf spot in common beans, and estimates the effects of climate change on common bean development.

Development of Pythium root rot on three cultivars of poinsettia inoculated at different ages

E. LOOKABAUGH (1), B. Shew (1)

(1) North Carolina State University, Raleigh, NC, U.S.A.

Phytopathology 104(Suppl. 3):S3.72

Pythium aphanidermatum is the predominant species associated with Pythium root rot of poinsettia (Euphorbia pulcherrima) in North Carolina. In this study, 3 cultivars of poinsettia (Prestige Red, Freedom Red, and Advent Red) were inoculated using rice grains colonized with $P$. aphanidermatum at 3 stages in production $(1.5,3.5$, or 6 weeks after transplanting). Plants were grown in a greenhouse with no light interference (trial 1) or a greenhouse with light interference (trial 2) for an additional 12 weeks after inoculation. Light conditions in trial 1 resulted in bract color change for all cultivars whereas plants in trial 2 did not change colors. Root rot ratings (RRR) and plant health ratings (PHR) were recorded on a 1 to 5 scale. Inoculated plants in trial 1 had more severe RRR (mean, 2.9) than plants in trial 2 (2.1). Advent Red had the highest RRR (3.3) and plants inoculated 1.5 weeks after transplanting had higher RRR (3.4; $\mathrm{P}=0.05)$ than plants inoculated 3.5 or 6 weeks after transplanting. PHR did not differ among groups. RRR and PHR did not differ among cultivars or age groups in trial $2(\mathrm{P}>0.05)$. The results suggest that early infections by $P$. aphanidermatum are more damaging to poinsettia than infections that occur later. They also suggest that the stress of color change could play a role in symptom expression. It is possible that asymptomatic winter stock plants harbor $P$. aphanidermatum since they do not undergo color change.

Macrophomina phaseolina and Heterodera glycines reducing soybean performance through early infection

H. D. LOPEZ-NICORA (1), B. W. Diers (2), A. E. Dorrance (3), T. L. Niblack (1)
(1) Ohio State University, Columbus, OH, U.S.A.; (2) University of Illinois, Urbana, IL, U.S.A.; (3) Ohio State University, Wooster, OH, U.S.A. Phytopathology 104(Suppl. 3):S3.72

Macrophomina phaseolina is affecting soybean production in areas where charcoal rot has not previously been a problem. Heterodera glycines is the most economically important soybean pathogen. Ample studies have been conducted to understand M. phaseolina and H. glycines in soybean, but almost nothing is known of soybean performance when these soil-borne pathogens infest soybean fields concomitantly. The objective of this study was to evaluate soybean production under high population densities of both pathogens. Soybean back-cross lines $\left(\mathrm{BC}_{4} \mathrm{~F}_{2}\right.$ derived), half with resistance to H. glycines and the other half susceptible, were planted in a field infested with $H$. glycines. In a split-plot experimental design, the same soybean genotypes were planted in adjacent plots, one infested with M. phaseolina and the other without. Fifteen to twenty soil cores were collected at each plot at planting and harvest for initial $(\mathrm{Pi})$ and final $(\mathrm{Pf}) H$. glycines egg counts and $M$. phaseolina colony-forming units. Stand counts, nematode penetration, and soybean yield were also collected. Soybean yield was reduced as $H$. glycines $\mathrm{Pi}$ increased. Yield was not affected by $M$. phaseolina; however, plant emergence was reduced by the fungus in infested plots. In this study, there was no evidence of interaction between the two pathogens. The use of soybean cultivars with similar genetic background will help unravel the complicated relationship between multiple pathogens affecting a crop.

Ralstonia solanacearum degrades the key plant immune signal, salicylic acid, during tomato infection

T. M. LOWE (1), J. M. Jacobs (2), F. Ailloud (3), C. Allen (1)

(1) University of Wisconsin, Madison, WI, U.S.A.; (2) IRD, Montpellier, France; (3) CIRAD, Reunion Island, France

Phytopathology 104(Suppl. 3):S3.72

Salicylic acid (SA) regulates plant defenses and primes plants to resist the bacterial wilt pathogen Ralstonia solanacearum. During infection of tomato plants, $R$. solanacearum expresses nag genes that encode an SA degradation pathway. Further, in response to SA, $R$. solanacearum decreased expression of several known virulence factors. To test the hypothesis that SA degradation contributes to pathogenic success of $R$. solanacearum, we created a nagGH deletion mutant strain, which lacks the first enzyme in the SA degradation pathway. Preliminary results indicate that the $\triangle n a g G H$ mutant had reduced virulence on tomato plants. Several non-exclusive mechanisms could explain the in planta role of SA degradation: 1) carbon acquisition in plant xylem vessels; 2) protection from the antimicrobial properties of SA; 3) suppression of SA-dependent plant immune responses; and 4) regulation of the bacterium's SA-responsive virulence factors. In vitro growth assays demonstrated that SA degradation allows $R$. solanacearum to use SA as a sole carbon source, and an in vitro MIC inhibition assay demonstrated that SA degradation protects the pathogen from SA toxicity. Thus, SA degradation may contribute to $R$. solanacearum virulence through multiple mechanisms. Bioinformatic analyses of the $R$. solanacearum species complex shows that 21 of 24 strains have SA degradation capacity; this broad conservation suggests that it plays a key role in pathogenic success.

\section{Complete genome sequence of Clavibacter michiganensis subsp. insidiosus} R1-1

Y. LU (1), D. A. Samac (2), C. A. Ishimaru (1), J. Glazebrook (1)

(1) University of Minnesota, St. Paul, MN, U.S.A.; (2) USDA ARS, St. Paul, MN, U.S.A.

Phytopathology 104(Suppl. 3):S3.72

Clavibacter michiganensis subsp. insidiosus ( $\mathrm{Cmi}$ ) causes bacterial wilt disease of alfalfa (Medicago sativa L.) and can also infect the model legume plant $M$. truncatula. The virulence mechanisms of $\mathrm{Cmi}$ are yet to be identified, hampered by the lack of efficient mutagenesis tools as well as by the lack of a reference genome sequence. Here we describe use of Illumina sequencing to obtain the complete genome sequence of one $\mathrm{Cmi}$ field isolate from Minnesota, Cmi R1-1. Cmi R1-1 has a genome average $\mathrm{G}+\mathrm{C}$ content of $73 \%$, which is similar to other sequenced subspecies of C. michiganensis. The genome of Cmi R1-1 is comprised of one presumably circular chromosome of $3,206 \mathrm{kbp}$ and two circular plasmids, pCI1 and pCI2, of $48 \mathrm{kbp}$ and $153 \mathrm{kbp}$, respectively. pCI2 appears to be required for virulence, as sequencing of an avirulent strain, Cmi ATCC10253, revealed the loss of this plasmid. Cmi R1-1 has 23 copies of the repetitive insertion element IS1122 in the chromosome and two copies on each of the plasmids. Whole genome rearrangement events compared to C. michiganensis subsp. michiganensis were detected at four of the IS1122 loci. A total of 3,355 genes are predicted from the genome of $\mathrm{Cmi}$ R1-1. Reciprocal BLAST analysis between subspecies of C. michiganensis reveals that many genes are unique to $\mathrm{Cmi}$. These $\mathrm{Cmi}$ unique genes are 
candidates for identifying virulence factors contributing to colonization and symptom development of $\mathrm{Cmi}$ infecting alfalfa.

Draft genome sequence of Xanthomonas citri pv. malvacearum strain MSCT1 and development of pathovar specific PCR primers

S. E. LU (1), K. Showmaker (1), X. Wang (1), H. Wang (1), C. Y. Hsu (1), P. Deng (1), S. M. Baird (1), T. Allen (1), B. Golden (1), D. Peterson (1), R. Nichols (2)

(1) Mississippi State University, Mississippi State, MS, U.S.A.; (2) Cotton Incorporated, Cary, NC, U.S.A.

Phytopathology 104(Suppl. 3):S3.73

Xanthomonas citri pv. malvacearum $(\mathrm{Xcm})$ causes bacterial blight of cotton. Strain MSCT1 was isolated from cotton in the Mississippi Delta in 2011 and subsequently confirmed to be $\mathrm{Xcm}$ by sequence typing and pathogenicity assays. The aim of the project was to develop a pathovar specific PCR protocol to detect the pathogen from plant materials based on genomic analysis. Illumina Truseq PCR-Free and Illumina Nextera mate-pair DNA libraries were constructed and sequenced for MSCT1. Illumina reads were assembled into a single $5 \mathrm{Mb}$ circular scaffold composed of 27 contigs with a N50 of $332 \mathrm{~Kb}$. The Prodigal gene-finding algorithm predicted 4,269 genes within the MSCT1 draft assembly. Mapping and variant analysis of the Illumina reads to 113 Xanthomonas complete and draft genomes deposited in the NCBI Genome database showed that the MSCT1 genomic sequence is more similar to $X$. citri pv. malvacearum strain GSPB1386 (Race 18) than any other Xanthomonas species or strain sequenced to date. Ten PCR primer pairs were designed from variable regions of Xanthomonas genomes, identified from genomewide comparison with related xanthomonads. Preliminary tests indicated three of the primer pairs exhibited specificity in detecting the Xcm grown on culture plates. Evaluation of functionality and specificity of the PCR primers to detect $\mathrm{Xcm}$ from plant material is under way.

Characterization of a putative serine protease effector from Clavibacter michiganensis subsp. sepedonicus in non-host interaction

Y. LU (1), N. Hatsugai (1), F. Katagiri (1), C. A. Ishimaru (1), J. Glazebrook (1)

(1) University of Minnesota, St. Paul, MN, U.S.A.

Phytopathology 104(Suppl. 3):S3.73

Clavibacter michiganensis is a group of Gram-positive phytopathogenic bacteria causing diseases on major crops. Genome sequencing and functional analyses enable the study of virulence strategies used by some subspecies of C. michiganensis. Comparative genomics analysis revealed that the pat-1/chp gene family, which encodes putative serine proteases known to be required for full virulence, is only present in the two subspecies infecting Solanaceae hosts, C. michiganensis subsp. michiganensis ( $\mathrm{Cmm}$ ) and C. michiganensis subsp. sepedonicus (Cms). One member of this gene family, chp-7 (CMS_2989), has been shown to be important for symptom development in $\mathrm{Cms}$ infected potato and eggplant, and is also required for $\mathrm{Cms}$ to trigger a hypersensitive response (HR) in Nicotiana tobacum. Further study showed that purified Chp-7 triggers HR in N. tabacum leaves in the absence of the pathogen, indicating Chp-7 itself is the HR elicitor from Cms. Mutation of the putative catalytic serine 232 in Chp-7 abolished the HR induction activity, suggesting that enzymatic activity is required and a plant-derived substrate of Chp-7 is cleaved to initiate the HR signaling. Ectopic expression of intron containing chp-7 constructs in $N$. tabacum leaves revealed that the Chp-7 fused to a plant secretion signal targeting the apoplast triggered HR, while the cytoplasmic Chp-7 did not trigger HR. We conclude that Chp-7 initiates HR in the apoplast of $N$. tabacum leaves.

Interaction studies of Pratylenchus penetrans and Fusarium verticillioides on corn seedlings

T. LUNT (1), A. MacGuidwin (1)

(1) UW Madison, Madison, WI, U.S.A.

Phytopathology 104(Suppl. 3):S3.73

Pratylenchus penetrans $(\mathrm{Pp})$ and Fusarium verticillioides $(\mathrm{Fv})$ are common pathogens of corn. Both Pp and Fv infect plant roots and mesocotyl as soon as the plant begins to grow, but evidence of an interaction has been mixed. The objective of our study was to determine if the two pathogens interact for the growth of corn seedlings. Pregerminated seed of Pioneer P99117XR was planted into pots containing $500 \mathrm{~cm}^{3}$ loamy sand soil inoculated with pathogen growth media only (control), $2000 \mathrm{Pp}, 50 \mathrm{~cm}^{3}$ of a sand/cornmeal culture of $\mathrm{Fv}$, or both pathogens together. Plants were grown at $27^{\circ} \mathrm{C}$ in a growth chamber in a randomized complete block design with six replications, with one repeat. After 25 days, plant height, dry shoot and root weights, and root length were determined. Root length and weight for the nematode + fungus treatment was reduced as compared to the noninoculated control in one experiment, but no differences were detected between the co-inoculated and single pathogen treatments for either experiment. Pathogens were detected only in pots receiving inoculum, and population densities of Pp were not affected by the presence of the fungus. We failed to detect an interaction of Pp and $\mathrm{Fv}$, but studies are ongoing to determine if changing the duration, inoculum levels, and environmental conditions affect experimental outcomes.

Finding an optimal spatial scale for citrus health management in California

W. LUO (1), T. R. Gottwald (2)

(1) Center for Integrated Pest Management, Raleigh, NC, U.S.A.; (2) USDA ARS, Fort Pierce, FL, U.S.A.

Phytopathology 104(Suppl. 3):S3.73

Citrus huanglongbing (HLB), spread by its vector Asian Citrus Psyllid (ACP), is a devastating disease in all infected citrus growing areas worldwide. The disease is endemic in FL, and the recent finds of HLB in Los Angeles residential basin and ACP in the CA central valley underscores the urgency of citrus health management. Citrus Health Management Areas (CHMAs) facilitate the coordinated control of ACP populations, the clean-up of abandoned groves, and the removal of infected trees. Growers cooperating within a CHMA have been effective in suppressing ACP populations and slowing the spread of HLB in FL, indicating CHMAs are a viable management strategy. However, the boundaries of CHMAs in FL are not optimized in size, but constructed primarily on arbitrary boundaries with an attempt to combine resources and garner neighbor participation. Improving from CHMA design deficiency in FL, we constructed CHMA boundaries in CA based on estimated HLB/ACP risk level under 1-mile ${ }^{2}$ grid resolution. Through cluster analysis and spatial statistics, 13 CHMAs are proposed for central valley $(230,423$ acres), 5 for coastal area (32,439 acres) and 10 for southern CA (43,780 acres), in representing citrus areas as homogenous as possible for $\mathrm{HLB} / \mathrm{ACP}$ risk. In addition to $\mathrm{HLB} / \mathrm{ACP}$ epidemiological factors, other abiotic factors (e.g. total citrus acreage and spray facility logistics) will also be considered to refine CHMAs with manageable size to better optimize cost-effective control.

High-throughput molecular screening tools for detection of Tobacco rattle virus in crude plant extracts

L. LUTES (1), S. Zhang (1), D. Groth-Helms (1)

(1) Agdia, Inc., Elkhart, IN, U.S.A.

Phytopathology 104(Suppl. 3):S3.73

Tobacco rattle virus (TRV), the type member of the genus Tobravirus in the family Virgaviridae, is transmitted naturally by soil-inhabiting nematodes. The virus infects more than 400 plant species in over 50 families including economically important fabaceous, solanaceous, and herbaceous ornamental crops. There have been numerous TRV-caused disease outbreaks worldwide in the past decade resulting in tremendous crop damages and economic losses. Most recent reports include: potatoes in North Dakota (2010), spinach in California (2010), pepper in Japan (2011), and flax and onion in Egypt (2012). Since treatments to eliminate TRV from plants are non-existent, sensitive and cost-effective diagnostic assays are essential for plant disease management. Due to the non-enveloped nature of some strains, serological assays are not always a reliable method of detection for TRV. In this study, a TRV-specific Real-time PCR assay was developed and validated using a cost-effective, efficient crude plant extraction method. Additionally, a TRV-specific dot blot hybridization assay was developed and validated using the same crude extraction method. Both the Real-time PCR and the dot blot hybridization assays were used to screen the same collections of TRV-free and TRVinfected plants and the assay specificities and sensitivities were determined. These sensitive and high-throughput qualitative assays are useful tools in a comprehensive disease management and screening program.

Evaluation of chemical and cultural tactics in reducing Xanthomonas gardneri populations and bacterial spot disease in tomato seedlings X. MA (1), M. Lewis Ivey (2), S. A. Miller (1)

(1) Ohio State University, OARDC, Wooster, OH, U.S.A.; (2) Agcenter, Louisiana State University, Baton Rouge, LA, U.S.A.

Phytopathology 104(Suppl. 3):S3.73

Xanthomonas gardneri $(\mathrm{Xg})$ is the primary pathogen causing bacterial leaf spot (BLS) of processing tomatoes in the Midwest. Environmental conditions during seedling production are conducive to BLS development in the greenhouse. Experiments were performed to determine the effect of agrochemicals, seedling density and strobe lights on BLS development in tomato seedlings. Tomato seeds were sown into 288 and 338-cell plug trays. Agrochemicals were applied twice after inoculation with $\mathrm{Xg}$. The effects of agrochemicals and seedling density on epiphytic populations of $\mathrm{Xg}$ were both significant. Epiphytic populations of $X g$ were lower in lower density (288cell) than in higher density (338-cell) trays. However, there were no 
differences among agrochemicals in foliar lesion numbers. In 338-cell plug trays, Cuprofix plus Citrex and Actigard significantly reduced epiphytic $X g$ populations. In 288-cell trays, Actigard and Kasumin reduced Xg populations. In a separate experiment, the effects of red, amber, blue and green strobe lights on tomato seedling growth and BLS development were evaluated. Stem thickness was reduced in seedlings exposed to green and red strobe lights compared to the natural light control. All strobe lights reduced plant shoot length compared to natural light for two observation points. However, strobe light exposure did not result in reduced foliar lesion number or epiphytic $\mathrm{Xg}$ populations.

Characterization of genes associated with antifungal activity of Pseudomonas sp. strain MS82 against the mushroom pathogen Mycogone perniciosa

L. MA (1), X. Wang (2), P. Deng (2), S. M. Baird (2), S. E. Lu (2)

(1) Jiangsu Academy of Agricultural Sciences, Nanjing, China; (2) Mississippi State University, Mississippi State, MS, U.S.A.

Phytopathology 104(Suppl. 3):S3.74

Wet bubble disease caused by the fungus Mycogone perniciosa is the main constraint in production of white button mushroom (Agaricus bisporus) worldwide. Biologically-based disease management is in demand. Bacterial strain MS82, isolated from agricultural soil, exhibits significant antifungal activity against $M$. perniciosa but no detectable inhibitory effect on the mushroom. The 16S rRNA gene sequence of strain MS82 shared the high identities (99\%-98\%) with strains of Pseudomonas spp., suggesting that the strain belongs to this genus. Phylogenetic analysis of the 16S rRNA sequences revealed that strain MS82 was grouped with the strains of $P$. fluorescens. Further sequence analyses are under way to determine its identity. Mutants MS82MT10 and MS82MT19 obtained through Tn5 random mutagenesis lost their antifungal activity on plate bioassays. The targeted genes of the mutants were cloned using the plasmid rescue procedure. The genes mutated in MS82MT10 and MS82MT19 shared significant homology with an ABC transporter (Pfl01 5478) and a GGDEF/EAL domain protein (Pfl01_4876), respectively, of $P$. fluorescens Pf0-1. Further work is under way to identify additional genes required for production of the antifungal activity. Findings of the study will provide insights to understand molecular mechanism of Pseudomonas sp. strain MS82 against the mushroom fungal pathogen.

Comparative genomics in the boxwood blight system: Insights into the global diversity of the mating-type locus

M. MALAPI-WIGHT (1), J. Hébert (2), Y. Rivera (2), E. Ismaiel (1), N. Saied (1), B. Gehesquière (3), K. Heungens (3), J. A. Crouch (1)

(1) USDA ARS, Beltsville, MD, U.S.A.; (2) USDA ARS / Rutgers University, Beltsville, MD, U.S.A.; (3) Institute for Agricultural and Fisheries Research, Merelbeke, Belgium

Phytopathology 104(Suppl. 3):S3.74

The ascomycete Calonectria pseudonaviculata, a destructive fungal pathogen responsible for boxwood blight disease, was first reported in the U.S. in 2011. In a matter of months, North American boxwood blight outbreaks occurred across the eastern seaboard and Pacific Northwest. In order to understand the sexual reproductive potential of $C$. pseudonaviculata, we have applied next generation sequencing to assess the primary determinant of mating (MAT1) locus. The genome of fifteen C. pseudonaviculata isolates were sequenced, used to create a local BLAST databases in CLC Genomics, and then searched for homologues of the idiomorphic genes MAT1-1 and MAT1-2. Only a single MAT1 idiomorph was identified in each of the fifteen genome assemblies, indicating that $C$. pseudonaviculata is a heterothallic fungus. Subsequently, we designed specific primers to amplify differently sized regions for each MAT1 locus. Over 200 isolates originating from four continents were analyzed by a duplexed PCR reaction. PCR analyses revealed that the mating types correspond with the genetic lineages G1 and G2. MAT1-2 genotype is globally distributed, but exclusively in the G1 lineage. The MATI-1 genotype is solely present in five European countries from isolates of the G2 lineage. These results indicate that the fungus is currently unable to sexually reproduce in North America, since all the U.S. isolates belonged to the MATI-2 genotype.

\section{Characteristics of resistance to Goss's wilt of corn in Minnesota caused} by Clavibacter michiganensis subsp. nebraskensis (CMN)

D. MALVICK (1), R. D. Curland (1), C. A. Ishimaru (1), C. Floyd (1), R. R. McNally (1)

(1) University of Minnesota, St. Paul, MN, U.S.A.

Phytopathology 104(Suppl. 3):S3.74

Goss's wilt (GW) is an important disease of corn that was first confirmed in Minnesota (MN) in 2009 and has been spreading in the U.S. and Canada. Genetic resistance is central to management of GW. The goals of this study were to determine if hybrids rated for resistance in other states provide resistance in $\mathrm{MN}$ and to determine how plants respond after using different inoculation and evaluation methods. Studies were done in 2012 and 2013 to examine resistance to $\mathrm{GW}$ for 12 hybrids ranging from susceptible to resistant. Three replicated field plots were inoculated with CMN at the V8 growth stage using a leaf inoculation tool, and three plots of each hybrid were noninoculated. Disease severity and spread in plants, lodging, and grain yield were measured. GW reduced yields for all hybrids. For the most susceptible hybrid, severe GW developed and yield was $\sim 55 \%$ lower in inoculated vs. noninoculated plots. Yield loss decreased for most hybrids as their reported resistance increased. Yield was poorly correlated $\left(\mathrm{R}^{2}=0.3\right)$ with disease severity, suggesting tolerance in some hybrids. Stalk lodging was most severe in plots with GW. The same hybrids were grown in replicated pots in a greenhouse and inoculated via stalk injection at the V3 stage. In the greenhouse, all hybrids were susceptible, suggesting that the stalk inoculation method may circumvent resistance. GW can significantly reduce yield of maize and diligent efforts to monitor and manage this disease are warranted.

Screening and identification of resistance to Leptosphaeria maculans in Brassica napusaccessions

S. M. MANSOURIPOUR (1), L. del Rio-Mendoza (1)

(1) North Dakota State University, Fargo, ND, U.S.A.

Phytopathology 104(Suppl. 3):S3.74

Blackleg caused by the fungus Leptosphaeria maculans is a devastating disease of canola (Brassica napus L.) and causes extensive yield loss worldwide. In North Dakota the most prevalent strains belong to pathogenicity group (PG) 4 and all commercial canola cultivars are susceptible to them. Greenhouse trials are being conducted to identify sources of resistance against this PG. So far, 187 B. napus accessions have been evaluated. All seedlings were inoculated at the cotyledon stage using a mixture of five isolates. Ten seedlings per entry in each of three replications have been evaluated. Disease severity was rated ten days after inoculation using a scale of 0 to 9 . Cultivar Westar was used as control. The median for each accession was used to estimate the relative treatment effect and to identify the most resistant materials. Ten accessions were identified as highly resistant to PG-4 and the reaction of two of them was confirmed in two additional trials. Doubled haploid plants will be developed from these materials to study the genetics of their resistance. Additional accessions are currently being evaluated.

RNAseq and histological analysis of the canola - Sclerotinia pathosystem S. MAO (1), M. F. Belmonte (1), D. Fernando (1), T. De Kievit (1)

(1) University of Manitoba, Winnipeg, MB, Canada

Phytopathology 104(Suppl. 3):S3.74

The fungal pathogen Sclerotinia (Sclerotinia sclerotiorum) impacts production and yield in one of Canada's number one crops, canola (Brassica napus). Unfortunately, few cultivars show tolerance to this devastating fungal pathogen. Thus, understanding the plant's response to this aggressive fungus is paramount in the identification of resistance genes. While our understanding of the host pathogen interaction is becoming clearer, there is remarkably little information available for Sclerotinia-canola pathosystem. Moreover, we know nothing about how this interaction is specified at the genetic or cellular level. Therefore, we have taken an initiative to compare all of the genes expressed both a susceptible cultivar (Westar) and a partially tolerant cultivar [Zhongyou821 (ZY821)] when challenged with Sclerotinia using global RNAseq strategies. We complement the global RNA profiling data with detailed anatomical studies and validations using qRT-PCR. At the cellular level, Sclerotinia penetrates both cultivar leaves directly through the cuticle and epidermis rather than through stomata openings. While Sclerotinia growth is prevalent on the epidermis of ZY821, pathogen entry into the subtending layers is slowed. Genes associated with defense response signaling pathways including AOS and PRI express differently at the later infection stages. Collectively, our data provides both anatomical and predicted molecular mechanisms underlying canola's response to Sclerotinia.

GATA-dependent nitrogen acquisition strategies promote rice infection by the blast fungus Magnaporthe oryzae

M. R. MARROQUIN-GUZMAN (1), R. A. Wilson (1)

(1) University of Nebraska, Lincoln, NE, U.S.A.

Phytopathology 104(Suppl. 3):S3.74

In Magnaporthe oryzae the nitrogen regulator NUT1, a GATA-family transcription factor, has been shown to be dispensable for disease but has been important in elucidating two processes essential for infection: An NADPHdependent genetic switch and carbon catabolite repression. These findings make NUTI part of a critical nutrient response network that integrates carbon and nitrogen metabolism during $M$. oryzae infection. Using gene functional analysis, cell biology techniques, transcriptional and phenotypic analysis we 
demonstrate here that $N U T 1$ is required for metabolizing limiting amounts of the preferred nitrogen source ammonium, but is not needed for utilizing some common plant transporter nitrogen sources that are likely found in host cells during biotrophy. Several genes needed to assimilate ammonium into amino acid are under NUT1 control during growth on high and low ammonium concentrations and also during in planta infection, such as the glutamine synthetase-encoding gene $G L N 2 . \Delta g \ln 2$ gene deletion mutants are impaired for plant infection. These results lead us to conclude that NUT1 is needed for assimilating nitrogen under starvation conditions and that some of the genes under NUT1 control are required for full virulence.

Evidence for host preference of Macrophomina phaseolina on strawberry F. N. MARTIN (1), R. Arias (2), S. Koike (3), C. Hogan (1)

(1) USDA-ARS, Salinas, CA, U.S.A.; (2) USDA-ARS National Peanut Research Laboratory, Dawson, GA, U.S.A.; (3) University of California Cooperative Extension - Monterey County, Salinas, CA, U.S.A.

Phytopathology 104(Suppl. 3):S3.75

With the transition from broadcast preplant fumigation to individual bed treatment using alternative fumigants $M$. phaseolina has become an emerging disease problem in California strawberry production, causing serious losses in all production districts. Population analysis using SSR markers of 68 isolates recovered from across the state reveals a distinct grouping of strawberry isolates separate from isolates recovered from other crops (primarily watermelon, cantaloupe, alfalfa, thyme; 24 isolates). There were two exceptions to this grouping, one strawberry isolate grouped with isolates recovered from other hosts from the Central Valley (CV) and a cantaloupe isolate from the $\mathrm{CV}$ grouped with the strawberry isolates. Thirty of the strawberry isolates were from the same field and exhibited several different genotypes. Pathogenicity tests with a subset of isolates recovered from strawberry representing each subclade were virulent on this host but did not cause disease on the other host species tested; however, the strawberry isolate from the CV exhibited low virulence on strawberry. A more detailed analysis of host range and virulence on strawberry are in progress and will be discussed.

\section{Mitochondrial genomes of Bremia lactucae and development of haplotype} markers for population and genetic studies

F. MARTIN (1), J. Gil (2), L. Derevnina (2), O. Ochoa (2), R. Michelmore (2) (1) USDA ARS, Salinas, CA, U.S.A.; (2) University of California, Davis, CA, U.S.A.

Phytopathology 104(Suppl. 3):S3.75

Bremia lactucae, the causative agent of lettuce downy mildew, is the most important pathogen of lettuce in the US and worldwide. In order to identify cytoplasmic markers for use in population and genetic studies the reference mitochondrial genome of $B$. lactucae isolate SF5 was assembled from Illumina and Pacific Biosystems reads that had been generated for sequencing the nuclear genome. The SF5 mitochondrial genome is 40,513 bp in size, encodes the 2 rRNAs, 34 genes, 5 putative ORFs and tRNAs common in Pythium and Phytophthora spp.; it also has two unique putative ORFs, a 212 bp inverted repeat encoding duplicate $\operatorname{trn} K$ genes, and has 21 copies of an 80 bp repeat. An unusual feature of its organization is the position of the rns coding region; this is in a similar location as in Phytophthora spp. but on the strand opposite to the $r n l$ gene. The mitochondrial genomes have now been assembled for 28 isolates from geographically diverse origins. This revealed polymorphisms due to both SNPs and indels. Network analysis grouped the isolates approximately paralleling their geographic distribution. The relationship between mitochondrial haplotypes and nuclear genotypes and virulence phenotypes will be discussed.

\section{Genotype-isolate interactions for resistance to Sclerotinia homoeocarpa in} seashore paspalum

A. D. MARTINEZ-ESPINOZA (1), C. J. Steketee (1), P. Raymer (1)

(1) University of Georgia, Griffin, GA, U.S.A.

Phytopathology 104(Suppl. 3):S3.75

Seashore paspalum (Paspalum vaginatum) is a warm-season turfgrass specie utilized on golf courses and athletic fields in the southeastern USA and in other tropical regions of the world. It is best known for its high salt tolerance, but available cultivars can be sensitive to dollar spot disease. Dollar spot, caused by Sclerotinia homoeocarpa, is a major fungal pathogen of turfgrasses. A management strategy is host plant resistance, but more needs to be known about the pathogen and host interaction to incorporate this resistance into elite genotypes. Five genotypes of seashore paspalum with dollar spot resistance differences were artificially inoculated with five isolates of $S$. homoeocarpa from several turfgrass species, and evaluated for percent disease visually and with digital image analysis in the field over two years. The experiment was a two-factor, split-plot design, with seashore paspalum genotypes as the main plot, and eight isolate and control treatments as subplots. Using area under the disease progress curve (AUDPC) values, there were statistical differences among the seashore paspalum genotypes and treatments. However, there was not a significant genotype by isolate interaction between the seashore paspalum genotypes and the dollar spot isolates. Using this information, breeders should be able to use one highly virulent $S$. homoeocarpa isolate to screen for host plant resistance in seashore paspalum.

\section{Incidence of bacterial decline of bentgrass in Georgia}

A. D. MARTINEZ-ESPINOZA (1), R. Alexander (1), B. Dutta (2)

(1) University of Georgia, Griffin, GA, U.S.A.; (2) University of Georgia, Tifton, GA, U.S.A.

Phytopathology 104(Suppl. 3):S3.75

Diseased bentgrass (Agrostis palustris) plants were submitted to the UGA Plant Disease Clinic from a golf course in northeast GA. Symptoms were unique and included an abnormal plant elongation, general yellowing, wilt, and necrosis. Bacteria were found associated with the symptomatic tissue. The causal agent was determined to be Acidovorax avenea subsp avenea (Aaa). Here, we report a spatial and temporal incidence of this bacterial disease. Suspected bentgrass diseased samples collected in northeast Georgia, as well as samples from two additional sites located in north and northwest parts of the state, tested positive for Aaa using specific antibodies in ELISA. All of the selected colonies, which tested positive using ELISA, had 96-99\% similarity to Aaa using Biolog ${ }^{\mathrm{TM}}$. Universal primers were used to amplify the $16 \mathrm{~S}$ rRNA gene from four isolates and sequencing of amplified product showed 97 to $99 \%$ nucleotide similarity to Aaa. Further confirmation was achieved by amplifying bentgrass isolates with Aaa-specific primers in a nested PCR assay. The bacterial isolates were inoculated into healthy bentgrass $\mathrm{cv}$ Penncross plants. Healthy plants were successfully infected with Aaa when inoculated plants were incubated at high humidity (RH 90-100\%) and high temperatures $\left(30-35^{\circ} \mathrm{C}\right)$. Re-isolated bacterial colonies were characterized as Aaa using morphological features, ELISA specific antibodies and speciesspecific DNA primers.

Effects of climate change on the components of wheat leaf rust disease on winter wheat

A. M. MASHAHEET (1), D. S. Marshall (1), K. O. Burkey (1)

(1) North Carolina State University, Raleigh, NC, U.S.A.

Phytopathology 104(Suppl. 3):S3.75

Understanding climate change effects on wheat leaf rust (caused by Puccinia recondita) is important for maintaining yield. Four winter wheat genotypes known to differ in their response to leaf rust were grown under combinations of $\mathrm{CO}_{2}(400,570 \mathrm{ppm})$ and $\mathrm{O}_{3}(10,50 \mathrm{ppb})$, major components of climate change. Plants were inoculated with leaf rust spores at Zadoks growth stage of 39-40. Plant growth, ozone injury and disease development were documented throughout the growing season. Results showed that elevated levels of both gases, singly and in combination, accelerated plant development. Foliar injury from $\mathrm{O}_{3}$ was observed in all genotypes and elevated $\mathrm{CO}_{2}$ ameliorated this effect. Ozone concentrations utilized were not high enough to affect yield, plant height, or vegetative dry weight, but elevated $\mathrm{CO}_{2}$ and the combination of gases $\left(\mathrm{O}_{3}+\mathrm{CO}_{2}\right)$ increased each of these parameters. Rust infection decreased plant yield, height and the vegetative dry weight. Elevated $\mathrm{CO}_{2}$ partially reduced yield loss from rust infection, but did not alter rust effects on height and vegetative dry weight. In terms of gas effects of disease progression, $\mathrm{O}_{3}$ increased disease severity and pustule size but $\mathrm{CO}_{2}$ effects were not significant. The genotype $\times$ rust $\times$ gas interaction was not significant.

Relative ability of chemical management tools to inhibit stem lesion development on pepper plants inoculated with $P$. capsici

M. E. MATHERON (1), M. Porchas (1)

(1) University of Arizona, Yuma, AZ, U.S.A.

Phytopathology 104(Suppl. 3):S3.75

The oomycete pathogen Phytophthora capsici can cause losses in pepper plantings due to stem and leaf lesions, crown and root rot, and fruit rot. Fungicides are important tools in an integrated disease management system. Several materials have been registered and some new compounds are being developed with activity against $P$. capsici. Two trials were conducted to evaluate 14 different products for their ability to inhibit stem lesion development. Three-month-old chile pepper transplants were wounded on the stem with a $5-\mathrm{mm}$ diameter cork borer about $8 \mathrm{~cm}$ above roots. Foliage and stems or roots of plants were submerged in a fungicide solution for 2- or 5seconds, respectively, placed on paper towels until dry, then replaced into transplant trays and maintained in a greenhouse. After 1- and 3- weeks, plants were placed horizontally in trays containing moist paper towels, each stem wound was inoculated with a drop of $P$. capsici zoospore suspension, then trays were covered with clear plastic film and maintained under lights at 25 to 
$26^{\circ} \mathrm{C}$ in the laboratory. The length of stem lesions was recorded after 7 days. Whether applied to foliage and stems or to roots of transplants inoculated 1or 3-weeks after treatment, ametoctradin+dimethomorph, dimethomorph, ethaboxam, fenamidone, fluopicolide, mandipropamid, and oxathiapiprolin reduced stem lesion growth by at least $80 \%$ compared to nontreated plants.

Pathotype diversity in single zoospore isolates from a race 1 isolate of Phytophthora sojae

R. MATTHIESEN (1), A. Robertson (1)

(1) Iowa State University, Ames, IA, U.S.A.

Phytopathology 104(Suppl. 3):S3.76

Phytophthora sojae causes pre- and post-emergence damping-off of soybean. The pathogen is predominantly managed by using resistant soybean varieties with specific Rps genes, however $P$. sojae evades this resistance by changing its arsenal of corresponding Avr genes. Although P. sojae is homothallic, changes in the $A v r$ gene range (pathotype) of single zoospore isolates (SZIs) has been reported. The objective of this study was to determine the pathotypic diversity among SZIs from a race 1 isolate (virulent of Rps 7 only) of $P$. sojae, PR1, grown on dilute V8 agar over three generations. Pathogenicity of 100 SZIs from PR 1 was tested using a standard 14-line differential set and a susceptible soybean variety. In the first generation of SZI, two of the 100 isolates were virulent on Rps 1a (78\% and 72\%) and one isolate was virulent on $R p s$ 1d (73\%) changing pathotype from race 1 to race 3 and race 54 , respectively. All three SZIs remained virulent on Rps7. Second and third generation progenies showing virulence on Rps 1a, 1d, and 7, and SZIs that did not change in pathotype are currently being screened for pathogenicity. An improved understanding of how $P$. sojae evolves to evade Rps gene resistance in soybeans could lead to the development of novel management practices.

\section{Identification of new Rathayibacter species using multi-locus sequence} analysis

S. J. MAUZEY (1), W. L. Schneider (2), D. G. Luster (2), M. A. McClure (3), T. D. Murray (1), B. K. Schroeder (1)

(1) Department of Plant Pathology, Washington State University, Pullman, WA, U.S.A.; (2) USDA, Agricultural Research Service, Foreign DiseaseWeed Science Research Unit, Ft. Detrick, MD, U.S.A.; (3) The School of Plant Sciences, University of Arizona, Tucson, AZ, U.S.A.

Phytopathology 104(Suppl. 3):S3.76

The genus Rathayibacter currently is composed of seven bacterial plant pathogens that infect grass and cereal hosts in association with nematode vectors in the genus Anguina. Two species, $R$. rathayi and $R$. agropyri, are currently found in the United States as pathogens of orchard grass and western wheat grass, respectively. A new grass host for Rathayibacter in the genus Sporobolus was identified based on samples collected in a 2013 survey of the Pacific Northwest U.S. In addition, a Rathayibacter sp. was isolated from $A$. pacificae galls collected from golf course grasses (Poa апnиa) in California. Based on analysis of the 16S rRNA sequence, the isolates from Sporobolus and Poa are hypothesized to represent two new Rathayibacter species. A multi-locus sequence analysis (MLSA) for Rathayibacter using four housekeeping genes $(r p o B, \operatorname{gyr} B, \operatorname{rec} A$, and $p p k)$ is being used to test the hypothesis. Preliminary analysis of the $16 \mathrm{~S}$ rRNA sequence demonstrates that the isolates from Sporobolus and Poa form unique clades distinct from the seven known Rathayibacter species. The isolates from Sporobolus and Poa form the same unique clades in the rроB gene analysis. These data support the hypothesis that the bacteria from Sporobolus $s p$. and $P$. апnиa are new Rathayibacter species. Further analyses of the individual housekeeping genes are in progress, and a concatemer will be used in a final analysis if congruency is upheld among the four housekeeping genes.

Distribution of Rathayibacter spp. and their nematode vectors (Anguina spp.) in the United States Pacific Northwest

S. J. MAUZEY (1), W. L. Schneider (2), D. G. Luster (2), B. K. Schroeder (1), T. D. Murray (1)

(1) Department of Plant Pathology, Washington State University, Pullman, WA, U.S.A.; (2) USDA, Agricultural Research Service, Foreign DiseaseWeed Science Research Unit, Ft. Detrick, MD, U.S.A.

Phytopathology 104(Suppl. 3):S3.76

The genus Rathayibacter is an obscure group of bacteria vectored by seed gall nematodes that are not widely studied because the diseases they cause are generally limited to forage grasses and non-agricultural hosts. R. toxicus is probably the most well-known species, because in addition to causing gumming disease on ryegrass in Australia, it also produces a toxin that causes Annual Ryegrass Toxicity. Consequently, $R$. toxicus was added to the USDA APHIS list of Select Agent plant pathogens, but diagnosis is complicated by the presence of $R$. rathayi and $R$. agropyri, which cause similar gumming diseases on other grass hosts in the U.S. Pacific Northwest (PNW). In 2013, a survey was conducted to determine the distribution of $R$. rathayi and $R$. agropyri in the PNW. Roadside pasturelands were visually surveyed for signs of Rathayibacter and/or nematode seed galls. A total of 34 samples were collected from Idaho and Montana. Bacteria was successfully isolated from 16 samples; five of these samples also yielded nematodes, isolation from three other samples yielded nematodes but no bacteria. All of the bacteria isolated were identified as a Rathayibacter spp. based on the 16S rRNA sequence. Nematodes have been identified preliminarily as Anguina species based on morphological characteristics. These data suggest that Rathayibacter and Anguina species are more widely distributed in the PNW and possibly throughout the U.S. than previously known. Surveys will continue in 2014.

Pennsylvania Ag Resource Centers: A joint initiative between Penn State University and the Pennsylvania Department of Agriculture

S. R. MAY (1)

(1) Penn State University, University Park, PA, U.S.A.

Phytopathology 104(Suppl. 3):S3.76

The development of three Ag Resource Centers focused on plant health, food safety, and animal care was initiated in 2013 as a partnership between Penn State College of Agricultural Sciences (PSU) and the Pennsylvania Department of Agriculture (PDA). Through these Centers, PSU and PDA collaborate and leverage resources to target high-priority issues by fostering research and programs that work to provide solutions to the agricultural challenges facing Pennsylvania producers and consumers. The Centers are currently addressing a broad spectrum of issues and topics in response to the needs of industry. Current focus areas include Pennsylvania's readiness to respond to food and agricultural crises, and preparing the food and feed industries for regulatory changes associated with the Food Safety Modernization Act. As the Centers continue to develop, there will be continued opportunities for Pennsylvania's regulatory agency and the University to collaborate in order to meet the changing needs of agriculture.

\section{Breeding for root lesion nematode resistance in Montana winter wheat}

D. MAY (1), A. Dyer (1), P. Bruckner (1), J. Berg (1)

(1) Montana State University - Bozeman, Bozeman, MT, U.S.A.

Phytopathology 104(Suppl. 3):S3.76

Root lesion nematodes (Pratylenchus spp.) present a serious challenge to dryland wheat production worldwide. Development of resistant cultivars would provide great economic benefit to farmers. From 2012-2013, a set of backcross lines (MT08185//MT08184/Persia 20) were screened for resistance to $P$. neglectus. Progeny and parent lines were grown in infested soil for 16 weeks. Nematodes were extracted from roots of individual plants and counted to obtain per plant final populations. ANOVA results from the 2013 screen provided convincing evidence of a difference in mean $P$. neglectus populations among lines $(p<0.01)$. The median final population of susceptible parent MT08184 was an estimated 4.0 times greater than that of resistant parent Persia 20. A prior screen using inoculum obtained from a different Montana region provided no evidence of a difference in control means, suggesting the existence of pathotypes. A 2013 yield trial provided no evidence of correlation between a line's average final population and grain yield $\left(R^{2}<0.01, p=0.57\right)$. Similar results were obtained for test weight, protein, plant height and heading date. Identification of QTL for resistance to root lesion nematodes will facilitate marker-assisted introgression of resistance genes in a backcross-breeding program. To that end, 140 genome-wide SNPs were used to genotype progeny lines, and single-marker analysis was performed to identify genomic regions associated with resistance to $P$. neglectus.

Characterizing geographic footprint of Ophiosphaerella species causing spring dead spot of bermudagrass in Virginia

D. MCCALL (1), E. Bush (1), J. Derr (2), A. Nichols (2)

(1) Virginia Tech, Blacksburg, VA, U.S.A.; (2) Virginia Tech, Virginia Beach, VA, U.S.A.

Phytopathology 104(Suppl. 3):S3.76

Spring dead spot (SDS) is the most common and destructive disease of bermudagrass turf throughout the Mid-Atlantic. In addition to being unsightly, the depressed voids left behind can potentially be an injury hazard on athletic playing surfaces. Cultural management strategies of SDS are dependent on speciation of the causal agent. Two species of Ophiosphaerella are known to incite disease in the transition zone region of the United States. O. korrae is considered the dominant species in the lower transition zone (upper Southeastern states), while $O$. herpotrica is more prevalent in more northern regions of the zone (Maryland, Kentucky, and throughout portions of the Midwest). Localized sampling in Virginia has indicated that both species are present, but that one species tends to dominate a site-specific population. We hypothesize that while both species are present in Virginia, geography-based 
control recommendations can be made to minimize the severity of SDS. Symptomatic plant tissue was collected from locations in Virginia and surrounding states. Presence or absence of each species was determined using published primer sets specific to internal transcribed spacer regions of each species using real-time polymerase chain reaction (qPCR). Geo-referenced qPCR speciation results were used to provide geographical delineations of the causal agents, resulting in more accurate SDS suppression recommendations.

\section{Training and education to increase early NPDN detections of high-} consequence threats

R. L. MCCARTHY (1), R. W. Hoenisch (2), S. D. Stocks (3), G. K. Douce (4), J. J. Dombroskie (1), J. Allen (5), R. M. Bostock (2), J. B. Jones (3), M. Fuchs (6)

(1) Cornell University, Ithaca, NY, U.S.A.; (2) University of California, Davis, CA, U.S.A.; (3) University of Florida, Gainesville, FL, U.S.A.; (4) University of Georgia, Tifton, GA, U.S.A.; (5) University of Connecticut, Storrs, CT, U.S.A.; (6) Cornell University, Geneva, NY, U.S.A.

Phytopathology 104(Suppl. 3):S3.77

Since 2003, the National Plant Diagnostic Network (NPDN) Training \& Education team has created resources for First Detectors to facilitate early detections of high-consequence plant pests and pathogens that cost the United States billions of dollars annually. Members of the NPDN Training and Education team have expanded the types, quality and quantity of diagnostic resources for the First Detector audience in an effort to improve the number of quarantine significant samples being submitted to NPDN laboratories, increase the number of people actively looking for high-consequence threats and keep them engaged in the First Detector program. The new "Sharpening your Observation Skills" series was created to highlight key taxonomic features so First Detectors can improve their field observations and increase the number of significant samples being submitted. The newly developed photographic field guides can be customized and downloaded to smartphones and tablets for scouting in the field. The materials created through this project were translated to Spanish to encourage Hispanic and Latino groups to become First Detectors. Educators and First Detectors will be able to access all materials at www.firstdetector.org. New diagnostic resources are essential to keep First Detectors engaged and apprised of imminent threats and may increase the number of early detections.

\section{Characterization of a regional population of Phytophthora nicotianae}

K. MCCORKLE (1), K. Ivors (1), D. Shew (1)

(1) North Carolina State University, Raleigh, NC, U.S.A.

Phytopathology 104(Suppl. 3):S3.77

Black shank, caused by the oomycete Phytophthora nicotianae, is an important disease of tobacco in most tobacco growing regions. Several disease management strategies are available for disease control, but they often place selection pressure on the pathogen population to adapt. Phytophthora nicotianae has a high level of genotypic diversity that allows the population to quickly adapt to changes in the host and environment. In this study, the level of genetic diversity and the geographic patterns associated with genotype distribution were determined for populations of $P$. nicotianae in tobacco regions of $\mathrm{NC}, \mathrm{KY}, \mathrm{VA}$, and GA. A total of 453 isolates were collected from infested tobacco fields. Five genes, ITS46, beta-tubulin, elongation factor, $\mathrm{NADH}$, and COXII, were sequenced in each isolate and used to find single nucleotide polymorphisms (SNPs) among individuals. The greatest numbers of SNPs were found in the COXII region for isolates from NC and VA, and in the EF region for KY and GA isolates. Genotypic variability was high, with multiple genotypes recovered in each state. The level of detected genetic variation was highest in GA with one new clade for every 10 isolates, one new clade for every 14 isolates in VA, and a new clade for every 22 isolates in KY. The high level of diversity found among isolates may help the pathogen population quickly shift to adapt to changes in host and environment and influence management decisions.

\section{The genome of the wheat pathogenic fungus Tilletia caries encodes all the} molecular components necessary for RNA interference

S. W. MCCOTTER (1), J. L. Humann (1), D. Main (1), L. M. Carris (1), L. Castlebury (2)

(1) Washington State University, Pullman, WA, U.S.A.; (2) USDA ARS, Beltsville, MD, U.S.A.

Phytopathology 104(Suppl. 3):S3.77

RNA-silencing (RNAi) is a gene-expression regulatory and cell-defense mechanism found throughout Eukarya. While the Ustilaginomycetes Malassezia globosa and Ustilago maydis lack RNAi-genes, most studied smut fungi contain intact RNAi systems. The genome sequence of Tilletia caries, the smut responsible for common bunt of wheat, was queried for the presence of the molecular components required for RNAi. These include the RNA- dependent RNA polymerase and the nucleases Dicer and Argonaute. Putative orthologs to all essential RNAi genes were annotated in silico, revealing three Dicer and six Argonaute orthologs, considerably more than in other smuts. Maximum likelihood phylogenies were carried out comparing predicted protein sequences of hypothetical T. caries RNAi proteins to each other and to orthologs from other basidiomycetes. Interestingly, not all RNAi-related genes in $T$. caries group with those of related Ustilaginomycetes, in some cases exhibiting more similarity to members of Pucciniomycotina and Agaricomycotina. Analyses of the genomes of related Tilletiales will be necessary to verify these phylogenetic placements and functional studies are necessary to check RNA-silencing activity. The large family of putative RNAi-gene orthologs in $T$. caries suggests the evolution of unique silencing functions and that RNAi plays a multifaceted role in the biology of $T$. caries, likely related to regulation of gene expression in its morphologically distinct life stages.

\section{Drippy vine - a new disease of cucurbits}

M. MCCULLOUGH (1), C. Nischwitz (1), B. Schroeder (2), C. Dhiman (1)

(1) Utah State University, Logan, UT, U.S.A.; (2) Washington State University, Pullman, WA, U.S.A.

Phytopathology 104(Suppl. 3):S3.77

During the 2011 growing season, cantaloupes were observed to have a ring of foam at the point of vine attachment. After removing the melons from the vine, foam continued to exude the vines in a dripping fashion for another four to five days. In the fall of 2011, a winter squash sample was submitted with a small hole from which foam was dripping. Bacteria were isolated from the foaming melons and identified through biochemical tests and 16S sequencing as Brenneria sp. (syn. Lonsdalea sp.). There are species in the genus Brenneria that cause similar symptoms on lupines (Lupinus sp.) and acorns (Quercus spp). Koch's postulates were completed and the bacteria were confirmed as the causal agent of the disease. The bacteria are only able to infect the melons and cause symptoms when the flesh is orange but the fruit does not smell like melon. Symptomatic fruit cannot be sold and vines dripping foamy sap eventually turn yellow and die back. The economic impact of this disease is unknown but it is possible that this disease is more widely spread than realized as melons are frequently discarded as 'overripe' without being checked for ripeness.

Fungal and insect-associated branch damage of eastern black walnut in Indiana and Tennessee

M. E. MCDERMOTT-KUBECZKO (1), J. Juzwik (2), M. Paschen (3), W. E. Klingeman (4), M. D. Ginzel (3)

(1) University of Minnesota, Minneapolis, MN, U.S.A.; (2) U.S. Forest Service, St. Paul, MN, U.S.A.; (3) Purdue University, West Lafayette, IN, U.S.A.; (4) University of Tennessee, Knoxville, TN, U.S.A.

Phytopathology 104(Suppl. 3):S3.77

Thousand Cankers Disease (TCD), caused by interaction of the walnut twig beetle (WTB) and the fungus Geosmithia morbida, threatens the health of high-value eastern black walnut. However, other fungi and insects may also cause dieback and death of the species. Baseline data on fungi and insects colonizing the bark was obtained from branches of black walnut in urban areas with (Tennessee) and without TCD (Indiana). A drawknife was used to expose insect and non-insect related damage on 1 girdled $(\mathrm{G})$ and 1 nongirdled (NG) branch from 29 visually healthy trees and 2 NG branches from 3 visually-healthy trees (controls, Indiana only). Branches (NG) from three trees with TCD also were examined. Six types of bark damage (including two insect-related) were characterized for Indiana branches, while seven types (including two insect-related) were described for Tennessee branches. Buprestid-like damage was the only type common to both states. For Indiana, damage frequencies were similar for $\mathrm{G}$ and $\mathrm{NG}$ branches but lower for NG branches of control trees. Typical G. morbida cankers were found on branches of both visually healthy and TCD trees in Tennessee. Fungal isolations were attempted from representatives of all damage types from each state. To date, isolates obtained represent 21 genera. In Tennessee, G. morbida was isolated from three non-insect damage types and from $>50 \%$ of WTB damage on G branches. Results are of value to diagnosticians receiving TCD-suspect samples.

Potential for metam sodium to eradicate Plasmodiophora brassicae in field soils

M. R. McDonald (1), B. D. GOSSEN (2), S. F. Hwang (3)

(1) University of Guelph, Guelph, ON, Canada; (2) Agriculture and AgriFood Canada, Saskatooon, SK, Canada; (3) Alberta Agriculture and Rural Development, Edmonton, AB, Canada

Phytopathology 104(Suppl. 3):S3.77

Canola (Brassica napus L.) is a major crop in western Canada, grown on about $8 \mathrm{M}$ ha with a value $>\$ 19 \mathrm{~B}$ per year. Clubroot caused by Plasmodio- 
phora brassicae Wor., was first identified on canola in western Canada in 2003 and has already been confirmed in more than 1450 fields. Although treatment with a soil fumigant is not economical for commercial crop production, it may have a role in containing or eradicating infestations at sites that have only recently become infested. Fumigation may also have a role in reducing the transmission of the pathogen to new sites associated with industrial activities such as oil and gas exploration / development, construction of roads, and provision of utilities. Under controlled conditions, application of high rates of metam sodium (Busan 1236 at 85 and $170 \mathrm{~kg}$ ai $\mathrm{ha}^{-1}$ ) to naturally-infested soil reduced clubroot severity (DSI) from $38 \%$ in the untreated check to 1.8 and 0 in Shanghai pak choy, a highly susceptible indicator crop, in a soil with high organic matter. Application of Vapam, another formulation of metam sodium, at a moderate rate $\left(21 \mathrm{~L}\right.$ ai ha $\left.{ }^{-1}\right)$ reduced, but did not eliminate, clubroot severity in canola in a mineral soil (31 DSI compared to 82 DSI in the untreated check). Treatment of specific sites with high rates of metam sodium may prevent or reduce the spread of $P$. brassicae.

\section{Suppression of late blight by resistant tomato cultivars}

M. T. MCGRATH (1)

(1) Cornell University, Riverhead, NY, U.S.A.

Phytopathology 104(Suppl. 3):S3.78

Tomato cultivars bred to be resistant to late blight and tomatoes reported to be less susceptible to this disease were evaluated in two replicated experiments conducted outdoors on Long Island, NY. Seedlings were transplanted on 5 July 2012 and 17 June 2013. Phytophthora infestans genotype US-23 occurred naturally. Symptoms were first observed on 31 July 2012 and 16 Aug 2013. Degree of suppression achieved in 2013 based on AUDPC values relative to Mountain Fresh Plus (no known resistance) was 79\% for Mountain Merit and $87 \%$ for Defiant PHR (both heterozygous for $\mathrm{Ph} 2$ and $\mathrm{Ph} 3$ resistance genes; red slicer fruit type), $88 \%$ for Iron Lady (homozygous $\mathrm{Ph} 2$ and $\mathrm{Ph} 3$; red slicer), 93\% for Mountain Magic (heterozygous $\mathrm{Ph} 2$ and $\mathrm{Ph} 3$; campari), $81 \%$ for Mr Stripey (unknown resistance; slicer), $89 \%$ for Lemon Drop (unknown; cherry), $91 \%$ for Jasper (unknown; cherry), and $98 \%$ for Matt's Wild Cherry (unknown; cherry). New Yorker (Ph1), West Virginia 63 (Ph2), and Juliet (reported resistant) did not suppress late blight relative to Mountain Fresh Plus. Legend $(\mathrm{Ph} 2)$ and Plum Regal $(\mathrm{Ph} 3)$ provided limited suppression. Their foliage was significantly less severely affected than that of Mountain Fresh Plus only in 2012 when an organic fungicide program with Badge X2 copper fungicide, Actinovate, and Regalia was applied to all plots. Applications were started on 14 Aug when late blight was already severe on Mountain Fresh Plus (75\% severity on 20 Aug), thus limiting achievable control.

Evaluation of virulence potential and host resistance to the Goss's wilt pathogen Clavibacter michiganensis subsp. nebraskensis

R. R. MCNALLY (1), R. D. Curland (1), C. A. Ishimaru (1), D. K. Malvick (1)

(1) University of Minnesota, St. Paul, MN, U.S.A

Phytopathology 104(Suppl. 3):S3.78

Goss's wilt is a disease of corn caused by the Gram-positive coryneform bacterium Clavibacter michiganensis subsp. nebraskensis (Cmn). Goss's wilt was first diagnosed in 1969 but resistance breeding reduced Goss's wilt disease pressure and geographically relegated Cmn to central USA. In 2009 Goss's wilt was identified for the first time in Minnesota. The emergence of Goss's wilt in Minnesota was part of a national trend and Cmn distribution now extends from Manitoba to Louisiana. While Goss's wilt epidemics can reduce corn yields as much as $50 \%$, much remains to be learned about the ecology, epidemiology and virulence of Cmn. To explore variations in virulence potential across a diverse range of $\mathrm{Cmn}$ isolates, more than $145 \mathrm{Cmn}$ strains were collected from Colorado, Illinois, Kansas, Minnesota, North Dakota, Nebraska, South Dakota and Texas. Isolates were inoculated into a susceptible corn hybrid and assayed for virulence development in the greenhouse. Cmn exhibited dramatically different levels of aggressiveness. In addition, to explore the reemergence of Goss's wilt, the virulence of Cmn isolates was determined in a range of different corn hybrids as well as inbred lines. Our results confirm that $\mathrm{Cm} n$ is a highly virulent bacterial pathogen and reinforce the need for contemporary control measures for Goss's wilt of corn.

\section{Characterization of Phomopsis spp. involved in spruce decline in Michigan}

C. K. MCTAVISH (1), D. W. Fulbright (2), A. M. Jarosz (2)

(1) Michigan State University, Rochester, MN, U.S.A.; (2) Michigan State University, East Lansing, MI, U.S.A.

Phytopathology 104(Suppl. 3):S3.78

Landscape spruce trees throughout Michigan have experienced a sharp increase in the incidence and severity of branch death, tip blight, and needle drop symptoms. While traditional spruce diseases have a subset of these symptoms, no known pathogen causes the full range of symptoms that characterizes the current epidemic on both juvenile and mature trees that we are calling spruce decline. A 2013 survey of spruce cankers throughout the Lower Peninsula of Michigan revealed Phomopsis spp. as the pathogen most correlated with spruce decline. However, isolates obtained from cankers were highly variable in regards to morphology and virulence. Therefore, the aim of this study was to identify to species the Phomopsis associated with spruce decline. Sequences from the internal transcribed spacer region (ITS) revealed five different groups based on substitutions and insertions at 11 variable positions along the 600 base pair sequences. Three of the groups differed by only one or two base-pair changes, while the fourth group differed by three unique changes. The last group differed from the other four by an additional 8 differences. BLAST searches on GenBank were performed on the shortest sequence of each spruce decline Phomopsis ITS group and preliminary analyses suggest they are part of a poorly resolved lineage involving several species. At least one additional gene will be sequenced in order to characterize the Phomopsis spp. involved in spruce decline.

Spruce decline: Phomopsis spp. may be the main pathogen in the Lower Peninsula of Michigan

C. K. MCTAVISH (1), D. W. Fulbright (2), A. M. Jarosz (2)

(1) Michigan State University, Rochester, MN, U.S.A.; (2) Michigan State University, East Lansing, MI, U.S.A.

Phytopathology 104(Suppl. 3):S3.78

In the last decade, spruce trees throughout Michigan have experienced a sharp increase in the incidence and severity of branch death, tip blight, and needle drop symptoms. While there are known pathogens of spruce that cause a few of these symptoms, no known pathogen causes the full range of symptoms that characterizes the current epidemic on both juvenile and mature trees found in the landscape, on tree farms, and in naturalized areas. There are no visual symptoms other than needle loss and eventual branch death. However, cankers can be found by scraping away the bark of dying stems. A survey of these cankers was performed at 24 sites across the lower peninsula of Michigan in 2013. Eight trees per site were sampled by taking one single branch per tree, and isolates were obtained from two cankers per branch for a total of more than 400 cankers. Phomopsis spp. were most commonly isolated from cankers ( $48 \%$ of isolates that are potentially pathogenic), followed by Diplodia sp. (26\%). In contrast, Cytospora kunzei, a pathogen known to kill branches on spruce trees, was isolated from only $2 \%$ of the cankers. Future work includes defining the species of Phomopsis and Diploidia involved in spruce decline, as well as disease screening trials to determine host susceptibility to this emerging disease.

Genetic variance of Xanthomonas spp. in Begonia, Pelargonium and poinsettia

E. T. MEEKES (1), M. Hooftman (1), B. S. Koenen (1), J. Westerhof (1), M. J. Ebskamp (1)

(1) Naktuinbouw, Roelofarendsveen, Netherlands

Phytopathology 104(Suppl. 3):S3.78

Many Xanthomonas species are able to cause destructive diseases in ornamentals. Information on genetic variability within the Xanthomonas pathovars is scarce, but essential for the development of reliable detection techniques. Here we report on variance of different Xanthomonas pathovars based on BOX-ERIC PCR fingerprinting, gyrase-B sequence analysis and bioassays for three ornamental plant species and five related xanthomonads. For Pelargonium information on two Xanthomonas spp. will be discussed: $X$. hortorum pv. pelargonium, the causal agent of Pelargonium wilt ( $>7 \mathrm{BOX}$ ERIC groups), and a new leaf pathogen which belongs to $X$. campestris based on GyrB. Although the latter is of minor importance, it can cause serious symptoms on Pelargonium in combination with overhead watering. For poinsettia (Euphorbia pulcherrima) information on two Xanthomonas spp. will be provided, $X$. axonopodis pv. poinsettiicola (3 BOX-ERIC groups) and $X$. arboricola pv. poinsettiicola, both foliar diseases. On poinsettia leaf also many look-a-like xanthomonads are present, most belong to $X$. arboricola and are non-pathogenic. For Begonia, data will be presented on X. axonopodis pv. begonia, a foliar disease (4 BOX-ERIC groups). The genetic variability within these Xanthomonas pathovars and across various Xanthomonas species in relation to current and new detection techniques will be discussed.

Evaluation of foliar-applied products' effects on Goss's wilt severity on corn K. M. MEHL (1), K. A. Ames (1), C. A. Bradley (1)

(1) University of Illinois, Urbana, IL, U.S.A.

Phytopathology 104(Suppl. 3):S3.78

In 2010, Goss's wilt (GW), caused by Clavibacter michiganensis subsp. nebraskensis $(\mathrm{Cmn})$, damaged many corn fields in Illinois. Although no data were available to support or oppose them, some recommendations provided to 
farmers with severely affected fields that year were to apply different products as "rescue treatments". To better understand the effect of different products on GW, a field study was conducted in 2012 at 1 location and 2013 at 2 locations, which totaled 3 environments. Products that contained either citric acid or copper hydroxide were applied at different timings relative to inoculation with Cmn (1 d prior, $1 \mathrm{~d}$ after, or 5-7 d after) and were compared with an inoculated, non-treated control. Non-inoculated treatments also were included. In 2013, the citric acid treatment applied $1 \mathrm{~d}$ after Cmn inoculation also included copper sulfate pentahydrate. At 2 of the 3 environments, the only differences in GW severity were between inoculated and non-inoculated treatments, but in 1 of the environments, copper hydroxide for all application timings and citric acid for the post-inoculation timings significantly reduced severity compared to the inoculated, non-treated control. Although reductions were observed in 1 environment, GW was still at damaging levels in these treatments. The products tested did not reduce GW severity to below damaging levels; therefore, host resistance and crop rotation should be utilized to manage this disease.

Association between brown marmorated stink bug (Halymorpha halys) injury and mycotoxin contamination in Virginia field corn

H. L. MEHL (1), D. A. Herbert (1)

(1) Virginia Tech Tidewater AREC, Suffolk, VA, U.S.A.

Phytopathology 104(Suppl. 3):S3.79

During the 2013 Virginia Soybean Insect Pest Survey, large populations of brown marmorated stink bug (BMSB), a recent invasive from Asia, were observed on edges of corn fields. Feeding by BMSB in these fields damaged developing kernels. To determine if BMSB damage increases mycotoxins in Virginia field-corn, ears were collected from edges and interiors of 8 corn fields; kernels were evaluated for BMSB injury, fungal infection, and mycotoxin contamination. Proportions of BMSB-injured kernels were higher from ears collected at the edge (23\%) compared to the interior of fields $(2 \%)$. Fusarium verticillioides was the fungus most frequently isolated from kernels. Other fungi included $F$. graminearum, Aspergillus flavus, A. niger, and Penicillium spp. Kernels from field edges had greater levels of Fusarium infection than kernels from the interior $(P=0.02)$. Fumonisin, zearalenone, deoxynivalenol, and aflatoxin were all detected, but only fumonisin exceeded FDA advisory levels. Concentrations of all four mycotoxins varied among locations, but fumonisin was consistently higher in kernels collected from field edges (4 to $37 \mathrm{ppm}$ ) compared to interiors $(<2 \mathrm{ppm})$. Fumonisin levels were positively correlated with Fusarium infection $\left(\mathrm{r}^{2}=0.33, P<0.0001\right)$ and BMSB damage $\left(\mathrm{r}^{2}=0.26, P=0.0004\right)$. BMSB-injured kernels separated from uninjured kernels had over $100 \mathrm{ppm}$ fumonisin. BMSB damage at the edge of corn fields has the potential to increase overall mycotoxin levels in the crop.

Using comparative genomics to develop a new model of understanding Verticillium wilt resistance in potato

A. MEIER (1), D. Halterman (2)

(1) University of Wisconsin, Madison, Madison, WI, U.S.A.; (2) USDA/ARS, Madison, WI, U.S.A.

Phytopathology 104(Suppl. 3):S3.79

Verticillium wilt (VW) is a persistent and costly disease affecting potato production. VW is caused by the soilborne fungi Verticillium dahliae and $V$. albo-atrum. Because of the difficulty in dealing with this disease, host resistance offers a low input, effective method of control. Most potato cultivars grown in the US are very susceptible, but sources of resistance have been identified in several wild relatives. In tomato, resistance to $\mathrm{VW}$ is conferred by Ve1, a member of a gene family encoding two membrane-bound, extracellular receptors. Homologs of Ve have been identified in wild and cultivated potato, including DM1-3, from the S. tuberosum group Phureja and reference for the potato genome sequence. Comparison of the Ve locus on chromosome 9 of VW susceptible DM1-3 and the resistant diploid hybrid C287 identified structural variation that could determine $V e$ gene function. Using comparative genomics and gene-expression analyses, we have developed a model for VW resistance and susceptibility in potato.

\section{Survey on sweet potato viruses in Brazil}

A. F. MELLO (1), A. K. INOUE-NAGATA (1), F. R. Fernandes (2)

(1) Brazilian Agricultural Corporation, Embrapa Vegetables, Brasília, Brazil; (2) Embrapa Plant Quarantine, Brasília, Brazil

Phytopathology 104(Suppl. 3):S3.79

Sweet potato is a staple crop in many tropical and subtropical countries and its cultivation usually requires low inputs to produce reasonable yields. Most of Brazilian growers use vine cuttings from previous crops to establish new fields. This practice increases the spread and accumulation of pathogens in the field, leading to a gradual reduction on yield and root quality. In Brazil, very little is known about the prevalence and impact of different viruses on sweet potato productivity. The goal of this work is to assess the occurrence of ten sweet potato viruses on plants of the Embrapa Vegetables Sweet Potato Germplasm Collection (SPGC), kept in Brasília, Brazil. Stem samples from symptomatic and asymptomatic sweet potato plants from the SPGC are being grafted on the indicator plant Ipomoea setosa. Grafted plants are visually evaluated and tested for the presence of viruses by Dot-ELISA. Since the SPGC is formed by plants collected in different regions of Brazil, these results will provide valuable information on the major viruses present in the country. New tools of virus identification will be developed in symptomatic plants and negative for the detection tests. This study will enable the identification of the major diseases in sweet potato in Brazil and will help on the development of management strategies. In addition, it will show which viruses should be targeted on the establishment of a virus-free tissue culturing program for sweet potato in Brazil.

Cytotoxic effects of chemical seed treatments on soybean seedlings

F. Melnichukf (1), O. Marchenko (1), G. Fekak (2), A. XUE (2)

(1) Institute of Water Problems and Land Reclamation NAA, Kiev, Ukraine; (2) Agriculture and Agri-Food Canada, Ottawa, ON, Canada

Phytopathology 104(Suppl. 3):S3.79

Soybean plants are sensitive to a large number of pathogens which can significantly reduce the yield. Seed treatment by pesticides controls the development of many diseases in the early stages of growth. However, a number of modern chemical treatments have some negative effects on plants such as damage to the cellular components, expressed as inhibition of mitotic processes in apical meristems. Seeds of the soybean variety Annushka were treated with a total of five different seed treatments at commercially recommended rates and germinated for 120 hours under laboratory conditions at ambient temperatures. After germination, $10 \mathrm{~mm}$ sections of upper portions of petioles were excised and fixed for 24 hours in a $3: 1$ mixture of $95 \%$ ethanol: glacial acetic acid. Apical portions of the petioles were stained in acetocarmine, then squashed in $45 \%$ acetic acid under coverslips and examined microscopically for determination of mitotic indexes. Three seed treatments, captan + carbendazim, metalaxyl + fludioxonil and flutriafol + imazalil + thiabendazole produced reduced mitotic indexes of 4.5, 4.4 and $4.3 \%$, respectively, compared to water-treated controls at $4.9 \%$. Two other formulations produced marked increases in mitotic indexes. These were fludioxonil + kiralaxyl and thiophanate-methyl + azoxystrobin + kiralaxyl that produced mitotic indexes of 9.3 and $8.5 \%$, respectively. Thus seed treatments can have variable effects on subsequent plant growth, development and performance.

Partial characterization of a novel emara-like virus from Cordyline fruticosa $(\mathrm{L}$.) with ti ringspot disease

M. MELZER (1), J. Sugano (2), J. Uchida (3), M. Kawate (3), W. Borth (3), J. $\mathrm{Hu}(3)$

(1) University of Hawaii At Manoa, Honolulu, HI, U.S.A.; (2) University of Hawaii, Kaneohe, HI, U.S.A.; (3) University of Hawaii, Honolulu, HI, U.S.A. Phytopathology 104(Suppl. 3):S3.79

Ti ringspot disease is an emerging virus-like disease of Cordyline fruticosa (L.) reported only in the Hawaiian Islands where it is quickly moving through the island chain. Disease symptoms include small, circular ringspots $2-10 \mathrm{~mm}$ in diameter that can coalesce into amorphous lesions. Transmission electron microscopy revealed double-membrane bodies (DMBs) approximately $85 \mathrm{~nm}$ in diameter in the cytoplasm of cells at the edge of the ringspot lesion but not in cells outside of the lesion or in leaf tissue from asymptomatic plants. These DMBs resembled those associated with infection by members of the genus Emaravirus, a taxon of negative sense ssRNA viruses unassigned to a family. Reverse-transcription PCR using degenerate primers targeting the polymerase gene of emaraviruses amplified a $390 \mathrm{bp}$ product from ten symptomatic plants collected from five locations on the islands of Oahu and Hawaii, but not from five asymptomatic plants collected from two locations on Oahu. When translated into amino acid sequence, identity values of $68,53,52,49,48$, and $47 \%$ to the tentative or member emaraviruses Raspberry leaf blotch virus, European mountain ash ringspot-associated virus, Redbud yellow ringspot virus, Rose rosette virus, Fig mosaic virus, and Pigeon pea sterility mosaic virus were obtained, respectively. These results suggest this novel emara-like virus is the causal agent of ti ringspot disease.

The pathogen and its mycotoxin effect of maize seedling blight in Hexi Corridor in China

Y. MENG (1), L. Luo (1), M. Hu (1), C. Liang (1), O. Mtung'e (1), C. Mortensen (2), J. Li (1)

(1) China Agricultural University, Beijing, China; (2) University of Copenhagen, Copenhagen, Denmark

Phytopathology 104(Suppl. 3):S3.79 
Maize is the key crops in China. Hexi Corridor in Gansu province is the largest seed production basement with an area of more than 10,000 ha and about $50 \%$ supply of all the hybrid maize seeds in China. In Hexi Corridor, maize seedling blight (MSB) was first observed in 2000 and the seriousness has increased rapidly in recently years. Previous studies verified that Fusarium verticillioides, $F$. oxsporiun, $F$. graminearum, $F$. subglutinans and Rhizoctonia zeae could be the pathogen of MSB. However, different results compared to the previously reported in Hexi corridor were found in this study. 30 diseased maize plants from Hexi corridor were collected in April 2013 for pathogen isolation and diagnosis. Based on fungal morphological identification and ITS sequence analysis, a total of 37 identified strains, including $23 F$. graminearum, $6 F$. chlamydosporum, $5 F$. oxysporum and $3 F$. equiset were identified. The pathogenicity test on male parent of variety Zhengdan 958 confirmed Koch's postulates. In this test, only 7 strains of $F$. graminearum showed high virulence on maize seedlings and were identified as the key pathogen of the MSB. The fungal metabolite in Czapek liquid media of $F$. graminearum caused significant root rot on maize seedlings in $4 \mathrm{~d}$ and decreased root activity up to $20 \%$ in $72 \mathrm{~h}$. The results suggest that the mycotoxin produced by $F$. graminearum could weaken the nutrient absorption and metabolism, as well as reduce the disease resistance of maize root.

Control of Colletotrichum acutatum on infected strawberry transplants

J. MERTELY (1), N. A. Peres (1)

(1) University of Florida-GCREC, Wimauma, FL, U.S.A.

Phytopathology 104(Suppl. 3):S3.80

Strawberry runner plants infected with Colletotrichum acutatum grow poorly after transplanting. Old roots are dark and die prematurely; new structural roots are lesioned or fail to develop. In severe cases, the basal crown is invaded and the plant dies. Disease control consists of dipping plants in solutions or suspensions of fungicides or biologicals prior to planting. Runner plants naturally infected with $C$. acutatum were dipped in selected products for $5 \mathrm{~min}$, transplanted, and assessed for mortality and yield in a replicated trial conducted during the 2013-14 growing season. Of eight products tested, only three significantly reduced plant mortality and significantly increased berry yield. Cyprodinil + fludioxonil (Switch), penthiopyrad (Fontelis), and Streptomyces lydicus (Actinovate) each reduced mortality from $15 \%$ in the water-treated control to less than $5 \%$. Early season yields were increased from $2000 \mathrm{~kg} / \mathrm{ha}$ in the control to 3300,2900 , and 3100 in the respective treatments. The yield increases were primarily due to reduced plant mortality and increased fruit numbers, plus a slight increase in average fruit weight. Other products such as azoxystrobin (Abound), Bacillus subtilis (Serenade Soil), hydrogen dioxide (Oxidate), iprodione (Rovral), knotweed extract (Regalia), and a mixture of Abound and Actinovate and did not provide significant control.

\section{Mustard seed meal amendments for suppression of Meloidogyne incognita} on tomato

S. L. F. MEYER (1), I. A. Zasada (2), S. M. Rupprecht (1), C. R. Hooks (3), M. J. Morra (4), K. L. Everts (5)

(1) USDA-ARS, Nematology Laboratory, Beltsville, MD, U.S.A.; (2) USDAARS Horticultural Crops Research Laboratory, Corvallis, OR, U.S.A.; (3) Department of Entomology, University of Maryland, College Park, MD, U.S.A.; (4) Division of Soil and Land Resources, University of Idaho, Moscow, ID, U.S.A.; (5) University of Maryland College Park, Salisbury, MD, U.S.A.

Phytopathology 104(Suppl. 3):S3.80

Mustard seed meal is applied to soil as a fertilizer and for suppressing weeds and pathogens. Brassica juncea (Bj) 'Pacific Gold' and Sinapis alba ( $\mathrm{Sa}$ ) 'IdaGold' seed meals were tested for suppression of Meloidogyne incognita on tomato 'BHN 444'. In greenhouse trials these treatments (all 0.25\% weight meal:weight soil) were evaluated (eight pots in each of two trials): untreated controls \pm plastic tarp; linseed controls \pm tarp; $S a ; B j ; 3: 1 S a: B j ; 1: 1 S a: B j$; and $1: 3 \mathrm{Sa}: B j$. Soil in each pot was inoculated with 5,000 nematode eggs and planted with tomato seedlings two weeks later. After five weeks, the highest and lowest egg counts (Trial 1) were 1,283 eggs/g root (tarped control) and 7 eggs/g root $(B j ; P \leq 0.05)$. In Trial 2 , eggs/g root were similar in all mustard seed meal amendments, but 3:1 Sa:Bj suppressed egg numbers compared with the untreated or linseed controls (174 eggs/g root vs. 824-1,099 eggs/g root). In a 2013 tomato field study, treatments were ( $\mathrm{kg} / \mathrm{ha}): 560$ 20-03-18 NPK fertilizer + 5 0-44-0 NPK fertilizer; 1,904 Sa; 1,904 Bj; $952 \mathrm{Sa}$; $952 \mathrm{Bj} ; 1,904$ (3:1 $S a: B j) ; 1,904$ (1:1 $S a: B j) ; 1,904$ (1:3 Sa:Bj); and 1,904 Bj (finely ground), five replicates per treatment. At harvest, there were no significant differences among treatments in fresh root weights or number of eggs/g root (two root systems per plot). While some mustard seed meals suppressed M. incognita in the greenhouse, the rates applied in the field were not sufficient to reduce $M$. incognita on tomato.
Validation of a TaqMan diagnostic assay for the systematic development of Phytophthora genus and species specific markers

T. D. MILES (1), F. N. Martin (1), G. J. Bilodeau (2), M. D. Coffey (3)

(1) USDA/ARS, Salinas, CA, U.S.A.; (2) Canadian Food Inspection Agency, Ottawa, ON, Canada; (3) University of California - Riverside, Riverside, CA, U.S.A.

Phytopathology 104(Suppl. 3):S3.80

The genus Phytophthora contains many species that are not native to the USA and have the potential to cause significant damage to agriculture and native ecosystems. A genus and species-specific diagnostic assay was developed based on mitochondrial gene order differences that allowed for the systematic development of species-specific TaqMan probes for their identification. Previous research validated the marker system for 14 Phytophthora spp. Here we will discuss $130+$ unique probes developed in silico for additional Phytophthora spp., with the focus on non-native species. Additional speciesspecific TaqMan probes (including $P$. austrocedrae, $P$. cajani, $P$. parvispora, $P$. melonis, $P$. multivesiculata, $P$. pinifolia, $P$. pistaciae, $P$. primulae, $P$. quercina, and $P$. vignae) were validated with 96 valid Phytophthora spp. and 14 provisional species. All probes were found to be species-specific and could be multiplexed with a genus-specific probe and a plant internal control (IC). The lower limit of linear detection using purified DNA was $100 \mathrm{fg}$ in all assays. An IC was developed to check for PCR inhibitors common in soil DNA extractions. It should be possible to develop probes capable of distinguishing $>85 \%$ of the Phytophthora spp. investigated; we are currently in the process of validating an additional 18 probes. This system represents a comprehensive, hierarchal approach to increase detection capability and reduce the introduction of these non-native Phytophthora species.

Rapid isothermal detection and species-specific assay of Phytophthora in plant samples using recombinase polymerase amplification

T. D. MILES (1), F. N. Martin (2), M. D. Coffey (3)

(1) USDA/ARS, East Lansing, CA, U.S.A.; (2) USDA/ARS, Salinas, CA, U.S.A.; (3) University of California - Riverside, Riverside, CA, U.S.A.

Phytopathology 104(Suppl. 3):S3.80

Recently several isothermal amplification techniques have been developed that are extremely tolerant towards inhibitors present in many plant extracts. Recombinase polymerase amplification (RPA) assays for the genus Phytophthora have been developed to provide a simple and rapid method to macerate plant tissue and detect target DNA using primers and a labeled probe. Four RPA assays were developed, a Phytophthora genus-specific assay, $P$. ramorum and $P$. kernoviae species-specific assays and a plant internal control. Assays were tested for sensitivity and specificity using DNA extracted from multiple plant species, 96 Phytophthora and 22 Pythium spp. The lower limit of linear detection using purified DNA was 1 pg in all pathogen RPA assays. Six different extraction buffers were tested for use during plant tissue maceration and the assays were validated in the field with 222 symptomatic plant samples from over 51 different hosts throughout California. Ninety-one samples were positive using the Phytophthora genus specific RPA test and the same samples were also positive using TaqMan PCR and traditional isolation techniques. A technique for the generation of sequencing templates from positive samples to confirm species identification has been developed. These RPA assays have added benefits over traditional technologies because they run in less than $25 \mathrm{~min}$, don't require DNA extraction, are field portable and are more specific than current immunologically based methods.

Capacity of compost with high content of particulate organic matter to suppress root rot disease of tomato plants

P. A. MILLAS (1)

(1) Inst de Investigaciones Agropecuarias, Chillan, Chile

Phytopathology 104(Suppl. 3):S3.80

Compost is often reported as a substrate that is able to suppress soil-borne pathogens, however the capacity to reduce disease is not consistent and mechanisms associated are not clearly identified. Particulate organic matter (POM) is organic matter present in compost that recently has been associated to disease suppression. The objetive was determine the effects of incorporating compost rich in POM to potting mix on severity of root rot caused by Pythium ultimum and Rhizoctonia solani in tomato plants, on microbial activity, and populations of antagonist. Five tomato seed was planted on a series of potting mixes prepared by blending compost with perlite $(0: 100 ; 25: 75 ; 50: 50 ; 75: 25$, and 100:0 vol/vol). Pathogenic inoculums were subsequently added. Root rot severity was rated on 0 to 4 scale at $40 \mathrm{~d}$ after sowing. Microbial activity was estimated in fluorescein diacetate (FDA) hydrolysis assay. To determinate antagonistic populations serial dilutions were plated on selective media for isolation of Trichoderma, Actinomycetes and Pseudomonads, total culturable copiotrophic and oligotrophic bacteria. Compost percentage had effects on disease severity, mixes containing 100, 75 
and $50 \%$ compost did not have significant differences with the control without inoculums, showed severity less than 0.5 . In contrast, the mix $0 \%$ compost reached severity of 2 . Add compost in potting mixes suppress root rot and increase significantly FDA and Actinomycetes population.

A new gene in the Luteoviridae essential for systemic infection

W. A. MILLER (1), E. Smirnova (2), A. E. Firth (3), D. Scheidecker (2), A. Rakotondrafara (4), B. Chung (3), V. Ziegler-Graff (2)

(1) Iowa State University, Ames, IA, U.S.A.; (2) Institut de Biologie Moléculaire des Plantes, Strasbourg, France; (3) University of Cambridge, Cambridge, United Kingdom; (4) University of Wisconsin, Madison, WI, U.S.A. Phytopathology 104(Suppl. 3):S3.81

The Luteoviridae family includes many important plus strand RNA viruses, e.g. Barley yellow dwarf, Potato leafroll, and Turnip yellows (TuYV) viruses. These viruses generate a subgenomic mRNA (sgRNA1) for translation of (i) the coat protein (CP), (ii) a C-terminally extended form of the CP (CP-RTD), and (iii) open reading frame 4 (ORF4). ORF4 is nested within the CP ORF in a different reading frame. Computational analysis of $>200$ Luteovirid genomes revealed a new ORF (ORF3a) in what was thought to be the 5' untranslated region of sgRNA1. ORF3a (i) contains about 50 codons, (ii) lacks an AUG start codon, (iii) is in a different reading frame from either the CP ORF or ORF4, and (iv) terminates between the CP ORF start codon and the ORF 4 start codon. In vitro translation showed that ORF3a of TuYV initiates at an ACG codon. Changing this codon to AUG or AGC greatly increased or eliminated translation of ORF3a, respectively. Immunoblots detected the protein product of ORF3a (P3a) in infected cells. Previous studies of deletion mutants unwittingly showed that ORF3a is unnecessary for virus replication in single cells. However, Agroinfection with TuYV containing the above mutations in ORF3a start codons showed that ORF3a is required for viral systemic movement in plants and that overexpression of ORF3a can increase symptoms in inoculated leaves of Nicotiana benthamiana. Thus, P3a is a new participant in the long distance spread of luteoviruses within their host.

Impact of watered-in preventive DMI fungicide applications and paclobutrazol on foliar disease severity on golf putting greens

G. L. MILLER (1), D. T. Earlywine (1)

(1) University of Missouri, Columbia, MO, U.S.A

Phytopathology 104(Suppl. 3):S3.81

Preventive applications of DMI fungicides provide control of several soilborne turfgrass diseases on golf putting greens. The objectives of this twoyear field study were to determine the impact of these watered-in applications on foliar diseases, and evaluate the impact of application timing of paclobutrazol, a plant growth regulator, on disease severity. Plots were $1.5 \mathrm{~m}$ $\times 1.5 \mathrm{~m}$, and arranged in a randomized complete block with four replications. The experimental design was a split-plot with creeping bentgrass cultivar ('Penncross' and 'Penn A-4') as the main plot, and fungicide and paclobutrazol application as subplots. Triadimefon $\left(0.16 \mathrm{~g} \mathrm{~m}^{-2}\right)$, tebuconazole $\left(0.08 \mathrm{~g} \mathrm{~m}^{-2}\right)$, metconazole $\left(0.04 \mathrm{~g} \mathrm{~m}^{-2}\right)$, and triticonazole $\left(0.06 \mathrm{~g} \mathrm{~m}^{-2}\right)$, were applied twice in late April and May. Paclobutrazol $\left(0.03 \mathrm{~g} \mathrm{~m}^{-2}\right)$ was applied alone, in a tank-mix with the fungicide application, $7 \mathrm{~d}$, or $14 \mathrm{~d}$ after the fungicide application. Fungicide and fungicide + paclobutrazol tank-mix applications were immediately watered-in with $5 \mathrm{~mm}$ of irrigation. Dollar spot severity was significantly lower in fungicide-treated plots than untreated plots and plots treated with paclobutrazol alone. Triadimefon-treated plots had lower AUDPC values than other fungicide treatments. Short-lived phytotoxicity (bronzing) was observed in plots treated with both paclobutrazol and fungicide. Spring preventive fungicide applications targeted at soilborne disease control also provided control of a foliar disease.

Pseudomonas syringae benefits from host-derived carnitine during spermosphere colonization

M. MILLICAN (1), A. Klein (1), Y. J. Lee (1), G. A. Beattie (1)

(1) Iowa State University, Ames, IA, U.S.A.

Phytopathology 104(Suppl. 3):S3.81

Knowledge of choline and related compounds, including carnitine, is useful for predicting and limiting the growth of phytopathogens during their colonization of plants. Here we investigated the ability of Pseudomonas syringae to utilize these compounds as osmoprotectants and as carbon and nitrogen sources in the plant host environment. $P$. syringae contains orthologs of the $P$. aeruginosa genes caiX, $c d h A B C$, and $d h c A B$, which are involved in the transport and breakdown of carnitine. Although a mutant lacking the first step in the catabolic pathway $(\triangle c d h A)$ was not altered in epiphytic fitness, a mutant lacking the second step $(\triangle d h c A B)$ showed reduced fitness, consistent with elevated $d h c A B$ but not $c d h A$ expression on leaves; these results suggest a dual function for $P$. syringae $d h c A B$ genes on leaves. Due to the role of carnitine in fatty acid metabolism, we hypothesize that cartinine is abundant when seedlings begin to utilize their lipid stores. P. syringae $\triangle c d h A$ established smaller populations on seeds than the wild type did, two days postgermination. Mass spectrometry analysis of seed exudates showed a transient increase in carnitine levels during this two-day period, consistent with the window of carnitine exploitation by $P$. syringae. We showed that $P$. syringae specifically utilizes carnitine in the spermosphere, thus identifying a potential factor contributing to the early establishment of $P$. syringae populations.

Characterization of race specific adult plant resistance to stripe rust in soft red winter wheat

E. MILUS (1), D. Moon (1)

(1) University of Arkansas, Fayetteville, AR, U.S.A.

Phytopathology 104(Suppl. 3):S3.81

Although there has been little research on race specific adult plant resistance (RSAPR), this type of resistance appears to be the most important type of resistance protecting soft red winter wheat (SRWW) cultivars in the United States from stripe rust. Seedlings are susceptible, but adult plants are moderately to highly resistant. The race specific nature of the resistance becomes evident when different isolates of the pathogen are used to inoculate flag leaves of adult plants. Based on inoculations with isolates collected in the field from 1990 to 2013, at least seven different sources of RSAPR have been identified in cultivars Progeny 166, Arcadia, Mason, AGS 2035, LA 821, 26R61, and a breeding line VA07W-415. Interestingly, the Mason source was susceptible to isolates collected before 2000 but has been highly resistant to all isolates collected since 2000 . Nearly all contemporary SRWW cultivars in the United States have stripe rust resistance that appears to be due to RSAPR, but the number and identity of the resistance genes are unknown. RSAPR may be an effective strategy for controlling stripe rust in other regions. More research is needed to identify the RSAPR genes in adapted and exotic germplasm and to identify races of the stripe rust pathogen based on reactions to RSAPR genes.

Use of composted pig manure separated solids for the control of Potato Early Dying in Manitoba

O. I. MOLINA (1), M. Tenuta (1), F. Daayf (1)

(1) University of Manitoba, Winnipeg, MB, Canada

Phytopathology 104(Suppl. 3):S3.81

Application of compost to control soil-borne plant pathogens is of increasing interest. In Manitoba, we previously observed reduction of Potato Early Dying (PED) in plots treated with composted cattle manure and composted pig manure separated solids (CSS). In 2013, a study was established on two commercial fields planted to Russet Burbank (RB) in Manitoba to determine the effect of CSS at fresh weight rates 20,40 , and $80 \mathrm{Mg} \mathrm{ha}^{-1}$ on yield and reduction of PED. Fields had $1.5 \%$ and $5.9 \%$ of soil organic matter (SOM), respectively. The effect of CSS in the two fields was compared against a high fertility treatment and the standard fertility producer-management (control). The incidence and severity of PED, soil inoculum of Verticillium dahliae, plant-available soil nitrogen and phosphorus and marketable yield were evaluated. The area under the disease progress curve in plots treated with high fertility and CSS was lower than the control. However this effect was only found in the site with the lowest SOM (1.5\% compared to $5.9 \%)$. In the site with lower SOM, CSS increased yield when added at $40 \mathrm{Mg} \mathrm{ha}^{-1}$. In contrast, the higher rate of compost encouraged high production of aboveground biomass instead of tuber yield. The effect of the high rate of CSS on lower tuber yield was particularly noticeable on the field with higher SOM. In general, single applications of CSS can reduced PED and increase potato yield in RB, however the potential effect seems dependent upon SOM content.

Clematis chlorotic mottle virus, a novel virus occurring in clematis in the USA

D. MOLLOV (1), B. Lockhart (2), A. Phibbs (3), T. Creswell (4), G. Ruhl (4), E. Dorman (5), G. Kinard (1), R. Jordan (1)

(1) USDA ARS, Beltsville, MD, U.S.A.; (2) University of Minnesota, St. Paul, MN, U.S.A.; (3) Wisconsin Department of Agriculture, Madison, WI, U.S.A.; (4) Purdue University, Lafayette, IN, U.S.A.; (5) Michigan Department of Agriculture, East lansing, MI, U.S.A.

Phytopathology 104(Suppl. 3):S3.81

Clematis is a genus of temperate climbing vines that are popular as ornamentals. Samples from domestic and international sources showing symptoms of yellow mottling and veining, chlorotic ring spots, line pattern mosaics, and in some cases flower distortion and discoloration were received at several diagnostic clinics in the mid-western USA. Spherical virus particles $(28-30 \mathrm{~nm})$ were observed by transmission electron microscopy (TEM) in samples associated with symptoms. The virus was detected by TEM and/or RT-PCR in 35 of 80 samples including Clematis sp. 'Barbara', 'Bees Jubilee', 
'Dancing Dorien', 'Dancing Smile', 'Gypsy Queen', 'Henryi', 'Jackmanii', 'Natascha', 'Niobe', and 'Still Waters'. This previously undescribed virus was provisionally named Clematis chlorotic mottle virus $(\mathrm{ClCMoV})$. The $\mathrm{ClCMoV}$ ssRNA is approximately $4 \mathrm{~kb}$ with four putative open reading frames (ORFs) having similarity to members of Tombusviridae. These ORFs have the highest amino acid sequence similarities (38-59\%) to Rosa rugosa leaf distortion virus (RrLDV), Rose yellow leaf virus (RYLV), Pelargonium line pattern virus (PLPV), Pelargonium chlorotic ring pattern virus (PCRPV), Elderberry latent virus (ELV) and Pelargonium ringspot virus (PRSV). Based on these preliminary results $\mathrm{ClCMoV}$ appears to be a novel virus in Tombusviridae. Phylogenetic analyses group ClCMoV with RrLDV, RYLV, PLPV, ELV, PRSV, and PCRPV into a distinct group, suggesting a new genus in Tombusviridae may be warranted.

Effects of temperature and leaf wetness duration on antifungal activity of Bacillus subtilis MBI600 for management of sheath blight in rice

L. MONCAYO (1), X. G. Zhou (2), L. Wilson (2), Y. K. Jo (1)

(1) Texas A\&M University, College Station, TX, U.S.A.; (2) Texas A\&M AgriLife Research and Extension Center, Beaumont, TX, U.S.A.

Phytopathology 104(Suppl. 3):S3.82

Sheath blight caused by Rhizoctonia solani is the most important disease in rice in the southern U.S. Timely application of fungicides is effective but can cause negative impacts on the environment and human health. Bacillus subtilis strain MBI600 is the promising biological control agent that may provide an alternative option for management of sheath blight. In this study, effects of temperature and leaf wetness duration on the antimicrobial efficacy of strain MBI600 were evaluated under controlled environmental conditions. Seedlings of the two rice cultivars Presidio and Jasmine 85, susceptible and moderately resistant to sheath blight, respectively, were spray treated with strain MBI600 and followed by inoculation with $R$. solani. Treated plants were exposed to the combinations of temperature $(10,15,20,25,30,35$, or $\left.40^{\circ} \mathrm{C}\right)$ and leaf wetness duration $(4,9,14,19$, or $24 \mathrm{~h})$. Sheath blight severity was measured at 8 days after inoculation. The efficacy of strain MBI600 in reducing sheath blight development was significantly affected by temperature and leaf wetness duration. Its efficacy generally increased with the increase of temperature but decreased with the increase of leaf wetness duration. The results of this study will help to develop an optimized method to improve the efficacy of strain MBI600 for management of sheath blight in rice.

Validating forensics tools for crop biosecurity: Simple sequence repeat typing of Fusarium proliferatum associated with salmon blotch of onions I. MONCRIEF (1), C. Garzon (1), S. Marek (1), A. Gamliel (2), J. Stack (3), Y. Issac (2), J. Fletcher (1)

(1) Oklahoma State University, Stillwater, OK, U.S.A.; (2) Inst. Agricultural Engineering ARO, The Volcani Center, Bet Dagan, Israel; (3) Kansas State University, Manhattan, KS, U.S.A.

Phytopathology 104(Suppl. 3):S3.82

Ensuring the security of agricultural resources is critical to national security. Microbial forensics technologies can help to identify potential pathogen sources. Since 2008, unprecedented outbreaks of salmon blotch of onion, caused by $F$. proliferatum $(F p)$, have occurred in southern Israel. Though presumed to be of natural causes, the disease provided an opportunity to validate plant pathogen forensic tools in the field. To test whether onion sets grown in northern Israel and shipped to southern farms for planting could be a source of the fungus. The diversity and structure of $F p$ populations from different locations and sample types were investigated. Ninety-one $F p$ isolates from onion sets, field soil, nearby date palm orchards, weeds and other local vegetation, and symptomatic onion bulbs were characterized using simple sequence repeat (SSR) typing. Of 17 SSR loci screened, 6 were informative. SSR analysis revealed two distinct $F p$ populations. Onion set strains, from northern Israel, clustered together. Southern strains, whether from soil, weeds, onion bulbs, or adjacent palm trees, also clustered together and were genetically distant from the northern population. The data indicate that the sets were unlikely to have been the source of the fungus responsible for the outbreaks of salmon blotch and demonstrate that SSR typing can be useful in investigations of pathogen origin.

Symptom expression and detection of Grapevine red blotch associated virus

J. MONIS (1), L. A. Miles (1)

(1) Eurofins/STA Laboratories Inc, Gilroy, CA, U.S.A.

Phytopathology 104(Suppl. 3):S3.82

Grapevine red blotch associated virus (GRBaV) is a newly described virus associated with red blotch disease. Due to the similarity of foliar symptoms the effect of GRBaV infection was confused with leafroll disease and/or other disorders that cause reddening in red fruited grape varieties. In spite of its name, GRBaV was detected in white fruited varieties. Besides the typical foliar symptoms, GRBaV has been reported to affect sugar accumulation in grapevines reducing the brix values and delaying the harvest of fruit. To determine if the detection of virus is seasonal, our lab tested GRBaV-infected vines throughout the year (2012 fall winter/2013 spring/summer) using tissue from different sections of the vine. Vine samples were collected from basal and apical sections of the vine, green or lignified canes, codon and trunk were tested. In addition, inflorescence from infected samples from vines were dissected and tested. The results showed that the detection of GRBaV was equally sensitive throughout the season in all grapevine tissues tested. The presentation will also describe symptoms of white and red fruited varieties infected with GRBaV.

Tospovirus-vector interactions in Costa Rica: Establishing the research system

M. MONTERO-ASTÚA (1), L. Garita (1), E. Vásquez-Céspedes (1), W. Villalobos (1), L. Moreira (1)

(1) Universidad de Costa Rica, San Jose, Costa rica

Phytopathology 104(Suppl. 3):S3.82

Tospoviruses are plant-infecting viruses transmitted by thrips. Transmission efficiency varies across thrips and tospovirus species. Our research objectives were to standardize a molecular taxonomy protocol to aid in thrips identification, and to determine the efficiency of thrips-mediated transmission of Impatiens necrotic spot virus (INSV) in Costa Rica. To this end, a thrips colony was established on green beans from field samples. Individual thrips were used to test DNA extraction by salting-out and resin-based protocols. The mitochondrial cytochrome $\mathrm{c}$ oxidase subunit I was PCR-amplified and sequenced. Blast similarity search in NCBI identified the samples as Frankliniella schultzei $(96 \%$ identity), a known Tospovirus vector. Transmission assays were thus conducted for this vector, with a modified protocol in which the feeding periods for tospovirus acquisition (AAP) and inoculation (IAP) were increased to $72 \mathrm{~h}$. Field collected, INSV-positive sweet pepper fruits were used as a virus source for AAP and Emilia forsbergii leaf discs were used for IAP. INSV presence in the leaf discs was tested by DAS-ELISA and $11(18 \%)$ and $5(8 \%)$ of leaf discs $(n=60)$ per experiment were INSV-positive, suggesting a low vector capacity for the $F$. schultzei population. In contrast, $F$. occidentalis adults collected from an INSV-infected field showed a $53 \%(\mathrm{n}=60)$ transmission. INSV control strategies should consider differential contribution of thrips species to virus epidemiology.

Inhibition of Ophiognomonia clavigignenti-juglandacearum by Juglans species bark extracts

M. J. MOORE (1), M. E. Ostry (1), A. D. Hegeman (2), A. C. Martin (2)

(1) USDA Forest Service, NRS, St. Paul, MN, U.S.A.; (2) University of Minnesota, St. Paul, MN, U.S.A.

Phytopathology 104(Suppl. 3):S3.82

A rapid and reliable technique is needed for selecting butternut trees (Juglans cinerea) with resistance to butternut canker. We investigated the potential of a bark extract bioassay to detect levels of resistance to Ophiognomonia clavigignenti-juglandacearum $(O c-j)$, the causal agent of butternut canker. Both reagent grade naphthoquinones and crude bark extracts of Juglans species inhibited germination of $O c-j$ conidia. The disc diffusion bioassay revealed that the level of germination inhibition varied by selection of butternut and species of Juglans tested. Over a three year period, butternut trees selected for disease resistance could be distinguished from unselected trees, depending on the month of bark collection. The levels of inhibition of conidia germination roughly correlated to the level of resistance observed in field inoculations of the trees. Quantification of the naphthoquinone compounds juglone and plumbagin in butternut bark was performed using ultra-performance liquid chromatography mass spectrometry. Concentrations of these two compounds varied by month and by individual tree, and juglone levels correlated with the bark extract bioassay in some months. Juglone may account in part for the observed range of inhibition levels in the bioassay and variation in canker resistance among selections of butternut exhibited in the field. This bioassay may have potential use for selecting butternut with resistance for conservation and restoration purposes.

Modeling the progress of ceratocystis wilt on mango through a Bayesian approach

W. B. MORAES (1), L. Maffia (1), V. C. Garnica (1), A. G. C. Souza (2), F. F. Silva (3)

(1) Departamento de Fitopatologia, Universidade Federal de Viçosa, Viçosa, Brazil; (2) EMBRAPA Milho e Sorgo, Sete Lagoas, Brazil; (3) Departamento de Estatística, Universidade Federal de Viçosa, Viçosa, Brazil

Phytopathology 104(Suppl. 3):S3.82

Ceratocystis wilt of mango, caused by $C$. fimbriata, is a destructive disease. To help understanding disease epidemiology, we studied its temporal 
dynamics and compared epidemics from two production areas. Disease progress was followed over 15 months in five orchard units, two in ItaocaraRio de Janeiro State (RJ1 and RJ2) and three in Frutal-Minas Gerais State (MG1, MG2, and MG3). The logistic, Gompertz, and monomolecular models were fitted to data of incidence, severity, and death through the Bayesian analysis. Best fit was with the Gompertz, assuming binomial distributions for incidence and dead plants, from which samples of the marginal posterior parameters distribution were used to compare the epidemics. Higher values of initial and maximum of the incidence, severity, and dead plants were found in the marginal posterior distribution of the Itaocara epidemics. The marginal posterior distribution of RJ2 epidemics had a higher incidence rate; the distributions of the RJ1 and MG1 - 3 epidemics did not differ. Lower severity and mortality rates were found in the marginal posterior distribution of the Itaocara epidemics; there were no differences between the distributions of the Frutal epidemics. It was concluded that the temporal dynamics of ceratocystis wilt in Itaocara and Frutal differed most likely because of differences on the initial inoculum and pathogen dispersal, and the Bayesian analysis is potentially useful to describe and compare epidemics.

\section{Epidemiological description of the syndromes associated with the} Ceratocystis wilt of mango

W. B. Moraes (1), A. G. C. SOUZA (2), L. A. Maffia (1), V. C. Garnica (1) (1) Departamento de Fitopatologia, Universidade Federal de Viçosa, Viçosa, Brazil; (2) EMBRAPA Milho e Sorgo, Sete Lagoas, Brazil

Phytopathology 104(Suppl. 3):S3.83

Although Ceratocystis wilt, caused by C. fimbriata, is a destructive disease, its epidemiology is poorly understood. There are two types of syndromes associated with the disease differentiated by the progression of symptoms: root rot and crown rot. The objective of the study was to describe and compare the types of syndromes associated with the disease from two production areas. Epidemics of ceratocystis wilt were followed over 15 months in five experimental units, two in Itaocara-Rio de Janeiro State (RJ1 and RJ2) and three in Frutal-Minas Gerais State (MG1, MG2, and MG3). The pattern of occurrence of the disease in each plant differed between the regions. In Itaocara, the severity values varied in a continuum from 0.1 to $100 \%$, whereas in Frutal the severity in a diseased plant was always 100\%. When the disease was first detected, the severity values in each plant in Itaocara varied from 0.1 to $60 \%$, whereas the severity value in Frutal was always $100 \%$. Itaocara diseased plants initially exhibited a darkening of the wood tissues in branches, and the darkening progressed towards the trunk. In Frutal, the wood darkening was concentrated in the roots and towards the base of the trees. Additionally, in Itaocara, it took longer for the diseased plants to die than in Frutal. It was concluded that the dynamics of ceratocystis wilt in Itaocara and Frutal resembled the crown rot and root rot syndromes, respectively.

Cytokinin levels correlate with virulence in Ustilago maydis dikaryon and solopathogenic strain infections

E. N. MORRISON (1), R. Emery (1), B. J. Saville (1)

(1) Trent University, Peterborough, ON, Canada

Phytopathology 104(Suppl. 3):S3.83

Ustilago maydis is a causative agent of common smut of corn. This biotrophic fungal plant pathogen is being developed as a model for investigating the role cytokinins play during biotrophic fungal infection of plants. Cytokinins [CKs] are a group of phytohormones that are commonly associated with actively dividing tissues. Infection of corn by $U$. maydis stimulates uncoordinated cellular division, resulting in tumor formation on aerial portions of the plant including the cob. Previous studies have noted differences in virulence between $U$. maydis dikaryon and diploid infections. The current study examines two $U$. maydis strains, a dikaryon and solopathogen that are frequently used in genetic and pathogenic analysis. Samples, from infected cob tissue, were taken at various timepoints post infection and biochemical profiling conducted using liquid-chromatography-electrospray ionizationtandem mass spectrometry, LC- (ESI) MS/MS. This analysis revealed that there are altered levels of major CKs between the uninfected control and the $U$. maydis infected tissue as well as differences between the dikaryon and solopathogen infection. The increased cytokinin levels in the more virulent dikaryon relative to the solopathogenic strain are consistent with a role for cytokinins in moderating virulence during biotrophic infection. Furthermore, this analysis provides a base for investigating the control of phytohormone dynamics during biotrophic infection of plants.

Capacity of Chilean Andisol soils to induce the phenomenon of take-all decline associated with presence of 2,4-DAPG producing Pseudomonas spp.

E. A. MOYA-ELIZONDO (1), N. Brellenthin (1), N. L. Arismendi (1), H. A. Doussoulin (2)
(1) Universidad de Concepcion, Chillan, Chile; (2) Universidad Austral de Chile, Valdivia, Chile

Phytopathology 104(Suppl. 3):S3.83

Take-all disease, caused by Gaeumannomyces graminis var. tritici, can be managed by monoculture-induced take-all decline (TAD). TAD is a phenomenon associated with the natural suppressiveness of the disease and the presence of 2,4-diacetylphloroglucinol (2,4-DAPG)-producing Pseudomonas in some soils. Pseudomonas spp. that produce 2,4-DAPG were recently determined in commercial wheat fields in Chile, but their influences to induce TAD is unknown. Natural Chilean Andisol soils from Perquenco, CajónTemuco, Gorbea (Araucania Region), Valdivia (Los Ríos Region), and Osorno (Los Lagos Region) were studied in their capacities to induce TAD in a microscosm assay under glasshouse conditions during five wheat-growing cycles. Isolation and detection of 2,4-DAPG-producing rhizobacteria from the microcosms were conducted in diluted plates and use of PCR at seedling and mature spike stages in each growth cycle. Soils from Perquenco, CajónTemuco, and Osorno showed presence of bacterial populations with the gene $p h l D+$ and after three cycle of wheat crop reduced notoriously the severity of take-all disease in the inoculated microcosms. Soils from Valdivia and Gorbea did not show presence of 2,4-DAPG-producing bacteria and wheat plants were severely affected by the disease. Results suggest that inducing TAD in presence of 2,4-DAPG-producing Pseudomonas spp. in Chilean soils could be explored to reduce take-all disease in wheat crops. FONDECYT Nº11110105.

Prevalence and fungal isolates associated with Korogwe leaf spot disease (KLS) of sisal

O. G. MTUNG'E (1), L. Luo (1), X. Liu (1), R. B. Mabagala (2), Y. Diao (1), Y. Meng (1), J. Li (1)

(1) China Agricultural University, Beijing, China; (2) Sokoine University of Agriculture, Morogoro, Tanzania

Phytopathology 104(Suppl. 3):S3.83

Sisal (Agave sisalana Perrine) is a hard fibre producing plant grown mainly in tropical and subtropical countries. Korogwe leaf spot disease of sisal is characterised by chocolate brown concentric scab like eruption that was first observed in 1951 in Korogwe District, Northern Tanzania. The disease causes difficulty in fibre extraction leading to darkened, low quality fibre. To date there is no report of its distribution within and out of Tanzania sisal fields. The actual cause of the disease has not been confirmed, and the isolated pathogens have been poorly described in few reports. A total of 64 affected leaf samples from 5 sisal farms located in Guangxi and Hainan provinces in the Southern China, and 13 farms located in the North Eastern Tanzania were collected in July to September 2013 for pathogen isolation and identification. Result showed that the disease is wide spread to all locations visited in a varying severity. Incidence up to $100 \%$ was recorded in some fields. Through a combined fungal morphological identification and ITS sequence analysis, a total of 11 fungal species in 110 isolates from both China and Tanzania were identified. Among these, Fusarium equiseti had the highest frequency of occurrence covering all locations, followed by Alternaria alternata, A. tenuissima and Phoma herbarum. The findings suggest the possibility of association of a disease with several fungal pathogens in combination with other factors.

Impact of white pine blister rust on resistant and previously immune cultivated Ribes and neighboring Eastern white pine in New Hampshire I. A. MUNCK (1), P. Tanguay (2), K. Lombard (3), J. Weimer (3), S. Villani (4), K. Cox (4)

(1) USDA Forest Service, Durham, NH, U.S.A.; (2) Canadian Forest Service, Quebec, QC, Canada; (3) New Hampshire Division of Forests and Lands, Concord, NH, U.S.A.; (4) Cornell University, Geneva, NY, U.S.A.

Phytopathology 104(Suppl. 3):S3.83

White pine blister rust (WPBR) has been a threat to both forest resources and agricultural commodities since its introduction to North America in the early 1900s. The objective of this study was to determine the impact of WPBR following the recent breakdown in immunity. During 2013, 255 plants of 19 Ribes cultivars and 445 neighboring white pines (Pinus strobus) in 43 sites were evaluated. Disease severity defined as percentage of leaf area affected was assessed for 18 leaves per Ribes plant. Infected leaves of each cultivar were collected for PCR analyses. Immune Ribes from Canadian Clonal Genebank were inoculated with samples of immune black currants from NH. At each site, WPBR incidence for the 12 nearest white pines within $300 \mathrm{~m}$ of cultivated Ribes was recorded. Incidence of WPBR ranged from $0 \%$ to $60 \%$ and 0 to $100 \%$ for immune and resistant Ribes cultivars, respectively. Mean WPBR severity on resistant Ribes was limited to $<6 \%$ of leaf area compared to $14 \%$ for previously immune Ribes. All infected Ribes cultivars were PCR positive for Cronartium ribicola. Immune Ribes from the Canadian Clonal Genebank were successfully infected with samples from NH. It was more 
likely to find infected pines neighboring infected immune black currants (probability $=0.18$ ) than neighboring infected resistant Ribes $(0.09)$, or WPBR-free Ribes (0.02). The breakdown in of WPBR immunity poses a threat to the white pine resource and to cultivated Ribes production.

Effect of SHAM and azoxystrobin on germination of Sclerotinia sclerotiorum ascospores

C. L. MUNOZ (1), L. E. del Rio Mendoza (1)

(1) North Dakota State University, Fargo, ND, U.S.A.

Phytopathology 104(Suppl. 3):S3.84

Sclerotinia sclerotiorum is a pathogen that affects crops of economic importance in North central US. Azoxystrobin is a QoI fungicide registered for use against this pathogen and has a history of inducing resistance in other target pests. The objective of this study was to determine the sensitivity $\left(\mathrm{EC}_{50}\right)$ of S. sclerotiorum isolates collected from the north central region in the US to azoxystrobin. Here we report results of preliminary trials conducted to identify the appropriate salicylhydroxamic acid (SHAM) concentration to be used. SHAM blocks the alternate respiration pathway that fungi use when exposed to QoI fungicides. In this study, SHAM and azoxystrobin were evaluated alone or in combinations at $0,70,119$, and $202 \mathrm{ug} / \mathrm{ml}$ and at $0,0.05$, $0.092,0.166,0.30 \mathrm{ug} / \mathrm{ml}$, respectively. Spores were incubated on amended water agar in dark for $24 \mathrm{~h}$ at $25^{\circ} \mathrm{C}$. Results indicated SHAM reduces spore germination significantly even when used at $70 \mathrm{ug} / \mathrm{ml}$ and its impact on the sensitivity of $S$. sclerotiorum to azoxystrobin varies with its concentration. $S$. sclerotiorum isolates were more sensitive to azoxystrobin when SHAM was used at $70 \mathrm{ug} / \mathrm{ml}$ than at higher concentrations. At $70 \mathrm{ug} / \mathrm{ml}$ of SHAM, the sensitivity $\left(\mathrm{EC}_{50}\right)$ of 16 other $S$. sclerotiorum isolates to azoxystrobin averaged $0.11 \mathrm{ug} / \mathrm{ml}$ and ranged between 0.08 and $0.21 \mathrm{ug} / \mathrm{ml}$. The interaction between SHAM and azoxystrobin and their combined effect on S. sclerotiorum needs to be studied in more detail.

Biocontrol formulations but not an inter-row cover crop of rye reduced Tomato spotted wilt virus (TSWV) incidence in pepper and tomato

J. F. MURPHY (1), T. Morawo (1), T. Monday (1), H. Fadamiro (1), W. Foshee (1)

(1) Auburn University, Auburn, AL, U.S.A.

Phytopathology 104(Suppl. 3):S3.84

We evaluated 2 production practices and 2 biological treatments to reduce TSWV incidence in pepper and tomato. Pepper (cv Calwonder) and tomato plants (cv FL47) were grown in plastic mulch with either (1) a bare soil, weed-free inter-row space or (2) a rye inter-row soil cover. Minor treatments included a non-treated control, Bio treatment 1 (BT1, from Pathways Biologic), experimental Bio treatment 2 (BT2, from Agricen Sciences) and the combined treatment of BT1+BT2. Early in the season (3-5 weeks posttransplant), significantly greater thrips densities occurred on pepper and tomato plants grown with the rye inter-row soil cover. At week 5, a terminal leaf sample was collected from each plant and tested for TSWV by ELISA. A significantly greater TSWV incidence occurred in pepper and tomato plants grown in the rye than in the bare soil treatment. This corresponds with the greater thrips densities in the rye treatment. TSWV incidence in the bare soil treatment was low leading to limited detectable differences among minor treatments; however, for pepper and tomato plants grown in the rye treatment, significantly less TSWV occurred among plants in each of the Bio treatments compared with the non-treated control. When differences occurred among the Bio treatments, BT2 and BT1+BT2 tended to have significantly less TSWV than BT1 alone. Pepper and tomato yields differed significantly only between major treatments with significantly less yield in the rye treatment.

Effects on bell pepper fruit yield by infection of each of three strains of Tobacco etch virus (TEV)

J. F. MURPHY (1)

(1) Auburn University, Auburn, AL, U.S.A.

Phytopathology 104(Suppl. 3):S3.84

Three TEV strains, HAT, Mex21 and N, (along with a mock-inoculated control) were evaluated for their effects on bell pepper fruit yield under greenhouse growth conditions. The experiment was performed in a greenhouse in order to avoid cross contamination of the different virus strains by aphid vector transmissions. Pepper plants were individually grown in 3 gallon pots in a soilless growth medium. Each virus strain was applied to leaves 1-3 ( 3 oldest leaves along the stem) when plants were at the 9-10 leaf stage. There were 14 plants for each TEV strain and each plant expressed systemic symptoms by $7 \mathrm{dpi}$. Yields were similar for HAT-infected plants and the healthy control. Mex21-infected plants produced significantly less fruit than healthy control plants and those infected with HAT and, none of the fruit from Mex21-infected plants was marketable, i.e., fruit size and weight were similar to marketable fruit from healthy and HAT treatments but the fruit was discolored and misshapen. Pepper plants infected with the $\mathrm{N}$ strain produced fruit late in the experiment and the fruit were significantly smaller in number, size and weight and were misshapen. None of the fruit from $\mathrm{N}$-infected plants were marketable.

Gene regulatory networks for resistance in maize to ear rots and to aflatoxin accumulation

B. MUSUNGU (1), G. O'Brien (2), R. Brown (3), D. Bhatnagar (3), G. Payne (2), M. Geisler (1), A. M. Fakhoury (1)

(1) Southern Illinois University, Carbondale, IL, U.S.A.; (2) North Carolina State University, Raleigh, NC, U.S.A.; (3) USDA, New Orleans, LA, U.S.A. Phytopathology 104(Suppl. 3):S3.84

An interactome is the genome-wide roadmap of protein-protein interactions that occur within an organism. Interactomes for humans, the fruit fly, and now the plant Arabidopsis thaliana have been generated using high throughput experimental methods. It is possible to use these experimentally derived interactomes to predict interactomes for other species based on the orthology of genes between species. To build an interactome for Zea mays (maize), orthologs were identified to determine a one-to-one orthology between genomes of maize and reference species where both maize orthologs occurred for an interaction in the reference species; this implied that the proteins likely interacted in maize. Using the interactome, mining for key genes suspected to be involved in resistance to A. flavus and other ear rots is possible. Since it has been shown that interacting proteins tend to be co-expressed, RNA-seq data was added as another layer of information to network. RNA-seq looked at infection of A. flavus and Z. mays to develop static networks. The static network allows for visualization of key regulatory genes in A. flavus and $Z$. mays for determining positive correlation and anti-correlations of genes. The maize static expression networks will provide researchers with a tool for studying interactions of key proteins in Z. mays and A. flavus. This will facilitate identifying biomarkers linked to resistance to pathogens and linking of key regulators in A. flavus.

Temporal changes in Pseudoperonospora cubensis field populations in MI cucumber

R. P. NAEGELE (1), J. Kurjan (1), L. M. Quesada-Ocampo (2), M. K. Hausbeck (1)

(1) Michigan State University, East Lansing, MI, U.S.A.; (2) North Carolina State University, Raleigh, NC, U.S.A.

Phytopathology 104(Suppl. 3):S3.84

Cucurbit downy mildew (CDM) incited by Pseudoperonospora cubensis affects cucurbits worldwide. When left untreated, the pathogen causes rapid defoliation and plant death. In Michigan, approximately 46,000 acres of susceptible crops are grown annually, including cucumbers with an estimated value of \$28.7 million. To manage CDM, growers rely on frequent fungicide applications but pathogen resistance to systemic fungicides is an increasing concern. This obligate, wind-dispersed pathogen arrives in MI each growing season from overwintering sites. During the production season, isolates can undergo multiple cycles of infection and reproduction, with new isolates potentially appearing throughout the season. To evaluate temporal changes in $P$. cubensis populations, sporangia from CDM lesions were collected from cucumber foliage growing in four MI field locations; samples were collected on two dates in 2011. Population structure and genetic diversity were assessed using eight single-copy simple sequence repeat (SSR) markers against 277 isolates.

Development and formulation of the most complimentary fungicide combination for row crops

D. NARVAEZ (1), M. Bradley (2)

(1) Makhteshim Agan of North America MANA, Wildwood, MO, U.S.A.; (2) Makhteshim Agan of North America MANA, Raleigh, NC, U.S.A.

Phytopathology 104(Suppl. 3):S3.84

Fungicides have been used in field crops production since the 1700s. Producer desire to push crops to extract their maximum yield in recent years has changed "typical" production practices. This change in practice has dramatically increased the number of fungicides available for use in field crops. Most of these newly available fungicides products belong in one of the two classifications of fungicides: quinine outside inhibitors and Demethylation Inhibitors. Unfortunately, fungicide resistance is developing in many fungi worldwide due to the intensive use and reliance on a single fungicide with an effect on one specific site or function. Therefore, using fungicides that combine products with different modes of action is one of the most effective ways to reduce the risk of fungicide resistance. ADAMA has a broad fungicide portfolio and through its formulation, development and efficacy screening program developed a highly effective fungicide mixture: Custodia ${ }^{\circledR}$. Custodia's unique premix offers the most complementary multi- 
mode of action fungicide providing preventive and curative disease control on corn, soybeans, peanuts, wheat and several other crops. Data from replicated field trials demonstrate the disease control efficacy of Custodia ${ }^{\circledR}$ when applied in Corn at V5 and VT growth stages under high disease pressure locations.

Potential of artichoke as a trap crop for control of the cyst nematodes Globodera ellingtonae and G. pallida

D. Navarre (1), I. Zasada (2), L. M. DANDURAND (3)

(1) USDA-ARS, Prosser, WA, U.S.A.; (2) USDA-ARS, Corvallis, OR, U.S.A.; (3) University of Idaho, Moscow, ID, U.S.A.

Phytopathology 104(Suppl. 3):S3.85

The efficacy of artichoke (Cynara cardunculus var. scolymus) as a trap crop to control the potato cyst nematodes Globodera ellingtonae and G. pallida was examined. In one study, whole root diffusate from artichoke roots induced hatch of G. ellingtonae (44\%) and G. pallida (46\%) eggs, which was equivalent to or higher than hatch in potato root diffusate. No nematode reproduction occurred on artichoke plants exposed to either G. ellingtonae or G. pallida (initial rate $=5 \mathrm{eggs} / \mathrm{g}$ soil) for $16 \mathrm{wk}$. Under the same conditions, potato ('Desiree') had a reproductive factor $(\mathrm{RF}=$ final nematode population/initial population) of 41.6 for G. pallida and 35.9 for $G$. ellingtonae. In a separate experiment, 4-wk-old artichoke and potato plants were inoculated with nematodes at a rate of $5 \mathrm{eggs} / \mathrm{g}$ soil. After $6 \mathrm{wk}$, roots were stained with acid fuschin, and the different life stages of G. pallida were determined. Second-stage juveniles (J2) and third-stage males and females were found in roots of potato but not in artichoke roots. Both nematode species continued development in potato to form adult females, males and cysts with viable eggs, but no nematode development occurred in artichoke. In a separate experiment, after 14 days artichoke roots were infected with $G$. ellingtonae $\mathrm{J} 2$ but at significantly lower levels than potato. These results indicate that artichoke might provide another practice as part of an eradication program for these important nematode parasites of potato.

\section{Assessment of foliar fungicides on soybean diseases and yield advantage during 2011, 2012 and 2013 in Iowa}

S. S. NAVI (1), L. Jing (2), X. Yang (1), X. Li (1)

(1) Iowa State University, Ames, IA, U.S.A.; (2) Inner Mongolia Agricultural University, Hohhot, China

Phytopathology 104(Suppl. 3):S3.85

Diseases of soybean are significant components of yield loss for crop producers. Use of fungicides is one of the management options of soybean diseases. Ten trials at Nashua and one at Ames were set up in a RCBD with 4 replications each with $10^{\prime} \times 45.5^{\prime}$ plots in $30^{\prime \prime}$ row spacing. Triazoles (4), Strobilurin (5), their premixes (3), and other fungicides (7), insecticides (3) and herbicides (2) were tested. As per companies' protocols, products were sprayed and pre- \& post-spray foliar, stem and root diseases were recorded weekly through 1-week before the harvest. After harvest, yields were measured in bu/ac (at 13\% grain moisture). Some treatments showed significant effect $(\mathrm{P}<0.05)$ on diseases and yields in 2011. The 2012 and 2013 were dry, consequently, diseases were low, and were difficult to detect evidence of fungicide effect on diseases except in one trial. Significant $(\mathrm{P}<0.05)$ yield differences of sprayed vs. unsprayed were noted in 3 trials in 2011 and 1 in 2013. In 2011, QoI mixed with insecticides resulted higher yields than QoI alone and one of the herbicides tested showed yield penalty. Across 3-seasons with 123 treatments, average yield advantage was $2.5 \mathrm{bu} / \mathrm{ac}$ (range -4.6 to 10.1), the mean yield advantage of sprayed vs. control was 3.9 bu/ac in 2011 (range -1.1 to 10.1), $2.7 \mathrm{bu} / \mathrm{ac}$ in 2012 (range -4.6 to 8.7 ) and $1.9 \mathrm{bu} / \mathrm{ac}$ in 2013 (range 0.5 to 5.4). Variation in yields may be due to weather, crop rotation, diseases, varieties planted and products efficacy.

Importance of soil moisture and isolate origin on disease severity of three Rhizoctonia solani AG 2-2 IIIB isolates

O. T. NEHER (1), E. J. Wenninger (2), H. Neibling (2)

(1) The Amalgamated Sugar Company LLC, Boise, ID, U.S.A.; (2) University of Idaho, Kimberly, ID, U.S.A.

Phytopathology 104(Suppl. 3):S3.85

Differences in irrigation practices, environmental conditions and Rhizoctonia root rot (RRR, Rhizoctonia solani) severity in two sugar beet growing regions in Idaho raised questions about potential interactions between soil moisture and isolate origin. A study was conducted to investigate the interaction between irrigation (irrigation levels: 40, 70, $100 \& 130 \%$ evapotranspiration (ET)) and $R$. solani AG 2-2 IIIB isolates (F521 - related to $R$. solani isolate R9, F551 - found in the southern and F517 - from the western production area of Idaho). The study was conducted using drip irrigation to ensure consistent and precise water delivery. Statistical analysis for RRR disease index (DI) based on a 1-9 rating scale $(1=$ no disease on beet root, $9=$ root completely dead) showed significant differences for $R$. solani isolates $(\mathrm{Pr}>\mathrm{F}$
$<0.0007)$. F517 was not significantly different from the non-inoculated control, whereas root rot increased significantly by a factor of 2.8 and 2.3 for F521 and F551, respectively, compared to the control. Pooled data for $R$. solani isolates across tested ET levels and the interaction between ET and $R$. solani isolates were not statistically different $(P>F 0.094$ and 0.2335$)$. Root rot was not severe enough to affect root quality. Pooled data across ET levels showed significant differences for root yield and sugar quality. Plots kept at $40 \%$ ET had significant lower clean root yield and estimated recoverable sucrose (ERS) than other tested ET levels.

New severe symptoms caused by Watermelon mosaic virus on squash and pumpkin in Utah

C. NISCHWITZ (1), C. Dhiman (1), M. McCullough (1), B. Rhoads (1), J. Barnhill (2), M. Pace (3)

(1) Utah State University, Logan, UT, U.S.A.; (2) Utah State University, Ogden, UT, U.S.A.; (3) Utah State University, Brigham City, UT, U.S.A.

Phytopathology 104(Suppl. 3):S3.85

In the last two years, leaves of squash and pumpkin with severe deformations resembling herbicide damage and fruit showing color breaking and warts have been found in numerous fields in Utah. Economic losses for individual growers reached $20 \%$ due to reduced yield and quality. Leaf and fruit samples were screened by ELISA tests for Potyviruses, TSWV, TMV, CMV and SqMV. In addition, they were screened with PCR for Curtoviruses. All samples were positive for Potyviruses and subsequent ELISA testing showed they were positive for Watermelon mosaic virus but not Papaya ringspot virus. The virus was confirmed with RT-PCR and DNA sequencing. The symptoms were more severe than what has been described in the literature. To rule out other viruses, RT-PCR was conducted for the Tobamovirus, Tospovirus, Comovirus and Fabavirus groups. The samples were negative for all other viruses. Weeds and alfalfa also tested positive with ELISA for the virus. Moving winter squash to fields that have not been in cucurbits for several years is currently the best option for Utah growers to reduce yield losses and efforts are under way to develop additional management options.

Involvement of a rice heme activator protein gene (OSHAP2E) in defense to fungal and bacterial infections

M. NISHIGUCHI (1), M. M. Alam (1), T. Tanaka (1), K. Kobayashi (1), T. Yaeno (1), N. Yamaoka (1)

(1) Ehime University, Matsuyama, Japan

Phytopathology 104(Suppl. 3):S3.85

During the course of microarray analysis of rice treated with probenazole (PBZ), a chemical inducer of disease resistance, we found the up-regulation of rice heme activator protein (HAP) gene (OSHAP2E) by PBZ. HAP, also known as nuclear factor $\mathrm{Y}$ or CCAAT binding protein, (HAP/NF-Y/CBP) has important functions in regulating plant growth, development, and abiotic and biotic stress responses. Thus we further characterize this gene by RT-PCR. As a result, $O S H A P 2 E$ was induced not only by the infection of Magnaporthe oryzae or Xanthomonas oryzae pv. oryzae but also by salicylic acid (SA), isonicotinic acid (INA), abscisic acid (ABA) and hydrogen peroxide $\left(\mathrm{H}_{2} \mathrm{O}_{2}\right)$. Furthermore rice was transformed with this gene driven by CaMV $35 \mathrm{~S}$ promoter and the overexpressed (ox) lines were selected. When inoculated with $M$. oryzae or X. oryzae pv. oryzae, the ox-lines showed a high resistance to these pathogens, even higher than that in each resistant variety of rice. Microarray analysis showed that some defense related genes were upregulated in the ox-lines. These results showed that $O S H A P 2 E$ is involved in defense response to fungal and bacterial pathogens. This work was supported by the Program for Promotion of Basic and Applied Researches in Biooriented Industry (BRAIN).

Screening of fungicides for the control of ripe rot on grapes caused by Colletotrichum acutatum and $C$. gloeosporioides

M. NITA (1), C. Oliver (1), S. Hartley (1)

(1) Virginia Tech, Winchester, VA, U.S.A.

Phytopathology 104(Suppl. 3):S3.85

Ripe rot of grape, caused by Colletotrichum acutatum and C. gloeosporioides species complexes, is a wide-spread disease throughout Virginia vineyards, and proposes serious risks to crop yield and wine quality. There are only a few fungicides currently recommended for the management of this disease, thus, we conducted two studies to screen ten different mode of action groups. In the first study, the surface sterilized table grape berries were sprayed with a treatment using an airbrush. After 24 hours of drying, 5 microliter of the inoculum suspension $\left(5 \times 10^{5}\right.$ spores per $\left.\mathrm{ml}\right)$ was placed on the surface of the berry. Following 24 hours of incubation under a high $\mathrm{RH}$ condition at $30^{\circ} \mathrm{C}$, the berry surface was dried for long-term incubation, and disease assessments were conducted up to 17 days after the inoculation. Results from four experimental replications demonstrated the two species, C. acutatum, and $C$. 
gloeosporioides, reacted differently to the same fungicide. For example, $C$. acutatum was less sensitive to a SDHI (boscalid) than C. gloeosporioides with $44 \%$ and $95 \%$ control, respectively. A DMI (tetraconazole) and a phosphite resulted in significant reductions of disease incidence of both species (83$100 \%$ control). In addition to the detached berry study, results from fungicide amended plate assay will be discussed.

\section{Distribution and prevalence of Sclerotinia sclerotiorum and $S$. subarctica} in Norwegian lettuce

B. NORDSKOG (1), H. Eikemo (1), E. Gauslaa (1), V. H. Le (1), R. Warmington (2), J. Clarkson (2)

(1) Bioforsk Norwegian Inst of Agric \& Env Res, Aas, Norway; (2) Warwick Crop Centre, School of Life Sciences, University of Warwick, Warwick, United Kingdom

Phytopathology 104(Suppl. 3):S3.86

Lettuce drop caused by Sclerotinia spp. is one of the most common diseases in field production of lettuce in Norway. During spring and autumn, fields are covered with agricultural fleece to prolong the season, often resulting in crop losses of $20-30 \%$ due to the increased humidity under such covers. As part of a new project aimed at improving control of lettuce drop in Norway, we carried out a survey to determine the prevalence and distribution of Sclerotinia species pathogenic to lettuce. S. sclerotiorum has previously been considered the only Sclerotinia sp. affecting lettuce in Norway but our preliminary results have identified both S. sclerotiorum and S. subarctica with a 50:50 distribution. Both species have been found within the same lettuce fields and from several regions of the country. S. subarctica is difficult to distinguish morphologically from $S$. sclerotiorum, and was identified by sequencing of the rDNA internal transcribed spacer (ITS) region and from the lack of an intron in the LSU rDNA compared with S. sclerotiorum. The practical implications of the discovery of $S$. subarctica in relation to control strategies, as well as the epidemiology of this pathogen, is now being studied at Bioforsk in Norway and at the University of Warwick in the UK.

\section{VIPS - an open source technology platform for implementation of IPM} tools, aimed at international collaboration and local adaptations

B. NORDSKOG (1), T. E. Skog (1), H. Eikemo (1), H. Hole (1), A. F. Schjøll (1), J. Netland (1), N. Trandem (1), T. Rafoss (1)

(1) Bioforsk Norwegian Inst of Agric \& Env Res, Aas, Norway Phytopathology 104(Suppl. 3):S3.86

VIPS is a technology platform developed for international collaboration on IPM, where forecasting models can be easily distributed to users worldwide. The model output views are flexible and simple to incorporate in existing web sites or distribute on smart phones and tablets. Cooperation on development, implementation, testing and validation of forecasting models is made easy in VIPS, and the source code for the platform is released under an Open Source License. The VIPS-system is based on 13 years of experience with a webbased forecasting and information service for integrated management of pests and diseases in cereals, vegetables, and fruit crops in Norway. A totally reconstructed and internationally flexible version of VIPS will be tested in Sweden and Bosnia and Herzegovina in 2014. The system allows local adaptations, including language, incorporation of models and other services. Our aim is to create a technology platform for international collaboration on IPM. The rapid worldwide adaptation of mobile telecom technology creates new opportunities for information flow and interactive forecasting of pests and diseases. We are seeking partners interested in cooperation on developing the system, for example through joint $\mathrm{R} \& \mathrm{D}$ projects that include implementation of forecasting models and development of applications.

\section{In field resistance to asiatic citrus canker of Citrus spp. genotypes}

W. M. Nunes (1), S. A. Carvalho (2), J. Belasque (3), A. M. O. GonçalvesZuliani (1), M. A. Machado (4), J. Croce Filho (5), Z. Abdo (6), C. H. BOCK (7)

(1) Universidade Estadual de Maringa, Maringa, Parana, Brazil; (2) Instituto Agronomico de Campinas, Cordeiropolis, Brazil; (3) Universidade de Sao Paulo, Piracicaba, SP, Brazil; (4) Instituto Agronomico de Campinas, Cordeiropolis, SP, Brazil; (5) Agencia de Defesa Agropecuaria do Parana, Maringa, Parana, Brazil; (6) USDA, ARS, SAA, Athens, GA, U.S.A.; (7) USDA-ARS- SEFTNRL, Byron, GA, U.S.A.

Phytopathology 104(Suppl. 3):S3.86

Asiatic citrus canker (ACC), caused by Xanthomonas citri ssp. citri causes yield loss and renders fruit unfit for the fresh market. A 6-year study in Paraná state Brazil was conducted to compare the susceptibility of 186 genotypes of Citrus representing 9 species (C. aurantifolia, C. aurantium, C. clementina, C. deliciosa, C. limon, C. paradisi, C. reticulata, C. sinensis, and C. unshui, and) and 4 hybrids $(C$. reticulata $\times$ C. sp, C. reticulata $\times$ C. paradisi, C. reticulata $\times$ C. sinensis, and C. unshui $\times$ C. sinensis). The number of lesions per leaf was assessed eighteen times from 2005 to 2010 ( $\leq 4$ times per year). Mixed model analysis of fixed and random effects showed six resistance-susceptibility groupings of species and hybrids. The most resistant species grouping included C. unshui and C. limon (mean lesions per leaf of 4.32 and 4.26, respectively), and the most susceptible species were $C$. paradisi and $C$. aurantifolia, with 14.84 and 10.96 lesions per leaf, respectively. Other species (C. reticulata, $C$. aurantium, C. deliciosa, and $C$. sinensis) had intermediate severity (5.48 to 9.56 lesions per leaf). Hybrids had a range of ACC severity, and were in the more resistant groupings (5.26 to 7.35 lesions per leaf). These results of the relative susceptibility of different citrus genotypes can be used in future research, and to assist in varietal selection or for breeding purposes both within Brazil and other locations where ACC is an issue.

Comparison of greenhouse inoculation methods for Fusarium root rot of dry pea

J. ODOM (1), J. Pasche (1)

(1) North Dakota State University, Fargo, ND, U.S.A.

Phytopathology 104(Suppl. 3):S3.86

Fusarium root rot of dry peas is caused mainly by Fusarium solani f. sp. pisi and $F$. avenaceum in the Midwest region of the US and in Canada. The availability of genetic resistance to one or both of these pathogens is extremely limited. While field evaluations under natural inoculum conditions are the most effective way to evaluate germplasm for disease resistance, reliable greenhouse inoculation methods can accelerate the development of disease resistant cultivars. Little information exists to determine if current inoculation methods accurately identify resistance to $F$. solani and $F$. avenaceum in dry peas. Additionally, evidence exists to indicate that resistance to these two pathogens may not be related. Three isolates each of $F$. solani and $F$. avenaceum were compared across 10 dry pea cultivars with varying levels of resistance to $F$. solani using three inoculation methods, vermiculite layering, soil drench and seed soak, under greenhouse conditions. Preliminary results indicate that the vermiculite layer method resulted in the highest disease severity, however, differential responses across cultivars was more easily observed using the seed soak assay. A differential response also was observed across isolates, confirming the importance of both isolate and method selection on the identification of resistance. The results from these inoculation methods will be confirmed in field trials.

Sudden death syndrome of soybeans caused by Fusarium virguliforme can be controlled by the Syngenta seed treatment fungicide A10466G

G. OLAYA (1), D. Ireland (2), C. Watrin (3), P. Pedersen (4)

(1) Syngenta Crop Protection, Vero Beach, FL, U.S.A.; (2) Syngenta, Greensboro, NC, U.S.A.; (3) Syngenta, Basel, Switzerland; (4) Syngenta, Stanton, MN, U.S.A.

Phytopathology 104(Suppl. 3):S3.86

Control of sudden death syndrome of soybeans caused by Fusarium virguliforme has been achieved with Syngenta fungicide A10466G and registration is expected in July 2014. The activity of this fungicide against $F$. virguliforme has been characterized under laboratory, greenhouse and field conditions. The mean sensitivity (ED50 values) of different isolates tested is $0.463 \mathrm{mg} / \mathrm{L}$. Efficacy studies have been conducted by treating seed with A $10466 \mathrm{G}$ at the rates of 5,10 and $20 \mathrm{gai} / 100 \mathrm{~kg}$ alone and in combination with CruiserMaxx Beans. In inoculated studies in the greenhouse disease severity on the hypocotyls and root area has been reduced to very low levels. In one study for example, disease severity was reduced from $71.5 \%$ in the untreated control to $3.5 \%$ with $\mathrm{A} 10466 \mathrm{G}$ alone at $20 \mathrm{gai} / 100 \mathrm{kgseeds}$ and to $0.5 \%$ when A10466G at the same rate was in combination with CruiserMaxx Beans. Foliar disease symptoms were also controlled. Field studies have been conducted over multiple years on susceptible, moderate resistant, and resistant varieties to $F$. virguliforme with consistent yield advantage over current soybean seed treatment offers (CruiserMaxx Beans and Avicta Complete Beans) when the disease is present. No phytoptoxicity problems have been detected with A10466G and it has shown that it is going to be an excellent addition to the CruiserMaxx Beans seed treatment package to broaden the spectrum of control of Fusarium affecting soybeans at early growth stages.

Strawberry nursery plants as a source of Botrytis cinerea isolates resistant to fungicides

M. S. OLIVEIRA (1), N. A. Peres (1)

(1) University of Florida, Wimauma, FL, U.S.A

Phytopathology 104(Suppl. 3):S3.86

Nurseries across Canada, the northern U.S., and California produce strawberry transplants that are shipped annually to other strawberry growing regions including Florida. Botrytis cinerea, the causal agent of gray mold, produces latent infections on transplants which are suggested as a potential source of primary inoculum in annual strawberry producing regions. In this study, we 
isolated B. cinerea from strawberry plants from various nurseries in U. S. and Canada and evaluated their sensitivity to most fungicides used to control gray mold in Florida. During the 2013 strawberry season, 40 plants from 14 different nurseries from five different regions were sampled. The overall frequency of infection was $53,63,77,74$, and $70 \%$ from nurseries in North Carolina $(n=74)$, Nova Scotia $(n=120)$, California $(n=83)$, Ontario $(n=87)$ and Quebec $(\mathrm{n}=116)$, respectively; resulting in a total of 480 isolates. Twenty isolates from each nursery were tested for their sensitivity to seven different fungicides: boscalid, penthiopyrad, pyraclostrobin, fenhexamid, pyrimethanil, fluopyram, and fludioxonil. The percentage of $B$. cinerea isolates resistant to the respective fungicides was $91.8,0,94.5,53.5,98.8,0.3$, and $0 \%$, showing an increase in the resistance to fenhexamid and pyrimethanil compared to isolates collected in 2012. These findings reinforce the need for an integrated approach between strawberry nurseries and production fields for the management of $B$. cinerea.

\section{The adoptive use of biological clocks in crop protection}

\section{O. F. OLOTUAH (1)}

(1) Adekunle Ajasin University, Akungba-Akoko, Ondo, Nigeria

Phytopathology 104(Suppl. 3):S3.87

The efficacy of applications of Hyptis suaveolens at three different times, morning, afternoon and evening was considered in the control of insect pests of cowpea. The crude oil of Hyptis suaveolens which was extracted using soxhlet apparatus and petroleum ether was tested on insect pests of cowpea at field conditions. The extracted oil was applied to three different plots of a local variety of cowpea, Oloyin which was identified at The International Institute of Tropical Agriculture, Ibadan (IITA). Each treatment plot was specified for a periodic spray treatment application of morning, afternoon or evening and the spray treatment was administered using a conventional handsprayer. This application commenced at two weeks after planting, progressing forthnightly till marturity. Data were collected from the three different treatment plots on the available insects in situ prior to treatment application, the extent of foliar, bud and floral damages as well as yield of the crop based on seed count. Means of data collected were subjected to analysis of variance. The results obtained showed the potential use of Hyptis suaveolens in pest control. Consequently, the application of Hyptis suaveolens at the three different times of the day gave an insight to the probable adoption of the circadian or biological rhythm in pest control.

Student perception of peer review for written and oral exercises as a feature of student learning style and multiple intelligences L. M. ORTIZ-RIBBING (1), D. C. Zlesak (2), S. L. Maki (2)

(1) Minnesota Dept of Agriculture, St. Paul, MN, U.S.A.; (2) University of Wisconsin-River Falls, River Falls, WI, U.S.A.

Phytopathology 104(Suppl. 3):S3.87

Improving student communication skills can be challenging. The peer review process has provided positive results; however, the perceived value of peer review and whether it links to students' thinking style and multiple intelligence is largely unknown. Students from three courses (Plants and Society, Plant Propagation, and Weed Science) at the University of Wisconsin-River Falls participated in a study evaluating intelligence and thinking styles in relation to their perceived value of peer review for improving written and oral assignments. Many models and instruments have been used to characterize learning styles. We used an easy online version of the Gregorc Style Delineator and Multiple Intelligences for Adult Literacy and Education. At the end of the semester, students filled out a survey regarding their perception of the value of different aspects of the peer review process. Our hypothesis is that some learning styles would have stronger perceived benefits regarding the peer review process than others and that associations may exist with student gender and major. All students agreed that the peer review process was valuable. Preliminary data shows significant differences among courses and assignments. Understanding associations of student perception and learning styles could result in instructors exploring alternatives to reach and benefit those students who perceived they benefitted least from the current peer review model.

Assessing the effect of vernalization on the detection of Phytophthora ramorum in native soil, potting media, and cull piles

N. OSTERBAUER (1), S. Lane (1), A. Trippe (1), S. Navarro (1)

(1) Oregon Dept of Agriculture, Salem, OR, U.S.A.

Phytopathology 104(Suppl. 3):S3.87

The USDA APHIS PPQ Official Regulatory Protocol For Nurseries Containing Plants Infected With Phytophthora ramorum requires composite samples $(1,000 \mathrm{~g})$ be collected from a nursery's native soil, potting media, and cull piles that may have been exposed to the pathogen. Each sample is tested for pathogen presence with a baiting bioassay using susceptible leaf baits plated onto a selective medium. For the bioassay, samples were thoroughly mixed, then divided in half; $500 \mathrm{~g}$ was baited immediately while the rest was vernalized for 30-d at 4C prior to baiting. From 2007 to 2013, we processed 282 samples for $P$. ramorum; 148 were from native soil, 96 from potting media, and 38 from cull piles. In total, 40 samples tested positive for $P$. ramorum, with 14 testing positive pre- and post-vernalization, 16 prevernalization only, and 10 post-vernalization only. A chi-square analysis of dependent paired observations indicated there was no significant difference between pathogen detection in samples baited pre- and post-vernalization. Similar analyses of the data by sample type showed no significant difference in pathogen detection for native soil and potting media samples baited preand post-vernalization. However, the pathogen was detected significantly more often $(\mathrm{p}<0.05)$ in cull pile samples baited pre-vernalization. This suggests vernalization does not provide a significant benefit to pathogen detection. Other factors, such as sample size, may play a greater role.

tepR: A new luxO-type regulatory gene of the rice pathogenic bacterium, Burkholderia glumae

S. OSTI (1), I. K. Barphagha (1), J. H. Ham (2)

(1) Louisiana State University Agricultural Center, Baton Rouge, LA, U.S.A.; (2) Louisiana State University, Baton Rouge, LA, U.S.A.

Phytopathology 104(Suppl. 3):S3.87

Burkholderia glumae, which causes bacterial panicle blight in rice, has several virulence factors associated for the disease development. Toxoflavin, lipase and flagella are important virulence determinants of B. glumae. A genomewide search for regulatory factors of $B$. glumae virulence identified tepR, which encodes a sigma 54-dependent response regulator, as a negative regulator for toxoflavin production. TepR is homologous to LuxO, a quorumsensing signaling component of Vibrio spp. A markerless tepR deletion mutant of B. glumae 336gr-1, LSUPB401, produced larger amount of toxoflavin and lipase as compared to the wild type, $336 \mathrm{gr}-1$. The phenotype of LSUPB401 was complemented by a functional tepR clone, indicating that tep $R$ is a novel negative regulator for toxoflavin and lipase production in $B$. glumae. LSUPB401 was more aggressive than the wild type in causing symptoms on rice panicles and onion bulb scales in virulence assay studies. Currently, the regulatory effect of tepR in other virulence-related phenotypes of B. glumae such as flagella-dependent motility and biofilm assay are under investigation along with the study of effect on transcription of other genes regulating the virulence factors.

Molecular characterization of genes regulating conidiogenesis and metabolism in Fusarium graminearum

H. A. OSUNGA BUYU (1), R. L. Hirsch (1), Z. O. Bilton (1), A. B. Lum (1), C. M. Rivera (1), J. E. Flaherty (1)

(1) Coker College, Hartsville, SC, U.S.A.

Phytopathology 104(Suppl. 3):S3.87

Fusarium graminearum is an important phytopathogenic fungus that causes wheat head blight and maize ear and stalk rot in most agricultural production areas of the world. F. graminearum persists in the soil and disseminates onto susceptible plants in the spring with specialized asexual spores called conidia. However, the molecular regulation of conidiogenesis is poorly understood. In order to further elucidate the genetic mechanisms underlying conidiation, putative regulatory genes were identified in a previous gene expression study and selected for functional characterization. The genes targeted for deletion were predicted to be involved in protein transport, DNA modification, transcriptional activation, or had unknown functions based on similarity to previously characterized proteins. In order to characterize the regulatory function of the targeted genes, the deletion strains were assayed for phenotypic abnormalities compared to the wild-type strain. Results from the assays revealed that many of the genes regulated aspects of conidiogenesis, conidium morphology, and both primary and secondary metabolism during growth on media and plant tissue. The development of a collection of targeted mutants of $F$. graminearum impaired in important biological functions lays a foundation for future molecular research in this system and promises to facilitate the discovery of novel regulatory pathways involved in conidiation and pathogenesis.

\section{Phyllosphere envirosphere}

A. OTTESEN (1), J. R. White (1), S. Gorham (1), E. Reed (1), M. Newell (2), E. Burrows (1), M. Mahovic (1), E. Brown (1)

(1) FDA CFSAN, College Park, MD, U.S.A.; (2) Wye Research and Education Center, University of Maryland, Queenstown, MD, U.S.A. Phytopathology 104(Suppl. 3):S3.87

Culture independent data describing phyllosphere microflora has advanced our understanding of this complex and far reaching environment. A question that remains unanswered however, is which of the many microbes described 
in phyllosphere research are actually plant driven. Collection of microflora from plant surfaces has often been performed without an environmental control, so the opportunity to examine which members of phyllosphere microflora are driven by environmental forces and which members are driven by biology and physiology of plants, has not been fully realized. To examine this question, we placed plastic plants adjacent to tomato plants in the field and sampled both sets of leaves at same time-points. DNA was extracted from a wash of tomato leaves and plastic leaves and examined using metagenomic methods. The vast majority of OTUs (operational taxonomic unites) were shared between both environments however, there was also a compositional effect observed in PCoA plots, suggesting that an OTU from an envirosphere sample is more likely to be shared with another envirosphere sample, and the same is true for phyllosphere. The differences indicated that composition plays a factor, although only in the low frequency OTUs. Among these OTUs, there were taxa that were observed in enivrosphere but not in phyllopshere. Almost no organisms were observed in phyllosphere and not envirosphere, forcing us to reconsider which abiotic and ecological forces play the most important roles as drivers of phyllosphere microbial ecology - if not the plants. These findings have important implications for the direction of food safety research aimed at describing the most important risk factors associated with production of fresh produce.

Variation in pathogen suppression among indigenous soil Streptomyces from natural and agricultural habitats

L. OTTO-HANSON (1), M. Dedrick (1), L. Kinkel (1)

(1) University of Minnesota, St. Paul, MN, U.S.A.

Phytopathology 104(Suppl. 3):S3.88

Indigenous soil antagonists can play a significant role in mediating plant diseases. However, while all soils support antagonistic populations, there is little information on the variation in or correlates of antagonist densities and inhibitory activities in natural habitats. We collected soil samples $(\mathrm{n}=82)$ from 6 habitats at the Cedar Creek Ecosystem Science Reserve. For each sample, total bacterial, Streptomyces, and antagonist densities as well as the mean inhibition zone produced in response to a target overlay were determined. In addition, soil edaphics were determined for every sample. Agricultural soils had significantly higher total bacterial $(\mathrm{p}=<0.0001)$ and Streptomyces densities $(\mathrm{p}<0.0001)$ than the other habitats. However, antagonist densities in agricultural soils were not different from those in other habitats, except for wetland soils, which had significantly lower antagonist densities $(\mathrm{p}=0.0137)$ than any other habitat. Edaphic characteristics also varied significantly among habitats, and were sometimes significantly correlated with microbial community characteristics across all samples, though in other cases, they varied among habitats. Variation in these relationships among habitats suggests that plant communities may mediate the effects of soil edaphics on microbial communities and selection for antagonists. Further study of indigenous communities in natural and agricultural systems may shed light on important predictors of pathogen suppression.

Development and validation of multiplex real-time PCR and conventional PCR tests for the detection of Phytophthora austrocedri

K. J. OWENS (1), Z. G. Abad (1), J. C. Bienapfl (1), S. Green (2), M. K. Nakhla (1)

(1) USDA-APHIS-PPQ-S\&T, Beltsville, MD, U.S.A.; (2) Centre for Forestry and Climate Change, Roslin, United Kingdom

Phytopathology 104(Suppl. 3):S3.88

Phytophthora austrocedri was described in Argentina causing dieback on Chilean Cedar in 2007 and in late 2010 was found in northern England causing a serious decline of native juniper and at two sites in Scotland in 2011 on Lawson's cypress and Nootka cypress. Further concern for this pathogen is due to its potential to be disseminated through shipments of contaminated nursery stock. P. austrocedri is ranked \# 21 in the list of 29 Phytophthora spp. of concern for the USA published by CPHST-Plant Epidemiology and Risk Analysis Laboratory in 2009. Due to the importance of the pathogen denoted by the recent discoveries and its inclusion in the Cooperative Agricultural Pest Survey List (2014), we consider $P$. austrocedri as a priority for USDA regulatory programs. A real-time PCR assay that targets the ITS region published by the Centre for Forestry and Climate Change (CFCC) in Scotland was validated and paired with the COX gene to detect host plant DNA in a multiplex assay. In addition, a multiplex conventional PCR was developed using species-specific primers for the L10 gene and paired with the plant gene NAD5 as the internal control. Both methods have been tested with DNA from cultures and environmental samples from the UK provided by the CFCC. In addition, both methods are under evaluation for cross-reactivity with other Phytophthora species in clade 8d, as well as other Oomycetes and fungal pathogens.
Effect of Fusarium species isolated from switchgrass plants and seeds on foliar disease and stand establishment

B. H. OWNLEY (1), S. B. Collins (1), M. M. Dee (1)

(1) University of Tennessee, Knoxville, TN, U.S.A.

Phytopathology 104(Suppl. 3):S3.88

Fusarium spp. have been reported from switchgrass plants and seeds, but information on their potential effects on stand establishment is lacking. Studies are needed to distinguish between fungi isolated from switchgrass that have minimal impact on stand establishment and biomass yield, from those that negatively impact the health of seeds, seedlings and mature plants. The aim of this study was to determine the pathogenicity and virulence of Fusarium spp., isolated from lesions of switchgrass field plants or commercial seed. Thirty isolates of Fusarium spp. were evaluated for pathogenicity and virulence on switchgrass with cut leaf assays. For each isolate, five 10- $\mu 1$ droplets with $10^{4}$ spores $/ \mathrm{ml}$ were applied to replicate cut leaf sections, maintained in a moist chamber for 5 days, and percent lesion area was determined with Assess software. Isolates of $F$. graminearum were the most virulent with lesion area of 38 to $42 \%$. Virulent isolates were evaluated further with a seed disease assay. Two rates of inoculum, $10^{1}$ spores $/ \mathrm{ml}$ and $10^{5}$ spores $/ \mathrm{ml}$, were applied to 100 seeds and incubated for $24 \mathrm{~h}$. Twenty inoculated seeds were planted in soil mix in five replicate pots. Plant stand was significantly reduced by most isolates at the high inoculum rate; while $F$. equiseti and $F$. acuminatum isolates did reduce stand significantly, $F$. graminearum isolates resulted in $<10 \%$ plant stand. These results suggest that Fusarium spp. play a role in poor stand establishment of switchgrass.

Regional temporal variations in potato psyllid haplotypes trapped in natural vegetation ecosystem

L. PAETZOLD (1), A. Rashed (2), F. Workneh (1), C. M. Rush (1)

(1) Texas A\&M AgriLife Research, Bushland, TX, U.S.A.; (2) University of Idaho, Aberdeen R\&E Center, Aberdeen, ID, U.S.A.

Phytopathology 104(Suppl. 3):S3.88

Since its first discovery in 2000 , potato zebra chip has become an economically important disease in most US potato production regions. The potato psyllid (Bactericera cockerelli) has been identified as a vector of the causal agent 'Candidatus Liberibacter solanacearum'. Recently, four haplotypes of the insect (central, northwest, southwestern, and western) were identified as occurring in the US. Knowledge of the dynamics of the different haplotypes in different locales is useful in understanding insect ecology, survival, and movement across regions. In 2012-2013, potato psyllids were collected from the Texas Panhandle (Bushland, Dalhart, and Olton) and southwest Texas (Andrews, Fort Stockton, and Kermit), using yellow sticky traps placed in pastures (surrounded by natural vegetation) and collected and replaced biweekly. DNA from the psyllids was extracted and tested for haplotype specificity using high resolution melting point analysis (HRM). Each of the psyllid haplotypes were detected in our samples over the two-year period in different proportions, except the northwestern type. The proportion of individual haplotypes varied among locations and over time within a location. For example, psyllid samples from Andrews were of the central type in one collection, but the southwestern type predominated in the following collection. This finding suggests that a long-term investigation is required to understand population dynamics of the haplotypes within a region.

The USDA huanglongbing (HLB) multiagency coordination (MAC) initiative

M. E. PALM (1)

(1) USDA APHIS PPQ PHP, Riverdale, MD, U.S.A.

Phytopathology 104(Suppl. 3):S3.88

Huanglongbing (HLB or citrus greening) is one of the most serious diseases of citrus. Because HLB continues to devastate citrus production in Florida and threatens the crop in other citrus producing States, in December 2013 USDA established a new, multi-agency coordination response framework to better position the Department to respond in a concerted, direct way to both the immediate and long-term needs of the citrus industry. The HLB Multi-Agency Coordinating (MAC) Group, made up of industry, state and federal representatives, is charged with facilitating near term practical solutions and tools for use by the citrus industry to combat HLB. Additionally, the Group coordinates and prioritizes Federal research with that of industry, in order to complement, fill research gaps, and reduce unnecessary duplication. The Group is focused on identifying applied research projects that, if funded, will produce tangible near term tools for the industry. USDA committed $\$ 1 \mathrm{M}$ and Congress appropriated $\$ 20 \mathrm{M}$ to support these efforts in January 2014. In addition to the direct-funded research projects, a Stakeholder Suggestion process will be used to identify additional promising ideas which, with funding, would likely produce near term results for the industry. An overview of process, projects, preliminary results and opportunities will be presented. 
Fungicide impact on in vitro germination of Puccinia horiana, the causal agent of Chrysanthemum white rust

C. L. PALMER (1), S. E. Nester (2), J. M. Revell (2), M. R. Bonde (2)

(1) IR-4, Princeton, NJ, U.S.A.; (2) USDA ARS, Frederick, MD, U.S.A. Phytopathology 104(Suppl. 3):S3.89

Puccinia horiana is an actionable pathogen which upon diagnosis triggers an eradication protocol combining destruction of symptomatic chrysanthemums with a strict fungicide regime for symptomless plants. Appearance of symptoms typically occurs during the fall just as growers are preparing to ship their chrysanthemum crops. To expand the list of fungicide tools in the eradication protocol and develop fungicide sensitivity baselines, we screened in vitro germination of $P$. horiana basidiospores in $0.05 \%$ water agar amended with varying concentrations of 12 fungicides: azoxystrobin, fluoxistrobin, mancozeb, mandestrobin, metconazole, myclobutanil, propiconazole, pyraclostrobin + boscalid, tebuconazole, trifloxystrobin, trifloxystrobin + triademefon, and triticonazole. Leaves with pustules ready to sporulate were affixed to petri plate lids over bases containing fungicide-amended agar. After at least 16 hours in the dark at $17^{\circ} \mathrm{C}$, percent germination was assessed. Concentrations required for $50 \%$ germination $\left(\mathrm{LC}_{50}\right)$ grouped according to fungicide mode of action class. Benzimidazole fungicides (FRAC 3) exhibited $\mathrm{LC}_{50} \mathrm{~S}$ ranging from 20 to $72 \mathrm{ppm}$, while strobilurin fungicides (FRAC 11) ranged from 2 to $16 \mathrm{ppb}$. Mancozeb (FRAC M3) exhibited an $\mathrm{LC}_{50}$ of $7 \mathrm{ppm}$. Combinations of FRAC 11 fungicides with other modes of action exhibited $\mathrm{LC}_{50} \mathrm{~s}$ in the same range as the strobilurins. These data provide a baseline for monitoring development of fungicide resistance in $P$. horiana over time.

Detection and identification of four Aspergillus section Nigri species by PCR

J. PALUMBO (1), T. O'Keeffe (1)

(1) USDA ARS WRRC, Albany, CA, U.S.A.

Phytopathology 104(Suppl. 3):S3.89

Species of Aspergillus section Nigri are not easily or quickly distinguished by traditional morphological techniques, and typically are identified by DNA sequencing methods. This complicates routine screening to detect mycotoxigenic species in section Nigri, including ochratoxin-producing $A$. carbonarius and $A$. niger, and fumonisin-producing $A$. niger and $A$. welwitschiae. We developed four PCR primer sets to distinguish between $A$. niger, A. welwitschiae, A. carbonarius and A. tubingensis, based on speciesconserved differences in the calmodulin gene sequence. PCR amplification from total DNA using these primers was species-specific; no amplification occurred from non-target Aspergillus section Nigri species DNA for each primer pair. Species-specific PCR could distinguish among the four target species in mixed DNA templates, indicating a utility in determining culture uniformity of isolated Aspergillus strains. In addition, with these primer sets, each species could be detected in soil following mixed-species inoculation with Aspergillus spores. This indicates that PCR with these species-specific primers may be useful in determining the distribution of Aspergillus species in environmental samples without the need for species identification from isolated strains, as well as detecting species that may be infrequently isolated by culture-based methods.

Function, evolution, and interaction of the coupled genes responsible for the $P i k$ - $h$ encoded blast resistance of rice

Q. PAN (1), C. Zhai (1), W. Wu (1), Y. Zhang (1), L. Wang (1)

(1) South China Agricultural University, Guangzhou, China

Phytopathology 104(Suppl. 3):S3.89

$P i k-h$, which is an allele of $P i k$, confers resistance against certain races of rice blast. Its positional cloning showed that it comprises a pair of NBS-LRR genes, Pikh-1 and Pikh-2. The allele is distinguishable from other known blast resistance genes on the basis of key variable nucleotides, and SNP diagnosis among the five rice populations implies that it appears to be the most recently evolved of the set of Pik alleles. Comparisons between the sequences of Pik-h and other $P i k$ alleles showed that the functional K haplotype exists as two subhaplotypes, $\mathrm{KM}$ and $\mathrm{KH}$, which both evolved prior to the domestication of rice. While Pikh-1 appears to be constitutively transcribed, the transcript abundance of $P i k h-2$ responds to pathogen challenge, suggesting that while Pikh-1 may well be involved in elicitor recognition, Pikh-2 is more likely to be responsible for downstream signalling. In vitro, the CC domain of Pikh-1 was shown interact directly with both AvrPik-h and Pikh-2. Transient expression assays demonstrated that Pikh-2 mediates the initiation of the defence response. Moreover, nucleocytoplasmic partitioning of both Pikh-1 and Pikh-2 is required for the functionality of Pik-h. In the proposed Pik-h resistance pathway, it is suggested that Pikh-1 acts as an adaptor between AvrPik-h and Pikh-2, while Pikh-2 transduces the signal to trigger Pik-hspecific resistance.
Three dimensional structural prediction of the silencing suppressor protein coded by Tomato spotted wilt virus (Tospovirus:Bunyaviridae)

H. PAPPU (1), Y. Zhai (1), B. Adhikari (2), J. Cheng (2)

(1) Washington State University, Pullman, WA, U.S.A.; (2) University of Missouri, Columbia, MO, U.S.A.

Phytopathology 104(Suppl. 3):S3.89

It is generally accepted that most plant viruses code for a silencing suppressor gene to overcome the host defense response. Tospoviruses (genus Tospovirus, family Bunyaviridae) cause significant crop losses worldwide. Tomato spotted wilt virus (TSWV), the most prolific tospovirus, was used in our study to better understand the structure and function relationships of the silencing suppressor gene, NSs, coded by the small RNA. Using mutational analysis, we recently identified two motifs that are critical for the suppressor activity of the NSs protein: GKT at positions 191-193 and YL at positions 429-430. We utilized in silico 3D modeling algorithms to predict the secondary and tertiary structures of the TSWV NSs protein and to localize these two amino acid motifs. 3D modeling of the structure of the protein showed that it contained both beta sheets and alpha helices, even though an accurate tertiary structure still could not be constructed due to lack of a known protein structure template that is homologous to the NSs protein. The computational prediction of solvent accessibility suggested that the two motifs were located in the relatively buried regions of the protein. Identification and characterization of specific sequences of NSs that are critical for its activity would provide targets for engineering virus resistance that is potentially both broad spectrum and durable.

Comparative analysis of virus-specific small RNA profiles of three biologically distinct strains of Potato virus $\boldsymbol{Y}$

H. PAPPU (1), K. Naveed (1), N. Mitter (2), A. Harper (1), A. Dhingra (1)

(1) Washington State University, Pullman, WA, U.S.A.; (2) University of Queensland, St. Lucia, Australia

Phytopathology 104(Suppl. 3):S3.89

Potato virus $Y$ (PVY) is an economically important viral pathogen of potato worldwide. We investigated the nature and relative levels of virus-derived small interfering RNAs (vsiRNAs) in potato cv. Russet Burbank separately infected with three biologically distinct strains of PVY, the ordinary strain (PVY-O), tobacco veinal-necrotic strain (PVY-N) and tuber necrotic strain (PVY-NTN). Analysis showed vsiRNAs of 20-24 nt in PVY-infected plants. Considerable differences were present in the distribution of vsiRNAs as well as total small RNAs. The $21 \mathrm{nt}$ class was the most prevalent in PVY-infected plants irrespective of the virus strain, whereas in healthy potato plants, the 24 nt class was the most dominant. vsiRNAs were derived from every position in the PVY genome, though certain hotspots were identified for each of the PVY strains. Among the three strains used, the population of vsiRNAs of different size classes was relatively different with PVY-NTN accumulating the highest level of vsiRNAs, whereas PVY-N infected plants had the least population of vsiRNAs. The highest number of total vsiRNAs was from the cytoplasmic inclusion protein gene (CI) in PVY-O and PVY-NTN strains, whereas from PVY-N, the NIb gene produced maximum total vsiRNAs. These findings indicate that the three PVY strains interact differently in the same host genetic background and provided insights into virus-host interactions in an important food crop.

Design and evaluation of microsatellites from whole-genome transcript sequences in Phytophthora capsici

C. H. PARADA ROJAS (1), L. M. Quesada-Ocampo (1)

(1) Department of Plant Pathology, North Carolina State University, Raleigh,

NC, U.S.A.

Phytopathology 104(Suppl. 3):S3.89

Phytophthora capsici is a devastating oomycete that affects solanaceous, cucurbitaceous, fabaceous and other crops in the United States (US) and worldwide. Research on the genetic structure of this pathogen has increased in recent decades; however, such studies have used limited markers due to the absence of genomic resources for $P$. capsici. The recent release of the $P$. capsici genome allows for design of whole-genome markers for genetic studies. Microsatellites or simple sequence repeats (SSRs) have been a preferred marker due to their co-dominant nature and high polymorphism. Identifying SSRs associated with transcript sequences is of interest since they constitute annotated markers with a predicted function. In this study, we used an in silico approach to identify SSRs in P. capsici whole-genome transcript sequences and to design primers for genetic analyses. Using the MIcroSAtellite identification tool (MISA), the total number of 1-6 bp perfect SSRs detected in $P$. capsici transcript sequences $(19,805)$ represented $9.36 \%$ $(1,855$ SSRs) of the transcripts. Identified SSRs mainly included trinucleotide repeats, of which repeat motif AAG was the most abundant (7.92\%). Primers were designed for detected SSRs; a subset was evaluated in a diverse set of $P$. 
capsici isolates, and several were found to be polymorphic. The identified SSRs improve the current resources of molecular markers for this pathogen, which will facilitate $P$. capsici genetic studies in the US.

Common bean lines with resistance to all known races of Uromyces appendiculatus and the strains of the BCMV, BCMNV, and BGYMV viruses

M. A. PASTOR-CORRALES (1), J. A. Beaver (2), J. C. Rosas (3), T. G. Porch (4), G. Godoy-Lutz (5), E. Prophete (6)

(1) USDA ARS, Beltsville, MD, U.S.A.; (2) University of Puerto Rico, San Juan, U.S.A.; (3) EAP/Zamorano, Tegucigalpa, Honduras; (4) USDA ARS SAA TARS, Mayaguez, U.S.A.; (5) University of Nebraska, Lincoln, NE, U.S.A.; (6) Service National Semencier, Port-au-Prince, Haiti

Phytopathology 104(Suppl. 3):S3.90

The objective of this research was to develop common bean lines with multiple genes with broad resistance to the rust pathogen, to the bean common mosaic (BCMV) and bean common mosaic necrosis (BCMNV) potyviruses, and to the bean golden yellow mosaic geminivirus (BGYMV). To develop broadly rust resistant lines, crosses were made with BelNeb-RR-2 having $U r$ 5, Ur-6, Ur-7, BelMiDak-RMR-10 with $U r-4, U r-11$, and Raven with $U r-3$ rust resistance genes, respectively. BelMiDak-RMR-10 also has the dominant $I$ gene and the recessive $b c-3$ genes for resistance BCMV and BCMNV. Another progenitor, PR0301-181, has the $I$ and the $b c-3$ genes, and the recessive allele bgm and the QTL SW12 for resistance to BGYMV. Molecular markers and specific races of the rust pathogen were used to identify the lines with multiple rust resistance genes. To identify lines with viral resistance genes, we used a molecular marker to identify the $I$ gene and a viral strain to detect the $b c-3$ gene. Molecular markers were also used to identify the $b g m$ gene and the SW12 QTL. We selected two lines with confirmed Ur-4, Ur-5, and $U r-11$ genes that confer resistance to all known races of $U$. appendiculatus. The identification of additional rust resistance genes in these breeding lines is in progress. These lines also combine the $I$ and $b c-3$ genes that provide resistance to all known strains of the BCMV and BCMNV potyviruses. In addition, these carry the recessive allele $b g m$ and the QTL SW12 for resistance to BGYMV.

Oxathiapiprolin: An effective new chemistry for control of downy mildew of basil

J. S. Patel (1), S. ZHANG (1), M. I. Costa de Novaes (1)

(1) Tropical REC-Homestead, University of Florida, Homestead, FL, U.S.A. Phytopathology 104(Suppl. 3):S3.90

Downy mildew of basil, caused by Peronospora belbahrii, has spread to more than 30 US states since it was first detected in Florida in 2007. A field study was conducted to investigate the efficacy of a new fungicide Zorvec (oxathiapiprolin, QGU42, DuPont) for control of basil downy mildew. Seeds of basil var. 'Genovese' were planted into raised beds in a randomized complete block design. Prior to planting, basil seeds were treated with QGU42 at six rates $(0.0625,0.125,0.25,0.5,1.0$ and $2.0 \mathrm{fl}$. oz/A) and the same six rates were applied as foliar sprays in experiment 1 . In experiment 2, QGU42 was applied alone as a foliar spray at $0.5 \mathrm{fl}$. oz/A, a tank mix and rotational applications with Quadris at $9 \mathrm{fl}$. oz/A and Prophyt at 3 pints/A, Timorex Gold at $1.25 \%$, Organocide at $2 \mathrm{fl}$. oz/G, and rotational applications of QGU42 with Timorex Gold and Organocide. Foliar sprays of tank mix containing Quadris and Prophyt were made weekly as a standard chemical control. In both experiments, basil plants were naturally infected with $P$. belbahrii. In experiment 1 , foliar sprays of QGU42 at all six rates had significantly lower AUDPC values compared to the untreated control. In experiment 2, the chemical control, and a tank mix of QGU42 with Prophyt had lower AUDPC values than other treatments. Results indicated that QGU42 as foliar sprays alone, a tank mix, and rotational applications with Quadris can effectively control downy mildew of basil in the field of South Florida.

Localization of a QTL for resistance to Phytophthora infestans in Solanum pennellii

S. PATEV (1), W. E. Fry (1)

(1) Cornell University, Ithaca, NY, U.S.A.

Phytopathology 104(Suppl. 3):S3.90

Resistance to late blight has been described in Solanum pennellii, a close relative of cultivated tomato (Solanum lycopersicum). A QTL for resistance was located on a section of chromosome 6 from $S$. pennellii and this section of chromosome 6 was introgressed into S. lycopersicum (M82) producing a near isogenic line (IL6-2) by Eshed and Zamir. In previous studies we demonstrated that IL6-2 had constitutive gene expression prior to infection with late blight indistinguishable from that of M82, however post-infection gene expression varied significantly. When assayed under disease conditions a number of genes were differentially expressed at a significant level in IL6-2 as compared to M82. This differential expression was comprised of genes directly introgressed from $S$. pennellii, and a number of genes not found on the chromosomal introgression but originating from the M82 background. To better localize the genes and regulatory elements from $S$. pennellii responsible for the resistance we investigated a series of 24 sub-introgression lines (developed by D. Zamir) for their reaction to late blight. Each subintrogression line contains a portion of the 6-2 chromosomal segment. These sub-introgression tomato lines were grown under greenhouse conditions and inoculated with $P$. infestans clonal lineage US-11. Disease assessments enabled a more precise location of the resistance in IL6-2.

\section{Disease response of soybean genotypes after application of resistance inducers} M. PAWLOWSKI (1), G. L. Hartman (2)

(1) Department of Crop Sciences, University of Illinois, Urbana, IL, U.S.A.; (2) Department of Crop Sciences, University of Illinois; USDA-Agricultural Research Services, Urbana, IL, U.S.A.

Phytopathology 104(Suppl. 3):S3.90

Soybean (Glycine max) is a major crop in the U.S. Like all crops, soybeans are under constant threat of disease from invading pathogens. One potential management method is to treat plants with elicitors to induce host resistance. The objective of this research was to determine if elicitors, previously shown to elicit defense responses in other pathosystems, reduce disease severity in soybean. Two soybean genotypes producing high and low amounts of reactive oxygen species after elicitation were selected for field experiments. In two consecutive growing seasons, five different elicitors and a water control were sprayed onto foliage of both genotypes at the Crop Research and Education Center, Urbana, IL under natural inoculation conditions. Foliage and stems were evaluated for disease incidence and severity weekly and post-harvest. Two elicitors reduced disease severity in the soybean genotype with high ROS but not in the genotype with low ROS, and the specific reduction of a particular disease was associated with elicitor type in each year. In year 1, significant differences between the high and low ROS responses occurred for anthracnose and charcoal rot severity ratings after treatment with benzothiadiazole. The same trend was found for pod and stem blight after elicitation by chitosan in year 2. Selected elicitors will be further evaluated in greenhouse studies to determine if application under controlled pathogen inoculations reduces disease severity.

Mating type distributions of Microsphaeropsis tanaceti isolated from pyrethrum fields in Tasmania, Australia

T. L. PEARCE (1), J. B. Scott (1), S. J. Pethybridge (2), F. S. Hay (1)

(1) Tasmanian Institute of Agriculture, University of Tasmania, Burnie, Australia; (2) The New Zealand Institute for Plant \& Food Research Limited, Christchurch, New Zealand

Phytopathology 104(Suppl. 3):S3.90

Tan spot disease, caused by the ascomycete Microsphaeropsis tanaceti, is a prominent disease in Tasmanian pyrethrum crops, which are grown for insecticide production. $M$. tanaceti reproduces profusely via asexual conidia, however ascospores haven't been observed and the role of sexual reproduction in the field unknown. In many ascomycete fungi, mating type (MAT) genes govern mating between individuals. In this study, two mating types, MAT1-1 and MAT1-2, have been identified within $M$. tanaceti in a heterothallic arrangement. A PCR assay was developed to identify mating type and used to screen two hierarchically sampled, field populations in August 2012. Each field consisted of two $50 \mathrm{~m}$ transects, sampled at $0.5 \mathrm{~m}$ intervals $(n=101$ per transect). Within each transect, $M$. tanaceti was isolated from 62 and 46 sampling units in field $\mathrm{A}$, and 21 and 45 sampling units in field $\mathrm{B}$. The ratio of mating types did not differ from a 1:1 ratio at either the field or transect scale $(P \geq 0.13)$. Ordinary runs analysis identified no spatial aggregation of $M$. tanaceti or of MAT1-2 genotypes $(P \geq 0.06)$ within transects. Spatially significant $(P<0.05)$ aggregation of MAT1-1 was only identified in a single transect in field B. This data indicates a heterothallic species with no evidence to preclude a sexual cycle occurring in the field. MAT gene functionality will be examined via in vitro crosses and RNA expression analysis.

Identification and aggressiveness of soybean seedborne Fusarium species in Kansas

R. PEDROZO (1), J. Fenoglio (1), C. R. Little (1)

(1) Kansas State University, Manhattan, KS, U.S.A.

Phytopathology 104(Suppl. 3):S3.90

To determine the identity and aggressiveness of seedborne Fusarium spp. of soybean seed in Kansas, seed was collected from 142 soybean samples produced in ten counties (Labette, Cherokee, Neosho, Shawnee, Franklin, Republic, Saline, Reno, Pottawatomie, Crawford) during two growing seasons $(2010,2011)$. Semi-selective media (PCNB) was used for fungal isolations 
and identification was based upon morphological characters and PCR. The rolled-paper towel approach was used to test the aggressiveness of the isolates to soybean seedlings under controlled conditions. The multi-location/year screening efforts showed that seed from $70 \%$ of the counties were infected with Fusarium spp. and a total of 70 isolates were obtained. Seeds from Labette and Cherokee counties (southeast KS) showed the highest incidence of Fusarium (43\% and 19\%, respectively), followed by Crawford $(13 \%)$, Republic $(10 \%)$, Neosho (9\%), Pottawatamie (7\%) and Franklin (1\%) counties. The most frequently encountered Fusarium spp. were: $F$. incarnatum $(50 \%), F$. proliferatum ( $24 \%), F$. equiseti $(9 \%), F$. oxysporum $(4 \%), F$. graminearum $(1 \%), F$. verticillioides $(4 \%), F$. tricinctum $(1 \%), F$. brachygibbosum (1\%) and $F$. thapsinum (1\%). Regarding their agressiveness, $F$. proliferatum, $F$. graminearum, $F$. verticillioides, $F$. tricinctum, $F$. brachygibbosum and $F$. thapsinum were pathogenic to soybean seedlings with moderate to high aggressiveness. Isolates from the $F$. incarnatum-equiseti complex showed no or low pathogenicity.

\section{Generation of disease resistance in tomato using Arabidopsis thaliana} defense-related genes

J. PEREIRA (1), G. V. Minsavage (1), J. B. Jones (1), Z. Mou (1)

(1) University of Florida, Gainesville, FL, U.S.A.

Phytopathology 104(Suppl. 3):S3.91

The United States is one of the world's biggest producers of tomatoes, which accounts for approximate $\$ 2$ billion in annual production. Tomato is a profitable but costly, crop, due to the labor and the amount of chemical inputs required to protect tomato from pests and diseases. Bacterial spot, caused by Xanthomonas perforans, is one of the most devastating diseases of pepper and tomato. Generation of transgenic plants resistant to X. perforans and other fungal and viral diseases of tomato would be a promising alternative. The aim of this work is to identify Arabidopsis thaliana defense-related genes that could confer broad-spectrum resistance to tomato. Two genes involved in the salicylic acid signaling pathway in A. thaliana were transformed into the tomato commercial cultivar Moneymaker. Sixty-one $\mathrm{T}_{1}$ tomato plants (31 transformed with one gene and 30 with the other) were inoculated with $X$. perforans and the symptoms were graded from 1 to 4 , according to its severity (1: no symptoms and 4: severe symptoms). Eight plants showed no symptoms. We are using real-time qPCR to test whether expression levels of the transgene is correlated with the disease severity. A second inoculation has been performed to confirm the disease results. Resistance of the transgenic plants to fungal and viral pathogens will be tested in $T_{2}$ and $T_{3}$ generations.

AtNPR1 confers resistance against Xanthomonas fragariae, the causal agent of angular leaf spot in strawberry

K. J. PEREIRA SILVA (1), A. Brunings (1), N. Peres (1), K. M. Folta (1), Z. Mou (1)

(1) University of Florida, Gainesville, FL, U.S.A.

Phytopathology 104(Suppl. 3):S3.91

Angular Leaf Spot (ALS) of strawberry, caused by the bacterial pathogen Xanthomonas fragariae, is a potentially destructive disease of strawberry worldwide. Management of ALS is extremely costly and inefficient, causing significant environmental pollution. To date, no commercial cultivars is resistant to ALS, making development of resistant varieties important. In this study, we have generated strawberry Hawaii-4 (H4) accession (Fragaria vesca L.) plants expressing the Arabidopsis NPRI (AtNPRI) gene, a key positive regulator of systemic acquired resistance. Fully expanded leaflets of transgenic strawberry plants expressing different levels (high, medium, and low) of AtNPRI were inoculated with suspensions of $X$. fragariae. Leaf tissues were collected for RT-qPCR analysis of AtNPRI expression. Inoculation sites were evaluated every week for 4 weeks, being described as susceptible or resistant, and at the end of the experiments, were processed for bacterial counts. We found that AtNPRI expression could be induced in transgenic plants after $X$. fragariae infection and that transgenic lines expressing high levels of AtNPR1 were resistant and/or developed hypersensitive response. In addition, bacterial growth was significantly reduced in the infected tissues of the transgenic plants. Our data demonstrate that overexpressing the AtNPR1 gene is able to increase disease resistance, which holds great potential for developing strawberry cultivars resistant to $X$. fragariae.

\section{Managing Ramularia leaf spot of barley in Uruguay}

S. A. PEREYRA (1), J. P. Viera (2), N. D. Havis (3)

(1) INIA - Natl Inst for Agric Research, Colonia, Uruguay; (2) FADISOL, Colonia, Uruguay; (3) SRUC-Scotland's Rural College, Edinburgh, Scotland Phytopathology 104(Suppl. 3):S3.91

Ramularia leaf spot (RLS) caused by Ramularia collo-cygni (Rcc), has become a predominant disease of barley in Uruguay since it was first reported in 2000. Yield losses as high as $70 \%$ have been reported in susceptible cultivars. In order to optimize disease control measures, cultivar resistance, seed inoculum and fungicides were investigated during 2011 to 2013. Commercial cultivars and advanced lines were characterized under intermediate to high disease pressure in nurseries and field trials. Most of the commonly grown cultivars were intermediate to susceptible to RLS. Based on Rcc-DNA quantification on 40 seedlots from 2012, representing diverse regions and cultivars, only $25 \%$ of the lots were below the recommended level of inoculum in seeds (1 pg Rcc-DNA/100 pg total DNA). Best timings for fungicide application for RLS control were at stem elongation (ZGS 32 to 38) and awns-peeping (ZGS 47-49). Fungicides containing SDHI (isopyrazam+azoxystrobin and fluxapyroxad+pyraclostrobin+epoxyconazole) were the most effective fungicides in controlling RLS improving yield and grain quality. Results from these studies emphasize the importance of analyzing seedlots for Rcc and suggest that both fungicide and timing are important factors in optimizing RLS control.

A Bayesian approach to estimation of error rates in detection of Heterodera glycines in processed soil samples with low egg concentrations O. PEREZ-HERNANDEZ (1), L. J. Giesler (1), P. E. Reyes (2)

(1) University of Nebraska-Lincoln, Lincoln, NE, U.S.A.; (2) Kansas State University, Manhattan, KS, U.S.A.

Phytopathology 104(Suppl. 3):S3.91

Heterodera glycines, soybean cyst nematode, is the most yield-limiting pathogen of soybean in the U.S. Crucial to research and management of this pathogen is the reliable detection and accurate estimation of population densities, which are determined from microscope readings of the egg number in processed samples. Traditionally, a single reading per sample is conducted on 1-ml subsamples, creating the possibility of faulty detection when sample egg concentration is low or inaccurate estimations when egg concentration is high. In this study we consider the former situation and used a hierarchical Bayesian model to determine the error rates in detection of $H$. glycines. In particular, we examine the case where a first reading (out of 3 ) was negative. A stochastic Poisson process with a Gamma prior was assumed and a sensitivity analysis for prior determinations conducted to estimate the error rates' posterior distributions. Under the model assumptions, the false negative rate would decrease exponentially with each additional reading. $78 \%$ of the samples were classified as positive after 3 readings compared with $70.0 \%$ after 1 reading only. The first reading missed $10.8 \%$ of the positive samples while the second missed 3.3\%. This study shows that faulty detection of $H$. glycines occurs in single readings, suggesting that if reliable diagnosis of a sample is desired, 2 or more readings will significantly minimize potential errors of faulty diagnostics.

Positional cloning and characterization of Fusarium resistance genes in melon

R. PERL-TREVES (1), G. Miller (1), M. Normantovich (1), I. Kovalski (1)

(1) Bar-Ilan University, Ramat Gan, Israel

Phytopathology 104(Suppl. 3):S3.91

Four races of Fusarium oxysporum f.sp. melonis (FOM) exist, that cause severe damage to melon (Cucumis melo L.) worldwide. A whole range of interactions - from fully susceptible to tolerant, quantitatively resistant and fully resistant - have been described between different melon genotypes and specific FOM races. We report the recent cloning of the Fom-1 gene conferring dominant resistance against races 0 and 2 of the pathogen. The gene encodes a TIR-NBS-LRR protein with multiple nucleotide substitutions among resistant and susceptible alleles. This paves the way to study the molecular interaction between the fungus and the cloned R-gene. A transgenic root system ("Composite Plants") served to detect vascular and root-tip expression of a reporter gene driven by the putative $R$-gene promoter in melon. Regarding FOM race 1.2 resistance, quantitative inheritance was proposed. Colonization by a FOM race 1.2 strain that expresses a GFP reporter protein was monitored in vivo in infected roots and stems, suggesting reduced vascular colonization of the quantitative-resistant genotype. On the other hand, stem colonization by FOM race 0 was completely prevented in resistant plants that carry either the Fom-1 or Fom-2 dominant genes.

Use of pre-plant soil concentrations of root knot nematode DNA as a predictor of potato tuber damage

S. J. PETHYBRIDGE (1), F. S. Hay (2), S. J. Jones (2), K. Ophel-Keller (3)

(1) The New Zealand Institute for Plant \& Food Research Limited, Christchurch, New Zealand; (2) Tasmanian Institute of Agriculture, University of Tasmania, Burnie, Australia; (3) South Australian Research and Development Institute, Adelaide, Australia

Phytopathology 104(Suppl. 3):S3.91

Root knot nematode (RKN; Meloidogyne spp.) is common in potato fields in Tasmania, Australia, and capable of substantial yield loss. Meloidogyne fallax 
also causes damage inside the tuber necessitating excessive peeling during processing. Pre-plant quantification of RKN DNA in soil can inform the need for management strategies. In 53 fields, RKN DNA was quantified in soil preplant using a service available to Australian growers, and tuber samples visually assessed for galling at harvest. Meloidogyne fallax and M. hapla were detected in 33 and 13, of 53 fields respectively, with co-occurrence in 12 fields. Receiver operating characteristic (ROC) analysis indicated pre-plant soil DNA of M. fallax was a good predictor of tuber damage $(P<0.0001$; area under the ROC curve $($ AUROC $)=0.838)$. The optimum cut point was $2.90 \mathrm{pg}$ DNA/g dried soil $(J=0.57)$, with sensitivity of 0.75 (i.e. predicting tuber galling in $75 \%$ of 33 fields) and specificity of 0.82 (i.e. predicting no tuber galling in $82 \%$ of 20 fields). The sum of pre-plant soil DNA of $M$. fallax and M. hapla was a good predictor of tuber damage $(P<0.0001$; AUROC $=$ $0.823)$. The optimum cut point was $0.90 \mathrm{pg}$ DNA/g air dried soil pre-plant $(J$ $=0.58$ ) with a sensitivity and specificity of 0.85 and 0.73 , respectively. Preplant soil RKN DNA concentration was a good predictor of tuber damage at harvest, and shows promise as a management tool for growers.

Antagonistic one-way interaction between Phytophthora capsici and Meloidogyne incognita on bell pepper

A. PETKAR (1), V. Parkunan (1), P. Timper (2), P. Ji (1)

(1) University of Georgia, Tifton, GA, U.S.A.; (2) USDA ARS, Tifton, GA, U.S.A.

Phytopathology 104(Suppl. 3):S3.92

The root-knot nematode, Meloidogyne incognita (Mi), and the Phytophthora blight pathogen, Phytophthora capsici (PC), cause serious root diseases on bell pepper. However, the interactions between these two pathogens on different bell pepper genotypes are not clear. Greenhouse experiments were conducted on cultivars of bell pepper with different levels of resistance to $P c$, by concomitant and sequential inoculation of both pathogens. The treatments included inoculating both pathogens simultaneously, inoculating $M i 7$ days before $P c$, inoculating $P c 7$ days before $M i$, and inoculating $M i$ or $P c$ alone. In cultivars Aristotle and Intruder, $P c$ inoculation with $M i$ simultaneously or 7 days before or after $M i$ reduced gall index significantly as compared to $M i$ alone. In Paladin, $P c$ inoculation 7 days before or after $M i$ lowered gall index compared to $M i-P c$ simultaneous inoculation. Phytophthora blight severity was not influenced by $M i$ irrespective of inoculation methods used in the three genotypes. In Aristotle, $M i$ inoculation alone did not reduce fresh root weight, but $P c$ alone or combined with $M i$ reduced fresh root weight as compared to the untreated control. However, none of the treatments reduced fresh root weight in Paladin or Intruder. The interaction between $P c$ and $M i$ seems to be one way in that $P c$ reduced galling of $M i$, but the nematode had no effect on Phytophthora blight severity.

Visual rating of bacterial disease severity as a threshold to time sweet onion harvest

E. PFEUFER (1), B. K. Gugino (1)

(1) Penn State, University Park, PA, U.S.A.

Phytopathology 104(Suppl. 3):S3.92

In Pennsylvania, sweet onion fields may suffer harvest yield losses up to $50 \%$ due to center and other bacterial rots. These pathogens infect the foliage, then move into bulbs resulting in one to several rotten scales. Upon observation of foliar bacterial symptoms, many growers will harvest early, forgoing onion size in favor of more asymptomatic bulbs. To improve harvest recommendations, two replicated trials were conducted in 2013 in which uninoculated, adjacent-to-inoculated, and inoculated onion plants were rated for foliar disease severity and lodging, then harvested and evaluated for disease incidence and marketable yield, which occurred weekly for five weeks. The severity scale ranged from $0=$ asymptomatic to $7=\geq 50 \%$ of leaves collapsed, with lodging recorded as percent of plot. The data suggest a harvest threshold approaching a foliar severity rating of 4 (single bleached and/or collapsed leaf), which coincided with harvest disease incidence of $23-28 \%$ but increased marketable yield with a greater proportion of larger bulbs. This threshold was projected to be reached $17-26,27-35$, and $28-50$ days post-inoculation and resulted in average marketable bulb weights of $0.18-0.24,0.24-0.43$, and 0.25 $0.39 \mathrm{~kg}$ for inoculated, adjacent-to-inoculated, and uninoculated plants, respectively. In order to maximize marketable yields, growers may use this visual foliar disease severity threshold to time their onion harvest.

Development of multiple molecular based assays for the improved detection of viruses, viroids and phytoplasmas infecting imported germplasm D. D. PICTON (1), G. W. Wei (1), K. J. Owens (1), L. Levy (2), M. K. Nakhla (1)

(1) USDA-APHIS-PPQ-S\&T, Beltsville, MD, U.S.A.; (2) USDA-APHISPPQ-S\&T, Riverdale, MD, U.S.A.

Phytopathology 104(Suppl. 3):S3.92
Foreign plant germplasm is a valuable source of novel genes not present in U.S. plant germplasm. Viruses, viroids and phytoplasmas infecting imported plant germplasm pose a risk to agricultural, rural and native plant systems. Since 2009, the CPHST Beltsville lab has developed and adapted assays for the detection of viruses, viroids and phytoplasmas of regulatory concern. The program has developed a portfolio of thirty-two PCR assays targeting viruses, viroids and phytoplasmas. Within this portfolio are real-time and conventional RT-PCR assays that will detect viruses from six families. The assays include conventional PCR group tests for the Pospiviroids, Nepovirus subgroups A and B, Potyviruses, Luteoviruses and Phytoplasmas. These assays can be used by programs such as the PPQ Plant Germplasm Quarantine Program and the National Clean Plant Network to determine whether plant germplasm is infected with a particular virus or virus group. In addition tests will supplement or reduce the time required by biological indexing that will significantly reduce the period of time necessary for quarantine of imported plant germplasm.

Evaluation of potential inhibitory peptides targeting the essential PhoP/Q two-component regulatory system in Xylella fastidiosa

B. PIERCE (1), B. Kirkpatrick (1)

(1) UC Davis, Davis, CA, U.S.A.

Phytopathology 104(Suppl. 3):S3.92

Xylella fastidiosa $(X f)$ is a gram-negative, xylem-limited plant pathogenic bacterium that causes disease in a variety of economically important agricultural crops including Pierce's disease of grapevine. $X f$ biofilms formed in xylem vessels play a key role in early colonization and pathogenicity by providing a protected niche and enhanced cell survival. Like many other bacteria, $X f$ possesses homologs to the $\mathrm{PhoP} / \mathrm{Q}$ two-component regulatory system, which differentially regulates genes in responses to divalent periplasmic cation concentration, antimicrobial peptides and other environmental stimuli. $X f$ knockout mutants deficient in the production of PhoP/Q exhibit phenotypic differences in cell dispersal and clumping when grown in liquid culture. Grapevine pathogenicity assays showed PhoP/Q mutants are nonpathogenic and are unable to successfully colonize or move within the xylem vessels. These results are likely due to an inability of $X f$ to successfully sense, respond and adapt to the nutrient-limited environment of the xylem. PhoQ, responsible for sensing the environment and essential for $X f$ survival in grapevines, is being evaluated as a novel disease control target through the use of inhibitory peptides. The PhoP regulon is also being explored to further our understanding of $\mathrm{PhoP} / \mathrm{Q}$ induced gene regulation in $X f$.

Resistant strains of Botrytis cinerea to boscalid in Chilean grapevines (Vitis vinifera)

C. M. PIQUERAS (1), B. A. Latorre (1), R. Torres (1)

(1) Pontificia Universidad Catolica de Chile, Santiago, Chile Phytopathology 104(Suppl. 3):S3.92

Gray mold (Botrytis cinerea) is the most important grapevine disease in Chile. Boscalid, a single mode of action fungicide, has been extensively used against $B$. cinerea, introducing a high risk of resistance. The sensitivity of $B$. cinerea to boscalid was studied using 50 isolates from commercial vineyards with history of continuous use of boscalid. On the basis of mycelium growth inhibition on minimal media $(\mathrm{MM})+50 \mu \mathrm{gmL}^{-1}$ boscalid, isolates were high resistant (HR, 2\%), moderate resistant (MR, 32\%), low resistant (LR, 64\%) and sensitive (S, 2\%). Nine selected isolates (1 HR, $5 \mathrm{MR}, 2 \mathrm{LR}$ and $1 \mathrm{~S}$ ) were studied further for conidial germination on $\mathrm{YBA}+5 \mu \mathrm{gmL}^{-1}$ boscalid, obtaining $1 \mathrm{HR}, 1 \mathrm{MR}$ and $7 \mathrm{~S}$ isolates. These results suggested the absence of correspondence between mycelium and conidial sensitivity to boscalid. PCRPIRA was used to detect $S d h$ B mutations. HR isolate, amplified with H272Lfw/H272-rev and digested by enzyme BglII, yielded two fragments ( 35 and 85 bp), but $\mathrm{S}$ and $\mathrm{MR}$ isolates remained undigested, confirming a mutation at condon 272 (H272L) in HR phenotype. At label rate, boscalid slightly controlled rot on apples inoculated with mycelium or conidia of a HR phenotype isolate. These results demonstrate that mycelium and conidia assays were useful for detection of resistance to boscalid using, respectively, $\mathrm{MM}+50 \mu \mathrm{gmL}^{-1}$ boscalid or $\mathrm{YBA}+5 \mu \mathrm{gmL}^{-1}$ boscalid. The presence of boscalid HR strains of $B$. cinerea in Chilean vineyards is reported in this study.

Characterization of basal resistance of sour orange (Citrus aurantium) against Xanthomonas citri subsp. citri and 'Candidatus Liberibacter asiaticus'

M. PITINO (1), Y. Duan (2)

(1) USDA ARS, Fort Pierce, FL, U.S.A.; (2) USDA, Fort Pierce, FL, U.S.A. Phytopathology 104(Suppl. 3):S3.92

Plant immunity consists of two levels of defense response against pathogens: pathogen-associated molecular patterns (PAMP)-triggered immunity (PTI) 
and effector-triggered immunity (ETI). In response to infection by the citrus canker bacterium, Xanthomonas citri subsp citri (Xcc), citrus plants will employ both PTI and ETI, while infection by the citrus huanglongbing (HLB) bacterium, Candidatus Liberibacter asiaticus (Las), may only elicit PTI in citrus plants due to the nature of Las genome and its intracellular lifestyle. In this study, we evaluated the level of basal resistance of a resistant/tolerant sour orange variety (Citrus aurantium) by testing for reactive oxygen species (ROS) by its seedlings challenged with the Xcc-flg22 and Las-flg22, respectively. The results showed a wide range of ROS production among these seedlings. Further investigation showed a positive correlation between ROS production and disease resistance in these seedlings when the plants were inoculated with the individual bacterial pathogens. When inoculated with $\mathrm{Xcc}$ at ca. $10^{5}$ to $10^{6} \mathrm{CFU} / \mathrm{ml}$, plants with higher ROS showed fewer lesions and lower titers of Xcc bacteria in young leaves than those with a low or absent ROS production. Similar results were obtained with graft-inoculation of HLB, plants with higher ROS showed lower titers of Las bacteria and vice versa. This is the first evidence of bacterial resistance and segregation associated with ROS production in citrus seedling plants.

Association between fumonisin B1 production, maize colonization and glucanase activity suppression in Fusarium verticillioides

J. PLASENCIA (1), E. Galeana-Sanchez (2), D. Sanchez-Rangel (2)

(1) Univ Nacional Autonoma de Mexico, Mexico DF, Mexico; (2) UNAM, Mexico City, Mexico

Phytopathology 104(Suppl. 3):S3.93

Fusarium verticillioides is frequently isolated from maize worldwide as it causes seedling blight, stem rot and ear rot. This ascomycete synthesizes several toxins and fumonisin B1 (FB1) is probably the most abundant. This mycotoxin has several targets in maize cells as it inhibits ceramide synthase, $\mathrm{H}^{+}$-ATPase and certain isoforms of beta-1,3-glucanases. FB1 production is highly variable among $F$. verticillioides strains thus we address whether this capacity was associated with fungal colonization, symptom induction and glucanase inhibition in maize plants. We employed four $F$. verticillioides strains and measured FB1 production in synthetic media and in maize embryos. These strains were also employed to infect maize seeds, seedlings and plants to assess fungal colonization and symptom development. We characterized two high-producing strains and two low-producing strains that show ed a similar FB1 level pattern in both substrates. In general, the highFB1-producing strains yielded higher conidia counts in infected tissue and caused more to the embryos leaf and stems than the low-producing strains. Beta-1,3-glucanase activity was significantly suppressed in germinating maize embryos 24 hours post-inoculation only by the two FB1 high-producing strains. Although no isogenic strains were employed in this work, our results support the role of FB1 in the infection and colonization of maize plants because they are infected in the field by a wide array of strains.

\section{Reproduction of Meloidogyne arenaria on flue-cured tobacco homozygous} for $R k 1$ and/or $R k 2$

J. POLLOK (1), C. Johnson (2), J. Eisenback (3), D. Reed (2)

(1) Virginia Tech Southern Piedmont AREC, \& Dept. Plant Pathology, Physiology, \& Weed Science, Blacksburg, VA, U.S.A.; (2) Virginia Tech Southern Piedmont AREC, Blackstone, VA, U.S.A.; (3) Virginia Tech Dept. Plant Pathology, Physiology, \& Weed Science, Blacksburg, VA, U.S.A. Phytopathology 104(Suppl. 3):S3.93

Meloidogyne incognita has historically been the most significant root-knot (RK) nematode species on tobacco (Nicotiana tabacum L.) in Virginia. However, most cultivars now contain the $R k 1$ resistance gene to races 1 and 3 of $M$. incognita, allowing $M$. arenaria to become the most common RK species found on tobacco. An increasing number of cultivars also currently possess $R k 2$ for RK resistance, but aspects of its efficacy are unknown. Four greenhouse experiments were conducted in $2012-2013$ to investigate whether possessing both $R k 1$ and $R k 2$ increases resistance to $M$. arenaria compared to either gene alone. Each trial was arranged in a RCBD with 6 replications, and compared galling, and numbers of egg masses and eggs from roots of six cultivars C371G (susceptible), NC 95 and SC 72 (homozygous for $R k 1$ ), T15-1-1 (homozygous for $R k 2$ ), and STNCB-2-28 and NOD 8 (homozygous for both $R k 1$ and $R k 2$ ). Each plant was inoculated with 5,000 M. arenaria eggs; data were collected 60 days post-inoculation. Plants with $R k 1$ alone, and $R k 1$ and $R k 2$ together, significantly reduced root galling and numbers of egg masses and eggs versus C371G. Compared to presence of $R k 1$ alone, combining $R k 1$ and $R k 2$ further reduced galling in 1 of 4 trials, egg masses in 2 of 4 trials, and eggs in 1 of 3 trials. A better understanding of the specific effects of $R k 1$ and $R k 2$ on root-knot nematode parasitism should help plant breeders improve resistance in tobacco to M. arenaria.
Exploration of phyllosphere fungal communities of Quercus macrocarpa using network analysis

R. POUDEL (1), A. Jumpponen (1), K. Garrett (1)

(1) Kansas State University, Manhattan, KS, U.S.A.

Phytopathology 104(Suppl. 3):S3.93

Network analysis of the co-occurrence of microbial taxa provides a dynamic portrait of community interactions. We studied the networks of co-occurrence of phyllosphere fungi in oak trees (Quercus macrocarpa) sampled within (urban) and outside (rural) Manhattan, Kansas. A DNA database describing the relative frequency of fungal taxa was evaluated, where a node represents a taxon and an edge exists between two taxa if their frequency is correlated across samples. The average node degree was 2.0 for both the sites, although the connectance was higher in the urban site $(0.2)$ compared to the rural site $(0.1)$. The number of interacting taxa was higher in the rural site than in the urban (21 vs. 10) with an average path length of 1.12 and 1.16 respectively. At the phylum level, Basidiomycota and Ascomycota frequency were correlated in both the sites, where Ascomycota was the dominant group. Three out of ten taxonomic groups that exhibited correlation links (Puccinales, Taphrinales and Microbotrymycetidae) were unique to the urban site, while the node degree for the shared taxa was higher in the rural site. Thus, in addition to confirming previous analyses of taxa specific to a site, we also identified how patterns of co-occurrence of taxa varied in urban and rural sites. Understanding microbial interactions using topological network parameters may ultimately contribute to understanding the risk of foliar infection, and the general dynamics of foliar fungal communities.

Pathogenetic characterization of Magnaporthe oryzae population in Colombia and identification of sources of resistance

G. A. PRADO (1), C. Vallejos (1), Y. Ospina (1), G. Aricapa (1), J. Cuasquer (1), M. C. Duque (1), C. Grenier (2), G. M. Mosquera (1)

(1) CIAT, Palmira, Colombia; (2) CIRAR, Palmira, Colombia

Phytopathology 104(Suppl. 3):S3.93

Rice blast caused by Magnaporthe oryzae is the most important disease worldwide, genetic resistance is the most effective practice for managing the disease. To characterize Colombian blast population, 172 isolates collected in 15 different locations including a "blast hot spot", were inoculated under greenhouse conditions using the differential system developed by JIRCASIRRI (2008). At the same time two commercial varieties were evaluated using the same population. Data from 20 plants per line with each isolate including at least two replications were analyzed by SAS 9.2. Results showed the presence of 75 races with a virulence spectra ranging from 34 to $97 \%$ in $24 \mathrm{R}$ genes tested, where Pi- 9 and Pi- 40 were resistant to $89 \%$ and $79 \%$ of the races identified respectively, and commercial varieties tested shown to be resistant to $86 \%$ of the races. Sixty eight percent of the races were formed by a single isolate and $32 \%$ of the races were represented by 2 to 19 isolates. Nearly $43 \%$ of the races were found in the blast hot spot showing the importance of this place as selection site for blast resistant germplasm. The broad pathogenic diversity found in Colombian blast population, and at the same confirm the suitability of using "hot spot" strategy in which varieties selected by this approach have yielded genotypes with good levels of resistance. Ideally Pi-9 and $\mathrm{Pi}-40$ should be introduced in breeding program to improve the resistance of newly developed varieties for the region.

Simultaneous detection and differentiation of three stone fruit viruses by a multiplex TaqMan real-time RT-PCR

L. PU (1), G. Kinard (1), M. Bateman (2), P. Lan (1), R. Li (1)

(1) USDA-ARS, Beltsville, MD, U.S.A.; (2) USDA-APHIS, Beltsville, MD, U.S.A.

Phytopathology 104(Suppl. 3):S3.93

Many viruses infect Prunus spp. and cause diseases in stone fruit production including American plum line pattern virus (APLPV), Prune dwarf virus (PDV) and Tomato ring spot virus (ToRSV) In this study, a one-tube reverse transcription (RT) TaqMan real-time PCR was developed for the simultaneous detection and differentiation of APLPV, PDV and ToRSV. Amplification and detection of a fluorogenic cytochrome oxidase gene (COX) was included as an internal control. Sensitivity of the multiplex assay was $10^{-4}$ for each of the positive controls and for the COX mRNA, which was similar to that of individual conventional RT-PCR and 10-fold less than that of the individual TaqMan real-time RT-PCR. The multiplex assay was evaluated using samples from 47 field trees that had been previously identified as infected with APLPV or PDV. Samples were also collected from another 152 previously untested trees, and APLPV and/or PDV was detected in 40 and 27 cherry and plum trees, respectively. ToRSV was not detected in any samples other than the positive controls. The results of the multiplex assay were further confirmed by individual TaqMan real-time RT-PCR assays, indicating the multiplex assay was sensitive and specific. The multiplex assay described here 
offers a valuable tool for rapid, sensitive and cost-effective detection and identification of the three target viruses. It will be useful for quarantine, certification and germplasm curation programs.

\section{A new Umbravirus possibly associated with papaya ringspot disease in Ecuador}

D. F. Quito-Avila (1), M. A. Ibarra (1), R. A. ALVAREZ (1), R. Martin (2)

(1) Centro de Investigaciones Biotecnologicas del Ecuador CIBE-ESPOL, Guayaquil, Ecuador; (2) USDA-ARS, Corvallis, OR, U.S.A.

Phytopathology 104(Suppl. 3):S3.94

Papaya is one of the most important agricultural commodities in Ecuador. Papaya ringspot virus (PRSV), the most economically important and widespread virus of papaya, causes a range of symptoms on the leaves and fruit. In Ecuador, the virus has been managed mainly by rouging infected plants as soon as they exhibit symptoms. In recent years, experienced field managers have failed to control the spread of the disease due to inconsistencies in the expression of "classic" symptoms. The presence of new viruses possibly associated with PRSV was suggested. Symptomatic leaves were collected from both commercial and non-commercial plantings in Los Rios province. Electrophoretic analysis of double-stranded RNA preparations showed a $\sim 10 \mathrm{~kb}$ band, corresponding to the PRSV genome. In addition, a prominent band of $\sim 4 \mathrm{~kb}$ was observed. Blast searches revealed the presence of a novel virus with $38 \%$ amino acid (aa) identity to Carrot mottle virus, a $4.2 \mathrm{~kb}$ Umbravirus found naturally in carrot. Specific primers were then used to detect the virus in leaves collected from individual symptomatic trees in two commercial fields. Of the sampled plants $45 \%(n=24)$ tested positive for the new virus. A set of degenerate primers developed to detect several umbraassociated luteoviruses failed to amplify the expected fragment. To the best of our knowledge, this is the first report an umbravirus infecting papaya worldwide.

\section{Pathogenicity of Pythium species isolated from Minnesota soybean fields} on soybeans and corn

L. RADMER (1), G. Anderson (1), D. Malvick (1), J. Kurle (1)

(1) University of Minnesota, St. Paul, MN, U.S.A.

Phytopathology 104(Suppl. 3):S3.94

Pythium species are a major cause of seedling diseases in soybean and corn, two crops often planted in rotation. Although they are known to cause seed decay, seedling blight, damping-off and root rot, their importance as seedling pathogens in Minnesota is unknown. Our objectives were to determine which species of Pythium are present in soybean fields in Minnesota and the pathogenicity of those species to soybean and corn. Pythium isolates were collected from 46 soybean fields with establishment problems in 22 Minnesota counties using one of two methods. In the first method, Pythium was isolated directly from symptomatic soybean seedlings collected in the field. In the second, soil from the field was collected, dried, and baited with soybean seeds in the laboratory. Isolates were identified using both morphological and molecular techniques. Using the seedling sampling method, 29 Pythium and one Phytopythium species were found in 12 fields in nine counties. Nine Pythium and two Phytopythium species were found in 12 fields in 10 counties by baiting. The pathogenicity of the most common Pythium and Phytopythium species was tested on both soybean and corn using plate and pot assays at a variety of temperatures. The Pythium species in general severely reduced root growth but differed widely in their effect on seedling development. The results confirm that Pythium species are a significant cause of seedling disease on soybean and corn in Minnesota.

Transcriptomic profiling of soybean-Fusarium virguliforme interactions suggests a role of the pathogen and its toxins during the disease development

\section{O. RADWAN (1)}

(1) University of Illinois at Urbana-Champaign, Urbana, IL, U.S.A. Phytopathology 104(Suppl. 3):S3.94

Fusarium virguliforme is a soil-borne fungus, which causes sudden death syndrome by colonizing soybean roots causing root rot, and also produces toxins leading to leaf scorching symptoms and defoliation. The objectives of this study were to i) identify the fungal genes that are induced during the root rot development, and ii) determine the role of fungal toxins during triggering the programmed cell death (PCD) in soybean leaves. Total RNA was extracted from roots and leaves collected from same plants at 5, 7 and 9 day post infection (dpi). A total of $40 \mathrm{cDNA}$ libraries were sequenced via Illumina HiSeq2500 sequencing using a paired-end read. Data from the susceptible genotype was compared with those from the partial resistant genotype to identify differentially expressed genes from both host and pathogen. Pathogen reads from the resistant genotype represented only $54-71 \%$ from the susceptible one reflecting a high abundance of the pathogen in susceptible roots. Functional annotations of fungal genes induced in susceptible genotype suggested multiple mechanisms might be developed by the pathogen to protect itself from the toxin and induce root rot. On the other hand, it appears that the fungal toxins manipulate HR and SA pathway in susceptible leaves to induce host cell death in the absence of the pathogen. Understanding the pathogen mechanisms to induce SDS disease will further help pathologists and breeders to develop an effective method to control this disease.

Fungicidal management of celery anthracnose caused by Colletotrichum acutatum

R. N. RAID (1), D. Klamer (2), J. Klamer (2)

(1) University of Florida, Belle Glade, FL, U.S.A.; (2) V. \& W. Farms, Byron Center, MI, U.S.A.

Phytopathology 104(Suppl. 3):S3.94

Since 2010, celery anthracnose has been detected in several northern U.S. states and Canada. Incited by the fungal pathogen Colletotricum acutatum, the disease is characterized by leaf curl, adventitious root initials, petiole necrosis, and in severe infections, total death and decay of the celery heart. During 2013, a trial was conducted in a commercial field exhibiting a history of anthracnose to evaluate fungicidal efficacy for management of this disease. The experiment consisted of twelve treatments arranged in a randomized complete block design with four replications. Fungicides were applied at weekly intervals using a $\mathrm{CO}_{2}$ backpack sprayer equipped with a handheld boom. A total of 10 applications were made over the course of the season. Environmental conditions were very conducive for anthracnose development, with frequent rain supplemented by overhead irrigation. Disease pressure was significant, resulting in a disease incidence of $41 \%$ in the untreated check. All fungicides with the exception of the broad-spectrum protectant chlorothalonil provided for significant reductions in disease incidence. In general, fungicides containing a strobilurin fungicide, either alone or in combination with a carboximide or triazole fungicide, provided for the greatest efficacy. Carboximides and triazoles applied solo were intermediate in control, significantly better than the untreated check, but significantly inferior to the strobilurins alone or in premixture.

The link between RNA structure, factor requirement and plant viral resistance

A. RAKOTONDRAFARA (1), J. Zhang (1), R. Roberts (1), L. Mayberry (2), S. Tatineni (3), K. Browning (2)

(1) University of Wisconsin-Madison, Madison, WI, U.S.A.; (2) University of Texas-Austin, Austin, TX, U.S.A.; (3) University of Nebraska-Lincoln, Lincoln, NE, U.S.A.

Phytopathology 104(Suppl. 3):S3.94

Genetic resistance remains the best long-term strategy for combating viral diseases. Despite the direct link between plant resistance genes against many RNA viruses to host translation factors, it is astonishing how little we know on how these viruses are translated and how these resistance genes affect viral infection. Of particular interest, are the internal ribosome entry site (IRES) RNA sequences previously reported in the members of the largest family of plant viruses Potyviridae, which encompasses $30 \%$ of the most damaging viral diseases. These RNA are characterized by their small size, structural simplicity and requirement for most of the host translation factors to mimic the function of a cap structure. Our objective is to dissect the mechanism of translation of a newly emerging Triticum mosaic virus (TriMV), a member of Potyviridae, in wheat. Our preliminary results reveal that TriMV RNA has an atypically long and structured IRES RNA element, which corresponds to its 5' leader sequence. Such features have not yet been described in plant viruses, while they are prominent in the animal counterparts. Remarkably, the element sustains 2 fold higher translation than a cellular-mimicking mRNA and 100fold higher translation of that of previously reported potyviral IRESes. Such strong level of translation driven by a plant viral IRES has not yet been reported and raises many important questions in efficiency of disease resistance targeting translation.

Wheat curl mite and Wheat streak mosaic virus dynamics on green bridge hosts in Montana

N. B. RANABHAT (1), M. E. BURROWS (1), F. D. Menalled (1), Z. J. Miller (1)

(1) Montana State University, Bozeman, MT, U.S.A.

Phytopathology 104(Suppl. 3):S3.94

One of the most challenging aspects of managing Wheat streak mosaic virus (WSMV) is the diversity of alternative hosts that can act as a "green-bridge." In addition, understanding the peak period of vector movement (wheat curl mite, WCM, Aceria tosichella Keifer) is crucial for the ecological management of this disease. WCM presence on winter wheat trap plants was monitored weekly for thirteen weeks from August 2013 to October, 2013. We 
compared the probability of mite infestation in controls (bare ground) and six green bridge hosts: pre- and post-harvest volunteer wheat (Triticum aestivum L.), corn (Zea mays L.), fall and spring emerged downy brome (Bromus tectorum L.), and green foxtail (Setaria viridis (L.) P. Beauv). The probability of mite infestation varied significantly by week $(\mathrm{p}<0.0001)$ and treatment $(\mathrm{p}$ $=0.0021$ ). Peak seasonal WCM infestation risk was observed between midaugust to mid-September. During this period the probability of risk of WCM infestation was statistically similar to control (mean $=77 \%$ ). However, downy brome and green foxtail increased risk of infestation relative to the controls prior to and following the peak in mite infestation risk, respectively. The risk of infestation was largely driven by seasonal dynamics of WCM immigration. Further information will be obtained on transmission of WSMV by the WCM and incorporated into a risk model for the Great Plains region of the United States.

Pistachio Bushy Top Syndrome; an emerging threat to the pistachio industry

J. J. RANDALL (1), R. Stamler (2), J. Kilcrease (1), J. Randall (1)

(1) New Mexico State University, Las Cruces, NM, U.S.A.; (2) NMSU, Las Cruces, NM, U.S.A.

Phytopathology 104(Suppl. 3):S3.95

The UCB1 rootstock widely used by the Pistachio industry is a valuable rootstock that has undergone years of experimental trials and consistently performs well in the San Joaquin valley in California (Ferguson et al., 2002; Kallsen 2011). In the last three years, a significant percentage of clonal UCB1 rootstocks planted in orchards both in California and in Arizona exhibited symptoms. These trees are short and stunted, have closely spaced internodes, and exhibit "bushy" growth that resembles witches broom. The roots of these plants are also twisted. The percentage of these abnormal rootstocks within affected fields varied from $30 \%$ to $63 \%$. Symptomatic rootstocks exhibited only $30 \%$ budding success in the field. Many of the trees that were t-budded also suffered from cracking around the bud-union. The cause of these symptoms is unknown and we are proposing the term 'Pistachio Bushy Top Syndrome' (PBTS) for describing these affected trees. Healthy as well as PBTS positive samples are currently being screened for pathogens using DNA sequence analyses and transmission electron microscopy. Micrographs reveal accumulation of p-proteins within sieve elements and sieve pores, collapsed phloem cells, accumulation of callose, and other histological abnormalities. Initial sequence analysis associates PBTS with a microbe that has been demonstrated to cause similar symptoms in other plant species.

The role of soybean NADPH oxidases in resistance to Sclerotinia stem rot A. RANJAN (1), D. L. Smith (1), M. Kabbage (1)

(1) University of Wisconsin Madison, Madison, WI, U.S.A.

Phytopathology 104(Suppl. 3):S3.95

Sclerotinia sclerotiorum is a necrotrophic fungal pathogen with a broad host range. On soybean it causes Sclerotinia stem rot (SSR), which can be a substantial yield limiting disease. Recent studies have indicated that $S$. sclerotiorum can induce apoptotic-like programmed cell death (PCD) in its host plant. Induction of PCD and disease is reliant on the generation of reactive oxygen species (ROS) in the host. Major sources of ROS in plants under pathogen attack include plasma membrane bound NADPH oxidases. They catalyze conversion of oxygen to superoxide anion $\left(\mathrm{O}_{2}^{-}\right)$, a reactive free radical. NADPH oxidases, also known as respiratory burst oxidase homologs (RBOHs), have been identified in various plant species including tomato, tobacco, Arabidopsis and maize but their role in disease response in soybean is currently unknown. Arabidopsis NADPH oxidases, AtRbohD and AtRbohF have been implicated in plant defense response to biotic and abiotic stress. To characterize the role of soybean NADPH oxidases involved in S. sclerotiorum infection, 19 putative $R b o h$ genes were identified from soybean databases that are homologous to Arabidopsis AtRbohD and AtRbohF. This study has shown that virus induced gene silencing (VIGS) of one homolog of NADPH oxidases, $G m R b o h F$, leads to enhanced resistance to $S$. sclerotiorum. Further characterization of these genes may provide a better understanding of their role in resistance, cell death and ROS production during $S$. sclerotiorum infection.

The role of lipopolysaccharide in the vector-microbe and plant-microbe interfaces of the bacterial phytopathogen, Xylella fastidiosa

J. N. RAPICAVOLI (1), H. J. Shugart (2), C. M. Johnston (1), T. M. Perring (1), A. Morales-Cruz (3), D. Cantu (3), M. C. Roper (1)

(1) University of California, Riverside, CA, U.S.A.; (2) University of Florida, Lake Alfred, FL, U.S.A.; (3) University of California, Davis, CA, U.S.A. Phytopathology 104(Suppl. 3):S3.95

Xylella fastidiosa $(\mathrm{Xf})$ is a gram-negative, xylem-limited bacterium that causes serious diseases in economically important crops, such as Pierce's Disease (PD) of grapevine. Lipopolysaccharide (LPS) is the dominant macromolecule displayed on the bacterial cell surface. LPS acts as a selective barrier, preventing entry of toxic substances into the cell, and as an anchor for superficial structures, which contributes to attachment and colonization of plant and arthropod hosts. Finally, LPS is a well-described pathogenassociated molecular pattern (PAMP) and is known to elicit host basal defense responses in model plant systems. LPS is composed of a conserved lipid Acore oligosaccharide component and a variable $\mathrm{O}$-antigen. Through mutations made in wzy (XP0836), which encodes an O-antigen polymerase, we have demonstrated that the $X f$ O-antigen contributes to plant colonization, and depletion of the O-antigen causes a severe reduction in overall virulence in planta. Here, we show that truncation of the O-antigen significantly affects the transmission of $X f$ by an efficient insect vector, the blue-green sharpshooter, Graphocephala atropunctata (Signoret). We are also investigating the role of the $X f \mathrm{O}$-antigen in modulation of the basal defense response in grapevine. Our goal is to determine if specific alterations to the LPS structure cause a change in the elicitation of the grapevine response to $X f$, therefore affecting critical, early stages of $X f$ establishment in planta.

Transgenic control of aflatoxin contamination in corn through hostinduced gene silencing

Y. RARUANG (1), Q. Wei (2), B. H. Bluhm (3), R. L. Brown (2), D. Bhatnagar (2), Z.-Y. Chen (1)

(1) Department of Plant Pathology \& Crop Physiology, Louisiana State University Agricultural Center, Baton Rouge, LA, U.S.A.; (2) Southern Regional Research Center, USDA-ARS, New Orleans, LA, U.S.A.; (3) Department of Plant Pathology, University of Arkansas, Fayetteville, AR, U.S.A. Phytopathology 104(Suppl. 3):S3.95

Maize (Zea mays L.) is an important agronomic crop that is susceptible to Aspergillus flavus infection and subsequent contamination with aflatoxin, the most potent carcinogenic secondary metabolite of the fungus. This pathogen has the potential to cause severe economic losses due to aflatoxin contamination. The A. flavus alkaline protease, a key enzyme involved in the colonization of maize kernels, was selected as a possible candidate for suppression through host-induced gene silencing (HIGS). In this study, an RNAi vector containing the alk gene was constructed and introduced into immature B104 maize embryos through Agrobacterium transformation. Eight transgenic plants from eight independent transformation events were produced. PCR analysis of the genomic DNA from leaf tissue confirmed the presence of the transgene in six of these plants. Real time PCR analysis of RNA isolated from transgenic leaf tissues showed a high variation in fungal target gene expression among the transgenic plants. Kernel screening assays were performed to determine aflatoxin level in the mature transgenic seeds seven days after inoculation with A. flavus conidia. Transgenic plants containing the alk gene had less aflatoxin than the plants that did not contain the gene. These results suggest that HIGS using the alk gene can reduce aflatoxin contamination in maize. Future studies will focus on detecting the presence of gene specific siRNA.

Real-time PCR detection of the kiwi canker pathogen, Pseudomonas syringae pv. actinidiae

J. E. RASCOE (1), M. K. Nakhla (1)

(1) USDA-APHIS-PPQ-S\&T, Beltsville, MD, U.S.A.

Phytopathology 104(Suppl. 3):S3.95

Bacterial canker of kiwifruit, or kiwi canker, was first observed in Hunan Province, China in 1954-85, and was later isolated and described in Japan in 1989 as being caused by Pseudomonas syringae pathovar actinidiae. It was subsequently discovered in Korea and Italy, but did not cause severe losses until 2008, when an outbreak of a new, more severe strain was discovered in Italy. This outbreak continues to this day, having reached kiwi producing areas in New Zealand, France, Portugal, Chile, Spain, Turkey, and Switzerland, threatening the sustainability of the kiwi industry in all major growing regions. This pathogen has not been detected in the US. Earlier molecular diagnostic techniques relied primarily on detection of $16 \mathrm{~S}$ rDNA sequences which caused false positive reactions with related pseudomonads such as pv theae. In the detection assay described here, an effector gene present in all virulent kiwi canker isolates is targeted in a duplex real-time polymerase chain reaction (PCR) in which a second internal control reaction targets plant cytochrome oxidase subunit 1 gene $(\mathrm{COX})$, providing a measure of quality of DNA extraction and lack of PCR inhibition. This method should permit the screening of large numbers of samples for field assays or for confirmatory diagnostics.

Prevalent sunflower rust races in Manitoba, Canada in 2004-2013 K. Y. RASHID (1)

(1) Agric \& Agri-Food Canada, Morden, MB, Canada

Phytopathology 104(Suppl. 3):S3.95 
Sunflower is grown in western Canada on $\sim 50,000$ ha mainly in Manitoba. Rust (Puccinia helianthi Schwein) is a major sunflower disease worldwide. Disease surveys were conducted in 30-60 fields in Manitoba, 2004-2013. Rust incidence and severity were assessed based on \% leaf area affected, and isolates were collected. Urediospores of each isolate were suspended in petroleum oil and inoculated onto seedlings of the international set of nine rust differential genotypes under controlled growth room conditions. The Resistant/susceptible reactions for each isolate/genotype determined the race identity of isolates. Rust incidence and severity varied annually with severe epidemics in 2008-2009, and moderate local epidemics in the rest of the 10year. In 2009 , rust resulted in $25 \%$ leaf area affected in $70 \%$ of the crops in Manitoba caused primarily by the virulent races 776 and 777 and yield losses were estimated at $25 \%$ in affected crops. Local rust epidemics were observed in some years predominantly with race groups 700 and 300 . A steady increase in prevalence of race group 700 and a decline in the race groups 500 and 300 have been observed. The most virulent race 777 appeared in $23 \%$ of 2009 isolates but not in 2010 or 2011 due perhaps to the lack of fitness and a sharp decline in sunflower acreage. However, 777 re-appeared in 5\% of the 2012 and 2013 isolates.

\section{Prevalent races of sunflower downy mildew in Manitoba, Canada 2004-} 2013

K. Y. RASHID (1)

(1) Agric \& Agri-Food Canada, Morden, MB, Canada

Phytopathology 104(Suppl. 3):S3.96

Sunflower is grown in western Canada on $\sim 50,000$ ha mainly in Manitoba. Downy mildew (DM) caused by the fungus Plasmopara halstedii is a major sunflower disease worldwide causing $10-20 \%$ yield reduction, and up to $40 \%$ in some crops. Disease surveys were conducted in 30-60 fields in Manitoba, 2004-2013. DM incidence and severity were recorded, and infected plants were collected, DM was isolated to identify races. Isolates were tested by inoculating 2-days-old seedlings of the international nine sunflower differential genotypes by immersing the seedling in spore suspension for 4 hrs. Infected seedlings were transplanted into a soil mixture and kept under $18^{\circ} \mathrm{C}$ and $18 / 6 \mathrm{~h}$ day/night for 14 days, then incubated at $100 \mathrm{RH}$ for $24 \mathrm{~h}$. Virulence was assessed based on resistant and susceptible reaction to identify races. Prevalence of DM ranged from $20-82 \%$ with an incidence range from trace to $30 \%$. New race-groups 700 and 300 were more prevalent than the 100, 200 and 500 groups previously reported in Canada and USA. The new races are virulent on most commercial hybrids in Canada. Prevalence and incidence of DM in 2006, 2008, 2010, 2012, and 2013 were lower than in 2005, 2007, 2009, and 2011 due perhaps to the variability in soil moisture levels and temperatures at the seedling stage. Recently released sunflower hybrids proved more resistant to DM than old hybrids.

\section{Aster yellows, a potential disease epidemic in flax}

\section{K. Y. RASHID (1)}

(1) Agriculture \& Agri-Food Canada, Morden, MB, Canada

Phytopathology 104(Suppl. 3):S3.96

Aster Yellows (AY) caused by a Phytoplasma was first reported in 1902. It is a common and destructive disease worldwide affecting $>300$ plant species. AY has been considered a minor disease in flax causing the transformation of flower buds into vegetative buds with star-shape appearance and no seed production. It is transmitted by several species of leafhoppers that migrate from south to north in the spring, especially the six-spotted leafhopper Macrosteles fascifrons. The phytoplasma overwinters in perennial host plants, weeds, winter crops, and in adult leafhoppers. Annual surveys have been conducted since 1988 of 60-100 flax crops in western Canada. AY was identified by symptoms, and the incidence and severity were recorded. In 2012, AY was prevalent and caused serious epidemics on most field crops in western Canada. $60 \%$ of flax crops had AY with trace to $5 \%$ plants affected in comparison with $5-30 \%$ of the crops and trace to $1 \%$ incidence in the previous 12 years. The last major epidemics of AY in flax were in 1953-1957 with $90-100 \%$ prevalence and $5-15 \%$ incidence. The moderate epidemic of AY in 2012 have been attributed to the early migration of the leafhopper from the south coinciding with the warm spring weather. All commercial flax cultivars are equally susceptible, thus early seeding is recommended to avoid the early migration of infected leafhoppers.

Pathogenicity of fungi from walnut branch cankers and fungi isolated from ambrosia beetles colonizing stressed walnut trees

S. Reed (1), J. ENGLISH (1), J. Juzwik (2)

(1) University of Missouri, Columbia, MO, U.S.A.; (2) U.S. Forest Service, St. Paul, MN, U.S.A.

Phytopathology 104(Suppl. 3):S3.96
Thousand Cankers Disease (TCD) of eastern black walnut (Juglans nigra) results from mass attack by the walnut twig beetle and coalescing of numerous cankers caused by the insect vectored fungus Geosmithia morbida. An array of fungi has been isolated from cankers on black walnut branches and from insects that had colonized stressed J. nigra. The potential for selected taxa of these fungi to contribute to decline of TCD trees was evaluated. Two 15week-long pot trials were conducted in shade houses to assess pathogenicity of representative isolates on stems of 1-year-old J. nigra. In June and July 2013, five seedlings were inoculated per fungal isolate. Lengths of resulting stem lesions or of xylem stains were measured and morphological characteristics noted. In both trials, stem cankers resulted from inoculations with Fusarium solani obtained from the ambrosia beetle Xylosandrus crassiusculus and with Fusarium-like isolates obtained from walnut branch cankers. In addition, stained woody tissue resulted from inoculations with Phaeoacremonium angustius, P. rubrigenum, P. mortoniae, Penicillium sumatrense and Graphium penicilloides. Neither cankers nor stain were found on sterile agar plug inoculated stems. All canker and stain-causing fungi were recovered from the fungus-inoculated stems. These results confirm the pathogenicity of $F$. solani and other Fusarium-like isolates obtained from branch cankers and from ambrosia beetles colonizing J. nigra.

Incidence of Botrytis cinerea and Sclerotinia sclerotiorum in seed alfalfa fields of southern Alberta

J. REICH (1), D. Johnson (1), S. Chatterton (2)

(1) University of Lethbridge, Lethbridge, AB, Canada; (2) Agriculture and Agri-Food Canada, Lethbridge, AB, Canada

Phytopathology 104(Suppl. 3):S3.96

In cool, moist conditions, blossom blight of seed alfalfa, caused by the fungal pathogens Botrytis cinerea Pers.:Fr. and Sclerotinia sclerotiorum (Lib.) de Bary, are the largest contributors to seed yield losses in western Canada. Recently, growers in southern Alberta, the largest seed alfalfa production area in Canada, have experienced reduced seed yields without obvious symptoms of blossom blight. To determine whether blossom blight pathogens may be associated with yield losses, surveys were performed every 2-3 weeks in 19 seed alfalfa fields in the region. Visual ratings of severity and incidence were assigned in the fields, and flower and/or pod samples were plated on selective media. Based on field observations, blossom blight was present at trace levels. Plated samples revealed that, in all fields surveyed, B. cinerea infected $65 \%$ of florets at the beginning of July, and levels increased steadily to nearly $100 \%$ infected pods at the end of August. In all fields surveyed, S. sclerotiorum infected $10 \%$ of florets at the beginning of July, levels peaked in pods at $20 \%$ at the end of July, and declined to $<10 \%$ by the end of August. $B$. cinerea infected $15 \%$ of harvested seeds, but no infection by S. sclerotiorum was observed. Results indicate that $B$. cinerea inoculum persists throughout the growing season on symptomless plant tissues. Greenhouse studies are under way to determine the impact of symptomless infection by $B$. cinerea on seed development and yield.

A practical scheme of RBSDV kept in small brown planthopper and massively propagated indoors with wheat seedling as a virus source host C. Ren (1), Z. CHENG (1), Y. Zhou (1)

(1) Institute of Plant Protection, Jiangsu Academy of Agricultural Sciences, Nanjing, China

Phytopathology 104(Suppl. 3):S3.96

Rice black-streaked dwarf virus (RBSDV) naturally infects gramineae plants through vector of small brown planthopper (SBPH) but cannot be transmitted to SBPH offsprings via transovarian transmission. Wheat that services as an important intermediate host in winter is essential for RBSDV to complete its annual cycle in farm ecosystem, and is susceptible to RBSDV. Being enlightened by that, we have developed a novel, in vivo, indoor method to preserve RBSDV in SBPH using wheat seedling as a bridge host. The specific program has been made according to a series of experiments. First of all, we choose $23-27^{\circ} \mathrm{C}$ to rear the insects and plants. Before to initiate the cycle, viruliferous SBPH obtained by feeding the virus-free 1st-2nd instar nymphs on RBSDV-infected rice plants and passing the incubation period on healthy rice seedlings. The first step of this program is that to put 4-6 RBSDVinfected SBPH per plant to inoculate wheat seedlings at 2-4 leaf stage. After $48 \mathrm{~h}$ of inoculation the viruliferous SBPH were removed. Then five mated and newly emerged, virus-free SBPH females were transferred onto each inoculated plant and allowed to lay eggs for $48 \mathrm{~h}$. The newly hatched SBPH on wheat seedlings were raised until the 2 nd instar nymph stage and transferred onto healthy rice seedlings for further development till 5th instar nymphs or adults. These new obtained viruliferous SBPH can be used to inoculate new wheat seedlings in further maintenance cycles or used for experiment. 
Silicon improves the photosynthesis on sorghum leaves infected by Colletotrichum sublineolum

R. S. RESENDE (1), C. E. Perez (1), C. A. Milagres (1), D. Rezende (1), F. A. Rodrigues (1)

(1) Universidade Federal de Viçosa, VICOSA, Brazil

Phytopathology 104(Suppl. 3):S3.97

This study investigated the effect of silicon (Si) on chlorophyll $a$ fluorescence imaging and on the photosynthetic gas exchange system on sorghum leaves infected by Colletotrichum sublineolum. Four photosynthetic gas exchange parameters (net $\mathrm{CO}_{2}$ assimilation rate $[A]$, stomatal conductance to water vapor $\left[g_{s}\right]$, internal $\mathrm{CO}_{2}$ concentration $\left[C_{i}\right]$ and transpiration rate $[E]$ ) and three selected conventional chlorophyll fluorescence $a$ parameters (maximum photosystem II quantum yield $\left[F_{\mathrm{v}} / F_{\mathrm{m}}\right]$, effective photosystem II quantum yield $\mathrm{Y}(\mathrm{II})$ and nonregulated energy dissipation $\mathrm{Y}(\mathrm{NO})$ ) were assessed in sorghum plants grown in a nutrient solution containing $0 \mathrm{mM}(-\mathrm{Si})$ or $2 \mathrm{mM}(+\mathrm{Si}) \mathrm{Si}$ and non-inoculated or inoculated with C. sublineolum. Anthracnose severity decreased due to higher foliar Si concentration. For the inoculated +Si plants, $A, g_{s}$ and $E$ were significantly higher in contrast to the inoculated -Si plants. The values of $F_{\mathrm{v}} / F_{\mathrm{m}}$ and Y(II) were significantly higher in non-inoculated plants compared to inoculated as well as for the $+\mathrm{Si}$ plants as compared to $-\mathrm{Si}$ plants upon inoculation with $C$. sublineolum. In contrast, $\mathrm{Y}(\mathrm{NO})$, which indicates inhibition of photosynthesis, showed higher values for inoculated $-\mathrm{Si}$ plants. In conclusion, the supply of Si to sorghum plants improved their photosynthetic performance during the infection process of $C$. sublineolum the supply of Si to sorghum plants improved their photosynthetic performance to during the infection process of $C$. sublineolum.

Colletotrichm species associated with tree tomato and mango in Colombia S. RESTREPO (1), A. M. Rincon (1), M. Cardenas (1), P. Jimenez (2)

(1) Universidad de los Andes, Bogota, Colombia; (2) Universidad Militar Nueva Granada, Bogota, Colombia

Phytopathology 104(Suppl. 3):S3.97

In a previous study, we characterized almost 100 Colletotrichum isolates collected from tree tomato (tamarillo) and mango in important production areas in Colombia. In that study, the use of two genic regions, GAPDH and ITS, allowed us to assign each isolate to a complex, Gloesporioides, Acutatum or Boninense. However, only 3 species could be identified. We decided to use 3 more genic regions to achieve a better resolution in the identifification. These regions were CHS-1 (chitin synthase I gene), ACT (actin gene) and TUB2 (tubulin 2 gene). Using these six molecular markers, we could assign almost all isolates to species. Our results showed that the most common species isolated from tree tomato was C. tamarilloi. We also confirmed our previous results that a new complex, Boninense, could be recovered from these hosts. From mango we obtained higher diversity and several species could be identified. We also evidenced again the lack of concordance between morphological identifications and molecular classifications.

\section{Species recognition and evolutionary history of Phytophthora infestans in} South America

S. RESTREPO (1), M. F. Mideros (1), M. Cardenas (1), S. Cañas-Duarte (1), D. Botero (1), L. Gonzalez (1), D. Riano-Pachon (2)

(1) Universidad de Los Andes, Bogota, Colombia; (2) Laboratorio Nacional de Ciencia e Tecnologia do Bioetanol (CTBE), Centro Nacional de Pesquisa em Energia e Materiais (CNPEM), Campinas, Brazil

Phytopathology 104(Suppl. 3):S3.97

Species recognition criteria are a central topic to evolutionary biology of plant pathogens. Among the Phytophthora group, species recognition criteria have been the subject of considerable debate and extensive revision. Species recognition has been based on morphological characteristics, host range and sequence analysis of mitochondrial and nuclear genes. However, recently, speciation started to be studied using novel genetic/genomic approaches. In South America, two major clonal lineages of Phytophthora infestans have been reported in Ecuador and Peru (EC-2 and EC-3), and recently new divergent $P$. infestans populations have been reported in Colombia. However, the phylogenetic relationship among these groups has not been clearly resolved. Here, we used sexual crosses tests and analysis of genotyping-bysequencing (GBS) to elucidate the evolutionary history of this group. Results of genotyping-by-sequencing (GBS) showed 147,694 putative SNPs for all isolated evaluated. Phylogenetic analysis of SNPs data confirmed the complexity of evolutionary relationships among Phytophthora species from Ic, and show important insights about the relationship of EC-2, EC-3 lineage, and the new divergent aggressive $P$. infestans populations in Colombia. In addition, pre and post mating isolating mechanisms were confirmed, highlighting the importance of mating-type tests for the species recognition on this group.
Hyphal growth through soil and colonization of organic matter by Fusarium proliferatum

A. REYES GAIGE (1), J. Stack (1)

(1) Department of Plant Pathology, Kansas State University, Manhattan, KS, U.S.A.

Phytopathology 104(Suppl. 3):S3.97

Fusarium proliferatum is a broad host-range pathogen and among the most common corn pathogens globally. It can be endophytic in corn seed, making it a high risk of importing it in corn or other grains. The ability of Fusarium proliferatum to colonize cured, non-viable maize seeds in unsterile Shellabarger sandy loam soil was investigated. A green fluorescent (GFP), hygromycin resistant, $F$. proliferatum transformant (Fp-G2) was developed to provide markers to track the fungus. Maize seeds were heat-killed in a $75^{\circ} \mathrm{C}$ water bath for 20 minutes, and re-colonized with Fp-G2 in a $1 * 10^{6}$ spores $/ \mathrm{ml}$ suspension overnight (16 hours). The re-colonized seeds were added to soil at $25^{\circ} \mathrm{C}$ in constant moisture conditions together with cured, non-viable maize seeds (used as baits), placed at different distances $(0 \mathrm{~cm}, 0.5 \mathrm{~cm}, 1 \mathrm{~cm}$ and 1.5 $\mathrm{cm})$. Controls included, non-viable corn seeds that were re-colonized with wild type $F$. proliferatum. After 14 days, the bait seeds were retrieved from soil and plated on Nash-Snyder medium with $1 \mu 1 / \mathrm{ml}$ hygromycin. Fp-G2 grew at least $1.5 \mathrm{~cm}$ through non-sterile soil and colonized the bait seeds at all the distances tested. In addition to pathogenic and endophytic potential, $F$. proliferatum may have an active soil inhabitant phase in its life history.

Modeling an observed shift towards resistance in distributions of pitch canker lesion lengths on Pinus radiata

G. J. REYNOLDS (1), N. McRoberts (1), T. R. Gordon (1)

(1) University of California, Davis, CA, U.S.A.

Phytopathology 104(Suppl. 3):S3.97

Pitch canker, caused by Fusarium circinatum, is a disease affecting Monterey pine (Pinus radiata) and many other pine species throughout the world. Susceptibility phenotypes, based on lesion lengths, are normally distributed in naïve populations but skewed toward shorter lesion lengths in populations previously exposed to the pathogen. We postulate that systemic acquired resistance (SAR) can explain this disparity. We have developed a stochastic process model to explain the shift in lesion length distribution as a consequence of exposure to the pitch canker pathogen. Data were collected from January 2012 through January 2014 on natural infection frequency in a native Monterey pine forest. Two plantations of $P$. radiata seedlings and two plantations of clones were established in Davis, CA from 2008 to 2012. These trees are being primed for SAR with various numbers of initial pitch canker infections and will subsequently be challenged to quantify how each infection contributes to future lesion length reduction. A saturation curve developed from this data set will be combined with a negative binomial distribution based on the natural infection frequency data to simulate numbers of pitch canker strikes in a contagious contact distribution. These two components of the model combined should explain the observed shift in lesion length distributions.

Identification and cloning of avr4 gene from Cercospora kikuchii

J. REZENDE (1), Z. Y. Chen (1)

(1) Louisiana State University, Baton Rouge, LA, U.S.A.

Phytopathology 104(Suppl. 3):S3.97

The importance of fungal effectors in disease development is getting increasingly recognized as more evidence accumulates. The Avr4 effector, secreted by Cladosporium fulvum, has been demonstrated to be involved in pathogen virulence. Moreover, it was suggested that chitin-binding lectins, such as Avr4, have evolved as core effectors in different fungal species. This is supported by the existence of Avr4 effector homologs in other dothideomycete pathogens such as Mycosphaerella fijiensis and various Cercospora species. However, there are no reports of whether Cercospora kikuchii, the causal agent of cercospora leaf blight (CLB) on soybean, contains an Avr4 homolog. Therefore, PCR primers were designed based on the avr4 gene sequences from related Cercospora species. A $0.3 \mathrm{~kb}$ PCR product was amplified using genomic DNA from C. kikuchii. Subsequent analysis of the deduced amino acid sequence showed that this product is homologous to the Avr4 protein sequences of C. apii, C. beticola, $C$. nicotianae and C. zeina. To clone the full length avr4 gene, several genomic libraries of $C$. kikuchii were constructed with restriction enzymes. Internal walking primers based on partial avr4 sequences were designed to walk upward and downward into unknown genomic regions. DNA fragments amplified by PCR were cloned and the sequence data confirmed the presence of an Avr4 homolog in C. kikuchii. This is the first report of an Avr4 effector in the fungal organism causing CLB. 
Salmonella contamination and persistence in tomato fields as affected by irrigation, fertilization and cultivation practices

S. L. Rideout (1), G. GU (2), M. S. Reiter (1), J. Zheng (3), E. W. Brown (3)

(1) Virginia Tech, Painter, VA, U.S.A.; (2) Virginia Tech, Ellicott City, MD, U.S.A.; (3) FDA, College Park, MD, U.S.A.

Phytopathology 104(Suppl. 3):S3.98

Salmonella enterica contamination on tomatoes is of interest due to recent outbreaks. Two field experiments were conducted in 2012 and 2013 to investigate the effects of irrigation, fertilization and planting practices on Salmonella contamination of tomatoes in the field. Exp. A was performed by split plot design with irrigation sources (pond and well) as main plots and planting practices as sub-plots. Subplot planting practices included staked with plastic mulch (SP), staked without mulch (SW) and non-staked without mulch (NW). For exp. B, a randomized complete block design was used with four fertilization/planting practices (conventional/SP, poultry litter ash/SP, poultry litter/SP and poultry litter/NW) in each of the four blocks. Irrigation water, poultry litter, plant rhizosphere, branches, and fruits were sampled for Salmonella detection. Bacterial population was measured by the most probable number method. Multiplex and rep-PCR were performed for further identification and sub-grouping of isolated Salmonella. In exp. A, there were significant positive correlations between Salmonella population in pond water and that in plant rhizosphere of pond water irrigated plots $(\mathrm{P}<0.01)$. In exp. B, the bacterial population in the rhizosphere of plants grown on poultry litter amended soils decreased during the growth season. In both experiments, rhizosphere Salmonella population was highest in SP plots, while Salmonella was only isolated from the branches of NW plants.

\section{Citrus health management areas and associated surveys in Florida}

T. D. RILEY (1), W. Lou (2), T. R. Gottwald (3)

(1) USDA, APHIS, PPQ, Orlando, FL, U.S.A.; (2) CIPM, NC State University, Fort Pierce, FL, U.S.A.; (3) USDA, ARS, US Horticultural Research Laboratory, Fort Pierce, FL, U.S.A.

Phytopathology 104(Suppl. 3):S3.98

Citrus Health Management Areas (CHMAs) have been created in Florida to facilitate and coordinate control strategies among neighboring growers to suppress Asian citrus psyllid (ACP) Diaphorina citri populations in commercial groves. ACP is the known vector responsible for the spread of Candidatus Liberibacter asiaticus, the causal agent of citrus huanglongbing (HLB) disease. To sustain citrus production in the near-term, while long-term HLB mitigation strategies are developed, priority has been given to ACP management and young tree protection. As of March 2014, there are 48 CHMAs statewide in Florida, representing 486,000 acres of the state's 531,000 total active commercial citrus acres. Each CHMA varies in area ranging from 1,323 to 32,164 acres. ACP populations are monitored within a CHMA on a 3-week basis utilizing up to 6000 citrus production blocks totaling approximate 150,000 acres. In each citrus block, 50 trees are monitored utilizing 10 trees each of 5 locations; center of the block, and northwest, northeast, southeast, and southwest corners. ACP counts and overall population trends are reported to the CHMA's lead coordinator and individual growers which are then used to monitor the effectiveness of their spray schedules and for other management decisions. The structured survey program was established in August 2011 and since its inception there has been more than a $60 \%$ reduction of ACP counts during peak season in the monitored area.

\section{Assessing the mismatch between incubation and latency for a vector- borne plant disease}

L. RIMBAUD (1), A. Delaunay (2), S. Dallot (2), S. Borron (2), S. Soubeyrand (3), G. Thebaud (2), E. Jacquot (2)

(1) Montpellier SupAgro - UMR BGPI, MONTPELLIER Cedex 5, France; (2) INRA - UMR BGPI, Montpellier, France; (3) INRA - UMR BioSP, Montfavet, France

Phytopathology 104(Suppl. 3):S3.98

The relative durations of incubation (the time between inoculation and symptom expression) and latency (the time between inoculation and infectiousness of the host) are poorly documented for plant diseases. However, the extent of asynchrony between the ends of these two periods (i.e. their mismatch) is a key determinant of the epidemic dynamics of many diseases and consequently is of primary interest in the design of disease management strategies. In this study, an experimental approach to assess this mismatch is presented and applied on sharka, a severe disease caused by Plum pox virus (PPV) on trees belonging to the Prunus family. Leaves of previously inoculated young peach trees were used individually as viral sources in aphidmediated transmission experiments carried out at three time points post inoculation to bracket symptom onset. By fitting a binomial generalized linear model to the obtained transmission rates, we demonstrated that the first symptoms appear on a leaf 1 day before it rapidly becomes infectious. Therefore, under our experimental conditions, latency and incubation of PPV infection are almost synchronized.

Potential role of oxalic acid in pathogenicity of Sclerotinia homoeocarpa on creeping bentgrass and various model hosts

R. RIOUX (1)

(1) NewLeaf Symbiotics, St. Louis, MO, U.S.A.

Phytopathology 104(Suppl. 3):S3.98

The fungal pathogen Sclerotinia homoeocarpa causes dollar spot, one of the primary diseases of amenity turfgrasses worldwide. S. homoeocarpa produces oxalic acid in vitro but it is not known if this phytotoxin is important for successful host infection by $S$. homoeocarpa. In the present research, whole plant infection assays were used to investigate the role of oxalic acid in pathogenesis of $S$. homoeocarpa on creeping bentgrass and various models hosts. Similar symptom severity was observed on all hosts but the relationship between symptom severity and oxalate content varied widely. Surprisingly, oxalate content was more related to symptom severity in hosts with low, as opposed to high, endogenous oxalate content. Time-course studies were used to compare the importance of oxalic acid for infection of creeping bentgrass and Brachypodium distachyon-hosts with low and high oxalate content, respectively - by $S$. homoeocarpa. These experiments revealed that oxalate content explained $75 \%$ of variability in symptom severity for creeping bentgrass but only $28 \%$ in $B$. distachyon. Moreover, an isolate of $S$. homoeocarpa with low in vitro oxalic acid production was less aggressive on creeping bentgrass but not on $B$. distachyon. Together, these results indicate that both host endogenous oxalate level and $S$. homoeocarpa-produced oxalic acid are important for pathogenicity of $S$. homoeocarpa.

Next generation sequencing of Plasmopara halstedii emergent race 734 causing downy mildew on sunflower and black-eyed Susan

Y. RIVERA (1), C. Salgado-Salazar (1), T. J. Gulya (2), N. Saied (3), J. A. Crouch (3)

(1) USDA-ARS Systematic Mycology and Microbiology Laboratory \& Rutgers University, Beltsville, MD, U.S.A.; (2) USDA-ARS Northern Crop Sciences Laboratory, Fargo, ND, U.S.A.; (3) USDA-ARS Systematic Mycology and Microbiology Laboratory, Beltsville, MD, U.S.A.

Phytopathology 104(Suppl. 3):S3.98

Plasmopara halstedii is a rapidly evolving pathogen that causes downy mildew disease on sunflower, black-eyed Susan and other Asteraceae. Management of downy mildew in commercial hybrid sunflower production largely relies on genetic resistance; however, most resistance genes are ultimately overcome by newly virulent races of $P$. halstedii. In 2009, the broad spectrum Pl6 resistance gene was defeated by a highly aggressive new race, designated 734 . More recently, we showed that race 734 was responsible for destructive downy mildew disease outbreaks affecting black-eyed Susan (Rudbeckia fulgida cv. Goldsturm). To understand the emergence and evolution of race 734 in the U.S., we used Illumina sequencing-by-synthesis technology to sequence the $P$. halstedii genome from a race 734 isolate collected from sunflower in 2009, and to re-sequence a race 734 isolate collected from black-eyed Susan in 2013. De novo read assembly estimated the genome size at $70 \mathrm{Mb}(8,573$ contigs; $\mathrm{N} 50=22,673$; avg. coverage $113 \mathrm{x})$. 570 microsatellite regions and $>200,000$ intragenomic SNPs were identified. Genomic DNA was extracted from 121 race typed isolates from sunflower, 46 black-eyed Susan isolates, and 425 herbarium specimens collected from Asteraceae (1881-1998). These resources underlie population-scale research currently underway, using a combination of RAD-Seq and microsatellite analyses. This work will help us to understand the evolution of $P$. halstedii races in the U.S. and worldwide.

Verticillium wilt, but NOT by Verticillium dahliae: Accurate detection and identification of other important pathogens in the genus Verticillium G. P. ROBIDEAU (1), F. N. Martin (2), G. J. Bilodeau (1)

(1) Canadian Food Inspection Agency, Ottawa, ON, Canada; (2) USDA-ARS, Salinas, CA, U.S.A.

Phytopathology 104(Suppl. 3):S3.98

The fungal genus Verticillium contains several destructive plant pathogens that typically have a broad host range. The most common and well-studied species is $V$. dahliae, but species such as $V$. albo-atrum and $V$. tricorpus are also important pathogens that cause damage on many different hosts. Proper detection and identification of Verticillium species is therefore essential in estimating their disease potential at certain locations and on certain hosts. A confounding issue is the recent taxonomic splitting of $V$. albo-atrum and $V$. tricorpus into multiple species. Now there is a need to assess the contribution 
of each species to plant disease in susceptible crops, and accurate identification will be crucial to this effort. Due to the persistence of Verticillium propagules in soil over time, growers will also benefit from tools that can accurately detect the presence of each species in the field prior to making planting decisions. Here we present newly developed real-time PCR assays that provide accurate and sensitive detection of several Verticillium species. Using DNA extraction methods optimized for soil, samples from California and Canada were analyzed, and the real-time PCR assays were tested for specificity and sensitivity on a large collection of isolates of all known Verticillium species. These assays may be used to evaluate disease pressure and to provide growers with valuable information concerning Verticillium wilt caused by species other than $V$. dahliae.

The impact of tissue type, growth stage and fertilizers on the community structure of cultured bacterial wheat endophytes in the Broadbalk experiment

R. R. ROBINSON (1), B. A. Fraaije (1), I. M. Clark (1), R. W. Jackson (2), P. R. Hirsch (1), T. H. Mauchline (1)

(1) Rothamsted Research, Harpenden, United Kingdom; (2) University of Reading, Reading, United Kingdom

Phytopathology 104(Suppl. 3):S3.99

Endophytes play an important role in plant growth promotion, bio-fertilization and pathogen control. We investigated the effects of tissue type, growth stage and soil fertilizers on the bacterial endophyte community of winter wheat (Triticum aestivum $c v$ Hereward). Culturable endophytes were isolated from roots and leaves across six fertilizer treatments, at two developmental stages, GS 37 and 83. In total 804 strains were grouped into 29 operational taxonomic units using RFLP. Endophyte abundance was higher in roots than leaves and tissue type affected relative phylum abundance $(\mathrm{p}<0.05)$. Proteobacteria dominated in the roots whereas Firmicutes and Actinobacteria dominated in the leaves. Growth stage was found to affect the endophyte community structure at the genus level. Higher abundance of endophytes was found in the treatment without fertilizer, a trend not found for soil bacterial abundances. Fertilizer treatment had no effect on phylum distribution in the roots but there were distinctions at the genus level. In the roots at GS 37 Plantibacter and Curtobacterium were linked with treatment $144 \mathrm{~kg} \mathrm{~N} \mathrm{ha}^{-1} \mathrm{yr}^{-1}$, whereas Arthrobacter and Agromyces showed strong associations with treatment 288 $\mathrm{kg} \mathrm{N} \mathrm{ha}{ }^{-1} \mathrm{yr}^{-1}$. These distinctions between treatments for the endophytic community did not reflect those observed in the underlying soil bacterial community, indicating that plant growth stage is a main driver of endophytic bacterial community structure in wheat.

Eicosapolyenoic fatty acids induce expression of 9-oxylipin pathway genes and resistance in tomato to Phytophthora capsici

S. M. ROBINSON (1), K. Dehesh (1), R. M. Bostock (1)

(1) University of California, Davis, CA, U.S.A.

Phytopathology 104(Suppl. 3):S3.99

Eicosapolyenoic fatty acids (EP) - arachidonic (AA) and eicosapentaenoic (EPA) acids - are common fatty acids in lipids and cellular components of plant pathogenic oomycetes, but are absent from higher plants and most other plant pathogens. EP released during infection of plants by Phytophthora species serve as elicitors or pathogen associated molecular patterns (PAMPs) that engage defense signaling networks. Tomato plants whose roots are pretreated with EP and subsequently inoculated with Phytophthora capsici exhibit increased lignification in the roots and crowns and decreased susceptibility to crown rot and collapse. Initial recognition and action of EP may be quite different than other studied PAMPs and involve specific disruption of host membrane integrity and/or perturbation of oxylipin metabolism. Specifically, treatment of tomato roots with EP elicits increased expression of 9-oxylipin pathway genes in tomato roots. RT qPCR analyses performed on tomato roots treated with EP reveal significantly increased expression of 9-lipoxygenase (9-LOX) and 9-divinyl ether synthase (9-DES) genes in AA-treated roots compared to controls. Investigation is ongoing to further characterize oxylipin metabolism and induced resistance responses in EP-treated tomato roots and crowns. This research will increase understanding of oxylipin regulation of plant defense responses and potentially elucidate new approaches for combating oomycete pathogens.

Dry edible bean exhibits variation in metabolic and hormone profiles associated with partial resistance to white mold, Sclerotinia sclerotiorum F. M. ROBISON (1), H. F. Schwartz (1), A. L. Heuberger (1), J. E. Prenni (1), M. A. Brick (1)

(1) Colorado State University, Fort Collins, CO, U.S.A.

Phytopathology 104(Suppl. 3):S3.99

Dry edible bean (Phaseolus vulgaris L.) is an important global food crop, and bean seed yields can be severely impacted by white mold (Sclerotinia sclerotiorum). The molecular processes that mediate bean resistance to Sclerotinia are largely unknown and identifying metabolites and hormones associated with Sclerotinia infection may provide novel targets to breed for enhanced resistance. The metabolic changes that occur during Sclerotinia infection of a detached leaf were characterized using a non-targeted metabolomics workflow spanning primary and secondary metabolism, and a targeted panel of 13 hormones. Partial resistant (A195) and susceptible (Sacramento) Andean bean lines were inoculated with Sclerotinia isolate S20 for non-targeted metabolite profiling at 16, 24, and 48 hours post inoculation (hpi), and at 8 and 16 hpi for hormones. Metabolites from healthy tissue adjacent to the necrotic lesion were extracted with methanol:water $(80: 20)$ and detected using non-targeted UPLC-MS and GC-MS workflows, and hormones were profiled using UPLC-MS/MS. The analyses detected 144 metabolites that varied between A195 and Sacramento, with the greatest metabolite variation occurring at 16 hpi. The diversity in metabolic changes observed point towards a multi-faceted mechanism for plant resistance to Sclerotinia in dry bean. The integration of metabolomics and genomic data can be used to discover functional markers of metabolic resistance to white mold.

Pathogen-induced alterations in the antioxidative system of mango cultivars with different levels of resistance to Ceratocystis fimbriata infection

F. A. RODRIGUES (1), W. M. S. Bispo (1), L. Araujo (1), M. B. B. Cardona (1), I. S. Cacique (1), F. M. DaMatta (1)

(1) Universidade Federal de Vicosa, Vicosa, Brazil

Phytopathology 104(Suppl. 3):S3.99

The mango wilt, caused by Ceratocystis fimbriata, is one of the most important diseases affecting mango production worldwide. Nevertheless, to date, few attempts have been made to elucidate the physiological mechanisms underlying host responses to fungal infection. The activities of a range of enzymes (SOD, CAT, POX, GST, APX, GPX and GR), metabolites (ascorbate, total glutathione and total phenolics) involved in the antioxidant system as well as some markers for oxidative stress (malondialdehyde and hydrogen peroxide) were evaluated for over 30 days on plants from resistant (Ubá) and moderately resistant (Tommy Atkins) cultivars. Disease severity was more pronounced on plants from cv. Tommy Atkins (higher values for the upward (34\%), downward (51\%) and radial fungal colonization of the stem tissues (44\%). Plants from cv. Tommy Atkins presented more prominent increases in the activities of enzymes and concentration of metabolites related to the oxidative stress responses while these increases were minimal on plants from cv. Ubá. It can be suggested that plants from cv. Tommy Atkins upon fungal infection were less efficient in restrict the fungal spread in the vascular tissues and needed to trigger their antioxidant system at the leaf level to cope with the more rapid stress development in comparison to plants from cv. Ubá.

Defense responses induced by acibenzolar-S-methyl in cape gooseberry (Physalis peruviana) against Fusarium oxysporum

M. X. RODRIGUEZ-BOCANEGRA (1), I. M. Ramos-Pena (1), C. E. Carvajal-Arias (1), J. S. Montana-Lara (1), P. Jimenez (2), S. Restrepo (3)

(1) Pontificia Universidad Javeriana, Bogota, Colombia; (2) Universidad Militar Nueva Granada, Bogota, Colombia; (3) Universidad de los Andes, Bogota, Colombia

Phytopathology 104(Suppl. 3):S3.99

The phytoactivator acibenzolar-S-methyl (ASM) was evaluated on cape gooseberry plants for its potential to induce resistance to Fusarium oxysporum, causal agent of vascular wilt, which is currently considered the main constraint in this crop production. Cape gooseberry plants were treated with ASM (15 mg/L, foliar application) at 0, 0-7, 0-7-14, 0-14 and 0-14-28 days and infected with a conidial suspension 4 days after first ASM application. Treated plants progressively reduced the severity of vascular wilt (up to $79.1 \%$ ) with ASM applications at 0-14-28 days), as well as vascular browning and fungal colonization. Parallely, the accumulation of signal molecules and defense related enzymes was evaluated in plants sprayed with ASM at 0 and 7 days; salicylic and jasmonic acid were quantified by HPLC and chitinase $(\mathrm{CHI})$, carbonic anhydrase $(\mathrm{CA})$ and phenylalanine ammonia lyase $(P A L)$ expression were determined by qRT-PCR. Salicylic acid accumulated at $0,1,7$ and 8 days, while no signal for jasmonic acid was detected; confirming SAR signaling induction by ASM. CHI and PAL significatively increased their expression after the second ASM application (89 and 9-10 days, respectively). Therefore, ASM has the ability to control vascular wilt in cape gooseberry by induction of defense genes, related to fungal cell wall hydrolysis and phenolic compounds synthesis. Consequently, the application of ASM might be established as an effective alternative for preventive disease control. 
Development of diagnostic assays for rapid detection of Phytophthora spp. causing soybean root rot

A. ROJAS (1), T. D. Miles (2), M. D. Coffey (3), M. Chilvers (1), F. N. Martin (4) (1) Michigan State University, East Lansing, MI, U.S.A.; (2) USDA/ARS, East Lansing, CA, U.S.A.; (3) University of California - Riverside, Riverside, CA, U.S.A.; (4) USDA/ARS, Salinas, CA, U.S.A.

Phytopathology 104(Suppl. 3):S3.100

Phytophthora root rot of soybean, caused by Phytophthora sojae is one of the most important diseases in the Midwest US, causing losses of up to 44 million bushels per year. Disease may also be caused by $P$. sansomeana, however the prevalence and damage caused by this species is not well known, partly due to limitations of current diagnostic tools. Efficient, accurate and sensitive detection of pathogens is crucial for management, thus a multiplex real-time PCR and isothermal (RPA: Recombinase Polymerase Amplification) assay were developed using a hierarchical approach. Both assays consist of a genusspecific probe and two species-specific probes that target a mitochondrial region that is highly specific for the genus Phytophthora. The qPCR approach multiplexes the three probes and a plant internal control. The RPA assay runs each probe independently, obtaining a result in 20 mins. The multi-copy mitochondrial genome provides sensitivity with sufficient variability to discern among different Phytophthora species. The assays were highly specific when tested against a panel of 96 Phytophthora spp. and range of Pythium spp. The detection level of the assay is $100 \mathrm{fg}$ for the qPCR assay and $10 \mathrm{pg}$ for the RPA assay. The assay was validated on symptomatic plants collected during the 2013 field season and greenhouse inoculated plants, showing correlation with isolation. The assays are valuable diagnostic tools for detection of Phytophthora spp. affecting soybean.

\section{Internship opportunities at Monsanto}

S. A. ROSENBERGER (1)

(1) Monsanto Vegetable Seeds, Woodland, CA, U.S.A.

Phytopathology 104(Suppl. 3):S3.100

Twenty years from now, the earth's population will need $55 \%$ more food than it can produce now. Today, Monsanto is working with farmers around the world to do something about it. We have professional development programs and a history of building careers in more than 60 countries. We have established industry-leading products because we give professionals like you the freedom to make real decisions. From the first day of your Monsanto internship, you'll be contributing as a member on a team and have meaningful responsibilities. Your internship will allow you to have a realistic look at the types of roles you can pursue in a career with Monsanto. You'll gain valuable professional experience and developmental feedback from Monsanto scientists. A Monsanto internship is a great way to get the inside track on how an industry leader like Monsanto works.

Parallel sequencing and GIS-based mapping of soil bacterial diversity in Michigan potato production systems

N. ROSENZWEIG (1), L. Steere (1), K. Steinke (1), W. W. Kirk (1)

(1) Michigan State University, East Lansing, MI, U.S.A.

Phytopathology 104(Suppl. 3):S3.100

Michigan (MI) ranks seventh in potato production (\$128 million) nationally. Recently a grower survey was conducted and identified that an increase in soil-borne disease complexes coupled with declining yields in parts of MI have raised concerns of inability to serve high-demand markets. Additionally growers in MI identified the activities of soil organisms as an important factor related to soil health and limiting yield. Moreover the amount of acreage affected by potato common scab (PCS) caused by Streptomyces spp. increased by $11 \%$ or more over last decade. PCS is an annual production concern for commercial potato production in MI, but the soil ecology related to PCS is not adequately understood. Twenty soil samples $(\mathrm{n}=520)$ were taken and GPS marked in the fall/spring of 2012-13 from 26 fields scheduled to be in potato production, and total genomic DNA was extracted. A parallel sequencing approach targeting the 16S rRNA gene, coupled with dual indexing allowed processing of 384 samples simultaneously. The total number of sequences identified to phyla, class, order, family and genus was $28,81,140,300$ and 814 respectively. Sequencing results and information gathered on yield and scab pressure from each point was used to generate multi-layer GIS-based maps. The results of this study provided comprehensive information on the distribution of bacterial diversity across potato production fields related to soil physical properties, scab disease severity and total yield.

Validation of the Gleason-Duttweiler warning system for sooty blotch and flyspeck management using a modified relative humidity threshold

H. B. ROSLI (1)

(1) Iowa State University, Ames, IA, U.S.A.

Phytopathology 104(Suppl. 3):S3.100
Sooty blotch and flyspeck (SBFS) fungi produce black blotches and blemishes that reduce marketability of fresh apples. The Gleason-Duttweiler warning system uses a threshold of 215 hours of relative humidity (RH) $>97 \%$ to help Midwest U.S. growers reduce the number of fungicide sprays they apply. A previous study revealed that there were high variations among RH sensors in recording hours of $\mathrm{RH}>97 \%$. In the present study, we modified the RH threshold to $>90 \%$ in order to minimize this variation and validated it in a 2013 field trial in Iowa. Treatments compared the warning system with conventional calendar-based spraying, using either thiophanate-methyl or reduced-risk fungicides plus captan. Treatment subplots were five-tree row segments, with five replications per treatment. A total of 50 fruits per treatment were assessed at harvest for SBFS incidence. Under low SBFS pressure, the warning system with $\mathrm{RH}>90 \%$ threshold saved five fungicide sprays compared to the conventional calendar-based spray treatment, and SBFS incidence did not differ significantly from the calendar-based spray control. The validation trial will be repeated in 2014 and 2015.

Bacterial isolates for biological control of selected fungal pathogens in Cornus florida

E. ROTICH (1), M. Mmbaga (2)

(1) Tennesee State University, Nashville, TN, U.S.A.; (2) TN State University College of AgricHuman \& Natural ci, Nashville, TN, U.S.A.

Phytopathology 104(Suppl. 3):S3.100

The objective of this study was to confirm the biological activity of three bacterial isolates on powdery mildew of C. florida and evaluate bacterial volatile compounds that may be associated with their bioactivity. The bacteria were grown in nutrient broth homogenate with $3 \times 10^{6} \mathrm{CFU} / \mathrm{mL}$ and dogwood seedlings were sprayed weekly. Plant growth promotion was measured at the end of the season when the study was terminated. Volatiles compounds produced by these bacteria were detected and identified by using Gas Chromatograph Mass-Spectrometry (GC/MS) using solid phase microextraction fiber with Helium as a carrier gas. The GC-MS spectra showed different peaks signifying different secondary metabolites including compounds that have been reported to have antimicrobial, antibiotic properties, and polymers to antibiotics. The GC/MS spectra also revealed heterocyclic compound oxyme methoxy phenyl that is an active ingredient in fungicides. Bioactivity of the three bacteria on other fungal pathogens was also evaluated; growth of Cercospora cornicola, Fusarium solani, and Macrophomina phaseolina was significantly inhibited in culture. These results suggest that in addition to suppressing Erysiphe pulchra powdery mildew, the three growth-promoting bacteria are potential biological control agents for several fungal pathogens.

Atmospheric variations of rust spore concentrations during sugarcane growing seasons in Florida

P. C. ROTT (1), M. Kanaan (2), N. Glynn (3), W. G. Gibson (4), J. Haudenshield (5), M. Irey (6), C. LaBorde (7), R. Raid (1), J. Shine (8), J. C. Comstock (2)

(1) University of Florida, Belle Glade, FL, U.S.A.; (2) USDA/ARS, Canal Point, FL, U.S.A.; (3) Syngenta Seeds Inc., Longmont, CO, U.S.A.; (4) BASF, Vero Beach, FL, U.S.A.; (5) USDA/ARS, Urbana, IL, U.S.A.; (6) United States Sugar Corporation, Clewiston, FL, U.S.A.; (7) Florida Crystals Corporation, South Bay, FL, U.S.A.; (8) Sugar Cane Growers Cooperative, Belle Glade, FL, U.S.A.

Phytopathology 104(Suppl. 3):S3.100

Orange rust and brown rust of sugarcane are two economically important diseases caused by the obligate biotrophic pathogens Puccinia kuehnii and $P$. melanocephala, respectively. Most of sugarcane cultivars grown in commercial fields in Florida are currently susceptible to one of these diseases, and fungicides are used to preserve yields. A real-time PCR assay for $P$. kuehnii and P. melanocephala was used in conjunction with weather-vane spore traps to monitor changes in atmospheric concentrations of spores (as pathogen DNA) through the growing seasons 2012 and 2013 in 6 different locations. Spores trapped on vaseline-coated glass slides were PCR-quantified twice a week. Spore concentrations varied according to the location and the month of the year, ranging from no DNA detected to DNA from the equivalent of thousands of spores. In 2012 and in 2013, high concentrations of $P$. kuehnii spores started to be trapped end of May or early June in 5 of 6 locations. High concentrations of $P$. melanocephala spores were also detected in these five locations during the same period in 2012. However, in 2013, high amounts of brown rust spores were already detected from mid-March to early May, suggesting that atmospheric rust spore concentrations vary not only according to climate but also to each disease. Spore data combined with disease and environmental data will be investigated in the future to predict sugarcane rust epidemics, and recommend fungicide applications only when needed. 
Genetic variability in the nucleocapsid genes of nuclear citrus leprosis virus reveals the presence of a distinct species of dichorhavirus

A. ROY (1), A. Stone (2), G. Otero-Colina (3), W. L. Schneider (2), J. S. Hartung (4), R. H. Brlansky (5)

(1) University of Florida, Fort Detrick, MD, U.S.A.; (2) USDA-ARS, Fort Detrick, MD, U.S.A.; (3) Colegio de Postgraduados, Campus Montecillo, Texcoco, Estado de Mexico, Mexico; (4) USDA-ARS, Beltsville, MD, U.S.A.; (5) University of Florida, Lake Alfred, FL, U.S.A.

Phytopathology 104(Suppl. 3):S3.101

The nuclear type of Citrus leprosis virus (CiLV-N) has been reported from Brazil, Panama, Mexico and very recently from Colombia. Citrus leprosis disease was once endemic in Florida but has not been seen since 1968. Leaf lesions of CiLV-N are small in size with a dark brown center surrounded by concentric orange and chlorotic halos. In 2013, we reported the complete genome sequence of a Mexican CiLV-N isolate, which shared $90 \%$ nucleotide sequence identity with Orchid fleck virus (OFV) genome sequences. The incidence of CiLV-N in diseased and symptomless plants from seven citrus cultivars obtained from the state of Querétaro in Mexico was determined. Nucleocapsid $(\mathrm{N})$ gene specific primers were used to amplify CiLV-N by reverse transcription-polymerase chain reaction. A total of $25 \mathrm{CiLV}$ infected symptomatic plants were tested to assess the variability in the $\mathrm{N}$ gene of the CiLV-N. The complete $\mathrm{N}$ gene (1353 nt) was amplified, cloned and sequenced for further analysis. Alignments of the $\mathrm{N}$ gene sequences showed low $(80 \%)$ to very high $(99 \%)$ sequence identity with the previously reported CiLV-N isolates. The $\mathrm{N}$ gene of five Mexican isolates had only $80-81 \%$ sequence identity with either CiLV-N or OFV, and formed a separate cluster in the dendrogram. This data suggests the presence of a previously unknown species of dichorhavirus may be present in the Mexican leprosis affected samples.

\section{Identification of host-microbe interaction factors in the soft rot pathogen,} Pectobacterium carotovorum, using supervised machine learning

I. RUBIO (1), B. Ma (1), A. O. Charkowski (1), N. T. Perna (1)

(1) University of Wisconsin-Madison, Madison, WI, U.S.A.

Phytopathology 104(Suppl. 3):S3.101

Host-microbe interactions are complex biological processes that involve a substantial number of genes from each organism. Genome sequencing provides many insights into the genes involved, but traditional bioinformatics approaches struggle with unknown genes and have high false positive rates, discouraging experimental verification. A supervised machine learning strategy was developed to overcome these limitations and generate a list of novel candidate genes involved with host-microbe interactions. To test this new approach, we selected the soft rot pathogen, Pectobacterium carotovorum, due to its worldwide importance, large amount of genomic data available, and tractability in laboratory environments. We constructed a miniTn5 transposon mutation library in P. carotovorum and will use a PCR-based strategy to identify strains with mutations in genes of interest. We are focusing on genes of unknown function that are predicted to either be important for plant-microbe interactions or not involved in them. The mutants will be tested with multiple plant assays to determine if they are impaired in survival, growth, persistence or disease development in potato or other plant hosts. Our long-term goal is to develop a generalized machine learning tool to aid in identification of microbial genes involved in complex traits. This tool would be particularly useful with host-microbe interactions that are difficult to manipulate through standard mutagenesis methods.

\section{Soybean vein necrosis virus in Mississippi}

S. SABANADZOVIC (1), R. C. Stephenson (2), N. AboughanemSabanadzovic (3)

(1) Department of Biochemistry, Molecular Biology, Entomology and Plant Pathology, Mississippi State University, Mississippi State, MS, U.S.A.; (2) Hancock County Extension Service, Mississippi State University, Mississippi State, MS, U.S.A.; (3) Institute for Genomics, Biocomputing and Biotechnology, Mississippi State University, Mississippi State University, MS, U.S.A. Phytopathology 104(Suppl. 3):S3.101

Soybean vein necrosis virus (SVNV) is a recently described virus, currently reported from all major soybean-producing states in the USA. In 2013 we initiated a focused study on this virus in Mississippi (MS) in the framework of the project funded by local Soybean Promotion Board. The project was designed to estimate SVNV incidence in production fields, study population profile and effects/symptoms of this virus on the host in single and in mixed infections. The virus was identified in 16 out of 28 counties scouted during the statewide survey. Curiously, SVNV was not found in any of the scouted counties in Southern MS in 2013. Twelve SVNV isolates were used for intraspecies diversity study based on nucleotide sequences of RNAs 2 and 3. In addition, yellow stick card-based monitoring, carried out in three different locations, revealed that $2 / 3$ of captured thrips were identified as soybean thrips, followed by flower- and tobacco thrips.

Association of a putative mycovirus with resurrection fern in Mississippi S. Sabanadzovic (1), A. Lawrence (2), J. Hill (1), N. ABOUGHANEMSABANADZOVIC (3)

(1) Biochemistry, Molecular Biology, Entomology and Plant Pathology, Mississippi State University, Mississippi State, MS, U.S.A.; (2) Institute for Imaging and Analytical Technologies, Mississippi State University, Mississippi State, MS, U.S.A.; (3) Institute for Genomics, Biocomputing and Biotechnology, Mississippi State University, Mississippi State, MS, U.S.A.

Phytopathology 104(Suppl. 3):S3.101

The resurrection fern (Pleopeltis polypodioides; syn. Polypodium polypodioides) is an epiphyte native to the southeastern United States and is characterized by extreme drought resistance. During the spring 2013, we collected resurrection fern samples from three distinct geographic locations in Northeastern Mississippi (MS) and examined them for the presence of viruses. Interestingly, all three samples contained dsRNA molecules of comparable sizes, ascertained to represent a genomic segments of a new putative virus related to extant chrysoviruses. Virus-specific primers have been designed and applied to study the presence of this virus in resurrection ferns collected from other locations in MS. The aim of the present study is to understand the nature of virus/fern association by investigating possible presence of endophytic fungus as a primary host of this virus.

\section{Viruses of muscadines in Mississippi}

S. SABANADZOVIC (1), N. Aboughanem-Sabanadzovic (2)

(1) Department of Biochemistry, Molecular Biology, Entomology and Plant Pathology, Mississippi State University, Mississippi State, MS, U.S.A.; (2) Institute for Genomics, Biocomputing and Biotechnology, Mississippi State University, Mississippi State, MS, U.S.A.

Phytopathology 104(Suppl. 3):S3.101

Muscadines (Vitis rotundifolia) are grapevines native to the southeastern USA where they thrive in warm and humid climate, hostile for European grapes. It is known that Vitis vinifera and related rootstocks are susceptible to more than 60 different viruses, whereas current knowledge on viruses affecting muscadines is very limited. This study, designed to identify viruses infecting this crop in Mississippi was carried out in 2012 and 2013. Results of our investigation indicate that muscadines are prone to natural infections by several viruses either known or novel species. Interestingly, some of viruses identified in this work are considered of economically important for European grapevines. Study is underway to estimate impact of these viruses on longevity and productivity of muscadines.

Fungicide resistance phenotypes in Botrytis cinerea populations from blueberries in California and Washington

S. SAITO (1), T. Michailides (2), C. L. Xiao (1)

(1) USDA ARS, Parlier, CA, U.S.A.; (2) University of California-Davis, Parlier, CA, U.S.A.

Phytopathology 104(Suppl. 3):S3.101

Gray mold caused by Botrytis cinerea is a major postharvest disease of blueberries grown in the Central Valley of California (CA) and western Washington State (WA). Understanding fungicide resistant phenotypes of $B$. cinerea is important to the development of preharvest fungicide programs for control of postharvest gray mold. Sensitivities to boscalid, cyprodinil, fenhexamid, fludioxionil, and pyraclostrobin, representing five fungicide classes, were examined on agar media for 251 and $106 \mathrm{~B}$. cinerea isolates recovered from decayed blueberry fruit in CA and WA, respectively. Seven and 17 fungicide resistant phenotypes were detected; 66 and $49 \%$ of the isolates were resistant to boscalid; 20 and $29 \%$ were moderately resistant to cyprodinil; 29 and $29 \%$ were resistant to fenhexamid; and 66 and $55 \%$ were resistant to pyraclostrobin in CA and WA, respectively. All isolates from CA were sensitive to fludioxonil, while $70 \%$ of the isolates from WA showed reduced sensitivity to fludioxonil. In CA, 26 and $30 \%$ of the isolates were resistant to two and three classes of fungicides, respectively. In WA, 31,16, and $9 \%$ of the isolates were resistant to two, four, and five classes of fungicides, respectively. Most fungicides failed to control gray mold on detached blueberry fruit inoculated with fungicide resistant phenotypes. Our results suggest that as preharvest treatments, alteration of different classes of fungicides will be needed to control postharvest gray mold in blueberries.

Inferring introduction pathways of the poplar pathogen, Mycosphaerella populorum using a population genomic approach

M. L. SAKALIDIS (1), N. Feau (1), R. C. Hamelin (1)

(1) The University of British Columbia, Vancouver, BC, Canada

Phytopathology 104(Suppl. 3):S3.101 
Mycosphaerella populorum Thompson (anamoph Sphaerulina musiva (Peck), Quaedvlieg, Verkley \& Crous) is a leafspot and canker causing pathogen of poplar and is endemic and widespread amongst poplar in North America. Increased reports of this pathogen, including an ability to colonise and cause cankers in woody tissue coincided with the explansion of poplar plantations. This pathogen was reported for the first time in BC in 2006 and in Alberta in 2009. In order to manage disease spread, prevent further incursions and ensure the sustainability of breeding programs, we characterised pathways of pathogen movement by examining the genomes of 84 individuals collected from planted poplar in five Canadian provinces and six American states. We found that $M$. populorum forms two broad groups in eastern and western North America and generally genetic clusters are linked to geographical populations. Modeling based approaches suggest that M. populorum originated in the US and has repeatedly spread or been introduced into Canada. The most likely vector enabling the recent introductions of this pathogen may be the movement of poplar material for commercial poplar culture or breeding programs. This finding has significance for the certification of plant material that relies only on observation-based pathogen screening as opposed to molecular based diagnostics.

Citrus leprosis and Brevipalpus mites in the Lower Rio Grande Valley, TX

B. SALAS (1), E. Braswell (1), M. B. Kunta (2)

(1) APHIS USDA, Edinburg, TX, U.S.A.; (2) Texas A\&M UniversityKingsville Citrus Center, Weslaco, TX, U.S.A.

Phytopathology 104(Suppl. 3):S3.102

Citrus leprosis virus (CiLV) causes one of the most economically damaging diseases for the citrus industry worldwide. The flat mite Brevipalpus phoenicis is the vector of CiLV. The fairly recent discovery of CiLV in Southern Mexico has increased the possibility that the disease and the mites carrying CiLV could reach the United States. Since 2006, citrus fruits and leaf samples from dooryards and groves in the Lower Rio Grande Valley-TX (LRGV) were collected and examined for the diagnostic symptoms of citrus leprosis. Brevipalpus mites were also extracted from the samples for identification and to determine its distribution. Thus far, Citrus leprosis was not detected, but Brevipalpus mites were widely distributed in LRGV and they were more frequently found in citrus in dooryards than in groves. Based on DNA sequence similarity three species were found: $B$. californicus, $B$. phoenicis, and $B$. obovatus. Two of these species, B. californicus and $B$. phoenicis, appear to be widespread occurring from Brownsville to McAllen and north to Raymondville. Further, $B$. californicus occurred on every sampled host except lemon and wild olive, whereas $B$. phoenicis and $B$. obovatus appeared to be more restricted. Currently, fast and more efficient methods of detection for CiLV and better identification of the vector are being pursued to limit CiLV spread if it is found.

\section{Studies on isolation of Elsinoe australis the causal agent of sweet orange scab}

B. SALAS (1), E. Braswell (1), M. B. Kunta (2)

(1) USDA APHIS, Edinburg, TX, U.S.A.; (2) Texas A\&M UniversityKingsville Citrus Center, Weslaco, TX, U.S.A.

Phytopathology 104(Suppl. 3):S3.102

The discovery of the exotic citrus disease sweet orange scab (SOS) caused by Elsinoe australis in Texas in 2010, led to the realization that information on techniques and methods for isolation of E. australis was lacking. Several techniques were examined in this study. Ultimately, we isolated E. australis by washing, surface sterilizing and drying fruit before scraping rind tissue onto culture plates. Several culture media and fungicide combinations were examined. Difco ${ }^{\mathrm{TM}}$ Rose Bengal Agar Base, Difco ${ }^{\mathrm{TM}} \mathrm{YM}$ agar, and Difco ${ }^{\mathrm{TM}}$ Potato Dextrose, all amended per liter with Dodine (Syllit FL Fungicide) (400 $\mathrm{ppm})$, streptomycin sulfate $(100 \mathrm{ppm})$, and tetracycline $(100 \mathrm{ppm})$ showed to be useful for the isolation of $E$. australis from citrus fruits. DNA from isolates that morphologically resembled E. australis was extracted and sequenced for confirmation. Out of 117 E. australis-like isolates collected from the Lower Rio Grande Valley-TX, 71 were identified as E. australis, 14 were inconclusive, and 32 were not E. australis. E. australis isolates were not always recovered from fruits testing positive for E. australis by PCR. The use of the genetic information improved our ability to identify SOS and $E$. australis based on morphology.

$\mathrm{CO}_{2}$ and Fusarium graminearium chemotype effects on Fusarium head blight and mycotoxin contamination in wheat

J. D. SALGADO (1), P. A. Paul (1), L. V. Madden (1)

(1) Ohio State University, Wooster, OH, U.S.A.

Phytopathology 104(Suppl. 3):S3.102
Elevated atmospheric temperatures and $\mathrm{CO}_{2}$ concentrations are projected consequences of climate change. However, it is unclear how these factors will affect plant diseases such as Fusarium head blight (FHB) of wheat, caused by Fusarium graminearum, and its associated toxins, deoxynivalenol (DON) and nivalenol (NIV). Research was conducted to evaluate the effects of temperature $\left(25\right.$ and $\left.30^{\circ} \mathrm{C}\right)$ and $F$. graminearum chemotypes (3-ADON, 15ADON and NIV) as influenced by $\mathrm{CO}_{2}$ levels (ambient [ $\left.390 \mathrm{ppm}\right]$ and elevated [660ppm]) on FHB and mycotoxins in wheat. Spikes were pointinoculated with a spore suspension of $F$. graminearium, rated at 6-7 day intervals, and mycotoxin was quantified. The rate of increase in FHB severity (percent spike area diseased) was higher at elevated $\left(0.8\right.$ to $2.1 \%$ day $\left.^{-1}\right)$ than at ambient $\mathrm{CO}_{2}$ level $\left(0.4\right.$ to $1.2 \%$ day $\left.^{-1}\right)$, with the highest values observed in spikes inoculated with the 3-ADON chemotype at both 25 and $30^{\circ} \mathrm{C}$, compared to the 15-ADON and NIV chemotypes. Mycotoxin content was consistently higher at elevated $\mathrm{CO}_{2}$ for all chemotypes, with 3-ADON ranging from 4.9 to $13,15-\mathrm{ADON}$ from 0.5 to 15.2 and NIV from 0.7 to $13.8 \mathrm{ppm}$. At ambient $\mathrm{CO}_{2}$, the ranges were 1.9 to $7.8,0.1$ to 5.4 and 0.4 to $3.1 \mathrm{ppm}$, for 3ADON, 15-ADON, and NIV, respectively. These results suggest that an increase in atmospheric $\mathrm{CO}_{2}$ concentration may increase the risk of FHB and mycotoxins in wheat, particularly if the 3-ADON chemotype of $F$. graminearum is predominant in the population.

Genome sequencing of Plasmopara obducens, the causal agent of impatiens downy mildew, for microsatellite marker development

C. SALGADO-SALAZAR (1), Y. Rivera (1), J. A. Crouch (1)

(1) USDA-ARS, Systematic Mycology \& Microbiology Lab, Beltsville, MD, U.S.A.

Phytopathology 104(Suppl. 3):S3.102

Impatiens downy mildew (IDM) is a destructive disease of Impatiens walleriana caused by the oomycete pathogen Plasmopara obducens. Although sporadic incidences of IDM have been reported in the United States since 2004, the disease is now widespread, leading to substantial losses for growers and the bedding plant industry. In this work, we generated a de novo assembly of the $P$. obducens genome using paired-end Illumina sequencing technology $(2 \times 250 ; 2 \times 300)$ to develop microsatellite markers. An assembly of $70.2 \mathrm{Mb}$ across 63,317 contigs was constructed from 13.9 million reads using CLC Genomics software $\left(\mathrm{N}_{50}=1284\right.$; average coverage $\left.=37 \mathrm{x} ; 47.7 \% \mathrm{GC}\right)$. Contigs were used as input file for microsatellite mining (repeat units of 2-12) using the MISA script in Perl. A total of 1,118 microsatellites were identified, with dinucleotides and trinucleotides being most abundant $(n=622$ and 408 respectively; larger repeats units $=88$ ). Microsatellite positions were mapped against the assembly, which included annotation of heterozygous positions. Candidate microsatellite markers were visually inspected for heterozygosity, suitable priming regions, and stepwise evolution. Primers have been synthesized and are being tested to assess polymorphism, population structure and distribution. Ongoing work with the de novo sequencing of the $P$. obducens genome will contribute to our understanding of the events underlying recent IDM disease outbreaks.

Characterization of the bacterial stem blight pathogen of alfalfa, Pseudomonas syringae pv. syringae ALF3

D. SAMAC (1), D. J. Studholme (2), S. Ao (3)

(1) USDA ARS, St. Paul, MN, U.S.A.; (2) University of Exeter, Exeter, United Kingdom; (3) University of Minnesota, St. Paul, MN, U.S.A.

Phytopathology 104(Suppl. 3):S3.102

Bacterial stem blight of alfalfa occurs sporadically in the central and western U.S. Yield losses of up to $50 \%$ of the first harvest can occur with some cultivars. Developing resistant cultivars is hampered by lack of information on the pathogen and a standard test for evaluating plant germplasm. Bacteria producing a fluorescent pigment were isolated on King's B agar from alfalfa with symptoms of bacterial stem blight from near Cheyenne, WY. The strain ALF3 was tentatively identified as Pseudomonas syringae pv. syringae based on 16S rDNA sequence and PCR amplification of $\operatorname{syr} B$ for lipodepsinonapeptide toxin production. Multilocus sequence analysis indicated that ALF3 falls within a clade containing strains of $P$. syringae pv. syringae with closest affinity to FF5 from pear. Comparison of a draft whole-genome sequence of ALF3 further confirmed that ALF3 most closely resembles FF5 ( $\sim 96 \%$ sequence identity) and P. syringae pv. aptata DSM50252 from beet. Approximately 60 genes were unique to ALF3, including several predicted genes in the T3SS cluster such as a type III helper protein HrpZ (Pto) and phage-associated genes. ALF3 was highly pathogenic to snapbean pods but caused only mild symptoms on leaves of snapbean, pear, and sugarbeet. A standardized method for evaluating disease resistance in alfalfa was developed. Cultivars with fall dormancy ratings of 1 and 2 had higher percentages of resistant plants than cultivars with fall dormancy ratings of $8-11$. 
A mineral seed treatment for control of seedling diseases of alfalfa suitable for organic production systems

D. SAMAC (1), S. Scraber (2), J. Blosberg (2), S. Barclay (3)

(1) USDA ARS, St. Paul, MN, U.S.A.; (2) University of Minnesota, St. Paul, MN, U.S.A.; (3) Summit Seed Coatings, Caldwell, ID, U.S.A. Phytopathology 104(Suppl. 3):S3.103

Most alfalfa seed is treated with the fungicide mefenoxam (Apron XL) for control of soilborne seedling diseases. However, Apron XL is not active against Aphanomyces euteiches and Apron XL-treated seed cannot be used in organic production systems. An aluminosilicate (natural zeolite) mineral seed treatment allowed under organic standards was compared to Apron XL as an alfalfa seed treatment. Alfalfa seedlings were inoculated with Phytophthora medicaginis, Aphanomyces euteiches, or a mixture of the two pathogens. The mineral seed treatment gave significantly greater control of $P$. medicaginis and $A$. euteiches at 14 days after inoculation than the Apron XL seed treatment. Treated seeds were plated with Pythium ultimum or $P$. paroecandrum in assays to evaluate control of seed rot and seedling damping off. The mineral seed treatment resulted in a greater percentage of protected plants than the Apron XL treatment. In growth chamber assays with naturally infested field soils the mineral treatment resulted in a similar or greater percentage of protected plants than the Apron XL treatment. Field experiments were established to compare the mineral treatment to Apron XL and Stamina fungicide seed treatments. Results of plant counts, nodule numbers, and initial forage yields will be presented. Controlled environment experiments indicated that the zeolite mineral seed treatment is a promising means of controlling seedling diseases in alfalfa production systems.

\section{Phenotypic and genotypic characteristics of Phytophthora infestansin} Wisconsin in 2013

A. SANCHEZ PEREZ (1), A. Gevens (1)

(1) University of Wisconsin, Madison, WI, U.S.A

Phytopathology 104(Suppl. 3):S3.103

Late blight caused by the oomycete Phytophthora infestans (Mont.) de Bary is a serious disease of potato and tomato worldwide. The pathogen is dynamic leading to the emergence of new population structures. Constant monitoring is required for management of the disease. The distribution, and phenotype and genotype characteristics of the $P$. infestans population collected from symptomatic plant tissues in Wisconsin during 2013 were analyzed. Mating type and sensitivity to the fungicide mefenoxam were used as phenotypic markers. Analyses of the Glucose-6-phosphate isomerase (Gpi) allozyme locus, and microsatellite and mitrocondrial DNA analyses were used for the genotypic analysis. A total of 55 isolates were collected from symptomatic potato and tomato crops from 15 counties of Wisconsin during the growing season of 2013. Allozyme analyses revealed that two $P$. infestans genotypes, US-23 (Gpi 100/100) and US-8 (Gpi100/111/122), were present with US-23 as the predominant type (53/55 isolates). Mating type tests indicated that isolates of the US-23 genotype were of the mating type A1 and isolates of the US-8 genotype were A2. All US-23 isolates were sensitive to mefenoxam and the two US-8 isolates were intermediately sensitive. As previously reported, there was correlation between genotype, mating type and sensitivity to mefenoxam in the isolates representing the population of $P$. infestans in Wisconsin in 2013.

Viability of Phytophthora infestans oospores from US-22, -23, and -24 clonal lineage crossings under overwintering conditions

A. SANCHEZ PEREZ (1), A. Gevens (1)

(1) University of Wisconsin, Madison, WI, U.S.A.

Phytopathology 104(Suppl. 3):S3.103

Phytophthora infestans, causal agent of potato and tomato late blight, is a heterothallic oomycete pathogen, with 2 mating types (A1 and A2) that can interact to form soil persistent oospores. We evaluated the effects of overwintering conditions on the viability of oospores produced by the progeny of US-22 (A2) x US-23 (A1) and US-22 x US-24 (A1) clonal lineages. Fivemonth duration soil overwintering conditions were modeled under controlled laboratory environments at 5 temperatures and under contained field environments for 2 winters (2011-12 \& 2012-13) in central WI. In field, a CRD with 4 replicates was applied to placement of buried 5-gallon buckets containing loamy sand soil surrounding 6-in-diam pots of loamy sand infested with 10,000 oospores/pot from each mating pair. Oospores were retrieved and subjected to a tetrazolium bromide stain and microscopy to determine $\%$ viability based on color. There was no significance difference in $\%$ viability between the oospores produced from the crosses of either mating pairs from the field or laboratory. On average, $26 \%$ of oospores were viable after exposure to field conditions. Incubation temperatures of $22,0,-5,-10$ and $-20^{\circ} \mathrm{C}$ resulted in average \% viability values of $33,27,23,16$ and $10 \%$, respectively; oospore viability decreased with increasing cold. Progeny oospores of the US-22, -23 , and -24 lineages may remain viable in field soils experiencing typical overwintering conditions of central WI.

Suppression of Fusarium wilt in commercially grown watermelons using selected fungicides and a plant defense activator

F. H. SANDERS (1), E. D. Beasley (2), J. Lanier (3), D. B. Langston (1)

(1) University of Georgia, Tifton, GA, U.S.A.; (2) University of Georgia, Nashville, GA, U.S.A.; (3) University of Georgia, Cordele, GA, U.S.A. Phytopathology 104(Suppl. 3):S3.103

Fusarium wilt (FW), caused by fungus Fusarium oxsysporum f.sp. niveum (FON), is a destructive soil borne disease of watermelon. Current pest management strategies do not provide adequate control of $\mathrm{FW}$, or they are cost prohibitive. Fungicides, until recently, have not been available to control this disease. In this experiment, selected fungicides and the plant defense activator, Actigard (acibenzolar-s-methyl), were tested for the control of FW in a naturally infested commercial watermelon field. Transplanted watermelons (var. Abbott and Cobb 790) resistant to FON race 1 were transplanted to bare ground beds. The trial was arranged in a randomized complete block design with 8 replications. Initial fungicide treatments were applied by drenching transplants immediately after planting, and initial Actigard treatments were applied by a 12 in banded spray. Some plots received a follow up banded spray treatment of fungicide or Actigard 2 weeks after planting. Incidence, stand loss, and yield were recorded and data were analyzed using Fisher's protected LSD $(\mathrm{P} \leq 0.5)$. Plots that received both the initial and the 2 week follow up applications of Proline (prothioconozole) or Actigard had significantly less FW incidence than the untreated plots 45 days after planting and incidence was 27,42 , and $67 \%$, respectively. Results from this trial indicate that Proline and Actigard may be beneficial tools in suppressing FW in commercially grown watermelons.

Magnetic resonance imaging for detecting viral infections in potato tubers S. Sankaran (1), C. Brown (2), H. PAPPU (1)

(1) Washington State University, Pullman, WA, U.S.A.; (2) USDA ARS, Prosser, WA, U.S.A.

Phytopathology 104(Suppl. 3):S3.103

Potato is an important crop in the Pacific Northwest (PNW) of the United States. Viral infections in potato tubers are a major concern to potato producers. Potato mop top virus (PMTV) and Tobacco rattle virus (TRV) could cause significant internal blemishes in infected potato tubers. PMTV is largely soil-borne, transmitted by the powdery scab "slime mold", and perpetuation through vegetative tuber seed. Both PMTV and TRV cause rusty brown arcs of discoloration and internal rings within the potato tubers, as well as less characteristic spots and blotches. As a result, cutting the tubers is necessary for diagnosis and laboratory testing. The absence of external symptoms and movement of infected tubers could introduce these viruses into new production areas. We evaluated the potential of magnetic resonance imaging (MRI) for detecting internal blemishes potentially caused by PMTV. Results from MRI-based imaging were compared with ELISA testing of the same tubers for the presence of PMTV. Preliminary findings indicated that MRI-based technique could detect the internals caused by PMTV with an accuracy of about $70 \%$. With further improvements, MRI-based non-invasive diagnosis could be useful for identifying viral infections in tubers and implementing control programs for limiting the further spread of these viruses.

Case studies of high school student success from plant pathology based extension program

L. SANTAMARIA (1), A. Bernert (2), A. Schauer (3)

(1) Oregon State University, Aurora, OR, U.S.A.; (2) Oregon State University, Corvallis, OR, U.S.A.; (3) West Linn - Wilsonville School District, Wilsonville, OR, U.S.A

Phytopathology 104(Suppl. 3):S3.103

Agricultural education will be an essential aspect of attracting youth to pursue careers in agriculture related fields. Using plant pathology as a theme for student outreach and education programs provides students with science research skills as well as agriculture education. The objective of our project was to engage high school students in plant pathology research thus empowering them to create an independent research project. These research projects allow students to learn about agriculture, to experience science, and to generate ideas for real world problem solving. This program provide students the opportunity to present their research projects at the multiple venues designed to showcase high school student research and inspire youth to pursue scientific careers. Our work here examines four case studies, their research projects, and the impact it has had on their future in academics. Their projects include testing antimicrobial compounds produced by lichens as treatment for Rhodococcus fascians, micropropogation of Vaccinium spp. to test Botrytis pathology in vitro, antagonism between endophytes and 
Phytophthora spp, and the use of Trichoderma spp to combat Pythium spp. This case studies analysis reveals that plant pathology based programs can be effective vectors of scientific and agricultural education to the high school student audience. Similar program designs can be applied for engaging future generations and as potential university recruitment tools.

\section{An online course "Simulation Modeling in Botanical Epidemiology and Crop Loss Analysis" in The Plant Health Instructor on the APSnet} Education Center

S. Savary (1), K. Bowen (2), K. L. Stevenson (3), L. WILLOCQUET (1)

(1) INRA, Castanet Tolosan Cedex, France; (2) Auburn University, Auburn, AL, U.S.A.; (3) University of Georgia, Tifton, GA, U.S.A.

Phytopathology 104(Suppl. 3):S3.104

This course was developed to highlight, illustrate, and implement the linkages between models and data. The course focuses on a mechanistic simulation approach, which is visual and involves as little calculus as possible in order to bridge the gap between 'observers' and 'modelers'. Simulation models are good educational tools; they can provide an intuitive, hands-on analysis of (plant-pathogen) systems. They are powerful tools to synthesize and integrate quantitative information in the biological sciences. The technique, which has been used for decades in various fields of ecological and agricultural sciences, is derived from the concepts of systems analysis and makes use of numerical integration. This approach allows identifying key processes that govern a dynamic system by analyzing the effects of the components of a system on its behavior (e.g., the dynamics of an epidemic), and exploring "futures" through scenario analyses. This course first introduces basic concepts and simple examples of systems analysis and simulation modeling. It then focuses on plant disease epidemics and crop yield losses. Simulation models are provided to explore model structures, their behavior, and the effect of key parameters (at the sub-process level) on system (process level) dynamics. This material is primarily intended for graduate students, but also for undergraduate students and any biologist who wishes to gain some exposure to simulation modeling applied to ecological systems.

\section{Evolutionary relationships and displacements of historic and present day} Phytophthora infestans

A. Saville (1), M. D. Martin (2), M. T. Gilbert (2), J. B. RISTAINO (1)

(1) North Carolina State University, Raleigh, NC, U.S.A.; (2) Centre for GeoGenetics, Natural History Museum of Denmark, University of Copenhagen, Copenhagen K, Denmark

Phytopathology 104(Suppl. 3):S3.104

Phytophthora infestans caused the historic potato famine and is an important constraint to potato production worldwide. The evolutionary relationships of modern clonal lineages of the pathogen and historic $P$. infestans in herbarium samples from old and new world collections were examined using nuclear and mitochondrial genomes and multilocus genealogies. Multiple distinct genotypes were present in historical Europe and a suite of infection-related genes were different from modern strains. Historic outbreaks were not caused by the US- 1 clonal lineage ( $1 \mathrm{~b}$ mtDNA haplotype) but by a closely related sister lineage of the Ia mtDNA haplotypes, Herb-1, that was found in modern New World populations from both Mexico and South America. The US-1 lineage formed a distinct cluster from most modern US lineages in PCA and STRUCTURE analysis of RFLP fingerprints. A maximum-likelihood phylogeny, coalescent analyses, and population subdivision statistics for the RXLR effectors PiAVR2 and PiAVR2-like showed four haplotypes that diverged into two lineages. PiAVR2 but not PiAVR2-like haplotypes were present in historic samples screened thus far. Six haplotypes were observed for IRRAS, and early 20th-century $P$. infestans shared haplotypes with modern lineages, while other haplotypes from 19th century outbreaks were distinct. Our data suggest multiple global introductions of $P$. infestans and the displacement of these lineages over time.

Relationship between Heterobasidion root disease severity and soil characteristics in Wisconsin red pine plantations

K. SCANLON (1), L. Haugen (2), J. Juzwik (2)

(1) Wisconsin Dept of Natl Resources, Fitchburg, WI, U.S.A.; (2) U.S. Forest Service, St. Paul, MN, U.S.A.

Phytopathology 104(Suppl. 3):S3.104

Heterobasidion irregulare, causal agent of Heterobasidion root disease (HRD) in Wisconsin, spreads from diseased trees to healthy ones either by aeriallydispersed basidiospores infecting stump surfaces and wood-exposing wounds following tree removal or by root contact between adjacent trees. The disease is most commonly found and severe on red pine, followed by white pine, in plantations in the state. The numbers of HRD foci and sizes of the foci were recorded during field surveys of 30 red pine plantations previously confirmed to have the disease and located in 15 counties. Data on soil texture and drainage class also were obtained for each stand. The numbers of disease foci found ranged from 0.09 to 11.53 per hectare. The numbers of cumulative affected trees varied from 1 to 22 trees per focus. The mean numbers of cumulative affected trees per focus in plantations on loamy sand/sand soils were higher than those on heavier sandy loam/loam soils $(\mathrm{P}<.05)$. However, the numbers of HRD foci per hectare were similar $(\mathrm{P}>0.30)$ for sites with loamy sand/sand soils and sandy loam/loam soils. All of the surveyed stands were classified as "well" to "excessively drained" and distance of at least 60 $\mathrm{cm}$ to the water table. The results suggest that in Wisconsin soil texture affects rate of pathogen spread via root contact, but not rate of initial infection through above-ground cut surfaces.

Evaluation of osmoprotectants and carriers for formulating Gramnegative biocontrol agents active against potato dry rot in storage

D. A. SCHISLER (1), P. J. Slininger (1), N. L. Olsen (2), M. A. Shea-Andersh (1), L. K. Woodell (2)

(1) USDA ARS MWA NCAUR, Peoria, IL, U.S.A.; (2) University of Idaho, Twin Falls R\&E Center, Twin Falls, ID, U.S.A.

Phytopathology 104(Suppl. 3):S3.104

The production of a dry formulation containing viable cells of a Gramnegative biological control agent is a challenging yet vital step in developing the agent into a commercial product. Pseudomonas fluorescens strains S11:P:12, P22:Y:05, and S22:T:04 reduce Fusarium dry rot of potatoes (causal agent, Gibberella pulicaris) in storage. The strains were evaluated in a high-throughput microtiter plate assay for viability after drying in differing amounts of carbohydrate-based osmoprotectants applied after $24 \mathrm{~h}$ of cell growth in a liquid medium. Fructose and trehalose at $20 \mathrm{~g} / \mathrm{L}$ were the most effective at maintaining the viability of the strains. Different grades of diatomaceous earth, perlite, fumed silica and clay were then combined with high titer suspensions of washed cells of each strain. After being dried for 18$22 \mathrm{~h}$ in a controlled RH atmosphere, several DE products including Kenite 700 were superior in maintaining cell viability. Combining cells suspended in osmoprotectant with Kenite 700 resulted in dried products with up to $10 \mathrm{X}$ higher cell survival. In initial laboratory assays, most dried products containing P22:Y:05 and S22:T:04 reduced dry rot by more than 50\% in laboratory assays $(\mathrm{P}<0.05$, FPLSD) but dried products containing S11:P:12 were less effective. Results of a small pilot-scale trial testing dried biocontrol products for efficacy in reducing dry rot on potatoes in storage will also be reported.

Developing a bioassay to predict and characterize Prunus replant disease in California orchards

L. S. SCHMIDT (1), M. T. Devengenzo (2), G. T. Browne (3)

(1) USDA ARS CPGRU, Davis, CA, U.S.A.; (2) UC Davis, Davis, CA, U.S.A.; (3) USDA ARS, Davis, CA, U.S.A.

Phytopathology 104(Suppl. 3):S3.104

Prunus replant disease (PRD) is a poorly understood soilborne disease complex that suppresses productivity of almond and stone fruit on replanted soils. The incidence and severity of PRD varies greatly among orchards, and predicting disease impact is challenging. Soil fumigation prevents PRD but is costly and increasingly restricted. We developed a greenhouse bioassay to predict PRD and examine microbial contributions to the disease among diverse California soils. Initially, Nemaguard peach seedlings were planted in replicate pots of replant soils (10-30 or 31-60 cm soil depths); mixed with sterile sand $(1: 0,5: 1,4: 1,3: 1,2: 1,1: 1)$; and exposed to fumigation, pasteurization, or autoclaving. After 6 weeks, plant weights revealed that soil from both depths and all soil:sand ratios induced PRD similarly, and that pasteurization and fumigation were more effective than autoclaving in preventing PRD. A refined bioassay (soil from 10-60 cm, 2:1 soil:sand mixture, and pasteurization vs. non-treated control) was then tested on 20 almond and stone fruit replant soils from the Central Valley, CA. Depending on the soil, pasteurization increased plant weight by $0 \%$ to $42 \%$ compared to the controls. Plant weight was negatively correlated with the incidence of Pythium spp. and Cylindrocarpon macrodidymum ( $P=0.01$ and 0.002 , resp.). Bioassay responses are being compared to orchard responses at 3 of the 20 sites. The bioassay has potential to predict and characterize PRD in CA.

Genomic sequencing of Rathayibacter toxicus and the associated phage reveals potential source of toxin production

W. SCHNEIDER (1), A. E. Sechler (2), D. J. Schneider (3), S. J. Mauzey (4), B. K. Schroeder (4), T. D. Murray (4), D. G. Luster (2)

(1) USDA ARS, Fort Detrick, MD, U.S.A.; (2) USDA ARS Foreign DiseaseWeed Science Research Unit, Fort Detrick, MD, U.S.A.; (3) USDA ARS, Ithaca, NY, U.S.A.; (4) Department of Plant Pathology, Washington State University, Pullman, WA, U.S.A.

Phytopathology 104(Suppl. 3):S3.104 
Rathayibacter toxicus (RT) is a gram-positive bacteria that affects multiple forage grass species. RT is of particular interest because of the production of a lethal toxin (tunicamycin) that affects livestock, a disease known as Annual Ryegrass Toxicity (ARGT). Not all members of the genus produce toxin, and the exact mechanisms of toxin control and production are not known. In RT, toxin production is associated with the presence of phage CS14Ф. The complete genomes of RT and CS14 $\Phi$ were sequenced. The RT genome is a single chromosome $2.5 \mathrm{Mb}$ in size, which is relatively small for the genus. Approximately 2340 hypothetical open reading frames (ORFs) were identified, and no additional plasmids were found. The genome of CS14Ф is $44,000 \mathrm{nt}$ in size with 73 hypothetical ORFs. A cluster of genes with similarity to tunicamycin production clusters from other bacteria was found in the genome of RT. Interestingly, the tunicamycin gene cluster (TGC) had a significantly lower GC content (54\%) than the rest of the RT genome $(61 \%)$, suggesting that the TGC may have originated as a mobile element. In addition, the TGC has the same GC content as CS14 1 and there are regions of shared sequence between CS14 $\Phi$ and the TGC, suggesting a possible role for the phage in control of TGC expression and toxin production.

Thermal inactivation of Phytophthora ramorum is a management option to treat infested plants, nursery equipment and soil

W. SCHWEIGKOFLER (1), K. Kosta (2), V. Huffman (1), K. Suslow (1)

(1) Dominican University of California, San Rafael, CA, U.S.A.; (2) California Department of Food and Agriculture, Sacramento, CA, U.S.A. Phytopathology 104(Suppl. 3):S3.105

Infected nursery plants play an important role in the spread of $P$. ramorum, the causal agent of Sudden Oak Death and Ramorum blight. In order to minimize the risk for disease transmission to new areas, nurseries are inspected for P. ramorum and if found positive, federal regulations require the destruction of infested plants and treatment of nursery soil and equipment. The National Ornamental Research Site at Dominican University of California (NORS-DUC) is a federally funded research nursery devoted to develop environmentally friendly management options for quarantine pathogens of ornamental plants. In the laboratory, we tested the effect of wet and dry heat on the survival rate of $P$. ramorum growing on Rhododendron leaf disks. Incubation at $30^{\circ} \mathrm{C}$ showed little effect on the survival rate. At $40^{\circ} \mathrm{C}$, growth rates started to decrease. Incubation at $50^{\circ} \mathrm{C}$ for 30 minutes (wet heat) inactivated $P$. ramorum completely, whereas dry heat was slightly less effective. At the research nursery, thermal inactivation of $P$. ramorum infested plant debris, soil and nursery equipment was achieved by steaming using a commercial steaming unit at a minimum temperature of $50^{\circ} \mathrm{C}$ for two hours (the extended time was chosen to ensure complete pathogen kill). Temperature increase was influenced by ambient temperature, soil depth, moisture content and compactness. Further tests at commercial nurseries are planned to optimize the use of steaming for the management of $P$. ramorum.

Effect of bioactive compounds on infection, growth rates and symptom expression of apple trees infected with the Apple Proliferation phytoplasma

W. SCHWEIGKOFLER (1), S. Schmidt (2), S. Baric (2), M. Massenz (2), C. Kerschbamer (2)

(1) Dominican University of California, San Rafael, CA, U.S.A.; (2) Research Centre of Agriculture and Forestry Laimburg, Auer/Ora, Italy

Phytopathology 104(Suppl. 3):S3.105

Apple Proliferation (AP), caused by 'Candidatus Phytoplasma mali', is a serious disease of apple trees in Europe. Current control of AP consists of the removal of symptomatic plants and insecticide treatments of the vectors. Resistance induction might be an alternative management option. Here we report on the effects of four commercial products on the infection rates, symptom expression and growth rates of potted apple trees (Golden Delicious) infected with the AP phytoplasma. Post-infection treatments using Bion ${ }^{\circledR}$ (active ingredient: Acibenzolar-S-Methyl), Messenger ${ }^{\circledR}$ (Harpin protein), Regalis $^{\circledR}$ (Prohexadione-Ca) and Dormex ${ }^{\circledR}$ (Cyanamid) had no significant effect on infection rates and phytoplasma titres. Terminal growth of apple trees was increased significantly by AP infection; Prohexadione-Ca was the only compound which had a significant inhibiting effect on the growth of both infected and control trees. Expression of typical AP symptoms followed a seasonal cycle with a peak in fall. Acibenzolar-S-Methyl and Harpin had no significant effects on symptom expression. AP symptoms were masked during summer by Prohexadione-Ca, which caused severe growth abnormalities. Cyanamide changed the seasonal appearance of AP symptoms: while symptoms were delayed the first two years (2008 and 2009), symptoms appeared earlier the third year (2010). Differences in symptom expression levelled off later in the vegetative season, and no significant difference was found in October.
In-vivo studies of the interactions between a GFP-transformed Trichoderma asperellum strain and Phytophthora ramorum

W. SCHWEIGKOFLER (1), S. Sharma (1), T. L. Widmer (2), K. Suslow (1) (1) Dominican University of California, San Rafael, CA, U.S.A.; (2) USDAARS-FDWSRU, Fort Detrick, MD, U.S.A.

Phytopathology 104(Suppl. 3):S3.105

Phytophthora ramorum, the causal agent of Sudden Oak Death, is an exotic, invasive plant pathogen. The spread in natural ecosystems and in commercial settings (such as forests and nurseries, respectively) is a leading cause of concern. The necrotrophic, mycoparasitic ability of Trichoderma spp. renders it as a potential biocontrol agent against soilborne pathogens. The aim of this study was to visualize the pathogen-biocontrol agent interaction using molecular and imaging methods. A T. asperellum strain was transformed with a hygromycin B (hyg B) and green fluorescent protein $(g f p)$ gene encoding plasmid by a protoplast $/ \mathrm{PEG} / \mathrm{CaCl}_{2}$ method. The transformation efficiency was determined and the transgene was detected by PCR with $g f p$ and $h p h$ (hygromycin phosphotransferase) primers. The successful transformants were subjected to stability tests. Cultures of $P$. ramorum and the transformed $T$. asperellum strain were placed at opposite ends of a prepared cover slip coated with $20 \%$ PDA and incubated for $24,48,72$ and $96 \mathrm{~h}$ at $22^{\circ} \mathrm{C}$ in a $100-\mathrm{mm}$ sterile Petri dish chamber. Confocal microscopy was used for direct observation of the interaction between the two microbes. Images of spore attachment, hyphal branching, swollen ends and various other phases of parasitism by the gfp:Trichoderma transformants on P. ramorum hyphae and propagules will be discussed. The confocal image data collected in this study is expected to reveal the fundamental mechanism of in vivo fungal interactions.

Symptomatology of crown rot of pyrethrum caused by Sclerotinia spp. in Tasmania, Australia

J. B. SCOTT (1), R. Arvier (2), T. L. Pearce (1), S. Pilkington (1), S. J. Pethybridge (3), F. S. Hay (1)

(1) Tasmanian Institute of Agriculture, University of Tasmania, Burnie, Australia; (2) Botanical Resources Australia-Agricultural Services Pty. Ltd., Ulverstone, Australia; (3) The New Zealand Institute for Plant \& Food Research Limited, Christchurch, New Zealand

Phytopathology 104(Suppl. 3):S3.105

Sclerotinia crown rot (SCR), caused by S. minor and S. sclerotiorum, is an important disease affecting the vegetative tissues of pyrethrum (Tanacetum cinerariifolium) during winter and spring. Symptoms of SCR include bleaching and wilting of leaves leading to necrosis of vegetative tissues. Under severe conditions plant death occurs. This study characterised the progression of SCR symptoms and the relative differences between the two species and forms of inoculums. The relative ability of sclerotia and ascospores to initiate SCR infection was tested under greenhouse conditions. Sclerotia of both species were demonstrated to infect the crown of pyrethrum when myceliogenically germinating sclerotia were in direct contact with the host plant. However, inoculation with ascosporic suspensions of either species failed to initiate SCR symptoms. Under favourable conditions, symptom expression was apparent at 2 to 3 days post inoculation (DPI) with the occurrence of water soaked lesions in leaf tissues. From 20 DPI, infected tissues typically appeared necrotic. Symptoms did not differ between the two species. However, isolates of $S$. minor were more aggressive with a mean proportion of necrotic leaves at 20 DPI of 0.34 , relative to $0.16(P=0.01)$ for $S$. sclerotiorum. Sclerotial formation by $S$. minor was also faster, producing sclerotia within 13 DPI, while $S$. sclerotiorum required 28 DPI. These results support field observations where $S$. minor is dominant.

Effect of temperature on disease progression of Rhizopus soft rot and Fusarium root rot on sweetpotato

A. SCRUGGS (1), L. Quesada (2)

(1) North Carolina State University, Cliffside, NC, U.S.A.; (2) North Carolina State University, Raleigh, NC, U.S.A.

Phytopathology 104(Suppl. 3):S3.105

Sweetpotatoes are an important food crop around the world, both economically and nutritionally. Sweetpotato production is limited by postharvest diseases including Rhizopus soft rot (RSR) and Fusarium root rot (FRR); however, little is known about the epidemiological conditions that promote disease progression. This study aimed to determine the susceptibility of sweetpotato to RSR and FRR when kept at commonly used storage or curing temperatures. Six wounded, 'Covington' sweetpotatoes were inoculated with an actively growing $5 \mathrm{~mm}$ diameter agar plug of Rhizopus stolonifer or Fusarium proliferatum, and incubated in the dark at $13 \mathrm{C}$ or at 29C with 97\% humidity. Control roots were included and the experiment was repeated twice. Disease progression was recorded for RSR and FRR as percent decay and lesion diameter, respectively, for two weeks. The first 
symptoms of RSR were observed at 1 day post inoculation (dpi) at 29C and 3 dpi at 13C, while FRR symptoms started at 2 dpi at 29C and 7 dpi at 13C. There were significant differences in disease progression at different temperatures for both pathogens. RSR decay and FRR lesion diameter were higher at $29 \mathrm{C}$ that at $13 \mathrm{C}$. Experiments also indicated that RSR progresses significantly faster than FRR at high temperatures. These findings will contribute in providing accurate recommendations to growers so that environmental conditions in storage and curing facilities may be optimized to reduce postharvest diseases.

\section{Sensitivity of Fusarium sambucinum to phenylpyrrole and SDHI fungicides}

G. Secor (1), V. V. RIVERA (1), N. C. Gudmestad (1)

(1) North Dakota State University, Fargo, ND, U.S.A.

Phytopathology 104(Suppl. 3):S3.106

Fusarium dry rot of potato continues to be a major disease of all market classes of stored potatoes. The primary cause of potato dry rot is Fusarium sambucinum, although other Fusarium species have been reported. The disease occurs when soil inoculum enters through a harvest wound and disease develops slowly in storage. Dry rot management in storage is difficult because of the lack of resistant cultivars, the inability to reduce soil inoculum and the paucity of post-harvest fungicides. The difficulty in management of seed borne inoculum has been exacerbated by the development of fludioxonil resistance, illustrating the need to identify additional fungicides for disease control. F. sambucinum isolates collected prior to 2002 and in 2013 were compared for sensitivity to phenylpyrrole and three SDHI fungicides, penthiopyrad, penflufen and sedaxane by reduction of radial growth as measured by $\mathrm{ED}_{50}$ values on half-strength PDA and water agar amended with technical grade fungicide. None of the SDHI fungicides effectively reduced the growth of $F$. sambucinum isolates collected prior to 2002 or collected in 2013. This data also serves as baseline data of $F$. sambucinum for these three SDHI fungicides. Isolates resistant to phenylpyrrole were found. Isolates from both collection dates were sensitive to difenoconazole and resistant to thiabendazole. The SDHI fungicides may not be effective as seed treatments or post-harvest fungicides for Fusarium dry rot management.

Etiology of soybean root rot caused by Fusarium solani and its effect on root and above ground plant development

F. J. SESSOMS (1), L. K. Otto-Hanson (1), J. Lencowski (1), D. K. Malvick (1), J. E. Kurle (1)

(1) Department of Plant Pathology, College of Food, Agriculture, and Natural Sciences, University of Minnesota, St. Paul, MN, U.S.A.

Phytopathology 104(Suppl. 3):S3.106

Fusarium solani is an important soybean root pathogen. However, its interaction with soybean and the etiology of root rot are poorly understood. Our objectives were to determine if $F$. solani is a pathogen of soybean at different growth stages and if it affects plant growth, development and yield. Experiments were conducted in sterile phytagel and several growth media. The effects of soil temperature and moisture conditions were also tested. In phytagel, greater spore concentrations led to reduced tap root length and lateral root number, and the highest concentration $\left(10^{4} / \mathrm{ml}\right)$ stopped seed germination. Root lesions, initiated where lateral roots emerged, were observed in the inoculated plants, and microscopy will reveal if hyphal presence is associated with these lesions. Results indicate that most $F$. solani isolates, 11 of 18 tested, were weakly pathogenic. $F$. solani could cause severe root rot in all moisture conditions at $15^{\circ} \mathrm{C}$ but not at $30^{\circ} \mathrm{C}$. Root biomass was often lower in the infected plants, but no clear negative relationship was found between discoloration percentage and shoot biomass or yield. $F$. solani DNA was detected at low levels in inoculated roots at the R1 and R4 stages using quantitative PCR, whereas it was detected at higher levels in vegetative stages. Results suggest $F$. solani infects young seedlings, causes root rot, and reduces root development that may lower the plant's ability to overcome abiotic and biotic stresses.

Developing host-specific bioherbicides for sustainable management of Orobanche crenata in Egypt

Y. M. SHABANA (1), M. El-Hawary (2), M. E. Sadek (1)

(1) Mansoura Univ, El-Mansoura, Egypt; (2) Agricultural Research Center, Giza, Egypt

Phytopathology 104(Suppl. 3):S3.106

A substantial damage is imposed on food and fodder plants by mass incident of the parasitic weed plants, Orobanche spp. Although a considerable number of herbicides have been tested as means for controlling broomrape, none of them had an effective control measure. In addition, high cost and toxicity of chemical herbicides also limit their application. Indigenous, weed-specific fungal pathogens can be used as safe and effective bioherbicides. As an alternative or adjunct to conventional weed control technology of chemical and mechanical controls, the bioherbicides offer excellent means of ecologically sound weed management. There is now a unique opportunity to develop fungal pathogens of Orobanche crenata as bioherbicides in legumes. Two strains of Fusarium oxysporum in addition to one strain of both $F$. semitectum and $F$. camptoceras have been shown to be effective bioherbicide candidates for $O$. crenata. Results from laboratory and greenhouse trials have confirmed the high feasibility of using these fungal strains to control broomrape of broad bean (Vicia faba). In an attempt to develop these bioherbicides, various formulations of the fungus and its impact on broomrape control were determined. Effect of various environmental factors including temperature, cultural media, $\mathrm{pH}$, light regime, and aeration will also be discussed.

A new caveat for blackberry: A new badnavirus identified in diseased plants

M. S. SHAHID (1), N. Aboughanem-Sabanadzovic (2), S. Sabanadzovic (3), I. E. Tzanetakis (1)

(1) Department of Plant Pathology, Division of Agriculture, University of Arkansas, Fayetteville, AR, U.S.A.; (2) Institute for Genomics, Biocomputing and Biotechnology, Mississippi State University, Mississippi State, MS, U.S.A.; (3) Department of Biochemistry, Molecular Biology, Entomology and Plant Pathology, Mississippi State University, Fayetteville, AR, U.S.A. Phytopathology 104(Suppl. 3):S3.106

In the last decade several new viruses have been discovered in symptomatic blackberry plants. Curiously, all new viruses identified had an RNA genome. Here we report a new dsDNA virus with significant similarities to the genus Badnavirus, family Caulimoviridae. The genome consists of 7,663 base pairs and codes for four genes, similar to other members of the genus. Phylogenetic analysis places the new virus in a clade alongside Cacao swollen shoot virus. The virus was detected in plants showing yellow vein diseases symptoms from Arkansas, Mississippi and North Carolina. This agent may pose a new threat to already virus-devastated blackberry industry, as possible incorporation in the host genome will not allow for the much sought-after, virus-free blackberry plant.

Molecular characterization of dsRNA in Heterobasidion irregulare and Heterobasidion occidentale and effects on growth and sporulation

S. F. SHAMOUN (1), I. Kassatenko (1), C. Hammett (2), G. Sumampong (1) (1) Canadian Forest Service, Victoria, BC, Canada; (2) UBC Forest Sciences and Conservation, Vancouver, BC, Canada

Phytopathology 104(Suppl. 3):S3.106

The root and butt-rot caused by Heterobasidion annosum sensu lato is one of the most destructive diseases of conifer and hardwood species in the Northern hemisphere. There are two species endemic to North America: H. occidentale (S-type) and H. irregulare (P-type). H. occidentale is found on the Pacific coast of North America from Alaska to Southern Mexico west of the Rocky Mountains infecting many tree genera, preferably Tsuga, Abies, and Pseudotsuga. H. irregulare is found in Pine forests across North America from Quebec, down through the USA and Mexico to the Northern Caribbean. Heterobasidion populations around the world have been isolated and surveyed for the presence of dsRNA mycoviruses. However North American mycoviruses have not been well studied, with only three previously surveyed and one confirmed to be from the partitivirus genus. We screened 140 isolates, $62 \mathrm{H}$. irregulare and $78 \mathrm{H}$. occidentale from around North America for dsRNA viruses. Analysis showed 26 dsRNA bands including 23 from $H$. irregulare and 3 from $H$. occidentale including five isolates with consensus sequences of the partitivirus HetPV1. Ongoing research into growth and sporulation is underway. Future sequencing of these viruses will reveal the family of virus and possibly identify new viruses present in North American Heterobasidion spp.

Molecular characterization of genomic components of Potato mop-top virus from Washington State

R. SHANMUGIAH (1), G. Raikhy (1), C. Brown (2), H. Pappu (1)

(1) Washington State University, Pullman, WA, U.S.A.; (2) USDA ARS, Prosser, WA, U.S.A.

Phytopathology 104(Suppl. 3):S3.106

Potato mop-top virus (PMTV) is the type species of genus Pomovirus, family Virgaviridae. The occurrence of PMTV was reported recently in the Pacific Northwestern USA. PMTV genome is characterized by three linear positive sense ssRNA molecules. As part of a study to better understand the virus biology and molecular biology, the genome of a WA isolate was characterized and we report the complete nucleotide (nt) sequence of the genomic components RNA-2, and RNA-3. RNA2 was 3134nt in length with an ORF between nt positions 314-844 that codes for the coat protein and the same 
ORF as a read-through extends further to nt position 2791 coding for the CPread through protein. RNA 3 was 2964 nt in length and potentially encodes four ORFs - three of them forming the triple gene bock (TGB) that was shown to be involved in cell to cell movement. RNA2 also contains a $206 \mathrm{nt}$ ORF present toward the 3' end that encodes a cysteine-rich $8 \mathrm{~K}$ protein. RFLP analysis of RNA2 and RNA 3 of the WA isolate revealed the combination of RNA2-II and RNA3-B types, the combination that is prevalent in Europe and elsewhere. The sequenced regions had $99 \%$ sequence identity with the corresponding RNA components reported from Europe. The high degree of sequence identity could be useful in developing virus detection tools and for designing RNAi-based virus management strategies.

Interaction map of Tomato spotted wilt virus-specific small RNAs and the tomato transcriptome

R. SHANMUGIAH (1), N. Mitter (2), H. Pappu (1)

(1) Washington State University, Pullman, WA, U.S.A.; (2) University of Queensland, St. Lucia, Australia

Phytopathology 104(Suppl. 3):S3.107

Tomato spotted wilt virus (TSWV; Tospovirus:Bunyaviridae) is one of the most prolific and economically important viruses of field and horticultural crops. In the plant-virus interactions, virus-derived siRNAs (vsiRNAs) are the result of activity of host-mediated silencing mechanism. We recently obtained the TSWV-specific small RNA profiles from virus-infected tomato. A subset of siRNAs derived from the TSWV genome hotspots was analyzed in silico for their propensity to down regulate tomato transcriptome. vsiRNAs were found to interact with a gamut of host genes involved in basal cellular activities such as nucleic acid metabolism, ribosomal turnover, translational factors, cytoskeletal proteins, phenylpropanoid biosynthesis, glycosyl transferases, peptidases, hormonal signalling, protein kinases, intercellular transporter genes, and stress-related proteins. Notably, vsiRNAs derived from the TSWV NSs gene binds with transcripts generally associated with stress signalling, whereas siRNAs from NSm were predominantly found to bind the transcripts involved in abiotic stress responses such as dehydration responsive protein, ion exchange transporters, and low temperature and salt responsive proteins. The predicted interactome scenario when validated through gene expression studies using RNAseq could provide a detailed picture on the molecular mechanism underlying the tospovirus-plant interactions.

Plant miRNAome and soybean-infecting Begomovirus genomes: Identification of innate plant small RNAs in antiviral resistance

R. SHANMUGIAH (1), H. Pappu (1)

(1) Washington State University, Pullman, WA, U.S.A.

Phytopathology 104(Suppl. 3):S3.107

Small non-coding RNAs (ncRNAs) have emerged as effector molecules in response to pathogen invasion in plants and animals. We conducted in silico analysis of the DNA genomes of two distinct species of genus Begmovirus (family Geminiviridae), Mungbean yellow mosaic India virus (MYMIV) and Mungbean yellow mosaic virus (MYMV), that infect soybean. Using an miRNA target prediction algorithm, plant small RNA target analyzer in silico analysis was carried out. The analysis revealed multiple targets for plant miRNAome across both viral genomes. Between the two viruses, MYMV displayed a greater vulnerability to plant-derived miRNAs with 33 plant miRNAs targeting its genome, whereas 24 small RNAs appeared to be targeting the MYMIV genome. However, no significant difference was observed in the number of miRNA target sites in the genomes of both viruses. The miRNA gamut of 17 other plants species also displayed propensity to target various genomic regions of the two begomoviruses. ORFs, $\mathrm{BC} 1$ and BV1 that code for proteins involved in movement functions had greater vulnerability for plant-derived miRNA binding. Soybean-derived six prospective miRNA families revealed that the miRNAs could potentially play a role in host-microbe interactions, nodulation process, and abiotic stress responsiveness. The study thus provides in silico evidence for the plantderived miRNAs in antiviral immunity in soybean small RNA (sRNA) milieu.

Global analysis of population structure, spatial and temporal dynamics of genetic diversity of Iris yellow spot virus (Tospovirus: Bunyaviridae)

R. SHANMUGIAH (1), R. Iftikhar (1), S. Bag (1), M. Ashfaq (2), H. Pappu (1)

(1) Washington State University, Pullman, WA, U.S.A.; (2) University of Guelph, Guelph, AB, Canada

Phytopathology 104(Suppl. 3):S3.107

Thrips-transmitted Iris yellow spot virus is an economically important viral pathogen of Allium crops worldwide. A global genetic analysis of known IYSV nucleocapsid gene ( $\mathrm{N}$ gene) sequences was carried out to determine the comparative population structure, spatial and temporal dynamics with reference to its genetic diversity and evolution. A total of 98 complete $\mathrm{N}$ gene sequences available in GenBank and reported from 23 countries were characterized by in silico RFLP analysis. Based on RFLP, 94\% of the isolates could be grouped into NL or BR types while the rest belonged to neither group. The relative proportion of NL and BR types was $46 \%$ and $48 \%$, respectively. A temporal shift in the IYSV genotypes with a greater incremental incidence of IYSV $_{\mathrm{BR}}$ was found over IYSV $\mathrm{NL}_{\mathrm{NL}}$ before 2005 compared to after 2005 . The virus population had at least one evolutionarily significant recombination event, involving IYSV $V_{\mathrm{BR}}$ and $\mathrm{IYSV}_{\mathrm{NL}}$. Genetic differentiation studies showed inherent differentiation and infrequent gene flow between IYSV $V_{\mathrm{BR}}$ and IYSV $\mathrm{NL}_{\mathrm{NL}}$ genotypes corroborating the geographical confinement of these genotypes. Taken together the study suggests that the observed diversity in IYSV population and temporal shift in IYSV $\mathrm{BR}_{\mathrm{BR}}$ genotype is attributable to genetic recombination, abundance of purifying selection, insignificant positive selection and population expansion.

Use of azoxystrobin and a new formulation of abamectin for managing Trichodorus obtusus in a zoysiagrass stand

J. B. SHAVER (1), P. Agudelo (1), S. B. Martin (2)

(1) Clemson University, Clemson, SC, U.S.A.; (2) Clemson University, Florence, SC, U.S.A.

Phytopathology 104(Suppl. 3):S3.107

Azoxystrobin is often used to control common fungal diseases in turfgrass. Anecdotal evidence has suggested that azoxystrobin may be useful for alleviating plant-parasitic nematode damage in turfgrass. Special local need registrations in several states have resulted in increased use of Avid $(2 \%$ abamectin) as a nematicide. Field trials were conducted in 2012 and 2013 to determine the effects of azoxystrobin and abamectin, applied alone and in combination, on the population densities of stubby root nematode Trichodorus obtusus. Multiple soil cores $(5 \mathrm{~cm}$ diameter, $20 \mathrm{~cm}$ depth $)$ were collected within each plot and nematodes were extracted using sugar centrifugal flotation from a $100 \mathrm{~cm}^{3}$ subsample from each core. In 2012, all treatments including azoxystrobin, reduced $T$. obtusus densities relative to the control. Further, the combination of azoxystrobin and abamectin as well as abamectin alone reduced $T$. obtusus levels comparably to oxamyl. However, in 2013 azoxystrobin significantly increased $T$. obtusus populations. During the second year, the only treatment that significantly reduced $T$. obtusus population densities was an experimental formulation of abamectin applied alone. More work is needed to fully understand the effects of azoxystrobin on T. obtusus population densities on zoysiagrass.

Mechanisms of drought-induced susceptibility of Austrian pine to Diplodia pinea

P. SHERWOOD (1), C. Villari (1), P. Capretti (2), P. Bonello (1)

(1) The Ohio State University, Columbus, OH, U.S.A.; (2) Università degli Studi di Firenze, Firenze, Italy

Phytopathology 104(Suppl. 3):S3.107

Water limitation is an important abiotic factor that often predisposes plants to infection. However, the molecular mechanisms of this induced susceptibility are poorly understood, particularly in trees. Here we studied the molecular responses of Pinus nigra and its susceptibility to the pathogen Diplodia pinea. Drought increased host susceptibility, and resulted in the accumulation of proline and reactive oxygen species (ROS) in the shoots before inoculation. However, ROS levels were lower and proline concentrations higher around infection sites relative to non-inoculated shoots on droughted trees. Proline has several beneficial roles in stress responses including an ability to scavenge ROS and act as a nutrient source. We hypothesized these functions may be contributing to $D$. pinea's success in stressed pines, because proline enhanced the in vitro growth of $D$. pinea by functioning as a preferred $\mathrm{N}$ source while also protecting the fungus from $\mathrm{H}_{2} \mathrm{O}_{2}$ damage. Furthermore, hyperactive proline metabolism can trigger apoptosis, which may facilitate $D$. pinea's necrotrophic lifestyle. When challenged in vitro with $\mathrm{H}_{2} \mathrm{O}_{2}, D$. pinea's catalase and peroxidase activities were induced, suggesting that the pathogen has substantial ROS detoxifying abilities. We propose that $D$. pinea is particularly successful in droughted trees because it is well adapted to water stress-induced shifts in host $\mathrm{N}$ and ROS metabolism that can lead to apoptosis.

Effect of areated compost tea on the microbial diversity and control of plant disease in organic cucumber cultivation

C. K. SHIM (1), M. J. Kim (1), Y. K. Kim (1), H. J. Jee (1), J. C. Yun (1), S. J. Hong (1), J. H. Park (1), E. J. Han (1), M. H. Lee (1)

(1) National Academy of Agricultural Science, Suwon, South Korea Phytopathology 104(Suppl. 3):S3.107

The aim of this study was to assess the effect of aerated compost tea on the organic cucumber and the soil microbial diversity. The compost tea was rich in bacteria and fungi which may act as antagonists to the pathogens. A field experiment was carried out at organic cucumber cultivation farm. Foliar 
application of the compost tea significantly reduced powdery mildew and downy mildew incidence on leaflets in cold season cultivation of organic cucumber. The compost tea was significantly improved cucumber growth and microorganism density and diversity compared to the untreated control. The results showed that the average cucumber yield was higher with $35 \%$ yield increasing and $70 \%$ income compared to conventional farm. The results recommended that the compost tea may be useful ways as part of an integrated plant health management strategy to control of foliar plant disease and to improve the microbial density in organic cucumber cultivation field.

Growth-promoting effects of microorganisms and control of bakanae disease by organic material

G.-H. SHIN (1), Y.-W. Seo (1), H.-J. Kim (1), H.-W. Kim (1), J. Song (2)

(1) JARES, Naju, South Korea; (2) NAAS, RDA, Suwon, South Korea

Phytopathology 104(Suppl. 3):S3.108

Three species of microorganisms, Bacillus subtilis (P91281), Pseudomonas sp. (P91282) and Kluyvera sp. (P91283), growth-promoting effects such as phosphate solubilization were drenched in soil six times at 7-day intervals in Chinese cabbage growing season. Yield of Chinese cabbage increased 9-13\% in the microbial agent treatments compared to control. Bakanae disease caused by Gibberella fujikuroI is common and serious disease in rice and occurs from nursery stage to paddy fields. This study was conducted to improve the environment-friendly disinfection method in nursery for control bakanae disease on rice. The seed disinfection methods of organic culture, hot water soaking reported that were ineffective on the bakanae disease severely infected rice seed because the hot water did not transmit the pericarp layer of rice seed. The stepwise disinfection of rice seed, the seed soaking for 24 hours on lime sulfur mixture solution (50 times diluted) at 30 Celsius after hot water soaking ( 60 Celsius for $10 \mathrm{~min}$.) controlled bakanae disease by $99.4 \%$ in control value compared to control at 30 days after sowing in the nursery box. Disease incidence of the control was $15.1 \%$. In rice field the seed treatment by lime sulfur mixture reduced bakanae disease by $96.9 \%$ in control value compared to hot water treatment. Thus, the mixture of hot water soaking and lime sulfur would be used as a method to control bakanae disease in environmentally friendly rice cultivation.

Disease control using Fe-enriched sheath produced by an Fe-oxidizing bacterium, Leptothrix sp. (I) Sheath exudate blocks fungal infection

T. SHIRAISHI (1), K. Toyoda (2), H. Kunoh (2), J. Takada (2)

(1) Research Inst for Biological Science, Okayama Pref, Kaga-gun, Japan; (2) Okayama University, Okayama, Japan

Phytopathology 104(Suppl. 3):S3.108

The iron-oxidizing bacteria, Leptothrix spp., produce microtubular hollow sheath extracellularly in aquatic environments where groundwater outwells. The basic matrix of sheath is a unique Fe-rich organic/inorganic hybrid comprising organics released from bacterial cells and aquatic phase of inorganics such as $\mathrm{Fe}, \mathrm{Si}$, and $\mathrm{P}$. Approximate ratio (atomic \% determined by SEM/EDX) of these elements in sheath was Fe: $\mathrm{Si}: \mathrm{P}=75: 20: 5$ irrespective of sampling sites and times. The proteinaceous and saccharic exopolymers secreted from the bacterial cell compose the core of fiber matrix of sheath which attracts aqueous phase of particular inorganic ions to make a variety of chemical bonds such as Fe-O-Si, Fe-O-P etc. The supernatant of powdered sheath which was mixed with spore suspension of Mycosphaerella pinodes suppressed the symptom development when applied to pea leaves. The sonication-prepared colloidal suspension of powdered sheath prominently blocked formation of infection structure of Botrytis cinerea, resulting in suppression of penetration. The suspension $(5 \mathrm{mg} / \mathrm{ml})$ prepared from powdered sheath often caused burst of its germtube. A similar effect was seen against another pathogen, Alternaria alternata. Although further studies on the suppression mechanism are required, it is noteworthy that the natural product of bacterial origin opened an encouraging approach avenue to develop a novel protectant for fungal diseases.

Identification of fungi associated with and evaluation of seedling plant reactions to root rot diseases in spring wheat of North Dakota

S. SHRESTHA (1), S. Zhong (1)

(1) North Dakota State University, Fargo, ND, U.S.A.

Phytopathology 104(Suppl. 3):S3.108

Wheat Crown Rot (CR) and common root rot (CRR) are mainly caused by Fusarium Species and Bipolaris sorokiniana, respectively. The diseases can cause average yield losses of $3-5 \%$ in a year. However, few studies have been conducted for these diseases in the last thirteen years in North Dakota (ND). To assess incidence and severity of these diseases in ND, we collected wheat root samples from fields distributed all over the state in 2012 and 2013. The collected root samples were scored for CRR severity using a 0-4 scale, then fungi associated with the CRR and CR symptoms were isolated and identified.
Higher incidence and severity of CRR were observed in 2012 (warm and dry year) than in 2013 (extended cold year), and more isolates were recovered from Fusarium species than from B. sorokiniana in both years. One $F$. culmorum isolate and one $B$. sorokiniana isolate were used to evaluate seedling reactions of ten spring wheat lines to CR and CRR in plastic cups filled with sand at the bottom, cornmeal and inoculum mixture in the middle and autoclaved vermiculite on top. Disease severity was rated at tenth day after inoculation using a 0-5 scale. Results showed Amidon was less susceptible to $B$. sorokiniana, while Faller and Reeder were less susceptible to $F$. culmorum than other wheat lines. Steele-ND failed to germinate when inoculated with both $B$. sorokiniana and $F$. culmorum isolates, indicating it is highly sensitive to the fungal infections.

Effects of organic amendment C:N ratio on Fusarium oxysporum f. sp. lycopersici populations following anaerobic soil disinfestation

U. SHRESTHA (1), A. Bruce (1), B. H. Ownley (1), D. M. Butler (1)

(1) University of Tennessee, Knoxville, TN, U.S.A.

Phytopathology 104(Suppl. 3):S3.108

Organic amendments (OA) of dry molasses (DM) and wheat bran (WB) have been used in anaerobic soil disinfestation (ASD) to provide labile $\mathrm{C}$, which stimulates soil microbial activity and can be an effective control against Fusarium oxysporum $(F o)$. The aim of this study was to evaluate effectiveness of these OAs at $4 \mathrm{mg} \mathrm{C/g}$ soil, and varying C:N ratios of 40:1, 30:1, 20:1 and 10:1, to reduce $F o$ propagules. Two polyethylene mesh bags with $2 \mathrm{~g}$ Fo rice inoculum were buried at 5- and $15-\mathrm{cm}$ depths in pots with soil/sand mix at $15-$ $25^{\circ} \mathrm{C}$. Soil $\mathrm{pH}$ and cumulative redox potential (CEh) below a critical value were assessed. After ASD, Fo survival was assessed by dilution plating of recovered inoculum on Snyder-Nash agar. Data were analyzed with mixed model ANOVA, and Fisher's P-LSD at $P=0.05$. Across both OAs, soil $\mathrm{pH}$ was lowest for the 10:1 C:N ratio, but there were no soil $\mathrm{pH}$ differences among other treatments. For OA, CEh was greater (more anaerobic) and $F_{O}$ colonies were fewer for DM than WB. The interaction of OA and C:N ratio was significant for CEh and Fo counts. For DM, CEh was similar for all C:N ratios and lowest in the untreated control; there were fewer $F_{O}$ colonies recovered from the lower $\mathrm{C}: \mathrm{N}$ ratios and colonies were highest for the 40:1 ratio and the control. For $\mathrm{WB}, \mathrm{CEh}$ was similar for all $\mathrm{C}: \mathrm{N}$ ratios and the control; Fo counts were high across all C:N ratios and lowest in the control. Across all treatments, Fo populations were lower at the $15-$ than $5-\mathrm{cm}$ depth.

Genome-wide identification of molecular markers for partial resistance of rice to bacterial panicle blight using high-throughput sequencing data B. K. SHRESTHA (1), H. S. Karki (2), J. H. Ham (1)

(1) Louisiana State University Agricultural Center, Baton Rouge, LA, U.S.A.; (2) Purdue University, West Lafayette, IN, U.S.A.

Phytopathology 104(Suppl. 3):S3.108

Bacterial panicle blight (BPB), an economically important rice disease, is caused by the Gram-negative bacteria, Burkholderia glumae and B. gladioli. There is no completely resistant cultivar available for BPB, but a few cultivars show partial resistance. Comparative genomics study with partially resistant and susceptible cultivars will be helpful in identifying new molecular markers associated with BPB resistance. Whole genome sequence data of Jupiter (a partially resistant medium-grain cultivar) and Trenasse (a susceptible longgrain cultivar) from Illumina GAIIx platform were assembled using the Nipponbare reference sequence, with the DNASTAR software package. More than $800 \mathrm{~K}$ of single nucleotide polymorphisms (SNPs) were identified in each of Jupiter and Trenasse. From the comparison of SNPs between the two cultivars, 685 non-synonymous (ns)-SNPs were identified. Allele-specific SNP markers were designed for those SNPs, and a sub-set of these markers (corresponding to 24 nsSNPs in 24 genes) were randomly selected for validating their polymorphisms between those two cultivars. Eleven of the 24 SNP markers tested showed polymorphisms in agarose gel electrophoresis, suggesting that more than $45 \%$ of SNP markers designed based on the highthroughput sequence data can be used for genetic mapping with a mapping population derived from those two cultivars. SNP markers developed in this approach will be useful for fine genetic mapping of the partial resistance to $\mathrm{BPB}$

Nematode resistance screening of upland cotton using microplots

R. B. Sikkens (1), K. S. LAWRENCE (1)

(1) Auburn University, Auburn, AL, U.S.A.

Phytopathology 104(Suppl. 3):S3.108

Thirteen upland cotton ( $G$. hirsutum) germplasm lines with introgressed resistance to reniform nematode ( $R$. reniformis), belonging to 4 groups depending on source of resistance and developmental background, were tested in microplots. Susceptible cultivars FM966 and SG747 functioned as controls. Each microplot, 24.6 liter containers filled with soil infested with reniform 
nematode, was seeded with one seed of each resistance group, along with one seed of a control. At 60 days after planting, seedlings were extracted, shoot heights (SH), shoot fresh weights (SFW) and root fresh weights (RFW) determined. Nematode eggs were extracted and counted (eggs/gRFW). Germplasm lines from the BARBREN and M713 groups, both incorporating resistance from accession GB 713 of G. barbadense, combined excellent tolerance with good resistance. They outperformed the susceptible controls by $45 \%(\mathrm{SH}), 150 \%$ (SFW) and $133 \%$ (RFW), while reducing eggs/gRFW by $90 \%$. Lines from the LONREN group, with resistance derived from $G$. longicalyx, and the MT2468 group, with resistance derived from accession TX2468 of G. hirsutum, lowered eggs/gRFW by $71 \%$ and $65 \%$, respectively, compared to the controls. LONREN lines showed poor tolerance with stunted seedlings and 35\% less RFW, whereas SH and RFW of MT2468 lines were $19 \%$ and 34\% higher than the controls. Microplot results correlated well with those obtained in field and greenhouse tests, indicating that they can efficiently be used for resistance screening.

Behaviour of rust in new and in old inner leaves in conilon coffee M. B. SILVA (1), F. L. Partelli (1), R. Sambugaro (1), L. S. Oliari (1) (1) Universidade Federal do Espírito Santo, São Mateus, KS, Brazil Phytopathology 104(Suppl. 3):S3.109

The coffee rust (Hemileia vastatrix) epidemic is well studied in C. arabica, where a single lesion is usually enough to cause defoliation, in contrast to the disease progress in C. canephora, in which the infected leaves remain on the plant. The objective of this study was to quantify the progress of coffee rust based on incidence in young and in old inner leaves in different genotypes of conilon coffee. For this purpose, a field experiment was performed in Vila Valério, state of Espírito Santo. The genotypes 143 (ENCAPA 8131), 02 (ENCAPA 8111), G35 (Verdebrás) and Verdinho HP were evaluated. Rust progress was evaluated for 12 months. Incidence data were used to determine the area under the disease progress curve (AUDPC) for each genotype, both in young and old leaves, and these data were then subjected to analyses of variance at $5 \%$ probability. There was a greater occurrence of rust on old inner coffee leaves. Incidence was used to fit disease-progress models, and the Gompertz model was the one with the best fit. Genotype 143 was the most resistant in the field. Infected leaves remaining on the plant could be an important inoculum source to be considered in the management of rust in conilon coffee.

Foliar applications of iron suppress Cercospora leaf blight and increases yield in soybean

E. C. SILVA (1), A. K. Chanda (1), T. G. Aroca (1), C. L. Robertson (1), B. M. Ward (1), R. W. Schneider (2)

(1) Louisiana State University, Agricultural Center, Baton Rouge, LA, U.S.A.; (2) Louisiana State University, Baton Rouge, LA, U.S.A.

Phytopathology 104(Suppl. 3):S3.109

Cercospora leaf blight (CLB) caused in soybean by Cercospora kikuchii is a devastating disease. Losses in Louisiana fields can range from $20 \%$ to complete crop failure. Preliminary work testing foliar applications of reagent grade micronutrients showed that Fe decreased CLB severity. The objective of this work was to test the effects commercial formulations of Fe, Manny Plex $\mathrm{Fe}$ and Fe EDTA (Brandt Consolidated, Springfield, IL) on leaf colonization by $C$. kikuchii, symptom development (blight and purple leaves), and yield. Four rates of each formulation were applied to field plots at the R3 and R5 growth stages. Leaf tissue analyses for microelements and qPCR testing for the pathogen were performed. Disease severity was assessed quantitatively for leaf blight and purple leaf symptoms, and yield was measured. Results showed there was no correlation between leaf colonization and symptoms (neither purple nor blight). In addition, Fe concentration in leaves did not affect biomass of C. kikuchii. Purple leaf symptoms increased in severity as Fe concentration in leaves increased, but severity decreased as Fe concentrations surpassed $200 \mathrm{ppm}$. Blight symptoms were completely suppressed above the threshold of $280 \mathrm{ppm}$. There was no correlation between purple leaf and blight symptoms. There was a positive relationship between concentrations of $\mathrm{Fe}$ in leaves and yield and negative relationships between yield and Cercospora biomass and severity of blight and purple leaf symptoms.

Effects of climate changing on the development of rice diseases

V. L. SILVA-LOBO (1), J. T. Aguiar (2), D. S. d'Afonseca (3), S. L. Lopes (4), E. Del Ponte (5), M. L. Junior (4)

(1) Embrapa Rice and Beans, Santo Antonio De Goias, Brazil; (2) Graduate program on Agronomy, Universidade Federal de Goiás, Goiânia, Brazil; (3) Informatics Institute, Universidade Federal de Goiás, Goiânia, Brazil; (4) Embrapa Rice and Beans, Goiania, Brazil; (5) Universidade Federal de Viçosa, Viçosa, Brazil

Phytopathology 104(Suppl. 3):S3.109
To estimate the risks and possible impacts of climate change on rice diseases, current activities of an ongoing project investigate the influence of weather variables on the development of disease epidemics, in Brazilian states. Research activities include the recovery of severity and yield records from dozens of field trials carried out since the 80's, by Embrapa's rice breeding programs. These time series were organized in a database, and the historical data has being compiled and gathered to climate data. Climate data, by its turn, consists of maximum and minimum temperatures, precipitation and number of rainy days provided by INMET and includes the identification of years with the ENSO (El Niño - Southern Oscillation) phenomenon. Local and broad-scale estimates of disease and climate impacts on crop yield were achieved with descriptive and multivariate statistics. Furthermore, maps of current and future climate scenarios for Brazil for the 2020s, 2050s, and 2080s were prepared for the diseases targeted in this study, based on models defined by the IPCC. These maps anticipate shifts on disease relevance through the country, with huge consequences on cropping systems, yield expectations, breeding programs and new challenges on integrated disease management.

\section{Mapping bacterial wilt resistance in an F2 tomato population developed from a cross of CLN1466EA x NC84173}

E. SILVERMAN (1), F. J. Louws (1), D. Panthee (1)

(1) North Carolina State University, Raleigh, NC, U.S.A.

Phytopathology 104(Suppl. 3):S3.109

Ralstonia solanacearum (Rs) is the causal agent of Bacteria wilt (BW), a devastating disease of over 200 plant species including members of the Solanaceae family such as tomato. $R s$ is a soil-borne bacterium prevalent in the southeastern USA. Management of BW is limited and requires an integrated approach to reduce the impact of disease. Host resistance has proven effective against BW with great focus on quantitative resistance. The goal of the tomato breeding project is to develop an early screening method to assist in selection based on phenotypic observations and single nucleotide polymorphism (SNP) genotypic analysis to map quantitative trait loci (QTLs) associated with BW resistance within a segregating tomato population. Two parents, CLN1466EA and NC84173, were selected based on contrasting traits to develop the segregating $\mathrm{F} 2$ population, $\mathrm{NC11212}$, for resistance to $\mathrm{BW}$, decent fruit quality, and other traits. Individuals (140) were randomly selected from the F2 population for phenotypic observations, genotypic analysis, and F3 family development via the single seed descent breeding method. Observations of BW disease incidence and symptom severity were collected from routine BW inoculations of asexually propagated cuttings from F2 plants, parental lines, Cherokee Purple susceptible control, and HI7997 resistant control. This project will provide tomato breeders with a new, useful tool for rapid selection of $\mathrm{BW}$ resistance and future line release for $\mathrm{NC}$ growers.

\section{Managing bacterial wilt of tomatoes in North Carolina through grafting with disease resistant rootstocks}

E. J. SILVERMAN (1), J. Driver (1), J. Kressin (1), F. Louws (1), D. Panthee (1)

(1) North Carolina State University, Raleigh, NC, U.S.A.

Phytopathology 104(Suppl. 3):S3.109

Bacterial wilt, caused by Ralstonia solanacearum, $R s$, is endemic to many temperate regions around the world causing enormous economic loss in crops like potato, tobacco and tomato. Management of BW, is difficult and is centered on extended crop rotation, cultural practices, host resistance and grafting. The goal of this project was to examine the efficacy of grafting with $\mathrm{BW}$ resistant tomato rootstocks in $\mathrm{NC}$ on-farm trials and develop better recommendations for growers to reduce the impact of $\mathrm{BW}$. Ten $\mathrm{BW}$ rootstocks grafted to the common scion 'FL47' were evaluated over two growing seasons in a high BW pressure field in western NC. Rootstock selection impacted disease incidence. In 2012, rootstock varieties 'Cheong gang', BHN1054, DP106, and CRA66 exhibited the least number of diseased plants with $25,33,35$, and $43 \%$ respectively. Non-grafted and self-grafted 'FL47' controls reached $100 \%$ plant death by 75 days after planting (DAP). Similar results were observed in 2013 with rootstocks CRA66, DP106, HI7997 and 'Cheong gang' possessing the lowest BW disease incidence with $4,8,9$, and $10 \%$ wilt, respectively. Non-grafted and self-grafted controls reached 70 and $63 \%$ disease by 77 DAP. Marketable yields were dramatically impacted with high yields from plots with superior resistance compared to the susceptible controls. Based on regional experiments, several large commercial farms have adapted grafting as an IPM tool to manage BW.

\section{Wheat curl mite populations under deficit irrigation}

A. R. SIMMONS (1), F. Worneh (1), S. O'Shaugnessy (2), S. Evett (2), C. M. Rush (1)

(1) Texas A\&M Agrilife Research, Amarillo, TX, U.S.A.; (2) USDA-ARS, Bushland, TX, U.S.A.

Phytopathology 104(Suppl. 3):S3.109 
Wheat streak mosaic virus, transmitted by the wheat curl mite (Aceria tosichella), causes extensive reductions in crop water-use efficiency and wheat production across the Great Plains region of the United States. Wheat streak severity has been observed to increase during years of severe drought, possibly due to higher mite populations. Populations of other mite species also have been reported to increase in numbers in drought stressed plants. In the Texas Panhandle, much of the wheat crop is irrigated, but due to depletion of the Ogallala Aquifer less than full irrigation often is applied and little is known about the effect of deficit irrigation on wheat curl mite populations and disease severity. Therefore, field studies were conducted with two cultivars, Karl 92 and TAM 112, at three different irrigation levels. Mite population counts, disease severity and soil moisture content were evaluated for each cultivar and water treatment. Overall, mite populations were found to increase as irrigation decreased $\left(R^{2}=0.5077, P=0.0009\right)$. Also, there was a low but significant positive correlation between disease severity and soil moisture content $\left(R^{2}=0.3307, P=0.0125\right)$. These results show that with deficit irrigation the wheat curl mite population increases, which in turn results in increased disease severity and reduced water use efficiency leaving unused water in the soil.

\section{Seed transmission of Zucchini yellow mosaic virus (a potyvirus) in transgenic wild gourds}

H. E. SIMMONS (1), H. R. Prendeville (2), J. P. Dunham (3), M. J. Ferrari (4), J. D. Earnest (5), D. Pilson (2), G. P. Munkvold (1), E. C. Holmes (5), A. G. Stephenson (5)

(1) Iowa State University, Ames, IA, U.S.A.; (2) University of Nebraska, Lincoln, NE, U.S.A.; (3) University of Southern California, Los Angeles, CA, U.S.A.; (4) Pennsylvania State University, College Park, PA, U.S.A.; (5) Pennsylvania State University, University Park, PA, U.S.A.

Phytopathology 104(Suppl. 3):S3.110

Resistance to Zucchini yellow mosaic virus (ZYMV) has been genetically engineered in Cucurbita pepo, resulting in reduced symptom expression in transgenic plants. ZYMV can be seed-transmitted in C. pepo but there is no prior information about the potential for seed transmission in transgenic, resistant cultivars. We determined if seed to seedling transmission was possible for ZYMV in seeds harvested from inoculated transgenic C. pepo and compared these transmission rates to those of inoculated non-transgenic $C$. pepo. The overall seed to seedling transmission rate of ZYMV $(\sim 1.4 \%)$ was consistent with previous studies. Interestingly, ZYMV seed-to-seedling transmission was not significantly different between the transgenic and nontransgenic squash. Although the transgene reduces viral symptom development, seeds of resistant plants can be infected and can transmit ZYMV to seedlings at a frequency similar to that of susceptible plants, suggesting that this may contribute to the spread of ZYMV via the movement of ZYMVinfected seeds from apparently healthy, resistant plants. Illumina sequencing of the seed-transmitted viral populations was undertaken and results were compared to previously sequenced, geographically separate, populations of ZYMV. Twenty-three percent of the variants found in this study were also found in the other populations, suggesting that these variants may be necessary for seed transmission, or that they may be distributed geographically via seeds.

\section{A survey of plant-parasitic nematodes associated with corn (Zea mays) in Ohio}

A. C. M. SIMON (1), T. L. Niblack (1), P. A. Paul (2)

(1) The Ohio State University, Columbus, OH, U.S.A.; (2) The Ohio State University, Wooster, OH, U.S.A.

Phytopathology 104(Suppl. 3):S3.110

A survey of corn fields in Ohio was conducted in 2013 to determine the frequency and abundance of plant-parasitic nematodes (PPN). A total of 184 soil samples were collected at a depth of $40-50 \mathrm{~cm}$ from 5 soil regions across 12 counties. Samples were collected between the V3-V6 growth stages, with 15-16 fields sampled per county, representing 3 cropping systems (continuous-corn, corn-soybean and corn-soybean-wheat). Standard laboratory techniques were used for processing and extraction, and nematodes were identified to genus. Helicotylenchus and Pratylenchus were found in 92 and $84 \%$ of samples, respectively, followed by Tylenchorhynchus $(63 \%)$, Paratylenchus (55\%), Hoplolaimus (51\%) and Xiphinema (44\%). Averaged across fields, the most abundant nematode genus was Helicotylenchus, with a mean of 69 nematodes $/ 100 \mathrm{~cm}^{3}$ soil. PPNs were more abundant in continuouscorn fields than in fields with corn-soybean rotation. Nine soil textures were recorded, all of which had high abundance of Helicotylenchus, with the exception of sand, which had the lowest abundance. Tylenchorhynchus, Xiphinema and Pratylenchus were most prevalent in loamy-sand and Hoplolaimus in loam soil. The presence of economically important PPN in Ohio corn fields requires more attention by researchers and further work is needed to determine whether nematicides are impacting corn yield and if nematicide seed treatments are warranted in Ohio.

Factors dictating outcomes of competition between two morphotypes of Aspergillus flavus: In vitro versus in vivo observations

P. SINGH (1), P. J. Cotty (2)

(1) University of Arizona, Tucson, AZ, U.S.A.; (2) University of Arizona/ ARS-USDA, Tucson, AZ, U.S.A.

Phytopathology 104(Suppl. 3):S3.110

Aflatoxins are hepatotoxic carcinogens produced by Aspergillus flavus. These mycotoxins contaminate crops in warm regions worldwide. A. flavus has two major morphotypes, the ' $\mathrm{S}$ ' and ' $\mathrm{L}$ ' strains. $\mathrm{S}$ strain isolates produce more consistent high levels of aflatoxins than L strain isolates, which frequently produce no aflatoxins at all and may be used as biological control agents to prevent contamination. Fungi with S strain morphology are the primary causal agents of contamination in certain regions. Differences in distribution between the $\mathrm{S}$ and L strains may reflect adaptations to different agroecosystems and/or niches. The current study sought to determine roles of temperature in competition during colonization, sporulation and contamination of crops with aflatoxins. Levels of aflatoxin produced by S strain isolate AF70 in maize increased with temperature from $20^{\circ} \mathrm{C}$ to $35^{\circ} \mathrm{C}\left(\mathrm{y}=21734 \mathrm{X}-428137, \mathrm{R}^{2}=\right.$ 0.96 ) with $315,727 \mathrm{ppb}$ aflatoxin $\mathrm{B}_{1}$ at $35^{\circ} \mathrm{C}$. No aflatoxin was detected at $40^{\circ} \mathrm{C}$. Highest inhibition of aflatoxin by the biocontrol isolate AF36 occurred at $30^{\circ} \mathrm{C}$. Preliminary results suggest the S strain is most competitive with the $\mathrm{L}$ strain in both growth and sporulation at $40^{\circ} \mathrm{C}$. Pyrosequencing assays quantifying frequencies of $\mathrm{S}$ and $\mathrm{L}$ strain isolates in A. flavus populations were developed and used to dissect competition during maize kernels infection. These assays will be useful for monitoring competition among the morphotypes both during crop production and after harvest.

Linkage disequilibrium-based association mapping of Red rot resistance in sugarcane

R. K. SINGH (1), S. Khan (2), N. Banerjee (2), S. Kumar (2), S. K. Dattamajumder (2)

(1) Indian Institute of Sugarcane Research (ICAR); Presently working at: Plant Genome Mapping Laboratory, University of Georgia, Lucknow, India; (2) Indian Institute of Sugarcane Research, Lucknow, India

Phytopathology 104(Suppl. 3):S3.110

Red rot, caused by the fungus Colletotrichum falcatum, is one of the most important diseases of sugarcane in India and several other south Asian countries. Yield loss due to red rot may go up to 100 percent. Development of red rot resistant varieties is one of the important measures to manage this disease. The high variability of red rot pathogen poses serious challenge in breeding for stable red rot resistant varieties. Association mapping, an emerging alternative approach to conventional linkage mapping, is a method for detecting correlation between genotypes and phenotypes in a sample of individuals on the basis of linkage disequilibrium. In the present study an association panel of 124 genotypes was screened with a virulent race of red rot pathogen for two years. Genotyping was carried out with 100 SSR primer pairs (genomic SSRs and EST-SSRs). The genotypic and phenotypic data have been used for deciphering their linkage disequilibrium based association with red rot resistance. When the association panel was analyzed for population structure using model-based approach, seven genetically distinct groups or admixtures thereof were observed. Further, marker-trait association by mixed linear model (MLM) using TASSEL identified four putative SSR markers associated with red rot resistance in sugarcane. These associated markers after validation can be used in marker aided selection for developing red rot resistant sugarcane varieties.

Control of Radopholus similis in anthurium with spinosad, spirotetramat, and thiophanate-methyl

B. SIPES (1), J. Y. Stephens (1), R. Myers (2), J. Lichty (1), K. Sewake (1) (1) University of Hawaii At Manoa, Honolulu, HI, U.S.A.; (2) USDA ARS, Hilo, HI, U.S.A.

Phytopathology 104(Suppl. 3):S3.110

Radopholus similis is an endoparasitic migratory nematode that causes anthurium decline. Our objective was to evaluate the efficacy of spinosad, spirotetramat, and thiophanate-methyl for the control of $R$. similis in anthurium. Plants were weighed, transplanted into $15-\mathrm{cm}-\mathrm{d}$ clay pots filled with cinders and inoculated with 3000 R. similis. Six months later, treatments of $4.8 \mathrm{mg}$ spinosad, $4.8 \mathrm{mg}$ spirotetramat, $0.01 \mathrm{~g}$ thiophanate-methyl, or nothing were applied to the leaves in $50 \mathrm{ml}$ water. Two months later, leaf number and leaf area of the youngest mature leaf were recorded. The average number of leaves on uninoculated plants treated with spinosad, spirotetramat, thiophanate-methyl, or water was $9,10,9$, and 10 , respectively. The average number of leaves on inoculated plants treated with spinosad, spirotetramat, 
thiophanate-metyl, or water was $11,9,11$, and 9 , respectively. The average leaf area for uninoculated plants treated with spinosad, spirotetramat, thiophanate-methyl, or water was 90.6, 101.2, 96.9, and $77.5 \mathrm{~cm}^{2}$, respectively, On inoculated plants treated with spinosad, spirotetramat, thiophanate-methyl, or water leaf area was $91.7,84.5,76.2$, and $92.5 \mathrm{~cm}^{2}$, respectively. Anthurium treated with spinosad, thiophanate-methyl, spirotetramat, or water increased weight 17, 16, 17, and 14 fold whereas those inoculated with nematodes increased 16, 14, 14, and 15 fold, respectively. Spinosad controls anthurium decline and maybe a treatment option for growers.

\section{Framework to align plant pathology curricula and training to} phytopathology workforce needs

S. N. SIVAKUMAR (1)

(1) Michigan State University, East Lansing, MI, U.S.A

Phytopathology 104(Suppl. 3):S3.111

Aligning workforce expectations with educational curricula and training can be achieved by performing evidence-based research on academic pedagogy and by analyzing workforce needs and expectations by hiring authorities. This study will focus to test how plant pathology curricula is aligned to workforce needs, by first identifying what knowledge, training and skills do hiring authorities expect from plant pathology workforce. Job listings pertaining to plant pathology will be analyzed to obtain information about required qualifications and skills and this information will be complemented by collecting data through surveys and by interviewing hiring authorities. Knowledge and skills that are defined by hiring authorities will be transformed into plant pathology lexis using expertise in plant pathology. Current curricula in plant pathology at higher institutions will be analyzed by the investigation team to identify and create an inventory of knowledge and skills provided by teaching plant pathology courses. This would be subdivided further into plant pathology-specific knowledge and skills and other general skills such as problem solving, critical thinking, and communication \& presentation skills. Once a clear understanding about workforce needs in plant pathology is established and have generated a comprehensive list of skills, one could examine and report how curricula, teaching and training programs in plant pathology are aligned to workforce needs.

Microbiological examination of exopolysaccharide ooze produced by Erwinia amylovora

S. SLACK (1), G. W. Sundin (1)

(1) Michigan State University, East Lansing, MI, U.S.A.

Phytopathology 104(Suppl. 3):S3.111

Fire blight, caused by Erwinia amylovora, is the most damaging bacterial disease of pome fruit trees. The main dispersal method of E. amylovora is through ooze, a mass of bacterial cells and exopolysaccharide that is exuded from infected tissue. In this study, we characterized E. amylovora populations associated with ooze droplets from field-infected trees and used scanning electron and confocal laser-scanning microscopy techniques to analyze the extrusion process of ooze to the plant surface. We examined 201 ooze droplets emerging from inoculated shoots of apple (cv 'Jonathan'); these droplets averaged 2.7 microliters in volume and harbored an average of $7.7 \times 10^{11}$ and a median size of $7.2 \times 10^{10}$ E. amylovora cells per microliter. The color of ooze drops ranged from white to dark red, with white drops containing slightly smaller E. amylovora populations than colored ooze drops. The size of E. amylovora populations was also assessed from one-centimeter stem sections on either size of an ooze drop. Microscopic examination of apple stem tissue at sites of ooze extrusion revealed that bacteria were not escaping the plant through natural openings. Instead, erumpent mounds, indicative of internal pressure buildup, were visualized suggesting that stem wounds exuding ooze were initiated by E. amylovora cells. Through these studies, the base-knowledge of this dispersal method is expanding, which could have implementations in future disease control methods.

\section{Expressed bioactivity in endosymbionts of Prunus virginiana}

\section{T. B. SMART (1)}

(1) Brigham Young University, Provo, UT, U.S.A.

Phytopathology 104(Suppl. 3):S3.111

Virtually every plant maintains populations of endosymbionts. These endosymbionts commonly produce secondary metabolites possessing antibacterial, antifungal and anticancer properties. These characteristics are more prevalent in poisonous plants. In Prunus virginiana, commonly named chokecherry, plant metabolites exhibit pesticidal traits in both root and stem. Likewise, plant metabolites are poisonous to large range feeding mammals. Despite these indicators, the endosymbionts of $P$. virginiana have been previously overlooked. In this study, antifungal and antibacterial assays were performed to assess the bioactivity of fungal endosymbionts of $P$. virginiana.
To conduct bioassays, $6 \mathrm{~mm}$ mycelial plugs were placed 3.5 centimeters apart on a $6 \mathrm{~cm}$ diameter petri dish. Bioactivity was assessed by measuring inhibition zones at intervals of 24, 48, 72 and 96 hours. Those with successful inhibition were cultured in 6 liters of 12 grams/L potato dextrose broth. After one week of growth, the fungus underwent liquid-liquid extraction. Assays using the extract confirmed inhibition. Chemicals were further separated using normal-phase column chromatography. Controls included Maxim 4FS, Subdue Maxx (FV), as well as $20 \mu \mathrm{L}$ of pure $\mathrm{MeOH}$. Results indicate that several endosymbionts of $P$. virginiana express inhibition against pathogenic fungi including sclerotinia, fusarium and botrytis. This expressed antifungal activity indicates a possible application in agriculture.

To the brink of extinction: Disease, population decline and conservation efforts of the Florida torreya (Torreya taxifolia)

J. SMITH (1), T. Spector (2), R. Determann (3), J. Cruse-Sanders (3), R. Pruner (4), M. Friel (4), K. O'Donnell (5)

(1) University of Florida, Gainesville, FL, U.S.A.; (2) US Fish and Wildlife Service, West Valley City, UT, U.S.A.; (3) Atlanta Botanical Garden, Atlanta, GA, U.S.A.; (4) Florida Parks Service, Panama City, FL, U.S.A.; (5) NCAUR-ARS-USDA, Peoria, IL, U.S.A.

Phytopathology 104(Suppl. 3):S3.111

Florida torreya, Torreya taxifolia, has experienced a dramatic and precipitous population decline in its native habitat in ravines along the Apalachicola River. Recent studies have demonstrated that a novel fungal pathogen, Fusarium torreyae, is causing a severe canker and dieback that is decimating the remaining extant individuals. The status and condition of the current wild population has recently been evaluated and in addition to very high disease incidence, the average stem lengths are declining, few seed bearing trees were observed and the population is estimated at less than 1500 individuals. Efforts are underway to better determine methods to improve health of the population in situ, propagate disease-free trees and expand an ex situ germplasm conservation program at the Atlanta Botanical Garden. In addition, studies to assess genetic diversity within the population are underway. Recommendations for future in situ and ex situ conservation strategies for this endangered conifer will be discussed.

Effect of foliar fungicides on yield, quality, and disease of alfalfa for dairy production in Wisconsin

D. SMITH (1), P. D. Esker (2), B. Jensen (1), B. Halfman (3), G. Blonde (4)

(1) University of Wisconsin, Madison, WI, U.S.A.; (2) University of Costa Rica, San Jose, Costa Rica; (3) Monroe County - UW Extension, Sparta, WI, U.S.A.; (4) Waupaca County UW-Extension, Waupaca, WI, U.S.A.

Phytopathology 104(Suppl. 3):S3.111

Alfalfa (Medicago sativa) is an important feed crop for dairy cows in Wisconsin. Recently use of fungicides on alfalfa has increased in an effort to reduce disease, improve quality, and increase yields. Field trials were conducted during the 2011-2013 field seasons at multiple locations and on 23 cuttings per location. A total of 26 separate field trials (years $\times$ sites $\times$ cuttings) were sprayed with fungicides or not sprayed. Disease was assessed in plots were pathogen activity was observed. Plots were harvested using a small-plot forage harvester and dry matter yields were determined. Quality parameters were also assessed. Only 6 trials (23\%) resulted in significant $(P<0.05)$ yield differences among treated and non-treated plots. Average yield increase in these trials was $538 \mathrm{~kg} / \mathrm{ha}$. Only 8 trials $(31 \%)$ resulted in significant $(P<0.05)$ quality differences between treated and non-treated plots. In most cases no differences in treated vs. non-treated plots were observed with few difference in disease control. Across all 26 trials, the average yield increase was just $201 \mathrm{~kg} / \mathrm{ha}$ when fungicide was used. This yield increase did not cover the cost of applying fungicide in these trials.

Phytophthora root rot mortality of container-grown rabbiteye and southern highbush blueberry plants

B. J. SMITH (1), M. Miller-Butler (1), K. J. Curry (2)

(1) USDA ARS Thad Cochran Southern Horticultural Lab, Poplarville, MS, U.S.A.; (2) Dept. Biological Sciences, University of Southern Mississippi, Hattiesburg, MS, U.S.A.

Phytopathology 104(Suppl. 3):S3.111

Two studies evaluated the effect of Phytophthora cinnamomi Rands isolates and inoculum delivery methods on mortality of blueberry plants grown in a pine bark:sand:peat medium in 2.5 liter pots. P. cinnamomi isolates were obtained from the root zone of blueberry plants with symptoms of Phytophthora root rot. In the first study, rabbiteye cultivar Tifblue and southern highbush cultivar Biloxi blueberry plants were grown in medium infested with five isolates of $P$. cinnamomi. Inoculum was prepared as a mycelial slurry or grown on rice grains or vermiculite. After two years, $3 \%$ of the Tifblue and $80 \%$ of the Biloxi plants were dead. There was no significant 
difference in plant mortality due to method of inoculum delivery. In the second study plants of southern highbush cultivars, Biloxi and Star, were grown in medium infested with four $P$. cinnamomi isolates. Inoculum was prepared as a mycelial slurry, zoospore suspension, or grown on rice grains. Plants were subjected to a 48 hour flood treatment every six weeks. After five months, $31 \%$ of 90 Biloxi and $2 \%$ of 90 Star plants were dead; $31 \%$ of 50 plants grown in media infested with $P$. cinnamomi grown on rice were dead, but only $12 \%$ of plants grown in media infested with $P$. cinnamomi mycelium or zoospores were dead. Results of these trials will be used to develop procedures for evaluating chemical and biological root rot controls and studying environmental effects on the development of Phytophthora root rot on blueberries.

Identification of Fusarium oxysporum f. sp. vasinfectum races present in Alabama cotton fields

A. SMITH (1), K. Lawrence (1), J. Hu (1)

(1) Auburn University, Auburn, AL, U.S.A.

Phytopathology 104(Suppl. 3):S3.112

Fusarium oxysporum f. sp. vasinfectum (FOV) is the causal agent of Fusarium wilt in upland cotton (Gossypium hirsutum) in Alabama and throughout the world. The objective was to identify FOV races present in the 2013 season. Symptomatic plants were collected from the Commercial Cotton Variety Trial located at E.V. Smith Research Center near Tallassee, Alabama. The fungus was isolated from each infected plant onto half-strength acidified potato dextrose agar (APDA). Plugs from each isolate were transferred to $10 \mathrm{ml}$ of potato dextrose broth (PDB) and grown for 7 days. Grinding was used to extract DNA from the mycelium using a DNA extraction kit. PCR products were amplified and sent for sequencing. Four pairs of primers were used: elongation factor (EF), beta-tubulin (BT), phosphate permease (PHO), and nuclear intergenic spacer region (IGS). 71 percent of all samples isolated tested positive for FOV. Races present in Alabama were 1, 2, 3, and 8. Race 4 was also present, and was genetically similar to the extremely pathogenic race 4 from California when using elongation factor DNA; however, the race 4 in Alabama is different from the CA race 4 based on pathogenicity tests and nuclear IGS DNA. LA isolates 112, 108, 140, and 127 were previously identified as new pathogenic races to the southeastern United States. Only LA 112 has been found in Alabama to date.

A feedback regulatory loop between G3P, DIR1 and AZI1 mediates azelaic-acid-induced systemic immunity

J. M. SOARES (1), K. Yu (1), M. K. Mandal (1), A. Kachroo (1), P. Kachroo (1)

(1) University of Kentucky, Lexington, KY, U.S.A.

Phytopathology 104(Suppl. 3):S3.112

Systemic acquired resistance (SAR) is a form of defense response that provides broad-spectrum immunity against diverse pathogens. A number of chemical signals and proteins contributing to SAR have been identified and characterized. We show that SAR inducer dicarboxylic acid, azelaic acid (AzA) is derived from unsaturated $\mathrm{C} 18$ fatty acids (FA) that contain a double bond on carbon 9. Furthermore, AzA serves upstream of the mobile SAR inducer glycerol-3-phosphate (G3P), since AzA induced biosynthesis of G3P and was unable to confer SAR in mutants that are impaired in G3P biosynthesis. The lipid transfer-like proteins, DIR1 and AZI1, both of which are required for G3P- and AzA-induced SAR, were also essential for G3P accumulation. Conversely, reduced G3P led to transcriptional instability of AZI and DIR1 genes. Our results demonstrate that an intricate feed-back regulatory loop between G3P, DIR1, and AZI1 regulates SAR and that AzA and $\mathrm{C} 18$ unsaturated FAs functions upstream of G3P in this pathway.

Chromobacterium vaccinii as a potential biocontrol agent

S. SOBY (1), A. Fetz (1), A. Holmberg (1)

(1) Midwestern University, Glendale, AZ, U.S.A.

Phytopathology 104(Suppl. 3):S3.112

Chromobacterium vaccinii (Neisseriaceae), a newly described species of betaproteobacteria isolated from wild and cultivated cranberry bogs and irrigation ponds in eastern Massachusetts, was evaluated for its activity against nematodes and insect larvae, and for its ability to produce biologically active compounds against common agricultural pests in the cranberry ecosystem, including filamentous fungi and the oomycete Phytophthora cinnamomi. C. vaccinii produces the pigments violacein and deoxyviolacein, as well as cyanide and several siderophores. Violacein and deoxyviolacein were active against the soil organisms Thanatophytum and $P$. cinnamomi, but not against the cranberry fruit-rot pathogens Fusicoccum, Phomopsis, Colletotrichum or Coleophoma. Despite their production by Chromobacterium, which is normally found in soils and limnic environments, violacein and deoxyviolacein have not previously been tested for toxicity against plant tissues. We have found that violacein and deoxyviolacein are not toxic to roots, root border cells or germinating seeds, indicating that native strains of C. vaccinii producing violacein and deoxyviolacein have potential as biocontrol agents.

\section{Phytophagous hemipterans as vectors of Salmonella enterica}

J. P. SOTO-ARIAS (1), R. L. Groves (1), J. D. Barak (1)

(1) University of Wisconsin-Madison, Madison, WI, U.S.A.

Phytopathology 104(Suppl. 3):S3.112

Contamination of fresh produce with Salmonella enterica most likely occurs pre-harvest. Despite the general perception of synanthropic insects as vectors of foodborne pathogens, the possibility that phytophagous insects could be vectors of $S$. enterica remains untested. Here, two commonly occurring hemipteran insect pests (Macrosteles quadrilineatus and Myzus persicae) were fed on $S$. enterica inoculated lettuce leaf discs or artificial liquid diets confined in parafilm sachets for a $24 \mathrm{~h}$ acquisition access period (AAP). Individual insects were then transferred to two, consecutive non-inoculated leaf discs or liquid diets for a $48 \mathrm{~h}$ post-AAP, and internalization, excretion, and transmission of the pathogen were evaluated. Ingested S. enterica was detected within the body of $M$. quadrilineatus and $M$. persicae upon feeding on an inoculated diet. Likewise, S. enterica was detected in honeydew excreted by both species, but recovered from a higher proportion of honeydew samples from M. quadrilineatus at $48 \mathrm{~h}$ post-AAP. Additionally, both species were able to transmit $S$. enterica to non-inoculated leaf discs and liquid diets, demonstrating that transmission of the pathogen might not be limited to physical passage. Overall, these results suggest that both internally and externally contaminated insects may increase the risk of dispersal of the pathogen within or among plants.

A novel viral satellite from cassava plants showing Cassava frogskin disease symptoms

A. N. SOUZA (1), F. N. Silva (1), C. M. Carvalho (1)

(1) Universidade Federal de Viçosa, Viçosa, Brazil

Phytopathology 104(Suppl. 3):S3.112

Cassava Frogskin Disease (CFSD) is an important disease affecting the production of cassava (Manihot esculenta) due to the presence of severe symptoms on tubers. Different organisms have been found associated with cassava plants showing CFSD. A phytoplasma belonging to a 16SrIII-A subgroup and a probable reovirus were found in plants showing CFSD in Minas Gerais, Brazil. To investigate the presence of some other RNA virus in these plants, dsRNA was extracted from tubers showing CFSD symptoms and the dsRNA was sequenced by 454 GS-FLX platform. Reads were assembled using the software Geneious and contigs were analyzed using Blastn, Blastp, ORF Finder and CDD. The presence of a previously unknown sequence was identified in a contig of 946nt in length, derived from 10 reads. This contig was found to have two potential open reading frames (ORFs), one encoding a structural protein, and another encoding a putative protein. These two ORFs were most similar to ORF 1 and 2 of Grapevine satellite virus (NC_021480.1), showing $87 \%$ and $96 \%$ of coverage and $60 \%$ and $38 \%$ of amino acid identity, respectively. Analysis of conserved domains has shown that the structural protein belongs to a Potex-coat superfamily, a superfamily that consists of several Potexvirus coat proteins. According to our analysis, this contig is probably a novel viral satellite from cassava plants.

Quantifying grain losses caused by leaf anthracnose in different sorghum genotypes

A. G. SOUZA (1), L. V. Cota (1), D. D. Silva (1), F. E. Lanza (1), R. V. Costa (1), L. A. Maffia (2)

(1) EMBRAPA CNPMS, Sete Lagoas, Brazil; (2) Univ Federal de Viçosa, Vicosa, Brazil

Phytopathology 104(Suppl. 3):S3.112

This study aimed to quantify the effect of sorghum anthracnose (Colletotrichum sublineolum) on grain yield of sorghum plants. The field experiments were carried out with sorghum lines (BR009 and BR008) and hybrids (BR304, BR308, BR310, AG1060, DKB599, and MR43). The experiment was carried out in the EMBRAPA experimental area located in Sete Lagoas-MG, Brazil, during the years 2009 to 2011. In all experiments, the disease was assessed weekly from flowering to harvest by using a severity scale range from $1(0 \%)$ to $9(>75 \%)$. The regression analysis was used to estimate the damage caused by anthracnose on sorghum yield. The best variable to infer about the disease damage on the yield was the severity on 100 days after plating. The anthracnose severity affected the sorghum yield and the disease intensity ranged depending of the sorghum genotype. The yield on AG1060 and BRS308 hybrids were not affected by leaf anthracnose. Other hand the BR009 showed to be highly susceptible to the damage caused by disease. The biggest production reduction was observed on the BR009 line 
(losses up to $86 \%$ of yield). In the BR008 and BR310 genotypes were observed production losses values up to $72 \%$ and $35 \%$, respectively. Therefore, we can conclude that losses caused by C. sublineolum on sorghum production is dependent of the sorghum genotype and that our results will be useful to the Integrated disease Management program.

\section{New insights into the systematics of Pythium Pringsheim}

C. F. SPIES (1), A. W. de Cock (2), S. L. Glockling (3), C. Y. Chen (4), C. A. Lévesque (1)

(1) Agriculture and Agri-Food Canada, Ottawa, ON, Canada; (2) Centraalbureau voor Schimmelcultures, Utrecht, Netherlands; (3) Eastbourne, United Kingdom; (4) National Chung Hsing University, Taichung, Taiwan Phytopathology 104(Suppl. 3):S3.113

The genus Pythium has been increasingly under taxonomic scrutiny since the first sequence based phylogenies of the genus became available 15-20 years ago. Some of the taxonomic milestones reached in this period include the phylogenetic division of the genus into 11 clades (A-K) that grouped together based on sporangial morphology, the establishment of the new genus Phytopythium to include all species of clade $\mathrm{K}$, and the division of the remainder of the genus into four distinct genera: Pythium (clades A-D), Elongisporangium (clade H), Globisporangium (clades E-G, I and J), and Pilasporangium (not in any of the previously identified 11 clades). While this division of Pythium Pringsheim is generally supported by phylogenies of commonly used markers such as ITS, 28S, Cox2, and beta-tubulin, support for some of the important nodes are lacking and Pythium-related taxa such as Pythiogeton and Lagena are not accounted for in the phylogenies used for the division. Inclusion of such taxa in a large-scale phylogeny of concatenated 18S-ITS-28S, beta-tubulin, and Cox2 sequences suggest that paraphyly persists in the revised genus Pythium, but support for important nodes remain elusive. Addressing the problem with novel phylogenetic markers selected from genome sequences validates most of the genera previously proposed and clarifies the relationships among these genera as well as Pythiogeton, Lagena, and Pythium-associated strains of Lagenidium.

\section{Spatiotemporal distribution of Thielaviopsis basicola after a ten year} cotton monoculture

T. N. SPURLOCK (1), A. M. Greer (1), A. C. Tolbert (1), T. L. Kirkpatrick (2), C. S. Rothrock (3), S. Monfort (4)

(1) University of Arkansas Division of Agriculture, Monticello, AR, U.S.A.; (2) University of Arkansas Division of Agriculture, Hope, AR, U.S.A.; (3) University of Arkansas, Fayetteville, AR, U.S.A.; (4) Clemson University, Blackville, SC, U.S.A.

Phytopathology 104(Suppl. 3):S3.113

Thielaviopsis basicola causes black root rot of cotton, Gossypium hirsutum. When cool and wet soil temperatures exist $\left(<26^{\circ} \mathrm{C}\right)$, black root rot results in stunting of growth and reduced vigor that can negatively impact yield. In 2001, T. basicola was quantified at planting and harvest for two consecutive years and at planting in 2003 in 512 contiguous plots in a texturally diverse cotton field near Portland, AR, USA. The field was planted in cotton for ten consecutive years before being planted in soybean in 2013. In fall 2013, T. basicola was quantified again. The objective was to determine the spatial distribution of T. basicola and if these distributions had changed over time or with crop. Data were subjected to exploratory spatial analysis and then rigorous spatial regression to determine the distribution and correlations for each sampling time. For each year sampled, the distributions of $T$. basicola were aggregated. In all years, a significant negative correlation existed between $T$. basicola and percent sand fraction and a significant positive correlation existed between the fungus and percent silt. Areas corresponding to the highest clay content also had the highest $T$. basicola populations from 2001 and $2013(P<0.0001)$. Over the time of the study, the spatial distribution of $T$. basicola was relatively stable and dependent on soil texture.

\section{An alternative route for bacterial internalization in tomato fruit}

S. L. Stahl (1), J. A. BARTZ (1), D. J. Huber (1), D. Spiceland (1), J. H. Lee (1), M. T. Elkahky (1)

(1) University of Florida, Gainesville, FL, U.S.A.

Phytopathology 104(Suppl. 3):S3.113

Tomato fruit have a waxy, gas-impermeable cuticle that develops well prior to harvest. Routes for gas exchange required by normal fruit development and ripening are generally attributed to the stem attachment area. Gas egress and dye infiltration experiments reveal one to six putative lenticels located in the area normally covered by the calyx. Egress is vigorous even with relatively minor pressure differentials. These structures are found near the rim of the stem attachment and over pericarp-endocarp junctions. As such, direct impact of water droplets from rainfall, overhead irrigation or spray application could force external water into tissues. Additionally, internal vacuums created as fruit are cooled by rainfall, irrigation or air temperature change could draw external water into tissues. Tomato fruit internal free-space is most prevalent in the shoulder area of fruit and extends to placental tissues holding developing seeds. Bacterial or yeast cell-suspensions flooding these apertures can be physically forced into fruit as previously shown by postharvest decay studies. This route of microbial internalization has important implications concerning the siting of tomato fields and sourcing of irrigation or spray make-up water. Dusts and aerosols from nearby confined animal operations or use of contaminated surface water for irrigation or spray applications can lead to tomato fruit internalization of plant as well as human pathogens.

Investigation into the mechanism of resistance to azoxystrobin in Cercospora sojina, the causal agent of frogeye leaf spot

J. STANDISH (1), M. Tomaso-Peterson (1), T. W. Allen (2), S. Sabanadzovic (1), N. Aboughanem-Sabanadzovic (3)

(1) Mississippi State University, Mississippi State, MS, U.S.A.; (2) Delta Research and Extension Center, Stoneville, MS, U.S.A.; (3) Institute for Genomics, Biocomputing \& Biotechnology, Mississippi State University, Mississippi State, MS, U.S.A.

Phytopathology 104(Suppl. 3):S3.113

Frogeye leaf spot (FLS) is a foliar disease of soybean caused by Cercospora sojina Hara. Circular to angular lesions develop and may coalesce to form larger, irregular spots. When lesions cover more than $30 \%$ of the leaf surface blighting occurs leading to potential yield loss. Quinone outside inhibitor (QoI) fungicides are often used to manage FLS, but resistance to this class of fungicides has been documented in various phytopathogens. Resistance to QoIs is generally associated with the presence of amino acid substitutions within the cytochrome $\mathrm{b}($ cyt $b$ ) gene. A glycine to alanine substitution at position $143(\mathrm{G} 143 \mathrm{~A})$ is known to confer complete resistance to QoI fungicides. Two other notable substitutions that confer moderate resistance are phenylalanine to leucine at position 129 (F129L) and glycine to arginine at position 137 (G137R). The objective of this study was to evaluate C. sojina isolates collected from Mississippi soybean fields for resistance to QoIs by identifying point mutations within $c y t b$. Genomic DNA was extracted from C. sojina isolates collected during the 2013 growing season as well as from a baseline isolate collected prior to the release of QoI fungicides. A 238-bp fragment of the cyt $b$ gene was amplified using $C$. sojina-specific primers that were developed in our laboratory. Nucleotide sequences of $C$. sojina isolates from Mississippi were compared to a known sensitive baseline isolate to identify potential point mutations.

Developing inoculation methods for screening wheat for reaction to Xanthomonas translucens pv. undulosa (Bacterial Leaf Streak)

J. L. STANTON (1), R. D. Curland (1), C. A. Ishimaru (1), M. J. Smith (1), R. Dill-Macky (1)

(1) University of Minnesota, St. Paul, MN, U.S.A.

Phytopathology 104(Suppl. 3):S3.113

This study aimed to develop effective methods to screen wheat to bacterial leaf streak (BLS). Inoculum of $X$. translucens pv. undulosa $\left(1 \times 10^{7} \mathrm{cfu}^{-\mathrm{ml}^{-1}}\right)$ was applied to the spring wheats Blade, Knudson and RB07 in three field experiments. The inoculation methods examined included $\mathrm{CO}_{2}$ - and gaspowered (Solo) sprayers, timing of inoculation, and the use of carborundum. Disease development was assessed 15 and 9 days after the early and late inoculations, respectively. Assessments included whole plot score (1-9 scale), flag leaf infected (\%; 10 plants/plot), and plant score (1-9 scale; 10 plants/plot). Disease severities ranged from 5 to $70 \%$ across experiments, inoculum treatments and cultivars. Of the cultivars tested, RB07 was most susceptible and Blade most resistant: results being consistent across inoculation methods. Disease was greatest in plants inoculated twice rather than once. Differences in disease ratings between resistant and susceptible hosts were consistent across the irrigated and dryland experiments. Carborundum improved inoculation effectiveness in many, but not all treatments. Both types of sprayers were effective in delivering inoculum and generating measurable levels of BLS, however the Solo sprayer was better suited to covering large field areas. Based on these findings, we concluded that inoculum prepared with carborundum and applied using a Solo sprayer provides an effective method for inoculating field nurseries for screening germplasm to BLS.

Using geostatistics to study soil borne pathogens in Michigan potato fields L. STEERE (1), N. Rosenzweig (1), W. W. Kirk (1), K. Baker (2)

(1) Michigan State University, East Lansing, MI, U.S.A.; (2) Western Michigan University, Kalamazoo, MI, U.S.A.

Phytopathology 104(Suppl. 3):S3.113

In 2012, growers and university researchers came together to address the issue of declining yields and decreased tuber quality in Michigan potato (Solanum 
tuberosum) production. The goals of the research were: 1. To better understand soil-borne pathogen inoculum levels in potato fields; 2 . To better understand the soil biology and quantify soil microbial diversity and 3. To make correlations between yield and soil biological factors. In addition to interactions among soil properties and microbial diversity, the research team incorporated the use of geostatistics to create predictive maps of diversity, soil pathogen populations and yield of entire fields from the sample points. Twenty soil sample points were collected from 26 fields prior to potato planting in 2013 and each sample point was assessed for soil characteristics, Verticillium dahliae colony forming units, and soil microbial diversity. At harvest, yield was measured at each location and tubers were evaluated for incidence and severity of potato common scab (Streptomyces scabies). Each field was assessed for spatial continuity and variability of the sample points using geostatistical parameters. Information was then interpolated using various mapping methods and statistical correlations were made. The procedures and methods developed during this study will become a useful tool for understanding microbial interactions as well as visualizing pathogen levels as part of an integrated pest management system.

Gene clusters $F D B 1$ and $F D B 2$ in Fusarium verticillioides were acquired through multiple horizontal gene transfer events

J. E. Stewart (1), Z. Abdo (2), A. E. GLENN (3)

(1) University of Georgia, Department of Plant Pathology, Athens, GA, U.S.A.; (2) USDA, ARS, South Atlantic Area, Athens, GA, U.S.A.; (3) USDA, ARS, Toxicology \& Mycotoxin Res. Unit, Athens, GA, U.S.A. Phytopathology 104(Suppl. 3):S3.114

The corn pathogen Fusarium verticillioides is of significant importance because of its deleterious effects on plant and animal health and on the quality of their products due to mycotoxin contamination. The fungus is known to metabolize antimicrobial compounds produced by corn using genes within two gene clusters, $F D B 1$ and $F D B 2$. Preliminary phylogenetic and genomic synteny data show that the two clusters share an evolutionary history, though not driven by gene duplication and divergence, but rather through horizontal gene transfer (HGT) events between known corn pathogens. We hypothesize that host preference and associated exposure to phytochemicals are driving factors in the evolution of these genomes. Results suggest that $F$. verticillioides acquired the FDB1 cluster via HGT from Colletotrichum graminicola with near perfect synteny conservation. A separate HGT event is postulated whereby Aspergillus kawachii (or its progenitor) also acquired the $F D B 1$ cluster from $C$. graminicola but with gene rearrangements. Phylogenetic and synteny data also support that the A. kawachii gene cluster was transferred to $F$. verticillioides to constitute the $F D B 2$ cluster. Thus, the data suggest that $F$. verticillioides acquired both clusters from two different fungi by HGT, that the $F D B 2$ genes are themselves derived in part from the $F D B 1$ genes via an earlier, intermediary HGT event involving Aspergillus, and that $C$. graminicola is the progenitor of all the common FDB1 and FDB2 genes.

Incidence and impact of dual infection by Panicum mosaic virus and its satellite virus in switchgrass breeding fields

C. L. Stewart (1), C. Jochum (1), G. Y. YUEN (1), K. Vogel (2), J. D. Pyle (3), K. B. G. Scholthof (3)

(1) University of Nebraska, Lincoln, NE, U.S.A.; (2) USDA-ARS (retired), Lincoln, NE, U.S.A.; (3) Texas A\&M University, College Station, TX, U.S.A. Phytopathology 104(Suppl. 3):S3.114

Panicum mosaic virus (PMV) and satellite panicum mosaic virus (SPMV) were detected in field-grown switchgrass (Panicum virgatum) in Nebraska in 2012. Mixed infection by PMV+SPMV in other grasses heightens symptom expression over PMV alone. This study aimed to assess PMV- or PMV+SPMV-infection incidence and associated symptoms in biofuel switchgrass breeding fields in Nebraska. PMV and SPMV were detected by ELISA and RT-PCR, respectively, in leaf samples from randomly selected plants. Symptom severity was assessed on these plants using a 1 to 5 scale (1 $=$ no symptoms; $5=$ plants stunted and $>50 \%$ foliage with mottling). PMVinfection incidence varied widely among fields and switchgrass populations within fields, e.g. 11\% and 77\% PMV infection occurred in 'Kanlow' and 'Summer', respectively, in one field. Common among sampled populations was dual infection by PMV and its satellite virus. Plants infected with PMV alone exhibited none to moderately severe symptoms (1 to 3 rating). There also were many PMV+SPMV-infected plants that exhibited none to mild symptoms, although other plants infected with both viruses displayed severe symptoms (ratings of 4 or 5). These results suggest that while SPMV might enhance PMV transmission and establishment in switchgrass, it heightens symptom expression in a host-dependent manner. Furthermore, resistance to PMV within current switchgrass populations may be useful in developing more productive biofuel cultivars.
Vernalization affects the reservoir capacity for perennial hosts of Plum pox virus

A. Stone (1), W. SCHNEIDER (1)

(1) USDA ARS, Fort Detrick, MD, U.S.A.

Phytopathology 104(Suppl. 3):S3.114

Many plant viruses use perennial plants as a reservoir for overwintering. Despite this, very little is known about the effects of vernalization (cold induced dormancy) on plant viruses. Plum pox virus (PPV) is a serious pathogen of Prunus worldwide, with a broad experimental host range. The experimental host range of Pennsylvania PPV isolates has many wild Prunus species, including black cherry (BC), chokecherry (CC) and American Plum (AP). However, the ability to support immediate viral replication and movement does not necessarily correlate with being a good reservoir. Continued persistence and the capacity to move back into the original host (peach) are critical. $\mathrm{BC}, \mathrm{CC}$ and $\mathrm{AP}$ were tested for reservoir capacity for two Pennsylvanian PPV isolates. PPV was capable of infecting all three species. In $\mathrm{BC}$ the virus persisted through vernalization cycles, but back transmission to the original host was inconsistent and correlated with the presence of symptoms. In CC the virus did not persist prior to or after vernalization, and back transmissions were unsuccessful. In AP the initial infection did not persist prior to vernalization, nor could the virus be transmitted from these trees. Interestingly, after the first vernalization, PPV was detectable in AP, and was consistently transmitted back to peaches. This suggests that vernalization affects the viral population in ways that affect infection and transmission phenotypes.

Effect of anaerobic soil disinfestation and vermicompost on soilborne phytopathogenic agents under tree-crop nursery conditions

S. STRAUSS (1), D. Kluepfel (2), G. Browne (1)

(1) USDA ARS, Davis, CA, U.S.A.; (2) USDA ARS CPGRU, Davis, CA, U.S.A.

Phytopathology 104(Suppl. 3):S3.114

Anaerobic soil disinfestation (ASD) is a fumigation-independent management strategy for controlling soilborne pathogens. Walnut nurseries currently employ preplant fumigation to control soilborne phytopathogens and weeds, and may be amenable to use ASD instead. We investigated the potential of ASD and post-ASD vermicompost applications to manage seed-borne populations of Agrobacterium tumefaciens and other soilborne phytopathogens in walnut nursery conditions. Rice bran at 20 metric tons/ha was applied to ASD plots, irrigated for $24 \mathrm{~h}(13 \mathrm{~cm}$ of water, 1 drip emitter per $930 \mathrm{~cm}^{2}$ ), and covered by TIF for 6 weeks. Mesh bags of sterile soil inoculated with either A. tumefaciens or Pythium ultimum were buried prior to ASD treatments. Anaerobic conditions were reached at 18 " depth within 1 week and maintained for 6 weeks. A. tumefaciens and P. ultimum populations were reduced below detection limits during ASD as determined by dilution plating. Next-generation sequencing of DNA extracted from soil revealed significant differences in the soil microbial community after ASD. To examine effects of ASD efficacy and post-ASD vermicompost application on disease incidence, Paradox walnut seeds were dipped in A. tumefaciens inoculum prior to planting. The abundance of $A$. tumefaciens on walnut seeds planted in ASD and control plots with vermicompost was significantly less than plots without vermicompost.

Multiplex qPCR assay for detecting the four causal agents of bacterial spot of tomato

A. STRAYER (1), M. L. Paret (2), J. B. Jones (1), A. Jeyaprakash (3)

(1) Department of Plant Pathology, University of Florida, Gainesville, FL, U.S.A.; (2) NFREC, University of Florida, Quincy, FL, U.S.A.; (3) Division of Plant Industry, Florida Department of Agriculture \& Consumer Services, Gainesville, FL, U.S.A.

Phytopathology 104(Suppl. 3):S3.114

Bacterial spot is one of the most detrimental diseases of tomato that occurs worldwide. It is caused by four species; Xanthomonas euvesicatoria (Xe), X. vesicatoria $(\mathrm{Xv}), X$. perforans $(\mathrm{Xp})$, and $X$. gardneri $(\mathrm{Xg})$. Current identification methods can take several days to determine which species is present in a sample. Previously, the $h r p B$ region was shown to be useful in differentiating the four species using conventional PCR. A 420bp fragment of the $h r p B$ was amplified from a total of 75 strains representing the four species using PCR. The PCR products were sequenced and aligned. The alignments and phylogenetic analysis revealed that the $h r p B 2$ is highly conserved amongst the species, but a SNP exists amongst the Xv strains. Xe and Xp only varied by 2 SNPs. Four species-specific and two group-specific Xv probes were created based on the $h r p B 2$ sequences. We designed a multiplex qPCR to specifically detect each of the four bacterial spot pathogens. The four species-specific probes and their two primer sets were then optimized together for the $\mathrm{qPCR}$ assay. The two $\mathrm{Xv}$ group-specific probes and their primer set 
were optimized separately. Each probe was determined to be highly specific to their respective strains. This multiplex qPCR is a reliable method to detect each bacterial spot species and it will be a great addition to current diagnostic techniques.

\section{Daylily leaf streak found on Hemerocallis spp. in Norway}

G. M. STRØMENG (1), M. B. Brurberg (1), E. Vike (2), V. Talgø (3)

(1) Bioforsk, Ås, Norway; (2) Norwegian University of Life Sciences, Ås, Norway; (3) Bioforsk Norwegian Inst of Agric \& Env Res, Aas, Norway Phytopathology 104(Suppl. 3):S3.115

In the last five years, distinct leaf spots have been observed on daylily (Hemerocallis spp.) in plantings at the Norwegian University of Life Sciences at Ås, Akershus county, Norway. Initial leaf spots in the spring are small, circular with a water-soaked appearance, but turn brown as they enlarge, and eventually the spots become grey in the middle. Leaf spots develop faster longitudinally than transversely and they often coalesce. By mid-summer, the appearance of the plants is often severely affected. Incubation of symptomatic leaves in high moisture chambers resulted in growth of a fungus that was morphologically identified as Kabatiella microsticta (syn. Aureobasidium microstictum). Pure cultures of the fungus were obtained from leaf spots of yellow daylily ( $H$. lilioasphodelus) and three hybrid cultivars. On potato dextrose agar the isolates were pale pink with a shiny appearance. Sequencing of the ITS region of nuclear rDNA using primers ITS4 and ITS5 confirmed the morphological identification. Inoculation tests were carried out on potted plants of yellow daylily and a hybrid cultivar. Plants were sprayed with spore suspensions $\left(10^{6}\right.$ conidia per $\left.\mathrm{ml}\right)$ to runoff on intact leaves and on leaves wounded with a needle prior to inoculation. At a mean temperature of $18^{\circ} \mathrm{C}$, symptoms appeared on wounded leaves after one week and on leaves that had not been artificially wounded after three weeks. The pathogen was reisolated from leaf spots on inoculated plants.

\section{Characterization of Xylella fastidiosa pear leaf scorch strain in Taiwan} through whole genome sequence analyses

C. C. Su (1), W. L. Deng (2), F. J. JAN (2), C. J. Chang (3), H. Huang (4), J. Chen (5)

(1) Taiwan Agricultural Chemicals and Toxic Substances Research Institute, Taichung, Taiwan; (2) National Chung Hsing University, Taichung, Taiwan; (3) University of Georgia, Griffin, GA, U.S.A.; (4) University of South Florida, Tampa, FL, U.S.A.; (5) USDA ARS PWA, Parlier, CA, U.S.A. Phytopathology 104(Suppl. 3):S3.115

Xylella fastidiosa is a Gram-negative bacterium causing diseases on many economically important crops mostly in the Americas but also in Asia. A strain of $X$. fastidiosa was found to cause pear leaf scorch (PLS) disease in Taiwan in 1992. Because of nutritional fastidiousness, characterization of $X$. fastidiosa through traditional cultivation-based methodologies yielded limited information. Whole genome sequencing is a powerful tool to generate biological information for fastidious prokaryotes. In this whole genome sequencing project, a PLS strain (PLS229) was isolated from a symptomatic pear cultivar Hengshan (Pyrus pyrifolia) at Houli $\left(24^{\circ} 20^{\prime} 14^{\prime \prime} \mathrm{N}\right.$, $\left.120^{\circ} 44^{\prime} 11^{\prime \prime} \mathrm{E}\right)$, Taiwan. PLS229 was triple-cloned and cultured in PD2 medium at $28^{\circ} \mathrm{C}$ for 5-6 days. Total genomic DNA was extracted and sequenced using the 454 GS-FLX system. The bacterium has a genome size of $2,733,013$ bp with a $\mathrm{G}+\mathrm{C}$ content of $53.1 \%$. The PLS229 genome was annotated to have 3,259 open reading frames and 50 RNA genes. Compared to whole genome sequences in GenBank database, PLS229 shared 99\% similarity to $X$. fastidiosa subsp. fastidiosa (Temecula1 and M23), subsp. multiplex (M12) and subsp. pauca (9a5c) at locus rrs (16S ribosomal RNA gene). In contrast, strain PLS229 was more distantly related (88-89\% nucleotide sequence similarity) to all three subspecies at loci $g y r \mathrm{~B}, d n a \mathrm{~K}$, and rpoD, suggesting a possible new subspecies.

\section{Overview of impatiens downy mildew in Florida}

S. N. SUAREZ (1), A. J. Palmateer (1)

(1) University of Florida, Homestead, FL, U.S.A.

Phytopathology 104(Suppl. 3):S3.115

Downy mildew of impatiens, caused by Plasmopara obducens, is a devastating disease affecting nurseries and landscapes across the country. Introduction of the pathogen into Florida seemingly occurred by movement of diseased plants from the nursery to landscape, rather than by windblown sporangia. Scattered occurrences of the disease were reported in the winter of 2012 and caused a rapid and dramatic change in landscapes throughout Florida. Observations over the past two years in South Florida indicate that the disease remains active year round on gardening impatiens (Impatiens walleriana). Subsequently, gardening impatiens have been replaced by other downy mildew resistant impatiens species such as New Guinea Impatiens and its hybrids, but a desire for the shade tolerant vibrant colored gardening impatiens remains in high demand. Fungicide efficacy trials have identified products for controlling the disease in greenhouse and nursery production. The greater challenge is managing the disease in landscapes where fungicide use is legally limited and cost prohibitive. Thus, current research efforts have focused on identifying host resistance in I. walleriana and evaluating more short term integrated disease management strategies in the landscape such as irrigation management, nutritional amendments, impact of the growing environment and limited use of cost effective fungicides.

Residual efficacy of fungicides for impatiens downy mildew control S. N. SUAREZ (1), I. Maguire (1), P. Lopez (1), A. J. Palmateer (1) (1) University of Florida, Homestead, FL, U.S.A.

Phytopathology 104(Suppl. 3):S3.115

Fungicide efficacy trials have identified products for controlling downy mildew in greenhouse and nursery production. The greater challenge is managing the disease in landscapes where fungicide use is legally limited and cost prohibitive. Thus, current efforts have focused on identifying products applied in the greenhouse or nursery that provide long residual activity for downy mildew control in the landscape. To compare residual fungicide efficacy, impatiens received one of seventeen fungicide treatments including inoculated and non-inoculated controls. All fungicides were applied to visibly disease-free, 7-wk old, seed-raised impatiens as foliar sprays to runoff using a hand-pressurized sprayer. Applications delivered approximately $3.0 \mathrm{fl} \mathrm{oz}$ of spray solution per plant. Each treatment was applied once. The experimental design was a randomized complete block with ten replications per treatment. Each replicate consisted of a single plant grown in a 4-in. pot containing Pro Mix BX. The average daytime temperature was $78^{\circ} \mathrm{F}$ with $74 \%$ average relative humidity and average nighttime temperature was $71^{\circ} \mathrm{F}$ with $91 \%$ average relative humidity. Plants were spray inoculated with a suspension of $P$. obducens sporangia $\left(3 \times 10^{6}\right.$ sporangia/ $/ \mathrm{l} \mathrm{oz}=1 \times 10^{5}$ sporangia $\left./ \mathrm{ml}\right) 24 \mathrm{hr}$ post treatment. Plants were monitored daily to record the first evidence of sporulation on leaf tissue. Disease pressure remained high throughout the duration of the trial.

Resistance of a worldwide collection of resistant tomato, eggplant and pepper lines to South Asian strains of Ralstonia solanacearum

N. SUBEDI (1), S. A. Miller (1)

(1) Ohio State University, Wooster, OH, U.S.A.

Phytopathology 104(Suppl. 3):S3.115

Bacterial wilt, caused by Ralstonia solanacearum is one of the most important diseases of solanaceous crops in South Asia. Use of host resistance is the most effective and practical approach to manage this disease. However, diverse $R$. solanacearum populations may breakdown host resistance. The wide geographical distribution of $R$. solanacearum drives the diversity of this pathogen. To characterize diversity of South Asian populations, we collected over one hundred strains of $R$. solanacearum from Bangladesh, Nepal, and India. These strains were phylotyped and genomically fingerprinted by Rep PCR. We selected one representative strain of $R$. solanacearum from each of six Rep PCR clonal groups to screen twelve tomato, thirteen eggplant, and nine pepper lines being used in different parts of the world as sources of bacterial wilt resistance. Resistant phenotypes observed were categorized as highly resistant ( $0-5 \%$ wilt), partially resistant $(6-50 \%$ wilt $)$, and highly susceptible to bacterial wilt. Five tomato, seven eggplant, and two pepper lines were identified as highly resistant against South Asian strains. All other resistant lines tested, except one tomato line, were partially resistant. The outcomes of this study will support farmers and plant breeders in South Asia to manage bacterial wilt by identifying the lines most resistant to local $R$. solanacearum populations. Resistant lines can also be considered for use as rootstocks in grafting programs.

Detection of Pseudoperonspora cubensis and $P$. humuli using 5' nuclease probes, specific primers and high resolution melt curve analysis C. F. SUMMERS (1), D. H. Gent (2), C. D. Smart (1)

(1) Cornell NYSEAS, Geneva, NY, U.S.A.; (2) USDA-ARS, Corvallis, OR, U.S.A.

Phytopathology 104(Suppl. 3):S3.115

Pseudoperonospora cubensis, the causal agent of cucurbit downy mildew, results in much economic loss worldwide. The closely related pathogen, $P$. humuli, causes hop downy mildew, one of the most serious diseases of hop. Both pathogens produce wind-blown sporangia capable of spreading disease rapidly. Thus, detection of sporangia would be useful in early management of the disease. However, the genetic similarity between the two pathogens has prevented differentiation, and consequently successful detection, of either pathogen. Our goal was to utilize conserved single nucleotide polymorphisms in the cox 2 gene of each pathogen to develop methods that allow for their differentiation and detection. 5' nuclease assay probes were developed that 
permit concurrent detection of both pathogens from one reaction with great specificity. Specific primers developed for conventional PCR offer a lower cost option for detection. High resolution melt curve analysis was a third approach developed that allowed for differentiation and diagnosis of these pathogens. These three methods have been tested for use in diagnosis of diseased plants from the field as well as detection of DNA extracted from air samples collected using a roto-rod spore trap.

Latent infection biology of Cladosporium species associated with brown spot, a post-harvest disease of late harvest table grapes in California

C. L. SWETT (1), C. A. Young (1), W. D. Gubler (1)

(1) University of California - Davis, Davis, CA, U.S.A.

Phytopathology 104(Suppl. 3):S3.116

In California table grapes, Cladosporium species causing brown spot are becoming one of the major contributors to post harvest losses during exportation in late harvest grapes. Central to control is the assumption that most infections occur after harvest, but very little is known about pathogen infection biology. Studies were conducted in 2013 to evaluate the pre and post harvest association of Cladosporium with Red Globe and Crimson varieties in California. Surveys of symptomless berries indicate that at the green berry stage, $100 \%$ of clusters sustain latent infections, with $20-75 \%$ of berries infected / cluster; infection incidence post veraison was similar, with $65-78 \%$ of berries infected / cluster. In post harvest studies, internal infections alone contributed to disease development in $90 \%$ of clusters, compared to $100 \%$ of clusters when the surface population was present $(\mathrm{P}>0.05)$. The fungus colonized the style scar, ventral and ovular vascular bundles near the cap stem end, dorsal vascular bundles, epidermal tissue, and mesocarp tissue. A high incidence of recovery from cap stem tissue $(35-83 \%$ of stems / cluster) suggests that the fungus may be able to enter the berry through the stem tissue; if similar to other latent pathogens such as Botrytis cinerea, Cladosporium species may also colonize ovary tissue. Studies examining modes of entry, peak infection periods, and early season chemical treatments are underway.

Shoot infection biology of Fusarium circinatum: Wound-free infection and symptomless colonization

C. L. SWETT (1), T. R. Gordon (1)

(1) University of California Davis, Davis, CA, U.S.A.

Phytopathology 104(Suppl. 3):S3.116

Fusarium circinatum causes pitch canker, a disease that affects many pine species in managed plantings and native forests worldwide. Central to our understanding of the epidemiology of pitch canker is that wounds are required for branch infections, which lead rapidly to lesion formation. However, recent studies suggest that un-wounded branches may also sustain infections. In these studies, $70 \pm 13 \%$ of Pinus radiata branches sprayed with a suspension of $F$. circinatum spores $\left(10^{6}\right.$ spores $\left./ \mathrm{ml}\right)$ became infected, and most infections were symptomless. Similar inoculations of seedlings resulted in an infection rate of $97 \pm 9 \%$, with most showing no symptoms. Wounding at the time of inoculation increased the incidence of lesion formation by $90 \%$ in both branches and seedling stems. Surfactant addition increased the incidence of symptom development by $30 \%$ and the extent of colonization in asymptomatic stems by $15 \%$. In addition, the proportion of non-wound-inoculated plants that developed symptoms was significantly higher in seedlings supplied with 200 or $400 \mathrm{ppm}$ nitrogen compared to $25 \mathrm{ppm}$ nitrogen. These results are consistent with the symptomless association described for the fungus in root tissue of $P$. radiata, and may indicate that wounding is less important for shoot infection than for stimulating lesion formation following infection.

Reverse transcription loop-mediated isothermal amplification of Iris Yellow Spot Virus

S. SZOSTEK (1), H. Schwartz (1)

(1) Colorado State University, Fort Collins, CO, U.S.A.

Phytopathology 104(Suppl. 3):S3.116

Iris Yellow Spot Virus (IYSV) affects onion production worldwide. IYSV symptoms are typically confirmed by DAS-ELISA or RT-PCR, which can be time intensive and/or costly. This work demonstrates the detection of IYSV directly from small amounts $(10.0 \mathrm{mg})$ of affected onion tissue by Reverse Transcription Loop-Mediated Isothermal Amplification (RT-LAMP). Reverse transcription and amplification of IYSV by RT-LAMP occurs in a single microcentrifuge tube held at $65^{\circ} \mathrm{C}$ for 2.5 hours. Amplified products are visualized by gel electrophoresis. This technique is very sensitive and great care must be taken to avoid contamination. Because IYSV is amplified directly from plant tissue, thus circumventing RNA extraction and cDNA production, 100 RT-LAMP reactions cost approximately $25 \%$ that of 100 RTPCR reactions in reagents alone. Results from RT-LAMP can be obtained in less than half the time it takes to complete DAS-ELISA or RT-PCR. RT-
LAMP of IYSV may be of value to diagnostic clinics that need to confirm results obtained by other methods, for detecting IYSV from a small number of samples, or as a research tool.

Diseases and pests are threatening boxwood in Scandinavian landscape plantings

V. Talgø (1), C. Magnusson (1), I. M. Thomsen (2), H. P. Ravn (2), G. M. STRØMENG (3)

(1) Bioforsk Norwegian Inst of Agric \& Env Res, Aas, Norway; (2) University of Copenhagen, Frederiksberg C, Denmark; (3) Bioforsk, Ås, Norway

Phytopathology 104(Suppl. 3):S3.116

During the last decade, several disease and pest problems have occurred on boxwood (Buxus sempervirens) in Scandinavia, likely due to increased trade of symptomatic or latently infected plants. There are clear evidences that imported, infected plants have been distributed. Leaves and shoots on boxwood are damaged by the fungi Volutella buxi (Volutella leaf and stem blight) and Cylindrocladium buxicola (boxwood blight), the latter is also associated with death of whole plants. In Denmark, the insects Psylla buxi (boxwood psyllid) and Monarthropalpus buxi (boxwood leaf miner) are commonly found on damaged leaves, but more serious are the injuries caused by the boxwood nematode, Rotylenchus buxophilus. In 2013, very high densities of this nematode were detected on dying boxwood plants in a baroque garden in Copenhagen. The soil contained up to 60 times more $R$. buxophilus than the damage threshold. A Rhizoctonia sp. was isolated from the dying roots, but no Phytophthora spp. or other serious root pathogens were found. In general, parasitic nematodes are known to wound roots and thereby make entry points for pathogens, among them Rhizoctonia spp. In 2013, $R$. buxophilus was detected in symptomatic boxwood from Sweden. It was also found in containerized, seemingly healthy, imported plants in Oslo, but not on boxwood from a nursery in southern Norway.

Occurrence of sudden death syndrome of soybean caused by Fusarium virguliforme in South Dakota

C. TANDE (1), B. Hadi (1), R. Chowdhury (1), S. Subramanian (1), E. Byamukama (1)

(1) South Dakota State University, Brookings, SD, U.S.A.

Phytopathology 104(Suppl. 3):S3.116

In the 2013 soybean growing season, a soybean survey targeting SDS symptomatic plants was carried out in 20 arbitrary selected eastern-most South Dakota counties between July and August when soybeans were at the beginning seed and beginning seed maturity. Soybean plants (up to 15 plants per field) that had symptoms resembling those of SDS were sampled and fungal isolations were made. Leaf symptoms of the plants included some necrosis and slight interveinal chlorosis. The tap roots also had areas of necrosis and the vascular system was brown. Soybean plants with SDS-like symptoms were found in 8 counties at very low incidence $(<3 \%)$. Isolations were made from the symptomatic tap root sections on an acidified potato dextrose agar. Slow growing isolates of $F$. virguliforme with characteristic blue sporodochia were isolated from these symptomatic plant roots. Koch's postulates were performed using a modified layer test method in the greenhouse and $F$. viguliforme was re-isolated. SDS was further confirmed by PCR using primers designed from FvToxl gene. The PCR product size matched that of expected size. The PCR product was sequenced and a BLAST search matched $(100 \%)$ only the sequences of $F$. virguliforme FvTox 1 gene. To our knowledge, this is the first report of SDS in South Dakota. The confirmation of SDS in 8 counties in South Dakota indicates that SDS may be wide spread and a concern for soybean production when conditions are conducive for SDS to develop.

Effect of soybean cyst nematode (SCN) on age-related susceptibility of soybean plants to sudden death syndrome (SDS) caused by Fusarium virguliforme

N. TATALOVIC (1), L. F. Leandro (1), G. L. Tylka (1)

(1) Iowa State University, Ames, IA, U.S.A.

Phytopathology 104(Suppl. 3):S3.116

Fusarium virguliforme (Fv) is a fungal pathogen that infects soybean roots and causes SDS. Young seedlings have been shown to be more susceptible to SDS than older plants at vegetative growth stages. Our objective was to determine if SCN infection affects age-related resistance to SDS. Soybean cultivars AG2403 (susceptible to $\mathrm{Fv}$ and $\mathrm{SCN}$ ), and MACO2 4754 (susceptible to $\mathrm{Fv}$ and resistant to $\mathrm{SCN}$ ) were used. Seeds were planted in SCN-infested or non-infested soil for 4, 8, 12, 16, or 20 days, after which they were transplanted to Fv-infested or non-infested soil. Plants were incubated for 21 days, and evaluated for SDS foliar severity, root rot, and root anatomy. Older plants infected with Fv developed less severe foliar symptoms than 
younger plants $(\mathrm{p}<0.0001)$. Co-infected plants consistently had greater foliar severity than plants infected with $\mathrm{Fv}$ alone $(\mathrm{p}<0.0001)$, but the effect was dependent on plant age. In younger co-inoculated plants, SDS severity was 2 to 5- fold greater than in Fv-infected plants, while co-infected 20-day-old plants had up to 2 times more foliar symptoms than Fv-infected plants, depending on cultivar. Root rot decreased $(\mathrm{p}<0.05)$ with plant age. Infection with SCN increased the number of root tips compared to both non-infested and $\mathrm{Fv}$-infested plants. Our findings suggests that $\mathrm{SCN}$ infection increases age-related susceptibility to SDS by enhancing the availability of rootbranching wounds that serve as preferred entry points for $\mathrm{Fv}$ infection.

\section{Results of the soybean cyst nematode survey for Manitoba, Canada}

M. TENUTA (1), M. Madani (2), D. Lange (3)

(1) University of Manitoba, Winnipeg, MB, Canada; (2) Department of Soil Science, University of Manitoba, Winnipeg, MB, Canada; (3) Manitoba Agriculture, Food and Rural Development, Altona, MB, Canada

Phytopathology 104(Suppl. 3):S3.117

We report the findings of a survey conducted in 2012/2013 of soybean fields for the soybean cyst nematode (SCN; Heterodera glycines) in Manitoba. In total, 48 fields were sampled with fields prone to infestation selected. Fields were sectioned into areas that could be responsible for introduction of the pest. In total, 282 composite soil samples were analyzed from areas of the 48 fields. Overall, 37 of the composite samples from 22 fields had nematode cysts. Sixty cysts were recovered. Most were heavily damaged but 26 sufficient for examination. Of these, 23 had circumfenestrate vulval cone structures. Three cysts had bifenestrate vulval cone structures characteristic of the genus Heterodera. Most cysts were empty except for 15 circumfenestrate and one bifenestrate cysts. These were analyzed by ITS sequencing, three SCN species-specific PCR approaches, and in one case, by 28 s rDNA gene sequencing. ITS sequencing was possible for 14 of the circumfenestrate cysts. These best matched Cactodera. Only one of the bifenestrate cysts yielded DNA and identification was ambiguous for SCN and Cactodera. Cactodera is not a pest of soybean or other crop plants in Manitoba. These cyst nematodes are likely naturally present. The findings indicate SCN has still not been detected in the Province.

Determining the presence and identification of the stem nematode (Ditylenchus) on grain pea and creeping thistle in the Canadian prairie provinces

M. TENUTA (1), S. Briar (2), M. Madani (2), S. Subbotin (3)

(1) University of Manitoba, Winnipeg, MB, Canada; (2) Department of Soil Science, University of Manitoba, Winnipeg, MB, Canada; (3) Plant Pest Diagnostic Center, California Department of Food and Agriculture, Sacramento, CA, U.S.A.

Phytopathology 104(Suppl. 3):S3.117

This study examined if Ditylenchus weischeri and not $D$. dipsaci is present in yellow pea grain from harvest samples in 2009 and 2010 and from creeping thistle in Saskatchewan, Alberta and Manitoba. A total of 538 pea harvest samples (mainly yellow) were obtained from 151 growers. Two percent of the samples were positive indicating a low frequency of the nematode. Counts ranged from 4-1500 nematodes $\mathrm{kg}^{-1}$ pea. The nematode seemed morphologically dissimilar to $D$. dipsaci. Thistle plants were collected in fall 2011 from fields of yellow pea in MB, SK and AB. All plants from SK and MB were infested with Ditylenchus. Individuals from each sample were used for molecular identification (ITS-rRNA regions ITS1, 5.8S and ITS2 and fragment of hsp90). The results mainly match those published for $D$. weischeri. PCR with a species-specific primer for D. weischeri was also designed and successfully tested using the nematode from thistle.

\section{The influence of nitrogen and calcium fertilizers on septoria late blight} and yield of celery

M. Tesfaendrias (1), C. Trueman (2), B. Gossen (3), A. McKeowen (1), M. R. MCDONALD (1)

(1) University of Guelph, Guelph, ON, Canada; (2) Ridgetown campus, University of Guelph, Ridgetown, ON, Canada; (3) Agriculture and Agr-Food Canada, Saskatoon, SK, Canada

Phytopathology 104(Suppl. 3):S3.117

Late blight (Septoria apiicola) reduces quality and up to $70 \%$ yield of celery. Field trials were conducted over four site-years to evaluate the effect of nitrogen $(\mathrm{N})$ rate and foliar calcium $(\mathrm{Ca})$ fertilization on the development of late blight and yield on celery grown on high organic matter (O.M.) soils. A treatment with the protectant fungicide chlorothalonil was included to determine if fertilizer could replace fungicide application in celery production. There was a cultivar $\times$ site-year interaction for each of the disease and yield variables. Repeated application of chlorothalonil fungicide reduced late blight. Late blight severity decreased with increasing rate of $\mathrm{N}$ at the low O.M. site in
2008. An increasing rate of $\mathrm{N}$ also resulted in an increase in yield at the same site. Application of $\mathrm{Ca}$ did not affect late blight severity and increased yield in only one site-year. Negative correlations were observed between late blight severity and yield. Tissue $\mathrm{N}$ concentrations varied among the treatments at the low O.M. sites, but not at the high O.M. sites. No effect on Ca tissue concentrations was observed. Application of $\mathrm{N}$ and $\mathrm{Ca}$ alone did not adequately control late blight. However, a slight reduction in late blight severity was observed. Thus, $\mathrm{N}$ may improve late blight management practices in combination with fungicides and other integrated crop management practices and both $\mathrm{N}$ and Ca may increase celery yield especially on low O.M. soils.

Efficacy assessment of demethylation inhibitor (DMI) fungicides against Guignardia citricarpa, the causal agent of Citrus black spot (CBS)

N. THAPA (1), M. M. Dewdney (1)

(1) Citrus Research and Education Center, University of Florida, Lake Alfred, FL, U.S.A.

Phytopathology 104(Suppl. 3):S3.117

Copper-based and strobilurin fungicides are the recommended fungicides to manage CBS, a new fungal disease in Florida. DMI fungicides could be a potential alternative mode of action for rotation against CBS. The project objectives are to determine the in vitro fungal population response and the baseline sensitivity to the potentially registered DMIs. Tetraconazole, propiconazole, difenoconazole, fenbuconazole, and imazalil were evaluated in a mycelial growth inhibition assay with various ranges of concentrations for each fungicide. One $\mathrm{ml}$ of each stock solution, or water as a control, was mixed with the media to obtain the final concentrations. Inoculated plates were incubated at $24^{\circ} \mathrm{C}$, under $12 \mathrm{hr}$ light/dark conditions. The plates were scanned after 2 weeks and mycelial growth area was measured with Image-J. Mycelial growth on amended media was compared to the control. There were three replicates per concentration and the whole experiment was done twice. Regression with 3-parameter sigmoidal curves was used to calculate the half maximal effective concentration $\left(\mathrm{EC}_{50}\right)$. The mean $\mathrm{EC}_{50} \mathrm{~s}$ for difenoconazole, propiconazole, and tetraconazole were $0.0013,0.011$, and $0.0401 \mathrm{mg} / \mathrm{ml}$, respectively. Preliminary results for the mean $\mathrm{EC}_{50} \mathrm{~s}$ of fenbuconazole and imazalil were 0.0073 and $0.1 \mathrm{mg} / \mathrm{ml}$, respectively. Results obtained from this study will aid the management of CBS and to understand the fungal response pattern towards DMI fungicides.

Molecular characterization and genetic variation of an ophiovirus associated with blueberry mosaic

T. THEKKE-VEETIL (1), J. Polashock (2), I. M. Plesko (3), K. E. Keller (4), R. R. Martin (4), A. Schilder (5), T. Ho (1), I. E. Tzanetakis (1)

(1) Department of Plant Pathology, Division of Agriculture, University of Arkansas System., Fayetteville, AR, U.S.A.; (2) Marucci Center for Blueberry and Cranberry Research \& Extension, Chatsworth, NJ, U.S.A.; (3) Agricultural Institute of Slovenia, Hacquetova 17, Ljubljana, Slovenia; (4) USDA-ARS, Corvallis, OR, U.S.A.; (5) Department of Plant, Soil and Microbial Sciences, Michigan State University., East Lansing, MI, U.S.A. Phytopathology 104(Suppl. 3):S3.117

Blueberry mosaic is reported from several blueberry growing areas around the world and the causal agent has not been characterized to date. In an attempt to identify the disease agent, nucleic acids extracted from infected plants were subjected to deep sequencing, revealing the presence of a new ophiovirus. PCR assays were designed, confirming the association of virus and disease, in 59 symptomatic samples collected from various blueberry growing areas in the North America and Europe. The new virus, tentatively named Blueberry mosaic associated virus $(\mathrm{BlMaV})$, has a genome organization resembling that of Citrus psorosis virus, the representative member of the genus Ophiovirus with three negative-sense single-stranded RNAs. Evolutionary analyses performed on the movement protein and nucleoprotein revealed significant negative selection pressure on both genes. A universal PCR detection assay was developed based on conserved region of the movement protein; able to detect all the observed variants of the $\mathrm{BlMaV}$ population.

Development and use of root-knot nematode resistant rootstocks for managing Meloidogyne incognita in grafted watermelon

J. A. THIES (1), S. Buckner (1), R. Hassell (2), A. Levi (1)

(1) USDA, ARS, Charleston, SC, U.S.A.; (2) Clemson University, Charleston, SC, U.S.A.

Phytopathology 104(Suppl. 3):S3.117

Since the ban of methyl bromide, the southern root-knot nematode (RKN), Meloidogyne incognita, has re-emerged as a serious pest of watermelon worldwide. Bottle gourd (Lagenaria siceraria) and hybrid squash (Cucurbita maxima $\times C$. moschata) rootstocks are often used to control soil-borne diseases such as Fusarium wilt in grafted watermelon in Asia, Europe, and the 
Mediterranean. However, bottle gourd and hybrid squash rootstocks are extremely susceptible to RKN and are not suitable rootstocks for grafted watermelon in RKN-infested fields. Wild watermelon Citrullus lanatus var. citroides breeding lines were compared to 'Emphasis' bottle gourd and 'Strong Tosa' hybrid squash for their performance as rootstocks for 'Tri-X 313' seedless watermelon in M. incognita-infested fields in Charleston, SC. The experimental design was a randomized complete block with six replicates. 'Tri-X 313' seedless watermelon scions grafted on wild watermelon rootstock breeding lines (Citrullus lanatus var. citroides RKVL301, RKVL-316, and RKVL-318) produced $211.9 \%$ and $648.7 \%$ heavier $(P<0.05)$ fruit yields with $63.2 \%$ and $62.8 \%$ less $(P<0.05)$ root galling than 'Emphasis' bottle gourd rootstock and 'Strong Tosa' hybrid squash rootstock, respectively. These wild watermelon rootstock lines should be useful in breeding programs aiming to develop RKN-resistant rootstocks for grafted watermelon.

Factors influencing Erysiphe necator ascocarp overwintering and ascospore release of in the Willamette Valley of Oregon

L. THIESSEN (1), W. F. Mahaffee (2)

(1) Oregon State University, Corvallis, OR, U.S.A.; (2) USDA ARS, Corvallis, OR, U.S.A.

Phytopathology 104(Suppl. 3):S3.118

Exotic plant hosts and pathogens often display asynchronous development outside their native range, which may influence disease forecasting and management. In the Willamette Valley of Oregon, the development and maturation of $E$. necator ascocarps occurs asynchronously to the host, as the discharge of ascospores often occurs prior to bud break or much later in the host development. Cleistothecia were collected from commercial vineyards in the Willamette Valley prior to precipitous weather (October 9, 2012 and October 24, 2013) and were embedded onto artificial-bark substrates or grape trunk pieces. Infested bark substrates were then suspended above impaction spore traps. In addition, spore traps were placed adjacent to grapevine trunks naturally infested with E. necator ascocarps. Traps were collected biweekly and were processed using quantitative polymerase chain reaction specific for E. necator. Airborne E. necator DNA was detected from November through late April during periods considered favorable for ascospore release (e.g. $>2.5 \mathrm{~mm}$ precipitation and average temperature during wetness events $>$ $7^{\circ} \mathrm{C}$ ) until late April. These data indicate that $E$. necator ascocarp maturation needs to be further examined to better predict disease development in subsequent seasons.

Comparative genomic analysis of Pseudoperonospora cubensis to elucidate the genetic basis of host specialization

A. THOMAS (1), I. Carbone (1), P. Ojiambo (1)

(1) NCSU, Raleigh, NC, U.S.A.

Phytopathology 104(Suppl. 3):S3.118

Pseudoperonospora cubensis, the causal agent of cucurbit downy mildew is a destructive pathogen of cucurbits worldwide. Five pathotypes of $P$. cubensis were identified earlier in the US based on the differential compatibility of the pathogen with Cucumis spp., Citrullus lanatus and Cucurbita spp. and thus, provided evidence for host specialization in the population. However, it is still unclear how either the host or the pathogen influences host specialization. The goal of this study was to investigate the genetic basis for host specialization in this pathosystem. Genomic DNA extracted from nine isolates collected from Cucumis sativus, C. melo var. reticulatus, Cucurbita maxima, C. moschata, C. pepo and Citrullus lanatus were subjected to whole genome sequencing using Illumina's HiSeq platform. Comparative analysis of these nine P. cubensis genomes revealed the presence of two distinct evolutionary lineages. Many fixed polymorphisms separated one lineage that included isolates from Cucurbita pepo, C. moschata and Citrullus lanatus from a second lineage that comprised isolates from Cucumis spp. and Cucurbita maxima. The strong genetic differentiation observed genome-wide between these two lineages suggests that host specialization and possibly cryptic speciation may have been the important drivers of diversity in the evolutionary history of $P$. cubensis. Further studies will be conducted to elucidate genetic signatures of cryptic speciation within these two lineages.

\section{Expert elicitation: A complement to traditional experimental data}

S. THOMAS-SHARMA (1), G. Forbes (2), K. Garrett (1)

(1) Kansas State University, Manhattan, KS, U.S.A.; (2) International Potato Center, Beijing, China

Phytopathology 104(Suppl. 3):S3.118

The use of expert opinion to fill information gaps has gained momentum in many complex systems where decisions have to be made despite severe data limitations. Formal experiments or surveys to study system-level processes can be time-consuming and resource-intensive. A wealth of published and unpublished information, however, has been integrated into scientists' opinions through the course of studying particular systems for many years. This insight can be systematically collected through expert elicitation and synthesized to reduce knowledge gaps, provide preliminary data to hone hypotheses, and prioritize research and development goals. We describe a case study where expert opinion was collected from over twenty researchers working on vegetatively-propagated crops in developing countries through inperson and phone interviews. The distribution of expert estimates on parameters that affect pathogen build-up in planting material was characterized. Experts expressed greater uncertainty about estimates involving intricate biotic interactions such as (a) yield loss based on end-of-season infection levels, and (b) disease incidence after varying starting-levels of infection. In contrast, they had lower uncertainty about management traits such as effectiveness of individual management practices, and farmer selection skill. Challenges in expert elicitation, potential sources of bias and usage of the elicited data are also discussed.

Improving the health of vegetatively-propagated crops: Better integration of disease management strategies for seed produced on-farm

S. THOMAS-SHARMA (1), J. Andrade-Piedra (2), M. Carvajal Yepes (3), J. Hernandez Nopsa (1), P. Kromann (2), J. Legg (4), J. Yuen (5), G. Forbes (6), K. Garrett (1)

(1) Kansas State University, Manhattan, KS, U.S.A.; (2) International Potato Center, Quito, Ecuador; (3) International Center for Tropical Agriculture, Cali, Colombia; (4) International Institute of Tropical Agriculture, Dar es Salaam, Tanzania; (5) Swedish University of Agricultural Sciences, Uppsala, Sweden; (6) International Potato Center, Beijing, China

Phytopathology 104(Suppl. 3):S3.118

Pathogen build-up in vegetative planting material (seed degeneration) is a major problem for food staples in many developing countries. Since certified seed of good health status continues to remain scarce and/or expensive for much of the immediate future in these regions, improving the health of onfarm seed is critical. We developed a framework that integrates various management strategies available to minimize seed degeneration in five major food security crops: potato, sweetpotato, cassava, yam, and banana. Variability in biological and environmental elements was incorporated through stochastic model components and the likelihood of a range of potential outcomes was studied. As expected, the application of disease management strategies mitigated the risks associated with season-to-season variability in disease-conducive weather. We identified scenarios in which positive selection can be as effective as certified seed usage, especially if the rate of success in selecting healthy plants is high. Also, when choosing between within-season management strategies, those that directly reduce the proportion of infected seeds/plants can be particularly effective. Models are being validated with experimental results for the five crops, globally. By integrating results from field experiments and expert elicitation in Bayesian belief networks, we are now developing decision support systems to maximize efficacy of management strategies in crop- and region-specific scenarios.

Characterization of host resistance and Asian soybean rust (ASR) pathogen variability for durable resistance

P. TIAN (1), S. Smith (1)

(1) University of Georgia, Athens, GA, U.S.A.

Phytopathology 104(Suppl. 3):S3.118

Phakopsora pachyrhizi is the causal agent of Asian soybean rust (ASR) and one of the most aggressive soybean diseases. Resistance to ASR is controlled by five resistance genes (Rpp1-5) shown to be ineffective when challenged with different isolates of $P$. pachyrhizi. Knowledge of the genetics of resistance to ASR and the variability of ASR populations are essential in dissecting the relationship between the host and pathogen, and developing effective breeding strategies for resistance. This project has three major research components: 1) characterization of soybean and its wild progenitors host resistance, 2) analysis of Asian soybean rust pathogen variability, and 3) integration of host and pathogen datasets to establish pipelines for the release of resistant cultivars. We collected 8 ASR field isolates from 5 different locations in Georgia from 2011-2013. 20 ASR genotypes were identified at the 5 locations. Additionally, the resistance of 16 released Georgia cultivars and 8 soybean progenitors were tested in response to inoculation with the ASR Georgia field isolates. The soybean progenitors demonstrated different levels of resistance. Conversely, all of the released soybean cultivars were susceptible. By using RNA sequencing techniques, we will identify candidate pathogenicity genes in the ASR isolates and specific resistance genes that can potentially be used to develop durable broad-spectrum disease resistance strategies for soybean cultivars grown in Georgia. 
Characterization of Pseudomonas cichorii and $P$. viridiflava causing bacterial speck-like symptoms on tomato in Florida

S. TIMILSINA (1), H. Adkison (2), J. B. Jones (1), G. E. Vallad (2)

(1) Department of Plant Pathology, University of Florida, Gainesville, FL, U.S.A.; (2) Gulf Coast Research and Education Center, University of Florida, Wimauma, FL, U.S.A.

Phytopathology 104(Suppl. 3):S3.119

Since 2010, 19 putative Pseudomonas cichorii strains and 18 putative $P$. viridiflava strains have been isolated from tomato foliage and fruit exhibiting bacterial speck-like symptoms. These strains were characterized based on multilocus sequence analysis of four housekeeping genes namely gap $A$, gyrB, rpoD and gltA. Seventeen of the $P$. cichorii strains isolated from Florida shared identical sequence identity and varied slightly from the other two Florida strains; all shared lower identity with the type strain. Phylogenetic analysis of the 19 putative $P$. cichorii strains suggests that they are most closely related to $P$. cichorii. Similar results were observed in phylogenetic relationships within the putative $P$. viridiflava strains, where the 18 strains clustered in two distinct but closely related groups. Interestingly, these two phylogenetic groups varied in levan activity, since one phylogenetic group of $P$. viridiflava showed potential positive levan activity and associated yellow pigment on nutrient agar amended with $5 \%$ sucrose, while the other phylogenetic group showed no levan activity.

Applied practicums bring real-world experience to students enrolled in Turfgrass Diseases, a split-level course taught at Mississippi State University M. TOMASO-PETERSON (1)

(1) Mississippi State University, Mississippi State, MS, U.S.A. Phytopathology 104(Suppl. 3):S3.119

Turfgrass Diseases is a split-level course designed to serve students enrolled in the Golf and Sports Turf Management curriculum at Mississippi State University. The course encompasses the study of disease cycles and associated symptoms, pathogen identification, turf diagnostics, economic importance, and management strategies of turfgrass diseases. The overall objectives included an in-class and field practicum. The in-class practicum was designed to raise awareness of fungicide trade names, common names, phytomobility, and biochemical mode of action by assembling a fungicide database. The students had the freedom to design the database according to their personal preference with the intent that the fungicide database serve as a reference in the work place. The effectiveness of this exercise was determined by a questionnaire each student completed at the end of the semester. All students strongly agreed the database would serve as a valuable reference tool. In the field, students were involved in the plot establishment and ratings of a fungicide evaluation for control of leaf spot in a bermudagrass putting green. The field practicum was designed to teach students critical evaluation methods to identify leaf spot symptoms, the impact of disease on turfgrass quality, efficacy of commercial fungicides for leaf spot control, and research methodology. Seventy percent of the students strongly agreed on the overall effectiveness of participating in a fungicide evaluation.

Understanding genome-wide defense response gene regulation to improve crop disease resistance

B. TONNESSEN (1), J. E. Leach (1)

(1) Colorado State University, Fort Collins, CO, U.S.A.

Phytopathology 104(Suppl. 3):S3.119

Rice productivity is hindered by plant disease, which can diminish a farmer's harvest by about one third. To mitigate this issue, breeders and engineers focus on single resistance genes that are known to directly inhibit pathogen spread. The method is effective, but the pathogen is able to overcome this selective pressure after only a few generations. A more sustainable resistance could be attained by selecting for enhanced expression of plant defense response (DR) genes that elicit basal defense against a broad spectrum of pathogens. This project focuses on elucidating the genome-wide activation of DR genes based on de novo identification of transcription factor binding sites (TFBSs). These TFBSs show conserved organization into cis-regulatory modules that facilitate coexpression of DR genes in resistant varieties. The presence of these motifs could be a functional element for efficient DR gene expression across the genome. These cis-modules may be used as reliable markers in a novel breeding strategy for effective DR gene expression and durable plant defense. To attain the goals of this project would mean advancement in our abilities to develop sustainably resistant rice varieties.

Disease control using Fe-enriched sheath produced by an Fe-oxidizing bacterium, Leptothrix sp. (II) effects of sheath on pathogens and plant seedlings K. TOYODA (1), T. Shiraishi (1), H. Kunoh (1), J. Takada (1)

(1) Okayama University, Okayama, Japan

Phytopathology 104(Suppl. 3):S3.119
The iron-oxidizing bacteria, Leptothrix spp., produce microtubular sheaths extracellularly in aquatic environments where groundwater outwells. The sheath is primarily composed of iron oxides $(\mathrm{Fe} / \mathrm{Si} / \mathrm{P}=75 / 20 / 5$ in the atomic ratio). To evaluate the availability of the naturally-produced sheath for developing plant disease protectant, its antifungal activity was examined against several phytopathogenic fungi including Botrytis cinerea, Colletotrichum orbiculare and Mycosphaerella pinodes inoculated on ethanolkilled onion epidermis. The colloidal suspension of powdered Fe-enriched sheath effectively suppressed fungal pathogenesis, especially causing penetration failure from appressoria, when mixed with the spore suspension on onion epidermis. For eliminating $\mathrm{Fe}$ from the natural sheath, the $\mathrm{Fe}$ enriched sheath was heat-treated at $500^{\circ} \mathrm{C}$ in a $\mathrm{H}_{2}$ gas flow, followed by acid treatment. This $\mathrm{Fe}$-depleted sheath did not show any suppressive effects on the fungal pathogenesis, suggesting that the Fe constitute in the sheath could hold a key for the antifungal activity. Interestingly, on Arabidopsis seedlings exposed to the colloidal suspension of the Fe-enriched sheath, expression of defense-related genes encoding pathogenesis-related protein1 (PR1) and Pad3, the enzyme in the biosynthetic pathway to the phytoalexin, were prominently induced. On the basis of these results, the Fe-enriched sheath from Leptothrix spp. is expected to be used as a new protectant for plant diseases.

Extracellular DNases modulate biofilm formation of $R$. solanacearum

T. TRAN (1), M. Hawes (2), C. Allen (1)

(1) University of Wisconsin-Madison, Madison, WI, U.S.A.; (2) University of Arizona, Tucson, AZ, U.S.A.

Phytopathology 104(Suppl. 3):S3.119

Ralstonia solanacearum is a soil-borne vascular pathogen that causes bacterial wilt disease in more than 200 plant species by colonizing and blocking host xylem vessels. The bacterium's two extracellular DNases are required for full virulence on tomato following soil-soak inoculation of unwounded plants, likely because they degrade the DNA-containing traps that protect plant root tips from pathogens. Surprisingly, the DNases also contributed to bacterial wilt virulence after direct inoculation into tomato stems, possibly because these enzymes are required for normal biofilm formation. The bacterium forms biofilm-like aggregates on plant xylem vessel walls, but their biological importance is unexplored. Several factors contribute to $R$. solanacearum biofilm formation in vitro, including motility and aerotaxis. However little is known about factors involved in in planta biofilm formation and how it contributes to the pathogen's dispersion within and across xylem vessels. A double deletion mutant strain lacking both extracellular DNAses formed abnormally thick biofilms in vitro. We tested the hypothesis that extracellular DNases modulate bacterial biofilms in vitro, possibly by digesting the extracellular DNA content in biofilm structure. This may contribute to bacterial nutrition as well as bacterial spread within and between xylem vessels.

The novel Gr29D09 effector family from potato cyst nematode Globodera rostochiensis suppresses plant immunity to promote nematode parasitism

T. T. TRAN (1), S. Chen (1), X. Wang (2)

(1) Department of Plant Pathology and Plant-Microbe Biology, Cornell University, Ithaca, NY, U.S.A.; (2) USDA-ARS, Robert W. Holley Center for Agriculture and Health, Ithaca, NY, U.S.A.

Phytopathology 104(Suppl. 3):S3.119

Sedentary endoparasitic nematodes rely on secreted effectors to initiate and maintain specialized feeding sites within host plant roots which are essential for their growth and development. Although effectors from other plant pathogens have been amply demonstrated for a role in suppressing plant defense, studies confirming an involvement of nematode-secreted effectors in host defense suppression are only scarcely reported. We cloned a family of highly polymorphic effector genes (named Gr29D09) from G. rostochiensis, a potato pest of quarantine significance. These Gr29D09 effector genes were found to be expressed exclusively within the gland cells and dramatically upregulated during nematode infection, pointing to a role of this effector family in promoting nematode parasitism. Transgenic potato lines overexpressing individual Gr29D09 gene showed an increased susceptibility not only to $G$. rostochiensis but also to an unrelated potato pathogen Streptomyces scabies, suggesting that the Gr29D09 family may involve in suppressing plant basal defense. Using Agrobacterium-mediated transient expression assays in Nicotiana benthamiana, members of the effector family were found to suppress flg22-triggered defense responses as well as cell death triggered by a couple of resistance genes. Current focus is to identify interacting targets in potato to discover the potential defense pathways that are regulated by this important effector family. 
Searching for sources of resistance to Verticillium wilt of olive in seedlings from the genus Olea

C. TRAPERO (1), C. M. Diez (1), L. Rallo (1), F. J. Lopez-Escudero (1), D. Barranco (1)

(1) University of Cordoba, Cordoba, Spain

Phytopathology 104(Suppl. 3):S3.120

Verticillium wilt of olive (Olea europaea), caused by the fungus Verticillium dahliae, is the most serious disease of this crop and affects it worldwide. The use of plant material resistant to the disease is one of the most efficient measures, although nowadays there is a lack of resistant genotypes. As $V$. dahliae is a soilborne fungus, the resistance to this pathogen could be provided by either the rootstock or the scion. Since 2008, a breeding program aimed to obtain plant material resistant to the disease is carried out in the University of Córdoba, Spain. It has been developed a method to screen a great number of olive genotypes for resistance to $V$. dahliae in the first step of this breeding program. This method involves the root-dip inoculation of young seedlings with a highly virulent isolate of the fungus, and their assessment under controlled conditions. Up to date, using this test more than 10,000 seedlings obtained from crosses between olive cultivars, wild olives genotypes and other Olea species and $O$. europaea subspecies have been screened for resistance to $V$. dahliae. Most of the evaluated genotypes were susceptible to the disease, but some of them have been selected as resistant. Interesting sources of resistance have been found in some wild olive genotypes and $O$. exasperata species, which could be used as resistant rootstocks to control the disease. Resistance to Verticillium wilt of olive appears to be quantitatively inherited.

Identification of interacting regions of the movement protein and nucleocapsid protein of two distinct tospoviruses

D. Tripathi (1), G. Raikhy (1), H. PAPPU (1)

(1) Washington State University, Pullman, WA, U.S.A.

Phytopathology 104(Suppl. 3):S3.120

Tospoviruses (Tospovirus: Bunyaviridae) cause annual crop loss of over US \$ one billion per year in a wide range of field and horticultural crops. Some of the tospoviruses are known to exist as mixed infections in the same host plant. When present together in mixed infections, Tomato spotted wilt virus (TSWV) has been shown to facilitate the movement of Iris yellow spot virus (IYSV). Recently, we reported that $\mathrm{N}$ and NSm proteins of IYSV and TSWV multimerize and interact with each other in infected Nicotiana benthamiana plants. To identify the specific interacting regions of $\mathrm{N}$ and $\mathrm{NSm}$ proteins, $\mathrm{N}$ and NSm genes were divided into $\mathrm{N}$-terminal, middle, and $\mathrm{C}$-terminal regions and cloned into LexA-based Yeast-2-hybrid ( $\mathrm{Y} 2 \mathrm{H})$ vectors. $\mathrm{Y} 2 \mathrm{H}$ results were confirmed by beta-galactosidase assay of interacting partners. Identified interacting $\mathrm{N}$ - and $\mathrm{C}$-terminal regions have been reported to play critical roles in virus movement and pathogenesis. Findings of this research could facilitate a better understanding of the virus-virus interactions within host plants and could help develop efficient and durable control strategies for these viruses.

A novel rice resistance phenotype to Xanthomonas oryzae TAL effectors does not require the effector transcriptional activation domain

L. TRIPLETT (1), V. Verdier (2), M. Alexander (3), S. Cohen (1), J. Craven (1), A. Bogdanove (3), J. Leach (1)

(1) Colorado State University, Fort Collins, CO, U.S.A.; (2) IRD, Montpelier, France; (3) Cornell University, Ithaca, NY, U.S.A.

Phytopathology 104(Suppl. 3):S3.120

Strains of the rice pathogen Xanthomonas oryzae express between eight and 26 secreted Transcriptional Activator-Like (TAL) effectors that activate host susceptibility genes to favor bacterial growth. Known mechanisms of rice gene-for-gene resistance against TAL effectors depend on TAL gene activation activity; these include resistance (R) genes transcriptionally activated by a specific TAL effector, or promoter mutations preventing TAL effector function. We used a TAL effector-free X. oryzae strain, X11-5A, to determine the effects of individual TAL effectors on various rice cultivars. In one variety, $10 \mathrm{TAL}$ effectors from different $X$. oryzae strains and pathovars triggered resistance to X11-5A, with eight of the effectors abolishing symptoms. A truncated TAL effector lacking the transcriptional activation domain also triggered resistance, but resistance was not triggered by a TAL effector lacking the central repeat domain, nor by two TAL effectors from other Xanthomonas species. The rice variety was not resistant to most $X$. oryzae strains that natively express TAL effectors, although resistance was observed against all tested strains of $X$. oryzae pv. oryzicola from Africa. This suggests that plasmid expression from the X11-5A genetic background could affect the range of effectors that trigger resistance. Together, these results point to a novel mechanism of resistance triggered by the central repeat region of diverse TAL effectors.
Discriminatory concentration assay to detection of low and high benzimidazole resistant isolates of Cercospora beticola

N. R. TRKULJA (1), N. S. Dolovac (1), A. G. Milosavljevic (1), E. L. PfafDolovac (1)

(1) Institute for Plant protection and environment, Belgrade, Serbia Phytopathology 104(Suppl. 3):S3.120

The appearance of populations of Cercospora beticola resistant to benzimidazoles is recorded wherever sugar beet grows. Designing methods which have the ability to provide faster data of the benzimidazole sensitivity of $C$. beticola is very useful to improve the management of disease. We have selected five isolates from each sensitivity group, sensitive, low-resistant (167-ß-tubulin mutation) and high-resistant (198-ß-tubulin mutation). Each isolate was grown for 10 days at $25^{\circ} \mathrm{C}$ in dark on potato dextrose agar medium amended with carbendazim and thiophanate-methyl at $0,0.1,1,10,50,100$, 500 , and $1000 \mu \mathrm{g} \mathrm{ml}^{-1}$, afterwards colony growth was measured. The discriminatory concentration (DC) was defined as the concentration at which C. beticola isolates could clearly be separated into two groups, inhibited or not inhibited by the carbendazim or thiophanate-methyl. Appropriate DC for separation of sensitive from resistant isolates was $1 \mu \mathrm{g} \mathrm{m}{ }^{-1}$ for both carbendazim and thiophanate-methyl. Determined DC adequate to separate low-resistant from high-resistant isolates was $10 \mu \mathrm{g} \mathrm{ml}^{-1}$ and $100 \mu \mathrm{g} \mathrm{m} l^{-1}$ of carbendazim and thiophanate-methyl respectively. DC is very quick and useful method for resistance detection for large number of isolates, especially in case of single point mutation as well as resistance to benzimidazole fungicides. We propose using defined DC as method for detection of benzimidazole resistance of $C$. beticola.

Role of the NarXL regulatory system in Ralstonia solanacearum denitrification

A. N. TRUCHON (1), B. L. Dalsing (1), C. Allen (1)

(1) University of Wisconsin Madison, Madison, WI, U.S.A.

Phytopathology 104(Suppl. 3):S3.120

The bacterial wilt pathogen Ralsonia solanacearum grows to high cell densities in host plant xylem, a low oxygen, low nutrient environment that contains inorganic nitrogen species such as $\mathrm{NO}_{3}^{-}$. Under low oxygen conditions some bacteria switch from aerobic to denitrification-driven anaerobic respiration using a chain of nitrogen species as terminal electron acceptors (TEAs). This major metabolic change is mediated in many bacteria by the NarXL two-component sensor-regulator, wich senses nitrate and activates genes encoding denitrification enzymes. The $R$. solanacearum genome apparently encodes a complete denitrification pathway, which is highly expressed during tomato pathogenesis. We hypothesize that as $R$. solanacearum enters the plant xylem it encounters low oxygen conditions and uses NarXL to activate denitrification for anaerobic respiration. We constructed a narXL-deficient mutant of $R$. solanacearum to expore the role(s) of the NarXL regulatory system in pathogenic success. We compared the ability of the narXL and wild-type strains to grow under anaerobic and aerobic conditions, in culture and in host plant stems. Analyzing the impact of NarXL on $R$. solanacearum growth during infectioin will increase understanding of the overall metabolic network of the pathogen, and how it is adapted to the resources in the plant environment.

Progress in developing two-rowed barley for resistance to spot blotch J. R. TUCKER (1), W. G. Legge (1), A. D. Beattie (2), W. D. Voth (2)

(1) Brandon Research Centre, Agriculture and Agri-Food Canada, Brandon, MB, Canada; (2) Crop Development Centre, University of Saskatchewan, Saskatoon, SK, Canada

Phytopathology 104(Suppl. 3):S3.120

Spot blotch, caused by Bipolaris sorokiniana is a foliar disease of barley (Hordeum vulgare) which can significantly reduce yield and seed quality. There is growing concern about enhanced incidence and severity of spot blotch observed on barley in the eastern prairie region of Canada. Recent studies have also documented substantial virulence diversity in the Canadian B. sorokiniana population, and have described isolates from Manitoba with novel virulence patterns. While effective fungicides are available, the use of resistant cultivars provides a more economical and environmentally sustainable means of control. Disease nurseries at Brandon, MB, Saskatoon, SK and Melfort, SK have been used to evaluate barley breeding lines for spot blotch resistance. A differential set was grown in all locations over years 2007-2013 which indicated that local pathogens showed similar virulence patterns, with some level of differentiation on specific lines. While six-rowed barley generally possesses better resistance to this disease than two-rowed barley, recently two-rowed cultivars with excellent spot blotch resistance have been registered (Cerveza and AAC Synergy). Resistance exhibited by these two-rowed cultivars is comparable to that observed in resistant six-rowed 
barleys. Cultivars with improved resistance will provide producers in the eastern prairie region with a more suitable option for barley production.

Physiologic races explained the recent outbreaks of orange rust disease on major sugarcane cultivars in Brazil

A. URASHIMA (1), C. Sakuno (1), F. Piotto (2)

(1) Universidade Federal de Sao Carlos, Araras, Brazil; (2) Esalq/USP, Piracicaba, Brazil

Phytopathology 104(Suppl. 3):S3.121

Orange rust of sugarcane (Puccinia kuehnii) was first reported in Brazil in 2009. The most important measure to control this new disease was the identification of susceptible cultivars and their discontinuation. Nevertheless, severe epidemics were observed on SP81-3250 in 2012, the second most important cultivar in the Country, and in 2013/14 on CTC15, the most promising one, showing the success of resistant varieties is dependent of the knowledge of pathogen diversity. Because existence of physiological races in $P$. kuehnii in Brazil has not been examined yet the cause of recent breakdown of resistant cultivars is still a mystery. Therefore, this work aimed to examine the existence of races of $P$. kuehnii in Brazil. Pathogen isolates were obtained from RB72454, SP81-3250, SP84-2025, CTC 15, and RB995271. Inoculations were done by spraying $50 \mathrm{ml}$ suspension $\left(1 \times 10^{5}\right.$ spores $/ \mathrm{ml}$, germination 40\%) on four weeks plants of RB72454, RB855156, RB867515, RB92579, RB935744, RB975201, SP813250, and SP891115 and keeping them in water saturated atmosphere for $20 \mathrm{~h}$ at $25^{\circ} \mathrm{C}$. Cultivar reactions were evaluated 14 days after inoculation through leaf area damaged by image analysis with Assess software. Our work identified physiologic races because different reaction occurred between isolates from SP84-2025 and CTC15, RB72454, and SP81-3250. Moreover, diverse races were detected between $P$. kuehnii from SP81-3250 and CTC15 because the first caused symptoms on RB72454 while CTC15 could not.

Characterization of soybean resistance to Pythium aphanidermatum

K. E. URREA (1), J. C. Rupe (1), C. S. Rothrock (1), P. Chen (1)

(1) University of Arkansas, Fayetteville, AR, U.S.A.

Phytopathology 104(Suppl. 3):S3.121

Pythium spp. are important seed and seedling disease pathogens of soybean in Arkansas. Control of these pathogens is primarily through fungicide seed treatments, but resistance to seedling diseases caused by Pythium spp. has been identified in the soybean cultivar 'Archer'. To characterize and genetically map this resistance to Pythium aphanidermatum, $84 \quad \mathrm{~F}_{2: 7}$ recombinant inbreed lines (RIL) from an 'Archer' $\mathrm{X}$ 'Hutcheson' cross were evaluated for resistance to $P$. aphanidermatum by using seed plate and greenhouse assays. In the seed plate assay, ten seed were placed on water agar plates with $P$. aphanidermatum and incubated at room temperature. After seven days, the percent seed germination was determined. In the greenhouse assay, vermiculite was infested with $130 \mathrm{cfu} / \mathrm{g} \mathrm{or}^{3}$ of $P$. aphanidermatum and placed in $16 \mathrm{oz}$. pots and planted with ten seeds. After 14 days in the greenhouse at $28^{\circ} \mathrm{C}$, plant stands were taken. Both tests were repeated. In the seed plate assay, germination ranged from 4 to $85 \%$ and, in the greenhouse assay, stands ranged from 21 to $94 \%$. Archer and Hutcheson averaged germinations of 37 and $4 \%$, and stands of 73 and $21 \%$ for the seed plate and greenhouse assays, respectively. Results of single nucleotide polymorphisms (SNP) analysis using a 6K SNP chip will be presented.

Evaluation of new non-fumigant nematicides for control of root-knot nematodes in Ontario, Canada

D. J. VAN DYK (1), K. Jordan (1), M. R. McDonald (1)

(1) University of Guelph, Guelph, ON, Canada

Phytopathology 104(Suppl. 3):S3.121

Due to the loss of registered fumigants in Canada, damage to carrots (Daucus carota L., subsp. sativus (Hoffm) Arcang) caused by northern root-knot nematode (Meloidogyne hapla Chitwood) has become a constraint to production. Further regulations for soil fumigant applications will come into force in 2014, so it is important to identify new non-fumigant nematicides. A growth room trial was conducted using muck soil ( $\mathrm{pH} \sim 6.8$, organic matter $69.4 \%$ ) inoculated with Meloidogyne hapla at 43,750 eggs per experimental unit. The treatments were metam sodium at $210 \mathrm{~L}$ a.i./ha, Dazitol at $59 \mathrm{~L}$ a.i./ha, MustGrow at $840 \mathrm{~kg}$ a.i./ha, spirotetramat at $685 \mathrm{~g}$ a.i./ha, abamectin at $2 \mathrm{~L}$ a.i./ha, and fluensulfone at $4 \mathrm{~kg}$ a.i./ha. Inoculated and non-inoculated checks were included. Carrots cv. Cellobunch, were seeded into conetainers in a randomized complete block design with 20 plants per rep and grown at $24^{\circ} \mathrm{C}$ with a 10 hour photoperiod. Dazitol, MustGrow, and foliar-applied spirotetramat did not reduce nematode damage compared to the inoculated check. The grower standard metam sodium effectively reduced nematode damage $(13.0 \%)$ on carrots and had a larger average root weight $(4.6 \mathrm{~g})$ than the untreated, inoculated check $(92.3 \%, 2.6 \mathrm{~g}$ respectively). Fluensulfone and aba- mectin also reduced gall severity $(1.3,1.2)$ compared to the untreated, inoculated check (3.0). Field trials were conducted in 2013 and will be repeated in 2014 to determine the efficacy of these products under field conditions.

Occurrence of different pathotypes of Synchytrium endobioticum in Europe, and detection by molecular methods

G. C. VAN LEEUWEN (1), P. C. van Rijswick (1), B. T. van de Vossenberg

(1), M. Westenberg (1)

(1) NPPO the Netherlands, Wageningen, Netherlands

Phytopathology 104(Suppl. 3):S3.121

Synchytrium endobioticum causes potato wart disease, and a number of pathotypes have been reported. Identification of pathotypes relies on a bioassay using a set of differential cultivars as described in the EPPO Diagnostic Protocol (DP). At the moment there are four dominating pathotypes recognised in Western Europe : pathotype 1(D1), 2(G1), 6(O1) and 18(T1). In recent years the standardised set of differentials was re-evaluated in a ring test using the Glynne-Lemmerzahl method including five laboratories in three different countries (Poland, Germany, the Netherlands). For the pathotypes 2(G1) and 6(O1), in some labs the cultivars Miriam and Delcora reacted not according to the rating in the EPPO DP. Recent results of an international European project (Euphresco) showed that the cultivar Talent is suitable to replace cv Miriam (to separate pathotype 6(O1) from 18(T1)). As results from the bio-assay are only available after $6-8$ weeks, a PCR test was developed to discriminate pathotype 1(D1) from the other pathotypes. Within the Euphresco-project this test was ring-tested in a test performance study (TPS) including ten labs in Europe. The results will be presented in August in the meeting in Minneapolis (results not yet available).

Development of efficient and reliable Candidatus Liberibacter asiaticus detection methods in citrus

O. VAZQUEZ (1), S. Chavez (1), M. Kunta (1), E. Braswell (2), M. L. Keremane (3), R. F. Lee (3), E. S. Louzada (1)

(1) Texas A\&M University Kingsville, Weslaco, TX, U.S.A.; (2) USDA APHIS, Edinburg, TX, U.S.A.; (3) USDA ARS National Clonal Germplasm Repository for Citrus \& Dates, Riverside, CA, U.S.A.

Phytopathology 104(Suppl. 3):S3.121

Huanglongbing (HLB), a disease with no known cure, has crippled citrus industries worldwide, and currently poses a serious threat to the survival of Texas citrus. HLB is associated with the phloem-limited Gram negative alphaproteobacteria, Candidatus Liberibacter spp. The bacterium is unevenly distributed in the tree canopy and disease symptoms are commonly confused with general nutrient deficiencies and environmental stress which contribute to difficulty in identification. We found that roots serve as a more reliable diagnostic sample compared to leaves. The purpose of this study is to develop an early and reliable detection method for $\mathrm{Ca}$. L. asiaticus (CLas) using root samples as well as to understand the distribution of CLas within the host plant roots. Fibrous roots were collected from five HLB-infected trees at many points; the samples include surface roots which run horizontally up to 6 meters from the trunk and roots growing vertically down up to $1.5 \mathrm{~m}$ in depth. Several DNA extraction and DNA amplification methods including conventional PCR (cPCR), quantitative PCR (qPCR), and Loop mediated isothermal amplification (LAMP) were employed in root and leaf samples to determine their efficacy in CLas early detection. Results obtained to date shows that sample preparation and analysis of roots can be high throughput. Comparison of qPCR and LAMP for their efficacy in CLas detection in washed versus non-washed roots showed no difference.

Baseline sensitivity of tangerine-infecting Alternaria alternata to boscalid and molecular characterization of the SDH complex

B. VEGA (1), M. M. Dewdney (1)

(1) University of Florida, Lake Alfred, FL, U.S.A.

Phytopathology 104(Suppl. 3):S3.121

Altenaria brown spot (ABS) affects leaves, twigs, and young fruit, reducing fruit quality of fresh fruit tangerines in Florida. Disease control has depended on fungicide applications, but with the emergence of quinone outside inhibitor (QoI) resistance in A. alternata populations, the use of new chemical options is paramount. Boscalid, a succinate dehydrogenase inhibitor (SDHI), was registered in 2011 to control ABS. The objective of this study was to determine baseline sensitivity to boscalid in A. alternata isolates collected over multiple years from 14 Florida counties. When assessed, conidia germination and mycelial growth were not completely suppressed even at high concentrations. The most consistent effective concentration to inhibit $50 \%$ growth $\left(\mathrm{EC}_{50}\right)$ was found with the resazurin-based microtiter test. The mean and median $\mathrm{EC}_{50}$ values of 419 isolates were 0.60 and $0.44 \mu \mathrm{g} / \mathrm{ml}$, respectively. The mean $\mathrm{EC}_{50}$ values of isolates from different cultivars were not significantly different $(P=0.1350)$. Similarly, the mean $\mathrm{EC}_{50}$ values of 
isolates from different virulence classes were not significantly different $(P=$ $0.1888)$. The SDH complex ( $S d h B, S d h C$, and $S d h D$ genes) was partially sequenced. Polymorphism in all SDH subunits was found, but especially in SDHD where 12 mutations were found in 13 of the 15 isolates analyzed but no mutations conferring resistance were found. It is concluded that $A$. alternata populations of Florida are currently sensitive to boscalid.

Regulation of the mevalonate pathway by symbiotic receptor-like kinases and its role in early symbiotic signaling

M. VENKATESHWARAN (1), D. Jayaraman (2), B. K. Riely (3), E. Larrainzar (3), D. R. Cook (3), J. M. Ane (2)

(1) School of Agriculture, University of Wisconsin-Platteville, Platteville, WI, U.S.A.; (2) Department of Agronomy, University of Wisconsin-Madison, Madison, WI, U.S.A.; (3) Department of Plant Pathology, University of California-Davis, Davis, CA, U.S.A.

Phytopathology 104(Suppl. 3):S3.122

HMGRs (3-hydroxy-3-methylglutaryl coenzyme A reductases) are key enzymes in the mevalonate pathway controlling isoprenoid biosynthesis. Surprisingly, one of these enzymes (HMGR1) was found to interact with the symbiotic receptor-like kinase DMI2 and is required for legume nodulation in the model legume Medicago truncatula. Using split-ubiquitin assays, interactions between HMGR1 and two other symbiotic receptor-like kinases, NFP and LYK3, were found. In vitro kinase assays revealed that HMGR1 is phosphorylated by DMI2 but not by NFP or LYK3. Enzymatic assays revealed that HMGR1 activity is affected by interaction with DMI2. Mimicking phosphorylation by serine to aspartic acid substitutions at the phosphorylation sites also affected HMGR1 enzymatic activity. HMGRIsilenced roots were impaired for nuclear calcium spiking and symbiotic gene expression. Reciprocally, application of mevalonate, the product of HMGR1 activity, was sufficient to induce calcium spiking and symbiotic gene expression in wild-type and $H M G R 1$-silenced roots. Mevalonate was able to induce nuclear calcium spiking and ENOD11 expression in $d m i 2$ but not in dmil mutants. These results indicate that HMGR1 plays an early role in the symbiotic signaling cascade after DMI2 and before the nuclear cation channel DMI1. We hypothesize that HMGR1 connects signaling events at the plasma membrane level to nuclear ones by generating second messengers controlling downstream symbiotic signaling.

Seasonal variation in ascospore release by Oculimacula yallundae and $\boldsymbol{O}$. acuformis in the U.S. Pacific Northwest

D. I. VERA (1), T. D. Murray (1)

(1) Washington State University, Pullman, WA, U.S.A.

Phytopathology 104(Suppl. 3):S3.122

Eyespot disease of wheat is caused by Oculimacula yallundae (OY) and $O$. acuformis (OA). The teleomorphs of these fungi were recently reported in the U.S. Pacific Northwest, but their role in eyespot epidemiology is unknown. Ascospores of OY and OA were monitored in two inoculated field plots near Pullman, WA in 2012 and 2013. Winter wheat was sown in alternate plots in Sep 2010 and 2011, respectively, and inoculated with conidia of compatible mating types of both species in Nov 2010 and 2011. Plots were kept in stubble for 1 year after harvest and then replanted with winter wheat. Ascospores were monitored with Burkard volumetric spore samplers; DNA was extracted from sticky tapes and real-time PCR used to quantify DNA which was converted to number of ascospores. In both 2012 and 2013, the greatest number of Oculimacula ascospores was detected in Jun with 3,360 and 1,640 ascospores, respectively, followed by Apr-May 2013 with 625 ascospores, and Oct-Nov 2012 and 2013, with over 300 ascospores collected each year. More OA than OY ascospores were trapped in both years, with 3,282 and 3,954 OA and 583 and 2,808 OY ascospores in 2012 and 2013, respectively. There was no difference in number of ascospores captured between fields in crop or stubble. Based on these data, two main ascospore release periods occur; one each in spring and fall during and after rainfall events. Ascospores released in fall may have an important role as primary inoculum in addition to conidia.

Optimized infestation control and mycotoxin reduction strategies against Fusarium diseases of corn and wheat

J. A. VERREET (1), T. Birr (1), H. Klink (1)

(1) University of Kiel, Kiel, Germany

Phytopathology 104(Suppl. 3):S3.122

Epidemic and damage dynamics of 13 Fusarium species on wheat (3 different susceptible varieties, 2008-2013) and corn (4 different susceptible varieties, 2011-2013) were recorded at 9 locations in Germany. In addition to yield factors, disease severity were analysed by q-PCR and the $F$. toxins deoxynivalenol (DON) and zearalenone (ZEA) were analysed by LC/MS. The same $F$. spp. spectrum was detected in wheat and corn. Based on year-long national results, a head blight forecast model for the optimized control was derived for wheat. There was a close relationship between rainfall and temperature at flowering (infection parameters) on the one hand and Fusarium DNA $\left(R^{2}=0.78\right)$, DON $\left(R^{2}=0.80\right)$, and ZEA content $\left(R^{2}=0.76\right)$ of the grain on the other. The correlation between mycotoxin contents and Fusarium DNA at harvest and predicted values which were calculated on temperature and precipitation at flowering document the high quality of the model $\left(\mathrm{DON} \mathrm{R}{ }^{2}=\right.$ 0.85 , ZEA R ${ }^{2}=0.86$, Fusarium DNA R ${ }^{2}=0.83$ ). Both in susceptible and tolerant corn varieties, maximum values of $>26000$ micrograms DON and $>$ 2000 micrograms ZEA per kg dry mass were measured in no-till cropping systems with monoculture. Plowing reduced DON content by $82 \%$ and ZEA content by $65 \%$. The triazole fungicides metconazole, prothioconazole, and tebuconazole combined with strobilurines reduced mycotoxin levels in wheat by $\geq 60 \%$, and in corn by up to $90 \%$ when applied at flowering and panicle stage, respectively.

The impact of difenoconazole application timing on the difenoconazole sensitivity of a DMI-resistant Venturia inaequalis population

S. M. VILLANI (1), K. D. Cox (1), Z. A. Frederick (2)

(1) Cornell University, Geneva, NY, U.S.A.; (2) Washington State University, Pullman, WA, U.S.A.

Phytopathology 104(Suppl. 3):S3.122

Application of highly specific fungicides has traditionally been reserved for controlling apple scab and powdery mildew during critical infection periods in the northeastern United States. Heightened concerns over summer diseases on apple, however, has prompted growers to apply recently released fungicides such as difenoconazole later in the growing season. Unfortunately, the use of difenoconazole for summer disease control may select for Venturia inaequalis populations with decreased sensitivity to DMIs the following season. From 2010 to 2012, difenoconazole was applied to single tree replicates of 'Empire' during the scab season (4 applications, tight cluster -2 nd cover) and/or the summer season (4 applications, 3rd - 6th cover). The year following application, in vitro sensitivity to difenoconazole was evaluated for $V$. inaequalis isolates $(\mathrm{n} \geq 25)$ collected from each fungicide treatment block. Isolates exposed to difenoconazole only during the scab season were significantly less sensitive to difenoconazole in vitro than untreated trees in 2011 and $2012(P<0.05)$. In 2013, isolates from plots treated with difenoconazole during the scab season were significantly less sensitive to difenoconazole than isolates from both the untreated treatment and from the summer season treatments. These results suggest that summer applications of difenoconazole are no more likely to promote selection towards difenoconazole resistance than traditional scab season applications.

Multilocus analysis reveals phylogenetic placement of novel ERI fungi within Magnaporthaceae and Phaeosphaeriaceae of Ascomycota P. L. VINES (1), M. Tomaso-Peterson (1), T. W. Allen (2), F. Hoffmann (1)

(1) Mississippi State University, Mississippi State, MS, U.S.A.; (2) Delta Research and Extension Center, Stoneville, MS, U.S.A.

Phytopathology 104(Suppl. 3):S3.122

Ultradwarf bermudagrasses, in the low latitudinal region of the United States, often exhibit symptoms of decline during late summer and early fall months. Root systems appear diminutive, brittle, and discolored and are frequently colonized with dark, runner hyphae that are characteristic of ectotrophic rootinfecting (ERI) fungi. Currently, spring dead spot (Ophiosphaerella spp.) and bermudagrass decline (Gaeumannomyces graminis var. graminis) are the only recognized diseases of ultradwarf bermudagrasses caused by ERI fungi. Observations from this study led to the theory that additional ERI fungi are accountable for compromised ultradwarf bermudagrass roots. Due to laborious and inconclusive identification efforts associated with traditional diagnostic techniques, molecular-based strategies were employed to test the hypothesis. Multilocus sequence analysis was performed on representative Mississippi State University (MSU)-ERI isolates and related taxa. Phylograms were erected using combined consensuses from maximum parsimony, maximum likelihood, and Bayesian inference analyses. These assessments indicate that MSU-ERI isolates share common ancestry with the known ultradwarf bermudagrass pathogens, Gaeumannomyces graminis var. graminis and Ophiosphaerella spp. In addition, evolutionary analyses define relatedness between MSU-ERI isolates and Magnaporthiopsis spp., indicating the discovery of novel fungi associated with decline of ultradwarf bermudagrasses.

Distribution of ectotrophic root-infecting fungi associated with declining ultradwarf bermudagrass putting greens

P. L. VINES (1), M. Tomaso-Peterson (1), T. W. Allen (2), C. Balbalian (1), B. R. Stewart (1)

(1) Mississippi State University, Mississippi State, MS, U.S.A.; (2) Delta Research and Extension Center, Stoneville, MS, U.S.A.

Phytopathology 104(Suppl. 3):S3.122 
Samples of ultradwarf bermudagrasses, exhibiting symptoms of decline during late summer and early fall months, were collected in 2012 and 2013 throughout the southern region of the United States. Root systems appeared blackened, rotted, and diminutive in size and were frequently colonized with dark, runner hyphae that are characteristic signs of ectotrophic root-infecting (ERI) fungi. Colonized roots were cut into $1-\mathrm{cm}$ sections, surface disinfested with a $0.6 \% \mathrm{NaOCl}$ solution, rinsed three consecutive times with steriledistilled water, plated on modified potato dextrose agar (PDA), and incubated for 7 days at room temperature under 24 hour fluorescent light. Hyphal tips were transferred to PDA, resulting in sterile, pure cultures, from which genomic DNA was extracted. Multilocus sequence analyses revealed novel phylogenetic placement of MSU-ERI isolates within the Magnaporthaceae and Phaeosphaeriaceae families of the Ascomycota. Geographical distribution assessments demonstrated the presence of a single ERI fungal species at multiple locations throughout the southern United States. Additional assessments confirmed the underlying hypothesis that multiple ERI fungal species are present at a given geographical location.

\section{Construction of a bioluminescent Erwinia tracheiphila}

C. M. VRISMAN (1), S. A. Miller (1), G. Rajashekara (1)

(1) Ohio State University, OARDC, Wooster, OH, U.S.A.

Phytopathology 104(Suppl. 3):S3.123

Erwinia tracheiphila is the causal agent of bacterial wilt of cucurbits. It is considered one of the most destructive diseases of cucurbits in the Midwestern United States. Young plants can be killed within one week after infection. We developed bioluminescent $E$. tracheiphila strains to study the routes of entry of the pathogen into plants and its colonization of the host. E. tracheiphila strain TedCu10 was transformed by electroporation, using the previously constructed transposon vectors $\mathrm{pXX}-2$ and $\mathrm{pXX}-3$ carrying lux operon optimized for Gram positive and Gram negative bacteria, respectively, as well as the plasmid pBBRMCS-1/lux. The pXX-2 and pXX-3 vectors carry the

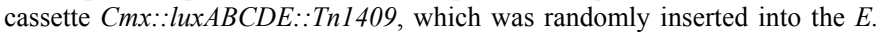
tracheiphila chromosome. In total, 259 chloramphenicol resistant colonies were recovered. Transformation of E. tracheiphila TedCu10 with the plasmid pBBRMCS-1 was efficient but not all transformants were stable for bioluminescence. The presence of the lux genes in E. tracheiphila will allow real time imaging of the pathogen colonization of host plants. Virulent and constitutively bioluminescent strains will be selected. Epiphytic survival of bioluminescent $E$. tracheiphila and its active colonization of cucurbits through leaf wounds and nectaries will be visualized using real time in planta biophotonic imaging system equipped with a sensitive CCD camera.

The effect of spirotetramat (Movento ${ }^{\circledR}$ ) against reniform nematode, Rotylenchulus reniformis, on pineapple, Ananas comosus

P. WAISEN (1), B. Sipes (1)

(1) University of Hawaii at Manoa, Honolulu, HI, U.S.A.

Phytopathology 104(Suppl. 3):S3.123

Reniform nematode, Rotylenchulus reniformis is an economically important plant-parasitic nematode of pineapple. This pathogen can reduce pineapple marketable yield by $26.8-50 \%$. Lack of host resistance in pineapple raises a need for nematode management. Spirotetramat is a group 23 insecticide, a lipid biosynthesis inhibitor with ambimobile translocation. Lipids play a significant physiological role in embryogenesis and molting of plant-parasitic nematodes therefore inhibition of lipid biosynthesis may provide a critical management tool. The objective of this study was to determine if spirotetramat is active against reniform nematode. Greenhouse bioassays were conducted where pineapple crowns were planted in steam-sterilized soil in 20$\mathrm{cm}-\mathrm{d}$ biodegradable pots. Approximately 3,000 R. reniformis eggs were inoculated onto 4-month-old plants. Four levels $(0,88,100$ and $175 \mathrm{~g}$ a.i/ha) of spirotetramat were applied 1 month post inoculation. Plants were harvested 6 months later for shoot and root weights, and a $250 \mathrm{~cm}^{3}$ soil sample collected. Data were normalized by $\log 10$ transformation and subjected to analysis of variance. The maximum nematode reduction was $64 \%$ at $88 \mathrm{~g}$ a.i. spirotetramat/ha rate with a corresponding reduction in dry root weight of $14.4 \%$ and an increase in shoot weight of $1.4 \%$ compared to the water treatment control. The $88 \mathrm{~g}$ a.i. spirotetramat/ha rate gave promising reductions in nematode numbers while promoting plant growth.

Whole genome sequencing and comparative genomics of multiple $F$. graminearum isolates

S. WALKOWIAK (1), R. Subramaniam (2), L. Wang (2), O. Rowland (1)

(1) Carleton University, Ottawa, ON, Canada; (2) Agriculture and Agri-Food Canada, Ottawa, ON, Canada

Phytopathology 104(Suppl. 3):S3.123

The $F$. graminearum species complex is the principal cause of Fusarium head blight in North America and results in severe losses in the yield and quality of cereals. New challenges are beginning to develop in the management of these pests; different field isolates have variations in both the toxins they produce as well as their pathology in wheat. There is growing concern surrounding the emergence and spread of some highly virulent isolates that produce the toxin 3 -acetyldeoxynivalenol (3ADON), which may be displacing the less virulent 15 -acetyldeoxynivalenol producing isolates $(15 \mathrm{ADON})$. In addition to these differences, our HPLC results indicate that culture conditions used to induce $15 \mathrm{ADON}$ production are unable to induce $3 \mathrm{ADON}$ production. Although some of the genetic mechanisms concerning these differences among isolates are known, many of them remain to be elucidated. This project focuses on characterizing the genetic differences between isolates within the $F$. graminearum species complex to identify the lineage specific regions that lead to differences in toxin production and pathology. We performed whole genome sequencing (Illumina) and comparative genomics of eight different isolates from the $F$. graminearum species complex, which have reported differences in toxin production and pathology in wheat. The genomes have been assembled with a coverage $>35$ fold. Several unique genomic features as well as regions of high nucleotide variability have been identified.

In silico identification and analysis of microsatellite location and frequency in downy mildew transcriptomes

E. C. WALLACE (1), L. M. Quesada-Ocampo (1)

(1) North Carolina State University, Raleigh, NC, U.S.A.

Phytopathology 104(Suppl. 3):S3.123

The group of oomycetes causing downy mildew (DM) includes several genera of economically important plant pathogens. Microsatellites, or Simple Sequence Repeats (SSRs), are commonly used to characterize populations and determine genetic variation. Identifying and characterizing SSR markers in coding regions would facilitate genetic studies in DMs by providing annotated polymorphisms. Whole-genome transcripts for Pseudoperonospora cubensis and Hyaloperonospora arabidopsidis are publicly available. The MIcroSAtellite identification tool (MISA) was used to extract locations and frequencies of 2-6 bp repeats from the transcriptomes of both pathogens. The percentage of SSRs in examined sequences was significantly different between $P$. cubensis (12\% of 23,522 transcripts) and H. arabidopsidis $(15 \%$ of 14,548 transcripts). Most identified SSRs were trimers, making up $61 \%$ and $71 \%$ of total SSRs from P. cubensis and H. arabidopsidis, respectively. The percentage of di- and tri- nucleotide SSRs was significantly different between species, but the percentage of tetra-, penta- and hexa- nucleotide SRRs were not. In addition, $73 \%$ of the fifteen most common motifs were the same in both transcriptomes. The most common repeat motif in both species was AGC/CTG. This SSR analysis revealed shared as well as species-specific transcriptome features of analyzed DMs. As further DM genomes and transcriptomes are released, detailed comparative genomics studies will be possible.

Response of blueberry and cranberry fruit rotting fungi to host plant floral extracts

T. J. WALLER (1)

(1) Rutgers University, Chatsworth, NJ, U.S.A.

Phytopathology 104(Suppl. 3):S3.123

Open flowers and concentrated bloom periods are critical times for fruit rot control in blueberries and cranberries as well as several other horticultural crops. It is reasonable to hypothesize that plant signals produced during bloom play a critical role in the infection processes of, at least, some fruit rotting fungi. In this project, we investigate the effects of water-soluble flower extracts (FE) on the development of disease in the fruit rotting fungi Coleophoma empetri, Colletotrichum acutatum and Colletotrichum gloeosporioides. Conidial suspensions treated with FE demonstrated increased appressorium formation in vitro as well as increased virulence on detached fruit. A comparison of FE derived from susceptible and resistant cultivars of blueberry suggests a significant difference in the rate of appressorium formation that correlates with field resistance. Time course experiments show that inclusion of FE in an infection droplet can significantly increase the rate of spore germination and appressorium formation. Therefore, FE may provide a key stimulus that reduces the minimum wetness period needed to initiate infection. Given these data, we hypothesize that FE are a viable target for disease control and trials aimed at evaluating bacteria to offset the stimuli of $\mathrm{FE}$ and reduce disease incidence are underway.

Phylogenetic assessment of Colletotrichum species associated with bitter rot and Glomerella leaf spot in the northeastern U.S.

M. WALlHEAD (1), G. Broders (1), E. Beaudoin (1), C. Peralta (2), K. Broders (1)

(1) University of New Hampshire, Durham, NH, U.S.A.; (2) Universidad del

Tolima, Durham, NH, U.S.A.

Phytopathology 104(Suppl. 3):S3.123 
Three taxa, Colletotrichum gloeosporioides, C. acutatum, and Glomerella cingulata, are currently associated with bitter-rot of apple (Malus domestica) with $C$. acutatum typically being the dominant species found in the northeastern United States. However, a recent phylogenetic study demonstrated that both $C$. gloeosporioides and $C$. acutatum are species complexes and over 10 distinct species used in these study were recovered from apple. Therefore, the objective of this study was to complete a phylogenetic analysis to determine how many and which species of Colletotrichum are associated with bitter-rot and Glomerella leaf spot in the northeastern U. S. Isolates were recovered from apple leaves and fruits collected between the 2011-2013 growing seasons from various locations in Massachusetts, New Hampshire and New York. The multi-gene phylogenetic analysis was completed using ITS, $\beta$-tubulin and GADPH gene sequences. Preliminary results indicate that species diversity on apple was limited as $C$. fioriniae was the dominant species recovered from both apple leaves and fruit in the northeastern U. S. This study demonstrates that C. fioriniae is the primary pathogen causing both bitter rot and Glomerella leaf spot in the northeast. Future research will focus on the epidemiology and ecology of the infection process of $C$. fioriniae as all current data was generated for $C$. acutatum, which is composed of multiple species.

Comparison of vegetation indices produced by two spectrometers: A hyperspectral assessment of Malus domestica leaves

M. WALLHEAD (1), K. Broders (1)

(1) University of New Hampshire, Durham, NH, U.S.A.

Phytopathology 104(Suppl. 3):S3.124

Despite the presence of various molecular techniques for the detection of plant pathogens there remains a demand for a fast, sensitive, and selective method for the detection of plant pathogens during the early stages of infection in order to minimize economic losses and maximize crop yield. This demand may be met through ground-based and low-altitude aerial-based remote sensing of crops. The objective of this research was to evaluate the usefulness of field spectrometers for monitoring orchard health by comparing the vegetation indices produced by scanning sets of apple leaves (Malus domestic) using the ASD FieldSpec4 and VIRIS side-by-side using a fixed light source under laboratory settings. A $5^{\circ}$ optic was attached to the ASD FieldSpec4 and mounted next to the optical sensor of the VIRIS and leveled. Three stacks of seven healthy leaves were scanned with three scans per leaf taken at 0,90 and $270^{\circ}$. Field experiments are in progress to ground truth laboratory results. The results of this study indicate that the ASD FieldSpec4 and the VIRIS are capable of producing similar vegetation index values when used in a standardized laboratory setting, which indicates that the ASD should be able to generate the same spectral indices when used outdoors in addition to being easily transported and convenient to use under field conditions.

Effects of a selenium-laden soil amendment on grapevine metabolism and progression of Pierce's disease

C. WALLIS (1), T. Centofanti (2), G. Banuelos (1)

(1) USDA-ARS San Joaquin Valley Agricultural Sciences Center, Parlier, CA, U.S.A.; (2) California State University- Fresno, Center for Irrigation Technology, Fresno, CA, U.S.A.

Phytopathology 104(Suppl. 3):S3.124

Selenium containing soil amendments might be beneficial to growers as selenium may increase resistance to certain plant pathogens and pests. Therefore, grapevines growing in soil with different amounts of seleniumladen amendment were evaluated for selenium effects on plant metabolism and susceptibility to Pierce's disease (caused by the bacterium Xylella fastidiosa). The selenium amendments had few effects on grapevine amino acid and sugar levels. Levels of the phenolic compounds caftaric acid, procyanidin $\mathrm{B} 2$, epicatechin gallate, and catechin gallate were significantly greater in plants grown in soil with greatest selenium levels than those with less. However, phenolic levels were similar between grapevines grown with the most selenium and those grown with no added selenium. Grapevines with low and moderate levels of added selenium had greater amounts of the terpenoids camphene and linalool than grapevines grown with no added selenium. PD symptom severity was not significantly different among grapevines grown in soils with different levels of selenium. These results exhibit the ability of selenium soil amendments to change grapevine host metabolite levels. However, selenium soil amendment did not affect PD symptom progression.

Potatoes tolerant of 'Candidatus Liberibacter solanacearum' infection do not undergo changes in tuber physiology associated with zebra chip disease

C. WALLIS (1), J. Munyaneza (2), R. Novy (3)
(1) USDA-ARS San Joaquin Valley Agricultural Sciences Center, Parlier, CA, U.S.A.; (2) USDA-ARS Yakima Agricultural Research Laboratory, Wapato, WA, U.S.A.; (3) USDA-ARS Small Grains and Potato Germplasm Research Unit, Aberdeen, ID, U.S.A.

Phytopathology 104(Suppl. 3):S3.124

Zebra chip disease (ZC), caused by the bacterium 'Candidatus Liberibacter solanacearum' (Lso), is of increasing concern to potato growers throughout the western United States and elsewhere. New potato cultivars are being developed that are tolerant of Lso and therefore infected tubers do not express ZC symptoms. Previous studies have observed that ZC symptoms are associated with increased tuber levels of amino acids, sugars, and phenolics. Therefore, this study examined whether or not increases of amino acids, sugars, and phenolics occurred in Lso-infected commercial or potentially ZCtolerant potato cultivars. Lso-infected tubers from the commercial, susceptible potato variety 'Atlantic' exhibited strong ZC symptoms. Levels of many amino acids, sugars, and phenolics were greater in infected 'Atlantic' tubers compared to those that were non-infected. By contrast, Lso-infected tubers from three tolerant cultivars, designated ' $\mathrm{M} 5$ ', 'ZC73', and ' $\mathrm{ZC} 74$ ', did not exhibit ZC symptoms. Levels of amino acids, reducing sugars, and phenolics were not significantly different between non-infected and Lso-infected tubers from the three tolerant cultivars. These results provide evidence that potato cultivars that do not have physiological changes in response to Lso infection are unlikely to develop ZC symptoms. Thus, tolerance, rather than immunity, may be sufficient to mitigate effects of ZC disease.

\section{Root-knot nematode parasitism suppresses host gene silencing}

E. WALSH (1), C. G. Taylor (1)

(1) Ohio State University, Wooster, OH, U.S.A.

Phytopathology 104(Suppl. 3):S3.124

Root-knot nematodes (RKN, Meloidogyne spp.) cause significant damage to several crops worldwide. After entering a root, RKN modify normal root cells into giant cells, from which they acquire all the nutrition necessary to complete their lifecycle. The ability of RKN to develop these giant cells raises interest into which of the host's pathways are influenced to make this interaction possible. Gene silencing pathways in plants serve many roles, from directing proper growth and development to functioning in antiviral defense. It is now known that gene silencing is involved in many other plant-microbe interactions [Katiyar-Agarwal and Jin, 2010]. Based on recent findings, we hypothesize that RKN actually suppress host gene silencing pathways in order to successfully parasitize their host. In order to test this hypothesis we first generated multiple transgenic $N$. tabacum lines expressing a silenced reporter gene. It is evident that the silenced reporter genes are recovered specifically within giant cells, indicating the disruption of these pathways. We then tested the hypothesis that if this disruption were important, a plant that is compromised in these pathways will be more susceptible to RKN infection. Using a transgenic $N$. tabacum expressing a viral suppressor protein, which disrupts these pathways, we observe an increase in parasitism. Better insight into how RKN influence host gene silencing will help elucidate their role during parasitic interactions.

Resistance the ultimate solution for bacterial panicle blight disease of rice Y. WAMISHE (1), Y. Jia (2), M. Rasheed (1), C. Kelsey (3), S. Belmar (3), T. Gebremariam (1)

(1) University of Arkansas Coop Ext Svc, Stuttgart, AR, U.S.A.; (2) USDA ARS, Stuttgart, AR, U.S.A.; (3) University of Arkansas, Stuttgart, AR, U.S.A. Phytopathology 104(Suppl. 3):S3.124

Bacterial panicle blight (BPB) has been observed in rice production fields of Arkansas and other southern states with increasing frequency since 1995. BPB was severe in Arkansas in 2010 and 2011 causing up to 50\% yield loss. A study was initiated to understand Burkholderia glumae, the major causal agent and to evaluate rice germplasm for resistance. B. glumae was frequently isolated from symptomatic panicles on CCNT. B. glumae isolates found to produce a hypersensitive reaction on tobacco leaves and pathogenic on rice seedlings were used to inoculate rice in the greenhouse and to screen germplasm for resistance in the field. Seedlings in the greenhouse were needle inoculated whereas foliage spray inoculation was employed between boot split to flowering stage in the field. Lesion sizes were not consistent to differentiate resistance levels among varieties using needle inoculation in the greenhouse. In 2012, among 300 entries of the Uniform Regional Rice Nursery (URRN) and Arkansas Rice Performance Trials (ARPT) tested in field, 14 entries showed no symptom of the disease and 30 entries showed moderate resistance. In 2013, within URRN and ARPT test, 15 entries showed no symptom of the disease and 53 entries showed moderate level of resistance. In the absence of effective chemical and cultural methods currently, the use of resistance would be the best option to combat such a sporadic disease that reduces yield and quality of rice. 
Stripe rust epidemics of wheat and barley and races of Puccinia striiformis identified in the United States in 2013

A. WAN (1), X. Chen (2)

(1) Washington State University, Pullman, WA, U.S.A.; (2) USDA ARS, Pullman, WA, U.S.A.

Phytopathology 104(Suppl. 3):S3.125

In 2013, stripe rust of wheat, caused by Puccinia striiformis f. sp. tritici (Pst), occurred widely in the U.S., but generally at relatively low levels in most areas. The disease was severe in the south-central states causing fairly significant yield losses, but had limited development in the Pacific Northwest due to unfavorable spring and summer conditions plus early fungicide application in the pathogen winter-survived areas. Stripe rust of barley, caused by $P$. striiformis f. sp. hordei $(P s h)$, occurred in California, Oregon and Washington continually at low levels. Stripe rust samples collected from 28 states were tested on 18 wheat and 12 barley differentials for identifying Pst and $P$ sh races, respectively. Six previously existing $P s h$ races were detected, of which PSH-48 (virulent only on Topper of the barley differentials) and PSH-33 (virulent only on Topper and Abed Binder 12 of the differentials) were predominant with frequencies of $42 \%$ and $35 \%$, receptively. A total of 34 Pst races were detected including 8 new races. PSTv-52 (39\%), PSTv-37 (13\%), PSTv-11 (8\%), PSTv-73 (8\%), and PSTv-14 (5\%) were the top five frequent races. PSTV-52, PSTV-37 and PSTV-73 were detected in every epidemiological regions, while PSTV-11 and PSTV-14 were detected only in the western U.S. High virulence frequencies were detected for $\operatorname{Yr} 6, \operatorname{Yr} 7, \operatorname{Yr} 8$, $\operatorname{Yr}$ 9, $Y r 17, Y r 27, Y r 43, Y r 44$, and $Y r E x p 2$; moderate for $Y r 1, \operatorname{YrTr} 1$, and YrTye; low for Yr10, Yr24, Yr32, and YrSP; and none for Yr5 and Yr15.

Regional differences in genetic structure of Puccinia striiformis f. sp. tritici, the wheat stripe rust pathogen, in the U.S. revealed by SSR makers A. WAN (1), M. Wang (1), X. Chen (2)

(1) Washington State University, Pullman, WA, U.S.A.; (2) USDA ARS, Pullman, WA, U.S.A.

Phytopathology 104(Suppl. 3):S3.125

Understanding the population structure and genetic diversity in the Puccinia striiformis f. sp. tritici (Pst) population is important for developing large-scale stripe rust management strategies. A total of 322 isolates collected from various epidemiological regions throughout the U.S. in 2010 were characterized using 17 co-dominant simple sequence repeat (SSR) markers. The population in the western U.S. (regions 1- 6) has higher genetic differentiation $\left(F_{S T}=0.17\right)$ than the population in the eastern U.S. (regions 712) $\left(F_{S T}=0.04\right)$. The number of effective migrants, $N m$, was 1.3 and 6.0 in the western regions and the eastern regions, respectively. The data suggest that the population in the west is more diverse than the population in the east, or the population in the east has more frequent inoculum exchange among subpopulations than the population in the west. Analysis of Nei's genetic distances indicated that the subpopulations of regions $1,2,3,4$, and 6 were more related to each other, those of regions $7,8,9,10,11$ and 12 formed another group, while the subpopulation of region 5 (northwestern Washington) was distinct from the other two groups. The results could be attributed to the differences in resistance genes, geographic landscapes, and climatic conditions. The information should be useful for deploying different stripe rust resistance genes in different regions.

\section{Adaptation genomics of a small colony variant (SCV) in the biofilm of} Pseudomonas chlororaphis 30-84

D. WANG (1), C. S. Han (1), C.-C. Lo (1), A. E. Dichosa (1), P. S. Chain (1), J. M. Yu (2), R. J. Dorosky (2), L. S. Pierson III (2), E. A. Pierson (2)

(1) LANL, Los Alamos, NM, U.S.A.; (2) TAMU, College Station, TX, U.S.A. Phytopathology 104(Suppl. 3):S3.125

The rhizosphere-colonizing bacterium Pseudomonas chlororaphis 30-84 is an effective biological control agent against fungal take-all disease of wheat. In this study, we characterize a small-colony variant (SCV) isolated from a $P$. chlororaphis 30-84 biofilm. The SCV exhibited pleiotropic phenotypes including small cell size, slow growth and motility, low levels of phenazine production, but increased biofilm formation and resistance to antimicrobials. To better understand the genetic alterations underlying these phenotypes, RNA and whole-genome sequencing analyses were conducted by comparing a SCV mutant to the wild-type strain. Of the genome's 5,971 genes, transcriptomic profiling indicated that 1,098 (18.4\%) have undergone a significant reprograming of gene expression in the SCV mutant. In accordance with observed phenotypes, transcript levels of genes involved in metabolism, motility and phenazine production were reduced; whereas, transcripts for genes involved in oxidative stress and iron uptake were significantly increased. Whole-genome sequence analysis revealed the deletion of a $70 \mathrm{~Kb}$ genomic island in the $\mathrm{SCV}$ strain. Mutations in $y f i R$ (cyclic-di-GMP production), cyoE (heme synthesis) and fus $A$ (elongation factor) were also detected in the SCV mutant. Taken together, our data suggest that the rise of $\mathrm{SCV}$ is linked to profound genomic and transcriptomic changes. Genes identified in this study will provide novel targets for future functional genomic studies.

First detection of 'Candidatus Phytoplasma pyri' from a pear tree in Nevada

S. WANG (1), R. A. Bomberger (1), J. Rolshoven (1)

(1) Nevada Department of Agriculture, Sparks, NV, U.S.A.

Phytopathology 104(Suppl. 3):S3.125

To survey for exotic phytoplasma species such as 'Candidatus Phytoplasma australiense', 'Ca. Phytoplasma AP-MLO', ' $\mathrm{Ca}$. Phytoplasma prunorum' and other ' $\mathrm{Ca}$. Phytoplasma' species, a total of 141 plant samples were collected from diverse locations and from a range of host plant species showing symptoms that resemble those caused by phytoplasmas. To detect phytoplasma from suspected plant samples, petioles and midribs from each sample were disrupted, homogenized, and then proceeded to genomic DNA extraction using a Qiagen DNeasy plant mini kit. DNA was then subjected to a nested polymerase chain reaction (PCR) using universal primer pairs $\mathrm{P} 1 / \mathrm{P} 7$ and $\mathrm{R} 16 \mathrm{~F} 2 \mathrm{n} / \mathrm{R} 16 \mathrm{R} 2$. DNA fragments of approximately $1.2 \mathrm{~kb}$ were amplified from 64 plant samples. To confirm the identity of PCR products, amplified DNA was subcloned into $\mathrm{pGEM}^{\circledR}$-T vector and then sequenced. The majority of amplified DNA fragments were confirmed to be non-phytoplasma DNA. One sample collected from a Bartlett pear tree (Pyrus communis) exhibiting leaf yellowing in a nursery generated a positive PCR product of $1,241 \mathrm{bp}$ (Accession No. KJ542562). BLASTn search and sequence alignments revealed $99 \%$ homology to the corresponding regions of Candidatus Phytoplasma pyri isolate D764-08 reported from Slovenia (Accession No. KF849463.1). This phytoplasma is a member of apple proliferation group (16SrX) subgroup $\mathrm{C}$ according to iPhyClassifier. To our knowledge, this is the first detection of pear decline phytoplasma from Nevada.

PifHDAC7, a Class II histone deacetylase, is involved in sexual behavior determination of Phytophthora infestans

X. W. WANG (1), K. Shan (1), M. Han (1), L. Y. Guo (1)

(1) China Agricultural University, Beijing, China

Phytopathology 104(Suppl. 3):S3.125

Gene expression and cellular phenotype can be regulated by Epigenetic mechanisms, which mainly include DNA methylation and histone modification. Histone acetylation occurs at the amino groups of lysine residues on histone N-terminal tails and non-histone proteins, which alters the chromatin structure and affect the gene transcription and cell cycle progression. Phytophthora infestans, which is the causal agent of late blight, is a heterothallic Oomycete that requires the presence of both A1 and A2 mating types to undergo the sexual reproduction. In this study, we identified a Class II histone deacetylases in P. infestans, the PifHDAC7 (PITG_15415), which is a 390 amino acid protein with a catalytic Hist_deacetyl domain. We found that PifHDAC7 was up-regulated at cyst stage, and at 10 and 14 days after pairing between $\mathrm{A} 1$ and $\mathrm{A} 2$ isolate, but was down-regulated at four days after pairing. The PifHDAC7-silenced mutants were then generated from a wild type A1 isolate, using PEG-mediated protoplast stable transformation. These mutants showed slower hyphal growth, less sporangia formation, and formed many defective oospores in single culture, which indicated the change of sexual behavior of these PifHDAC7-silenced mutants. These results suggest that PifHDAC7 is involved in sporangia and oospores development. Moreover, it might also associates with sexual behavior determination. Further studies are needed to illuminate the role of PifHDAC7 in sexual reproduction.

Identification of major blast resistance genes in the southern U.S.

X. WANG (1), Y. A. Wamishe (2), T. Bianco (3), M. Lin (3), B. Valent (4), Y. Jia (3)

(1) University of Arkansas, Stuttgart, AR, U.S.A.; (2) University of Arkansas Coop Ext Svc, Stuttgart, AR, U.S.A.; (3) USDA ARS, Stuttgart, AR, U.S.A.; (4) Kansas State University, Manhattan, KS, U.S.A.

\section{Phytopathology 104(Suppl. 3):S3.125}

Resistance $(R)$ genes in rice play important roles in preventing the infections of rice blast fungus Magnaporthe oryzae. In order to identify more $R$ genes for different rice growing areas in the Southern US, an extensive field survey of blast fungus was performed from 2012 to 2013. A total of 500 isolates were purified from diseased rice leaves, panicles, and grains from commercial and experimental fields in Arkansas, Louisiana, Missouri, and Texas. A total of 100 isolates were selected based on geographical location and host genotype for race identification using international rice differentials with the addition of Katy (with Pita/Pita2) and M202 (without Pita/Pita2). Based on race identity, 20 isolates were selected for sequencing to examine the alleles of avirulence 
(AVR) genes including AVR-Pital, AVR-Pi9, and AVR-Pik. The same isolates were used to inoculate monogenic rice lines carrying 26 major blast $R$ genes developed by the International Rice Research Institute, the Philippines. Results of DNA sequence analysis of these $A V R$ genes and pathogenicity assays were combined to identify more effective blast $R$ genes for different rice production regions in the Southern US.

Characterization of mating-type (MAT) loci of Guignardia citricarpa, the citrus black spot pathogen and $\boldsymbol{G}$. mangiferae, the ubiquitous endophyte

N. Y. WANG (1), K. Zhang (1), J. A. Rollins (2), M. M. Dewdney (1)

(1) Citrus Research and Education Center, University of Florida, Lake Alfred, FL, U.S.A.; (2) Department of Plant Pathology, University of Florida, Gainesville, FL, U.S.A.

Phytopathology 104(Suppl. 3):S3.126

Guignardia citricarpa causing citrus black spot was first found in Florida in March 2010. Where the disease is endemic, G. citricarpa produces pseudothecia in decomposing leaf litter under periodic wetting and drying conditions. Previously, we partially characterized the MAT1-2 idiomorph of G. citricarpa through a draft genome approach. To complete the structural characterization of the MAT locus of G. citricarpa and to provide a resource for comparative analysis, we sequenced the genome of the closely-related, homothallic endophyte, G. mangiferae using Ion Torrent technology. Blastp queries revealed a fused MAT1-1-1 and MATI-2-1 locus on the same contig. From the NCBI database, an additional G. citricarpa genome from China (accession number AOTE00000000) was obtained and analyzed. This analysis identified the MAT1-1 idiomorph. Sequence alignments between MAT1-1 and MAT1-2 of G. citricarpa using Artemis revealed the shared and the unique sequences. The comparison between the G. citricarpa idiomorphs and the fused idiomorph of G. mangiferae revealed a high level of gene conservation between two species with one exception of two diverged PH domain-encoding genes in G. citricarpa. We are currently screening Florida isolates using MATspecific primers to understand the potential for outcrossing within this population. The information obtained could greatly impact our understanding of citrus black spot epidemiology in Florida and will inform disease control strategies.

QTL mapping Fusarium wilt race 1 and race 4 resistance in an interspecific cotton cross between Gossypium barbadense and G. hirsutum C. WANG (1), M. Ulloa (2), P. A. Roberts (3)

(1) Northeast Institute of Geography and Agroecology, Chinese Academy of Sciences, Harbin, China; (2) USDA, ARS, SPA, Cropping Systems Research Laboratory Plant Stress \& Germplasm Development Research, Lubbock, TX, U.S.A.; (3) University of California, Riverside, CA, U.S.A.

Phytopathology 104(Suppl. 3):S3.126

Fusarium wilt, caused by the soil-borne pathogen Fusarium oxysporum f. sp. vasinfectum (FOV), is a vascular disease of cotton (Gossypium spp.). FOV race 1 (FOV1) causes significant yield loss in G. hirsutum cultivars in coinfection with root-knot nematode (RKN, Meloidogyne incognita), while FOV race 4 (FOV4) causes plant damage in most $G$. barbadense cultivars without RKN co-infection. QTL analysis of the interspecific cross G. barbadense Pima S-7 $\times$ G. hirsutum Acala NemX revealed independent control of resistance to FOV1 and FOV4. Two major QTLs present in Pima S-7 linked to SSR marker Gh243 on chromosome (chr) 12 and marker CIR069 on chr11 which are associated with the $r k n 1$ resistance region contributed $10-11 \%$ of resistance response to FOV1 based on disease index at 13, 16, 20 and 23 days after inoculation (DAI), respectively. A major locus with additive effect on chr17 from Acala NemX allele was identified linked to two SSR markers (BNL2650 and CIR112), which accounted for $15 \%$ and $26 \%$ of resistance response to FOV4 at 10 and $13 \mathrm{DAI}$, increasing to $35-36 \%$ at $16,20,23 \mathrm{DAI}$. Interestingly, the region on chr14 homoeologous with chr17 accounted for 9$11 \%$ of resistance response to FOV4 disease index. The resistance-linked markers provide a valuable resource for marker-assisted selection of FOV resistance. Some F8 lines carrying resistance to RKN, FOV1 and FOV4 provide multiple resistance sources for breeding.

Resistance index of Pseudoperonospora cubensis to six systemic fungicides in Hebei and Shandong, China

W. WANG (1), R. Meng (2), X. Zhang (2), X. Han (2), J. Zhao (2), Z. Ma (2), J. Cui (2)

(1) Hebei Academy of Agric \& Forest Sciences, Baoding, Hebei, China; (2) Plant Protection Institute, Hebei Academy of Agric \& Forest Sciences, Baoding, Hebei, China

Phytopathology 104(Suppl. 3):S3.126

Pseudoperonospora cubensis (Pc) causes cucumber downy mildew and devastating loss of cucumber in China. The control of the disease is mainly dependent on application of fungicides. Sensitivity to six fungicides in Pc population from Hebei and Shandong was detected with leaf disc floating assay, and resistance index (RI) was calculated as Xiaojun Zhao et al reported in 2013. The result showed that RI to the six fungicides fluctuates over years of practical use, RI to metalaxyl declined from 0.6537 in 1986-1995 to 0.4690 in 2008 , then ascended to 0.5295 in 2010 and dropped to 0.4887 in 2013 . RI to azoxystrobin ascended from 0.1667 in 2001 to 0.5995 in 2009 , then dropped to 0.4732 in 2013 . RI to cymoxanil rose from 0.3889 in 2001 to 0.6432 in 2011 , then dropped to 0.5621 in 2013 . RI to dimethomorph increased from 0.2798 in 2004 to 0.3939 in 2007 , then descended to 0.3770 in 2009 and 0.3263 in 2013. RI to mandipropamid rose from 0.2341 in 2009 to 0.3229 in 2011, then declined to 0.1949 in 2013. The pathogen exhibits the highest sensitivity to flupicolide with RI rising from 0.1667 in 2010 to 0.1794 in 2013. Pc population developed resistance to metalaxyl, cymoxanil and azoxystrobin, but no resistance to dimethomorph, mandipropamid and flupicolide. The fungicides containing metalaxyl, azoxystrobin or cymoxanil became less effective in control the disease, the fungicides containing dimethomorph, mandipropamid or flupicolide maintain high controlling efficacy.

Characterization of genetic structure in Mexico populations of Phytophthora infestans using single sequence repeats

J. WANG (1), M. Larsen (2), R. Gregorio-Cipriano (3), G. RodríguezAlvarado (3), N. J. Grunwald (4), S. P. Fernandez-Pavia (3), E. M. Goss (1)

(1) University of Florida, Gainesville, FL, U.S.A.; (2) US Department of Agriculture-Agricultural Research Service, Corvallis, OR, U.S.A.; (3) Universidad Michoacana de San Nicolas de Hidalgo, IIAF, Morelia, Mexico; (4) Oregon State University, Department of Botany and Plant Pathology, US Department of Agriculture-Agricultural Research Service, Horticultural Crop Research Unit, Corvallis, OR, U.S.A.

Phytopathology 104(Suppl. 3):S3.126

Phytophthora infestans, the causal agent of the late blight in potato and tomato, is a devastating plant pathogen around the world. In the central highlands of Mexico, P. infestans undergoes regular sexual reproduction. The biology of these populations together with multilocus genetic data points to Central Mexico as its center of origin. The diversity and population structure of $P$. infestans in the Toluca Valley has been previously described. However, other populations in Central Mexico have not received as much attention and have not been characterized using the simple sequence repeat (SSR) markers currently being used to monitor $P$. infestans in the United States, Europe, and elsewhere. We genotyped $P$. infestans isolates sampled from several states in Mexico using a 12-plex SSR assay. We used these data to examine genetic diversity within and among populations using analysis of molecular variance, Bayesian clustering, and discriminant analysis of principal components, among other methods. We found significant genetic variation both within and between states. In particular, the Michoacán population appears to be distinct from the Toluca population. Characterization of the genetic structure and diversity of $P$. infestans in Mexico, using the same set of hypervariable markers as used to study $P$. infestans in other countries, will contribute to our understanding of the contemporary global diversity and migration of this problematic pathogen.

\section{Study on the mode of action of the novel fungicide SYP-14288 to Phytophthora capsici}

Z. Wang (1), X. Ni (1), P. Liu (1), C. Liu (2), X. LIU (1)

(1) China Agricultural University, Beijing, China; (2) Shenyang Research Institute of Chemical Industry, Shenyang, China

Phytopathology 104(Suppl. 3):S3.126

SYP-14288 is a novel fungicide developed by the Shenyang Research Institute of Chemical Industry in China. Preliminary studies show it has high activity and broad antifungal spectrum against 32 important plant pathogens, including oomycota, basidiomycota, ascomycota, zygomycota and anamorphic fungi. Until now, the mode of action of this compound is still unknown. In this study, we investigated the mode of action of SYP-14288 by exogenously adding ATP to determine the effect of ATP on the activity of SYP-14288, and extracting mitochondria from Phytophthora capsici to survey the effect of SYP-14288 on the respiration rate of mitochondria in vitro. As results, the antifungal activity of the fungicide on $P$. capsici was reduced after ATP added, which implied that SYP- 14288 might inhibit the ATP synthesis of the pathogen. By further measuring with oxygen electrode, the respiration rate of $P$. capsici was positive correlated with the concentrations of SYP-14288, which was consistent with the uncoupler fluazinam, and SYP-14288 was more effective on promoting respiration rate than fluazinam at the same concentration. These results suggested that SYP-14288 was functioned as an uncoupler. Our findings provided important information for the registration and practical application of SYP-14288. 
A loop-mediated isothermal amplification with bacterial enrichment assay for detection of Xanthomonas fragariae in strawberry

H. WANG (1), W. Turechek (2)

(1) University of Florida, Fort Pierce, FL, U.S.A.; (2) ARS-USDA, Fort Pierce, FL, U.S.A.

Phytopathology 104(Suppl. 3):S3.127

We developed a rapid, sensitive and cost-effective loop-mediated isothermal amplification (LAMP) assay for detection of Xanthomonas fragariae, the cause of angular leaf spot of strawberry. $X$. fragariae is difficult to detect when present at low densities, thus a bacterial enrichment procedure was developed to precede the assay that consists of an overnight incubation step followed by a quick sample concentration and a boiling step to extract DNA. Extracted DNA was amplified by LAMP and qPCR using a related set of primers for comparison. Results showed that the LAMP primers amplified $X$. fragariae at $65 \mathrm{C}$ within $30 \mathrm{~min}$ in reactions containing fluorescent dye for real-time detection or hydroxy naphthol blue dye for end-product detection. Primer specificity was determined by negative amplification with different pathogens and saprophytes isolated from strawberry and other Xanthomonas species. The LAMP detection limit was approx. 4 cells per reaction for pure bacteria culture and $3 \times 10^{2} \mathrm{cfu} / \mathrm{ml}$ for leaf and petiole samples. LAMP provided comparable detection sensitivity as qPCR, but was much faster. In addition, the whole assay required only basic instruments and reagents, thus making it a simple and cost effective procedure for identification of $X$. fragariae in strawberry leaf and petiole tissue.

Management of soybean sudden death syndrome by seed treatment with fluopyram

J. WANG (1), J. Jacobs (1), M. Chilvers (1)

(1) Michigan State University, East Lansing, MI, U.S.A.

Phytopathology 104(Suppl. 3):S3.127

Soybean sudden death syndrome (SDS), caused by Fusarium virguliforme, is one of the most yield limiting diseases in the US, and effective disease management options are limited. An experiment was conducted to test the efficacy of Fluopyram applied as a seed treatment to control the infection of $F$. virguliforme in soybean roots at a naturally infested soybean field. Plots were planted on May 1 with the cultivars 92 Y 60 and 92Y51. The experiment was a randomized complete block design with six treatments and five replicates. Treatments consisted of four rates of Fluopyram at $0.0375,0.075$, 0.15 , and $0.25 \mathrm{mg} \mathrm{a} / \mathrm{mg}$, which were combined with a standard seed treatment of Evergol Energy (0.019 mg), Allegiance FL (0.021 mg), and Poncho/Votivo $(0.13 \mathrm{mg}) \mathrm{a} / \mathrm{seed}$, standard seed treatment, and untreated seeds as controls. Plant samples were taken on 13 May, 13 June, 2 July, 11 July, and 3 September to determine root colonization of $F$. virguliforme with qPCR. SDS foliar disease rating based on disease severity and incidence was conducted on 16 August. No significant differences between treatments were detected in stand count, disease index, or yield. With the soybean samples collected 2 weeks after planting, no significant effect of seed treatment on $F$. virguliforme colonization was detected. In other trials located within the same field, significant reductions in SDS disease and corresponding yield increases were noted in other cultivars in response to Fluopyram treatments.

Population genetic analysis indicates genetic diversity of $F$. virguliforme within and between sampling locations

J. WANG (1), J. Jacobs (1), A. Rojas (1), M. Chilvers (1)

(1) Michigan State University, East Lansing, MI, U.S.A.

Phytopathology 104(Suppl. 3):S3.127

Soybean sudden death syndrome (SDS) is one of the most devastating diseases in the US. Since the first discovery of SDS in Arkansas in the early 1970s, SDS has been reported in most soybean producing states in the US, with apparent spread from this epicenter. In North America, Fusarium virguliforme is the only predominant pathogen that causes SDS, and its genetic diversity was not detected until a recent study using RFLP and RAPD markers. In this study, we examined the genetic diversity of $F$. virguliforme by genotyping $369 \mathrm{~F}$. virguliforme isolates collected from 4 states in 2012 and 2013 with 12 microsatellite markers. Based on the genetic distance threshold, 41 unique genotypes were identified. Genotype $1(\mathrm{n}=120)$ and genotype $2(\mathrm{n}$ $=120$ ) were the two dominant genotypes accounting for $74 \%$ of all isolates. In both Bayesian and multivariate cluster analyses, the genetic structure of $F$. virguliforme populations was grouped into two clusters $(\mathrm{K}=2)$. In addition to the significant population differentiation between the sampling locations $\left(\mathrm{F}_{\mathrm{st}}\right)$, the genetic diversity within the sampling location was also detected with regards to genotype richness and evenness. Although there is not enough evidence to support the dispersion of $F$. virguliforme from Arkansas, isolates collected from southern states were predominantly genotype 2 and isolates from northern states were mostly genotype 1, while the Arkansas population showed approximately equal composition of genotype 1 and 2 .

\section{Plant pathogenic Streptomyces: An annotated research collection}

L. A. WANNER (1)

(1) USDA Agicultural Research Service, Beltsville, MD, U.S.A.

Phytopathology 104(Suppl. 3):S3.127

A research collection of more than 2000 Streptomyces isolates has been established, preserved and partially characterized. About 12 out of more than 700 species of soil-borne Streptomyces are plant pathogens, causing common scab disease on potato and underground tuber and root crops. This isolate collection was derived from a wide range of geographic areas and potato cultivars. The collection is annotated for species (based on $16 \mathrm{~S}$ ribosomal RNA sequence) and presence of the $t x t A B$ gene encoding thaxtomin, the only known pathogenicity determinant. Most isolates $(70 \%)$ have the $t x t A B$ operon, and many also harbor two pathogenicity factors, necl and tomA (associated with but not required for pathogenicity). Other features genetically and physiologically haplotyped are presence and locations of repetitive sequence elements (insertion sequences) and phage integrases; secretion of hydrolytic enzymes (proteases, glycosyl hydrolases and sythases), utilization of cell wall substrates, and production/secretion of anti-microbials, characteristic of their roles as decomposers of biological materials and sources of many industrially useful antimicrobials and enzymes. In addition to genomic and epidemiological research on the collection, species with potential for biocontrol of plant disease, insects, and insect-vectored diseases have been identified. Many further uses can be imagined, and isolates from the collection are available from the author to qualified researchers.

A survey of major fungal pathogens that cause soybean seedling diseases in eight soybean-producing states

A. WARNER (1), P. O'Shea (1), R. Morgan (1), T. Behrens (1), J. Bond (1), A. Fakhoury (1)

(1) Southern Illinois University, Carbondale, IL, U.S.A.

Phytopathology 104(Suppl. 3):S3.127

Seedling diseases are both common and very destructive on soybean, Glycine $\max$. They are caused by a variety of plant pathogens including fungi, bacteria, and oomycetes. The goal of this project is to identify fungal pathogens that cause seedling diseases of soybean through a multi-state effort. We are using two different approaches for genus and species determination. The first approach relies on morphological features using microscopy and the second through the sequencing of specific genetic loci in the isolated fungal pathogens. In the sequencing approach, up to three different barcodes are sequenced in some of the isolates to confirm the identification. The barcodes include the internal transcribed spacer (ITS), the elongation factor EF- $1 \alpha$, and the intergenic spacer (IGS) region. The data produced from this project will include identification of fungal isolates from eight different states, six fields per state and at least 25 isolates per field. Data will also involve correlating species and genera distribution to factors including geographical distribution, cultural practices, soil type, and climatic conditions. Over 3,000 fungal isolates are being processed for identification to help determine the main causal agent of seedling diseases on soybean.

Identification of peptides that possess high binding affinities to bacterial polygalacturonases for potential control of phytopathogenic prokaryotes J. WARREN (1), B. Kirkpatrick (1)

(1) UC Davis, Davis, CA, U.S.A.

Phytopathology 104(Suppl. 3):S3.127

Polygalacturonase (PG) enzymes have shown to be virulence factors of a number of bacterial and fungal plant pathogens. PGs degrade pectic polymers within plants, providing nutrients and allowing movement within the infected plant for pathogens. Xylella fastidiosa (Xf) is a xylem-limited, gram-negative bacterium that causes Pierce's disease of grapevines (PD). Xf possesses a single polygalacturonase gene that was shown to be essential for Xf to cause PD. Similarly, a PG mutant of Agrobacterium vitis (Av), causal agent of crown gall of grapes, was also shown to be less pathogenic. Thus, identifying peptides or proteins that could inhibit the activity of polygalacturonases may provide a viable means for protecting plants against pathogens that require PG as a virulence factor. A phage-displayed combinatorial peptide library was used to isolate a number of unique peptides that showed a high binding affinity to PG enzymes of both Xf and Av PGs. Interestingly, these peptides show strong sequence similarities to polygalacturonase inhibiting proteins (PGIPs) suggesting they indeed bind to areas important for PG activity. Furthermore, in vitro enzyme inhibition assays show that one of the peptides is capable of reducing AvPG activity. 
Determining seed transmissibility of Sugarcane mosaic virus and Sugarcane yellow leaf virus in sugarcane

A. WASHINGTON-KEIZERWEERD (1), C. Maroon-Lango (2), M. Grisham (1)

(1) USDA ARS, Houma, LA, U.S.A.; (2) USDA APHIS PPQ CPHST, Beltsville, MD, U.S.A.

Phytopathology 104(Suppl. 3):S3.128

The importation of sugarcane germplasm is essential to diversify sugarcane germplasm used in United States breeding programs. Foreign germplasm is received primarily as vegetative cuttings. Current permit requirements for importing sugarcane seed into the United States are impractical and limit the acquisition of germplasm from some geographic sources. The objective of this study was to investigate potential seed transmissibility of Sugarcane mosaic virus (SCMV) and Sugarcane yellow leaf virus (SCYLV) in an effort to determine the risk of exotic pathogen introduction. Seeds were collected from four crosses involving a Sugarcane mosaic virus-infected female parent. The seeds were surface disinfested with commercial bleach, sown and germinated, and the seedlings grown in a quarantine facility for 3 mo prior to RNA extraction. The resulting 389 seedlings were screened for SCMV and SCYLV via RT-PCR. Three unique primer sets were used for each virus, with at least one being family or group-specific for a wider target range. After repeated testing, all seedlings were shown to be negative for both viruses. These results support earlier findings, which revealed no transmission of viral sugarcane pathogens through seed. Our ultimate goal is to provide research information in support of modifying permit conditions that currently prevent importation of foreign seed.

\section{Alfalfa endophytes as novel sources of antimicrobial compounds that inhibit the growth of human and plant pathogens}

B. WASS (1), A. Jordon (1), M. Louters (1), D. A. Samac (2), D. FosterHartnett (1)

(1) Saint Catherine University, St. Paul, MN, U.S.A.; (2) USDA ARS, St. Paul, MN, U.S.A.

Phytopathology 104(Suppl. 3):S3.128

Fungal endophytes may contribute to plant health and disease protection, yet little is known about their various roles in alfalfa. Also, endophytes from several plant species produce novel antimicrobial compounds that may be useful clinically. We isolated endophytic fungi from over 50 samples from six locations in Minnesota and Idaho; both healthy and diseased alfalfa displaying wilt symptoms were collected. Sequence and BLAST analysis of the ITS region revealed a variety of genera but were dominated by Alternaria spp. Of 20 isolates tested, 17 were positive for antimicrobial production against at least one test organism using streak plate assays against 12 bacterial and fungal strains including both human and alfalfa pathogens. Of these, three Alternaria isolates were grown for three weeks in solid substrate rice fermentation cultures that were subsequently extracted with ethyl acetate, dried and resuspended in methanol. Disc diffusion assays using $1 \mathrm{mg}$ of crude extracts produced clear zones of inhibition against two Gram - and six Gram + bacteria, including Staphylococcus aureus and Clavibacter michiganensis. Fungistatic and bacteriostatic activity was seen for Candida albicans and Pseudomonas syringae, respectively. Further studies will investigate the (1) structure of these compounds, (2) activity against drug-resistant bacterial strains and tumor cell lines, and (3) potential of Alternaria spp. and other alfalfa endophytes to inhibit alfalfa pathogens in vivo.

\section{Biological control of aflatoxin in corn}

M. A. WEAVER (1), H. K. Abbas (1), L. Falconer (2), T. W. Allen (2), H. Pringle (2), G. Sciumbato (2)

(1) USDA ARS, Stoneville, MS, U.S.A.; (2) Mississippi State University MAFES, Stoneville, MS, U.S.A.

Phytopathology 104(Suppl. 3):S3.128

Aflatoxin contamination of corn is a grain quality issue that can be a significant economic limiting factor to Mississippi farmers. Biological control products containing atoxigenic strains of Aspergillus flavus are commercially available to reduce aflatoxins in corn. Field trials were conducted at fourteen test sites over three years to evaluate the efficacy of these biocontrol products at eleven and twenty-two $\mathrm{kg} \mathrm{ha}^{-1}$ application rates and application times ranging from mid-vegetative (V5) to mid-reproductive (R2) corn growth stage. There was no significant difference between the two treatment rates. The greatest reduction in aflatoxins generally occurred with the earliest applications. Furthermore, several treatments were identified that were costeffective across fields and years. These observations support the use of atoxigenic strains of $A$ flavus to promote the safety and marketability of corn grown in areas susceptible to aflatoxin contamination.
Multilocus analysis using putative fungal effectors to describe a population of Fusarium oxysporum from sugar beet

K. M. WEBB (1), P. Covey (1), B. Kuwitzky (1), M. Hanson (1)

(1) USDA ARS NPA SBRU, Fort Collins, CO, U.S.A.

Phytopathology 104(Suppl. 3):S3.128

Sugar beet Fusarium yellows is caused by Fusarium oxysporum f. sp. betae and leads to reductions in sucrose yields. F. oxysporum $\mathrm{f}$. sp. betae can be highly variable in morphology and many isolates from symptomatic sugar beet are non-pathogenic. Identifying pathogenicity factors and their diversity in $F$. oxysporum f. sp. betae could lead to further understanding of how this pathogen causes disease and provide markers to identify pathogenic from nonpathogenic isolates. Methods used to characterize the genetic diversity of $F$. oxysporum have done little to describe regional populations and are unable to differentiate between pathogenic and non-pathogenic isolates. In this work, we obtained the sequence for thirteen previously described pathogenicity genes (Fmk1, Fow1, Pda1, PelA, PelD, Pep1, Prt1, Rho1, Sge1, Six1, Six6, Snfl, and Ste12) to use as markers to characterize the phylogenetic diversity of a population of 26 pathogenic and non-pathogenic isolates of $F$. oxysporum originally isolated from and determine if this could be correlated to pathogenicity. Of the genes investigated, 6 were present in all isolates from sugar beet (Fmk1, Fow1, PelA, Rhol, Snfl, and Ste12), and 7 were found to be dispersed within the population (Pda1, PelD, Pep1, Prt1, Sge1, Six1, and Six6). Of these Fmk1, Fow1, PelA, Rho1, Sge1, Snfl, and Ste12 were significant in describing clade designations while PelD and Prt1 were significant for describing pathogenicity in $F$. oxysporum f. sp. betae.

Differential proteomic profiles of sugarbeet resistance genes $R z 1$ and $R z 2$ during interactions with Beet necrotic yellow vein virus

K. M. WEBB (1), W. M. Wintermantel (2), L. Hldaky (2), P. Covey (1), C. J. Broccardo (3), J. E. Prenni (3)

(1) USDA ARS NPA SBRU, Fort Collins, CO, U.S.A.; (2) USDA ARS CIPRU, Salinas, CA, U.S.A.; (3) Colorado State University, Fort Collins, CO, U.S.A.

Phytopathology 104(Suppl. 3):S3.128

Rhizomania, caused by Beet necrotic yellow vein virus (BNYVV), is an economically important disease affecting sugarbeet production by reducing root quality and sugar yield. A number of resistance genes have been identified for control; however, new pathotypes that overcome resistance have emerged. Understanding sugarbeet proteins involved in resistant and susceptible interactions with BNYVV may lead to novel management strategies. Three near isogenic sugarbeet lines; one susceptible to all forms of BNYVV and resistant lines separately, carrying the $R z l$ and $R z 2$ resistance genes, were infected with two sources of BNYVV; standard BNYVV A-type, and the $R z 1$ resistance-breaking BNYVV-IV. Seedlings were grown in a growth chamber at $24^{\circ} \mathrm{C}$ with 16 hour days. At 3 weeks post-germination plants were harvested and foliar and root portions of the plant were separated and pooled. Root samples from each pot were tested by ELISA to confirm BNYVV infection and total protein was extracted from lyophilized leaf tissue. Protein extracts were processed via LC-MS/MS and subsequently separated on a reverse phase nanospray column. Peptide spectra were examined for protein identity using a Uniprot Amaranthaceae database. Differential protein expression was evaluated and several proteins were found to be more highly expressed during comparisons between compatible and incompatible interactions suggesting different biological pathways that may lead to novel management and breeding targets.

Identification of resistance to Fusarium oxysporum f. sp. tracheiphilum race 2 in the U.S. core collection of cowpea (Vigna unguiculata)

W. P. WECHTER (1), C. Conrad (2), M. McMillan (1)

(1) USDA-ARS, Charleston, SC, U.S.A.; (2) Clemson University, Charleston, SC, U.S.A.

Phytopathology 104(Suppl. 3):S3.128

Fusarium wilt of cowpea (Vigna unguiculata) is a worldwide problem. Although resistance to Fusarium wilt exists in the US germplasm collections, no significant characterization of this collection has been undertaken in many years. According to the Genetic National Resources Program (GRIN) database, only 158 accessions out of more than 8000 have been screened for resistance to Fusarium wilt caused by Fusarium oxysporum f. sp. tracheiphilum (Fot). In addition, it is unknown what race of the pathogen was used for that screen. We have characterized the resistance reaction of 495 cowpea accessions, from the core collection held at the Plant Genetic Resources Conservation Unit, Griffin, GA, when challenged with Fot race 2. Ratings were based on vascular discoloration and tests were set up as complete randomized blocks. One hundred and thirty-nine accessions were found not to be significantly different than the susceptible differential 'Chinese Red', while 365 accessions were found not to be significantly 
different than the resistant differential 'Iron'. This study shows that a large number of the accessions in the germplasm collection possess significant levels of resistance to Fot race 2 .

Field and greenhouse evaluations of fungicide effects on northern leaf blight of corn

J. D. WEEMS (1), K. A. Ames (1), C. A. Bradley (1)

(1) University of Illinois, Urbana, IL, U.S.A.

Phytopathology 104(Suppl. 3):S3.129

Quinone outside inhibitor (QoI) and demethylation inhibitor (DMI) fungicides have been widely adopted as management tools to control northern leaf blight (NLB) of corn, caused by Exserohilum turcicum (Et). In 2011 and 2012, Etinoculated field studies were conducted to evaluate QoI, DMI, and QoI + DMI fungicide mixtures at different rates applied at silking stage for their effect on NLB. Disease severity was recorded and yield responses were measured. The labeled rates of DMI fungicides and DMI + QoI fungicide mixtures significantly reduced NLB severity compared to the reduced rates of DMI fungicides and all rates of the QoI fungicides. The labeled rates of the QoI fungicides and the DMI + QoI fungicide mixtures had significantly greater yields than the non-treated control and reduced rate DMI treatments. A greenhouse study was conducted to evaluate fungicide application timing relative to the time of Et inoculation. Fungicides were applied to corn plants at 7 days, 3 days, 1 day, and 3 hours prior to Et inoculation and 3 hours, 1 day, 3 days, and 7 days after inoculation. The estimated percent leaf area affected by NLB was recorded and analyzed. Though treatment effects significantly varied between trials, fungicides applied prior to Et inoculation consistently decreased NLB across trials.

\section{Variation in response among Pythium species and isolates to Streptomyces} lydicus

J. WEILAND (1)

(1) USDA ARS, Corvallis, OR, U.S.A.

Phytopathology 104(Suppl. 3):S3.129

Consistent, effective biological control of soilborne pathogens has been difficult to achieve in the field. Most research regarding biological control efficacy has focused on the biological control agent itself (i.e. dose, formulation, survival) or on its interaction with the rhizosphere and edaphic environments: Very little research has focused on how pathogen diversity affects the outcome. A dual-culture, in vitro assay was used to test the responses of 16 Pythium species (148 isolates) from three forest nurseries to Streptomyces lydicus. Pythium isolate responses (percent growth inhibition, inhibition zone distance, and mortality) to $S$. lydicus were measured and found to differ significantly among Pythium species and isolates. Furthermore, the responses of three species, $P$. irregulare, $P$. sylvaticum, and $P$. ultimum, were different depending on which nursery they were isolated from. Isolate growth rates strongly biased percent growth inhibition, and to a lesser extent, the inhibition zone distance. Results indicate that growth rate must be accounted for when conducting in vitro, dual-culture biological control inhibition assays and suggest that pathogen diversity deserves further exploration as a potential component contributing to inconsistent biological control.

\section{Identification of Tobacco streak virus (TSV) in commercial cranberry} plantings and its association with berry scarring in Wisconsin

L. D. WELLS (1), J. J. Polashock (2), N. Vorsa (3), B. E. L. Lockhart (4), P. S. McManus (1)

(1) University of Wisconsin, Madison, WI, U.S.A.; (2) USDA ARS, Chatsworth, NJ, U.S.A.; (3) Rutgers University, Chatsworth, NJ, U.S.A.; (4) University of Minnesota, St. Paul, MN, U.S.A.

Phytopathology 104(Suppl. 3):S3.129

In 2012, we discovered Tobacco streak virus (TSV) in cranberry plants bearing disfigured, scarred fruit. This was the first confirmation of a virus in Wisconsin cranberries, and nothing is known about how TSV will impact yield of this woody, perennial plant. We conducted field trials in 2013 to investigate the epidemiology and impact of TSV on cranberries. TSV was detected in leaves, fruit, and pollen of cranberry plants by ELISA, reverse transcriptase PCR, and transmission electron microscopy. In 2013, we showed that TSV overwinters in cranberry plants in the field. Plants which had produced scarred, symptomatic, TSV-positive fruit in 2012 produced nonscarred, asymptomatic TSV-positive fruit in 2013. This is referred to as 'recovery' when observed in crops infected with viruses related to TSV. In 2013, we initiated experiments to determine the effects of TSV infection on cranberry yield components such as flower set, fruit set, and berry weight. In 2013 , berry weight and fruit set were significantly reduced $(p<0.05)$ in scarred, symptomatic, TSV-positive cranberry plants compared to nonscarred, asymptomatic TSV-negative or TSV-positive "recovered" plants. Further experiments are being conducted, but we tentatively conclude that after plants "recover," TSV does not negatively affect yield components in cranberry.

Sugarbeets as biofuel: Decline rates of California sugarbeet cyst nematode under nonhost crops

B. B. WESTERDAHL (1), E. P. Caswell-Chen (1), F. R. Kegel (2)

(1) University of California, Davis, CA, U.S.A.; (2) University of California

Cooperative Extension, Stockton, CA, U.S.A.

Phytopathology 104(Suppl. 3):S3.129

Since the 1950s, crop rotation has been a commercial practice for managing sugarbeet cyst nematode (Heterodera schachtii, $\mathrm{SBCN}$ ) on sugarbeets. Prior research conducted in southern California established that $\mathrm{SBCN}$ populations decline at the rate of 49 to 80 percent per year, leading to estimates of 3-to-4 year rotations to nonhost crops as necessary to reduce SBCN densities to nondamaging levels. Following grower reports that much longer rotations were needed in northern California, trials were conducted to establish the rate of decline of SBCN in the San Joaquin Valley. Ten commercial fields with a history of SBCN infestation were sampled periodically for 6.5 -years. In each field, 10 circular subplots located 30.5 meters apart (each with a 6 m radius) were established with reference to a permanent landmark. On each sampling date, 12 subsamples from each subplot were taken randomly from the top 0-30 $\mathrm{cm}$ of soil and composited into a single sample. Standard techniques were utilized to extract and enumerate cysts and eggs from soil samples. Yearly rates of population decline in the sampled fields ranged from 12.2 to 34.6 percent, decline rates lower than previously estimated. This finding has implications for SBCN management in California sugarbeets grown for biofuel, as the lower decline rates indicate that nonhost rotations of longer duration than previously anticipated may be necessary.

Population dynamics of Pythium irregulare sensu stricto under different agronomic conditions

H. D. WHiTTINGTON (1), A. Torres-Barragan (1), J. Sun (1), J. Driver (1), F. J. Louws (1)

(1) North Carolina State University, Raleigh, NC, U.S.A.

Phytopathology 104(Suppl. 3):S3.129

Pythium irregulare is one of the causal agents of strawberry Black Root Rot (BRR). For many years this disease was controlled using methyl bromide (MB). Their elimination has stimulated the search and evaluation of ecologically-based strategies to manage it. The objective of this work was to evaluate the effects of 5 environmentally friendly treatments and two chemical treatments, in the populations of $P$. irregulare. Experiments were done at NCDA\&CS Horticultural Crops Research Station at Castle Hayne, NC. Treatments included Methyl Bromide, PicChlor60, Anaerobic Soil Disinfestation (ASD), Cover Crops (Cowpea and Pearl Millet), Compost, Cover crops+Compost, and Mustard Meal. Soil and root samples were collected before setting up experiment, when cover crops were harvested, at strawberry transplanting, one month after transplanting, at peak blooming and at peak harvest. DNA was extracted from soil and roots and qPCR analysis was done from each sample. Population dynamics in soil varied according to the phenological stage of strawberry plants, reaching its peak lowest during peak blooming (1 cfu/gr of dry soil). Root infection was decreased by treatments during the first month after planting, but increased as plants grew, except for treatments that included compost. Our results are in accordance with well documented reports that indicate that the enrichment of the microbial community in soil decrease pathogen root infection.

Frogeye leaf spot management with timed fungicide applications in the Mississippi soybean production system

T. H. WILKERSON (1), T. W. Allen (1), B. R. Golden (1), J. T. Irby (2)

(1) Mississippi State University, Stoneville, MS, U.S.A.; (2) Mississippi State University, Starkville, MS, U.S.A.

Phytopathology 104(Suppl. 3):S3.129

Frogeye leaf spot (FLS), caused by Cercospora sojina Hara is quickly becoming a major concern for Mississippi's soybean farmers. In fields exhibiting QoI- (strobilurin) resistant strains of the fungus, FLS management will continue to be important, especially in situations where FLS susceptible soybean cultivars are planted. The objectives of fungicides trials were to evaluate the effect of $C$. sojina on yield and the efficacy of multiple active ingredients on FLS incidence and severity. In 2013 field trials were conducted in Starkville and at the Delta Research and Extension Center in Stoneville, MS using a FLS susceptible cultivar, Armor DK 4744. Fungicide products were applied as either stand-alone (QoI or triazole) or in combination (premix or tank mix) at several different growth stages (V6, R5, R5.5) and assessed at multiple locations. AUDPC values at three locations determined that all products provided equal or better FLS control and out yielded the nontreated. In general, the efficacy of QoI fungicides was reduced compared 
to products that contained two modes-of-action. Triazole containing products provided the greatest FLS control and yield benefit; however, phytotoxicity resulted with some products. Observations of phytotoxicity associated with triazole-containing products did not negatively affect yield. More research evaluating QoI resistance distribution across Mississippi is in progress and alternative management practices should be considered.

Sclerotinia sclerotiorum aggressiveness and oxalic acid production in soybean

J. F. WILLBUR (1), M. Kabbage (1), D. L. Smith (1)

(1) University of Wisconsin, Madison, WI, U.S.A.

Phytopathology 104(Suppl. 3):S3.130

Sclerotinia sclerotiorum, the causal agent of Sclerotinia stem rot of soybean, produces oxalic acid which is considered a key pathogenicity factor. Due to the broad host range of $S$. sclerotiorum and the presence of both weed and agronomic hosts in the same fields, host specificity may be a concern. Evaluating the performance of a diverse collection of S. sclerotiorum isolates on soybean would determine whether host specificity exists. Additionally, assessing host colonization and disease severity of these isolates will increase our understanding of the infection mechanism of $S$. sclerotiorum in soybean. Isolates collected from a variety of hosts across the north central United States and central Europe were screened for aggressiveness and oxalate production. Isolate aggressiveness was evaluated using lesion lengths from inoculated 'Williams 82 ' soybeans and colony radii in vitro. Oxalate production was quantified via a spectrophotometric assay. Mild and aggressive isolates of $S$. sclerotiorum were assayed on both resistant and susceptible varieties of soybean; the infection process was monitored using a combination of staining and microscopy tools. Surprisingly, isolates collected from non-soybean hosts were moderate to strongly aggressive on soybean. Our findings suggest that screening of $S$. sclerotiorum-resistant soybean germplasm should be performed with multiple isolates of the fungus to account for the overall diversity of $S$. sclerotiorum isolates in the field.

Characterization of an RTX-like toxin in virulence of Pantoea stewartii subsp. stewartii

K. M. WILLIAMS (1), M. C. Roper (1), L. Burbank (1), S. von Bodman (2) (1) University of California-Riverside, Riverside, CA, U.S.A.; (2) National Science Foundation, Arlington, VA, U.S.A.

Phytopathology 104(Suppl. 3):S3.130

Pantoea stewartii subsp. stewartii (Pnss), the causal agent of Stewart's wilt, is an important bacterial pathogen of sweet corn. Pnss colonizes the apoplastic space and xylem tissue, resulting in characteristic water-soaked (WS) lesions and wilting. A gene encoding a putative RTX-like toxin, $r t x 2$, has been identified in Pnss. RTX toxins belong to the pore-forming toxin family and have lytic properties in mammalian systems. Little is known about the role of RTX toxins in plant pathogenesis, but interestingly, a Pnss $\Delta r t x 2$ mutant was strikingly unable to incite WS lesions in planta implicating it in the cell lysis associated with lesion formation. In addition, $r t x 2$ is genetically linked to components of the Rcs phosphorelay, a global regulatory system of critical virulence factors in Pnss. This suggests a possible functional relationship, particularly because they are transcribed from a single promoter. The RTX2 domain structure is consistent with an autotransported protein and contains conserved transmembrane domains suggesting it translocates itself to the cell surface. The signal relay pathway that stimulates the Rcs phosphorelay is unknown, but membrane perturbation plays a key role in the sensing and transduction of the signal that stimulates the Rcs system. We hypothesize that RTX2 may contribute to the membrane dynamics that are being sensed by the Rcs system, thus modulating activation of signal transduction through the system.

Development, production and validation of proficiency test panels for the PPQ NPPLAP Plum pox virus certification

K. L. WILliAMS (1), V. A. Mavrodieva (1), G. G. Dennis (2), S. S. Negi (3), L. Levy (4), R. M. DeVries (1), D. D. Picton (1), P. J. Shiel (2), M. K. Nakhla (1) (1) USDA-APHIS-PPQ-S\&T, Beltsville, MD, U.S.A.; (2) USDA-APHISPPQ-S\&T, Raleigh, NC, U.S.A.; (3) USDA-APHIS-PPQ-PHP-QPAS, Riverdale, MD, U.S.A.; (4) USDA-APHIS-PPQ-S\&T, Riverdale, MD, U.S.A. Phytopathology 104(Suppl. 3):S3.130

The CPHST Beltsville Laboratory is the sole provider of quantitatively validated proficiency test (PT) panels for the National Plant Protection Laboratory Accreditation Program's (NPPLAP) annual certification for diagnostics of USDA regulatory plant pathogens. Certifications are offered for three organisms: Phytophthora ramorum, citrus greening and Plum pox virus (PPV). The PPV panel provides external assessment of DAS ELISA and reverse transcription (RT)-PCR laboratory results. Multiple batches of healthy and PPV infected tissue samples from different prunus hosts are produced and lyophilized to manufacture stable and homogeneous samples. Quantitative validation using PPQ approved work instructions is conducted on site to assess quality and to characterize tissue batches before assembling randomized blind panel sets. Testing over time has validated that tissue samples, as produced and stored by the Beltsville Lab, are stable (low degradation rate) for at least 2 years. The PPV pilot panel was produced and distributed on a limited basis in 2010. The number of certified diagnosticians has tripled by 2013 and now includes NPDN, State, Federal and privatelyfunded labs. NPPLAP certification is a requirement for laboratories conducting screening testing for the PPQ PPV Eradication Program in NY State. Analysis of previous and current panel results shows improved passing rates by repeat participants and improved overall performance among participating labs.

Interactive effects of Blueberry red ringspot virus and root damage on symptom severity, plant vigor, and yield in southern highbush blueberry L. WILLIFORD (1), H. Scherm (1), A. Savelle (1)

(1) University of Georgia, Athens, GA, U.S.A.

Phytopathology 104(Suppl. 3):S3.130

Blueberry red ringspot virus (BRRV) occurs commonly in blueberry plantings in Georgia, but there is no information about interactions of the virus with other factors affecting plant health and yield. Here, we studied interactive effects of BRRV and root damage of southern highbush blueberry in field plants affected by abiotic injury and greenhouse-grown plants infected by Phytophthora root rot (PRR). Field plants were either stunted (presumed herbicide injury) or not stunted, and infected or not infected with BRRV as determined by symptoms and BRRV-specific PCR. Flower bud set and berry yields were highest in BRRV-negative plants that were not stunted and lowest in stunted, BRRV-positive plants. BRRV alone reduced flower bud counts but not berry yields. The combined effects of BRRV infection and stunting were additive but not synergistic with regard to yield reduction. In greenhouse trials with controlled inoculations of BRRV-positive and -negative plants with Phytophthora cinnamomi, there was no significant interaction between presence of BRRV and PRR, indicating that plants with or without BRRV reacted similarly to $P$. cinnamomi infection with regard to foliar disease progression and plant fresh weight. Thus, experimental data so far do not support anecdotal observations suggesting exacerbation of BRRV symptoms and yield losses in plants affected by biotic or abiotic root damage.

Genetic and geographic diversity of Moroccan pepper virus

W. M. WINTERMANTEL (1)

(1) USDA ARS, Salinas, CA, U.S.A.

Phytopathology 104(Suppl. 3):S3.130

Moroccan pepper virus (MPV) is one of two tombusviruses responsible for the disease, lettuce dieback, which is responsible for severe losses in western U.S. lettuce production. MPV also affects vegetable and ornamental production, and is being found with increased frequency throughout the world. In order to determine relationships among isolates from diverse locations and relate genetic variability to virus epidemiology, we sequenced the coat protein (CP) genes of several MPV isolates from California and a recent isolate from escarole (Chicorum endivia) in New Jersey, and compared sequences with those of isolates from Europe, Africa, Iran, and Japan. Comparative genetics shows the CP gene to be one of the more variable regions of the tombusvirus genome. Results demonstrated a relatively close relationship among geographically diverse isolates with all viruses exhibiting at least $96 \%$ identity in the $\mathrm{CP}$ gene. Geographic proximity was only partially correlated with genetic similarity. The widespread but geographically isolated distribution of MPV likely reflects movement on agricultural materials and establishment of the mechanically transmitted soil- and water-borne virus in fields. Continuing studies are examining factors leading to environmentally influenced disease development.

Developing genomic resources for species-specific molecular diagnostics of cucurbit downy mildew

S. WITHERS (1), E. Gongora Castillo (2), M. J. Bowman (2), K. L. Childs (2), D. Gent (3), P. Ojiambo (1), L. Quesada-Ocampo (1)

(1) North Carolina State University, Raleigh, NC, U.S.A.; (2) Michigan State Univerisity, East Lansing, MI, U.S.A.; (3) Oregon State University, Corvallis, OR, U.S.A.

Phytopathology 104(Suppl. 3):S3.130

Cucurbit downy mildew (CDM), caused by Pseudoperonospora cubensis, severely affects several cucurbit crops and is controlled largely by fungicide applications. Growers initiate chemical control when an outbreak has occurred in their state or surrounding counties. Early detection of CDM to reduce fungicide use remains a challenge since species-specific markers for pathogen detection in spore traps are not available and early infections are commonly 
missed during scouting. To address this problem, we used Illumina next generation sequencing technology to identify candidate genomic regions for species-specific molecular diagnostic tools. We compared the transcriptomes of CDM isolates from different cucurbit hosts, as well as the closely related hop downy mildew pathogen ( $P$. humuli) originating from diverse hop cultivars. From these comparisons, we selected candidate genomic regions shared by all CDM isolates in our analysis and not present in closely related species. We are testing these candidates against a larger pathogen sample to confirm unique CDM markers that could be used to develop a molecular diagnostic tool for plant disease clinics, growers or Extension agents. Comparing these transcriptomes from the CDM and hop downy mildew pathogens can also increase our understanding of evolutionary relationships of these sister species, as well as pathways involved in host specificity.

\section{Root-knot nematodes in west Texas cotton: Reassessing an old problem}

J. E. WOODWARD (1), T. Wheeler (2), S. Fuchs (3), S. Daily (3)

(1) Texas AgriLife Extension Service, Lubbock, TX, U.S.A.; (2) Texas A\&M AgriLife Research, Lubbock, TX, U.S.A.; (3) Dow AgroSciences, Indianapolis, IN, U.S.A.

Phytopathology 104(Suppl. 3):S3.131

Recently, several high yielding cotton (Gossypium hirsutum L.) cultivars that possess partial resistance to the root-knot nematode (Meloidogyne incognita) have been released. In 2013, a survey was initiated in west Texas to increase producer awareness of root-knot nematodes. A total of 492 fields were sampled and geo-referenced to develop educational materials. Overall, $77.2 \%$ of the fields surveyed contained $M$. incognita. These results are slightly higher than those from previous surveys, and may have been confounded by the fact that sampling was concentrated in a 21 county area known to be infested with M. incognita. Populations in fields that were positive ranged from 40 to 42,680 second stage juveniles plus eggs per $500 \mathrm{~cm}^{3}$. Field studies were also conducted to assess $M$. incognita reproduction on PhytoGen (PHY) cultivars marketed for to management the nematode. Reproduction was lower for the tolerant cultivar 'PHY 367WRF' than the susceptible commercial standards evaluated. The cultivars 'PHY 417WRF' and 'PHY 427WRF', which contain two genes conferring resistance to $M$. incognita, supported the lowest levels of reproduction. These results confirm that the potential for yield loss from rootknot nematode in west Texas is high and that differences in population densities within an area can be substantial. Furthermore, the PhytoGen cultivars evaluated possess tolerance or partial resistance to $M$. incognita and should be utilized in fields that are severely infested.

Comparison of time-related wheat streak mosaic severity assessments for plant biomass and grain yield predictive abilities

F. WORKNEH (1), C. M. Rush (1)

(1) Texas A\&M AgriLife Research, Bushland, TX, U.S.A.

Phytopathology 104(Suppl. 3):S3.131

In the Texas Panhandle, winter wheat is cultivated as a dual-purpose crop for grain and forage productions. Fields destined for grazing are generally planted early (late August to early September) to maximize forage biomass for winter grazing. Wheat streak mosaic, caused by Wheat streak mosaic virus, which is vectored by the wheat curl mite (Aceria tosichella), is a major wheat disease in the Southern Great Plains including the Texas Panhandle. The disease often begins at the field edge progressing to the infield over time, and is usually more severe in early- than in late planted wheat. The primary objective of this project was to determine if the progressive nature of the disease could be exploited for prediction of its effects on plant biomass and grain yield. To accomplish this, a strip of wheat was planted along one side a wheat field early in the season for trapping mites and establishing infection source. The spatial and temporal progression of the disease in the wheat field was assessed weekly (mid-April to end of May 2013) in multiple $1-\mathrm{m}^{2}$ plots (including uninfected areas) using a hand-held hyperspectral radiometer. The successive spectral scores then were related to plant biomass and grain yield using regression. The spectral values varied among assessment dates in terms of biomass and yield predictions $\left(R^{2}=0.41\right.$ to 0.67 , and $R^{2}=0.59$ to 0.95 , respectively, $P<0.05$ ), but their predictive abilities didn't strictly follow the assessment date sequence.

Differential transmission of two strains of Wheat streak mosaic virus by five wheat curl mite populations

E. N. WOSULA (1), A. J. McMechan (1), C. de Olivera (1), G. Hein (1)

(1) University of Nebraska - Lincoln, Lincoln, NE, U.S.A.

Phytopathology 104(Suppl. 3):S3.131

Wheat streak mosaic virus (WSMV), type member of the genus Tritimovirus in the family Potyviridae, is an economically important virus causing annual average yield losses of approximately $2-3 \%$ in winter wheat across the Great Plains. The wheat curl mite, Aceria tosichella, transmits WSMV along with two other viruses found throughout the Great Plains. Two common genotypes of WSMV (Sidney 81 and Type) in the U.S. share 97.6\% nucleotide sequence identity, but their transmission relationships with the wheat curl mite are unknown. The objective of our study was to determine transmission of these two strains of WSMV by five mite populations (Nebraska, Montana, South Dakota, Type1 and Type2). Non-viruliferous mites from each population were reared on wheat source plants mechanically inoculated with either Sidney 81 or Type WSMV strains. For each source plant, individual mites were transferred to 10 separate test plants and virus transmission determined by using DAS-ELISA assay. Source plants were replicated 6 times for each treatment (60 individual mite transfers). Results indicate that four mite populations transmitted Sidney 81 strain at higher rates compared with Type strain. Two mite populations Nebraska and Type 2 transmitted Sidney 81 and Type strains at higher rates compared with the other three populations. Results from this study demonstrate interactions between virus strains and mite populations important to the epidemiology of WSMV.

The interaction between Phytophthora spp. and Candidatus Liberibacter spp. damage to citrus fibrous roots

J. WU (1), E. G. Johnson (1), D. B. Bright (1), J. H. Graham (1)

(1) University of Florida, Citrus Research and Education Center, Lake Alfred, FL, U.S.A

Phytopathology 104(Suppl. 3):S3.131

Phytophthora nicotianae (P. n.) causes root rot in citrus, damaging the fibrous roots which depletes carbohydrate reserves and reduces water and nutrient uptake capacity. Huanglongbing (HLB) is a systemic citrus disease that disrupts photosynthate transport, and is caused by phloem-limited Candidatus Liberibacter asiaticus (Las). High P.n. populations were reported as HLB spread though Florida. To understand the possible interaction between these two pathogens, P.n. tolerant Swingle citrumelo and susceptible Cleopatra mandarin rootstocks were inoculated with Las, P.n. or both. P.n. infection, root loss, carbohydrate content of citrus fibrous root were assayed. P.n. infection increased on Swingle indicating that Las reduced its tolerance to P.n. Different P.n. infection on HLB positive Cleo seedlings at 5 and 11 weeks after inoculation suggests the interaction changed over time. Both pathogens caused significant root loss alone, but in combination P.n. did not cause additional loss compared to the total root loss caused by each pathogen alone. Based on these results, we hypothesize that 1) early in disease development, Las increases susceptibility to P.n infection by increasing zoospore attraction or facilitating penetration; 2) Las induces root loss resulting in a temporary drop in P.n. population by reducing available food supply; 3) As new roots flushes occur, P.n. starts the infection cycle again and repeats until there is a complete loss of fibrous root system.

Biocontrol mechanism of Pseudomonas fluorescens strain HN58 on sugarcane whip smut in blocking the sexual mating of Ustilago scitaminea P. XI (1), H. Deng (2), X. Sun (2), M. Li (2), W. Shen (3), L. Zhang (4), Z. Jiang (2)

(1) Department of Plant Pathology, South China Agricultural University, Guangzhou, 510642, China; Department of Plant Pathology, North Dakota State University, ND, U.S.A; (2) Department of Plant Pathology, South China Agricultural University, Guangzhou, 510642, Guangzhou, China; (3) College of Agriculture, South China Agricultural University, Guangzhou, 510642, Guangzhou, China; (4) South China Agricultural University, Guangdong Province Key Laboratory of Microbial Signals and Disease Control, Guangzhou, 510642, Guangdong, China

Phytopathology 104(Suppl. 3):S3.131

Sugarcane whip smut caused by Ustilago scitaminea is a destructive disease that causes serious yield losses on sugarcane in China. Previous study indicated a Pseudomonas fluorescens strain HN58 has showed inhibitory activities on the sexual mating of $U$. scitaminea. To elucidate the biocontrol mechanism, we studied the effects of strain HN58 on hypha growth and pheromone formation of $U$. scitaminea. Haploid cells and pathogenic dikaryotic hypha of $U$. scitaminea were grown separately in YEPS liquid supplemented with extracellular substances from HN58, and then the $\mathrm{OD}_{600}$ value, germination, and morphological characteristics of the fungus were compared with controls. No significant morphological changes were observed for haploid cells and dikaryotic hypha, but conjugation tubes were reduced. Meanwhile, mRNA expression of two genes, pral and $b E$, associated with pheromone production was analyzed by quantitative real-time PCR using the actin gene as an internal control. We found that pral and $b E$ expression were significantly reduced in fungal cultures containing the HN58 extracellular substances. Our data suggest that HN58 affected genes related to pheromone formation instead of morphological growth of haploid cells. This study provides important information for developing new biocontrol strategies on management of sugarcane smut disease. 
Biological control and plant growth promotion of Bacillus spp. on Meloidogyne incognita on cotton

N. XIANG (1), K. S. Lawrence (1), J. W. Kloepper (1), J. A. McInroy (1)

(1) Auburn University, Auburn, AL, U.S.A.

Phytopathology 104(Suppl. 3):S3.132

Tests were designed to select potential biocontrol agents that paralyze rootknot (RK) nematodes in vitro, reduce RK populations on the roots, and promote plant growth in cotton. Eighteen Bacillus strains and a strain of Lysinibacillus xylanilyticus were evaluated. In vitro assays in 96-well plates with $30-40 \mathrm{~J} 2 \mathrm{~s}$ in each well were evaluated the paralysis of RK J2s. Greenhouse trials were conducted with pasteurized soil and cotton inoculated with $1 \mathrm{ml}$ of each bacterium and 5,000 eggs and RK J2s. All the experiments were RCBD with four to five replications and were repeated twice. In vitro tests indicated that all 19 bacterial strains reduced live RK J2s compared with the control $(P \leq 0.05)$. Eleven Bacillus strains ( $B$. amyloliquefaciens, $B$. mojavensis, $B$. safensis, and B. subtilis) paralyzed RK J2s similarly to thiodicarb and avermectin, which paralyzed more than $75 \%$ RK J2s. In the greenhouse, none of the strains reduced numbers of RK eggs, but some strains promoted plant growth. Compared with aldicarb, strains AP-301 (B. amyloliquefaciens subsp. plantarum) and AP-3 (B. safensis) increased root fresh weight, AP-301 also increased root dry weight $(P \leq 0.05)$. In addition, strains AP-301 and AP-18 (B. pumilus) increased shoot fresh weight and shoot dry weight $(P \leq 0.05)$. Plant height and stem girth were all similar. Strains that showed biocontrol potential in vitro will be studied for metabolite production. Plant growth-promoting strains will be studied for yield increases.

Control of speck rot in apple fruit caused by Phacidiopycnis washingtonensis with pre- and postharvest fungicides

C. L. XIAO (1), R. J. Boal (2)

(1) USDA-ARS, Parlier, CA, U.S.A.; (2) Washington State University, Tree Fruit Research and Extension Center, Wenatchee, WA, U.S.A.

Phytopathology 104(Suppl. 3):S3.132

Speck rot caused by Phacidiopycnis washingtonensis is a recently reported postharvest fruit rot disease of apple. Infection of apple fruit by the fungus occurs in the orchard, but symptoms develop during storage. In this study, selected pre- and postharvest fungicides were evaluated for control of speck rot. Fruit were inoculated with the conidial suspension of the pathogen in the orchard 3 weeks before harvest. Part of the fruit was sprayed with a premixed formulation of pyraclostrobin and boscalid (Pristine), thiophanate-methyl, or ziram at 7 or 14 days before harvest, and the remaining fruit were either not treated as controls or dipped in fludioxonil, pyrimethanil or thiabendazole solutions after harvest. Fruit were stored at $0^{\circ} \mathrm{C}$ and monitored periodically for speck rot symptoms for up to 9 months. Over the 3 seasons, Pristine, thiophanate-methyl and ziram reduced speck rot by $5-87 \%, 0-80 \%$, and $53-$ $82 \%$, respectively, compared to the nontreated control. Fludioxonil, pyrimethanil and thiabendazole reduced speck rot by $93-100 \%, 96-100 \%$, and $77-95 \%$, respectively. Postharvest fungicide treatments were more effective than preharvest fungicides tested in this study. The results indicate that although infection of apple fruit by $P$. washingtonensis occurs before harvest, a preharvest fungicide applied near harvest or a postharvest fungicide treatment applied prior to storage can be an effective strategy for control of speck rot in stored apple fruit.

Interaction between Cassava brown streak virus VPg and cassava eIF4Es Z. XIONG (1), S. Shi (1), M. A. Mandel (1), T. Amuge (2), M. Ferguson (2), P. Zhang (3), S. Rounsley (4)

(1) School of Plant Sciences, University of Arizona, Tucson, AZ, U.S.A.; (2) International Institute of Tropical Agriculture, Nairobi, Kenya; (3) Institute of Plant Physiology and Ecology, Chinese Academy of Sciences, Shanghai, China; (4) Dow Agrosciences, Indianapolis, IN, U.S.A.

Phytopathology 104(Suppl. 3):S3.132

Cassava (Manihot esculenta) is an important tropical subsistence crop that is severely affected by cassava brown streak disease (CBSD) in East Africa. CBSD is known to be caused by two distinct virus species: Cassava brown streak virus (CBSV) and Cassava brown streak Uganda virus (UCBSV). Both are whitefly-transmitted ipomoviruses with a (+)-sense single-stranded RNA genome of about $9 \mathrm{~kb}$. Covalently linked to the 5 '-terminus of the viral genomic RNA is a genome-linked viral protein ( $\mathrm{VPg}$ ), which functionally resembles the cap structure of mRNA and serves as the binding site for eukaryotic translation initiation factor $4 \mathrm{E}$ (eIF4E and eIF(iso)4E). This binding is a prerequisite for the translation of viral genomic RNA in host cells. To determine which eIF4E(s) interacts with CBSV VPg, Illumina resequencing data of three eIF4E genes and two eIF(iso)4E genes were analyzed from 17 cassava lines tolerant or susceptible to CBSD. Sequence variations were observed in the exons of these genes but there is no apparent correlation to tolerance or susceptibility. Coding sequences of the five eIF4E genes were cloned from cassava mRNA (TMS60444 line) and the VPg-coding sequences of CBSV and UCBSV have also been cloned. Potential interactions between CBSV VPg and the cassava eIF4E family of proteins are currently evaluated in the LexA yeast two-hybrid system. Preliminary results showed that CBSV VPg interacted with two eIF4Es in vivo. Further confirmation analyses are underway.

Races of Phytophthora sojae causing root rot of soybean in Ontario A. XUE (1), Y. Chen (1), G. Marchand (1), S. Zhang (2), Y. Meng (1), E. Cober (1), A. Tenuta (3)

(1) Agriculture and Agri-Food Canada, Ottawa, ON, Canada; (2) Soybean Research Institute, Northeast Agricultural University, Harbin, China; (3) Ontario Ministry of Agriculture, Guleph, ON, Canada

Phytopathology 104(Suppl. 3):S3.132

Phytophthora sojae is the causal agent of Phytophthora root and stem rot (PRSR), an economically important disease of soybean worldwide. To determine the pathogenic variation of $P$. sojae in Ontario, where most Canadian soybean is grown, a total of 359 single-zoospore $P$. sojae isolates were obtained from plant and soil samples collected from 207 soybean fields and two PRSR nurseries during 2010-2012. Twenty-four races and 17 pathotypes of $P$. sojae were identified from the 359 isolates on a set of eight soybean differentials, each containing a single resistance (Rps) gene. Races 25,3 , and 9 were the predominant races, comprising 49, 43, and 34 isolates and representing $13.6,12.0$, and $9.5 \%$ of the pathogen population, respectively. Of the 41 races and pathotypes, 33, including the predominant race 25, were identified for the first time in Ontario. These results suggest that the race profile of $P$. sojae in Ontario has changed and new sources of resistance are needed for the development of resistant cultivars. Eleven races and four pathotypes were identified from $52 \mathrm{P}$. sojae isolates obtained from the PRSR nursery in Woodslee, and 13 races and two pathotypes identified from 44 isolates from the PRSR nursery in Ottawa. The common races in these nurseries were similar to what was found in commercial soybean fields, suggesting that both PRSR nurseries are appropriate and effective for screening soybean germplasm for cultivar development for Ontario.

Drought-responsive protein profiles reveal diverse defense pathways in corn kernels under field drought stress

L. Yang (1), T. Jiang (2), B. Scully (3), D. Lee (4), R. Kemerait (1), S. Chen (5), B. GUO (3)

(1) University of Georgia, Department of Plant Pathology, Tifton, GA, U.S.A.; (2) Northeast Forestry University, Key Laboratory of Forest Tree Genetic Improvement and Biotechnology of Ministry of Education, Harbin, China; (3) USDA-ARS, Crop Protection and Management Research Unit, Tifton, GA, U.S.A.; (4) University of Georgia, Department of Crop and Soil Sciences, Tifton, GA, U.S.A.; (5) University of Florida, Department of Biology, Genetics Institute, and Plant Molecular \& Cellular Biology Program, Gainesville, FL, U.S.A.

Phytopathology 104(Suppl. 3):S3.132

Drought stress is a major factor to contribute to preharvest aflatoxin contamination in agricultural crops. To identify drought responsive proteins and explore protein metabolic pathways involved in corn tolerance to drought, two corn genotypes (B73 and Lo964) with contrasting sensitivity to drought were used with treatments of drought and well-water at the stage of 14 days after pollination (DAP), and the protein profiles were investigated in developing kernels (35 DAP) using iTRAQ. Proteomic analysis showed that 70 and 36 proteins were significantly changed under drought treatments in B73 and Lo964, respectively. These drought response proteins were involved in diverse biological process pathways. By comparison the changes of protein patterns, the numbers and levels of differentially expressed proteins were generally higher in the sensitive genotype B73, implying an increased hypersensitive suffering with specific activities of proteins involved in redox homeostasis, cell rescue/defense, hormone regulation, protein biosynthesis and degradation. However, the tolerant genotype Lo964 gave a relatively more stable status with less number of differentially expressed proteins. We propose that the sensitive corn genotype switch on the rapid signaling pathways in response to drought through adjusting diverse defense pathways. The changes of these proteins produce a drought stress-responsive network in corn kernels.

Use of nested PCR to detect Ceratocystis fagacearum in sapwood of diseased northern oak species

A. YANG (1), J. Juzwik (2), D. Mollov (3)

(1) Department of Plant Pathology, University of Minnesota, St. Paul, MN, U.S.A.; (2) Northern Research Station, US Forest Service, St. Paul, MN, U.S.A.; (3) National Germplasm Resources Laboratory, USDA ARS, Beltsville, MD, U.S.A.

Phytopathology 104(Suppl. 3):S3.132 
Early and accurate diagnosis of oak wilt, caused by Ceratocystis fagacearum (Cf), is important when disease control action is planned. When laboratory diagnosis is needed, standard isolation protocols that are used rely on high quality samples and require $\geq 14$ days for incubation. Use of a nested PCR protocol to detect $\mathrm{Cf}$ in diseased sapwood shavings was compared to detection success using published isolation methods. Assays were performed on samples from oak species common in the Lake States. Four subsamples of three branches collected from each tree were tested. In actively wilting red oaks, $97 \%$ of the subsamples from nine trees were positive for Cf using PCR compared to $82 \%$ using isolation techniques. For wilting branches from eight bur oaks, $92 \%$ of subsamples were positive for $\mathrm{Cf}$ versus $67 \%$ based on isolation. In eight white oaks, $93 \%$ of subsamples were positive for $\mathrm{Cf}$ using PCR compared to $47 \%$ with isolation. The fungus was not detected by either technique in subsamples of branches obtained from healthy oaks (controls). For bur and white oak branches dead for $\geq 1$ year, Cf was detected using PCR (55 and $87 \%$ of subsamples, respectively), but was not detected by isolation. Similarly, only the PCR assay detected the pathogen in sapwood samples underlying remnant $\mathrm{Cf}$ sporulation mats on three main stem locations of seven trees. These findings are of particular interest to diagnostic laboratories that process disease-suspect samples in the region.

Biological control of Pierce's Disease: Identification of the endophytic mycobiota inhabiting diseased and symptomless grapevines

J. YANG (1), C. Roper (1), J. Borneman (1), J. Gloer (2), K. Maloney (3), P. Rolshausen (1)

(1) University of California, Riverside, Riverside, CA, U.S.A.; (2) The University of Iowa, Iowa City, IA, U.S.A.; (3) Point Loma Nazarene University, San Diego, CA, U.S.A.

Phytopathology 104(Suppl. 3):S3.133

Pierce's Disease (PD) of grapevines is an economically important disease caused by the bacterium Xylella fastidiosa $(X f)$. Notably, the presence of healthy vines alongside heavily infected or dying vines can be observed in infected vineyards. Given the fact that all grapevines in a planting are clones, we hypothesized that this observed resistance to PD is driven by a non-genetic mechanism and can be attributed, instead, to differences in the microbiome within these symptomless grapevines. We utilized both culture-dependent and culture-independent approaches to identify the endophytic organisms inhabiting grapevines. Field sites with a range of PD incidence were selected in the Napa and Temecula Valleys in California and plants were sampled over a three year period. High throughput sequencing of the fungal ribisomnal gene ITS region amplified from the endophytic microbial community indicated differences in the mycobiota between diseased and symptomless grapevines. 180 fungal isolates were recovered using a culture-based approach. From this culturable pool, we identified eight fungi with antagonistic properties against $X f$, and four among them reduced $X f$ titer and $\mathrm{PD}$ symptom severity in greenhouse trials. In addition, two fungal natural products produced by two of the antagonistic fungi were purified and demonstrated to have anti- $X f$ activity.

\section{Constructing a new phylogeny for the genus Phytophthora}

X. YANG (1), B. Tyler (2), C. Hong (3)

(1) Virginia Tech, Virginia Beach, VA, U.S.A.; (2) Oregon State University, Corvallis, OR, U.S.A.; (3) Virginia Tech Hampton Roads AREC, Virginia Beach, VA, U.S.A.

Phytopathology 104(Suppl. 3):S3.133

The genus Phytophthora includes many agriculturally and ecologically destructive plant pathogens. Investigations into its phylogeny are critical to understanding of the evolutionary history of this important genus. The current 10-clade phylogeny was established based on sequences of seven nuclear genetic markers back in 2007 and it includes approximately 80 Phytophthora species. Thereafter, this genus has expanded rapidly and now the total number of its members has reached about 130. Among the new species is $P$. stricta that does not belong to any known ITS clade. In this study, we construct a new phylogeny incorporating $P$. stricta and the vast majority of other recently described species including many ex-type and authentic cultures based on sequences of the seven phylogenetically informative genes. We also analyze the phylogenetic relations in clade 9 , a rapidly expanding clade, based on both nuclear and mitochondrial sequences. An array of phylogenetic analysis methods is employed to develop this new framework. This study helps advance the understanding of the evolutionary relationships among the clades, subclades and species while providing the signature sequences to assist species identifications in this rapidly expanding genus.

Molecular characterization of a novel soybean-associated virus identified by high-throughput sequencing

T. YASMIN (1), H. A. Hobbs (1), B. D. Nelson (2), L. L. Domier (3)
(1) Department of Crop Sciences, University of Illinois, Urbana, IL, U.S.A.; (2) North Dakota State University, Fargo, ND, U.S.A.; (3) USDA ARS; Department of Crop Sciences, University of Illinois, Urbana, IL, U.S.A. Phytopathology 104(Suppl. 3):S3.133

High-throughput sequencing of mRNA from soybean leaf samples collected from North Dakota revealed the presence of a novel soybean-associated virus that has a genome structure similar to invertebrate viruses in the family Dicistroviridae. The assembled length of the virus genome is 8,693 nt and contains two large open reading frames (ORFs). The 5'-proximal ORF is predicted to encode a protein of $85 \mathrm{kDa}$ that shows similarity to structural proteins of dicistroviruses (e.g., Aphid lethal paralysis virus and Cricket paralysis virus). The 3'-proximal ORF is predicted to encode a protein of 201 $\mathrm{kDa}$ that shows similarity to nonstructural proteins of animal picornaviruses (e.g., Hepatitis A virus), invertebrate dicistroviruses (e.g., Solenopsis invicta virus 1), and plant nepoviruses (e.g., Maize chlorotic dwarf virus). Hence, the gene order of the new virus is the opposite of that of members of the Dicistroviridae where ORF1 encodes the nonstructural proteins and ORF2 encodes the structural proteins. In addition ORF2 of the new virus is predicted to initiate translation at an AUG codon, while dicistroviruses use noncanonical initiation codons for their 3'-proximal ORFs. The unique genome organization and the similarity of the products of ORFs 1 and 2 to insect and plant viruses suggests that the new virus belongs to a new taxonomic group.

Identification of Lr34/Yr18 gene in wheat germplasm in Kazakhstan

G. Yessenbekova (1), A. Kokhmetova (2), A. Madenova (1), O. Amanov (1), Y. Dutbayev (1), G. KAMPITOVA (1)

(1) Kazakh National Agrarian Univ, Almaty, Kazakhstan; (2) Kazakh IIstitute of Plant Biology and Biotechnology, Almaty, Kazakhstan

Phytopathology 104(Suppl. 3):S3.133

Resistance of wheat to stripe rust (Puccinia striiformis) and leaf rust (Puccinia recondite) in Kazakhstan is very important, as these rusts significantly affect yield and quality wheat. This study was conducted to evaluate wheat (Triticum aestivum) genotypes in 19 Kazakhstan cultivars AND 47 lines of the international center ICARDA for the presence of the Lr34/Yr18-linked csLV34 allele, using STS marker $c s L V 34$ and to determine the effect of this gene complex on the components of partial resistance in wheat to stripe and leaf rusts. Lr34/Yr18-linked csLV34 allele was detected only in 14 foreign genotypes, namely IC-CAC Line8/KR11-26, IC-CAC Line9/KR11-29, ICCAC Line10/11KR-13, IC-CAC Line12/KR11-9025, IC-CAC Line15/KR1209, IC-CAC Line17/KR12-14, IC-CAC Line19/KR12-9010, IC-CAC Line24/ KR12-9023, IC-CAC Line25/KR12-5001, IC-CAC Line28/KR12-5051, ICCAC Line30/KR12-5070, IC-CAC Line31/KR12-5075, IC-CAC Line41/Hazrati Bashir and IC-CAC Line47/Egana, and Kazakhstan cultivars Ramin, Nureke, Mereke70, Maira, Bezostaya1, Almaly. Eleven genotypes showing the amplified $L r 34 / Y r 18$-linked allele were further studied to assess the effect of $\operatorname{Lr} 34 / \mathrm{Yr} 18$ on components of partial resistance along with 9 genotypes lacking this gene complex. It was suggested that $\operatorname{Lr} 34 / \mathrm{Yr} 18$ gene complex is more effective against the rust at later stages of plant growth. The results are used in wheat breeding programs for yellow rust resistance with marker-assisted selection.

Novel mild strains of Citrus tristeza virus from California and Peru

R. K. YOKOMI (1), S. Hajeri (2), K. Bederski (3), A. Giampetruzzi (4), G. Loconsole (4), M. Saponari (4)

(1) USDA ARS PWA, Parlier, CA, U.S.A.; (2) Central California Tristeza Eradication Agency, Tulare, CA, U.S.A.; (3) Vivero Topara, Lima, Peru; (4) Istituto di Virologia Vegetale, Consiglio Nazionale delle Ricerche, Bari, Italy Phytopathology 104(Suppl. 3):S3.133

Citrus tristeza virus (CTV) has caused great economic losses to citrus worldwide. CTV isolates from California were identified which reacted to MCA13 but were mild in biological indexing tests. Molecular markers were developed to differentiate these isolates from established CTV genotypes and the isolates were designated as having a California non-standard mild (CaNSM) genotype. Full-length genomes of representative CaNS-M isolates were obtained through deep sequencing. Alignment of these full-length sequences indicated close relatedness to reference genome NZRB-G90 (New Zealand resistance breaking $[\mathrm{RB}])$. These California isolates were differentiated into two sub-groups: subgroup 1 (RB genotypes) included Fillmore and CCTEA115 that had $100 \%$ sequence identity with NZRB-90; sub-group 2 (non-RB genotypes) included S1 and CCTEA65 that had 81\% sequence identity with NZRB-G90. Characterization of CTV isolates obtained from 15year cross-protected citrus trees from Peru showed a mixture of VT and a nonstandard genotype similar to RB-CaNS-M. A similar RB-CaNS-M isolate was identified from surviving trees in California which were infected with a severe VT genotype of CTV. Therefore, CaNS-M genotypes may have potential to 
cross-protective against severe strains of CTV. In depth characterization of the minor variants related to these non-standard genotypes and biological tests are underway to validate this hypothesis.

Impact of cyclic lipopeptide of Bacillus sp. for disease suppression against Fusarium soil-borne disease

K. YOKOTA (1)

(1) Tokyo University of Agriculture, Tokyo, Japan

Phytopathology 104(Suppl. 3):S3.134

Iturin and surfactin are antimicrobial cyclic lipopeptides produced by antagonistic Bacillus spp. It has been reported that antimicrobial lipopeptide producing-Bacillus strain shows biological control activity against several kinds of plant diseases. Since, the lipopeptide production is the key role in biological control by Bacillus spp. However, Bacillus spp. also produce biologically active substrates (e.g. 2,3-butanediol) which elicit the induced disease resistance of host plants. Therefore, the mechanism of biological control by using of Bacillus spp. is complicated. In this study, we show that the disease suppressive activities of lipopeptides for a soil-borne disease caused by Fusarium oxysporum on Taasai, Chinese flat vegetable, by using of purified lipopeptide. Both of iturin and surfactin showed disease suppressive activities by soil amendments, respectively. Interestingly, there are optimal concentrations which shows disease suppression for each lipopeptides. Our data suggested that iturin also elicits the induced disease resistance on host plant as known on surfactin.

\section{Trichothecene mycotoxin export in Fusarium graminearum}

B. Yordem (1), M. J. BOENISCH (1), K. L. Broz (2), H. C. Kistler (3)

(1) University of Minnesota, St. Paul, MN, U.S.A.; (2) USDA ARS Cereal Disease Laboratory, St. Paul, MN, U.S.A.; (3) USDA ARS Cereal Disease Laboratory/ University of Minnesota, St. Paul, MN, U.S.A.

Phytopathology 104(Suppl. 3):S3.134

Accumulation of trichothecene (TRI) mycotoxins by $F$. graminearum in wheat may require not only induction of enzymes for TRI biosynthesis but also may involve activation of cellular pathways to efficiently export these toxic metabolites and deliver them to the plant. Tri12 is a gene within the TRI biosynthetic gene cluster of $F$. graminearum that encodes a predicted drug resistance protein shown to play a role in export of TRI mycotoxins. Deletion mutants of Tri12 result in decreased TRI accumulation and reduced virulence when inoculated on wheat compared to the wild type strain. Mutants also are more prone to self-inhibition under toxin inducing conditions in vitro suggesting a role for Tri12 in self-protection, toxin export and virulence of the fungus in planta. Strains expressing Tri12p linked to green fluorescent protein show localization of the protein to the plasma membrane as well as to the vacuole and small $(1 \mu \mathrm{m})$ motile vesicles. Motile Tri12p linked vesicles may fuse with the vacuole or plasma membrane suggesting that vesicular transport of TRI may play a role in cellular sequestration and export of the toxin. The T-SNARE protein directing subapical exocytosis (SSO1) may be involved in toxin export. Deletion mutants $(\Delta s s o l)$ are significantly reduced in the ability to accumulate TRI in infected plants. Our results suggest a role for vesicular trafficking and exocytosis in export of TRI mycotoxins.

\section{Transcriptional analysis of Banana bunchy top virus DNA-U3}

N. Yu (1), Y. Zhang (1), J. Wang (1), P. Zhou (1), Z. Liu (1), Z. XIONG (2)

(1) Institute of Tropical Bioscience and Biotechnology, Chinese Academy of Tropical Agricultural Science, Haikou, China; (2) School of Plant Sciences, University of Arizona, Tucson, AZ, U.S.A.

Phytopathology 104(Suppl. 3):S3.134

Banana bunchy top virus (BBTV) has a genome of at least 6 circular, singlestranded DNA molecules of $\sim 1 \mathrm{~kb}$ each. With the exception of DNA2 (DNAU3), each genome component encodes a single gene with known functions. Previous reports identified a transcript of $\sim 400$ nucleotides from DNA-U3 of an Australian BBTV isolate. This transcript was predicted to have a 264-nt ORF capable of encoding a $10-\mathrm{kDa}$ protein with unknown functions. However, subsequent analysis of numerous BBTV DNA-U3 sequences indicated the presence of an in-frame stop codon that essentially broke the predicted ORF in many isolates. To ascertain if the DNA-U3 transcript is produced and whether it encodes a protein product, we performed 5' and 3' rapid amplification of cDNA ends (RACEs) from two Hainan isolates of BBTV. A very small transcript of 229 nucleotides, starting at nucleotide 287 and terminating at nucleotide 516, was identified from the RACE data. Subsequently transcriptomic analysis of BBTV-infected tissues confirmed the presence of such transcript and furthermore indicated its high abundance relative to transcripts from other BBTV components. This transcript does not contain a conventional ORF, but a 96-nucleotide ORF can be predicted if GUG could serve as the translation initiation codon. However, repeated attempts have failed to detect this protein product in infected tissues. The implications of this finding and the potential roles of DNA2 in BBTV infection will be discussed.

\section{Infection of biofuel switchgrass populations by two rust species in} Nebraska

G. Yuen (1), Y. MA (1), C. Jochum (1), K. Vogel (2)

(1) University of Nebraska-Lincoln, Lincoln, NE, U.S.A.; (2) USDA, ARS (retired), Lincoln, NE, U.S.A.

Phytopathology 104(Suppl. 3):S3.134

Rust diseases potentially could impact yield and biomass quality of new switchgrass (Panicum virgatum) cultivars being developed for use as biofuel feedstock. Two rust species, Uromyces graminicola and Puccinia emaculata, are known to infect switchgrass, but recent surveys of switchgrass populations in southern states revealed $P$. emaculata to be the primary or sole rust pathogen. The objectives of this study were to evaluate rust resistance among 22 switchgrass populations planted in a variety trial in Nebraska and to identify the rust species infecting these populations. Ten leaves were collected from each plot in August 2013 and scored for rust severity on a 0 (no symptoms) to 9 (abundant sporulation) scale. Mean rust severity scores and associated resistance levels varied among the entries from 0 : highly resistant to 5: moderately susceptible, with most entries scoring lower than 3: moderately resistant. Based on morphology and size of teliospores found on leaf samples, both $U$. graminicola and $P$. emaculata were identified as infecting the entries exhibiting higher levels of rust susceptibility. The results indicate that if newly developed biofuel switchgrass cultivars are to be productive in the northern plains, they must be resistant to both rust species.

Effect of Macrophomina phaseolina inoculation, irrigation and cultivar on soybean yield

M. ZACCARON (1), R. Holland (1), A. Steger (1), J. C. Rupe (1)

(1) University of Arkansas, Fayetteville, AR, U.S.A

Phytopathology 104(Suppl. 3):S3.134

Soybean charcoal rot, caused by M. phaseolina, is often reported to be more severe under drought stress. To improve disease management it is necessary to understand the effect of irrigation regimes on soybean yield loss caused by charcoal rot. In the years of 2011 and 2013 four soybean cultivars, Osage, Ozark, Hutcheson, and R0158F, were grown in field plots with and without inoculum and either irrigated throughout the season (FSI), only irrigated until R5 (UR5I), or not irrigated (NI). In 2011, there were no significant differences in yield between the cultivars, but yields were significantly greater in noninoculated than inoculated plots, 1848 and $1723 \mathrm{~kg} \mathrm{ha}^{-1}$, respectively, and greater in FS than IUR5 or NI, 2770, 1487, and $1116 \mathrm{~kg} \mathrm{ha}^{-1}$, respectively. In 2013, a three-way interaction occurred between cultivar, irrigation and inoculation. In the non-inoculated plots, there were no differences between cultivars but yields in FS were much greater than UR5I and NI, 2498, 1648, and $1624 \mathrm{~kg} \mathrm{ha}^{-1}$, respectively. In the inoculated plots, Osage had the highest yield and Hutcheson had the lowest yield in the FS treatment, in UR5I, Ozark had the highest yield and Hutcheson had the lowest yield, but in NI, there were no differences between cultivars. Adding inoculum of M. phaseolina at planting had a significant effect on the plant resulting in lower yields each year. In 2013, this effect was greatest in FS.

Genome-wide identification of Hfq-regulated small RNAs in the fire blight pathogen Erwinia amylovora discovered virulence-regulating small RNAs

Q. ZENG (1), G. W. Sundin (1)

(1) Michigan State University, East Lansing, MI, U.S.A.

Phytopathology 104(Suppl. 3):S3.134

Erwinia amylovora is a phytopathogenic bacterium that causes fire blight disease in apples and pears. Although many virulence factors have been characterized, the coordination of expression of these virulence factors in $E$. amylovora is still not clear. Regulatory small RNAs (sRNAs) are important post-transcriptional regulatory components in bacteria. A large number of sRNAs require the RNA chaperone Hfq for both stability and functional activation. In E. amylovora, Hfq was identified as a major regulator of virulence and various virulence traits. However, information is still lacking about Hfq-regulated sRNAs on a genome scale, including the virulence regulatory functions of these sRNAs in E. amylovora. Using both an RNA-seq analysis and a Rho independent terminator search, 42 Hfq-regulated sRNAs were identified in E. amylovora. Sequence conservation analysis identified sRNAs conserved only in the Erwinia genus as well as E. amylovora speciesspecific sRNAs. In addition, a dynamic re-patterning of expression of Hfqregulated sRNAs was observed at 6 and 12 hours after induction in Hrpinducing minimal medium. Furthermore, small RNAs that control virulence traits were characterized, among which ArcZ positively controls the type III secretion system, amylovoran exopolysaccahride production, biofilm 
formation, and motility, and negatively modulates attachment while RmaA and $\mathrm{OmrAB}$ both negatively regulate amylovoran production and positively regulate motility.

Population structure of Geosmithia morbida, the causal agent of Thousand cankers disease of walnut trees in the United States

M. M. Zerillo (1), J. R. IBARRA CABALLERO (1), K. E. Woeste (2), A. D. Graves (3), C. Hartel (2), J. W. Pscheidt (4), J. Tonos (2), K. D. Broders (5), W. Cranshaw (1), S. J. Seybold (6), N. A. Tisserat (1)

(1) Colorado State University, Fort Collins, CO, U.S.A.; (2) Purdue University, West Lafayette, IN, U.S.A.; (3) USDA Forest Service, Albuquerque, NM, U.S.A.; (4) Oregon State University, Corvallis, OR, U.S.A.; (5) University of New Hampshire, Durham, NH, U.S.A.; (6) USDA Forest Service, Davis, CA, U.S.A.

Phytopathology 104(Suppl. 3):S3.135

Geosmithia morbida, and the walnut twig beetle, Pityophthorus juglandis, are associated with an insect/disease complex of Juglans (walnut) species called thousand cankers disease. The disease has been reported in the western USA on several Juglans species, and recently it has been found in the natural range of the highly susceptible Juglans nigra in the eastern USA. We performed a comprehensive population genetic study of 207 G. morbida isolates collected from 6 Juglans species and Pterocarya stenoptera (Chinese wingnut) from 17 geographic regions distributed across 12 states of the USA. Using multi locus sequence typing data, $195 \mathrm{G}$. morbida isolates were placed into one of 57 haplotypes. Multiple haplotypes were recovered from isolates collected on the same tree. Forty two \% of the haplotypes were represented by more than one isolate. The two most frequently occurring haplotypes, representing $36 \%$ of all G. morbida isolates tested, were not recovered from New Mexico and Arizona but were detected in all other geographic regions where more than 4 isolates were collected and were especially abundant in California. The G. morbida population in the USA was best explained by four genetically distinct groups that clustered into three geographic units. No evidence of genetic recombination was found. The scattered distribution of the genetic clusters indicated that $G$. morbida likely was probably disseminated to different regions several times and from several sources.

Exploration of the causal agent of citrus blight using metagenomic approaches

Y. ZHANG (1), N. Wang (1)

(1) University of Florida, Lake Alfred, FL, U.S.A.

Phytopathology 104(Suppl. 3):S3.135

Citrus blight is a mysterious disorder that causes thousands of citrus trees to become unproductive every year. The disease is a problem wherever citrus is grown in hot and humid areas including North America, the Caribbean, South America, South Africa and Australia. The causal agent remains unknown despite tremendous efforts on citrus blight research. Advance in next generation sequencing and metagenomics has significantly helped identify the causal agents of multiple diseases. In our study, metagenomic analyses were conducted to investigate the microbes in the roots of healthy and citrus blight diseased citrus. We also examined the transcriptome response of citrus to citrus blight, which may reveal novel insights to this disease. Transcriptome analysis indicated that the JA and ET biosynthesis and signaling pathways showed decreased transcript abundance while several SA-mediated defenserelated genes showed increased transcript abundance, suggesting biotrophic pathogen(s) is/are involved in this disease. We will present our results in microbial diversity associated with citrus. The putative cause for citrus blight will be discussed.

Host range evaluation of Cercospora sojina, causal agent of frogeye leaf spot of soybean

G. ZHANG (1), U. Reuter-Carlson (1), D. K. Pedersen (1), C. A. Bradley (1)

(1) University of Illinois, Urbana, IL, U.S.A.

Phytopathology 104(Suppl. 3):S3.135

Cercospora sojina causes frogeye leaf spot (FLS) of soybean, but little has been published on its host range. In a greenhouse trial, 20 legume species, 10 weedy plants, cotton, and sweet corn were inoculated with C. sojina and evaluated for infection by observing symptoms, by using microscopy, and by isolation onto culture media. Typical FLS symptoms appeared on the legumes mung bean, adzuki bean, winged bean, guar bean, snap pea, winged pea, pigeon pea, sunn hemp, and red clover, and on the weeds morning glory and poison hemlock. Symptoms atypical of FLS appeared on the legumes lima bean, cowpea, peanut, hairy vetch, fava bean, Chinese mosaic yard long bean, scarlet runner bean, lentil, and snap bean, on the weeds common ragweed, common waterhemp, palmer amaranth, morning glory, smooth pigweed, velvetleaf, common lambsquarters, and giant ragweed, and on cotton. No symptoms appeared on chickpea, alfalfa, horseweed, redroot pigweed and sweet corn. Inoculated leaf surfaces were sterilized with a $10 \% \mathrm{NaOCl}$ solution, and placed on culture media for C. sojina isolation. Cultures resembling $C$. sojina were evaluated for morphological characters and verified as $C$. sojina using ITS primer sequence. Out of all potential hosts tested, results of $C$. sojina and microscopy isolations indicated that only chickpea and sweet corn were nonhosts.

Advancing our understanding of mature plant resistance to Potato virus $Y$ in selected varieties towards improved seed potato certification

C. ZHANG (1)

(1) University of Wisconsin, Madison, WI, U.S.A.

Phytopathology 104(Suppl. 3):S3.135

Potato Virus Y continues to be an important pathogen in seed potato production due to the emergence of novel recombinant strains combined with asymptomatic infections in both plants and tubers. Mature plant resistance is a phenomenon defined by an observed reduction in disease incidence associated with later season inoculations in plants. This concept can potentially be a valuable component to add to our existing PVY management programs in seed potato, especially since the majority of aphids are active in late growing season. Replicated field experiments were established in both 2012 and 2013 to investigate the response of four potato varieties to infection by two strains of PVY at different stages of potato development. Replicate sets of potato varieties were mechanically sap-inoculated with $\mathrm{PVY}^{\mathrm{O}}$ and $\mathrm{PVY}{ }^{\mathrm{N}: \mathrm{O}}$ during pre-flower and several post flowering stages, and compared with untreated controls. Visual field evaluation of foliage symptoms and ELISA detection of collected leaf samples were used respectively to confirm the presence of PVY originating from seed tubers in post-harvesting test. Diseases reduction in later season infection were observed in all three varieties except the cultivar 'Russet Norkotah'. Notably, the cultivars 'Silverton' $(\mathrm{F}=5.5, \mathrm{df}=(4,15), \mathrm{p}$ value $=0.006)$ and 'Dark Red Norland' $(\mathrm{F}=31.85, \mathrm{df}=(4,15)$, $\mathrm{p}$-value $=3.6 \mathrm{e}-07)$ possessed the greatest overall differences between pre- and post-flower inoculations.

IRE1/bZIP60 mediated unfolded protein response plays an important role in TuMV infection in Arabidopsis

L. ZHANG (1), A. Wang (1)

(1) Agriculture and Agri-Food Canada, London, ON, Canada

Phytopathology 104(Suppl. 3):S3.135

The unfolded protein response (UPR) signaling network consists of two pathways in plants, one mediated by IRE1 (inositol-requiring protein-1) / bZIP60 (basic leucine zipper), and the other by bZIP17/bZIP28. Although the UPR has very recently been shown to be associated with virus infection in plants, the mechanism of initiating UPR pathway(s) under virus insult is unclear. Here, we showed that Turnip mosaic virus (TuMV), a member of the genus Potyvirus in the family Potyviridae, could initiate the UPR in Arabidopsis thaliana, indicated by the expression of ER molecular chaperones, such as ER luminal binding protein (BiP) 3 and 1/2, protein disulfide isomerase (PDI), and calreticulin (CRT). We further demonstrated that, prior to the induction of the ER molecular chaperones, TuMV infection could cause the IRE1 mediated unconventional splicing of bZIP60 mRNA. Transient expression of each viral protein alone in Nicotiana benthamiana revealed that TuMV $6 \mathrm{~K} 2$ is the inducer of the bZIP60 splicing. In addition, compared to wild type Arabidopsis, TuMV infection is significantly inhibited in the bzip60-2 mutant allele and the irelairelb double mutant, in which bZIP60 splicing is absent. Importantly, the inhibition of TuMV infection and the abolishment of bZIP60 splicing could be rescued by complementing the double mutant using IRE1A or IRE1B alone. Together, these results suggest that IRE1/bZIP60 mediated UPR pathway is activated under TuMV attack to serve for viral infection.

Transcriptome analysis to understand host defense mechanisms in Brassica napus - Leptosphaeria maculans pathosystem X. ZHANG (1), M. F. Belmonte (1), M. G. Becker (1), W. G. Fernando (1) (1) University of Manitoba, Winnipeg, MB, Canada Phytopathology 104(Suppl. 3):S3.135

Blackleg, caused by Leptosphaeria maculans, is among the major diseases of Brassica napus (canola) worldwide. Growing resistant cultivars remains the most economical and environmentally friendly method to manage this disease. In order to better understand the resistance mechanism, RNA sequencing was employed to uncover transcriptome changes and key genes in the host. A time course expression pattern was studied from several selected marker genes: pathogenecity related gene $P R-1$, WRKY transcription factor gene $B n W R K Y 75$, and lignin metabolism related genes. These genes were examined with a $L$. maculans isolate that was inoculated onto both resistant and susceptible Brassica napus accessions and the expression pattern of each gene was quantified by qPCR prior to RNA-seq. The expression of $P R-1$ increased 
rapidly 3 days post inoculation (dpi) in both resistant and susceptible accessions while the response of BnWRKY75 was earlier in the resistant accession. The relative expression of lignin metabolism related genes increased following fungal infection. RNA-seq results revealed a high degree of differential expression post infection between resistant and susceptible accessions. Transcripts also showed varied expression patterns relative to several time points ( $3 \mathrm{dpi}$ and $7 \mathrm{dpi}$ ). Differentially expressed genes were then characterised for their function, with a focus on identifying genes involved in the blackleg resistance pathways and plant defence mechanisms in general.

AmplifyRP $^{\circledR}$ Acceler8 $^{\mathrm{TM}}$ - a rapid isothermal test for plum pox virus using reverse transcription-recombinase polymerase amplification

S. ZHANG (1), P. Russell (1), N. McOwen (1), S. Bohannon (1), A. Vrient (1)

(1) Agdia, Inc., Elkhart, IN, U.S.A

Phytopathology 104(Suppl. 3):S3.136

Plum pox virus (PPV), a member of the Potyviridae family, causes the most destructive viral disease known as plum pox or Sharka disease of stone fruit species such as cherry, peach or plum. As an important regulated pathogen, detection of PPV is thus of critical importance to quarantine and eradication of the disease. In this study, we present the development and validation of a rapid and sensitive isothermal test, AmplifyRP ${ }^{\circledR}$ Acceler8 ${ }^{\mathrm{TM}}$, for PPV using reverse transcription-recombinase polymerase amplification (RT-RPA). The Acceler8 ${ }^{\mathrm{TM}}$ utilizes the RT-RPA technology to produce PPV-specific DNA amplicons at a single constant temperature of $39^{\circ} \mathrm{C}$ without thermal cycling and the results are visualized through lateral flow strip assay performed inside an amplicon detection chamber. Thus, all amplicons are contained with little concern about contaminations. The Acceler8 ${ }^{\mathrm{TM}}$ also shares a common plant extraction buffer with ELISA and ImmunoStrip ${ }^{\circledR}$ and the crude plant extracts are directly applied to Acceler8 ${ }^{\mathrm{TM}}$, ELISA or ImmunoStrip ${ }^{\mathbb{B}}$ for testing. The whole test from sample preparation to result can be completed in as little as 30 minutes. The Acceler8 ${ }^{\mathrm{TM}}$ detects all nine known PPV strains (An, C, CR, D, EA, M, Rec, T, or W). The detection of PPV by Acceler8 ${ }^{\mathrm{TM}}$ is as sensitive as that by RT-PCR and can be performed both in laboratories and in field. This is the first application of the RT-RPA technology for the detection of plant viruses.

Amplification of genomic DNA of Puccinia striiformis f. sp. tritici from urediospores for population genetic analysis

R. ZHANG (1), B. M. Wu (1), Z. Ma (1)

(1) China Agricultural University, Beijing, China

Phytopathology 104(Suppl. 3):S3.136

Wheat stripe rust pathogen (Puccinia striiformis f. sp. tritici) is an obligate biotroph. It cannot be cultured and its propagation has to be on living wheat seedlings. The time, space and labor consuming nature of pathogen propagation limited how many samples we can process for further molecular genetic analysis. In this study we compared genomic DNA obtained via CTAB extraction and DNA amplified from urediospores via multiple displacement amplification (MDA). The fragment size and total amount of DNA were compared via electrophoresis on $1 \%$ agarose gel. The purities of DNA products were further determined via real time PCR with a pair of primers specific for the $\beta$-tubulin gene of $P$. striiformis f.sp. tritici. The Amplified fragment length polymorphism (AFLP) fingerprints were also compared between the different DNA products. The results demonstrated that adequate genomic DNA at fragment size larger than $23 \mathrm{~Kb}$ could be amplified from 20-30 urediospores via MDA method. The real time PCR results suggested that although fresh urediospores collected from diseased leaves were the best, spores picked from diseased leaves stored for a prolonged period could also be used for amplification. AFLP fingerprints exhibited no significant differences between amplified DNA and DNA extracted with CTAB method. Therefore, we conclude that MDA can be used to obtain genomic DNA from a few urediospores for population genetic analysis of wheat stripe rust pathogen.

Study on the CR811 gene present exclusively in pathotype 5 of the canola clubroot pathogen Plasmodiophora brassicae

H. ZHANG (1), J. Feng (2), S. F. Hwang (2), S. E. Strelkov (3)

(1) The Institute of Vegetables and Flowers, Chinese Academy of Agricultural Sciences, Beijing, AB, China; (2) Alberta Agriculture and Rural Development, Edmonton, AB, Canada; (3) University of Alberta, Edmonton, $\mathrm{AB}$, Canada

Phytopathology 104(Suppl. 3):S3.136

Clubroot caused by Plasmodiophora brassicae is an important disease of crucifers worldwide. Isolates of the pathogen can be classified into pathotypes according to their pathogenicity on differential host genotypes. Pathotypes may differ at a single locus or a few loci as a result of host-pathogen coevolution, and the functional alleles of these loci should be related to the pathogenicity. Currently, most of the database-available gene sequences from $P$. brassicae are derived from cDNA libraries constructed by suppression subtractive hybridization between healthy and infected crucifers, thus they are likely to be related to pathogenesis. In this study, the presence or absence of all database-available non-housekeeping $P$. brassicae genes (118 in total) was assessed by PCR analysis in five $P$. brassicae pathotypes (2, 3, 5, 6 and 8). One gene designated CR811 was present exclusively in pathotype 5, which was further confirmed by using alternative primers and DNA preparations. Reverse transcription quantitative PCR analysis indicated that CR811is highly expressed during canola infection, with a 22 -fold of up-regulation in secondary zoospores relative to primary zoospores. These findings suggest that CR811 is a possible pathogenicity factor and that it also could serve as a molecular marker for differentiation of pathotype 5 from other pathotypes.

Laboratory and field evaluations of potato cultivars for resistance to potato stem canker caused by Rhizoctonia solani AG2-1

X. ZHANG (1), Z. Yu (1), J. Hao (2)

(1) College of Agronomy, Inner Mongolia Agricultural University, Hohhot, China; (2) School of Food and Agriculture, University of Maine, Orono, ME, U.S.A.

Phytopathology 104(Suppl. 3):S3.136

Rhizoctonia solani is the casual agent of stem canker of potato. Field and laboratory trials were conducted to evaluate potato cultivars in resistance to stem canker. In 2009 and 2010, seed pieces of 20 potato cultivars were planted in field soil infested with $R$. solani AG2-1. The inoculum of $R$. solani was produced in wheat bran, and 2 to $4 \mathrm{~g}$ of wheat bran inoculum per a seed tuber was used. In results, none of 20 potato cultivars was completely resistant to $R$. solani AG2-1, and the disease severity varied from disease index 16.69 to 60.00. The cultivar Desiree (16.69) showed the least percentage of infection by $R$. solani AG2-1, while the cultivar Atlantic (60.00), Favorita (58.57), and Shepody (59.77) showed a higher incidence. Under laboratory conditions, virus-free potato seedlings were grown in Murashige and Skoog medium until their height reached around $12 \mathrm{~cm}$, then transferred into $R$. solani AG2-1 produced toxin that the absorbance was 7.48 at wavelength 258.8 in a test tube. After incubation at $25^{\circ} \mathrm{C}$ with $16 \mathrm{~h}$ light (4000 lux)/day, the seedlings were observed for necrotic symptom from day 4 to 8 of incubation, and disease was measured. The disease severity was significantly different among potato cultivars, and the results were highly correlated with field data $\left(R^{2}=\right.$ $0.57, P<0.01$ ), which indicated that this laboratory method can be used as a fast and alternative procedure to field test in screening potato germplasm for stem canker resistance.

Fine mapping of novel QTL for Fusarium head blight resistance in PI 277012

M. ZHAO (1), S. S. Xu (2), S. Zhong (1)

(1) North Dakota State University, Fargo, ND, U.S.A.; (2) United States Department of Agriculture, Fargo, ND, U.S.A.

Phytopathology 104(Suppl. 3):S3.136

A hexaploid wheat line PI 277012 was previously shown to exhibit a high level of Fusarium head blight (FHB) resistance in both greenhouse and field experiments. QTL analysis of a doubled haploid (DH) population consisting of 130 lines from a cross between PI 277012 and 'Grandin' identified two major FHB resistance QTLs located on chromosome arms 5AS and 5AL, respectively. The 5AS QTL (Qfhb.rwg-5A.1) peaked at marker Xbarc40 between markers Xcfa2104 and Xgwm617, while the 5AL QTL (Qfhb.rwg$5 A .2)$ peaked at marker $X c f d 39$ between $X w m c 470$ and $X b a r c 48$, which is different from those found in other known sources of FHB resistance. To saturate the resistance QTL regions with more DNA markers, the DH population was further genotyped using the wheat $9 K$ SNP iSelect Assay. A total of 2,877 polymorphic SNPs were identified between PI 277012 and 'Grandin', and further mapped to the original genetic linkage map developed with SSR markers. Ten SNP markers within peaks of the two QTL regions were selected and used to genotype a larger population consisting of 1,050 recombinant inbred lines (RILs) from the cross between PI 277012 and 'Grandin'. Greenhouse evaluation using the single floret point inoculation method identified highly resistant and susceptible RILs in this population. Additional DNA markers are being developed from genome sequences of wheat and other related species and will be used for fine mapping of the novel QTL.

A single amino acid substitution in the movement protein alters the mechanical transmissibility of Tomato leaf curl New Delhi Begomovirus

Y. X. Zheng (1), C. H. Lee (1), Y. T. Lin (1), C. H. Chan (1), F. J. JAN (1)

(1) Department of Plant Pathology, National Chung Hsing University, Taichung, Taiwan

Phytopathology 104(Suppl. 3):S3.136 
Tomato leaf curl New Delhi virus (ToLCNDV) is one of the widely distributed bipartite begomoviruses that affect the production of many important crops in tropical and subtropical countries. A ToLCNDV oriental melon isolate (ToLCNDV-OM) identified in Taiwan is confirmed to be mechanically transmissible. To identify the viral factors involved in mechanical transmissibility of ToLCNDV, the viral genome was modified to generate the viral DNA infectious clones in the study. The DNA-A and DNA$B$ genomes from mechanical transmissible ToLCNDV-OM and nonmechanical transmissible ToLCNDV-CB were used in the genome replacement experiment. The results of pseudorecombination and recombination experiments showed that the movement protein (MP) gene was responsible for the mechanical transmissibility. The substitution mapping results indicated that the 19th amino acid residue (glutamate, $\mathrm{E}_{19}$ ) of ToLCNDV-OM MP gene determines the mechanical transmissibility. After mechanical inoculation, the detection of viral DNA accumulation by Southern blot hybridization indicated that the ToLCNDV-CB and individual mutants that don't carry wild type ToLCNDV-OM MP gene cannot replicate or accumulate in the inoculated leaves. Our results provide a likely explanation that the viral DNAs of nonmechanically transmissible viruses are not able to replicate in the initially invaded cells or unable to move to the adjacent cells through plasmodesmata after replication.

A whole genome sequence of 'Candidatus Liberibacter asiaticus' from Guangdong, China, where HLB was first described

Z. ZHENG (1), X. Deng (1), J. Chen (2)

(1) South China Agricultural University, Guangzhou, China; (2) USDA ARS

PWA, Parlier, CA, U.S.A.

Phytopathology 104(Suppl. 3):S3.137

Citrus Huanglongbing (HLB, yellow shoot disease) has been endemic in Guangdong Province, China, for $>100$ years and was confirmed to associate with "Candidatus Liberibacter asiaticus" (CLas) in 1996. Knowledge about CLas is critical for HLB control. Yet, the unculturable nature of CLas is highly challenging for biological research. Here, a draft whole genome sequence of CLas strain A4 from Guangdong is presented. Strain A4 was originally collected from an HLB mandarin citrus (Citrus reticulata cultivar Shatangju) in Sihui City of Guangdong Province and transmitted to healthy Shatangju plant through grafting in 2005. In 2007, CLas was transmitted from citrus to periwinkle (Catharanthus roseus) via dodder (Cuscuta campestris). For sequencing, DNA was extracted from CLas-infected periwinkle tissue and sequenced using Illumina technology after removing methylated DNA and multiple displacement amplification. Sequence reads of CLas were extracted referenced to currently available whole genome sequences of CLas of psyllid origins. After assembling, the A4 strain has the genome size of 1,208,625 bp, $\mathrm{G}+\mathrm{C}$ content of $36.4 \%, 1,107$ predicted open reading frames, and $53 \mathrm{RNA}$ genes. The A4 genome sequence has been deposited at DDBJ/EMBL/ GenBank under the accession no. JFGQ00000000. In addition to be from the historical HLB endemic region, the A4 whole genome sequence is also the first from a plant host.

\section{A Sec-dependent periplasmic protein VrpA is required for efficient secretion of type III secretion system and virulence in Xanthomonas citri X. ZHOU (1), N. Wang (1) \\ (1) University of Florida, Lake Alfred, FL, U.S.A. \\ Phytopathology 104(Suppl. 3):S3.137}

An efficient secretion of type III effector proteins from bacterial cytoplasm to host cell cytosol via type III secretion system is required to cause diseases for many phytopathogenic bacteria. In our previous study, a highly conserved hypothetical protein, here designed as $\operatorname{VrpA}$ (virulence-related periplasm protein A), was identified as a critical protein for Xanthomonas citri subsp. citri virulence by screening of a Tn5-transposon mutant library. Here we confirm that vrpA deletion mutant fails to cause visible citrus canker symptom. An N-terminal 26 residues signal peptide is identified and demonstrated to be required for VrpA's periplasm localization through Secdependent manner and $X$. citri virulence. The cyaA gene fusion analysis reveals that $v r p A$ mutant is reduced in its ability to inject type III effector proteins into plant cell. Yeast two-hybrid experiments show that VrpA physically interacts with periplasmic domain of $\mathrm{HrcJ}$ and $\mathrm{HrcC}$, two T3SS component proteins. Our data provide evidence that efficient secretion of type III effectors of $X$. citri requires VrpA.

Effects of two rice viruses on host preference and development of their vector and non-vector insects

G. ZHOU (1), B. Ling (1), D. Xu (1), M. Zhang (1)

(1) South China Agric University, Guangzhou, China

Phytopathology 104(Suppl. 3):S3.137
Southern rice black-streaked dwarf virus (SRBSDV) and Rice ragged stunt virus (RRSV) are two major viruses infecting rice in eastern and southeastern Asia, and are vectored by white-backed planthopper (WBPH, Sogatella furcifera) and brown planthopper (BPH, Nilaparvata lugens) in a persistent manner respectively. Using a Y-shape olfactometer-based device, we discovered that SRBSDV-free WBPHs significantly preferred SRBSDVinfected rice plants to healthy plants, whereas viruliferous WBPHs preferred healthy plants to infected plants; and RRSV-free BPHs significantly preferred healthy plants, whereas RRSV-carrying BPHs preferred SRBSDV-infected rice plants. These interactions may partially account for the rapid dissemination of SRBSDV and the co-infection of the two viruses in field. Meantime, we tested various biological parameters of WBPHs reared on SRBSDV infected rice plants under greenhouse condition. The results showed that both SRBSDV and infected plants were unfavorable for the expansion of WBPH population, leading to prolonged nymphal stages, reduced survival rates, shorter adult life spans and lower oviposition amounts. These findings give insight into the virus-vector-host plant relationship and are helpful for monitoring, forecasting, and controlling rice viral diseases.

Role of $r p o Z$ gene in regulating antibiotic synthesis and quorum sensing in Pseudomonas fluorescens

H. Zhou (1), Y. Wei (1), X. ZHANG (1), J. Hao (2), X. Wu (3)

(1) College of Agronomy, Inner Mongolia Agricultural University, Hohhot, China; (2) School of Food and Agriculture, University of Maine, Orono, ME, U.S.A.; (3) Department of Plant Pathology, China Agricultural University, Beijing, China

Phytopathology 104(Suppl. 3):S3.137

Pseudomonas fluorescens 2P24 produces secondary metabolites, such as 2,4diacetyphloroglucinol (2,4-DAPG), and has been used as an effective biocontrol agent to control damping-off (Rhizoctonia solani) of cotton. In this study, the regulation of genes phlA for synthesizing 2,4-DAPG and pcoI for synthesizing Pcol/PcoR quorum sensing signal was studied at transcriptional level. Mutants of $P$. fluorescens were derived by random mutagenesis on $P$. fluorescens strain 2P24. The lacZ marker gene was transferred and linked to gene phlA. Hybridization was performed between strain PM901 that contains the phlA-lacZ/970Km (receptor) and the plasmid pUT/Gm containing Tn5 transposon (provider) so that $\mathrm{pUT} / \mathrm{Gm}$ was randomly inserted into PM901. The mutants derived from $2 \mathrm{P} 24$ were modified by transferring with a kanamycin-resistant plasmid (pBBR), and either depleting rpoZ gene ($\Delta$ rpoZ) or inserting rpoZ gene (-rpoZ): $2 \mathrm{P} 24 / \mathrm{pBBR}, 2 \mathrm{P} 24-\Delta \mathrm{rpoZ} / \mathrm{pBBR}$, and $2 \mathrm{P} 24-\triangle \mathrm{rpoZ} / \mathrm{pBBR}-\mathrm{rpoZ}$. These strains were examined on their growth activity, mobility, N-Acyl homoserine lactone (AHL) production, 2,4-DAPG production, and extracellular polymeric substances. 2P24- $\triangle \mathrm{rpoZ} / \mathrm{pBBR}$ significantly reduced the production of antibiotic 2,4-DAPG and signaling molecules AHL, had slower growth rate, and almost lost its capability of disease controlling. The results indicated that the rpoZ gene plays an important regulatory role in the complex genes controlling the expression of biocontrol traits in P. fluorescens 2P24.

Effects of cultivar resistance and biocontrol agent on spatial spread of bacterial panicle blight of rice

X. G. ZHOU (1)

(1) Texas A\&M University System, AgriLife Research and Extension Center, Beaumont, TX, U.S.A.

Phytopathology 104(Suppl. 3):S3.137

Bacterial panicle blight caused primarily by Burkholderia glumae, a newly emerging rice disease in the southern U.S., can cause significant losses in grain yield and quality. The extent of damage caused by the disease largely depends on the secondary dissemination of the bacterium during a cropping season. Spread of the disease resulting from the rain splash and plant-to-plant contact of B. glumae was determined on Cocodrie and XL723 (susceptible and moderately resistant to the disease, respectively) in 2010 and 2011 by assessing disease severity on panicles at a range of distances from an introduced point source of infected panicles. Field plots were sprayed with or without a Bacillus subtilis biocontrol agent prior to the introduction of the inoculum. An exponential model was used to quantify the effects of distance, cultivar resistance and biocontrol agent on disease severity. At all assessment times, there was a significant decline in disease severity with increasing distance from the inoculum source. Cultivar resistance had a significant effect on the steepness of the disease gradient $(k)$ as well as the overall level $(a)$ of disease severity, with XL723 having a greater estimated $k$ and a smaller $a$ than Cocodrie. Application of the biocontrol agent significantly reduced $a$ but did not affect $k$. Use of cultivar resistance or the biocontrol agent can be an effective management option for limiting the spatial spread of bacterial panicle blight on rice. 
Rice main-crop cutting height affects severity of narrow brown leaf spot in the ratoon crop

X. G. ZHOU (1)

(1) Texas A\&M University System, AgriLife Research and Extension Center, Beaumont, TX, U.S.A.

Phytopathology 104(Suppl. 3):S3.138

Narrow brown leaf spot (NBLS) caused by Cercospora janseana is one of the major diseases limiting the yield potential of rice ratoon crop in Texas and Louisiana. No labeled fungicides are available for management of this disease in the ratoon crop. The objectives of this 2-year study were to evaluate the effects of main-crop harvest cutting height on ratoon crop NBLS severity and grain yield. In 2012 and 2013, the main-crop rice cultivars 'Presidio' and 'XL723' (susceptible and resistant to NBLS, respectively) were harvested at the 10-, 20- or 40-cm cutting height. Ratoon crop plots were applied with or without propiconazole (PropiMax) at the boot stage. When main-crop was harvested at 10 or $20 \mathrm{~cm}$, ratoon crop NBLS severity was significantly reduced on either cultivar compared to the traditional $40-\mathrm{cm}$ cutting height. Ratoon rice grain yield was increased by more than $16 \%$. Single application of the fungicide reduced NBLS severity on either cultivar in both years but did not improve yield in 2012. Lowering main-crop harvest cutting height from 40 to $20 \mathrm{~cm}$ can be an effective management option for NBLS in the ratoon crop.

Functional analysis of potential pathogenicity determinants in Verticillium dahliae

X. ZHU (1), M. R. Islam (1), L. Adam (1), F. Daayf (1)

(1) University of Manitoba, Winnipeg, MB, Canada

Phytopathology 104(Suppl. 3):S3.138

Verticillium dahliae causes major vascular wilts in many economicallyimportant plant species. Previous findings in Dr. Daayf's lab identified several proteins with differential abundance or absence/presence in highly versus weakly aggressive isolates of $V$. dahliae. They also showed that specific $V$. dahliae genes are specifically induced in highly aggressive isolates after elicitation with potato root extracts. The present work focuses on detecting expression levels of these genes in planta, and determining the key ones during infection. Using two $V$. dahliae isolates with different levels of pathogenicity, our results show that isochorismatase hydrolase and xanthine dehydrogenase are up-regulated in the highly aggressive isolate, downregulated in the weakly aggressive one, whereas myo-inositol 2-dehydrogenase expressed only in the highly aggressive one. We generated disfunctional mutants of isochorismatase hydrolase in $V$. dahliae, the virulence of these mutants towards different plant hosts is currently under analysis.

Evaluation of a quantitative (q)PCR assay as the basis for a stem rot of canola (Sclerotinia sclerotiorum) risk assessment tool

B. R. ZIESMAN (1), T. K. Turkington (2), U. Basu (1), E. J. deMilliano (3), S. E. Strelkov (1)

(1) University of Alberta, Edmonton, AB, Canada; (2) Agriculture and AgriFood Canada, Lacombe, AB, Canada; (3) Crop Production Services, Fort Saskatchewan, AB, Canada

Phytopathology 104(Suppl. 3):S3.138

Stem rot of canola, caused by Sclerotinia sclerotiorum, is a sporadic disease that can reduce yields by up to $50 \%$. In western Canada, stem rot is primarily managed with routine application of fungicides, often without any indication of disease risk. A S. sclerotiorum specific quantitative (q)PCR assay has been evaluated as the basis for a risk assessment tool to reduce non-economic application of fungicides. Canola petals collected from central Alberta in 2011, 2012 and 2013 at early bloom (EB) and late bloom (LB) were analyzed with the qPCR assay to provide an estimate of the amount of $S$. sclerotiorum DNA present. Non-linear regression was used to determine the relationship between qPCR estimates of petal infestation and final disease levels in the field. For all three years, LB qPCR estimates were more strongly related to final disease incidence than the EB estimates. The strength of the relationship for the LB samples in 2011,2012 and 2013 varied considerably $\left(R^{2}=0.4,0.7\right.$ and 0.2 respectively) which is consistent with different environmental conditions. In 2013, there appeared to be a significant effect of seeding date, when only early seeded crops were considered the regression became significant $\left(\mathrm{R}^{2}=0.9\right)$. Results indicate that the $\mathrm{qPCR}$ assay has potential as a risk assessment tool particularly if used at multiple flowering stages and if environmental conditions are taken into consideration to account for differences between years.

Pathogenicity of Pythium spp. isolated from soybean roots in North Dakota K. K. ZITNICK-ANDERSON (1), B. D. Nelson (1)

(1) Department of Plant Pathology, North Dakota State University, Fargo, ND, U.S.A.

Phytopathology 104(Suppl. 3):S3.138
The Oomycete Pythium comprises one of the most important groups of seedling pathogens affecting soybean, causing both pre- and post-emergence damping off. In a recent study of Pythium species in soybean seedlings in North Dakota, 26 species and several unknown species were recovered from roots. The species were tested for pathogenicity using Barnes soybean and an inoculum layer technique to determine which species cause seedling disease. The majority of Pythium species ( $P$. attrantheridium, $P$. debaryanum, $P$. diclinum, $P$. dissotocum, $P$. heterothallicum, $P$. hypogynum, $P$. inflatum, $P$. intermedium, $P$. irregulare, $P$. kashmirense, $P$. lutarium, P. minus, $P$. oopapillum, $P$. periilum, $P$. perplexum, $P$. sylvaticum, $P$. terrestris, $P$. viniferum, $P$. violae, and an unknown Pythium sp.) caused pre-emergence damping-off on soybean seedlings with less than $50 \%$ emergence and survival within 14 days. In contrast, $P$. orthogonon, $P$. nunn, and $P$. rostratifingens had approximately $80 \%$ or greater emergence and survival of soybean seedlings. The negative (no Pythium) and positive ( $P$. ultimum) controls had approximately $100 \%$ and $0 \%$, respectively, emergence and survival of seedlings. This is the first report of $P$. kashmirense, $P$. minus, $P$. periilum, $P$. perplexum, $P$. rostratifingens, $P$. terrestris, $P$. viniferum, and $P$. violae as pathogens of soybean seedlings in the United States.

Soil properties associated with the presence of Pythium communities in soybean roots

K. K. ZITNICK-ANDERSON (1), J. E. Norland (2), L. del Rio (1), A. M. Fortuna (2), B. Nelson (1)

(1) Department of Plant Pathology, North Dakota State University, Fargo, ND, U.S.A.; (2) School of Natural Resource Sciences, North Dakota State University, Fargo, ND, U.S.A.

Phytopathology 104(Suppl. 3):S3.138

The genus Pythium contains soil-borne pathogens that cause damping-off on numerous crops. The objective of this study was to examine associations between soil properties and Pythium communities in soybean roots in North Dakota. Pythium isolates were obtained and identified from 98 fields using morphological features and sequences of the ITS region and soil samples were analyzed for cation exchange capacity (CEC), $\mathrm{P}, \mathrm{K}, \mathrm{pH}$, electrical conductivity (EC), organic matter $(\mathrm{OM}), \mathrm{Zn}, \mathrm{Fe}, \mathrm{Cu}$, calcium carbonate exchange (CCE), sand, silt, and clay. Pearson correlation was performed for all 13 soil properties. Pythium communities were characterized using hierarchical clustering, indicator species analysis and multi-response permutation procedures and their association to soil characteristics evaluated using logistic regression analysis with stepwise selection. Three communities represented by $P$. ultimum, $P$. kashmirense and an unknown Pythium spp., and $P$. heterothallicum and $P$. irregular, respectively, were characterized. Statistically significant models $(P=0.05)$ were identified that associate $P$. ultimum with increasing levels of zinc, $P$. kashmirense and an unknown Pythium spp. with increasing CEC and $P$. heterothallicum and $P$. irregulare, with increasing CCE and decreasing CEC. Once validated, these models could have multiple applications as tools to study the ecology and distribution of these Pythium communities in larger ecosystems.

Soil fumigation with metam sodium as a management tool for clubroot (Plasmodiophora brassicae) in Canadian canola (Brassica napus)

K. A. ZUZAK (1), S. F. Hwang (2), G. D. Turnbull (2), V. P. Manolii (3), S. E. Strelkov (3)

(1) University of Alberta, Sherwood Park, AB, Canada; (2) Alberta Agriculture and Rural Development, Edmonton, AB, Canada; (3) University of Alberta, Edmonton, AB, Canada

Phytopathology 104(Suppl. 3):S3.138

Plasmodiophora brassicae, the causal agent of clubroot of crucifers, poses a serious threat to Canadian canola (Brassica napus L.) production, affecting both crop yield and quality. As of 2013, nearly 1500 fields in Alberta were confirmed to be clubroot-infested. Clubroot also is spreading to other regions, as isolated records of the disease have been identified in Saskatchewan and Manitoba. Soil fumigation may be one tool to manage localized infestations in specific fields or regions where the disease is not yet prevalent. Vapam is a metam sodium soil fumigation solution applied to the soil to suppress weeds, nematodes, insects and soil-borne diseases in various cropping systems. Field experiments were conducted in 2012-13 at two clubroot-infested sites in Edmonton, Alberta, Canada, to assess the efficacy of Vapam applied at varying rates for reducing clubroot severity and improving crop growth. Clubroot severity was reduced by approximately $50 \%$ at one site, and $30 \%$ at the second in 2012, although no residual activity was detected when the same sites were sown to clubroot-susceptible canola in 2013. The entire experiment was repeated in 2013, and treatment with Vapam resulted in an approximately $30 \%$ reduction in disease at one site from which data could be obtained. The results from the two site years also suggest significant differences between treatment and control plots for above ground canopy weights and pod numbers at one of the field sites. 


\section{Erratum}

\section{Vol. 104 (Supplement 3), No. 11, 2014}

The following abstract was presented at the APS-CPS Joint Meeting in Minneapolis, Minnesota, August 9-13, 2014.

It was withdrawn in error.

Strain-specific association of Soybean dwarf virus small subgenomic RNA vith virons is mediated by the $17 \mathrm{kDa}$ movement protein

T. THEKKE-VEETIL (1), N. K. McCoppin (2), L. L. Domier (3)

(1) Department of Plant Pathology, Division of Agriculture, University of Arkansas, Fayetteville, AR, U.S.A.; (2) USDA-ARS, Dept. Crop Sciences, University of Illinois, Urbana, IL, U.S.A.; (3) USDA ARS, Dept. Crop Sciences, University of Illinois, Urbana, IL, U.S.A.

Soybean dwarf virus (SbDV) belongs to the family Luteoviridae, members of which produce multiple subgenomic RNAs, but encapsidate only genomic (g) RNA. To study the encapsidation of SbDV RNAs, full-length infectious cDNA clones of soybean (W4) and red clover (ClIL2) isolates were constructed and introduced into Nicotiana benthamiana leaves by agroinfiltration. Both infectious clones replicated and formed virions in infiltrated tissues as evidenced by the presence of viral RNAs in northern blots and positive signals in DAS-ELISA of infiltrated tissues. Analysis of RNAs extracted from sucrose gradient purified SbDV virions revealed isolatespecific RNA contents. Virion fractions from the W4 isolate contained only gRNA while virion fractions from ClIL2 contained both gRNA and smal subgenomic (ssg) RNA. In contrast, virions of ClIL2 that were affinity purified using SbDV monoclonal antibodies, contained only gRNA. Sucrose gradient purified virions from a mutant of ClIL2 that did not express the 17$\mathrm{kDa}$ movement protein contained less ssgRNA. Mass spectrometry confirmed the association of the $17 \mathrm{kDa}$ protein with sucrose gradient-purified wild-type ClIL2 virions. Through a series of point mutations, we mapped sequences responsible for the association of ssgRNA with SbDV virions to four nucleotide positions in a stem-and-loop structure in the ClIL2 ssgRNA. These findings suggest that the $17-\mathrm{kDa}$ protein mediates strain-specific association of ssgRNA with SbDV virions. 